\title{
Spinal Cord Injury (SCI) Repair Strategies
}





\section{Spinal Cord Injury (SCl) Repair Strategies}

Edited by

\section{Giuseppe Perale, PhD}

Professor of Regenerative Medicine Faculty of Biomedical Sciences of the University of Southern

Switzerland (USI)

Lugano, Switzerland

Visiting Professor Ludwig Boltzmann Institute for Experimental and Clinical Traumatology Vienna, Austria

\section{Filippo Rossi, PhD}

Associate Professor Applied Physical Chemistry Laboratory

Department of Chemistry

Materials and Chemical Engineering

"Giulio Natta" of Politecnico di Milano

Milano, Italy 
Woodhead Publishing is an imprint of Elsevier

The Officers' Mess Business Centre, Royston Road, Duxford, CB22 4QH, United Kingdom 50 Hampshire Street, 5th Floor, Cambridge, MA 02139, United States

The Boulevard, Langford Lane, Kidlington, OX ${ }_{5}$ IGB, United Kingdom

Spinal Cord Injury (SCI) Repair Strategies

Copyright $\odot 2020$ Elsevier Ltd. All rights reserved.

No part of this publication may be reproduced or transmitted in any form or by any means, electronic or mechanical, including photocopying, recording, or any information storage and retrieval system, without permission in writing from the publisher. Details on how to seek permission, further information about the Publisher's permissions policies and our arrangements with organizations such as the Copyright Clearance Center and the Copyright Licensing Agency, can be found at our website: www.elsevier.com/permissions.

This book and the individual contributions contained in it are protected under copyright by the Publisher (other than as may be noted herein).

\section{Notices}

Practitioners and researchers must always rely on their own experience and knowledge in evaluating and using any information, methods, compounds or experiments described herein. Because of rapid advances in the medical sciences, in particular, independent verification of diagnoses and drug dosages should be made. To the fullest extent of the law, no responsibility is assumed by Elsevier, authors, editors or contributors for any injury and/or damage to persons or property as a matter of products liability, negligence or otherwise, or from any use or operation of any methods, products, instructions, or ideas contained in the material herein.

ISBN: 978-0-08-102807-0

Publisher: Matthew Deans Acquisition Editor: Sabrina Webber Editorial Project Manager: Emma Hayes Production Project Manager: Kiruthika Govindaraju Cover Designer: Alan Studholme 
To my parents Oreste \& Grazia, to my wife Maria Claudia and daughter Maria Ludovica, my first supporters

Filippo Rossi 



\section{Contents}

Contributors. $\mathrm{xV}$

Foreword xix

\section{CHAPTER 1 Introduction to spinal cord injury as clinical}

pathology................................................. 1

Simonetta Papa, PhD, Emanuele Mauri, PhD,

Filippo Rossi, PhD, Giuseppe Perale, PhD and

Pietro Veglianese, $P h D$

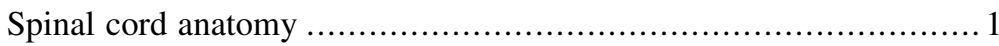

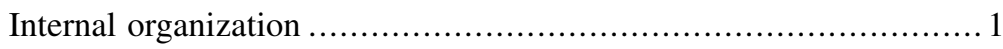

Spinal cord injuries ............................................... 2

SCI consequences ..................................................... 3

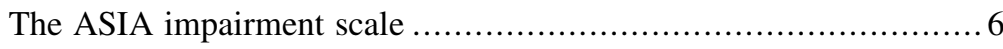

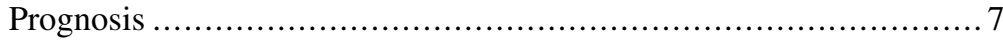

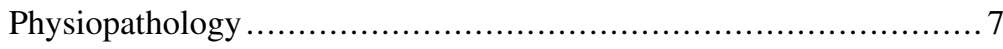

Limited central nervous system regeneration ........................ 7

Role of inflammation in posttrauma recovery ......................... 9

Role of microglia in SCI....................................... 10

References................................................... 11

CHAPTER 2 Spinal cord injury: role of neurophysiology ......... 13

Bruno Ferrero, MD and Alessandra Di Liberto, MD

Introduction......................................................... 13

Functional anatomy ................................................... 14

Neurophysiological techniques...................................... 16

Basic concepts of electrodiagnosis and electrical properties

of nerve and muscle .......................................... 16

Nerve conduction studies .......................................... 17

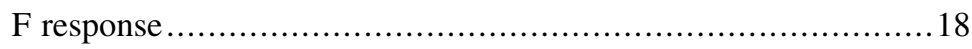

H reflex....................................................... 18

Electromyography................................................... 19

Nerve excitability studies ......................................... 19

Galvanic vestibular stimulation ....................................20

Sympathetic skin response....................................... 20

Bulbocavernosus reflex ....................................... 20

Standard transcranial magnetic stimulation........................21

Triple-stimulation technique ................................. 22 
Quantitative sensory testing and electrical perception

threshold.

Intraoperative neurophysiological monitoring .....................26

Application and prognostic value of neurophysiology ............... 26

Recording of peripheral pathways...............................26

Recording of spinal pathways ....................................28

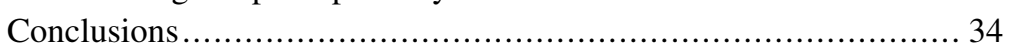

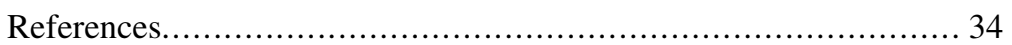

CHAPTER 3 Conventional treatments and surgical margins of maneuvering for spinal cord injury management.

Carlo Alberto Benech, MD, Alberto Tomatis, MD,

Rosa Perez, MD and Beatrice Boido, MD

Classification: Complete and Incomplete injuries ...................... 39

Management of spinal cord injury ................................... 43

Introduction .................................................... 43

Hospital management .......................................... 43

Radiological evaluation........................................ 43

Steroids in SCI management....................................44

Anticoagulation prophylaxis .....................................45

Blood pressure augmentation......................................45

Surgical options and timing ........................................ 45

Further reading.................................................. 47

CHAPTER 4 MRI as an imaging tool for in vivo noninvasive morphological and (partially) functional examination of injured spinal cord.

Fano Molinaro, MD, Pier Luigi La Zazzera, MD,

Marilena Ferraris, MD, Giulia Morbidoni, MSE,

Domenico Zaca', MSNS, PhD, Andrea De Rinaldis, MSE, PhD,

Fernando Carpanese, MSE, Armando Cioffi, MSE, Francesco

Naddeo, MSE, Leonardo Boccaccini, MSE and

Mauro Bergui, $M D, P h D$

Magnetic resonance image: key concepts 49

Magnetic resonance imaging: main sequences ....................50

Advanced techniques: diffusion tensor imaging and functional magnetic resonance imaging.................................51

Basic images and lesions classification ............................. 53

Clinical presentation......................................... 54 
Acute imaging .55

Postacute, including various evolutions.............................. 60

Clinical-magnetic resonance imaging correlations................... 61

Perspectives.................................................. 62

Diffusion tensor imaging and related techniques ..................62

Functional magnetic resonance imaging.........................6 63

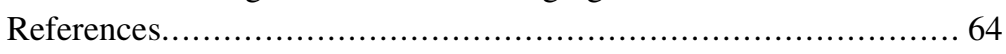

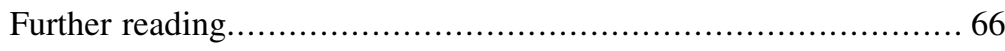

CHAPTER 5 Spinal cord injury: ethical issues ...................67

Virginia Sanchini, PhD and Giovanni Boniolo, PhD

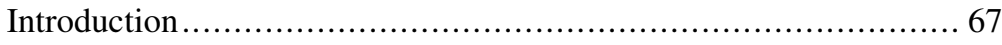

Ethical issues related to spinal cord injury ......................... 68

Spinal cord injury and justice .....................................6 68

Spinal cord injury and autonomy................................... 70

Spinal cord injury and beneficence ........................... 71

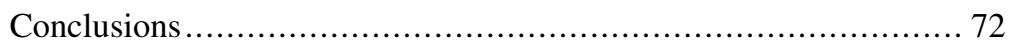

References..................................................... 73

CHAPTER 6 Elements of material science and polymeric

biomaterials ........................................... 77

Gianni Pertici, PhD

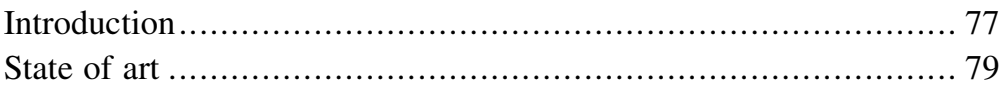

Aliphatic polyesters (poly(lactic acid), polycaprolactone, poly(glycolic acid), etc.) ..................................... 79

Natural biopolymers (dextran, chitosan, hyaluronic acid,

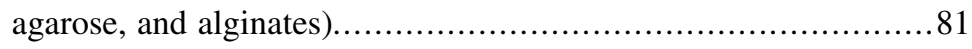

Poly- $\beta$-hydroxybutyrate ........................................ 84

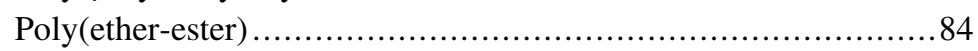

Poly(glycerol sebacic acid) .................................... 85

Poly(phosphoesters) ................................................. 85

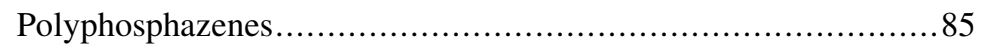

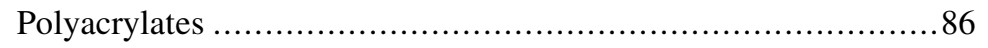

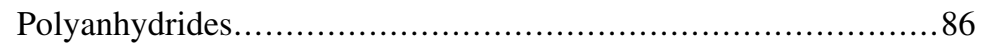

Poly(alkyl cyanoacrylates)..................................... 87

Poly(propylene fumarate) ........................................ 87

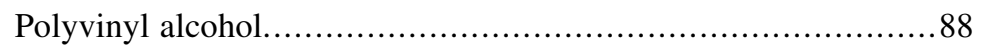

Biopolymers technology ......................................... 88

Properties (degradability, toxicity, biocompatibility, etc.) ...........88

Compounding, mechanical properties, and degradation time........89 
Extrusion and fiber manufacturing ............................. 90

Molding ............................................................ 92

Coating ............................................................ 94

Solvent casting ............................................... 94

Foaming........................................................ 95

Hydrogels manufacturing ..................................... 95

Micro- and nanoparticles manufacturing ...........................96

Additive manufacturing .............................................99

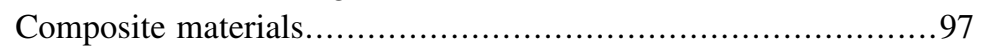

Sterilization ......................................................... 97

Future trends..................................................... 98

References........................................................ 98

Further reading...................................................... 103

CHAPTER 7 Soft and rigid scaffolds for spinal cord injury regeneration.............................................. 105

Šárka Kubinová, PharmD, PhD

Introduction .................................................... 105

Scaffold properties and characterization..............................106

Synthetic hydrogels based on PHPMA and PHEMA................. 109

Macroporous PHPMA hydrogel modified with RGD.............. 109

PHEMA hydrogels with different charges ......................... 110

Cholesterol-modified superporous PHEMA hydrogels.............. 112

Superporous PHEMA hydrogels modified by cell-adhesive peptides .................................................. 113

PHEMA hydrogels with oriented porosity ....................... 114

PHEMA hydrogel modified with serotonin....................... 117

Degradable PHEMA hydrogels .................................. 117

Natural-based materials ................................................ 118

Hyaluronic acid hydrogel modified with RGD ...................... 118

Extracellular matrix-based hydrogels........................... 119

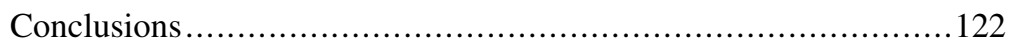

Acknowledgment................................................... 123

References........................................................ 123

CHAPTER 8 Nanomaterials for spinal cord injury (SCI) regeneration........................................... 129

Emanuele Mauri, PhD and Maurizio Masi, PhD

Nanotechnology and nanomedicine: an overview .....................129

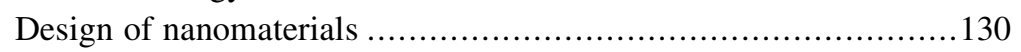

Key properties................................................... 131

Materials......................................................... 133 
Nanoparticles in SCI

Synthesis techniques ............................................. 136

Nanoparticle functionalization guidelines.......................... 138

Case study 1: design of PLGA nanoparticles for sustained

release of bioactive neurotrophin.............................. 140

Nanogels in SCI ................................................... 140

Synthesis techniques ............................................ 142

Nanogels functionalization principles............................... 143

Case study 2: nanogels as thiol-sensitive intracellular drug

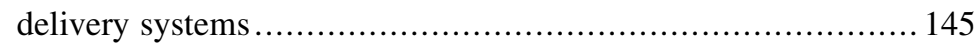

Nanochannels in SCI.......................................... 145

Nanotubes ................................................... 146

Nanowires .......................................................... 147

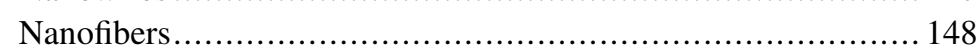

Conduits ........................................................... 148

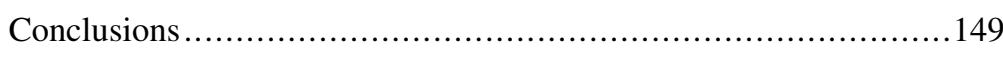

References......................................................... 149

\section{CHAPTER 9 Cell therapies for spinal cord injury}

\section{regeneration............................................. 157}

Eduardo D. Gomes, MSc, PhD, Luiś A. Rocha, MSc,

Rita C. Assunção-Silva, MSc, Rui Lima, MSc, N.A. Silva, BSc,

PhD, António J. Salgado, BSc, PhD, DSc

ESCs, iPSCs, and NSCs.................................................. 158

ESCs .......................................................... 158

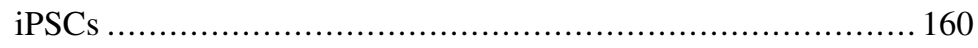

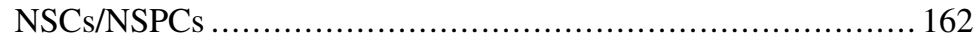

Mesenchymal stem cells ....................................... 166

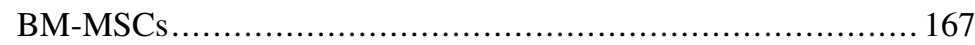

UC-MSCs ........................................................ 168

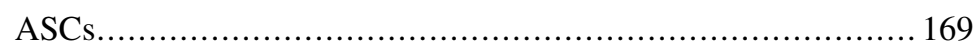

Glial cells (supportive cells) .................................. 171

Schwann cells ............................................... 171

Olfactory ensheathing cells.................................... 172

Conclusions ...................................................... 174

Acknowledgments.............................................. 174

References....................................................... 175 
CHAPTER 10 Functional properties of progenitor-like cells in the spinal cord: implications for self-repair .. 187 Raúl E. Russo, PhD

Introduction.

Membrane properties of cells in the CC stem cell niche ..............188

Signaling via connexins $(\mathrm{Cx})$ in the CC stem cell niche ..............190

Regulation of the CC stem cell niche by neurotransmitters ..........192

Concluding remarks ........................................... 195

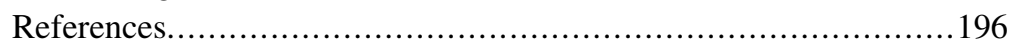

CHAPTER 11 Paracrine effects for spinal cord injury regeneration

Simonetta Papa, PhD, Irma Vismara, PhD and

Pietro Veglianese, $\mathrm{PhD}$

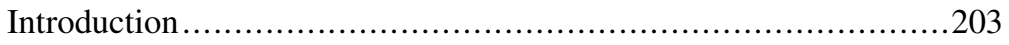

Stem cell-based therapies after SCI ..............................204

Stem cells can act in different ways ..................................204

Stem cells protect by paracrine release of therapeutic mediators ....205

Mesenchymal stem cells .............................................206

Embryonic stem cells ...........................................207

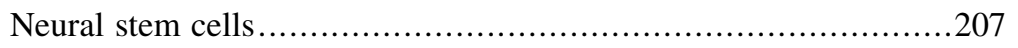

Induced pluripotent stem cells.....................................208

The paracrine release of therapeutic mediators.....................210

Engineered biomaterial for stem cell delivery in SCI ...............211

Conclusions ....................................................... 211

References..........................................................213

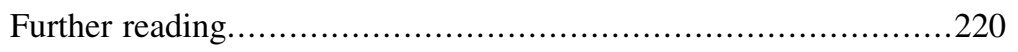

CHAPTER 12 Pharmacological therapies and factors delivery for spinal cord injury regeneration...... 223

Silviya Petrova Zustiak, PhD, Saahil Sheth and

Mozhdeh Imaninezhad, PhD

Introduction .....................................................223

Newer developments in spinal cord injury repair.....................224

Implantable and injectable hydrogels as permissive scaffolds

and depots for therapeutic delivery ................................225

Delivery of neurotrophic factors in SCI repair .....................228

Neurotrophic factors in SCI repair........................... 228

Strategies for sustained delivery of neurotrophic factors

to improve efficacy .............................................. 229 
Examples of delivery approaches for neurotrophic factors in SCI repair.

Neurotrophic factor delivery in combination therapies for SCI repair.

Delivery strategies for other therapeutic molecules in SCI ..........234

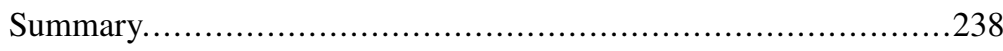

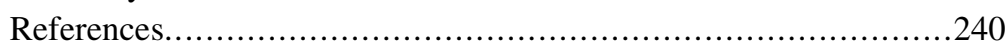

CHAPTER 13 Reliability on animal models..................... 249

María Permuy, DVM, PhD, Mónica López-Peña, PhD,

Antonio González-Cantalapiedra, DVM, PhD, and

Fernando Mariá Muñoz Guzón, DVM, PhD

Introduction...................................................249

Animal species used in experimental spinal cord injury repair......249

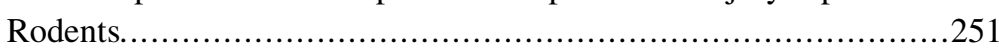

Large animals ................................................. 252

Nonmammals..................................................... 254

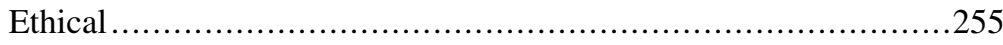

Regulatory affairs and design of procedures .......................256

Main experimental models ......................................257

Models based on spinal injury level ............................. 259

Models based on injury mechanism ................................. 259

Spinal cord injury evaluation tests ............................. 265

Quality control (Good Laboratory Practice, Good

Clinical Practice, and Good Clinical Laboratory Practice)............268

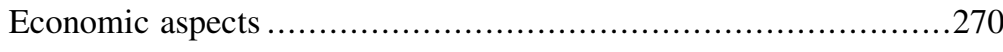

Publications ..................................................... 271

Remarks and recommendations .............................. 271

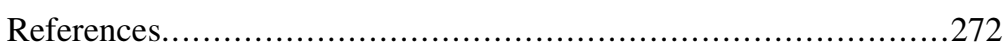

Further reading.....................................................276

\section{CHAPTER 14 Fundamentals and application of modeling} in support of spinal cord injury repair strategies................................................. 279 Tommaso Casalini, PhD

Introduction .........................................................279

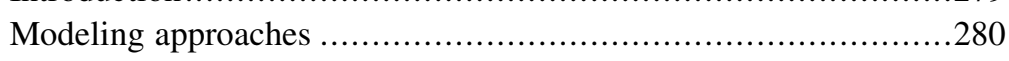

Molecular dynamics ............................................... 280

Coarse-grained models ............................................. 282

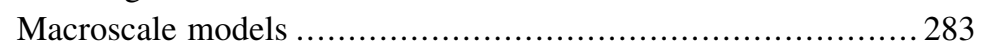

Applications .................................................. 291 


\section{xiv Contents}

Hydrogels....................................................... 291

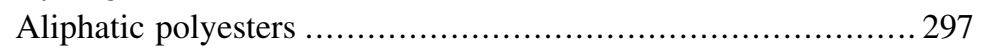

In vivo modeling..................................................... 301

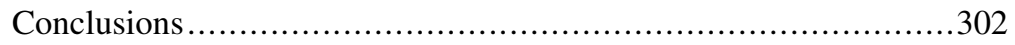

References..................................................... 303

Conclusions: Trends in regenerative therapies, combination approaches, and clinical highlights for spinal cord injury (SCI) regeneration.

Filippo Rossi, PhD, Mario Gimona and

Giuseppe Perale, PhD

References........................................................ 311

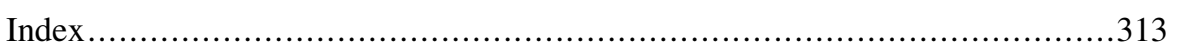




\section{Contributors}

\section{Rita C. Assunção-Silva, MSc}

Life and Health Sciences Research Institute (ICVS), School of Medicine, University of Minho, Campus de Gualtar, Braga, Portugal; ICVS/3B's - PT Government Associate Laboratory, Braga/Guimarães, Portugal

Carlo Alberto Benech, MD

Department of Neurosurgery, Clinica Fornaca di Sessant, Turin, Italy

\section{Mauro Bergui, MD, PhD}

Department of Neuroradiology, University of Turin, Torino, Italy

\section{Leonardo Boccaccini, MSE}

Siemens Healthcare, Milan, Italy

\section{Beatrice Boido, MD}

Department of Neurosurgery, Clinica Fornaca di Sessant, Turin, Italy

\section{Giovanni Boniolo, PhD}

Professor, Dipartimento di Scienze Biomediche e Chirurgico Specialistiche, Università di Ferrara, Ferrara, Italy

\section{Fernando Carpanese, MSE}

Siemens Healthcare, Milan, Italy

\section{Tommaso Casalini, PhD}

Institute for Chemical and Bioengineering, Department of Chemistry and Applied Bioscience, ETH Zurich, Zurich, Switzerland; Polymer Engineering Laboratory, Institute for Mechanical Engineering and Materials Technology, SUPSI, Manno, Switzerland

\section{Armando Cioffi, MSE}

Siemens Healthcare, Milan, Italy

\section{Alessandra Di Liberto, MD}

SC Neurologia 2 - ASL Città di Torino, Giovanni Bosco Hospital, Turin, Italy

\section{Marilena Ferraris, MD}

Neuroradiology Unit, Città della Salute Hospital, Torino, Italy

\section{Bruno Ferrero, MD}

SC Neurologia 2U- AOU Città della Salute e della Scienza di Torino- "Rita Levi Montalcini" Department of Neuroscience, University of Turin, Turin, Italy

\section{Mario Gimona, PhD}

GMP Laboratory, Spinal Cord Injury and Tissue Regeneration Center Salzburg (SCI-TReCS); Paracelsus Medical University (PMU), Salzburg, Austria 


\section{Eduardo D. Gomes, MSc, PhD}

Life and Health Sciences Research Institute (ICVS), School of Medicine, University of Minho, Campus de Gualtar, Braga, Portugal; ICVS/3B's - PT Government Associate Laboratory, Braga/Guimarães, Portugal

\section{Antonio González-Cantalapiedra, DVM, PhD}

Department of Anatomy, Animal Production and Veterinary Clinical Sciences, Universidade de Santiago de Compostela, Lugo, Spain

\section{Mozhdeh Imaninezhad, PhD}

Biomedical Engineering, Parks College of Engineering, Aviation and Technology, Saint Louis University, Saint Louis, MO, United States

\section{Šárka Kubinová, PharmD, PhD}

Department of Biomaterials and Biophysical Methods, Institute of Experimental Medicine of the Czech Academy of Sciences, Prague, Czech Republic

\section{Rui Lima, MSc}

Life and Health Sciences Research Institute (ICVS), School of Medicine, University of Minho, Campus de Gualtar, Braga, Portugal; ICVS/3B's - PT

Government Associate Laboratory, Braga/Guimarães, Portugal

\section{Mónica López-Peña, PhD}

Department of Anatomy, Animal Production and Veterinary Clinical Sciences, Universidade de Santiago de Compostela, Lugo, Spain

\section{Maurizio Masi, PhD}

Department of Materials, Chemistry and Chemical Engineering "G. Natta", Politecnico di Milano, Milan, Italy

\section{Emanuele Mauri, PhD}

Department of Engineering, Tissue Engineering and Chemistry for Engineering Unit, Università Campus Bio-Medico di Roma, Rome, Italy

\section{Fano Molinaro, MD}

Department of Neuroradiology, University of Turin, Torino, Italy

\section{Giulia Morbidoni, MSE}

Siemens Healthcare, Milan, Italy

\section{Fernando María Muñoz Guzón, DVM, PhD}

Department of Anatomy, Animal Production and Veterinary Clinical Sciences, Universidade de Santiago de Compostela, Lugo, Spain

\section{Francesco Naddeo, MSE}

Siemens Healthcare, Milan, Italy

\section{Simonetta Papa, PhD}

Department of Neuroscience, IRCCS Istituto di Ricerche Farmacologiche "Mario Negri", Milan, Italy 


\section{Giusepp Perale, PhD}

Department of Neuroscience, IRCCS Istituto di Ricerche Farmacologiche "Mario Negri", Milan, Italy; Faculty of Biomedical Sciences of the University of Southern Switzerland, Lugano, Switzerland; Ludwig Boltzmann Institute for Experimental and Clinical Traumatology, Vienna, Austria

\section{Rosa Perez, MD}

Department of Neurosurgery, Clinica Fornaca di Sessant, Turin, Italy

\section{María Permuy, DVM, PhD}

Department of Anatomy, Animal Production and Veterinary Clinical Sciences, Universidade de Santiago de Compostela, Lugo, Spain

\section{Gianni Pertici, PhD}

Researcher, Department of Technology Innovation, University of Applied Sciences and Arts of Southern Switzerland, Ticino, Switzerland; Executive VicePresident, Industrie Biomediche Insubri SA, Ticino, Switzerland

\section{Michael Reinert}

Faculty of Biomedical Sciences of the University of Southern Switzerland, Lugano, Switzerland; Department of Neurosurgery, Ente Ospedaliero Cantonale, Bellinzona, TI, Switzerland; Medical Faculty, University of Bern, Bern, Switzerland

\section{Andrea De Rinaldis, MSE, PhD}

Siemens Healthcare, Milan, Italy

\section{Luís A. Rocha, MSc}

Life and Health Sciences Research Institute (ICVS), School of Medicine, University of Minho, Campus de Gualtar, Braga, Portugal; ICVS/3B's - PT Government Associate Laboratory, Braga/Guimarães, Portugal

\section{Filippo Rossi, PhD}

Department of Neuroscience, IRCCS Istituto di Ricerche Farmacologiche "Mario Negri", Milan, Italy; Department of Chemistry, Materials and Chemical Engineering "Giulio Natta", Milan, Italy

\section{Raúl E. Russo, PhD}

Professor, Departamento de Neurofisiología Celular y Molecular, Instituto de Investigaciones Clemente Estable, Montevideo, Uruguay

\section{António J. Salgado, BSc, PhD, DSc}

Life and Health Sciences Research Institute (ICVS), School of Medicine, University of Minho, Campus de Gualtar, Braga, Portugal; ICVS/3B's - PT Government Associate Laboratory, Braga/Guimarães, Portugal; Dr, ICVS, School of Medicine, University of Minho, Braga, Portugal 


\section{xviii Contributors}

\section{Virginia Sanchini, PhD}

Faculty of Philosophy, Vita-Salute San Raffaele University, Milan, Italy; Department of Oncology and Hematology, University of Milan, Milan, Italy

\section{Saahil Sheth}

Biomedical Engineering, Parks College of Engineering, Aviation and Technology, Saint Louis University, Saint Louis, MO, United States

\section{N.A. Silva, BSc, PhD}

Life and Health Sciences Research Institute (ICVS), School of Medicine, University of Minho, Campus de Gualtar, Braga, Portugal; ICVS/3B's - PT Government Associate Laboratory, Braga/Guimarães, Portugal

\section{Alberto Tomatis, MD}

Neurosurgical Unit, Ospedale Spirito Santo, Pescara, Italy

\section{Pietro Veglianese, PhD}

Department of Neuroscience, IRCCS Istituto di Ricerche Farmacologiche "Mario Negri", Milan, Italy; Head of Unit, Neuroscience, Istituto di Ricerche

Farmacologiche Mario Negri, Milan, Italy

\section{Irma Vismara, PhD}

Department of Neuroscience, IRCCS Istituto di Ricerche Farmacologiche "Mario Negri", Milan, Italy

\section{Domenico Zaca', MSNS, PhD}

Siemens Healthcare, Milan, Italy

Pier Luigi La Zazzera, MD

Department of Neuroradiology, University of Turin, Torino, Italy

\section{Silviya Petrova Zustiak, PhD}

Biomedical Engineering, Parks College of Engineering, Aviation and Technology, Saint Louis University, Saint Louis, MO, United States 


\title{
Forewords
}

\author{
Giuseppe Perale ${ }^{1,2}$, Michael Reinert ${ }^{1,3,4}$, Filippo Rossi $^{5}$ \\ ${ }^{1}$ Faculty of Biomedical Sciences of the University of Southern Switzerland, Lugano, Switzerland; \\ ${ }^{2}$ Ludwig Boltzmann Institute for Experimental and Clinical Traumatology in Vienna, Austria; \\ ${ }^{3}$ Department of Neurosurgery, Ente Ospedaliero Cantonale, Bellinzona, TI, Switzerland; \\ ${ }^{4}$ Medical Faculty, University of Bern, Bern, Switzerland; ${ }^{5}$ Department of Chemistry, Materials and \\ Chemical Engineering "Giulio Natta”, Milan, Italy
}

Spinal cord injury (SCI) is a devastating trauma that results in motor and sensory disabilities with serious consequences for the lives of patients and their families. It is one of the major causes of irreversible nerve injury that indeed poses a significant health problem: it can be estimated that about 48000 people per year join the population of approximately over half a million current victims of SCI (12000/year only in the United States, with a 270000 spinalized population; similar figures for Europe and the rest of the world is estimated to be similar to the sum of United States + Europe). Furthermore, SCI has a high rate of prevalence in the younger population, characteristically, young men between 16 and 30 years of age, creating physical, emotional, and economic burdens on both the individual and also society.

This means that, dearest Reader, meanwhile you read just these foreword, statistically, a new case of spinal cord injury has occurred somewhere around the world (ca. 1 new case every $10 \mathrm{~min}$ ).

There are plenty of researchers around the globe tackling this pathology, from a wide spectrum of approaches, from those trying to regenerate the lesioned parenchyma to those trying to apply bioelectronics to jump over the gap.

Yet despite the extensive research and the number of ongoing clinical trials, there is still no effective clinical treatment that would regenerate the long-tract axonal damage and recover the lost neuronal function in patients.

Very recently, in October 2018, the team led by Prof. Gregoire Courtine (École polytechnique fédérale de Lausanne, EPFL, Switzerland) published their astonishing results on three patients with chronic paraplegia who were able to walk over ground, thanks to the precise electrical stimulation of their spinal cords via a wireless implant.

Surely neuroprosthetic systems, robotic interfaces, and advanced neurorehabilitation procedures represent a strongly relevant half of current research in treating chronic SCI patients. However, this book audaciously targets the complex field of SCI repair in a regenerative perspective.

The book structure is indeed meant to allow the reader getting firstly a quick glance on SCI as a clinical pathology (Ch. 1), on the state-of-the-art diagnostic tools from neurophysiology (Ch. 2), and on current options for surgical treatments (Ch. 3). The fourth chapter focuses on the potential offered by magnetic resonance imaging as a noninvasive tool for the in vivo monitoring of the lesioned cord (Ch. 4). Chapter 5 opens toward the regeneration research field, dealing with the essential but too 
often neglected issues on ethics related to designing and performing clinical studies on critical patients (Ch. 5). Following this, chapter 6 introduces biomaterial related concepts, as biomaterials play a pivot role in almost all regeneration strategies (Ch. 6). Chapters (7-12) points toward most recent highlights and future perspectives of possible therapeutical and regenerative solutions, from use of scaffolds (Ch. 7), to last generation nanomaterials (Ch.8), from different cell therapies (Ch. 9 and 10) to pharmacological therapies and in situ factors delivery (Ch. 11 and 12).

The book is closed by two final chapters, which present key methodological contributions on essential topics, also from a quality assurance perspective, such as animal model reliability (Ch. 13) and computational support tools, which are becoming a fundamental item to pursue the paradigm of safety by design.

Let us see in this sparkling arena which of these technologies will see its preclinical robust results give birth to clinical studies, finally proving to be effective in addressing the challenges of regenerating such a highly organized tissue as the spinal cord.

SCI is a multifactorial complex pathology, and it would not be probably wrong to imagine that the regeneration we are all aiming at is going to be based on a multidisciplinary approach for a multitherapeutical strategy, tackling inflammation, preventing the formation of the glial scar, supporting local regeneration, and finally benefitting from rehabilitation to stimulate the final recovery using CNS plasticity potential.

The centrality of the patients shall never be neglected as an ethical research encompasses also their positive physiological involvement, which is complementary to achieve recovery and health, hence honoring our Hippocratic oath. 


\title{
CHAPTER
}

\section{c0001 Introduction to spinal cord injury as clinical pathology}

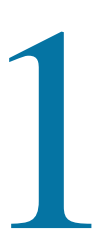

\author{
Simonetta Papa, PhD ${ }^{1}$, Emanuele Mauri, PhD ${ }^{2}$, Filippo Rossi, PhD ${ }^{1,3}$, \\ Giusepp Perale, PhD ${ }^{1,4,5}$, Pietro Veglianese, PhD ${ }^{1}$ \\ ${ }^{1}$ Department of Neuroscience, IRCCS Istituto di Ricerche Farmacologiche "Mario Negri", Milan, \\ Italy; ${ }^{2}$ Department of Engineering, Tissue Engineering and Chemistry for Engineering Unit, \\ Università Campus Bio-Medico di Roma, Rome, Italy; ${ }^{3}$ Department of Chemistry, Materials and \\ Chemical Engineering "Giulio Natta", Milan, Italy; ${ }^{4}$ Faculty of Biomedical Sciences of the \\ University of Southern Switzerland, Lugano, Switzerland; ${ }^{5}$ Ludwig Boltzmann Institute for \\ Experimental and Clinical Traumatology, Vienna, Austria
}

\section{s0010 Spinal cord anatomy}

p0010 The central nervous system, formed by brain and spinal cord, is the center in charge of reception, elaboration, and information transmission. The spinal cord comprises a bundle of neurons and has its place in the spinal canal that protects it. In humans, it has a cylindrical form, with $8-10 \mathrm{~mm}$ of medium diameter, starts at the base of the skull, but does not occupy the vertebral canal for his entire length, ending in the firsts lumbar vertebras. The spinal column and the spinal cord are divided into five regions: cervical, thoracic, lumbar, sacral, and coccyx (Fig. 1.1). From the spinal cord start the peripheral nerves, responsible for motor and sensory capabilities of the entire body. An external stimulus detected, for example, by the skin, is converted into chemical signals that are transported along the axons to the dorsal side of spinal cord and finally to the brain. In the brain, they are elaborated and the responsive motor signal goes back to the ventral side of the spinal cord and to the region that perceived the stimulus.

p0015 Three membranes, called meninges, of protective tissue cover the entire spinal cord. The external membrane has been named dura mater because of this strength, the intermediate one is the delicate arachnoid, which is thin and with web-like filaments, and the inner membrane is called pia mater. Between arachnoid and pia mater, there is a space filled with cerebrospinal liquid, and this gap contains arteries that supply blood to the spinal tissue: in case of trauma, there will be hemorrhaging.

\section{s0015 Internal organization}

p0020 The spinal cord is composed of two different types of tissue: the inner part appears grayish and is called gray matter; the outer instead is white and is called white matter (Fig. 1.2). White matter is subdivided into columns, each one containing neurons 
f0010 FIGURE 1.1

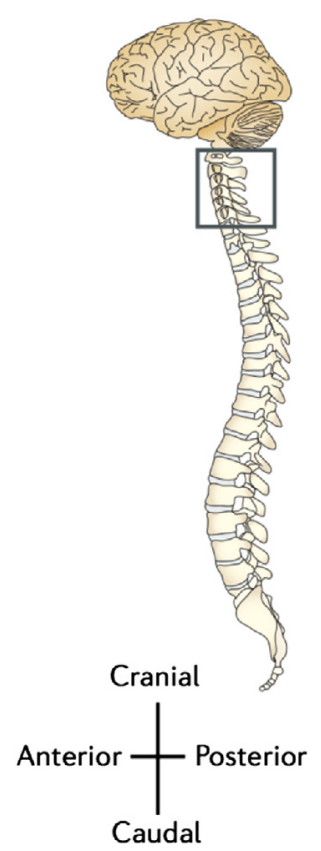

Spinal cord anatomy.

Reprinted with permission from Thuret S, Moon LDF, Gage FH. Therapeutic interventions after spinal cord injury. Nat Rev Neurosci 2006; 7:628-43.

related to specific functions. The lateral columns include axons that travel from the cerebral cortex to contact spinal motor neurons. In the dorsal zone, sensibility information are carried up way to the brain. The ventral columns carry both ascending information about pain and temperature and descending motor information. Gray matter is divided into dorsal and ventral horns. The dorsal ones are for skin stimulus reception; the ventral ones receive proprioceptive fibers, and the middle part receives sensorial inputs from muscles and viscera. The muscle's motor activity is the result of the cooperation that takes place between different groups of neurons put in series: if one of them is damaged, the information circuit would be broken and the movement results impeded.

\section{s0020 Spinal cord injuries}

p0025 Spinal cord injury (SCI) is a condition that implies a loss of motor abilities and results to be one of the first causes of noncongenic disability in the world, with an annual incidence between 40 and 80 cases per million people. About two and a 
f0015 FIGURE 1.2

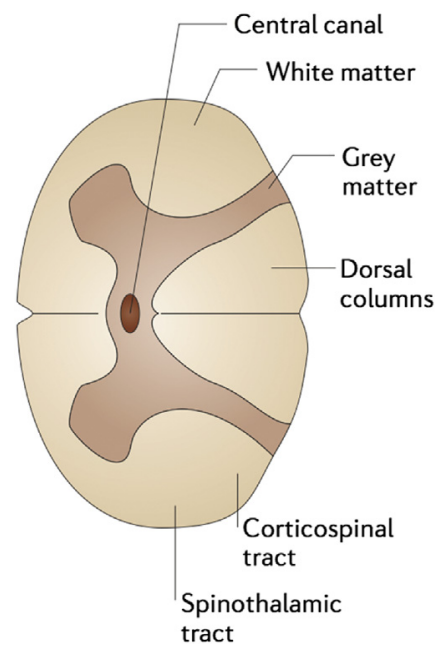

Spinal cord organization.

Reprinted with permission from Thuret S, Moon LDF, Gage FH. Therapeutic interventions after spinal cord injury.

Nat Rev Neurosci 2006; 7:628-43.

half millions of the people worldwide are affected by SCI [2-4]. Most of them are male ( $82 \%$ in the United States, $72.7 \%$ in India), and the mean age is 33 years, with a tendency to rise. The causes vary in the world differently from country to country and are mostly related to vehicular accidents, falls, violence, or sport activities. In addition, the incidence is region-dependent and visible in Fig. 1.3 [5]. The results of these traumatic events are immediate breakage, dislocation, or compression of one or more vertebras and afterward hemorrhage, inflammation, and infection contribute to worse the clinical situation. However, the latest statistics evidence on the increase of cases with nontraumatic origin (osteoporosis or a tumor) in the North America and European Union regions correlated to the progressively aging population. Due to the violence of the trauma, a high percentage $(15 \%-56 \%)$ of people victim of SCI dies before hospitalization. The ones who survived the trauma and the first $24 \mathrm{~h}$ had lower life expectancy compared to population without SCI, in dependence to the resulting condition (paraplegia, tetraplegia) and the capacity of the healthcare system.

\section{s0025 SCI consequences}

p0030 The most frequent SCI consequences are loss of sensibility and paralysis below the level of injury. Four cases are determined: paraplegia, tetraplegia, paraparesis, and tetraparesis (Fig. 1.4). Paraplegia is a syndrome that includes complete paralysis of lower limbs and sphincteric disorders; in tetraplegia are added also upper limbs 


\section{4}
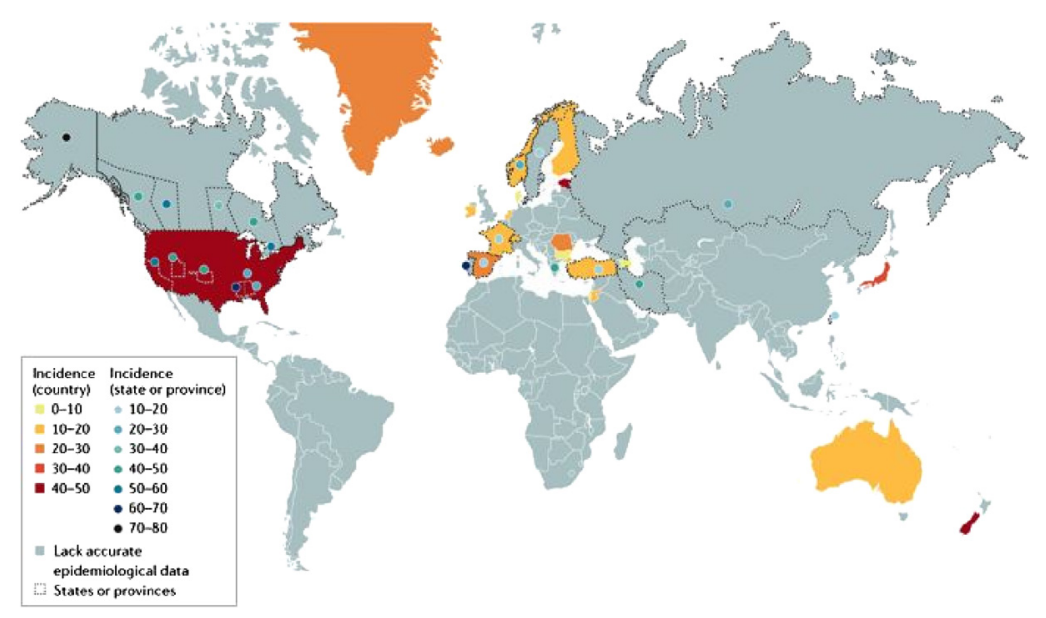

Relative annual incidences of countries, states/provinces, and regions. The red color scheme illustrates incidences of countries. The blue color scheme highlights incidences of states/provinces and regions (mil = million).

Reprinted with permission from Ahuja CS, Wilson JR, Nori S, Kotter MRN, Druschel C, Curt A, et al. Traumatic spinal cord injury. Nat Rev Dis Primers 2017;17018.

paralysis and, in the worst cases, autonomic nervous system's disorders. Paraparesis and tetraparesis are instead the incomplete paralysis of lower limbs or all of them.

p0035 The differences in outcomes are due to different injury levels along the vertebral column and by the lesion's completeness. Given the same neurological examination and findings, neurologists and physiatrists may not assign the same SCI level: neurologists define the level of injury as the first spinal segmental level that shows abnormal neurological loss.

p0040 In contrast, physiatrists or rehabilitation doctors tend to define the level of injury as the lowest spinal segmental level that is normal. For example, if a patient has normal $\mathrm{C} 3$ sensations and absent $\mathrm{C} 4$ sensations, a physiatrist would say the sensory level is $\mathrm{C} 3$ whereas a neurologist would call it a C4 injury level. A complete lesion implies the total and definitive inability of nervous information exchange between the two sides of the injury and therefore the loss of sensitive and motor abilities below the lesion level. In an incomplete lesion, some motor and sensitive capacities are maintained.

p0045 Consequences of SCI are as follows:

u0010 - Motion loss, with alteration of muscular tone and spastic contractions

u0015 - Skin sensibility loss

u0020 • Autonomic dysreflexia

u0025 • Urinary bladder loss of control 
Classification of spinal cord injury (SCl) severity using the American Spinal Injury Association (ASIA) Impairment Scale. The main categories of the Impairment Scale are as follows:

- A (complete): No motor or sensory function is preserved in the sacral segments S4-S5.

- B (incomplete): Sensory but not motor function is preserved below the neurological level and includes the sacral segments S4-S5.

- C (incomplete): Motor function is preserved below the neurological level, and more than a half of key muscles below the neurological level have a muscle grade of $<3$.

- D (incomplete): Motor function is preserved below the neurological level, and at least a half of key muscles below the neurological level have a muscle grade of $\geq 3$.

- E (normal): Motor and sensory functions are normal.

Extent of injury after damage to specific spinal segments is illustrated in the figure (see American Spinal Injury Association in Online links box for the complete standard neurological classification of $\mathrm{SCl})$.

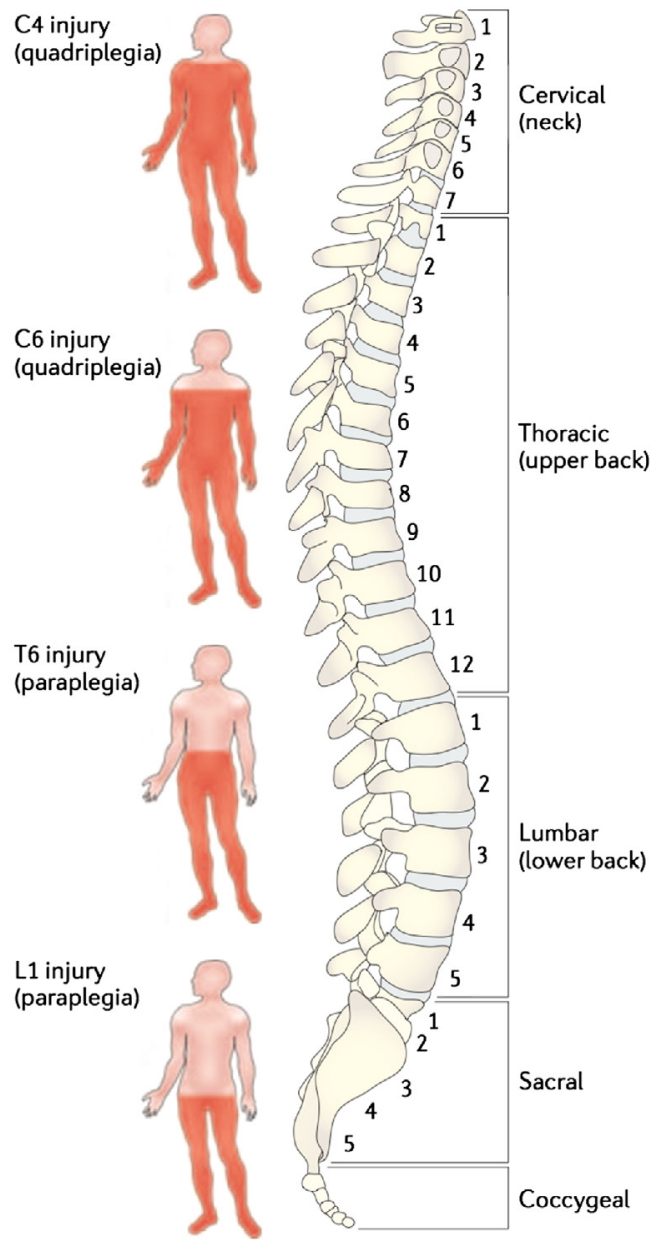

f0025 FIGURE 1.4

SCl levels.

Reprinted with permission from Thuret S, Moon LDF, Gage FH. Therapeutic interventions after spinal cord injury. Nat Rev Neurosci 2006; 7:628-43.

u0030 - Bowel loss of control

u0035 - Pain or burning sensation

u0040 - Breathing difficulties

u0045 • Circulatory problems 


\section{s0030 The ASIA impairment scale}

p0090 ASIA scale is a rating system used to define and describe the extent and severity of a patient's SCI and helps to determine future rehabilitation and recovery needs (Fig. 1.5). It is ideally completed within $72 \mathrm{~h}$ after the initial injury [7]. The patient's grade is based on how much sensation he can feel at multiple points on the body, as well as tests of motor function.

p0095 The scale has five degrees of seriousness:

u0050 A $=$ Complete lack of motor and sensory function below the level of injury (including the anal area)

u0055 $\mathbf{B}=$ Some sensation below the level of the injury (including anal sensation)

u0060 C $=$ Some muscle movement is spared below the level of injury, but $50 \%$ of the muscles below the level of injury cannot move against gravity

u0065 D = Most (more than 50\%) of the muscles that are spared below the level of injury are strong enough to move against gravity.

u0070 $\quad \mathbf{E}=$ All neurologic function has returned.

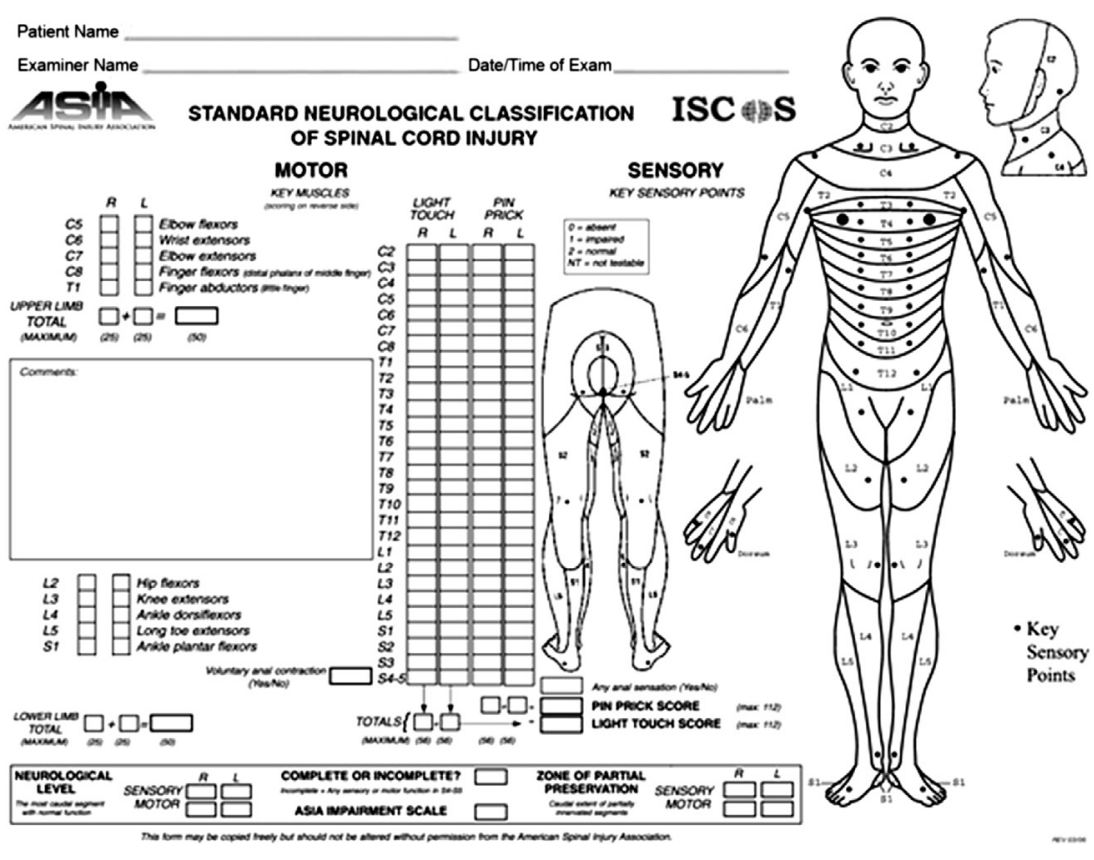

f0030 FIGURE 1.5

ASIA classification, used for the allocation of the neurological level and injury severity. 


\section{s0035 Prognosis}

p0125 When the SCI occurs, the spinal cord swells and some changes are seen in every system of the body. After days or weeks, the swelling begins to go down and some functioning may be regained. The improvement is not due to neuronal/axonal regeneration, but rather to hematoma reabsorption and reduction of the inflammation state. With many injuries, especially the incomplete ones, the patient may recover some functioning as late as 18 months after the injury, but usually the recovery ends in 6 months. Younger patients have major probabilities of motoric recovery, and two hypotheses attempt to explain this:

u0075 - Advanced age implies lower functional potential [8];

u0080 - Elder patients have more difficulty to translate neuronal recovery into physical activity [9].

\section{s0040 Physiopathology}

p0140 Three phases can be distinguished after SCI:

u0085 - Acute phase,

u0090 - Subacute phase,

u0095 - Chronic phase.

p0160 In the acute phase, starting immediately after the lesion, death of all types of cells (neurons, astrocytes, oligodendrocytes, and endothelia cells) occurs. Endothelial dying cells determine the breakage of arteries and hemorrhaging, with formation of edema near the injured region [10] (Fig. 1.6).

p0165 The subacute phase arises in few minutes from the injury and lasts for weeks. During this phase, usually patient conditions could be worsened by local inflammation state. Dead cells and the income and activation of microglia, macrophages, and lymphocytes modify the extracellular environment, intoxicating survived cells. When the inflammation diminishes, the chronic phase succeeds. A scar forms around the injury; it isolates and protects the damaged tissue but obstacles nerve regeneration. Near the scar states, a population of fibroblasts, activated macrophages and glial cells and often a cyst can be seen $[12,13]$.

\section{s0045 Limited central nervous system regeneration}

p0170 Different from peripheral nervous system (PNS), the Central Nervous System (CNS) has no regeneration skills. Research studies $[13,14]$ demonstrate that the different behaviors of PNS and CNS are more associated to environmental condition than to cell's different physiology.

p0175 Inflammation and glial scar's proteins create an inhibitory environment and in the same time the regeneration associated genes deregulation extinguishes innate 


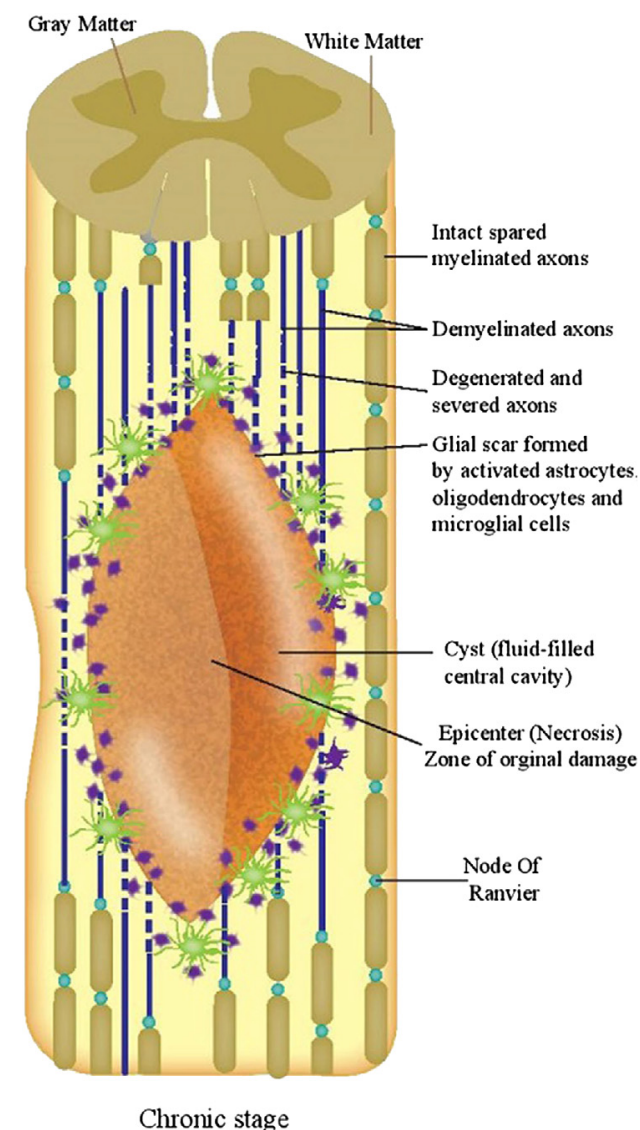

f0035 FIGURE 1.6

Spinal cord physiopathology after injury.

Reprinted with permission from Quadri SA, Farooqui M, Ikram A, Zafar A, Khan MA, Suriya SS, et al. Recent update on basic mechanisms of spinal cord injury. Neurosurg Rev 2018;7:1-17.

neuronal regeneration ability. Some days after the injury, astrocytes have morphological changes and become hypertrophic. They accumulate surrounding the lesion, incorporate microglia and oligodendrocytes, and link each other forming the scar. Microglia have a key role in recovery of CNS physical integrity and in closing up damaged brain blood barrier (BBB). Fixing BBB stops the income of unwanted peripheral cells and reduces cellular damage diffusion [15-17]. Nevertheless, glial scar produces axonal germination chemical inhibitors. 


\section{s0050 Role of inflammation in posttrauma recovery}

p0180 After primary lesion, the inflammatory state occurs: its activation is promoted by necrotic and apoptotic processes (Fig. 1.7). It comprises three steps:

00010 1. In the initial phase, BBB breakage allows hemorrhaging and inflammatory cells arrival meanwhile nervous system's cells dye and lost their myelin cover. Microglia and astrocytes arrive from all CNS and try to restore tissue homeostasis $[18,19]$. Glial cells release proteins, called cytokines and chemokines, which can act both as proinflammatory (TNF- $\alpha$, tumor necrosis factor, interleukins IL-1 $\beta$ and IL-6) and as antiinflammatory factors [20,21]. With hemorrhage, macrophages and other immune system's cells easily enter into the system showing two different contributions to lesion's evolution.

On the one hand, they cause the secondary damage after SCI [10,22], and a treatment for limit or eliminate their presence seems to be useful in the recovery [23]; on the other hand, in the lesion site, they differentiate into M2

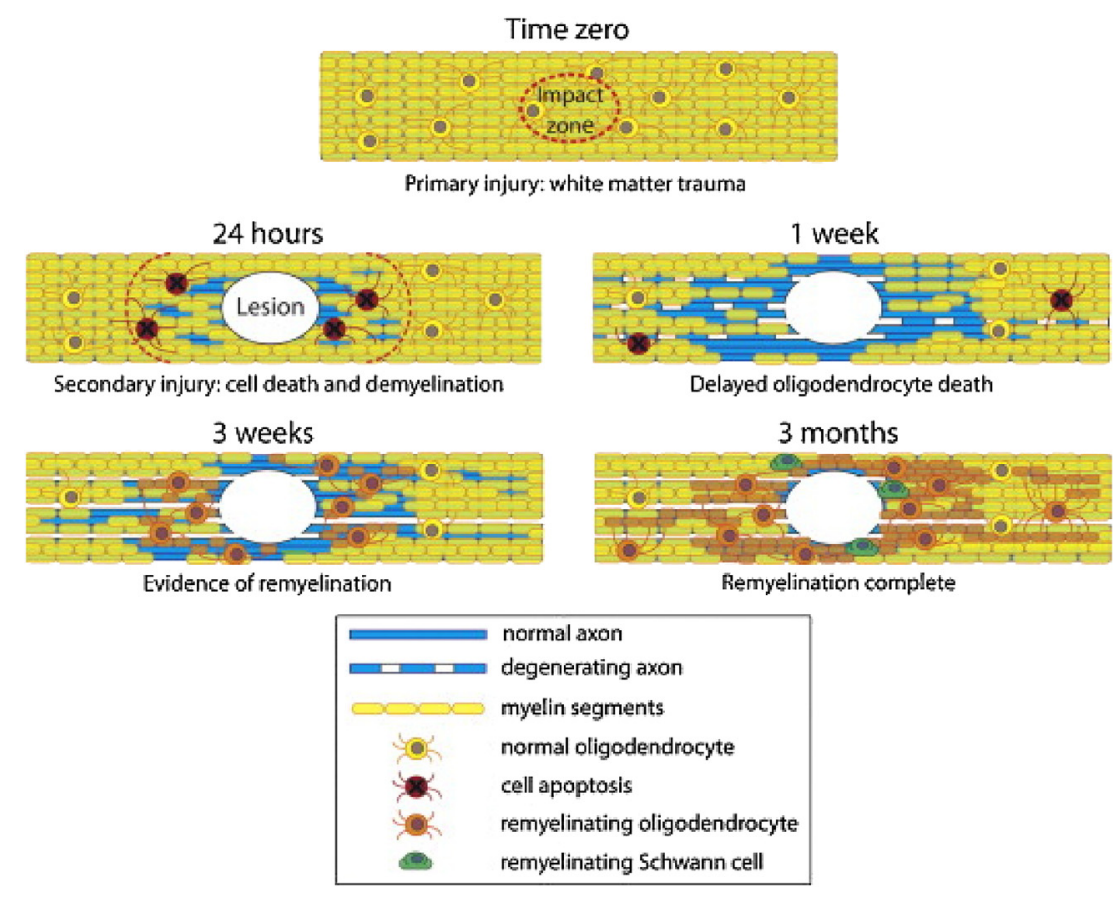

f0040 FIGURE 1.7

Primary and secondary injury.

Reprinted with permission from Plemel JR, Keough MB, Duncan GJ, Sparling JS, Yong VW, Stys PK, et al. Remyelination after spinal cord injury: is it a target for repair? Prog Neurobiol 2014;117:54-72. 
macrophages and emit antiinflammatory cytokines, arousing microglia deactivation $[24,25]$ and limiting damage diffusion.

o0015 2. Between 4 days and 2 weeks after injury, edema reduces and an increase of macrophages and microglia cells number can be observed [26,27]. Removing debris, macrophages create a cavity.

00020 3. The third phase, in which there is a spinal cord volume contraction, is called reactive gliosis. Astrocytes become the first cell population [28] and form the glial scar [29].

\section{s0055 Role of microglia in SCI}

p0205 Microglial cells are a particular type of immune system's cells that arrive in CNS during fetal period and assume its defense from infections and diseases [31].

p0210 Discovered at the beginning of XX century by Rio-Hortega, they represent $10 \%-20 \%$ of adult CNS cells. Microglia have macrophage abilities, provide to CNS homeostasis by digesting apoptotic bodies and noxious substances, and react to endogenous (cytokines, altered levels of neurotransmitters, and proteins) and exogenous factors (viral membrane glycoproteins) [32].

p0215 In normal conditions, microglia are in deactivated form, with dendritic morphology for better environmental control. After activation stimulus, it rushes to the lesion site and assumes first amoeboid and later phagocytic form [33]. There are two possible phenotypes of activated microglia (Fig. 1.8):

u0100 - M1, proinflammatory, produces cytokines responsible of body defense reaction but even of new damage to healthy cells [34]. It has been demonstrated that it is possible to reproduce in vitro this phenotype treating microglia with different stimuli, such as LPS, a lipopolysaccharide forming bacterial protective membrane $[35,36]$.

u0105 - M2 is instead antiinflammatory and activated, for example, by IL-3 and IL-4. It helps injury recovery with enzymes that enhance phagocytosis and tissue's remodeling [34]. In vitro study confirmed that M2 microglia present no toxicity and ability to promote axonal growth [37].

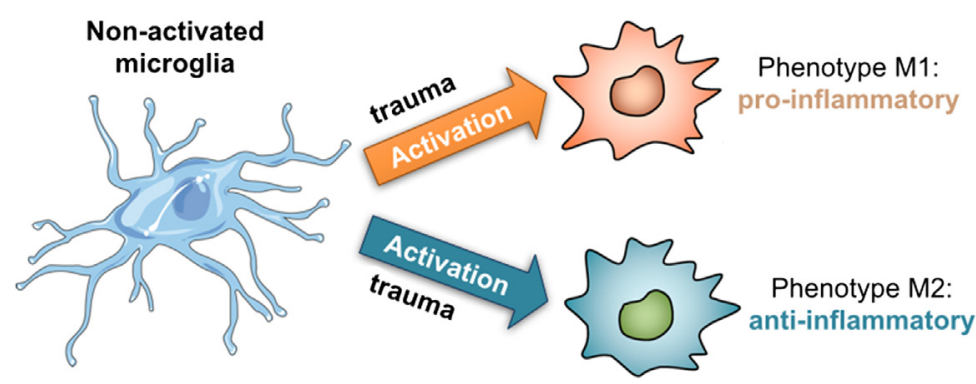

f0045 FIGURE 1.8

Microglia phenotypes. 


\section{References}

[1] Thuret S, Moon LDF, Gage FH. Therapeutic interventions after spinal cord injury. Nat Rev Neurosci 2006;7:628-43.

[2] Vismara I, Papa S, Rossi F, Forloni G, Veglianese P. Current options for cell therapy in spinal cord injury. Trends Mol Med 2017;23:831-49.

[3] Rossi F, Perale G, Papa S, Forloni G, Veglianese P. Current options for drug delivery to the spinal cord. Expert Opin Drug Deliv 2013;10:385-96.

[4] Veneruso V, Rossi F, Villella A, Bena A, Forloni G, Veglianese P. Stem cell paracrine effect and delivery strategies for spinal cord injury regeneration. J Control Release 2019;300:141-53.

[5] Singh A, Tetreault L, Kalsi-Ryan S, Nouri A, Fehlings MG. Global prevalence and incidence of traumatic spinal cord injury. Clin Epidemiol 2014;6:309-31.

[6] Ahuja CS, Wilson JR, Nori S, Kotter MRN, Druschel C, Curt A, et al. Traumatic spinal cord injury. Nat Rev Dis Primers 2017:17018.

[7] Herbison AE, Theodosis DT. Neurotensin-immunoreactive neurons in the rat medial preoptic area are oestrogen receptive. J Neuroendocrinol 1991;3:587-9.

[8] DiGiovanna AG. Human ageing: biological perspectives. New York, NY: McGraw Hill Companies; 2000.

[9] Jakob W, Wirz M, van Hedel HJ, Dietz V, Group E. Difficulty of elderly SCI subjects to translate motor recovery-"body function"-into daily living activities. J Neurotrauma 2009;26:2037-44.

[10] Donnelly DJ, Popovich PG. Inflammation and its role in neuroprotection, axonal regeneration and functional recovery after spinal cord injury. Exp Neurol 2008;209:378-88.

[11] Quadri SA, Farooqui M, Ikram A, Zafar A, Khan MA, Suriya SS, et al. Recent update on basic mechanisms of spinal cord injury. Neurosurg Rev 2018;7:1-17.

[12] B JH, N MD, K S, P W, M A, D D. Schwannosiss: role of gliosis and proteoglycan in human spinal cord injury. J Neurotrauma 2000;17:781-8.

[13] Chong MS, Woolf CJ, Turmaine M, Emson PC, Anderson PN. Intrinsic versus extrinsic factors in determining the regeneration of the central processes of rat dorsal root ganglion neurons: the influence of a peripheral nerve graft. J Comp Neurol 1996;370: 97-104.

[14] Yiu G, He Z. Glial inhibition of CNS axon regeneration. Nat Rev Neurosci 2006;7: 617-27.

[15] Reier PJ, Perlow MJ, Guth L. Development of embryonic spinal cord transplants in the rat. Brain Res 1983;312:201-19.

[16] Faulkner JR, Herrmann JE, Woo MJ, Tansey KE, Doan NB, Sofroniew MV. Reactive astrocytes protect tissue and preserve function after spinal cord injury. J Neurosci 2004;24:2143-55.

[17] Sofroniew MV. Molecular dissection of reactive astrogliosis and glial scar formation. Trends Neurosci 2009;32:638-47.

[18] Davalos A, Fisher M. Emerging therapies for cerebrovascular disorders. Stroke 2005; 36:208-10.

[19] Myer DJ, Gurkoff GG, Lee SM, Hovda DA, Sofroniew MV. Essential protective roles of reactive astrocytes in traumatic brain injury. Brain 2006;129:2761-72.

[20] Rice T, Larsen J, Rivest S, Yong VW. Characterization of the early neuroinflammation after spinal cord injury in mice. J Neuropathol Exp Neurol 2007;66:184-95. 
[21] Yang L, Blumbergs PC, Jones NR, Manavis J, Sarvestani GT, Ghabriel MN. Early expression and cellular localization of proinflammatory cytokines interleukin-1beta, interleukin-6, and tumor necrosis factor-alpha in human traumatic spinal cord injury. Spine 2004;29:966-71.

[22] Alexander JK, Popovich PG. Neuroinflammation in spinal cord injury: therapeutic targets for neuroprotection and regeneration. Prog Brain Res 2009;175:125-37.

[23] Stirling DP, Khodarahmi K, Liu J, McPhail LT, McBride CB, Steeves JD, et al. Minocycline treatment reduces delayed oligodendrocyte death, attenuates axonal dieback, and improves functional outcome after spinal cord injury. J Neurosci 2004;24:2182-90.

[24] Schwartz M, Kipnis J, Rivest S, Prat A. How do immune cells support and shape the brain in health, disease, and aging? J Neurosci 2013;33:17587-96.

[25] Shechter R, Miller O, Yovel G, Rosenzweig N, London A, Ruckh J, et al. Recruitment of beneficial M2 macrophages to injured spinal cord is orchestrated by remote brain choroid plexus. Immunity 2013;38:555-69.

[26] Ahn M, Lee C, Jung K, Kim H, Moon C, Sim KB, et al. Immunohistochemical study of arginase-1 in the spinal cords of rats with clip compression injury. Brain Res 2012;1445: $11-9$.

[27] Moon C, Heo S, Ahn M, Kim H, Shin M, Sim KB, et al. Immunohistochemical study of osteopontin in the spinal cords of rats with clip compression injury. J Vet Med Sci 2004; 66:1307-10.

[28] Fawcett JW, Asher RA. The glial scar and central nervous system repair. Brain Res Bull 1999;49:377-91.

[29] Popovich PG, Wei P, Stokes BT. Cellular inflammatory response after spinal cord injury in Sprague-Dawley and Lewis rats. J Comp Neurol 1997;377:443-64.

[30] Plemel JR, Keough MB, Duncan GJ, Sparling JS, Yong VW, Stys PK, et al. Remyelination after spinal cord injury: is it a target for repair? Prog Neurobiol 2014;117:54-72.

[31] Graeber MB, Streit WJ, Kiefer R, Schoen SW, Kreutzberg GW. New expression of myelo-monocytic antigens by microglia and perivascular cells following lethal motor neurone injury. J Neuroimmunol 1990;27:121-31.

[32] Monk PN, Shaw PJ. ALS: life and death in a bad neighborhood. Nat Med 2006;12: $885-7$.

[33] Vilhardt F. Microglia: phagocyte and glia cell. Int J Biochem Cell Biol 2005;37:17-21.

[34] Gordon S. Alternative activation of macrophages. Nat Rev Immunol 2003;3:23-35.

[35] Dean JM, Wang X, Kaindl AM, Gressens P, Fleiss B, Hagberg H, et al. Micro-glial MyD88 signaling regulates acute neuronal toxicity of LPS-stimulated microglia in vitro. Brain Behav Immun 2010;24:776-83.

[36] Pei Z, Pang H, Qian L, Yang S, Wang T, Zhang W, et al. MAC1 mediates LPS-induced production of superoxide by microglia: the role of pattern recognition receptors in dopaminergic neurotoxicity. Glia 2007;55:1362-73.

[37] Kigerl KA, Gensel JC, Ankeny DP, Alexander JK, Donnelly DJ, Popovich PG. Identification of two distinct macrophage subsets with divergent effects causing either neurotoxicity or regeneration in the injured mouse spinal cord. J Neurosci 2009;29: $13435-44$. 


\title{
Non-Print Items
}

\begin{abstract}
Spinal cord injury (SCI) remains one of the most devastating conditions among neurological diseases due to its pathologic consequences. It is estimated that this lesion affects about 330,000 European people as trauma from an incident (for example, a motor vehicle accident or a sport injury), or by violence such as gunshot wound, or because of medical treatments or diseases such as polio and split spine (http://www.eurostemcell.org). SCI effects can be classified as "primary injury" including the interruption of ascending and descending pathways due to a contusive, compressive, or stretch injury of the spinal cord, and as "secondary injury" that is related to posttraumatic degeneration of the tissue and leading to severe motor or sensory disabilities, often accompanied by paralysis of the arms or legs.
\end{abstract}

\section{Keywords:}

Neuroinflammation, Neuroregeneration, Spinal cord, Spinal cord injury. 


\title{
CHAPTER
}

\section{c0002 Spinal cord injury: role of neurophysiology}

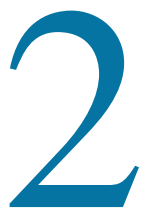

\author{
Bruno Ferrero, MD ${ }^{1}$, Alessandra Di Liberto, MD ${ }^{2}$ \\ ${ }^{1}$ SC Neurologia $2 U-A O U$ Città della Salute e della Scienza di Torino- "Rita Levi Montalcini" \\ Department of Neuroscience, University of Turin, Turin, Italy; ${ }^{2}$ SC Neurologia $2-A S L$ Città di \\ Torino, Giovanni Bosco Hospital, Turin, Italy
}

\section{s0010 Introduction}

p0010 Recent advances in medical and surgical management of spinal cord injury (SCI) have significantly improved diagnosis and survival rate of SCI patients. However, there have been only small progresses on treatment options to improve the neurological outcomes of SCI patients, mostly related to the etiological differences (traumatic or degenerative myelopathy), pathogenic variabilities (high-energy or low-energy mechanisms of injury, compression degree, vascular or infectious complications), and complexity of SCI repair physiopathology. The clinical sequelae of SCI are related to spinal cord lesion levels, severity of motor and sensory pathway involvement, and variable degrees of subsequent demyelination and axonal damage [1].

p0015 SCI severity is essentially based on a lesional level evidence and a combination of clinical signs/symptoms suggesting the involvement of autonomic system, spinal motor or sensory pathways, and gray matter [2]. Urinary retention or detrusor sphincter dyssynergia, stepsis, erectile dysfunction, and blood pressure dysregulation are often related to the dysautonomia after SCI. When sensory pathways are involved, limb ataxia and tactile, thermal, and pain hypo/anesthesia are evident, at various extent, under lesional level. Clinical evaluation of motor system damage after SCI is more complex. If the only part involved is the pyramidal tract (axonal myelinated fibers of frontal cortex upper motor neuron descending into anterolateral spinal cord columns down to lower motor neuron at spinal anterior horn), it is frequent to spot typical signs of cervical or dorsolumbar spinal cord injuries such as tetraparesis/tetraplegia or paraparesis/paraplegia, respectively. After spinal shock, whose resolution occurs over a period of days to months, spasticity slowly appears, related to the inhibitory tone decrease of the lower motor neuron exerted by the medullary interneurons no longer stimulated by collaterals of the damaged pyramidal bundle. Otherwise, if the SCI has also involved the lower motor neuron (lesion of the spinal anterior horns or the anterior root or the spinal nerve), a flaccid paralysis is maintained with the development of a more rapid muscular atrophy.

p0020 In 1992, the American Spinal Injury Association (ASIA) introduced a standardized clinical examination protocol for SCI patients, which aims to quantify the

Spinal Cord Injury (SCI) Repair Strategies. https://doi.org/10.1016/B978-0-08-102807-0.00002-8 Copyright $\odot 2020$ Elsevier Ltd. All rights reserved. 
extent of motor and sensory deficit. However, a higher diagnosis accuracy and an earlier prediction of functional outcome can be significantly increased when combining clinical examination, neuroradiological exams, and neurophysiology [3]. Computerized axial tomography and magnetic resonance imaging (MRI) can show vertebral lesions and signal abnormalities at the level of spinal cord damage but they are not always enough to come up with a complete prognosis, not assessing the functional involvement of the spinal cord precisely [4].

p0025 Neurophysiological techniques supplement clinical and neuroradiological exams, allowing the differentiation between peripheral nervous system lesions (radicular lesions, plexus, peripheral nerves) and spinal cord lesions, and giving a precise spinal cord functional damage localization. Moreover, neurophysiological techniques assess the severity of damage and quantify white or gray matter injury involvement [1], being able to differentiate between conduction velocity slowing (related to white matter demyelination) and amplitude reduction of sensory or motor evoked potentials (related to gray matter axonal damage). Altogether neurophysiological techniques are used to explore motor, sensory, and autonomic pathways.

\section{s0015 Functional anatomy}

p0030 The spinal cord is composed of white (myelinated fibers' prevalence) and gray (neurons and glial cells' prevalence) matter. The white matter, anatomically subdivided into anterior, posterior, and lateral columns, can be functionally differentiated into descending (corticospinal and extrapyramidal), ascending (spinothalamic and lemniscal), and propriospinal pathways. The gray matter is divided primarily into anterior horn (motor function) and posterior horn (sensory function), with a small lateral horn only from first dorsal to second lumbar spinal cord segments (autonomic function). The gray substance of the spinal cord, due to the morphology and density of neurons and glial cells present, can be anatomically divided into 10 large cellular groups known as Rexed laminae: laminae I-VI, lamina VII, and laminae VIII-X constitute the posterior, intermediate, and anterior horn, respectively. Somatotopic organization-the representation of individual body areas in well localized spinal cord areas-is evident for corticospinal, spinothalamic, and lemniscal pathways and for anterior horn neurons(Fig. 2.1).

o0010 1. The corticospinal motor system originates as upper motor neuron (UMN) from the precentral gyrus of the frontal cerebral cortex. UMN axons converge as corticospinal tract in the internal capsule, travel down the brain stem, and, after decussating at the medulla with a majority of its fibers, it reach the anterolateral columns of the spinal cord. At the anterior horn of the spinal cord, the UMN axons contract synapses with the lower motor neurons (LMN) directly or through intermediary excitatory and inhibitory neurons of the anterior gray matter of the spinal cord. The axons of LMN then leave the spinal cord as anterior nerve roots, becoming, once myelinated, spinal nerve roots in the 


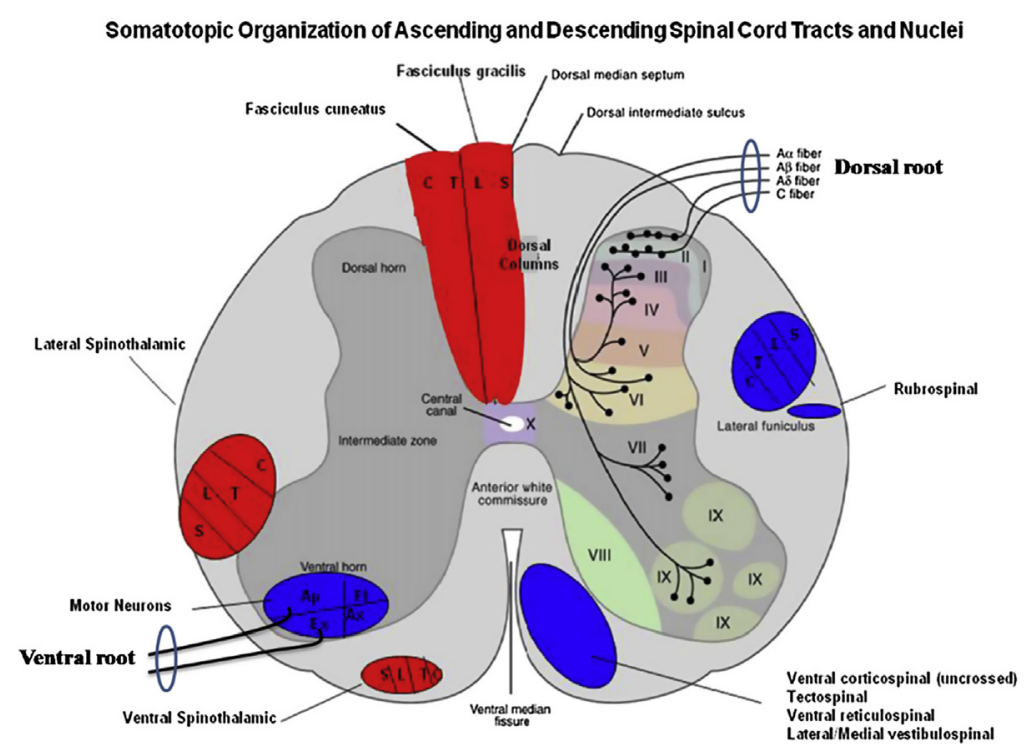

f0010 FIGURE 2.1

Spinal cord anatomy and somatotopic organization of nuclei and pathways. $A p$, Appendicular; $A x$, axial; $C$, cervical; $E x$, extensor; $F l$, flexor; I-X, Rexed laminae; $L$, lumbar; $S$, sacral; $T$, thoracic.

Adapted and modified from Squire LR, Bloom FE, McConnell SK, et al. Fundamental neuroscience. 2nd ed. San Diego: Academic Press; 2003. p. 681. Elsevier Science - USA.

neural foramina. Motor and sensory nerve roots converge to become a spinal nerve, and each spinal nerve divides into a dorsal and ventral ramus: the dorsal ramus supplies paraspinal muscles and sensory innervation to the skin over the spine; the ventral ramus in the thoracic region continues as an intercostal nerve, while in the cervical and lumbosacral region the ventral rami unite to form the cervical, brachial, and lumbosacral plexuses. Within each plexus, motor and sensory fibers from different nerve roots intermix to form peripheral nerves, supplying muscle or skin innervation: myotome and dermatome refer, respectively, to all muscle or cutaneous areas supplied by a single spinal segment. The axon of a single LMN innervates a number of muscle fibers, creating a motor unit.

00015 2. The sensory function is due to the cells of the root ganglia (situated along the posterior roots of spinal nerves, near the intervertebral foramen) that collect information from the peripheral receptors through fibers of the peripheral nerves, and transmit them through the posterior root of the spinal nerve. The sensory roots ascend in the posterior columns of spinal cord giving rise to the medial lemniscus that conveys tactile discrimination and vibration, or synapse with sensory neurons in the dorsal horn giving rise to spinothalamic tracts that 
convey nociceptive, temperature, and crude touch. Medial lemniscus and spinothalamic tracts project to neurons of the thalamus and of the postcentral gyrus of the parietal cerebral cortex.

00020 3. The autonomic nervous system is a component of the peripheral nervous system that provides the neural control of all parts of the body except for skeletal muscles. Sympathetic and parasympathetic systems, the two main anatomically distinct divisions, contain both afferent sensitive and efferent motor fibers. Noncranial autonomic nervous system motor pathways, up and down regulated by supraspinal structures, consist of a two-neuron series: a preganglionic neuron with a cell body at the intermediolateral columns of dorsolumbar (T1-L2) and at the sacral level (S2-S4) of spinal cord for sympathetic and parasympathetic system, respectively; a postganglionic neuron with a cell body at the paravertebral or peripheral ganglia. Unmyelinated postganglionic fibers pass back to the spinal nerves to innervate target organs.

\section{s0020 Neurophysiological techniques \\ s0025 Basic concepts of electrodiagnosis and electrical properties of nerve and muscle}

p0050 The common physics base for all the neurophysiological techniques is the possibility of recording extracellular potentials resulting from neuron or muscle intracellular events, both at rest conditions and following specific external stimuli. The process of an intracellular electrical potential being transmitted through extracellular fluid and tissue is known as volume conduction. Volume-conducted potentials can be modeled as either near-field potentials or far-field potentials. Near-field potentials, directly related to muscle or neuron transmembrane potential variations, can be recorded only close to their source, the features of the potential strictly depending on the distance between the recording electrodes and the electrical source: the closer the recording electrodes are to the current source, the higher potential amplitude is recorded. Potentials recorded during routine motor or sensory conduction and needle electromyography (EMG) studies are examples of volumeconducted near-field potentials. Far-field potentials, related to changes in the electric fields of underlying nervous structures, are electrical potentials distributed instantly over a wide area of the scalp, without significant amplitude variation. Most of the motor and sensory evoked potentials are examples of volumeconducted far-field potentials.

p0055 Among neurophysiological techniques, nerve conduction studies, late responses ( $\mathrm{H}$ reflex and $\mathrm{F}$ wave), needle electromyography, sympathetic skin response, motor evoked potentials or sensory evoked potentials play a key role in the evaluation of patients with spinal cord injury (Fig. 2.2). 


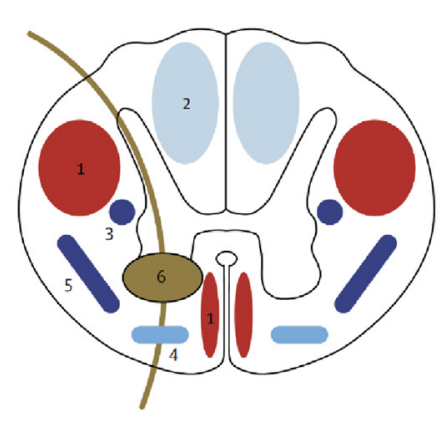

\begin{tabular}{|c|l|l|l|l|l|}
\hline & $\begin{array}{l}\text { Pathway/ } \\
\text { system }\end{array}$ & Method & Readout & Acceptance & $\begin{array}{l}\text { Clinical } \\
\text { correlation }\end{array}$ \\
\hline 1 & Corticospinal & MEP & Amp/lat & Routine & $\begin{array}{l}\text { Central } \\
\text { paresis }\end{array}$ \\
\hline 2 & $\begin{array}{l}\text { Dorsal } \\
\text { column }\end{array}$ & SSEP & Amp/lat & Routine & Proprioception \\
\hline 3 & $\begin{array}{l}\text { Sympathetic } \\
\text { SSR }\end{array}$ & Presence & Routine & $\begin{array}{l}\text { Cardiovascular } \\
\text { control }\end{array}$ \\
\hline 4 & Spinothalamic & $\begin{array}{l}\text { LEP } \\
\text { (laser-heat) }\end{array}$ & Amp/lat & Investigational & $\begin{array}{l}\text { Pain/temp } \\
\text { perception }\end{array}$ \\
\hline 5 & Vestibulospinal & GVS & Amp/lat & Investigational & $\begin{array}{l}\text { Postural } \\
\text { instability }\end{array}$ \\
\hline 6 & $\begin{array}{l}\text { Peripheral } \\
\text { System }\end{array}$ & $\begin{array}{l}\text { NCS/reflex/ } \\
\text { BMG }\end{array}$ & Amp/ncr & Routine & $\begin{array}{l}\text { Peripheral } \\
\text { paresis }\end{array}$ \\
\hline
\end{tabular}

f0015 FIGURE 2.2

Summary of the neurophysiological techniques analyzing the function of specific spinal tracts and peripheral nervous system. The location of the spinal pathways outlined in the table is numerically assigned in the schematic diagram. AMP, amplitude; EMG, electromyography; GVS, galvanic vestibular stimulation; $L A T$, latency; $L E P$, laser evoked potentials; MEP, motor evoked potentials; NCS, nerve conduction study; NCV, nerve conduction velocity; SSEP, somatosensory evoked potentials; SSR, sympathetic skin response.

From Dietz V, Curt A. Neurological aspects of spinal-cord repair: promises and challenges. Lancet Neurol. 2006; 5(8):688-94

\section{s0030 Nerve conduction studies}

p0060 Peripheral nerves usually can be easily stimulated and brought to action potential with a brief electrical pulse applied to the overlying skin: the electrical response is called compound muscle action potential (CMAP) or sensory nerve action potential (SNAP), placing surface recording electrodes over the muscle or the cutaneous nerve, respectively. The directly evoked CMAP, called M wave, represents the temporal summation of all underlying individual muscle fiber action potentials elicited by supramaximal stimulation of all slow and fast fibers of the nerve. Motor nerve is stimulated at more than one site along its course to evoke two or more CMAPs from the muscle: for each stimulation site, latency, amplitude, duration, and area of the CMAP are measured, and a motor nerve conduction velocity can be calculated by the differences in distance and latency between sites of stimulation. Standard studies of motor nerve conduction assess the ulnar, radial, and median nerves in the upper extremities and the femoral, tibial, and peroneal nerves in the lower extremities. Sensory nerve is stimulated at one site along its course to evoke an SNAP recorded over the cutaneous nerve: amplitude and duration of the SNAP, and sensory nerve conduction velocity is then calculated. SNAP is of much lower amplitude than CMAP, and averaging multiple responses sometimes is needed to provide measurable sensory potentials. Standard studies of sensory nerve conduction assess the ulnar, median, radial, medial, and lateral antebrachial cutaneous nerves in the upper extremities, and the superficial cutaneous peroneal, saphenous, and sural nerves in the lower extremities. Nerve conduction studies are fundamental to assess 
accompanying peripheral nerve lesions in polytraumatic SCI, and to define the pathophysiology and prognosis of nerve/radicular damage: an increase of distal motor latency and a reduction of motor or sensory nerve conduction velocity are related to a demyelination, a potentially reversible pathological process; a CMAP or SNAP amplitude reduction (or absence) is instead often related to an irreversible gray matter or axonal damage.

\section{s0035 F response}

p0065 The $\mathrm{F}$ response (or F wave) is a late motor response that occurs after the direct motor $\mathrm{M}$ wave, with a latency $25-32 \mathrm{~ms}$ or $45-56 \mathrm{~ms}$ when the nerves of upper or lower limbs are stimulated, respectively. The $\mathrm{F}$ waves represent responses observed following supramaximal electrical nerve stimulation causing antidromic motor neuron activation with backfiring of only a small population $(1 \%-5 \%)$ of anterior horn cells. As a result, $\mathrm{F}$ waves may not appear after each stimulus, they are also variable in configuration and are low in amplitude. F-wave detection, indicating a preserved conduction along the efferent peripheral motor pathway, is of diagnostic value in proximal nerve lesions, and it is related to the excitability of the segmental motoneuron pool [6]. Several measurements can be made on the F responses, including minimal and maximal latency, and persistence that is the percentage ratio between the number of F-wave responses and the number of supramaximal stimuli applied (normal value is up $70 \%$ ).

\section{s0040 H reflex}

p0070 The $\mathrm{H}$ reflex is a late motor response recorded from soleus and carpi radialis muscles by a submaximal electrical stimulation of posterior tibial or median nerve, respectively [7]. Lower limb H reflex is similar to the phasic myotatic ("deep tendon") reflex produced by muscle stretch, but involving a direct electrical stimulation of muscle spindle afferent Ia fibers. Such sensory large myelinated fibers have got a low stimulation threshold and can be selectively activated when a low submaximal electrical stimulus with a long duration is applied to a nerve. As the Ia afferents are stimulated, the sensory action potential travels orthodromically to the spinal cord, across the synapse, creating a motor potential that travels down the motor nerve, also giving rise to the $\mathrm{H}$ reflex. This reflex is then inhibited as the stimulus intensity is increased from submaximal to that required for eliciting a maximal direct $\mathrm{M}$ wave, related to the "collision" of orthodromic impulses with impulses conducted antidromically in motor axons [8]. The upper limit of normal latency for the $\mathrm{H}$ reflex of the soleus is $35 \mathrm{~ms}$, and that of the flexor carpi radialis is $21 \mathrm{~ms}$. The ratio of the peak-to-peak maximum $\mathrm{H}$ reflex to maximum $\mathrm{M}$-wave amplitude (H/M ratio) provides a measure of motor neuron pool activation and therefore excitability: an $\mathrm{H} / \mathrm{M}$ ratio higher than 0.7 can be related to a spinal cord inhibitory interneuron reduction (i.e., UMN damage). 


\section{s0045 Electromyography}

p0075 EMG is the neurophysiological technique to evaluate the electrical muscle activity, by inserting a disposable concentric needle recording electrode directly into the examined muscle, selected on the basis of the patient's symptoms and signs. The muscle is explored systematically with the electrode for the presence of any spontaneous activity: positive waves, fibrillation, fasciculation, and complex repetitive discharges are often found in denervated muscle, not before 3-5 weeks after an acute LMN lesion and persisting for months after injury. In particular, fibrillation potentials usually are detected previously when the lesion is close to the muscle rather than a more distant lesion. After assessment of spontaneous activity, the EMG examination moves on to the evaluation of motor unit action potentials (MUAPs), and their firing rate. During graded muscle contraction, the MUAP is a compound potential representing the sum of the individual action potentials generated in the muscle fibers of the same motor unit that are within the pick-up range of the needle recording electrode. Amplitude, duration, and phases are the most important parameters of MUAP potentials, depending on the firing synchronization of the muscle fibers and amplitude of the motor unit [9]. Any change in the number of functional muscle fibers related to motor unit pathological processes can affect the MUAP parameters. Indeed, when LMN or anterior motor roots are damaged during SCI, denervated muscle fibers of their motor units are reinnervated by collateral branches from the nerve fibers of surviving units: the recorded MUAP in such circumstances are of longer duration, more polyphasic and of greater amplitude than normal. During EMG evaluation, when a muscle is contracted weakly, a few of its motor units begin firing at a low rate. As the force of contraction increases, the firing rate of these active units increases until it reaches a certain frequency when additional units are recruited. The frequency at which a particular unit must fire before another is recruited (i.e., the recruitment frequency) depends on the muscle and motor unit being studied and on the number of units capable of firing. In general, the units recruited first are smaller in amplitude than those recruited later. When the maximum of voluntary contraction is reached, the baseline of the oscilloscope trace is interrupted continuously by the MUAPs, and individual potentials cannot be distinguished from each other: this is the normal interference EMG pattern. When the number of functional motor units is reduced, there is an increase in the rate at which individual units begin to fire and in the rate at which they must fire before additional units are recruited, not reaching the EMG interference pattern during maximal effort.

\section{s0050 Nerve excitability studies}

p0080 Measurements of nerve excitability provide complementary information to conventional nerve conduction studies. Membrane axonal excitability is determined by the activity of a variety of ion channels, energy-dependent pumps, and ion exchange processes activated during the process of impulse conduction. Talking about nerve 
excitability, the stimulus intensity required to produce a CMAP of a fixed size of maximal response is defined nerve "threshold." The threshold tracking techniques (stimulus-response curves; strength-duration properties; threshold electrotonus; current-threshold relationship and the recovery cycle) are used to measure the nerve threshold variation when other stimulation parameters are modified (i.e., duration or frequency of stimulation) or when the rest potential of the nerve is depolarized or hyperpolarized by direct current, allowing in vivo assessment of the properties of the axonal membrane [10-12]. In SCI patients, motor axons are of high threshold, stimulus-response curves are shifted to the right, and strength-duration time constant is reduced [13].

\section{s0055 Galvanic vestibular stimulation}

p0085 Galvanic vestibular stimulation (GVS) is used to investigate the integrity of vestibulospinal pathways (located in the anterior and lateral spinal cord) that are involved in posture and balance control [14]. This technique consists in stimulating vestibular apparatus by applying binaural bipolar galvanic currents with electrodes placed over the mastoid processes; typically it has evoked a biphasic electromyographic response consisting of a short and medium latency (ML) component, recorded in limb muscles of standing subjects. Most patients with SCI cannot stand, and so ML responses can be obtained in paravertebral (erector spinae) muscles in sitting patients [15].

\section{s0060 Sympathetic skin response}

p0090 The sympathetic skin response (SSR) is an electrophysiological test to examine the common efferent pathways of the sympathetic nervous system. The SSR studies a reflex that involves afferent myelinated sensory fibers, a complex central integrative mechanism, and sympathetic postganglionic unmyelinated $\mathrm{C}$ fibers to the sudomotor sweat glands. Surface electrodes placed on the hands, feet, and genital region detect the change in the electrical activity of sweat glands triggered by a supramaximal magnetic or electric stimulus of a mixed nerve [16]. SSR latency is about $1.5 \mathrm{~s}$ in the upper limb and about $2 \mathrm{~s}$ both in the lower limb and in the genital region: this long latency reflects the slow conduction velocity of unmyelinated postganglionic C fibers (approximately $1 \mathrm{~m} / \mathrm{s}$ ). Significant increase of SSR latency, its asymmetry, or SSR absence is held to be of pathologic significance for clinical purposes [17].

\section{s0065 Bulbocavernosus reflex}

p0095 Bulbocavernosus (BC) reflex is a sacral reflex electrophysiologically recordable as response of the pelvic floor and sphincter muscles to electrical stimulation of sensory fibers in the genital region [18]. Electrical stimuli are delivered via surface electrodes on dorsum of the penis/clitoris, and MUAPs are recorded by concentric needle electrodes placed into the bulbocavernosus or external sphincter of the 
anus muscles. Presence/absence or latency of the BC reflex is the only measured parameter. BC reflex is of value to assess patients with suspected lesions of cauda equine, conus medullaris, or LMN after SCI, although a normal latency of BC reflex does not exclude the possibility of a partial axonal lesion in its reflex arc.

\section{s0070 Standard transcranial magnetic stimulation}

p0100 Standard transcranial magnetic stimulation (TMS) is a noninvasive way to stimulate the human brain [19], and specifically the motor cortex (Fig. 2.3).

p0105 The rapid change of a magnetic field generated by the passage of a high-current pulse through a conducting coil placed tangentially on the scalp is able to induce such an electric field to depolarize the UMN of the motor cortex, resulting in multiple depolarizing volleys descending in the corticospinal tract. If corticospinal pathway is intact, UMN stimulation activates LMN and finally muscle: muscle twitch is therefore recorded as a motor evoked potential (MEP). MEP latency is the time between the delivery of the TMS pulse and the recorded muscle response. MEP due to transcortical stimulation can be recorded from different proximal and distal muscles of the upper and lower limbs, and can be used to assess the level and extent of SCI lesions. Indeed, MEPs from myotomes below lesional level of complete SCI are not recordable, or, if SCI is incomplete, MEPs can be recorded but the conduction time from motor cortex to the motor neuron pool in the spinal cord, known as the central motor conduction time (CMCT), is increased. CMCT

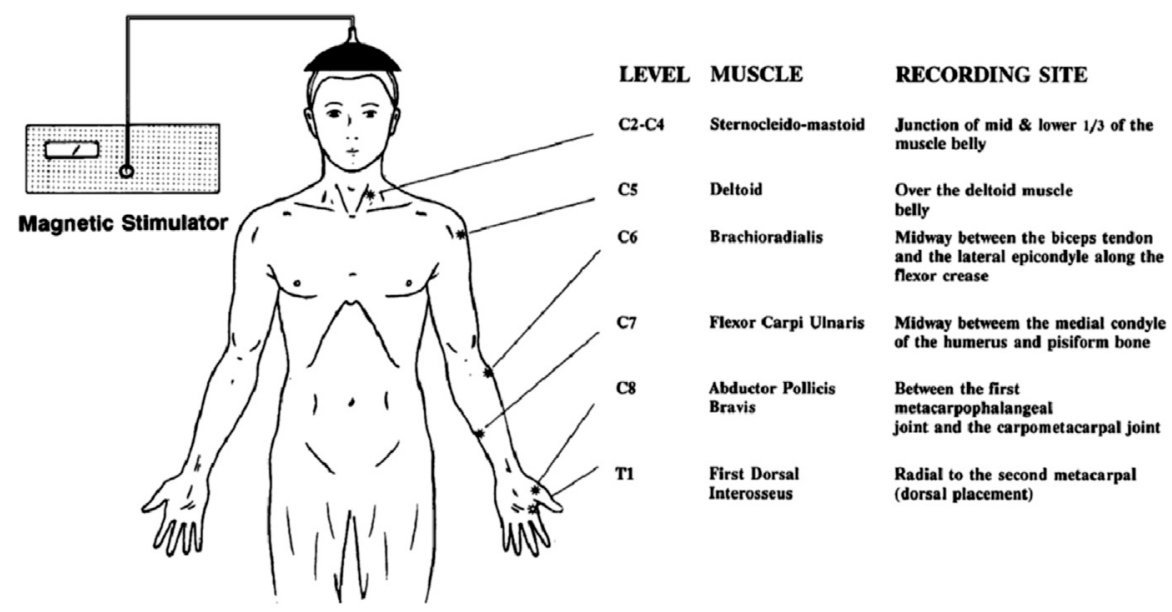

f0020 FIGURE 2.3

Position of the magnetic stimulating cap coil and recording electrodes used to obtain transcranial MEPs for the arms. The muscles represent the different myotomal levels. From Shields CB, Ping Zhang Y, Shields LB, Burke DA, Glassman SD. Objective assessment of cervical spinal cord injury levels by transcranial magnetic motor-evoked potentials. Surg Neurol. 2006;66(5):475-83; 
is obtained by subtracting the peripheral conduction time, calculated by F-wave method [(F-wave latency $+\mathrm{M}$-wave latency $-1 \mathrm{~ms}) / 2]$, from the MEP latency [21]. However, the CMCT increase is not directly related to the number of conducting corticospinal neurons and hence not strictly related to the motor deficit of the patient [22].

\section{s0075 Triple-stimulation technique}

p0110 By employing standard TMS technique, MEPs are variable in shape from one stimulus to the next, and show a smaller amplitude and a longer duration than the CMAPs evoked by peripheral nerve stimulation [23,24]. These MEP characteristics are due both to the multiple volleys after a single magnetic stimulus and to the various degrees of desynchronization of the spinal motor neuron discharges induced by TMS. Therefore, in a standard TMS study, the reduction of MEP/CMAP amplitude ratio, a measure of corticospinal fiber loss, is of little consideration. The triple stimulation technique (TST) is a method improving the study of MEPs, making reliable the reduction of MEP/CMAP amplitude ratio measure [25], and providing a quantitative electrophysiological measurement of the central motor conduction failure. TST is a collision method that synchronizes the depolarization of the motor neurons driven to discharge by the transcranial stimulus, thereby avoiding phase cancellation that accompanies the desynchronization of the biphasic motor unit potentials. Three stimuli are given in sequence and with appropriate delays. The first stimulus is a TMS applied to the scalp overlying the motor cortex, and the second stimulus, at appropriate delay from TMS, is an electrical supramaximal stimulus, given distally to the nerve supplying the target muscle: the action potentials descending from the cortex collide with the antidromic action potentials evoked distally. After another delay, a third electrical supramaximal stimulus is applied proximally to the nerve supplying the target muscle. The amplitude of the CMAP elicited by this third stimulus is then compared to the amplitude of the CMAP elicited by the only electrical supramaximal stimulation at proximal site of the nerve.

p0115 If the corticospinal pathway is intact, there is a complete collision between the first and second stimuli, and the amplitude of the CMAP elicited by the third stimulus will reach its maximum value, comparable to that obtained by the only electrical stimulation of the nerve. On the other hand, if a corticospinal lesion after SCI occurs, there is only a partial collision between the first and second stimuli, and the reduction in amplitude of the CMAP elicited by the third stimulus will be proportional to the extent of the corticospinal pathway damage.

\section{s0080 Sensory evoked potentials}

p0120 Evoked potentials (EPs) are low amplitude neuroelectric responses to sensory stimuli that can be noninvasively recorded using averaging techniques to reduce noise/ signal ratio and enhance the amplitude of signals generated by multiple stimulation sweeps. The EPs can be quantified by measuring their presence or absence, peak 
amplitudes, and latencies. EPs used in SCI studies have the advantages of being objective, often more sensitive than detailed neurological examination, recordable in sedated or unconscious patients, and almost not affected by spinal shock. Disadvantages of EPs in SCI clinical practice are that they are rarely disease specific and can be confounded by end organ disease (e.g., simultaneous presence of a polyneuropathy), they are affected by age, and they may sometimes require a degree of patient cooperation to obtain artifact-free recordings [26]. Almost any sensory modality may be tested, although for SCI studies somatosensory, contact heat, pain, and laser EPs are most frequently tested.

\section{s0085 Somatosensory evoked potentials}

p0125 Somatosensory evoked potentials (SEPs) [27,28] assess function of sensory nerves with their ganglion cells and dorsal column-lemniscal system that subserves mechanoreception and proprioception (Fig. 2.4).

p0130 SEPs consist of a series of waves that reflect sequential activation of neural structures along the somatosensory pathways. SEPs are recorded by surface or needle electrodes, and stimulated by electrical repetitive pulses of sufficient (usually to give a muscle twitch) but painless intensity applied on the skin over the selected nerve. The stimulation sites typically used for clinical diagnostic SEP studies are the wrist for median, ulnar, and radial nerves, and the ankle for tibial, peroneal, and sural nerves. Recording montages can be either "cephalic bipolar" (for nearfield potential detection), in which both electrodes are on the head, or "referential" (for far-field potential detection) with the reference electrode placed at a
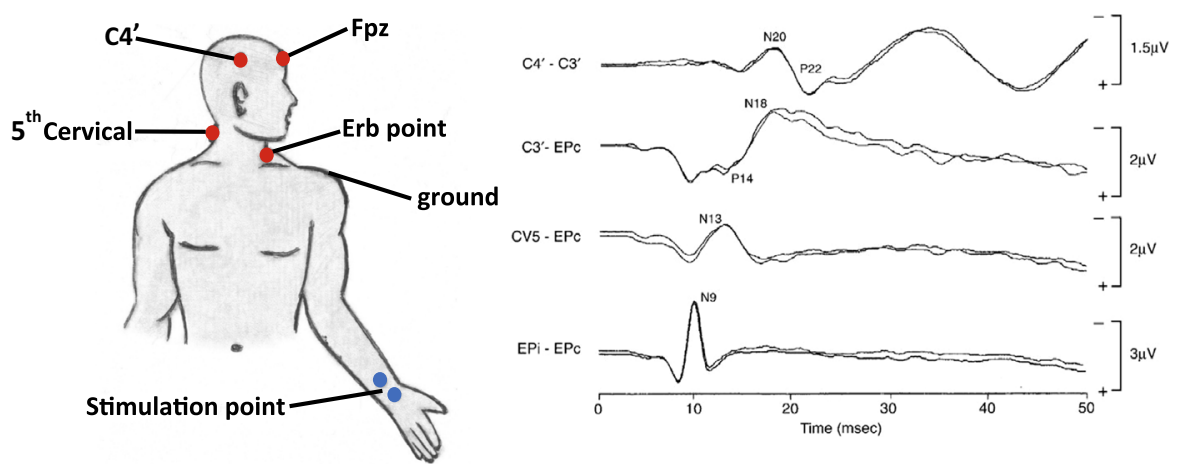

f0025 FIGURE 2.4

Normal SEP elicited by stimulation of the left median nerve and recorded over the Erb's point region (ipsilateral and contralateral), fifth cervical spine, and scalp $\left(\mathrm{C}^{\prime}-\mathrm{C} 4^{\prime}\right)$. The bottom trace shows the Erb's point potential (N9), the second channel the cervical potential, the third channel the subcortical far-field potentials (P14 and N18), and the top channel the N2O potential.

Adapted from Rabai F, Sessions R, Seubert CN. Neurophysiological monitoring and spinal cord integrity. Best Pract Res Clin Anaesthesiol 2016;30(1):53-68; Aminoff MJ, Eisen AA. AAEM minimonograph 19: somatosensory evoked potentials. Muscle Nerve 1998;21(3):277-90. 
noncephalic site. According to the international SEPs montage guideline [27], to study upper limb SEPs the recording electrodes are placed at bilateral Erb's point, at fifth/sixth cervical vertebra, and at scalp $\mathrm{C}^{\prime}$ or $\mathrm{C}^{\prime}{ }^{\prime}$ of International $10-20$ System placement; to study lower limb SEPs, the recording electrodes are placed at distal and proximal regions of the leg, at first/third lumbar vertebra, at scalp $\mathrm{Cz} / \mathrm{Cz}$ ' and at scalp Fz/Fpz or earlobe of International 10-20 System placement. The different SEP components, indicated with the letter $\mathrm{N}$ or $\mathrm{P}$ (referred to negative or positive polarity) followed by a number (referred to expected latency, in milliseconds), reflect sequential activation of specific neural generators excited by the ascending volley.

p0135 For upper limb, the main SEP components with their generators are as follows: N9 (brachial plexus), P11 (dorsal root entry zone), N13/P13 (dorsal horn of the spinal cord), P14 (cervico-medullary junction), N18 (upper pons and midbrain), and N20 (primary somatosensory cortex). N9, N13/P13, and N20 are near-field potentials; P11, P14, and N18 are far-field potentials. The presence or absence of one or more of the expected components must be assessed, and then the interpeak latencies (N9-N13; P14-N20; N9-N20; N13-N20) must be evaluated. The latency difference between N13 peak and the N20 cortical SEP gives a "central conduction time" (an abnormal interpeak latency suggests a defect above the lower medulla). An abnormal interpeak latency between the brachial plexus potential (N9) and N13/P13 suggests a conduction defect in the large-fiber sensory system, central to plexus and below the lower medulla. Absence or abnormally low amplitude of N13/P13 is also associated with a problem central to brachial plexus, below the lower medulla or involving the cervical dorsal horn.

p0140 For lower limb, the main SEP components with their generators are as follows: N8 (proximal peripheral nerve), N18 (cauda equine and posterior columns), N22 (postsynaptic activity in the lumbar gray matter), P30 (cervico-medullary junction), and P39 (primary somatosensory cortex). N8, N18, and P39 are near-field potentials; $\mathrm{N} 22$ and P30 are far-field potentials. The presence or absence of one or more of the expected components must be assessed, and then the interpeak latencies (N22-P30; P30-P39; N22-P39) must be evaluated. If spinal lesions involve the lumbar dorsal horn, the N22 component will be altered or abolished. The increase of N22-P39 interpeak latency is associated with an altered central conduction.

p0145 Adapting the nerve stimulated to the clinical problem can enhance the diagnostic value of SEP. A specific nerve based on a certain level must be chosen, thus for example, superficial radial and ulnar nerve stimulation should be preferred to median nerve to explore, respectively, $\mathrm{C} 6$ and $\mathrm{C} 8$ dermatomes.

\section{s0090 Dermatomal sensory evoked potentials}

p0150 To be more segmentally specific than mixed nerve or cutaneous nerve stimulation it is possible to study dermatomal SEPs (dSEPs) [31] (Fig. 2.5).

p0155 They are techniques of recording cerebral evoked responses to the stimulation of certain areas innervated by solitary nerve roots, such stimulation being intended to provide pure sensory input to the central nervous system through individual spinal 


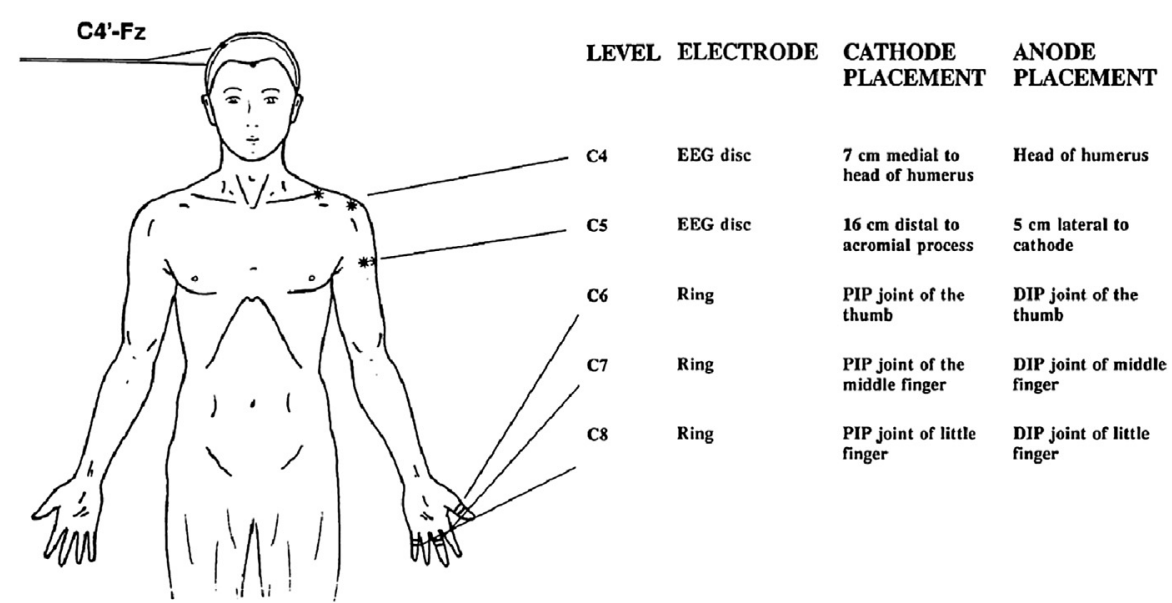

f0030 FIGURE 2.5

Standard position of placement of stimulating and recording electrodes used to obtain dermatomal SEPs.

From Shields CB, Ping Zhang Y, Shields $L B$, Burke DA, Glassman SD. Objective assessment of cervical spinal cord injury levels by transcranial magnetic motor-evoked potentials. Surg Neurol 2006;66(5):475-83;

discussion 483.

segments. This technique has been used especially to assess function of the lumbosacral roots. Stimulus specificity (avoiding spread to other dermatomes) is obtained by maintaining stimulus at 2.5 times the sensory threshold. Recording montage is the same as that used for SEPs.

\section{s0095 Pudendal sensory evoked potential}

p0160 In spinal cord injury, it is useful to evaluate pudendal SEP. The pudendal SEP is elicited by electrical stimulation of the dorsal penile nerve using ring electrodes in men and clitoral nerve with handheld bipolar-stimulating surface electrodes in women. Spinal responses were recorded at the first lumbar level by placing the reference electrode at iliac spine, and cortical evoked potentials (P40/N50 complex) at $\mathrm{Cz}^{\prime}$ against the reference $\mathrm{Fz}[32]$.

\section{s0100 Contact heat evoked potentials}

p0165 Contact heat evoked potentials (CHEPs) assess the function of spinothalamic tract. Its technology utilizes rapidly delivered heat pulses with adjustable peak temperatures to stimulate the differential warm/heat receptors expressed by $\mathrm{A} \delta$ and $\mathrm{C}$ fibers. The resulting evoked potentials can be recorded and measured from electrodes put on the midline of the head [33].

\section{s0105 Laser evoked potentials}

p0170 Laser evoked potentials (LEPs) are elicited by painful stimuli proved to be a useful tool to examine pain pathway. Radiant heat stimulations using laser allow selective 
activation of A-delta and $\mathrm{C}$ thermosensitive nociceptors, and corresponding cortical responses are recorded by electrodes placed on two midline sites ( $\mathrm{Fz}$ and $\mathrm{Cz}$ ) referred to the nose or earlobes and one controlateral temporal electrode referred to Fz. LEPs are used to investigate patients with neuropathic pain after SCI, and to probe the function of a single dermatome or dorsal root. $\mathrm{CO}_{2}$ laser stimulators deliver brief heat pulses that ensure synchronous activation of nociceptors. The highest-amplitude scalp signal after a laser stimulus is a complex named N2-P2, maximal at the vertex, which results from simultaneous activity of several cortical generators with major participation of cingulate gyrus, insular, and frontal operculum [27].

\section{s0110 Quantitative sensory testing and electrical perception threshold}

p0175 Quantitative sensory test (QST) methods evaluate thermal, light touch, and vibratory pathways along peripheral receptors, posterior spinal cord (light touch and vibration), and anterior spinal cord (nociceptive and thermal). QSTs are psychophysical evaluations, requiring patient cooperation. QST systems consist in multiple devices that generate specific physical vibratory or thermal stimuli applied to the skin, and then it is asked to the patient to report sensation of temperature change or heat pain. Algorithms for testing have been developed to facilitate sensory threshold determination [34]. Another QST method consists in electrical perception threshold (EPT) where electrical stimulations are delivered applying self-adhesive electrodes, with the advantages of reducing the variability associated with handheld thermodes and vibratory stimulators, although it is less selective than standard QST devices because EPT activates mixed populations of fibers in the periphery. EPT is determined on each dermatome by increasing the intensity of stimulus from $0 \mathrm{~mA}$ until the individual verbally reports first sensation or until $40 \mathrm{~mA}$ is achieved without sensation.

\section{s0115 Intraoperative neurophysiological monitoring}

p0180 Intraoperative neurophysiologic monitoring (IONM) during spinal surgery use MEP, SEP, and EMG techniques to provide continuous functional assessment of specific spinal cord anatomic structures, allowing rapid detection of neuronal compromise and early corrective action before an insult causes permanent neurological damage [29]. IONM is not used just as a diagnostic tool but also to improve surgical outcomes. Postoperative neurological status can be inferred from intraoperative signals but an exhaustive explanation goes beyond the aim of this chapter.

\section{s0120 Application and prognostic value of neurophysiology s0125 Recording of peripheral pathways}

p0185 In SCI patients, EMG and routine nerve conduction studies from upper and lower limb muscles are required to assess the site of LMN damage and accompanying 
anterior/posterior roots or peripheral nerve lesions in polytraumatic patients. Sensory conduction studies are normal until the dorsal root ganglia or sensory nerves are not involved in the injury. Motor conduction studies show M-wave reduction not only due to direct motor nerve damage but also when several adjacent roots are involved or when the recorded muscle has become atrophic. When LMN (anterior horn or root) is involved in SCI, signs of acute and chronic partial denervation are found in muscles supplied by the affected segments: denervation potentials, such as fibrillations, appear after 1-2 weeks after SCI, earlier in more proximal muscles (paraspinal muscles) and then in the distal limb muscles; the number of long and wide MUAPs increase, as does the number of polyphasic MUAPs; the interference pattern is reduced whereas the recruitment frequency and firing rate of individual MUAPs are increased. It is sometimes hard to distinguish a discrete spinal cord from a root lesion, but in SCI several segments, also bilaterally, are usually involved. Examining several myotomes, it is therefore possible to define the precise segmental distribution of a lesion involving LMN in SCI, also in areas of difficult clinical evaluation such as thoracic region. Indeed, when partial UMN and LMN disconnection due to spinal cord anterolateral columns lesion occurs, EMG shows a poor activation of motor units and a loss of their frequency increase during progressive muscle effort $[35,36]$. The combination of EMG and routine nerve conduction studies allows the differentiation between muscle paresis due to spinal anterior horn cell/anterior nerve root lesion or peripheral nerve damage (plexus, peripheral nerve): in the latter disorder, both the peripheral sensory and motor nerve fibers are affected, whereas in spinal lesions only the motor nerve fibers are affected [37]. Indeed, EMG and motor nerve studies are useful in predicting the development of a spastic or flaccid leg muscle paresis [65], which is of great influence for the rehabilitation program. As preservation of peripheral nerve function is critically important to the success of strategies aimed at regenerating the spinal cord, there has been increasing awareness over recent years of the importance of maintaining function below the level of the lesion [38]. For this purpose, nerve excitability studies provide interesting perspectives in monitoring the variation of nerve function after SCI. Initial axonal excitability studies in SCI patients [39] have identified significant functional changes in peripheral axons (high threshold and $\mathrm{M}$-wave amplitude reduction) coinciding with the period of spinal shock [13], perhaps related to the influence of decentralization and inactivity in such patients. These findings suggest that methods to monitor, preserve, or maintain peripheral nerve function must be warranted after acute SCI to improve the response to innovative therapies and therefore the prognosis. The use of the $\mathrm{F}$-wave response and $\mathrm{H}$ reflex studies are additional tools to diagnosis of concomitant spinal gray matter, anterior and posterior roots lesion after SCI, keeping also a prognostication value. F-wave latency increase and its persistence decrease can be related to LMN damage [40], while F-wave absence is related to spinal shock [41]. Clinical improvement has been associated with increased F-wave amplitudes and persistence [42]. H reflexes are affected by injury to either the posterior or anterior roots. $\mathrm{F} / \mathrm{M}$ and $\mathrm{H} / \mathrm{M}$ amplitude ratio increases are related to the anterior horn cell excitability and to developing spasticity; it can be used to monitor neuronal 
plasticity [43]. H-reflexes and bulbocavernosus reflex can be elicited during spinal shock, at a time when tendon reflexes of the lower limb are still lost. This confirms that the reflex arc is preserved and no accompanying peripheral nerve lesions below the level of lesion are present $[44,45]$. Therefore, the H/M amplitude ratio increases when UMN and LMN has been disconnected. An abolished bulbocavernosus reflex is usually associated with the development of bladder dysfunction due to an LMN lesion. However, similar to clinical and pudendal SEP examinations, urodynamic characteristics of the detrusor muscle (urine retention, renal reflux) cannot be predicted by this reflex [46]. Complete tetraplegic patients develop different syndromes of sympathetic failure with orthostatic hypotension, disturbance of the circadian blood pressure regulation (diminished modulation of blood pressure but preserved heart frequency modulation during the night periods due to intact vagal innervation), and can suffer from autonomic hyperreflexia [47]. In tetraplegic patients, the loss of SSR in the hands was associated with the development of autonomic hyperreflexia in $93 \%$ of patients.

\section{s0130 Recording of spinal pathways}

p0190 Motor and sensory evoked potentials play a crucial role in defining the extent and the severity of corticospinal and lemniscal pathway damage after SCI. Indeed, these methods can supplement clinical examination and neuroimaging findings in the assessment of SCI. MEP and SEP usefulness is also related to the possibility of analyzing motor and sensory pathways in uncooperative or unconscious patients, also during total intravenous anesthesia $[48,49]$.

p0195 In SCI patients, a good correlation is found between MEP findings and motor function [50]. By combining TMS, nerve conduction studies, and EMG, a precise quantification of UMN and LMN involvement can be differentiated [51,52]. Using standard TMS, the reliability is excellent for CMCT and good for TMS threshold, MEP amplitude, and duration [53]. Using triple stimulation technique, the reliability is excellent also for MEP amplitude. For careful assessment of the spinal cord function, MEPs must be recorded from multiple myotomes corresponding to different upper and lower limb muscles (Fig. 2.6).

p0200 However, the contribution of MEP evaluation in the assessment of SCI is greater for cervical than thoracolumbar spinal cord because MEP from lower limbs may be absent at rest even in a few normal subjects. Moreover, MEPs recorded from diaphragm or other inspiratory and expiratory muscles can be used routinely to monitor patients with impaired central respiratory drive due to high cervical lesions [54]. MEPs may be useful to predict functional outcome in the acute phase of SCI, follow the evolution of motor disturbances control and evaluate the effects of different therapeutic procedures (Fig. 2.7). Some limits of TMS are that prolonged CMCT has not disease specificity and that the facilitation of MEP responses usually induced by mild motor contraction is difficult or even impossible for the population of subjects with spinal cord disorders being partially or totally plegic. 
(A)

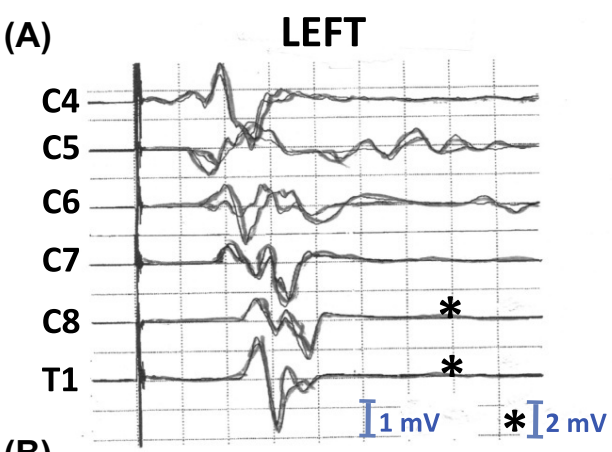

(B)

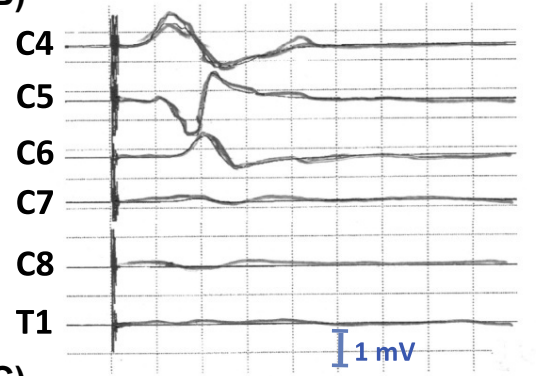

(C)

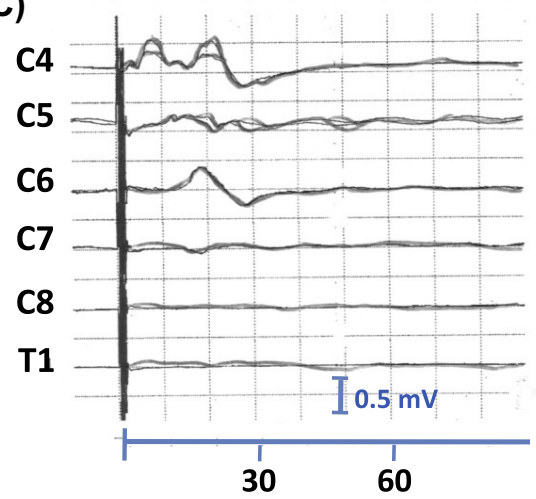

Time (msec)

\section{RIGHT}
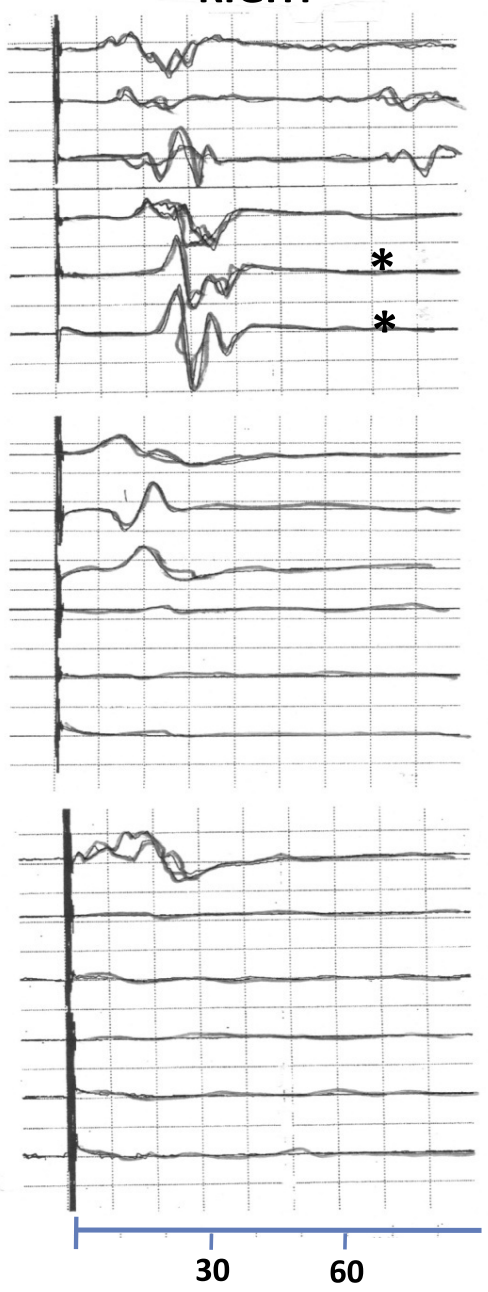

Time (msec)

\section{f0035 FIGURE 2.6}

MEPs bilaterally recorded from different myotomes. (A) Normal myotomal transcranial MEPs obtained from healthy volunteer. (B) MEPs obtained from a quadriplegic patient with absent responses caudal to $\mathrm{C} 6$ level. (C) MEPs from a patient with $\mathrm{C} 3$ cervical $\mathrm{SCl}$ on a respirator. The small amplitude potential recorded from C4 (sternocleidomastoid muscle) indicates that the lesion is above $C 4$.

From Shields CB, Ping Zhang Y, Shields $L B$, Burke DA, Glassman SD. Objective assessment of cervical spinal cord injury levels by transcranial magnetic motor-evoked potentials. Surg Neurol 2006;66(5):475-83; dis- 


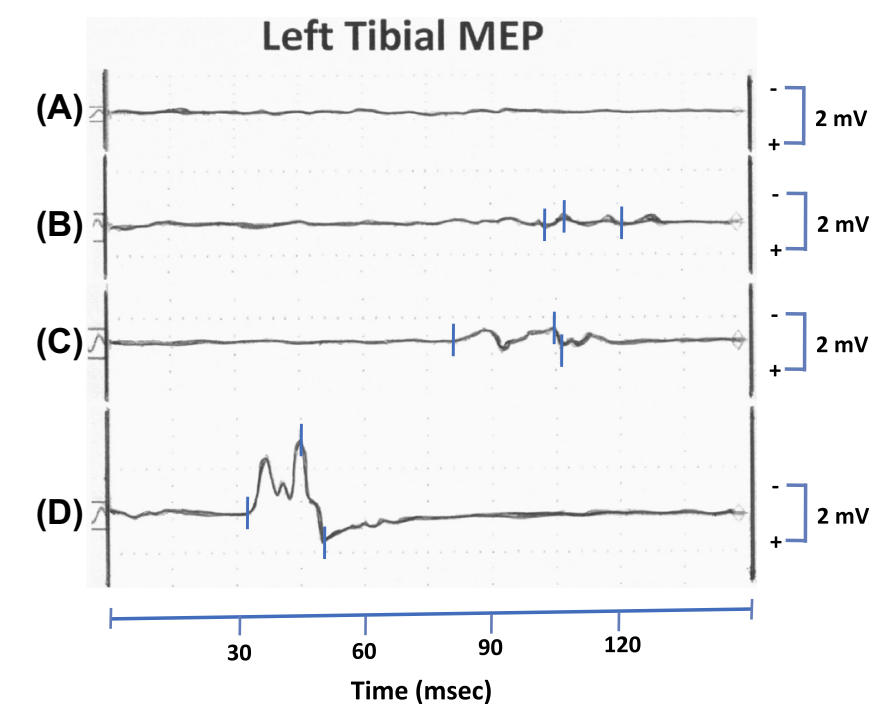

f0040

IGURE 2.7

Tibial MEP recorded from left flexor hallucis brevis at different time points. (A) Three days after $\mathrm{SCl}$ absent MEP response. (B) One week after $\mathrm{SCl}$ reappearance of very low amplitude MEP response. (C and D) One and three months after $\mathrm{SCl}$ progressive MEP response increase.

From our neurophysiology laboratory.

p0205 SEP recording tests the integrity of axonal tract conduction, evaluating impulse transmission of somatosensory fibers through central (conus medullaris, spine, and cortex) and peripheral (sensory nerves and plexus) nervous structures [55]. Abnormalities of SEPs include a reduction of amplitude and latency shifts of the early components. Some authors suggest that in the acute phase (1 week) amplitude changes predominate with little latency change but in the more chronic phase (6 weeks) the SEPs show both amplitude and latency changes. It must be considered that latencies seem to be more reliable in reflecting real damage, whereas amplitudes vary interindividually and depend more on the quality of the peripheral nerve stimulation. Therefore, amplitudes may rather be interpreted by bilateral comparison [56]. During the acute phase, patients with complete functional transection of the spinal cord can have no recordable SEPs (Fig. 2.8).

p0210 Transient abnormalities may depend on spinal cord edema. In some cases that appear clinically to be complete lesions, SEPs can persist suggesting some residual spinal cord function. In this situation, in the early hours or days after trauma, the accuracy of the clinical examination is limited and the SEPs may be able to differentiate between a totally transected cord and one that has the potential to improve [28]. SEPs are able to detect even subclinical abnormalities of central sensory pathways. In particular, an usual finding is the isolated loss or delay of the spinal near-field N13, while its recording can be considered a reliable diagnostic tool; the less frequent scalp 


\section{Left and right Median SEPs}

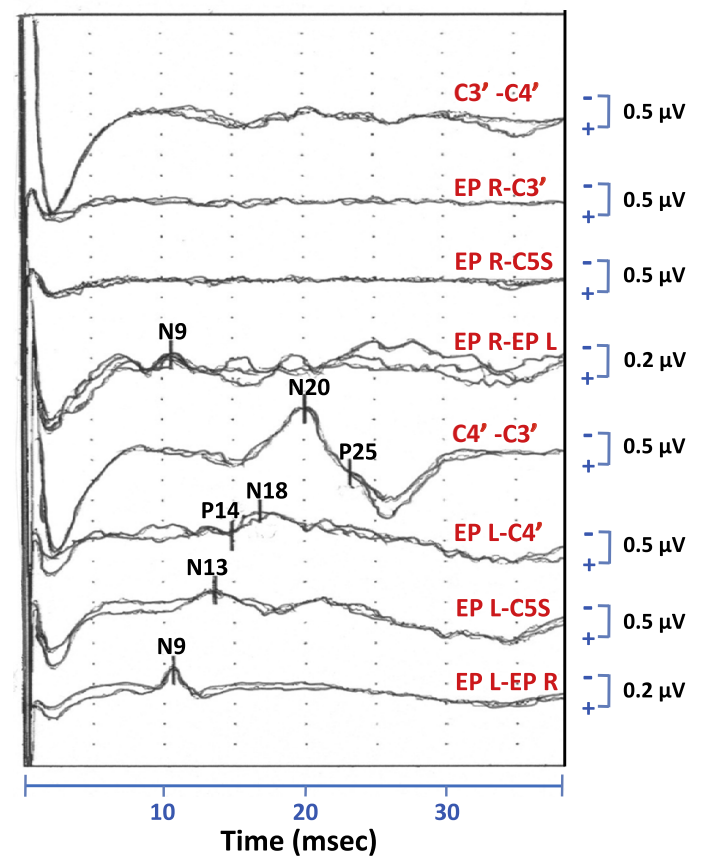

f0045 FIGURE 2.8

SEP elicited by stimulation of left and right median nerve and recorded over the Erb's point region (ipsilateral and contralateral), fifth cervical spine, and scalp $\left(\mathrm{C}^{\prime}-\mathrm{C} 4^{\prime}\right)$, 2 weeks after traumatic cervical cord left hemisection. Traces $1-4$ show only Erb's point potential (N9) with absence of spinal (N13) and cortical (N20) responses. Traces 5-8 show normal right spinal and cortical responses.

From our neurophysiology laboratory.

far-field P14 abnormalities are very useful in disclosing dorsal column dysfunction [66]. High intrasubject reproducibility makes SEPs suitable for longitudinal studies. Changes in SEP parameters may thus reflect the efficacy of new treatments in regeneration of damaged spinal pathways, because early persistence and progressive normalization may antedate clinical evidence of improvement. Consequently, SEP parameters could be of special value to track treatment-induced changes in the dorsal column during clinical trials [56]. Functional outcome about impairment or loss of hand function and ambulatory capacity is evaluated combining SEPs and MEPs. Patients with normal median and ulnar SEP develop an active hand function in more than $90 \%$ of cases. SEP recordings are of similar sensitivity $(P<.001)$ as the ASIA scores of the sensory functions in predicting the outcome of hand function. The tibial SEP can be used to predict recovery of lower limb function and is related to outcome of ambulatory capacity [57]. Most patients $(>80 \%)$ with normal tibial SEP latency 
develop a full ambulatory capacity [58]. The MEP recordings of the abductor digiti minimi muscle are significantly correlated to the outcome of hand function. Where abductor digiti minimi muscle MEP is absent, no patient regains an active hand function. Furthermore, MEP recording from proximal upper limb muscles (biceps and triceps muscle) predicts better patient's self-independence in daily activities [57]. The MEP values of lower limbs are similarly sensitive in predicting recovery of ambulatory capacity as the ASIA motor scores: patients with an elicitable MEP within the first 4 days posttrauma show the best recovery of motor function and achieve at least a functional ambulatory capacity [59].

p0215 SEPs are used to assess the level of SCI, for example SEP recordings by stimulation of upper limb nerves allow the assessment of cervical SCI level. In C3-C6 lesions, the median SEP is pathological in about half and ulnar SEP in most $(80 \%)$ of the patients. In C6-T1 lesions, the median SEP is less affected $(<20 \%)$ whereas the ulnar SEP is pathological in more than $85 \%$ of patients [57]. To enhance the ability to identify the lesion level, dermatomal somatosensory evoked potentials (dSEPs) that allow to track down changes in sensory function corresponding to individual dermatomes (a single spinal segment) above, at, and below the SCI level can be used [60] (Fig. 2.9).

p0220 dSEPs require that each dermatomal level to be tested individually on each side, which is time consuming and technically difficult because the responses are often

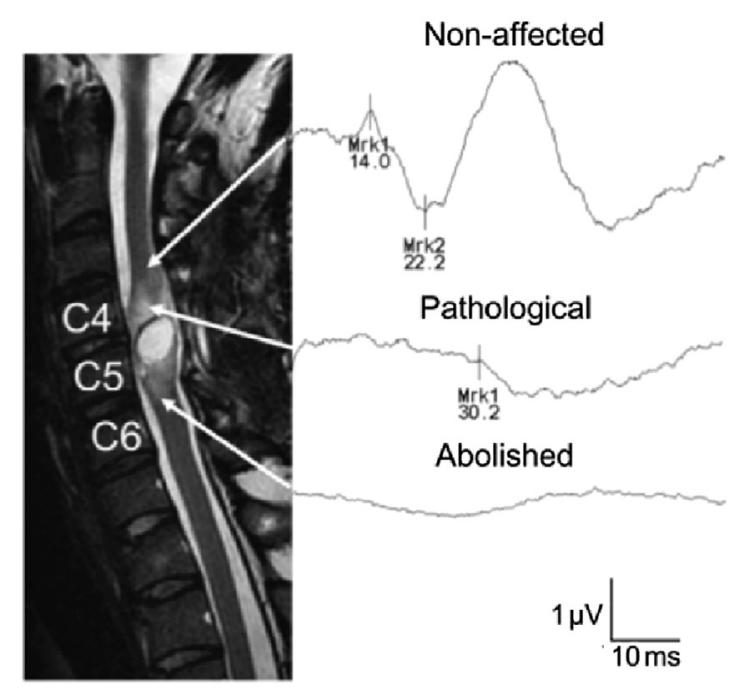

fO050 FIGURE 2.9

Magnetic resonance image (sagittal T2-weighted) and dermatomal somatosensory evoked potentials: nonaffected, pathological, and abolished recordings in a patient suffered from traumatic tetraplegia. Mrk1, onset latency; Mrk2, positive peak.

From Kramer JL, Moss AJ, Taylor P, Curt A. Assessment of posterior spinal cord function with electrical perception threshold in spinal cord injury. J Neurotrauma 2008;25(8):1019-26. 
low in amplitude. So dSEPs are often combined with MEPs that are present above the level of injury with severe impairment below. At the segmental levels where MEP and dSEP responses are elicited but delayed the injury could be reversible, thereby return of function is possible at that level even when an initial neurologic deficit is present. However, if MEP/dSEP responses are absent, the potential for return of electrophysiologic/clinical function at that level is low [20]. Some authors suggest that dSEPs may be potentially useful to monitor the safety of a therapeutic drug or cell transplant in early-phase clinical trials as well as document the potential efficacy of interventions where the standard neurological assessment might not detect subtle therapeutic effects [60].

p0225 Impaired bladder function is a major complication in patients with complete and incomplete SCI. Elicitable pudendal SEPs are indicative of a good recovery of bladder function and are significantly related to the functioning of the external urethral muscle.

p0230 SCI typically involves gross anatomical lesions of central gray and white matter but selective anterior, central, or Brown-Sequard (damage to one half of the spinal cord) lesions account for approximately $15 \%-20 \%$ and yield specific segmental sensory deficits due to segregated sensory modalities in the spinal cord [61]. CHEPs and quantitative sensory tests are not just used to assess sensory function in peripheral nerve, radicular, or plexus lesions, but, after SCI, they are used to find selective spinal cord sensory pathways' impairments. Thermal thresholds would predominantly test spinothalamic function, vibration would test posterior column function, and electrical threshold would test both sensory function. These tests can be also applicable in the thoracic region, for which SCI diagnosis currently relies mainly on clinical sensory testing.

p0235 By giving a continuous numeric threshold value, QST can better quantify the sensory impairment within the ASIA sensory grade 1 (defined as "impaired") and allow monitoring any change, so it is used in longitudinal monitoring of SCI especially for research purposes [62].

p0240 A common symptom following SCI is spontaneous and/or provoked neuropathic pain (NP) that may be associated with altered processing of somatosensory pathways in dermatomes rostral to the injury level. To explore this possibility, CHEPs and quantitative sensory testing are studied at and above the lesion level in patients with NP [63]. Pain and laser evoked potentials can detect conduction abnormalities at any point in the pain-temperature pathways (from periphery to cortex) that are associated or not to patient's heat-pain hypoesthesia [27]. Nonnoxious electrical stimuli used in SEPs recording activate the large myelinated A-beta fibers, which convey the nonnociceptive inputs, and so SEPs do not show any abnormality in case of an exclusive damage of the small-fiber system. Dissociation between normal SEPs and abnormal LEPs is the rule in pure spinothalamic lesions. LEPs can help to distinguish neuropathic pain (amplitude reduction) from nonneuropathic pain conditions (no amplitude change). It was shown also that spontaneous pain is related with LEP suppression, which is due to extensive axonal damage (A-delta fibers), while provoked pain corresponds to partially preserved LEPs, suggesting sensitized A-delta nerve fibers [64]. 
Limits in the diagnostic use of LEPs should be underlined: a laser stimulator for LEP recording is considerably more expansive than an electrical stimulator for SEPs and, despite LEP recording is harmless, reddening of the stimulated skin might occur and annoy the patient. Now, there has been development of LEPs with earth elements, including the largely used neodymium that has the least impact on the lesional skin, thus it is better tolerated compared to the $\mathrm{CO}_{2}$ stimulus [64].

\section{s0135 Conclusions}

p0245 SCI care keeps on improving every day. Clinical trials of neuroreparative agents are being tested, and new therapeutic approaches are being developed; it is very important to have valid prediction methods to forecast who may benefit from these therapies and to measure their neurological effects. The combination of both clinical and electrophysiological recordings in acute SCI patients represents a reliable predictor of spinal damage degree and of spontaneous or therapies-induced neurological recovery. Multimodal neurophysiological assessments may increase the ability to correlate injuries data and outcomes, especially regarding hand function, ambulatory capacity, bladder function, and autonomic dysfunction, improving the classification and quantification of SCI severity and prognosis. Electrophysiological recordings provide important information on the level and extent of spinal cord and peripheral nervous system lesions, representing a useful and noninvasive way to evaluate uncooperative patients, where the clinical examination is of restricted value. Using neurophysiological assessment makes it possible to better analyze therapies' effects on specific spinal cord and peripheral structures functioning. Putting together neurophysiological data, clinical signs, symptoms, and advanced resonance techniques enhances the ability to monitor patients during follow-up. Therefore, neurophysiological evaluation may be considered as a necessary "trait d'union" between clinical assessment and radiological investigations: advanced MRI methods and neurophysiological techniques, focusing on neuronal plasticity, may be able to provide new insights into the evolution of neural connectivity after SCI, particularly in clinical trials testing neuroreparative agents.

\section{References}

[1] Dietz V, Curt A. Neurological aspects of spinal-cord repair: promises and challenges. Lancet Neurol 2006;5(8):688-94.

[2] Petersen JA, Spiess M, Curt A, Weidner N, Rupp R, Abel R, EM-SCI Study Group, Schubert M. Upper limb recovery in spinal cord injury: involvement of central and peripheral motor pathways. Neurorehabilitation Neural Repair 2017;31(5):432-41.

[3] Hupp M, Pavese C, Bachmann LM, Koller R, Schubert M, EMSCI Study Group. Electrophysiological multimodal assessments improve outcome prediction in traumatic cervical spinal cord injury. J Neurotrauma 2018;35(24):2916-23. 
[4] Nové-Josserand A, André-Obadia N, Mauguière F. Cervical spondylotic myelopathy: motor and somatosensory evoked potentials, clinical and radiological correlation. Rev Neurol (Paris) 2002;158:1191-7.

[5] Squire LR, Bloom FE, McConnell SK, et al. Fundamental neuroscience. 2nd ed. San Diego: Academic Press; 2003. p. 681. Elsevier Science - USA.

[6] Kimura J, Yanagisawa H, Yamada T, Mitsudome A, Sasaki H, Kimura A. Is the F wave elicited in a select group of motoneurons? Muscle Nerve 1984;7(5):392-9.

[7] Christie AD, Inglis JG, Boucher JP, Gabriel DA. Reliability of the FCR H-reflex. J Clin Neurophysiol 2005;22(3):204-9.

[8] Stålberg E, Tronetlj J. Reflex studies. In: Single fiber electromyography: studies in healthy and diseased muscle. New York: Raven; 1994. p. 226.

[9] Daube JR, Rubin DI. Needle electromyography. Muscle Nerve 2009;39(2):244-70.

[10] Bostock H, Cikurel K, Burke D. Threshold tracking techniques in the study of human peripheral nerve. Muscle Nerve 1998;21:137.

[11] Kiernan MC, Burke D, Andersen KV, Bostock H. Multiple measures of axonal excitability: a new approach in clinical testing. Muscle Nerve 2000;23(3):399-409.

[12] Kiernan MC, Lin CS, Andersen KV, Murray NM, Bostock H. Clinical evaluation of excitability measures in sensory nerve. Muscle Nerve 2001;24(7):883-92.

[13] Boland RA, Lin CS, Engel S, Kiernan MC. Adaptation of motor function after spinal cord injury: novel insights into spinal shock. Brain 2011;134(Pt 2):495-505.

[14] Liechti M, Müller R, Lam T, Curt A. Vestibulospinal responses in motor incomplete spinal cord injury. Clin Neurophysiol 2008;119(12):2804-12.

[15] Iles JF, Ali AS, Savic G. Vestibular-evoked muscle responses in patients with spinal cord injury. Brain 2004;127(Pt 7):1584-92.

[16] Curt A, Weinhardt C, Dietz V. Significance of sympathetic skin response in the assessment of autonomic failure in patients with spinal cord injury. J Auton Nerv Syst 1996; 61:175-80.

[17] Maselli RA, Jaspan JB, Soliven BC, Green AJ, Spire JP, Arnason BG. Comparison of sympathetic skin response with quantitative sudomotor axon reflex test in diabetic neuropathy. Muscle Nerve 1989;12(5):420-3.

[18] Podnar S. Neurophysiologic studies of the penilo-cavernosus reflex: normative data. Neurourol Urodyn 2007;26(6):864-9.

[19] Barker AT, Jalinous R, Freeston IL. Non-invasive stimulation of the human motor cortex. Lancet 1985;1(8437):1106-7.

[20] Shields CB, Ping Zhang Y, Shields LB, Burke DA, Glassman SD. Objective assessment of cervical spinal cord injury levels by transcranial magnetic motor-evoked potentials. Surg Neurol 2006;66(5):475-83. discussion 483.

[21] Mills KR. Magnetic stimulation of the human nervous system. Oxford: Oxford University Press; 1999.

[22] Magistris MR, Rösler KM, Truffert A, Landis T, Hess CW. A clinical study of motor evoked potentials using a triple stimulation technique. Brain 1999;122:265-79.

[23] Hess CW, Mills KR, Murray NM. Responses in small hand muscles from magnetic stimulation of the human brain. J Physiol 1987;388:397-419.

[24] Magistris MR, Rösler KM, Truffert A, Myers JP. Transcranial stimulation excites virtually all motor neurons supplying the target muscle. A demonstration and a method improving the study of motor evoked potentials. Brain 1998;121:437-50.

[25] Magistris MR, Rösler KM. The triple stimulation technique to study corticospinal conduction. Suppl Clin Neurophysiol 2003;56:24-32. 
[26] Walsh P, Kane N, Butler S. The clinical role of evoked potentials. J Neurol Neurosurg Psychiatry 2005;76(Suppl. 2):ii16-22.

[27] Cruccu G, Aminoff MJ, Curio G, Guerit JM, Kakigi R, Mauguiere F, Rossini PM, Treede RD, Garcia-Larrea L. Recommendations for the clinical use of somatosensory-evoked potentials. Clin Neurophysiol 2008;119(8):1705-19.

[28] Chiappa KH. Evoked potential in clinical medicine. 3rd ed. Lippincott Williams; 1997.

[29] Rabai F, Sessions R, Seubert CN. Neurophysiological monitoring and spinal cord integrity. Best Pract Res Clin Anaesthesiol 2016;30(1):53-68.

[30] Aminoff MJ, Eisen AA. AAEM minimonograph 19: somatosensory evoked potentials. Muscle Nerve 1998;21(3):277-90.

[31] Dikmen PY, Oge AE. Diagnostic use of dermatomal somatosensory-evoked potentials in spinal disorders: case series. J Spinal Cord Med 2013;36(6):672-8.

[32] Tankisi H, Pugdahl K, Rasmussen MM, Clemmensen D, Rawashdeh YF, Christensen P, Krogh K, Fuglsang-Frederiksen A. Pelvic floor electrophysiology in spinal cord injury. Clin Neurophysiol 2016;127(5):2319-24.

[33] Chen AC, Niddam DM, Arendt-Nielsen L. Contact heat evoked potentials as a valid means to study nociceptive pathways in human subjects. Neurosci Lett 2001;316(2): $79-82$.

[34] Shy ME, Frohman EM, So YT, Arezzo JC, Cornblath DR, Giuliani MJ, Kincaid JC, Ochoa JL, Parry GJ, Weimer LH. Therapeutics and technology assessment subcommittee of the American Academy of Neurology. Quantitative sensory testing: report of the therapeutics and technology assessment subcommittee of the American Academy of Neurology. Neurology 2003;60(6):898-904.

[35] Leppanen RE. Intraoperative monitoring of segmental spinal nerve root function with free-run and electrically-triggered electromyography and spinal cord function with reflexes and F-responses. J Clin Monit Comput 2005;19:437-61.

[36] Wilbourn AJ, Aminoff MJ. AAEM minimonograph 32: the electrodiagnostic examination in patients with radiculopathies. American Association of Electrodiagnostic Medicine. Muscle Nerve 1998;21(12):1612-31.

[37] Parry GJ. Electrodiagnostic studies in the evaluation of peripheral nerve and brachial plexus injuries. Neurol Trauma 1992;4:921-34.

[38] Hubli M, Bolliger M, Dietz V. Neuronal dysfunction in chronic spinal cord injury. Spinal Cord 2011;49(5):582-7.

[39] Lin CS, Macefield VG, Elam M, Wallin BG, Engel S, Kiernan MC. Axonal changes in spinal cord injured patients distal to the site of injury. Brain 2007;130(Pt 4):985-94.

[40] Leis AA, Kronenberg MF, Stětkárová I, Paske WC, Stokić DS. Spinal motoneuron excitability after acute spinal cord injury in humans. Neurology 1996;47(1):231-7.

[41] Curt A, Keck ME, Dietz V. Clinical value of F-wave recordings in traumatic cervical spinal cord injury. Electroencephalogr Clin Neurophysiol 1997;105:189-93.

[42] Kim Y, Aoki T, Ito H. Evaluation of parameters of serially monitored F-wave in acute cervical spinal cord injury. J Nippon Med Sch 2007;74(2):106-13.

[43] Wolpaw JR. Spinal cord plasticity in acquisition and maintenance of motor skills. Acta Physiol 2007;189(2):155-69.

[44] Little JW, Halar EM. H-reflex changes following spinal cord injury. Arch Phys Med Rehabil 1985;66:19-22.

[45] Ashby P, Verrier M, Lightfoot E. Segmental reflex pathway in spinal shock and spinal spasticity in man. J Neurol Neurosurg Psychiatry 1974;37:1352-60. 
[46] Lucas MG, Thomas DG. Lack of relationship on conus reflexes to bladder function after spinal cord injury. Br J Urol 1989;63:24-7.

[47] Nitsche B, Perschak H, Curt A, Dietz V. Loss of circadian blood pressure variability in complete tetraplegia. J Hum Hypertens 1996;10:311-7.

[48] Sloan TB, Heyer EJ. Anesthesia for intraoperative neurophysiologic monitoring of the spinal cord. J Clin Neurophysiol 2002;19:430-43.

[49] Deiner S. Highlights of anesthetic considerations for intraoperative neuromonitoring. Semin CardioThorac Vasc Anesth 2010;14:51-3.

[50] Meyer B, Zentner J. Do motor evoked potentials allow quantitative assessment of motor function in patients with spinal cord lesions? Eur Arch Psychiatry Clin Neurosci 1992; 241:201-4.

[51] Clarke CE, Modarres-Sadeghi H, Twomey JA, Burt AA. Prognostic value of cortical stimulation in spinal cord injury. Paraplegia 1994;32:554-60.

[52] Tavy DL, Wagner GL, Keunen RW, Wattendorff AR, Hekster RE, Franssen H. Transcranial magnetic stimulation in patients with cervical spondylotic myelopathy: clinical and radiological correlations. Muscle Nerve 1994;17(2):235-41.

[53] Kalupahana NS, Weerasinghe VS, Dangahadeniya U, Senanayake N. Abnormal parameters of magnetically motorevoked potentials in patients with cervical spondylotic myeloapthy. Spine J 2008;8:645-9.

[54] Lissens MA, Vanderstraeten GG. Motor evoked potentials of the respiratory muscles in tetraplegic patients. Spinal Cord 1996;34:673-8.

[55] Curt A, Keck ME, Dietz V. Functional outcome following spinal cord injury: significance of motor evoked potentials and ASIA scores. Arch Phys Med Rehabil 1998;79: $81-6$.

[56] Spiess M, Schubert M, Kliesch U, EM-SCI Study group, Halder P. Evolution of tibial SSEP after traumatic spinal cord injury: baseline for clinical trials. Clin Neurophysiol 2008;119(5):1051-61.

[57] Curt A, Dietz V. Electrophysiological recordings in patients with spinal cord injury: significance for predicting outcome. Spinal Cord 1999;37:157-65.

[58] Taylor S, Ashby P, Verrie M. Neurophysiological changes following traumatic spinal lesions in man. J Neurol Neurosurg Psychiatry 1984;47:1102-8.

[59] Hirayama T, et al. Clinical assessment of the prognosis and severity of spinal cord injury using corticospinal motor evoked potentials. In: Shimoji K, Kurokawa T, Tamaki T, Willis WD, editors. Spinal cord monitoring and electrodiagnosis. Heidelberg: Springer; 1991. p. 503-10.

[60] Kramer JK, Taylor P, Steeves JD, Curt A. Dermatomal somatosensory evoked potentials and electrical perception thresholds during recovery from cervical spinal cord injury. Neurorehabilitation Neural Repair 2010;24(4):309-17.

[61] Kramer JL, Moss AJ, Taylor P, Curt A. Assessment of posterior spinal cord function with electrical perception threshold in spinal cord injury. J Neurotrauma 2008;25(8): 1019-26.

[62] Savic G, Bergström EM, Davey NJ, Ellaway PH, Frankel HL, Jamous A, Nicotra A. Quantitative sensory tests (perceptual thresholds) in patients with spinal cord injury. J Rehabil Res Dev 2007;44(1):77-82.

[63] Kumru H, Soler D, Vidal J, Tormos JM, Pascual-Leone A, Valls-Sole J. Evoked potentials and quantitative thermal testing in spinal cord injury patients with chronic neuropathic pain. Clin Neurophysiol 2012;123(3):598-604. 
[64] Valeriani M, Pazzaglia C, Cruccu G, Truini A. Clinical usefulness of laser evoked potentials. Neurophysiol Clin 2012;42(5):345-53.

[65] Curt A, Dietz V. Nerve conduction study in cervical spinal cord injury: significance for hand function. Neurol Rehabil 1996;7:165-73.

[66] Curt A, Dietz V. Traumatic cervical spinal cord injury: relation between somatosensory evoked potentials, neurological deficit and hand function. Arch Phys Med Rehabil 1996;77:48-53. 
Non-Print Items

\begin{abstract}
Clinical sequelae of spinal cord injury (SCI) are related both to the spinal cord lesion level and its severity, which is essentially based on a combination of clinical signs and symptoms suggesting the involvement of the autonomic nervous system, spinal cord motor or sensory pathways, and the gray matter, specifically lower motor neuron. A higher diagnosis accuracy and an earlier prediction of functional outcome can be significantly increased when combining clinical examination, neuroradiological exams, and neurophysiology. In this chapter, we review methods and usefulness of neurophysiological techniques most frequently used in SCI diagnosis and management: some of these techniques-nerve conduction studies, F-wave method, and electromyography - are able to investigate peripheral nervous system lesions, often associated to SCI; other neurophysiological techniques-motor evoked potentials and sensory evoked potentials-are mostly used to explore motor or sensory spinal cord pathways. Furthermore, neurophysiological techniques assess the degree of white or gray matter injury involvement, closely related to SCI prognosis. Electromyographic denervation or reinnervation signs and motor or sensory nervous potential amplitude reduction are typical finds of damage of lower motor neuron, radiculopathy, or nerve impairment. Absence or delay of motor evoked potential responses after transcranial magnetic stimulation of motor cortical area suggests corticospinal tract lesion. Absence or delay of expected spinal or cortical components of sensory evoked potentials after electrical or laser peripheral stimulation is related to dorsal column or spinothalamic tract lesion. Moreover, sympathetic skin response, bulbocavernosus reflex, and $\mathrm{H}$ reflex methods explore autonomic nervous system pathway and spinal cord reflex, frequently involved in SCI. Applying all these neurophysiological methods at various body sites makes it possible to precisely locate the level of functional damage after SCI.

In conclusion, neurophysiological methods, allowing a precise localization and quantification of neurological damage after SCI, are essential for early counseling and rehabilitation orientation, and their outcome prognostication is crucial to achieve meaningful stratification when conceiving clinical trials.
\end{abstract}

\title{
Keywords:
}

Evoked potentials, Functional outcome, Electromyography, Magnetic stimulation, Nerve conduction, Neurophysiological techniques, Spinal cord injury. 


\section{CHAPTER}

\section{c0003 Conventional treatments and surgical margins of maneuvering for spinal cord injury management}

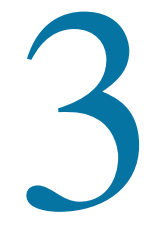

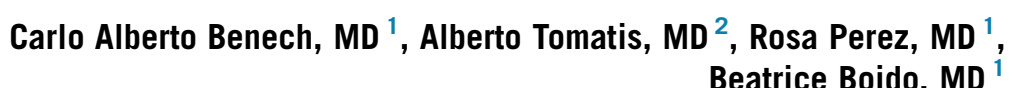

${ }^{1}$ Department of Neurosurgery, Clinica Fornaca di Sessant, Turin, Italy; ${ }^{2}$ Neurosurgical Unit,

Ospedale Spirito Santo, Pescara, Italy

p0010 Spinal cord injury (SCI) is a pathological event that results in disturbances to normal sensory, motor, or autonomic function. This devastating injury significantly compromises a patient's quality of life, functional status, and social independence. Moreover, in the United States, comorbidity cost due to SCI is estimated $\$ 9.7$ billion each year. Generally, costs in the first year were $\$ 121,600$ per person with a complete SCI, and $\$ 42,100$ per person with an incomplete injury. In the subsequent 5 years, annual costs were $\$ 5400$ and $\$ 2800$ for persons with complete and incomplete SCIs, respectively.

p0015 Usually, SCI results from traumatic causes: car accidents, falls, sports injuries, gunshots, etc. (Table 3.1). However, it can also result from nontraumatic causes such as tumors, arteriovenous malformations (AVM) bleeding, ischemia, herniated disc, or infection.

p0020 Two clinical presentations can be recognized: 1. Acute spinal cord injury: sudden onset damage or trauma to the spinal cord resulting in loss of tissue integrity, which can lead to impaired function, reduced mobility, or sensory dysfunction. 2. Chronic spinal cord injury: persistence of paralysis for $\geq 12$ months following injury.

p0025 The incidence and prevalence of acute SCI have been estimated at both national and regional levels in countries worldwide. Sekhon and Fehlings reported an annual global incidence of acute SCI of $14-40$ per million. There is a high male-to-female ratio of patient suffering from SCI and an age of peak incidence of younger than 30 years.

\section{s0010 Classification: Complete and Incomplete injuries}

p0030 The spinal cord is injured when there is damage to the functional unit of spine (vertebral bodies, discs, anterior and posterior longitudinal ligaments, and other). The severity of an injury depends on the part of the spinal cord that is affected. The

Spinal Cord Injury (SCI) Repair Strategies. https://doi.org/10.1016/B978-0-08-102807-0.00003-X Copyright $\odot 2020$ Elsevier Ltd. All rights reserved. 
t0010 Table 3.1 T-SCI trend grouped etiology by age-2017 Annual Statistical Report of National Spinal Cord Injury Statistical Center-University of Alabama at Birmingham.

\begin{tabular}{l|l|l|l|l|l|l|l}
\multicolumn{7}{l}{ Age at injury } \\
$\begin{array}{l}\text { Etiology } \\
\boldsymbol{n}(\mathbf{\%})\end{array}$ & $\mathbf{1 5}$ & $\mathbf{1 6 - 3 0}$ & $\mathbf{3 1 - 4 5}$ & $\mathbf{4 6 - 6 0}$ & $\mathbf{6 1 - 7 5}$ & $\mathbf{2 7 6}$ & Total \\
Vehicular & 362 & 7261 & 3332 & 1927 & 755 & 159 & 13,796 \\
& $(36.9)$ & $(46.3)$ & $(44.5)$ & $(37.7)$ & $(27.9)$ & $(20.5)$ & \\
Violence & 226 & 3735 & 1229 & 330 & $63(2.3)$ & $8(1.0)$ & 5,591 \\
& $(23.1)$ & $(23.8)$ & $(16.4)$ & $(6.5)$ & & & \\
Sports & 236 & 2224 & 544 & 232 & $73(2.7)$ & $7(0.9)$ & 3,316 \\
& $(24.1)$ & $(14.2)$ & $(7.3)$ & $(4.5)$ & & & \\
Falls & 78 & 1671 & 1717 & 1960 & 1404 & 509 & 7,339 \\
& $(8.0)$ & $(10.7)$ & $(22.9)$ & $(38.4)$ & $(51.9)$ & $(65.7)$ & \\
Medical/ & 27 & 107 & 122 & 315 & 298 & 62 & 931 \\
Surgery & $(2.8)$ & $(0.7)$ & $(1.6)$ & $(6.2)$ & $(11.0)$ & $(8.0)$ & \\
Other & 51 & 659 & 536 & 330 & 104 & 22 & 1,702 \\
& $(5.2)$ & $(4.2)$ & $(7.2)$ & $(6.5)$ & $(3.8)$ & $(2.8)$ & \\
Unknown & $0(0.0)$ & $16(0.1)$ & $11(0.1)$ & $11(0.2)$ & $6(0.2)$ & $8(1.0)$ & 52 \\
Total & 980 & 15,673 & 7,491 & 5,105 & 2,703 & 775 & 32,727 \\
\hline
\end{tabular}

higher is the SCI, the more it effect motor, sensory and autonomic function. Tetraplegia (a.k.a. quadriplegia) results from injuries to the cervical spinal cord region. Paraplegia is due to injuries interesting the spinal cord in the thoracic or lumbar areas.

p0035 Complete spinal cord lesion: no preservation of any motor and/or sensory function more than three segments below the level of the injury in the absence of spinal shock. About 3\% of patients with complete injuries on initial exam will develop some recovery within $24 \mathrm{~h}$. Recovery is essentially zero if the spinal cord injury remains complete beyond $72 \mathrm{~h}$ (Fig. 3.1).

p0040 SPINAL SHOCK: This term is often used in two completely different senses:

o0010 1. hypotension (shock) that follows spinal cord injury (SBP usually $\approx 80 \mathrm{~mm} \mathrm{Hg}$ ). Caused by multiple factors: (A) Interruption of sympathetics implies spinal cord injury above T1 (loss of vascular tone below level of injury; leaves parasympathetics relatively unopposed causing bradycardia). (B) Loss of muscle tone due to skeletal muscle paralysis below the level of injury results in venous pooling and thus a relative hypovolemia. (C) Blood loss from associated wounds.

o0015 2. transient loss of all neurologic function (including segmental and polysynaptic reflex activity and autonomic function) below the level of the SCI3,4 (flaccid paralysis and areflexia). 
f0010

FIGURE 3.1

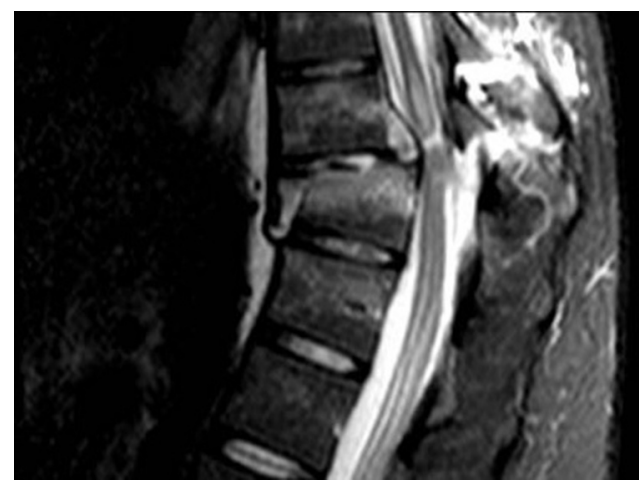

Example of complete spinal cord lesion (spinal transection).

p0055 This condition may abate in as little as $72 \mathrm{~h}$, but typically persists $1-2$ weeks, occasionally several months; it is accompanied by loss of the bulbocavernosus reflex; spinal cord reflexes immediately above the injury may also be depressed based on the Schiff-Sherrington phenomenon. When spinal shock resolves, there will be spasticity below the level of the lesion and return of the bulbocavernosus reflex. Anyway, it is a poor prognostic sign.

p0060 Incomplete spinal cord lesion: any residual motor or sensory function more than three segments below the level of the injury. Look for signs of preserved long-tract function and remember that an injury does not qualify as incomplete with preserved sacral reflexes alone (e.g., Bulbocavernosus).

p0065 Types of incomplete lesion (Fig. 3.2):

00020 1. Central cord syndrome (the most common pattern): The centermost region of spinal cord is a vascular watershed zone that renders it more susceptible to injury from edema. Long tract fibers passing through the cervical spinal cord are somatotopically organized such that cervical fibers are located more medially than the fibers serving the lower extremities. The clinical presentation is characterized by weakness of upper extremities with lesser effect on lower extremities; varying degrees of sensorial disturbance below level of lesion; and sphincter dysfunction. There is often an initial phase of improvement followed by a plateau phase and the late deterioration. Recovery is usually incomplete, and the amount of recovery is related to the severity of the injury and patient age. In this condition, surgery is often employed for ongoing compression, usually on a nonemergency basis except for rare cases of progressive deterioration.

o0025 2. Brown-Séquard syndrome: Spinal cord hemisection. Usually, as a result of penetrating trauma, it is seen in $2 \%-4 \%$ of traumatic spinal cord injuries. In addition, it may occur with radiation myelopathy, cord compression by spinal 


\section{4}

f0015 FIGURE 3.2
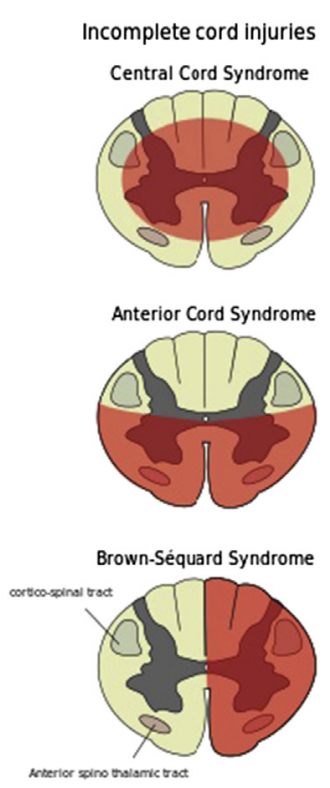

Anatomical illustration of incomplete spinal cord lesion.

epidural hematoma, large cervical disc herniation, spinal cord tumors, spinal AVMs, cervical spondylosis, and spinal cord herniation. Classical findings: 1. Ipsilateral findings: (A) motor paralysis below lesion; (B) loss of posterior column functions. 2. Contralateral findings: (A) loss of pain and temperature sensation inferior to lesion beginning 1-2 segments below; (B) preserved light touch due to redundant ipsilateral and contralateral paths. This syndrome has the best prognosis of any of the incomplete spinal cord injuries. About $90 \%$ of patients with this condition will regain the ability to ambulate independently as well as anal and urinary sphincter control.

o0030 3. Anterior cord syndrome: also known as anterior spinal artery syndrome results as infarction in the territory supplied by anterior spinal artery. It may result from occlusion of the anterior spinal artery, or from anterior cord compression (dislocated bone fragment, or by herniated disc). Clinical findings are paraplegia or quadriplegia, dissociated sensory loss below lesion (loss of pain and temperature with preservation of posterior column function). This condition has the worst prognosis of the incomplete injuries. Only 10\%-20\% recover functional motor control.

00035 4. Posterior cord syndrome (rare): Produces pain and paresthesia (often with a burning quality) in the neck, upper arms, and torso. Long tract findings are minimal. 


\section{s0020 Management of spinal cord injury s0025 Introduction}

p0090 The management of patients with SCI has drastically evolved over the past century as results of increasing knowledge on injury mechanisms (primary vs. secondary SCI injury), disease pathophysiology, and the role of surgery. Historically, the cornerstones of therapy for the acute spinal cord injury (ASCI) rested on principles of closed reduction, with prolonged courses of external immobilization, with little priority given to acute surgical care. Today, the advancements in rigid segmental fixation techniques and the acknowledgment on SCI pathophysiology changed the approach.

\section{s0030 Hospital management}

p0095 The major causes of death in SCI are aspiration and shock. Initial survey under ATLS protocol: assessment of airway takes precedence, then breathing, then circulation and control of hemorrhage (“ABC's"). NB: other injuries (e.g., abdominal injuries) may be masked below the level of SCI.

p0100 Any of the following patients should be treated as having an SCI until proven otherwise: (1) all victims of significant trauma; (2) trauma patients with loss of consciousness; (3) minor trauma victims with complaints referable to the spine (neck or back pain or tenderness) or spinal cord (numbness or tingling in an extremity, weakness, paralysis); (4) associated findings suggestive of SCI include abdominal breathing and priapism (autonomic dysfunction). This is followed by a brief neurologic exam. Clinical assessment will be evaluated with the American Spinal Injury Association (ASIA) scoring scale (Fig. 3.3), international standard for neurological and functional assessment of spinal cord injury.

\section{s0035 Radiological evaluation}

p0105 After neurological evaluation, if SCI is suspected radiological evaluation is needed. Imaging of the spine is an essential part of the initial management of acute SCI. The radiological diagnosis of SCI usually began with X-rays, but in some patients spine CT scan could be the first exam. CT scan is gold standard to evaluate bony anatomy, it is more accurate to see any type of fractures and allowed to determinate stability or instability of the spine column. However, these imaging modalities cannot visualize the spinal cord or the surrounding soft tissues to the same degree as MRI. Furthermore, some of the injury to the cord occur without any major radiological damage to the spinal column, described as spinal cord injury without radiological abnormality (SCIWORA). The potential benefits of MRI in the setting of acute SCI are that this type of imaging can identify ongoing spinal cord compression; shows soft tissue structures that are responsible for compression, including disc herniation, epidural hematoma, intramedullary hematoma, and preexisting canal stenosis; detects ligamentous instability at the level of the injury or at other spinal levels; and identifies 

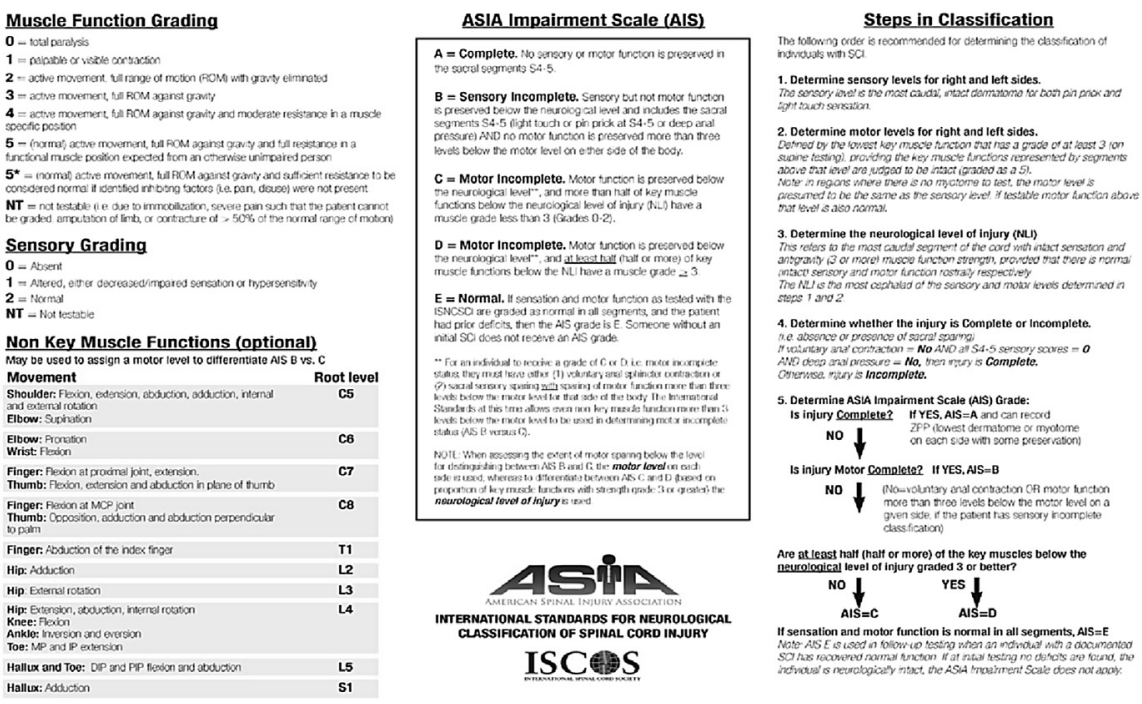

f0020

FIGURE 3.3

Description of ASIA impairment scale.

vertebral artery injury. Unfortunately, the evidence to support the role of MRI in clinical decision-making was largely indirect; specifically, MRI may help diagnose certain clinical entities that, if present, may influence management decisions, such as ongoing cord compression, disc herniation, and ligamentous injury. Moreover, the utility of MRI in prognostication is also becoming more apparent as validated prediction scores continue to be published.

\section{s0040 Steroids in SCI management}

p0110 Methylprednisolone sodium succinate (MPSS) is a corticosteroid that has been used for many diseases due to its potent antiinflammatory actions. Early preclinical studies demonstrated that glucocorticoids can have profound beneficial effects on the injured spinal cord. Several randomized controlled trials have investigated the potential neuroprotective efficacy and safety of MPSS in patients with acute SCI. The National Acute Spinal Cord Injury Study (NASCIS II) supported a small but clinically important improvement in neurological function for patients who received a $24-\mathrm{h}$ infusion of this drug within $8 \mathrm{~h}$ of injury. In the last decade, this article has been the target of intense criticism, especially with concerns at the potential for harmful and serious adverse events. Based on the current body of the literature, the last AOSpine guidelines suggest $24 \mathrm{~h}$ of IV MPSS be considered within $8 \mathrm{~h}$ of injury for patients without significant medical contraindication. 


\section{s0045 Anticoagulation prophylaxis}

p0115 Patients with SCI are at an increased risk of deep venous thrombosis (DVT) due to neurological dysfunction, immobilization, intimal injury, and hypercoagulability. Moreover, patients often underwent surgery and may be at risk of hemorrhage or bleeding around neural tissues. The prevention of DVT and pulmonary embolism with use of anticoagulation is critical in this high-risk population. So, prophylactic treatment in these patients is also associated with significant risks, including symptomatic hematoma formation, enlargement of spinal cord contusion, worsening of neurological deficits, bleeding, and mortality. The American Association of Neurological Surgeons (AANS)/Congress of Neurological Surgeons (CNS) published guideline for prevention on thromboembolic disease as follows: (1) prophylactic treatment in SCI patients with severe motor deficits; (2) use of low-molecular-weight heparins, rotating beds, or combination of modalities; (3) use of low-dose heparin in combination with pneumatic compression stockings or electrical stimulation; (4) early administration of VTE prophylaxis; (5) 3-month duration of treatment. In addition, it is strongly recommended to not use low-dose heparin therapy or oral anticoagulation alone, and the routine use of vena cava filters is not recommended.

\section{s0050 Blood pressure augmentation}

p0120 Vascular injury and localized edema contribute to ongoing ischemia in the perilesional region. Blood pressure augmentation has emerged as a viable strategy to neuro-protect at-risk tissue by enhancing perfusion. Current AANS/CNS guidelines recommend maintenance of mean arterial pressure (MAP) $\geq 85-90 \mathrm{~mm} \mathrm{Hg}$ for 7 days postinjury as this has been found to enhance long-term AIS grade outcomes patients.

\section{s0055 Surgical options and timing}

p0125 Over the past few decades, clinicians within the field have prioritized the early surgical management of SCI patients. Clinical evidence suggests that persistent compression of the spinal cord after the primary injury represents a reversible form of secondary injury, which, if ameliorated in an expeditious fashion, may lead to reduced neural injury and improved outcomes. This is the reason why: "Spine is time."

p0130 Although the optimal timing remains controversial, decompression of the spinal cord, stabilization of the vertebra, and maintenance of blood perfusion are well established as critical factors in generating optimal outcomes in traumatic SCI.

p0135 The Spinal Trauma Study Group identified the first $24 \mathrm{~h}$ as the most promising time window during which decompression may afford neuroprotection. With respect to clinical evidence on this topic, several comparative cohort studies have been published investigating the clinical impact of performing decompressive surgery before several thresholds. The Surgical Treatment of Acute Spinal Cord Injury Study 
(STASCIS) investigates the efficacy of early decompression before a 24-h threshold. Patients receiving early decompression experienced 2.8 times greater odds of experiencing an ASIA Impairment Scale (AIS) improvement of at least 2 grades at 6 months as compared with patients who underwent late decompression ( $\geq 24 \mathrm{~h}$ after SCI). A prospective Canadian cohort study confirmed the findings observed in STASCIS. Furthermore, an observational Canadian cohort study showed that AIS A (complete injury) and AIS B (complete motor injury with incomplete sensory injury) patients who received early decompression had shorter length of hospital stay, while AIS B, C, and D incomplete injury patients decompressed earlier demonstrated an additional 6.3 points in motor recovery as compared to those decompressed later.

p0140 Absolute indications for surgery in acute SCI patients:

00040 1. Progressive neurological deficit in the presence of cord compression.

o0045 2. Instability of spinal column.

p0155 Contraindications to emergent operation:

00050 1. Complete spinal cord injury $\geq 24 \mathrm{~h}$ (no motor or sensory function below level of lesion).

o0055 2. Medically unstable patient.

p0170 Depending on the type of injury causing SCI and level of the injury, surgery can be performed with the following:

00060 1. Anterior or posterior approach

00065 2. Traditional open or minimally invasive surgery

00070 3. With spinal stabilization or without.

p0190 The most rapid procedure to decompress the cord is often a multilevel laminectomy. Laminectomy consists of removal of vertebral lamina and spinous processes to covert the spinal canal from a closed structure to an open one. This is frequently accompanied by dorsal migration of the spinal cord that may be seen on MRI. With myelopathy, fused patients are better than those that are just decompressed without fusion. Fusion may be accomplished posteriorly (e.g., with lateral mass screws and rods) at the time of decompression, or anteriorly (e.g., multilevel discectomy, or corpectomy with strut graft and anterior cervical plating) at the same sitting as the laminectomy or staged later.

p0195 Many fusions procedures can be performed: anterior arthrodesis such as anterior cervical discectomy and fusion with implants of a graft (e.g., PEEK, cadaver bone, titanium cage) and optional anterior plate; posterior arthrodesis such as posterior lumbar interbody fusion characterized by a bilateral laminectomy and aggressive discectomy followed by the placement of a graft in into the decorticated disc space and supplemented with pedicle screws and rods; lateral lumbar interbody fusion-a minimally invasive lateral retroperitoneal transpsoas approach. Screws positioning is performed under X-ray guide or sometimes also with neuronavigation (e.g., O-arm). 
p0200 Talking about spine stability, it is easy to understand that patients who underwent surgical decompression + stabilization will be able to take part on rehabilitation programs earlier and with minor risk than patients only treated with decompression. Spinal instability can be the "primum movens" of further SCI. Rehabilitation commences once a patient is stable and focuses on preventing secondary complications and optimizing function with compensatory techniques.

\section{Further reading}

1. White AA, Panjabi MM. The problem of clinical instability in the human spine: a systematic approach. In: Clinical Biomechanics of the spine. 2nd ed. Philadelphia: J.B. Lippincott; 1990. p. 277-378.

2. Waters RL, Adkins RH, Yakura J, Sie I. Profiles of spinal cord injury and recovery after gunshot injury. Clin Orth Relat Res 1991;267:14-21.

3. Atkinson PP, Atkinson JLD. Spinal shock. Mayo Clin Proc 1996;71:384-9.

4. Chesnut RM, Narayan RK, Wilberger JE, Povlishock JT. Emergency management of spinal cord injury. Neurotrauma. New York: McGraw-Hill; 1996. p. 1121-38.

5. American Spinal Injury Association. International standards for neurological classification of spinal cord injury, Revised 2000. 6th ed. Chicago, IL: American Spinal Injury Association; 2000.

6. Ditunno Jr JF. New spinal cord injury standards, 1992. Paraplegia 1992;30:90-1.

7. Lucas JT, Ducker TB. Motor classification of spinal cord injuries with mobility, morbidity and recovery indices. Am Surg 1979;45:151-8.

8. Schneider RC, Cherry G, Pantek H. The syndrome of acute central cervical spinal cord injury. J Neurosurg 1954;11:546-77.

9. Levi L, Wolf A, Mirvis S, Rigamonti D, et al. The significance of dorsal migration of the cord after extensive cervical laminectomy for patients with traumatic central cord syndrome. J Spinal Disord 1995;8:289-95.

10. Meguro K, Tator $\mathrm{CH}$. Effect of multiple trauma on mortality and neurological recovery after spinal cord or cauda equina injury. Neurol Med Chir 1988;28:34-41.

11. Bracken MB, Shepard MJ, Holford TR, et al. Administration of methylprednisolone for 24 or 48 hours or tirilazad mesylate for 48 hours in the treatment of acute spinal cord injury. J Am Med Assoc 1997;277:1597-604.

12. Section on disorders of the spine and peripheral nerves of the American association of neurological Surgeons and the congress of neurological surgeons. Radiographic assessment of the cervical spine in symptomatic trauma patients. Neurosurgery 2002; 50(Supplement):S36-43.

13. Walter J, Doris P, Sha er M. Clinical presentation of patients with acute cervical spine injury. Ann Emerg Med 1984;13:512-5.

14. Dhall SS, Hadley MN, Aarabi B, Gelb DE, Hurlbert RJ, Rozzelle CJ, Ryken TC, Theodore N, Walters BC. Deep venous thrombosis and thromboembolism in patients with cervical spinal cord injuries. Neurosurgery 2013;72(Suppl. 2):244-54.

15. Schuster R, Waxman K, Sanchez B, Becerra S, Chung R, Conner S, Jones T. Magnetic resonance imaging is not needed to clear cervical spines in blunt trauma patients with normal computed tomographic results and no motor deficits. Arch Surg 2005;140: $762-6$. 


\section{0}

16. Benzel EC, Hart BL, Ball PA, Baldwin NG, Orrison WW, Espinosa MC. Magnetic resonance imaging for the evaluation of patients with occult cervical spine injury. J Neurosurg 1996;85:824-9.

17. Wagner FC, Chehrazi B. Early decompression and neurological outcome in acute cervical spinal cord injuries. J Neurosurg 1982;56:699-705.

18. Ahuja CS, Nori S, Tetreault L, Wilson J, Know B, Harrop J, Choi D, Fehlings MG. Traumatic spinal cord injury- repair and regeneration. Neurosurgery 2017;80(Suppl. ment): S9-22.

19. Rath N, Balain B. Spinal cord injury- the role of surgical treatment for neurological improvement. J Clin Orthop Trauma 2017;8:99-102.

20. Fehlings MG, Tetreault L, Wilson J, Know B, Burns AS, Martin AR, Hawryluk G, Harrop J. A clinical practice guideline for management of acute spinal cord injury: introduction, rationale, and scope. Glob Spine J 2017;7(3S):84-94.

21. Lee BB, Cripps RA, Fitzharris M, Wing PC. The global map for traumatic spinal cord injury epidemiology: update 2011, global incidence rate. Spinal Cord 2014;52:110-6. 


\title{
Non-Print Items
}

\begin{abstract}
Spinal cord injury (SCI) is a pathological event that results in disturbances to normal sensory, motor, or autonomic function. The severity of an injury depends on the part of the spinal cord that is affected. This devastating injury significantly compromises a patient's quality of life, functional status, and social independence. The aim of the neurosurgeon is to guarantee the best outcome for these affected patients. So, it is important to know the correct management, medical treatment, surgical indications and options for spinal cord injury.

Revision of the literature on treatments and surgical indications for spinal cord injury to perform an updated and schematized representation about actual SCI management.
\end{abstract}

\section{Keyword:}

Spinal cord injury, Medical treatment, Surgical treatment, Timing, Neuroprotection. 


\section{CHAPTER}

\section{c0004 MRI as an imaging tool for in vivo noninvasive morphological and (partially) functional examination of injured spinal cord}

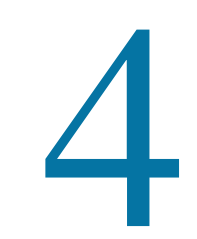

Fano Molinaro, MD ${ }^{1}$, Pier Luigi La Zazzera, MD ${ }^{1}$, Marilena Ferraris, MD ${ }^{2}$, Giulia Morbidoni, MSE ${ }^{3}$, Domenico Zaca', MSNS, $\mathrm{PhD}^{3}$, Andrea De Rinaldis, MSE, $\mathrm{PhD}^{3}$, Fernando Carpanese, $\mathrm{MSE}^{3}$, Armando Cioffi, $\mathrm{MSE}^{3}$, Francesco Naddeo, $\mathrm{MSE}^{3}$, Leonardo Boccaccini, MSE ${ }^{3}$, Mauro Bergui, MD, PhD

${ }^{1}$ Department of Neuroradiology, University of Turin, Torino, Italy; ${ }^{2}$ Neuroradiology Unit, Città della Salute Hospital, Torino, Italy; ${ }^{3}$ Siemens Healthcare, Milan, Italy

\section{s0010 Magnetic resonance image: key concepts}

p0010 Magnetic resonance imaging (MRI) is a medical application of nuclear magnetic resonance. Until the 1970s, MRI was used for chemical and physical analysis. With the advent of computed tomography (CT) (using computer techniques to develop images from MRI information) in 1973 and the advent of superconductors, the first human MRI examination was performed in 1977.

p0015 This imaging technique uses a static magnetic field (B0, usually $1.5 \mathrm{~T}$ ), which creates rotational motion of positively charged protons (spins), exposed to the external strong magnetic field in the bore of an MRI scanner. The static magnetization is then perturbed by a dynamic and short duration magnetic field (B1), which is defined as a radio frequency (RF) pulse, forcing the spins to tilt their magnetization M0 from the original longitudinal plane (Z-plane) to a transverse plane (XY-plane) with a relatively small force. When the magnetic resonance (MR) signal is tilted to the transverse plane, it takes different times (calculated in milliseconds, depending on tissue composition) to return to their initial longitudinal magnetization vector $(\mathrm{Mz})$ and/or to lose their transverse magnetization vector (Mxy). T1 time is defined as the time required for longitudinal MR magnetization $(\mathrm{Mz})$ to reach $63 \%$ of its original value. It is the time needed for the water protons to return to their initial state after the application of a $90^{\circ} \mathrm{RF}$ pulse. T1 relaxation strictly depends on the interaction of protons with their environment (spin-lattice interaction). T2 time is defined

Spinal Cord Injury (SCI) Repair Strategies. https://doi.org/10.1016/B978-0-08-102807-0.00004-1 Copyright $\odot 2020$ Elsevier Ltd. All rights reserved. 
as the time required for transverse MR magnetization (Mxy) to reach $37 \%$ of its initial value. It can be described as the time for spins to lose their due to their interactions among them (spin - spin interaction). Every tissue has its own T1 and T2 times. Because all protons in the region of interest are making almost the same precessional motion, to define their spatial location, it is necessary to analyze the measured MR signal frequency components. This frequency labeling is called spatial encoding and is done by applying a spatially changing magnetic field called gradients. The gradients are typically within $1-3 \%$ of the main static field and have a unit of milliTesla per meter $(\mathrm{mT} / \mathrm{m})$. Usually, three encoding gradients are used to define the protons' position properly.

p0020 To encode the gradient spatially in a two-dimensional plane (e.g., a single slice of MR image), it is necessary to combine at least two gradient encoding axes (frequency and phase encoding gradients) onto a row. If they are applied in a certain order, all protons in each row and column can be labeled accurately. Then, a mathematical transformation called Fourier transform is used to transform the frequencyencoded data from raw data domain or k-space to time domain or MR image domain. This process requires a certain time and computational power of electronics; nowadays MR images are reconstructed almost instantly at the end of acquisition.

\section{s0015 Magnetic resonance imaging: main sequences}

p0025 MR pulse sequences are performed to acquire MR images. For maximum simplification, the two main categories are spin echo (SE) based and gradient echo (GRE) based (depending on the type of echo created). The basic pulse sequence parameters are TR and TE, which stand for repetition time and echo time, respectively. They are typically measured in milliseconds. TE represents the time from the center of the RF pulse to the center of the echo. For pulse sequences with multiple echoes between each RF pulse, several echo times may be defined and are commonly noted TE1, TE2, TE3, and so on. TR is the length of time between corresponding consecutive points on a repeating series of pulses and echoes.

p0030 A typical SE pulse sequence applies a number of RF pulses and gradients in a certain order. Both $\mathrm{T} 1$ and $\mathrm{T} 2$ weighted images can be obtained. They provide overall good-quality image, optimal signal to noise ratio (SNR) and contrast to noise ratio. It is not sensitive to susceptibility artifacts. On the other hand, the acquisition time is long (12-20 min for T2 and 4-7 min for T1 contrast).

p0035 The other most commonly used sequences are the GRE family. The main difference between GRE and SE is the lack of the refocusing $180^{\circ} \mathrm{RF}$ pulse. They have increased sensitivity to blood flow and can be used for MR angiography, breath hold, and dynamic acquisitions. However, their SNR is usually lower than SE images, they produce noisier images, they are much more sensitive to field homogeneity and susceptibility effects, and they can only produce $\mathrm{T} 2 *$ contrast but not $\mathrm{T} 2$ contrast as in SE.

p0265 During the last years 3D high resolution T2 weighted protocols, either those employing the steady-state precession principle (with TEs shorter than T2 the transverse components of magnetization never fully dephase) as in CISS (constructive 
interference in steady state) or those based on fast-SE imaging as in SPACE (Sampling perfection with application optimized contrast evolution) have been increasingly used. These volumetric sequences allows an isotropic acquisition (no gap between slices) and consequently a reconstruction of orthogonal planes.

p0040 Inversion recovery is a technique employed in various sequence applications, using a conventional SE sequence preceded by a $180^{\circ}$ inverting pulse. The time between the $180^{\circ}$ inverting pulse and the second $90^{\circ}$ standard pulse is called the inversion time (TI). In fluid attenuation inversion recovery (FLAIR) sequences, the pulse width and RF TI are chosen so that the signal of fluids such as cerebrospinal fluid (CSF) is greatly attenuated. In this case, the TI (approximately $2500 \mathrm{~ms}$ to 1 $\mathrm{T}$ ) is far from the TI of cerebral parenchymal tissues; therefore, their signal will be affected to a negligible extent in the final images. The FLAIR sequence results in T2 images in which signals coming from the CSF and from other low-viscosity liquids are suppressed.

p0045 Diffusion-weighted imaging (DWI) sequences are based on echo planar imaging (EPI), which are fast GRE or SE sequences with alternate filling of k-space (zigzag) in one or a small number of excitations. The main difference is the presence of diffusion gradients. A first pulse sequence with diffusion gradients turned off generates a set of b0 (s/mm2) images that are T2-weighted and provide a baseline for calculated maps; then, diffusion gradients are turned on with different strengths and times (e.g., b500, b1000). Diffusion, also known as Brownian motion, is the random, microscopic movement of water and other small molecules caused by thermal collisions. Extracellular water diffuses more freely than intracellular water. Many tissues contain highly asymmetric structures, such as nerves. This leads to the concept of diffusion anisotropy, which is defined as the preferential diffusion of water molecules in certain directions more than others. Biological tissues are highly anisotropic: their diffusion rates are not the same in every direction. For routine DWI, this complexity is ignored by reducing diffusion to a single average value, the apparent diffusion coefficient (ADC).

p0270 DWI imaging is characterized by strong distortion artifact (related to the EPI read out) and a low resolution. Such artifacts are more evident in the sagittal plane and in regions with strong tissue inhomogeneities (bone/air/cartilage). A high resolution diffusion weighted imaging called RESOLVE [8] minimizes these problems, characterizing the diffusion properties of pathologies with higher spatial resolution, signal to noise ratio and lower EPI related artifacts.

\section{s0020 Advanced techniques: diffusion tensor imaging and functional magnetic resonance imaging \\ s0025 Diffusion tensor imaging}

p0050 A superior method to evaluate diffusion in complex materials is to use the diffusion tensor. This can be simplified with an example. A drop of ink in a glass of water expands over time in all directions without a preference; this kind of behavior is called isotropic diffusion, and a single number (ADC), which applies to all 
directions of space, is enough to define the average time frame of the phenomenon. When the molecules encounter obstacles, the diffusion becomes anisotropic, not equal in all directions, shaped as an ellipsoid. The images obtained by associating an ellipsoid with each point of space are called diffusion tensor imaging (DTI). One of the first and most interesting applications of DTI was to trace the axonal fibers of white matter (fiber tracking), deducing the trend based on the local estimated diffusivity. The mathematical tool that describes the distribution of diffusion in three dimensions is the diffusion tensor, which is nothing more than a $3 \times 3$ symmetric matrix (six independent parameters) corresponding to diffusion rates in each combination of directions. The three diagonal elements (Dxx, Dyy, and Dzz) represent diffusion coefficients measured along each of the principal ( $\mathrm{x}-, \mathrm{y}-$, and $\mathrm{z}-$ ) laboratory axes. The six off-diagonal terms (Dxy, Dyz, etc.) reflect the correlation of random motions between each pair of principal directions. The diffusion direction map assigns colors to voxels based on a combination of anisotropy and direction. It is also called the colored fractional anisotropy map, fiber direction map, or diffusion texture map. The first among them, the fiber assignment by continuous tracking, which is a deterministic method starting from a voxel of interest, identifies the direction of the fiber moving in the two adjacent voxels pointed by the main direction of the ellipsoid (seed voxels) and repeats the procedure in them. Automated software computes fiber trajectories in and out of that area. Advanced sequences can be combined with other acquisition strategies such as reduced field-of-view and parallel imaging to be used in spinal cord tractography.

\section{s0030 Functional magnetic resonance imaging}

p0055 Functional magnetic resonance imaging (fMRI) followed the technique of nuclear medicine (single photon emission CT and positron emission tomography) in the field of brain mapping techniques. fMRI does not use radioactive tracers and is characterized by better spatial and temporal resolution. However, tracers used in nuclear medicine (such as fluorodeoxyglucose) are direct markers of neuronal activity, whereas fMRI displays the change in hemoglobin status resulting from neuronal metabolism; it is therefore an indirect technique that exploits so-called neurovascular coupling, from which arises the contrast of a blood oxygenation level-dependent (BOLD) image [1]. Neuronal metabolism is based on aerobic glycolysis: neurons use glucose and oxygen to produce adenosine triphosphate and perform their cellular functions. Glucose reaches the brain with blood and reaches the neurons via specific transporters of the blood-brain barrier. After reaching the tissues, oxygen spreads freely in them, detaching from the sites of the hemoglobin molecule. The latter has a dual behavior within a magnetic field: it is diamagnetic (inert) when it is linked to oxygen and acquires paramagnetic properties when it is deoxygenated. Paramagnetism causes a signal drop in the $\mathrm{T} 2 *$ strain sequences.

p0060 Usually, rapid EPI T2* sequences are used, with relatively low spatial resolution but high acquisition speed (a volume of $25-28$ planes and $128 \times 128$ matrix can be acquired in about $3 \mathrm{~s}$ ). To obtain the activation maps, it is necessary to acquire numerous images of the entire brain volume: the set of all images is called a time 
series. During the acquisition, a protocol is run during which the subject under examination is asked to perform a task. There are two types of protocol (or paradigm) that can be practiced; one is a block design and the other is an eventrelated design. In the first one, there are alternating phases of activation in resting phases, with movement performed for about 20-30 s and same time stopping. In the case of an event-related paradigm, the individual stimulus or activity of the subject corresponds to a volume repeated several times during the time series with an irregular frequency.

\section{s0035 Basic images and lesions classification}

p0065 Because MRI is a multiparametric examination, every sequence is able to give a view of different findings:

u0010 - T1 turbo SE (TSE) images usually give a clear depiction of anatomy and fractures;

u0015 - T2 TSE images are excellent for detecting the cord edema, which will appear as an area of hyperintensity with focal cord enlargement at the level of trauma;

$\mathrm{u} 0020$ - T2* GRE images are helpful for detecting acute $(>2 \mathrm{~h})$ hemorrhage, which can easily be identified by a typical signal drop caused by susceptibility-induced distortions of local magnetic fields caused by the presence of hemosiderin degradation products (mainly deoxy-Hb); and

u0025 - Short $\tau$ inversion recovery (STIR) images are sensitive for detecting edema and soft tissue and ligamentous injuries (especially supraspinous or interspinous ligaments).

p0090 Spinal cord injury (SCI) is a dynamic process that may be grossly divided into different time-scale phases. In the first minutes, acute injury, depending mainly on forces delivered to the cord, immediately damages cells, axons, and myelin both directly and owing to vessel interruption. In the following minutes to hours, compression exerted on the cord by hematoma, bone, and eventually increasing cord edema may further worsen the damage; this immediate postacute damage is the main target for surgery and antiedema medications (steroids). In the following hours to days, a series of biochemical phenomena may further damage the injured cord, including excitatory amino acids, inflammatory cytokines, reactive oxygen species release, and ionic homeostasis disruption, finally leading to apoptosis and further cell death. After hours to days, fibrotic scars form, generated from glial cells and fibroblasts or cystic cavities. The final result is anatomical healing that achieves the generalist goal of separating damage from the remaining healthy tissues, but at the cost of forming a mechanical barrier to fiber regeneration, and finally hindering functional recovery. In some cases, spinal fluid disturbances may develop, leading to progressive myelopathy. Conventional MRI may show the vast majority of macroscopic damage in the acute phase; it may evaluate cord edema and compression, driving medical and surgical treatments; and it may show the various steps of 
anatomical healing. Its value in predicting prognosis remains gross and limited to major traumas. Advanced techniques are used mainly in the experimental setting to evaluate residual function and prognosis. A reliable marker of neurologic damage in the long term, to be used as a prognostic factor, is still lacking.

\section{s0040 Clinical presentation}

p0095 The severity of cord syndromes is classified using the American Spinal Injury Association (ASIA) scale, a system of tests used to define and describe the extent and severity of a patient's SCI and to determine future rehabilitation and recovery needs. It is ideally completed within $72 \mathrm{~h}$ after the initial injury. The patient's grade is based on how much sensation he or she can feel at multiple points on the body, as well as tests of motor function [1a].

p0100 Complete cord injury - In a complete cord injury (ASIA grade A), there will be a rostral zone of spared sensory levels, reduced sensation in the next caudal level, and no sensation in levels below. Similarly, there will be reduced muscle power in the level immediately below the injury, followed by complete paralysis in more caudal myotomes. In the acute stage, reflexes are absent, there is no response to plantar stimulation, and muscle tone is flaccid.

p0105 Incomplete injury-In incomplete injuries (ASIA grades B-D), there are various degrees of motor function in muscles controlled by levels of the spinal cord caudal to the injury. Sensation is also partially preserved in dermatomes below the area of injury. The relative incidence of incomplete versus complete SCI has increased over the past half century [1], mainly because of better initial care and proper immobilization after injury.

p0110 Central cord syndrome-An acute central cord syndrome, characterized by disproportionately greater motor impairment in upper compared with lower extremities, bladder dysfunction, and a variable degree of sensory loss below the level of injury, is described after relatively mild trauma in the setting of preexisting cervical spondylosis.

p0115 Anterior cord syndrome-These lesions more often represent direct injury to the anterior spinal cord by retropulsed disc or bony fragments rather than primary disruption of the anterior spinal artery.

p0120 Transient paralysis and spinal shock-Immediately after an SCI, there may be a physiologic loss of all spinal cord function caudal to the level of the injury, with flaccid paralysis, anesthesia, absent bowel and bladder control, and loss of reflex activity (cit. silver) [1b]. In males, especially those with a cervical cord injury, priapism may develop. There may also be bradycardia and hypotension not resulting from causes other than the SCI. This altered physiological state may last several hours to several weeks and is sometimes referred to as spinal shock. This functional impairment may be caused by the transient loss of potassium within the injured cells in the cord and its accumulation within the extracellular space. Clinical manifestations may normalize but are more usually replaced by a 
spastic paresis reflecting more severe morphologic injury to the spinal cord. A transient paralysis with complete recovery is most often described in younger patients with athletic injuries.

\section{s0045 Acute imaging}

p0125 The timing of baseline MRI is not clearly standardized; it varies from hours after hospital admission to more than 2 weeks after injury [2]. However, the best timing is within $72 \mathrm{~h}$ of injury because the T2 hyperintensity produced by edema improves the conspicuity and detectability of ligamentous injuries.

p0130 A standard trauma MRI protocol must include sagittal T2 images, which are the most important sequences for assessing the prognostic value [3,4]. Sagittal GRE sequences are useful for evaluating the presence of hemorrhage (the most important factor related to neurological status), although they are prone to motion artifacts (especially breathing) and might miss smaller amounts of blood or hemosiderin. Axial T2 sequences are useful for identifying clinically relevant lesions such as discal herniation and spinal cord compression that may alter management. Other sequences, such as sagittal and axial T1 or STIR (evaluation of ligaments), MR angiography (vertebral artery injury), or DTI (white matter tracts) can be used at the surgeon's discretion. Axial T2*protocol highlights contrast between white and gray matter with hypointense signal in case of hemorrhage. 2D MEDIC (Multi Echo Data Image Combination) [7] based on a multiple-echo protocol with high bandwidth is used to reduce chemical shift and flow artifacts in the spinal cord.3D sequences have the drawback of quite large acquisition time that made these sequences not suitable for non collaborative or injured patients. Recently paralled imaging techniques (CAIPIRINHA) [8] have been used in combination with SPACE leading to a $50 \%$ shorter acquistion time without loss in terms of image quality.

p0135 Sagittal T2 images can describe nearly the entire spectrum of SCI ([5,5a]):

u0030 • normal cord (Fig. 4.1),

u0035 - single-level edema (Figs. 4.1 and 4.2),

u0040 - multilevel edema, and

u0045 - mixed hemorrhage and edema (Figs. 4.3-4.7).

p0160 The limits to this four-pattern classification are the difficulty of representing the full continuum of acute SCI. [5] and that nonstandardized techniques for geometric measurements, such as hemorrhage and edema length, can be potential confounders. Patients with single-level edema had better initial neurological status than did patients with diffuse edema. They had an improvement of about two grades in ASIA score compared with an improvement of one grade for patients with diffuse edema. The prognosis of patients with edema is significantly better than for those with hemorrhage. Single-level hemorrhage alone often indicates a complete lesion (ASIA A). More severe abnormalities on MRI, especially hemorrhage, the number of levels of edema, a greater degree of cord compression, a greater degree of canal 


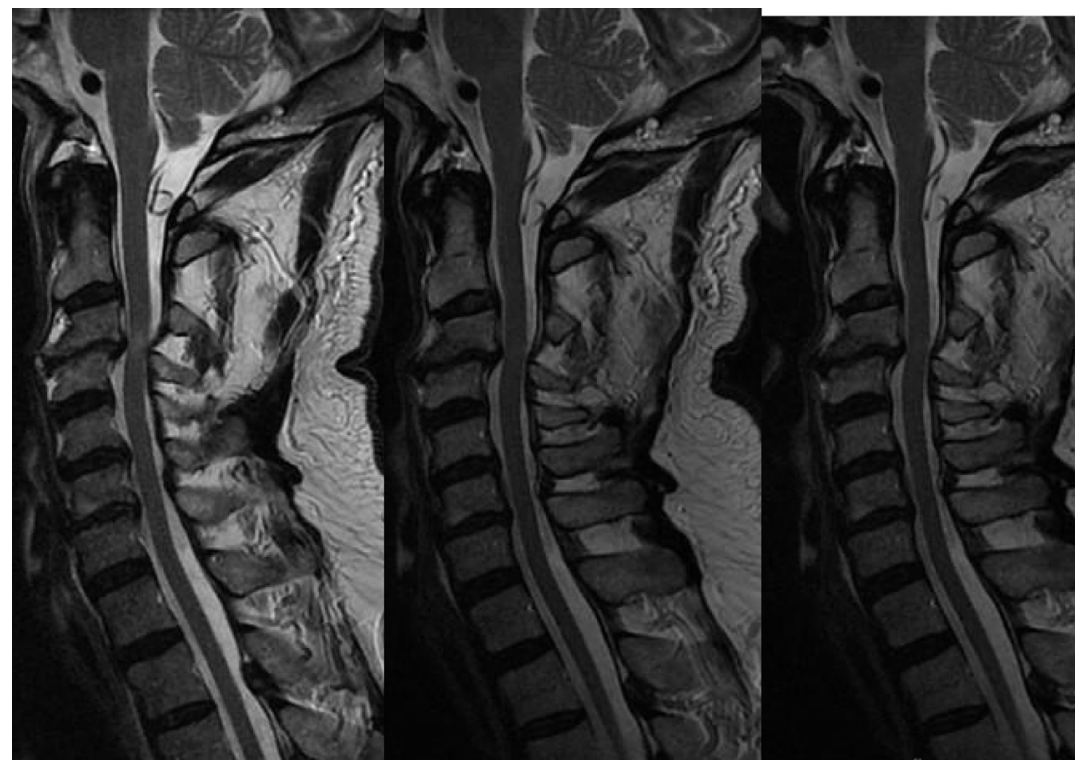

f0010 FIGURE 4.1

Sagittal turbo spin echo T2 weighted image of cervical spine. Despite negative

radiographs and computed tomography, magnetic resonance imaging reveals a T2

hyperintensity at the C3-C4 level corresponding to edema. This corresponds to an acute spinal cord injury without radiographic abnormality pattern. Images taken at 1 year 3 months' follow-up show anatomical healing with no pathological findings.

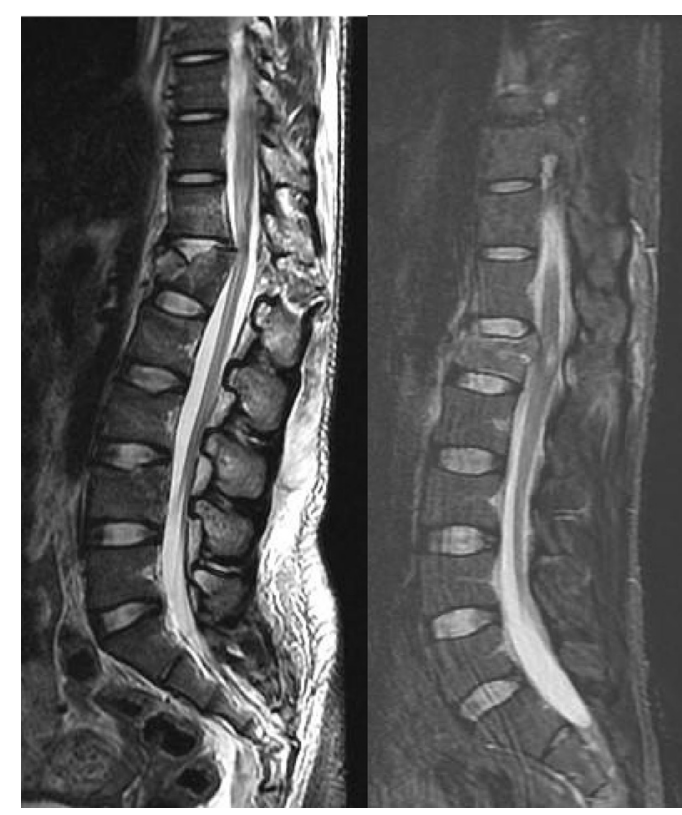

fO015 FIGURE 4.2

Sagittal turbo spin echo T2 weighted image and sagittal short $\tau$ inversion recovery of lumbar spine. Injury to the cord is revealed by the presence of a high T2 signal, which indicates edema or contusion. 


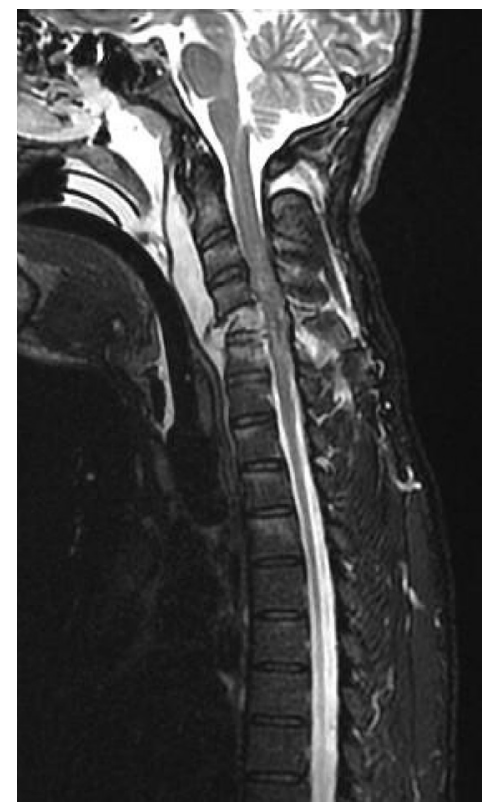

fO020 FIGURE 4.3

Sagittal short $\tau$ inversion recovery images of the cervical spine demonstrating a hemorrhage pattern (heterogeneous hypointensity caused by degradation of blood products, with internal regions of high signal intensities). The presence of this pattern is associated with worse neurologic outcomes.

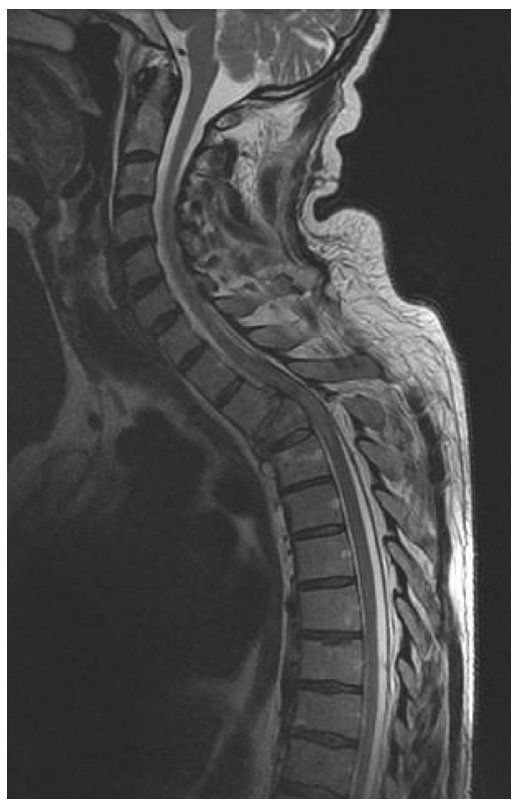

f0025 FIGURE 4.4

T2 turbo spin echo sagittal image: mixed edema-hemorrhage pattern. 


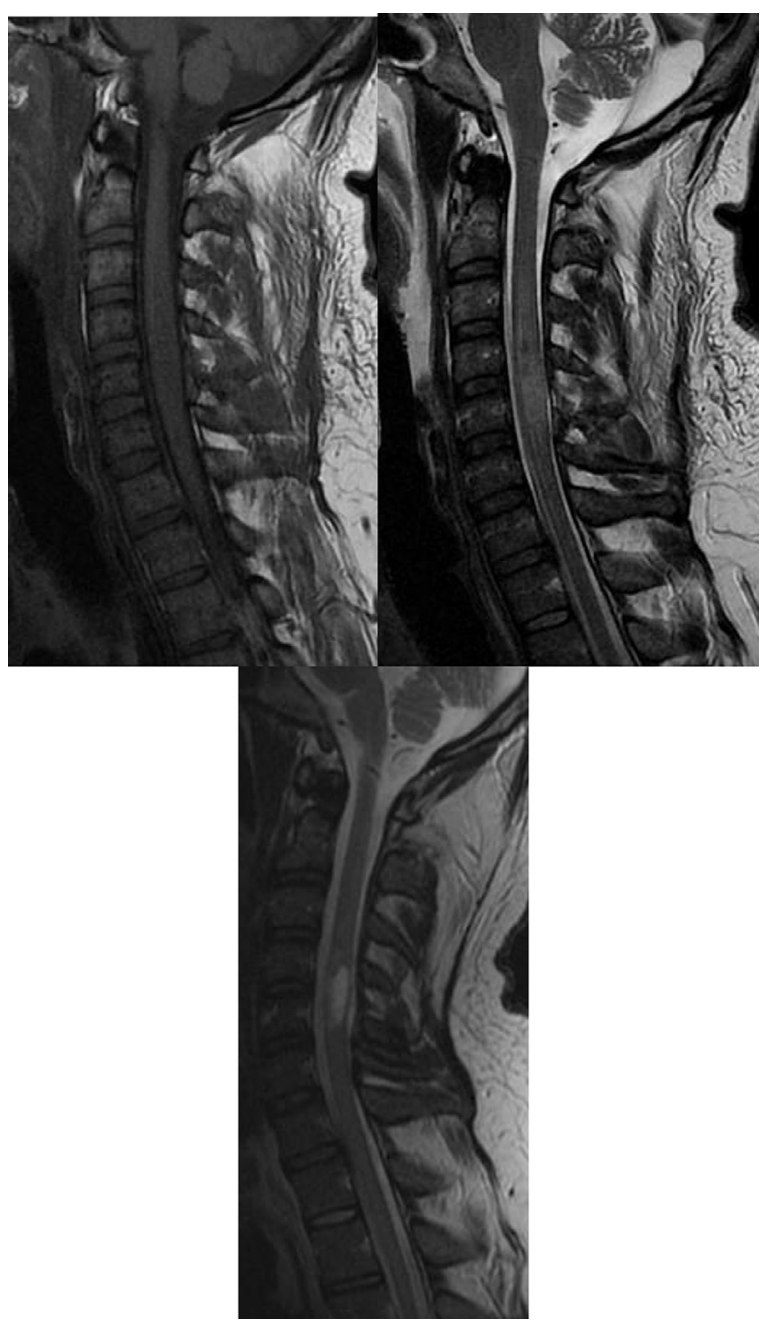

fO030 FIGURE 4.5

Sagittal turbo spin echo T1 and T2 weighted images: acute severe spinal cord injury (mixed pattern hemorrhage and edema) with 9 months' evolution with a residual medullary hyperintensity mimicking the presence of syrinx.

compromise, and the severity of soft tissue injury were shown to be associated with a worse neurological outcome [5b-e].

p0165 Complete cord transections and penetrating injuries, which are associated with different prognoses, do not fall within this classification owing to their different and easily differentiated patterns on MRI (Fig. 4.8).

p0170 A stand-alone category, called spinal cord injury without radiographic abnormality (SCIWORA), originated before the use of MRI and referred to patients with a 

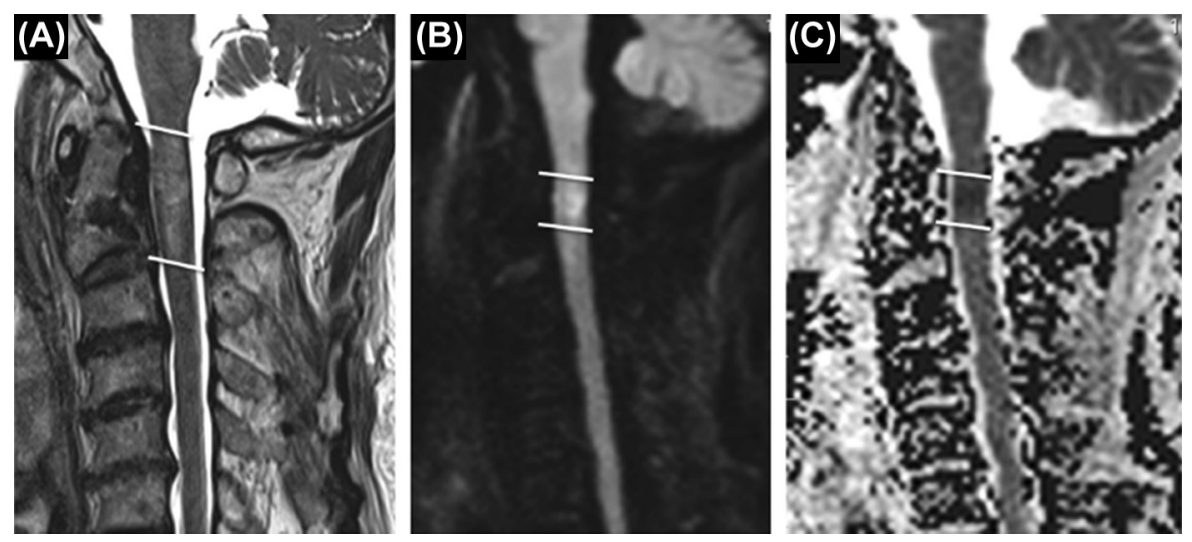

f0035 FIGURE 4.6

Cervical spine injury: mixed pattern. T2 FSE sagittal (A), DWI (B) and ADC map

(C) images.

(A)
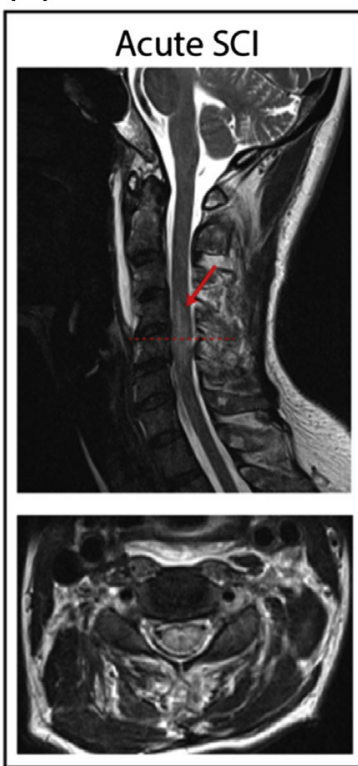

f0040 FIGURE 4.7

T2 sagittal and axial FSE images showing acute (A), sub-acute (B) and chronic spinal cord injury.

(B)
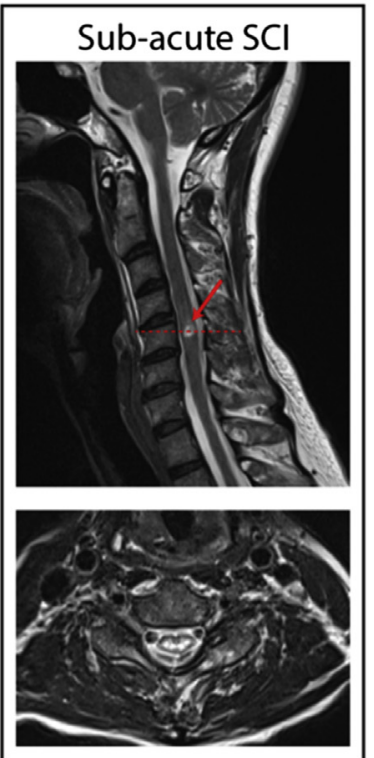

(C)

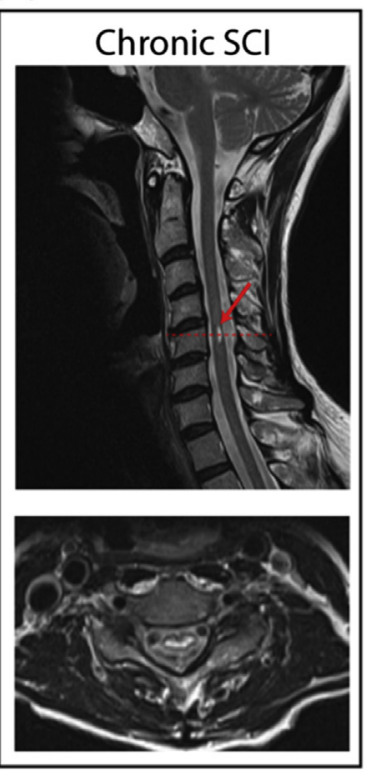


f0045 FIGURE 4.8

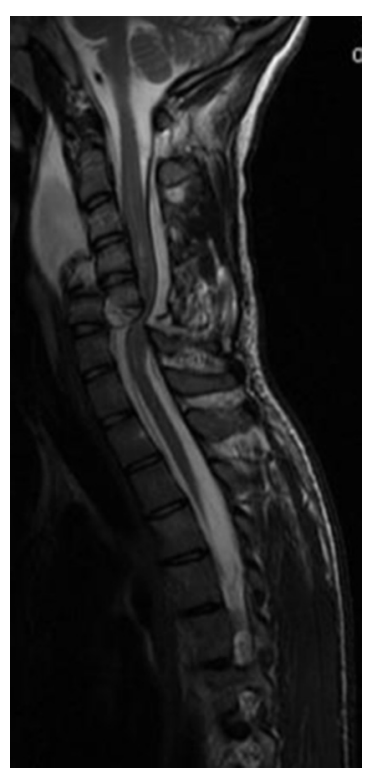

Spinal cord transection.

myelopathy with no radiographic evidence of fractures, dislocations, or malalignment of the spine on plain radiographs or CT. Because MRI provides superior imaging of the spinal cord, it can detect injuries to the cord that exist despite the apparent absence of bony abnormalities. Nevertheless, a number of patients with SCIWORA also have no detectable lesion on MRI.

p0175 A common explanation for this phenomenon is transient ligamentous deformation, which allows slight spinal bony deformation, followed by spontaneous reduction.

p0180 This is more often described in children who have weak paraspinal muscles, elastic spinal ligaments, and laxity of soft tissues, which fail to protect the spinal cord from force. Other possible mechanisms for SCIWORA include radiographically occult intervertebral disc herniation, epidural or intramedullary hemorrhage, radicular artery embolism caused by rupture of intervertebral disc, and spinal cord infarction (e.g., dissection of descending aorta).

p0185 MRI is invaluable for the diagnosing these conditions.

\section{s0050 Postacute, including various evolutions}

p0190 Clinical issues that arise in the subacute period (typically 2-6 weeks after acute injury) include neurological deterioration and infection. Pain is more commonly neuropathic and is assessed clinically rather than radiologically. 
p0195 Edema changes significantly over time. It increases in the first days after SCI [6], in the first $48 / 72 \mathrm{~h}$ after SCI, followed by a regression over the subsequent 2 to 3 weeks [7].

p0200 The behavior of hematoma is similar: it is usually identified on the initial MRI and remains present until 3 weeks after the injury [8]. It is reported, however, that hematoma can be identified later ( $12 \mathrm{~h}$ after the first scan). The mean length of the hematoma in the spinal cord increases in the first $48 \mathrm{~h}$ after injury and then gradually decreases in the 3 weeks after injury.

p0205 A rare complication is subacute progressive ascending myelopathy, also described as progressive posttraumatic myelomalacic myelopathy, with an incidence of $2 \%-6 \%$ (planner). It has an associated mortality of up to $10 \%$, although most patients spontaneously improve but rarely regain all of the lost function. It is caused by altered CSF flow resulting from dural adhesions (ischemic and inflammatory processes have also been proposed). On MRI, there is a characteristic progression of edema within the cord, remaining above the initial level. The signal abnormality is characteristically central [9]. Although its main clinical features are impairment in motor score with ascending sensory level, sometimes the condition can remain subclinical.

p0210 Progressive posttraumatic cystic syringomyelia is an uncommon but potentially clinically serious complication. It is defined as a fluid-filled intramedullary structure within cord parenchyma (therefore not lined by ependyma), with a signal isointense to CSF and usually expanding above the injured segment. The mechanism involved in its progressive enlargement remains uncertain and includes the presence of scarring and adhesions within the subarachnoid space, alterations in CSF flow dynamics, and fluid turbulences within the cavity. Its prevalence in chronic cord injury has been reported to be up to $20 \%$, although symptomatic syrinx-like manifestations occur in approximately $3 \%$. Progressive signs and symptoms can develop as early as 3 months after spinal cord trauma or can occur as late sequelae more than 30 years after SCI [10].

\section{s0055 Clinical-magnetic resonance imaging correlations}

p0215 Emergency MRI provides an invaluable tool for the accurate diagnosis of spinal cord compression and directly influences the initial clinical management in most protocol patients [10a]. Existing evidence suggests that certain MRI characteristics are important predictors of outcomes in acute SCI, including the length of intramedullary hemorrhage and the canal diameter at the level of maximum spinal cord compression (MSCC). Other imaging characteristics may also be useful for prognostication, such as alternative measures of canal stenosis, the presence of hemorrhage, and longer edema length. The length of the intramedullary hemorrhage spinal canal diameter at the location of MSCC and baseline neurological status are important predictors of worse neurologic outcomes. Conventional MRI may have only modest prognostic utility owing to its inability to distinguish recoverable from nonrecoverable tissue injury. 


\section{s0060 Perspectives}

p0220 A major drawback of conventional MRI is a lack of reliable markers for long-term neurological recovery [2]. This is particular critical within the perspective of treatment $[10 \mathrm{~b}]$. In the acute phase, medical and surgical therapies are basically cell-sparing strategies: the surgeon basically decompresses a compressed cord and medical treatment reduces edema or is directed to reduce secondary damage caused by inflammation, oxygen species, or excitatory amino acids. In later phases, regeneration of axons necessitates the elimination of barriers that biological healing processes have built over lesions (scars and cysts), with the goal of connecting unaffected and regenerative-prone fibers to a cozy medium, at the cost of probably damaging residual functioning fibers. A prognostic marker, in particular, an early marker of severe, permanent long-term deficit, will allow ethical application of such treatments earlier without the need to destroy before building. Within this context, MRI advanced techniques may be helpful. DTI may image fiber tracts directly [10c]. Fractional anisotropy has been shown to correlate with axonal damage and functional recovery. fMRI may show metabolic activity related to cell function (i.e., functional integrity) of spinal cord; longitudinal studies may reveal the time course of the damage, including reorganization of brain motor pathways in the long term.

\section{s0065 Diffusion tensor imaging and related techniques}

p0225 There are some issues in acquiring good-quality DTI images of the spine: the small size of spinal cord transverse diameters, artifacts caused by pulsatile flow of the CSF, swallowing, breathing, or motion of the internal organs (such as heart, esophagus, or descending aorta) during the examination, and artifacts caused by local magnetic inhomogeneity, metal implants, and chemical shift artifacts (vertebral fat and paravertebral soft tissues), especially in thoracic and upper lumbar segments. A 3 T machine may be required for the reliable measurement of DTI metrics necessary for clinical use; however, in most cases, $1.5 \mathrm{~T}$ is enough to obtain diagnostic results. A carefully planned study protocol includes field of view reduction (usually $12 \mathrm{~cm}$ ) [6] as well as a decreased reconstructive matrix, smaller slice thickness (compared with the brain DTI), not fewer than 15 diffusion directions, and proper $b$ value (e.g., $b=0$ and $b=1000)[11]$.

p0230 With those caveats, DTI is a sensitive and specific method for evaluating acute SCI and its chronic evolution. A significant decrease in fractional anisotropy (FA) and ADC values can be found at the level of direct trauma and adjacent to the normal-appearing parts of the spinal cord [11a], with ADC values correlated to the degree of acute cord injury. Detection of abnormalities in regions of the spinal cord that appear normal on plain MRI could be used to assess the true extent of injury and better correlate the neurologic deficit with MRI. In a canine model, differences between acute and chronic stages were detected and correlations were made between T2 weighted and DTI sequences: the ADC decreased and FA values 

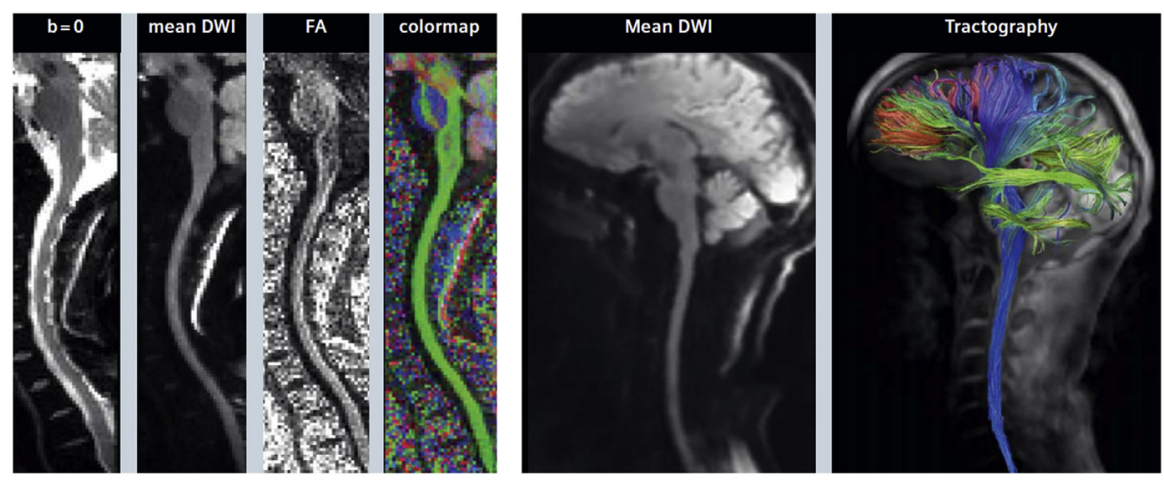

f0050

RESOLVE DTI data: b0 trace, mean DWI, FA, FA colormap, DTI tracts reconstruction.

increased in acute SCI, whereas in chronic SCI, lower FA and higher ADC were found compared with acute SCI, with a relation between FA values and the extent of lesion (Fig. 4.9) [12].

p0235 Sagittal or axial planes are used for DTI acquisitions and measurements, generating FA maps in the sagittal and axial planes, respectively; the axial plane is a more reliable method for measuring FA values than in the sagittal plane, because the regions of interest can be placed more precisely in the spinal cord without CSF contamination [6].

p0240 DTI can be useful as a functional as well as a prognostic tool because of its ability to explore the functional integrity and neural architecture of the spinal cord, which gives significant value to the prediction of potential functional recovery. There is a good correlation between DTI and functions of medial and lateral spinothalamic tracts assessed on spinal evoked potentials [13]. DTI is also useful for tracking the progression of SCI from the acute to the chronic stages and could be applied to monitor morphological changes in patients with chronic SCI [14].

\section{s0070 Functional magnetic resonance imaging}

p0245 As explained, several technical difficulties also impede fMRI generation from the spinal cord. Small size leads to low resolution and partial volume effect, surrounding bony structures enhance susceptibility artifacts, and pulsation of the CSF introduces spurious frequencies in signal. Moreover, a probabilistic map of the spinal cord was produced only recently; it enables standardization of spatial coordinates [15]. All of this makes this technique difficult and still experimental. fMRI images of the spinal cord in response to various stimuli were obtained in the past decade or so in both healthy subjects [16] and patients with several pathologies [17]. Evoked responses may assess the functional integrity of a fiber track above or below a lesion; moreover, evoked fMRI signals may be recorded more easily in the brain, assessing a certain functionality of the whole circuit [18]. Differently from a functional 
evaluation of spinal cord, i.e., clinical or electrophysiological, fMRI may eventually detect subclinical functioning pathways [18a], or cortical rearrangement in the mid or long term [18], both eventually involved in adverse symptoms, as spasticity or central pain and/or in long-term recovery.

p0250 It is even more difficult to extract resting state signals and requires high field devices from 3 to $7 \mathrm{~T}$. Resting state analysis was carried out only in healthy subjects [19] or in animal models of SCI [20].

p0255 Similar to evoked fMRI responses, cortical rearrangement of resting state signals was demonstrated after SCI lesion [21].

p0260 Owing to technical constraints, BOLD-based functional images of spinal cord, including both evoked fMRI and resting state images, are difficult to obtain. The most relevant feature is the possibility of studying the whole system, from the spinal cord to the brain, and in particular, of evaluating the cortical rearrangement. This latter is probably a key player in adverse symptoms and recovery after SCI.

\section{References}

[1] Ogawa S, Lee TM, Kay AR, Tank DW. Brain magnetic resonance imaging with contrast dependent on blood oxygenation. Proc Natl Acad Sci U S A 1990;87(24): 9868-72. https://doi.org/10.1073/pnas.87.24.9868.

[1a] Roberts TT, Leonard GR, Cepela DJ. Classifications in brief: American spinal injury association (ASIA) impairment scale. Clin Orthop Relat Res 2016;475(5): 1499-504. https://doi.org/10.1007/s11999-016-5133-4.

[1b] Ditunno JF, Little JW, Tessler A, Burns AS. Spinal shock revisited: a four-phase model. Spinal Cord42 2004:383-95. https://doi.org/10.1038/sj.sc.3101603.

[2] Kurpad S, Martin AR, Tetreault LA, et al. Impact of baseline magnetic resonance imaging on neurologic, functional, and safety outcomes in patients with acute traumatic spinal cord injury. Glob Spine J 2017;7(3 Suppl.):151S-74S. https://doi.org/10.1177/ 2192568217703666.

[3] Gillams AR, Soto JA, Carter AP. Fast spin echo vs conventional spin echo in cervical spine imaging. Eur Radiol 1997;7:1211-4.

[4] Katz BH, Quencer RM, Hinks RS. Comparison of gradient-recalled-echo and T2weighted spin-echo pulse sequences in intramedullary spinal lesions. AJNR 1989; 10:815-22.

[5] Bozzo A, Marcoux J, Radhakrishna M, Pelletier J, Goulet B. The role of magnetic resonance imaging in the management of acute spinal cord injury. J Neurotrauma 2011;28(8):1401-11. https://doi.org/10.1089/neu.2009.1236.

[5a] Andreoli C, Colaiacomo MC, Rojas Beccaglia M, Di Biasi C, Casciani E, Gualdi G. MRI in the acute phase of spinal cord traumatic lesions: Relationship between MRI findings and neurological outcome. Radiol Med 2005 Nov-Dec;110(5-6):636-45.

[5b] Dai LY, Jia LS. Central cord injury complicating acute cervical disc herniation in trauma. Spine 2000;25(3):331-5.

[5c] Flanders A, Schaefer D, Doan H, Mishkin M, Gonzalez C, Northrup B. Acute cervical spine trauma: correlation of MR imaging findings with degree of neurologic deficit. Radiology 1990;177:25-33. 
[5d] Miyanji F, Furlan J, Aarabi B, Arnold P, Fehlings M. Acute cervical traumatic spinal cord injury: MR imaging findings correlated with neurologic outcome-prospective study with 100 consecutive patients 1 . Radiology 2007;243:820-7.

[5e] Selden NR, Quint DJ, Patel N, d'Arcy HS, Papadopoulos SM. Emergency magnetic resonance imaging of cervical spinal cord injuries: clinical correlation and prognosis. Neurosurgery 1999;44(4):785-92.

[6] Sąsiadek MJ, Szewczyk P, Bladowska J. Application of diffusion tensor imaging (DTI) in pathological changes of the spinal cord. Med Sci Monit 2012;18(6):RA73-9. https://doi.org/10.12659/MSM.882891.

[7] Shimada K, Tokioka T. Sequential MR studies of cervical cord injury: correlation with neurological damage and clinical outcome. Spinal Cord 1999;37:410-5.

[8] Rutges JPHJ, Kwon BK, Heran M, et al. A prospective serial MRI study following acute traumatic cervical spinal cord injury. Eur Spine J 2017;26:2324. https:// doi.org/10.1007/s00586-017-5097-4.

[9] Chandra J, Sheerin F, Lopez de Heredia L, Meagher T, King D, Belci M, Hughes R. MRI in acute and subacute post-traumatic spinal cord injury: pictorial review. Spinal cord 2011;50:2-7. https://doi.org/10.1038/sc.2011.107.

[10] Agrawal A, Shetty MS, Pandit L, Shetty L, Srikrishna U. Post-traumatic syringomyelia. Indian J Orthop 2007;41(4):398-400. https://doi.org/10.4103/00195413.37006.

[10a] Fehlings Michael G, et al. Guidelines for the Management of Degenerative Cervical Myelopathy and Spinal Cord Injury: An Introduction to a Focus Issue. Global Spine Journal 2017;7(3_suppl):6S-7S.

[10b] Ahuja CS, Nori S, Tetreault L, et al. Traumatic spinal cord injury-repair and regeneration. Neurosurgery 2017;80:S9-22.

[10c] Zaninovich OA, Avila MJ, Kay M, Becker JL, Hurlbert RJ, Martirosyan NL. The role of diffusion tensor imaging in the diagnosis, prognosis, and assessment of recovery and treatment of spinal cord injury: a systematic review. Neurosurg Focus 2019;46(3):E7. https://doi.org/10.3171/2019.1.FOCUS18591.

[11] Lee JW, Kim JH, Kang HS, et al. Optimization of acquisition parameters of diffusiontensor magnetic resonance imaging in the spinal cord. Investig Radiol 2006;41(7): $553-9$.

[11a] Shanmuganathan K, Zhuo J, Chen HH, Aarabi B, Adams J, Miller C, Menakar J, Gullapalli RP, Mirvis SE. Diffusion tensor imaging parameter obtained during acute blunt cervical spinal cord injury in predicting long-term outcome. J. Neurotrauma 2017;28. https://doi.org/10.1089/neu.2016.4901.

[12] Wang-Leandro A, Hobert MK, Alisauskaite N, Dziallas P, Rohn K, Stein VM, et al. Spontaneous acute and chronic spinal cord injuries in paraplegic dogs: a comparative study of in vivo diffusion tensor imaging. Spinal Cord 2017;55(12):1108-16. https:// doi.org/10.1038/sc.2017.83.

[13] Ellingson BM, Kurpad SN, Schmit BD. Functional correlates of diffusion tensor imaging in spinal cord injury. Biomed Sci Instrum 2008;44:28-33.

[14] Czyz M, Tykocki T, Szewczyk P, Jarmundowicz W. Application of diffusion tensor imaging in the prognosis of outcome after traumatic cervical spinal cord injury. J Spinal Stud Surg 2017;1:25-8.

[15] Cadotte DW, Cadotte A, Cohen-Adad J, Fleet D, Livne M, Wilson JR, Mikulis D, Nugaeva N, Fehlings MG. Characterizing the location of spinal and vertebral levels in the human cervical spinal cord. AJNR 2015;36:803-10. 
[16] Stroman PW. Essentials of functional MRI. CRC Press; 2001. p. p278.

[17] Agosta F, Valsasina P, Caputo D, Rocca MA, Filippi M. Tactile-associated fMRI recruitment of the cervical cord in healthy subjects. Hum Brain Mapp 2009;30: 340-5. https://doi.org/10.1002/hbm.20499.

[18] Jurkiewicz MT, Mikulis DJ, McIlroy WE, Fehlings MG, Verrier MC. Sensorimotor cortical plasticity during recovery following spinal cord injury: a longitudinal fMRI study. Neurorehabilitation Neural Repair 2007;21:527-38.

[18a] Wrigley PJ, Siddall PJ, Gustin SM. New evidence for preserved somatosensory pathways in complete spinal cord injury: a fMRI study. Hum Brain Mapp 2018;39: 588-98. https://doi.org/10.1002/hbm.23868.

[19] Eippert F, Kong Y, Jenkinson M, Tracey I, Brooks JCW. Denoising spinal cord fMRI data: approaches to acquisition and analysis. Neuroimage 2017;154:255-66. https:// doi.org/10.1016/j.neuroimage.

[20] Chen LM, Mishra A, Yang PF, Wang F, Gore JC. Injury alters intrinsic functional connectivity within the primate spinal cord. Proc Natl Acad Sci USA 2015;112:5991-6. https://doi.org/10.1073/pnas.1424106112.

[21] Hawasli AH, Rutlin J, Roland JL, Murphy R, Song SK, Leuthardt EC, et al. Spinal cord injury disrupts resting-state networks in the human brain. J Neurotrauma 2018;35(6): 864-73. https://doi.org/10.1089/neu.2017.5212.

\section{Further reading}

[1] Kirshblum SC, Burns SP, Biering-Sorensen F, et al. International standards for neurological classification of spinal cord injury (revised 2011). J Spinal Cord Med 2011;34(6): 535-46. https://doi.org/10.1179/204577211X13207446293695.

[2] Planner AC, Pretorius PM, Graham A, Meagher TM. Subacute progressive ascending myelopathy following spinal cord injury: MRI appearances and clinical presentation. Spinal Cord 2008;46:140-4.

[3] Atesok K, et al. Posttraumatic spinal cord injury without radiographic abnormality. Adv Orthop 2018;2018:7060654. https://doi.org/10.155/2018/7060654. 


\title{
Non-Print Items
}

\begin{abstract}
Magnetic resonance imaging (MRI) is the state-of-the-art radiological tool used to visualize the spinal cord in both normal and pathological conditions. MRI enables visualization in nearly real time (within minutes), with submillimetric spatial resolution, and with high contrast between gray and white matter and surrounding cephalospinal fluid, and eventually ischemic, traumatic, and hemorrhagic lesions. The possibility of seeing the spinal cord directly changed the way surgeons and researchers approached spinal cord injury. A major drawback continues to be the limited size of the cord; major gray structures are visible, as well as major white matter tracts, but distinction between different tracts, and the vast majority of the vessels, including most of intramedullary vessels, are still not identifiable with current devices. Generally speaking, the spatial resolution and contrast of MRI are inversely related; in pathological conditions, one must choose to maximize one or the other. From the perspective of cellular damage, a step further was achieved by diffusion-weighted images, which achieve extremely high sensitivity for hyperacute cellular damage (at the cost of lowering spatial resolution). On the other hand, for axonal damage, diffusion-tensor images allowed researchers to produce synthetic images of white matter tracts (virtual tractography) and eventually to estimate the rate of fibers with coherent direction in a given voxel (fractional anisotropy). Also in this case, results come at a cost: the direct connection between signal acquisition and image generation is lost (images are interpolated, calculated a posteriori), which lowers spatial resolution for fractional anisotropy.
\end{abstract}

\section{Keywords:}

Magnetic resonance imaging, Radiology, Spinal cord injury, Spine. 


\title{
CHAPTER
}

\section{c0005 Spinal cord injury: ethical issues}

\author{
Virginia Sanchini, PhD ${ }^{1,2, *}$, Giovanni Boniolo, $\mathrm{PhD}^{3, *}$ \\ ${ }^{1}$ Faculty of Philosophy, Vita-Salute San Raffaele University, Milan, Italy; ${ }^{2}$ Department of Oncology \\ and Hematology, University of Milan, Milan, Italy; ${ }^{3}$ Professor, Dipartimento di Scienze Biomediche \\ e Chirurgico Specialistiche, Università di Ferrara, Ferrara, Italy
}

\section{s0010 Introduction}

p0010 A spinal cord injury (SCI) is generally defined as a damage to any part of the spinal cord or nerves at the end of the spinal canal, affecting some body functions, depending on the specific site in which the injury occurred and the severity of the injury itself. It may be complete or incomplete, and it may lead to a paraplegic paralysis or quadriplegic paralysis. SCI represents a very severe disease inasmuch as, as a consequence of partial or complete paralysis, several body functions may be compromised, therefore causing several problems [1-4], which may be partially overcome by an effective repairing strategy, dealing primarily around rehabilitation [5-10]. Among the most common problems, we may find bladder control problems - if the message carrier has been injured, the brain will not be able to control the bladder, which will anyway continue storing urine from kidneys; bowel control problems-although the injury does not affect the stomach and intestines, which will work much like they did before, control over bowel movements appears often altered; skin sensation problems - below the neurological site of the injury, part or all skin sensations may be compromised, thus preventing the brain from sending messages when the skin is injured; circulatory control problems of different degrees, ranging from low blood pressure to more serious syndromes, such as deep vein thrombosis and pulmonary embolus; respiratory problems, which mainly deal with breath difficulties, in case abdominal and chest muscles are affected; sexual life problems, inasmuch as sexuality, fertility, and sexual functions may be affected by the same injury. In addition to the aforementioned compromised functions, sedentary lifestyle-based diseases correlated to the limited mobility caused by SCI—namely obesity, cardiovascular disease, and diabetes-may affect injured individuals as well.

p0015 While specialized literature has been primarily devoted to the medical issues - in particular factors associated with (and body functions changed after) SCI [11-13] as

* The authors contributed equally to this chapter.

Spinal Cord Injury (SCI) Repair Strategies. https://doi.org/10.1016/B978-0-08-102807-0.00005-3 Copyright $\odot 2020$ Elsevier Ltd. All rights reserved. 
well as trends in mortality and causes of death of people affected by SCI [14-18]lesser attention has been paid to the related ethical questions. Against this backdrop and the lack of dedicated debates, this chapter indicates and explores both the main threats whose impact may be ethically sensitive and the possible solutions addressing them in the context of new repairing strategies. Concerning this last point, it is worth noting that there is virtually no ethical discussion about it (an exception is given by Ref. [19]. This could be a serious problem, especially now that novel cell therapies, drug delivery systems, and biomaterials are proposed almost every day.

\section{s0015 Ethical issues related to spinal cord injury}

p0020 Although undertheorized, ethical issues central to SCI have been referred to as related to the principle of justice on the one hand and the principle of autonomy on the other hand [20]. Indeed, inasmuch as SCI is an expensive disease, not only in terms of significant initial and direct costs for hospitalization and rehabilitation but also in terms of both direct and indirect costs related to the long-term disease management, strategies for granting equal access to care should be developed. Moreover, insofar as SCI is a complex, multidimensional disease potentially affecting the individual in its entirety, besides coping with the awareness that they will be (partially or completely) dependent for the rest of their lives, SCI-affected patients have to completely restructure their lives, being often also obliged to rethink their self-determination, i.e., reformulate their ends, as well as means to reach these ends. Finally, despite not always explicitly mentioned, being correlated with physical and psychological pain, SCI may be considered as dealing also with the ethical principle of beneficence. In the next paragraphs, these different ethical layers will be explored and discussed. In particular, we will present the challenges that SCI raises with respect to the three aforementioned ethical dimensions of justice, autonomy, and beneficence, as well as possible solutions to cope with them.

\section{s0020 Spinal cord injury and justice}

p0025 A first ethical dimension involved while approaching SCI is justice, which immediately appears potentially in danger while considering the costs needed to take care of an SCI-affected patient. In its original formulation, the principle of justice refers to the idea that all persons are equally entitled to a same degree of moral concern, thus promoting the notion that people in similar positions should be treated in a similar manner. Drawing from this assumption, it requires to distribute benefits, risks, and costs in a fair manner [21]. In its subsequent formulation, it is conceived in a broader manner, and it deals with the distributive stance of justice, thus requiring a fair distribution of goods within society [22]. A fundamental step in this direction is represented by the contribution of Priebe and colleagues, who estimated what it means for the healthcare system and private system to take charge of an SCI-affected patient 
and for the patient himself or herself to rearrange the whole life with the necessary supportive tools [20]. A first observation deserving consideration is that taking care of an SCI-affected patient requires significant direct costs throughout the whole life of the patient himself or herself, including both initial hospitalization and acute rehabilitation costs and recurring costs for medical support and personal assistancewhere "the level and extent of injury are important factors contributing to first-year costs. People with high tetraplegia incurred over 3 times the charges that people with incomplete, motor functional SCI incurred" ([20]; p. S84). Additionally, SCI is an expensive disease also in terms of direct significant costs related to home and vehicle modifications: since the vast majority of people affected by SCI are discharged at home, making their houses accessible is of vital relevance for allowing them to feel comfortable in their own environments. With respect to the latter and according to the National Spinal Cord Injury Statistical Center, the most common modifications required to improve house accessibility are building ramps (83\%), widening doors $(57 \%)$, and remodeling bathrooms $(46 \%)$ or other rooms in the house $(43 \%)$ [23]. Vehicle modifications-whose cost highly varies depending on the type of modification requested and the type of vehicle modified-are also very important to prevent SCI individuals to be marginalized and left alone in their own houses. Finally, there is an additional set of indirect costs related to underemployment and unemployment caused by SCI. With respect to overall rate of employment for people affected by SCI after the injury, relevant literature reports that this range from $13 \%$ to $58 \%$ [24,25], where the percentage of people employed after SCI becomes higher year by year, with $40 \%$ of people employed after 20 years by the moment of the injury itself ([20]; p. S85).

p0030 Drawing from these considerations, we may start figuring out what threats to justice may be and, conversely, what does it mean to comply with a principle of justice in an SCI-based scenario.

p0035 First, despite recognizing that SCI is an expensive disease, respecting justice seems to require allowing individuals affected by SCI to have an equal access to care, considered both in its more intuitive meaning of direct access to medical care during primary hospitalization and acute rehabilitation, and in its secondary meaning of allowing individuals to receive those apparently less important supportive equipment, such as funds for house modifications, which are nevertheless fundamental for letting the individual get acquainted with the new medical and life condition.

p0040 Moreover, as marginalization may be interpreted as a violation of justice, two other strategies should be put in place to respect this ethical principle. Besides (at least partially) funding vehicle modifications so as to allow individuals to drive or to be transported, thus reducing the risk for the injured individual to remain isolated, inasmuch as employment plays a core role in preventing people from being marginalized and in promoting social participation [26,27], strategies for helping injured people to find an employment should be among the priorities of an ethically sound rehabilitative strategy system for SCI-affected patients. Notably, although the rates of employment are correlated with functional status, evidence exists that the 
presence of a modified vehicle correlated highly with employment after SCI: "the employment rate for people who could drive and had a vehicle adapted for independent driving was $40 \%$, compared with $12 \%$ for those who did not" ([20]; p. S85).

\section{s0025 Spinal cord injury and autonomy}

p0045 A second ethical dimension involved in SCI debate is autonomy, interpreted in the threefold meaning of independence, self-determination, and absence from interference. The principle of autonomy derives from another principle-namely Principle of Respect for Persons. In its original formulation [21], it states that all persons have a fundamental right to self-determination. It incorporates at least two ethical convictions: first, that individuals should be treated as autonomous agents, and second, that persons with diminished autonomy are entitled to protection. In its more recent formulation [22], personal autonomy refers to self-governance, that is, to "self-rule that is free from both controlling interference by others and from limitations, such as inadequate understanding, that prevent meaningful choice" [22]. "The principle [of respect for autonomy] can be stated as a negative obligation and as a positive obligation. As a negative obligation: Autonomous actions should not be subjected to controlling constraints by others [...], whereas as a positive obligation, this principle requires respectful treatment in disclosing information and fostering autonomous decision-making" [22].

p0050 Besides the biomedical account developed by Beauchamp and Childress, another traditional interpretation of autonomy is what has been referred to as "executional autonomy," that is, being able to perform those activities that appear necessary for having a functioning daily life without being dependent upon others. This account of autonomy, if strictly interpreted, presents some challenges with respect to the context under investigation, insofar as it seems to be inapplicable for people with permanent disabilities such as SCI-affected patients [28]. However, recent literature based on qualitative investigation has shown that just after the end of hospitalization and at the beginning of the rehabilitation process, patients and professionals focus on regaining independence, as the latter is considered of fundamental importance by the side of the injured patient [29]. To what extent this is a reachable aim partially depends on the functional status of the patient. However, as being autonomous in this first account means not be dependent on others - which highly differs from not being equipped with artificial supports-some degrees of semiindependence may be individuated for quadriplegic patients as well [30-32].

p0055 After the end of the first phase (consisting in initial hospitalization and acute rehabilitation), "professionals should change their approach from paternalistic to a more coaching role, in which they provide room for planning and decision-making by the patient" ([29]; p. 253); in other words, autonomy as self-determination becomes relevant for the SCI-affected patient, once the very first and acute phase has passed. Van de Ven and colleagues report that, although the vast majority of patients with SCI experience a break in their own identities, which oblige them to completely restructure their goals-with this "repairing process," 
which can vary between individuals from several months to several years $[33,34]$ - in the long run do not consider their injury as something that negatively interfered with their capacity of setting goals, which was interpreted as a personal trait. Moreover, the recognition that personal choices in the context of SCI have a broader impact led some patients to become more aware of what they really wanted and in expressing their desires in a more explicit manner [33,34].

p0060 A more complex aspect of autonomous decision-making in SCI regards autonomy as noninterference with respect to life-sustaining treatments. This last meaning of autonomy plays a role in the ethical debate investigating to what extent advanced directives may be considered ethically legitimate in SCI-affected patients. With respect to this specific cohort of patients, recent literature suggests that a difference exists between advanced directives administered before SCI events, and those administered once the patient has already been injured, which are based on "a more realistic understanding of life with SCI and future needs" ([20]; S86). Therefore, while in the first context, advance directives are flawed, in the second one, these may be ideally considered as a legitimate option, as they are more likely to be supported by a valid informed consent.

\section{s0030 Spinal cord injury and beneficence}

p0065 A third ethically sound layer of SCI debate is represented by the discussion of those aspects of SCI dealing with the principle of beneficence, which traditionally refers to a normative statement of a moral obligation to act for the others' benefit. In particular, it claims that all the persons have a duty to advance the good of others where the nature of this good is in keeping with the fundamental and ethically defensible values of the affected party and where advancing their good does not entail disproportionate harm to oneself. Far from being conceived as a supererogatory act, as it happens within common morality, beneficence as a principle of biomedical ethics is understood in a stronger sense, as an obligation or, in technical terms, as a perfect duty-that is, as something for which a strict obligation exists. The obligations of beneficence affect both individual investigators and society at large, since they extend both to particular research projects and to the entire enterprise of research [22].

p0070 At first glance, beneficence is involved insofar as SCI-affected patients suffer both physical and psychological pain such as fatigue and depression, as a consequence of an everlasting condition. However, as broadly linked to quality of life (QoL), exploring what does it mean to comply with a principle of beneficence within the SCI context requires investigating what are the priorities of injured individuals, correlated, according to them, to an improvement of their QoL. Notably, despite the growing body of literature published over the past few decades [35-37], this remains a still unclear domain, where complexity is increased also as a consequence of the fact that after SCI individuals change their expectations and values. Simpson and colleagues recently published a systematic review in which they examined studies that directly surveyed people with SCI to ascertain their health priorities and life domains of importance [37a]. With respect to functional 
recovery priorities, the following areas were identified: motor function-in particular arm and hand function for individuals with tetraplegia, and mobility for individuals with paraplegia-bowel, bladder, and sexual function. Pain was considered as an important condition in those studies in which it was present, although it was considered less important than other areas such as recovering motor and bladder/bowel function. In addition, health and relationships were identified as salient factors in people's lives among the life area studies. Both psychological and physical aspects of health appeared to be endorsed by participants [37a]. As clearly emerged from these data, the concept of benefice that draws from QoL investigation refers to a set of simple - but still fundamental set of - functions and areas of human life, which, while representing the baseline in normal conditions, become hard to be reached objectives for SCI-affected patients. A repairing strategy willing to be defined as comprehensive should seriously take into consideration these evidences, so as set its effort in the right direction, toward a real improvement of the SCI-affected patient's QoL.

\section{s0035 Conclusions}

p0075 The present contribution aimed at analyzing the ethical issues related to, and originating from, SCI situations. By focusing on a still-to-be-developed debate, it was led by the attempt of showing that, besides medical and psychological concerns, less developed but nevertheless relevant ethical considerations may be raised as well. A promising strategy in this direction may be the one pursued in this chapter, i.e., looking at SCI condition through the lens of Principlism (even if this approach is here taken instrumentally and not as a founding one). When approached through this methodology, it clearly appears that all the four principles of biomedical ethics are somehow involved in SCI medical condition. The reference to justice pops up once recognized that SCI is an expensive disease, requiring a huge amount of monetary resources both in the preliminary and acute stage of the disease and in the ongoing management of the SCI-affected patient. Moreover, inasmuch as SCI is a disease potentially affecting the individual in its entirety, discourses around autonomy are deeply involved in this debate as well. Indeed, SCI-affected patients have to constantly cope with the potential risk of losing their self-determination, thus eventually realizing that autonomy should be rethought in a different manner, far from the traditional "executional autonomy" account. Finally, despite not always explicitly mentioned, being correlated with physical and psychological pain, SCI may be considered as dealing also with the dimension of beneficence, which requires professionals treating SCI-affected patients to deeply understand what matter for these patients, thus being open to completely restructure their QoL categories. An SCI repairing strategy, which aims to comprehensively take care of the SCI-affected patients, cannot avoid from considering also the ethical layer embedded in SCI conditions. Raising awareness among professionals dealing, on a daily basis, with 
SCI-affected patients about the ethical challenges-principles and their potential threats-present within these scenarios may actually represent a fruitful step toward the improvement of a condition which, besides its inherent and highly severe complications, may nonetheless be more responsive to the patients' actual needs and more respectful of the comprehensive dimension of the patients themselves.

p0080 There is another comment to add to conclude. Many times, the SCI-affected patients have to be categorized as critically ill patients (for a definition of critically ill patients, see Refs. $[38,39]$ ), and thus, another layer of ethical questions arises if they should be involved as potential subjects in clinical research dealing with regenerative techniques. Unfortunately, even on this topic, there is no specific ethical literature. And this is a serious problem, given its increasing relevance. However, it is to say that there could be a good starting point from which a serious analysis could begin. This is given by the very small ethical discussion concerning (in general) clinical research involving critically ill patients (see Refs. [40-47]). Thus, a new ethical field could and should be opened for the benefit of the SCI-affected patients and of the researchers dealing with this pathology.

\section{References}

[1] DeVivo MJ. Causes and costs of spinal cord injury in the United States. Spinal Cord 1997;35(12):809.

[2] Devivo MJ. Epidemiology of traumatic spinal cord injury: trends and future implications. Spinal Cord 2012;50(5):365.

[3] Kraus JF, Franti CE, Riggins RS, Richards D, Borhani NO. Incidence of traumatic spinal cord lesions. J Chronic Dis 1975;28(9):471-92.

[4] McKinley WO, Seel RT, Hardman JT. Nontraumatic spinal cord injury: incidence, epidemiology, and functional outcome. Arch Phys Med Rehabil 1999;80(6):619-23.

[5] Carter GT. Spinal cord injury rehabilitation. Phys Med Rehabil Clin 2014;25(3):xiii-xiv.

[6] Field-Fote E. Spinal cord injury rehabilitation. FA Davis; 2009.

[7] Gibson CJ. Spinal cord injury rehabilitation. Arch Neurol 1985;42(2):113.

[8] Guenther LA. Spinal cord injury rehabilitation. J Phys Ther Educ 2009;23(2):40.

[9] Hammell KW. Spinal cord injury rehabilitation. Springer; 2013.

[10] Somers MF. Spinal cord injury: functional rehabilitation. NJ: Prentice Hall; 2001.

[11] Donnelly DJ, Popovich PG. Inflammation and its role in neuroprotection, axonal regeneration and functional recovery after spinal cord injury. Exp Neurol 2008; 209(2):378-88.

[12] Hausmann ON. Post-traumatic inflammation following spinal cord injury. Spinal Cord 2003;41(7):369.

[13] Panter SS, Yum SW, Faden AI. Alteration in extracellular amino acids after traumatic spinal cord injury. Ann Neurol 1990;27(1):96-9.

[14] DeVivo MJ, Black KJ, Stover SL. Causes of death during the first 12 years after spinal cord injury. Arch Phys Med Rehabil 1993;74(3):248-54.

[15] Garshick E, Kelley A, Cohen SA, Garrison A, Tun CG, Gagnon D, Brown R. A prospective assessment of mortality in chronic spinal cord injury. Spinal Cord 2005;43(7):408. 
[16] Michael J, Krause JS, Lammertse DP. Recent trends in mortality and causes of death among persons with spinal cord injury. Arch Phys Med Rehabil 1999;80(11):1411-9.

[17] Soden RJ, Walsh J, Middleton JW, Craven ML, Rutkowski SB, Yeo JD. Causes of death after spinal cord injury. Spinal Cord 2000;38(10):604.

[18] Strauss DJ, DeVivo MJ, Paculdo DR, Shavelle RM. Trends in life expectancy after spinal cord injury. Arch Phys Med Rehabil 2006;87(8):1079-85.

[19] Rosenfeld JV, et al. The ethics of the treatment of spinal cord injury: stem cell transplants, motor neuroprosthetics, and social equity. Top Spinal Cord Inj Rehabil 2008; 14(1):76-88.

[20] Priebe MM, Chiodo AE, Scelza WM, Kirshblum SC, Wuermser LA, Ho CH. Spinal cord injury medicine. 6. Economic and societal issues in spinal cord injury. Arch Phys Med Rehabil 2007;88(3):S84-8.

[21] The National Commission for the Protection of Human Subjects of Biomedical and Behavioral Research. The Belmont report: ethical principles and guidelines for the protection of human subjects of research. Washington, D.C.: Department of Health, Education and Welfare; 1978. DHEW Publication OS 78- 0012.

[22] Beauchamp TL, Childress JF. Principles of biomedical ethics. USA: Oxford University Press; 2012.

[23] National Spinal Cord Injury Statistical Center. Spinal cord injury facts and figures at a glance. Birmingham: Univ Alabama; 2006.

[24] Jang Y, Wang YH, Wang JD. Return to work after spinal cord injury: the contribution of functional independence. Arch Phys Med Rehabil 2005;86:681-6.

[25] Valtonen K, Karlsson AK, Alaranta H, Viikari-Juntura E. Work participation among persons with spinal cord injury and meningomyelocele. J Rehabil Med 2006;38: 192-200.

[26] Anderson D, Dumont S, Azzaria L, Bourdais ML, Noreau L. Determinants of return to work among spinal cord injury patients: a literature review. J Vocat Rehabil 2007; 27(1):57-68.

[27] Fougeyrollas P, Noreau L. Long-term consequences of spinal cord injury on social participation: the occurrence of handicap situations. Disabil Rehabil 2000;22(4): 170-80.

[28] Cardol M, Jong BD, Ward CD. On autonomy and participation in rehabilitation. Disabil Rehabil 2002;24(18):970-4.

[29] Van De Ven L, Post M, De Witte L, Van Den Heuvel W. Strategies for autonomy used by people with cervical spinal cord injury: a qualitative study. Disabil Rehabil 2008; 30(4):249-60.

[30] Garber SL, Gregorio TL. Upper extremity assistive devices: assessment of use by spinal cord-injured patients with quadriplegia. Am J Occup Ther 1990;44(2):126-31.

[31] Lee MY, Kirk PM, Yarkony GM. Rehabilitation of quadriplegic patients with phrenic nerve pacers. Arch Phys Med Rehabil 1989;70(7):549-52.

[32] Welch RD, Lobley SJ, O'Sullivan SB, Freed MM. Functional independence in quadriplegia: critical levels. Arch Phys Med Rehabil 1986;67(4):235-40.

[33] Carpenter C. The experience of spinal cord injury: the individual's perspectiveimplications for rehabilitation practice. Phys Ther 1994;74(7):614-28.

[34] Duggan CH, Dijkers M. Quality of life-peaks and valleys: a qualitative analysis of the narratives of persons with spinal cord injuries. Can J Rehabil 1999;12:179-90.

[35] Dijkers MP. Quality of life of individuals with spinal cord injury: a review of conceptualization, measurement, and research findings. J Rehabil Res Dev 2005;42(3):87. 
[36] Hammell KR. Spinal cord injury rehabilitation research: patient priorities, current deficiencies and potential directions. Disabil Rehabil 2010;32(14):1209-18.

[37] Hill MR, Noonan VK, Sakakibara BM, Miller WC. Quality of life instruments and definitions in individuals with spinal cord injury: a systematic review. Spinal Cord 2010;48(6):438

[37a] Simpson LA, et al. The health and life priorities of individuals with spinal cord injury: a systematic review. J Neurotrauma 2012;29:1548-55.

[38] Boniatti MM, et al. Characteristics of chronically critically ill patients: comparing two definitions. Clinics 2011;66(4):701-4.

[39] Carson SS. Definitions and epidemiology of the chronically critically ill. Respir Care 2012;57(6):848-58.

[40] Bigatello LM, George E, Hurford WE. Ethical considerations for research in critically ill patients. Crit Care Med 2003;31(3 Suppl.):S178-81.

[41] Chenaud C, Merlani P, Ricou B. Research in critically ill patients: standards of informed consent. Crit Care 2007;11(1):110.

[42] Cook D, et al. Clinical research ethics for critically ill patients: a pandemic proposal. Crit Care Med 2010;38(4 Suppl.):e138-42.

[43] Luce JM, et al. The ethical conduct of clinical research involving critically ill patients in the United States and Canada: principles and recommendations. Am J Respir Crit Care Med 2004;170(12):1375-84.

[44] Morrow B. Ethical considerations for critical care research. Southern African Journal of Critical Care 2015;31:34. https://doi.org/10.7196/SAJCC.2015.v31i2.249.

[45] Rincon F, Lee K. Ethical considerations in consenting critically ill patients for bedside clinical care and research. J Intensive Care Med. 2015;30:141-50. https://doi.org/ 10.1177/0885066613503279.

[46] Ecarnot F, et al. Ethical challenges involved in obtaining consent for research from patients hospitalized in the intensive care unit. Ann Transl Med 2017;5(Suppl. 4):S41.

[47] Estella A. Ethics research in critically ill patients. Med Intensiva 2018;42(4):247-54. 



\title{
Non-Print Items
}

\begin{abstract}
A spinal cord injury (SCI) is generally defined as a damage to any part of the spinal cord or nerves at the end of the spinal canal, affecting some body functions, depending on the specific site in which the injury occurred and the severity of the injury itself. While specialized literature has been primarily devoted to the medical aspects -in particular factors associated with as well as trends in mortality and causes of death of people affected by SCI-lesser attention has been paid to the ethical issues related to, and originating from, SCI situations. Against this backdrop and the lack of dedicated debates on the ethical issues, this contribution indicates and explores both the main aspects whose impact may be ethically sensitive and the possible solutions addressing them in the context of repairing strategies.
\end{abstract}

\section{Keywords:}

Autonomy, Beneficence, Biotechnological repairing strategies, Ethical issues on spinal cord injury, Justice, Research ethics. 


\title{
CHAPTER
}

\section{c0006 Elements of material science and polymeric biomaterials}

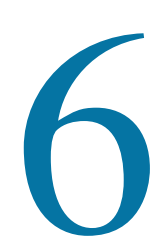

\author{
Gianni Pertici, PhD ${ }^{1,2}$ \\ Department of Technology Innovation, University of Applied Sciences and Arts of Southern \\ Switzerland, Ticino, Switzerland ${ }^{1}$; Industrie Biomediche Insubri SA, Ticino, Switzerland ${ }^{2}$
}

\section{s0010 Introduction}

p0010 Because physiopathology of spinal cord injury (SCI) is multiphasic and multifactorial, effective treatments will require the combinations of several strategies such as neuroprotection to oppose relapse, preparation of scaffolds for lost tissue replacement, and methods to stimulate axonal regrowth, synaptic plasticity, and inhibition of astrocytosis. Biomaterials show major advantages for spinal cord repair because of their structural and chemical versatility [1].

p0015 The term biomaterial was defined already in 1982 at the National Institutes of Health Consensus Development Conference (USA) as follows: "any substance (other than a drug) or combination of substances, synthetic or natural in origin, which can be used for any period of time, as a whole or as a part of a system which treats, augments, or replaces any tissue, organ, or function of the body [2].

p0020 Materials used in biomedical applications are in contact with the body and must satisfy strict requirements: they must be nontoxic and biocompatible and sometimes show adequate biomechanical properties and physical structure. The first biomaterials investigated were designed to achieve adequate mechanical strength and a relative state of "bioinertness." The results obtained were not satisfactory; therefore, second-generation materials are still today being developed. These materials are being designed specifically with the aim of being able to stimulate surfacespecific reactions or responses from the materials and particular cells within the body.

p0025 The most commonly used materials in clinical applications are natural and also metals, ceramics, synthetics, and composites.

p0030 Due to their mechanical properties, toughness, high strength, ductility, metals, and their alloys are used for implants in dental, orthopedic, and spinal applications. However, metallic implants may cause problems (irritation due to corrosion and erosion, second fracture of the operated bone caused by stress shielding, aseptic loosening of the implant) severe enough to lead to a second intervention to remove the device from the patient. 
p0035 Due to their chemical structure, ceramic and glasses (bioactive and non) have been used in several medical applications, in particular for bone replacement, but their brittleness makes them unsuitable for load-bearing applications.

p0040 Compared with the previous reported materials, biopolymers are relatively weak, but due to their versatility, ease of processing, and biocompatibility, many natural and synthetic polymers are widely and successfully used for replacement, support, augmentation, or fixation of living tissues.

p0045 Because single-phase materials sometimes do not possess specific properties, it is possible to create composites combining polymers, ceramics, and metals to obtain tailor-made devices for applications in various fields. In particular, polymeric composites are very attractive biomaterials because of their potentiality to become quite similar with most of the structural living tissues, composed of macromolecular composites [3].

p0050 According to their function in the biological environment, biomaterials can also be classified as stable, absorbable, bioactive materials and tissue engineering system.

p0055 The biostable materials such as metals, glasses, ceramics, nonbiodegradable polymers, and stable composites are designed to stay in the body for the maximum time maintaining their properties. They should be physiologically inert and cause only minimal response of the surrounding tissues. Biostable materials have wide application in permanent prostheses such as hip and tooth prostheses.

p0060 In many cases, the body shows sufficient regeneration capacity; in this case, it needs the temporary presence of a material to support, augment, and replace tissues and/or guide the regrowth: bioabsorbable (biodegradable or resorbable) polymers are adsorbed by the body and then disappear when; after healing, the device is no longer useful [4]. Biodegradable biomaterials are adopted to those medical devices in which tissue repair or remodeling is the target (as for example in artificial skin and cartilage repair) but not where long-term material stability is required (e.g., artificial heart, kidney, liver).

p0065 The term biologically active (bioactive) materials is referred to substances, which have the capability to conduct and induce the response to the biological system; they are designed to stimulate cell differentiation and proliferation, stimulate gene and tissue regeneration, and release bioactive molecules to respond body actively for restoring the compromised functionality of the organs [5]. This concept was first investigated during the bioglass development, glasses with the capability of bonding to living bone [6].

p0070 Biodegradable polymeric biomaterials are preferred candidates for developing therapeutic devices such as three-dimensional porous structures as scaffolds for tissue engineering and as controlled-release drug delivery vehicles. They are already widely adopted in different applications such as sutures, bone substitutes, and fixation screws. The reason is that they allow tissue growth into the matrix while eliminating the need for a second surgery to remove the implant [7-9]. 
p0075 During the past 35 years, the progressive transition from biostable prostheses to bioresorbable implants represents one of the most significant changing in biomaterials clinical research.

p0080 Biodegradable, bioresorbable, and bioabsorbable are often used for the same meaning; however, it could be better to clarify the different mechanism of action: the biodegradation process is connected to biological agents (as for example enzymes or microbes) as dominant components in degradation (disintegration, erosion, dissolution, etc.). The bioresorbable process includes polymers whose degradation products resorb in the body (metabolized). The bioabsorbable process involves polymers, which dissolve in biofluids and are eliminated without chain scission, such as poly(vinyl alcohol) and poly(ethylene glycol) [10].

\section{s0015 State of art}

p0085 In the body, several factors inhibit the spinal cord injury repair; thus, the regeneration of the central nervous system (CNS) was once thought to be unachievable. The standard approach, to repair damaged or diseased regions of the CNS, adopts tissue transplantation or peripheral nerve grafting; however, lack of donors and immunological problems are often occurred. Recently was demonstrated that axonal growth after spinal cord injury can be achieved when provided with the adequate substratum. Biomaterial scaffold is developed to create adequate substrates where cells are able to reproduce a specific tissue in a controlled way. The principal function of a scaffold is to manage the cell behavior, as for example migration, proliferation, differentiation, maintenance of phenotype, and apoptosis [11].

p0090 Main bioresorbable polymers adopted and investigated for the tissue regeneration in spinal cord injury are subsequently reported.

\section{s0020 Aliphatic polyesters (poly(lactic acid), polycaprolactone, poly(glycolic acid), etc.)}

p0095 This kind of polymers has a chemical structure determined by the presence of ester groups along the chains. The classification of these materials is derived by the production process [12]:

u0010 - naturally occurring polyesters;

$\mathrm{u} 0015$ - microbial polyesters;

u0020 - condensation polyesters;

u0025 - polyesters from ring-opening polymerization (ROP).

p0120 Shellac resin is the most representative inside the first group, is a mixture of monoesters and polyesters, and is scraped from the bark of the trees where the female lac bug (Kerria lacca) secretes it to form a tunnel [13]. This resin is used as coating for tablets, in particular in controlled-release capsules, since it stops the pill from breaking down in the stomach. 
p0125 In the second group, there are a large class of materials, known as "polyhydroxyalkanoates (PHAs)"; they are produced by bacterial fermentation of sugar and lipid [14]. This class is recently investigated for medical applications due to their high biocompatibility, mechanical properties, and biodegradability [15]. The first product arrived on the market is a suture made by poly(4-hydroxybutyrate) (P4HB) [16].

p0130 Poly-alpha-hydroxy acids such as poly(lactic acid) (PLA) and poly(glycolic acid) (PGA) are the most representative components of the next category. They are produced through polycondensation reaction involving one or more different monomers.

p0135 PLA, a hard and semicrystalline, high-molecular-weight polymer with thermoplastic properties, was introduced in 1955 [17]. It was recently shown that the implantation of a macroporous PLA hollow microfibers in the transected rat spinal cord promotes a modest axonal regeneration response. The use of macroporous PLA scaffolds for cell implantation into the injured spinal cord is promising as underlined by its biocompatibility with spinal nervous tissue [18].

p0140 Adopting the ring polymerization method, two enantiomeric forms of PLA, polyL-lactide (PLLA) and poly-D-lactide (PDLA), with opposite configurational structures, can be produced. PDLA is amorphous, resulting in a weaker and more rapidly degrading material [19].

p0145 Poly(L-lactic acid) (PLLA) is an aliphatic polyester with good biodegradability, biocompatibility, reasonably good mechanical properties, and processability in forming fibers. These fibers have been studied to reconnect transected peripheral nerves [20]. In vivo implantation of hollow fibers, made of high-molecular-weight PLLA seeded with cultured Schwann cells (SCs) (they have great potential to enhance axonal regrowth in nervous system), has shown abundant axonal growth into the tubes accompanied by wide vascularization. Moreover, grafts of poly(D,Llactic acid) scaffolds impregnated with brain-derived neurotrophic factors (BDNFs) enhanced axonal regeneration in rat spinal cord transection model [18]. For applications of biodegradable polymers, it is important to evaluate rates and mechanisms of their biodegradation. Different factors affect the degradation rate, such as molecular weight, enantiomeric composition, size and shape of the implant, environmental factors, and processing methods [21]. PLLA degrades in the slowest way of the different enantiomeric forms, and simple hydrolysis is the main degradation mechanism for PLLA [22].

p0150 PGA is the simplest linear aliphatic polyester, produced through polycondensation of glycolic acid. This material has a high melting point and high crystalline ratio, and it is insoluble in water. The degradation occurs by hydrolysis, producing glycolic acid, which is a natural metabolite that is recognized by the human body. Formaldehyde-cross-linked chitosan conduits containing PGA filaments were implanted into a 30-mm sciatic nerve gap in dogs; after 6 months, animals showed comparable functional histomorphometric recovery with the autograft control [23].

p0155 Poly ( $\mathcal{E}$-caprolactone) or simply polycaprolactone (PCL) is a flexible and easy-toprocess aliphatic polyester polymer. PCL is derived by chemical synthesis from crude oil. It has good water, oil, solvent, and chlorine resistance. PCL is degraded 
by hydrolysis of its ester linkages in physiological conditions. As described by Xu et al. (2006) [24], its hydrophobicity could lead to a surface erosion/degradation behavior, the complete breakdown of the polymer could occur into a period of 2 years depending on the degree of crystallinity [25]. It is widely diffused as a surgical material, as for example, in controlled drug delivery systems [26] and orthopedic implants [27]. PCL has a low glass transition temperature of around $-60^{\circ} \mathrm{C}$, a melting point of about $60^{\circ} \mathrm{C}$ and a high thermal stability. Due to this glass transition temperature, the amorphous phase displays high molecular mobility at body temperature. Schnell et al. produced PCL-based nanofibers as guidance to test their biocompatibility, cell adhesion, survival, migration, and also axonal growth and axonal guidance. Both types of electrospun fibers (100\% PCL and 25:75\% collagen/PCL) had demonstrated to support oriented neurite outgrowth and glial migration from dorsal root ganglion (DRG) explants (plucked from the spinal cords Sprague-Dawley rat embryos). SC migration, neurite orientation, and process formation of SCs, fibroblasts, and olfactory unsheathing cells were enhanced on collagen/PCL fibers, with respect to full PCL fibers [28].

\section{s0025 Natural biopolymers (dextran, chitosan, hyaluronic acid, agarose, and alginates)}

p0160 Polysaccharides are a group of biopolymers constituted by simple sugar monomers [29], obtained by animal, vegetal, or microbial extraction. These monomers (monosaccharides) are linked together by O-glycosidic bonds, which can be performed to any of the hydroxyl groups of a monosaccharide, giving polysaccharides the ability to form both linear and branched polymers. Physical properties, such as gelation and solubility, can be tailored through monosaccharide composition, chain shapes, and molecular weight. These materials show several interesting properties for medical applications such as hemocompatibility and nontoxicity [30].

p0165 Dextran is synthesized from sucrose by certain lactic acid bacteria, the best known being Leuconostoc mesenteroides, Lactobacillus, and Streptococcus mutans. The exact structure of each type of dextran depends on its specific microbial strain of origin. Dextrans are from the chemical point of view almost identical to amylose, but they have some branching of the polymer chain, which prevents this kind of stacking [31]. It is a complex branched glucan (polysaccharide derived from the condensation of glucose); its chains are of varying lengths (from 3 to $2000 \mathrm{kDa}$ ). In medicine, it is commonly used as an antithrombotic (antiplatelet) to reduce blood viscosity, as an antifibrin, and as plasma volume expansion in hypovolemia. It also enhances microcirculation by decreasing blood viscosity and impeding erythrocyte aggregation. Dextran hydrogels have been investigated for biomedical applications [32] and as drug delivery carriers [33]. It can be easily modified to add selective cell adhesion sites and growth factors [34]. Dextran-based bioactive coating technology was developed for silicon, polyimide, and gold, starting materials adopted to produce neural implants. In this study, the idea is to immobilize bioactive peptides to low cell-adhesive dextran monolayer coatings, to promote biospecific cell adhesion [35]. 
p0170 Chitosan is a linear polysaccharide composed of randomly distributed $\beta-(1-4)-$ linked D-glucosamine (deacetylated unit) and $N$-acetyl-D-glucosamine (acetylated unit). Chitosan (fully or partially deacetylated form of chitin) due to its properties was widely investigated for several applications such as skin, bone, and cartilage replacement. Chitin is a natural polysaccharide found in the shell of crustacean, cuticles of insects, and cell walls of fungi. This polysaccharide is biologically renewable, biodegradable, biocompatible, nonantigenic, nontoxic, and biofunctional [36]. The degree of deacetylation of commercial chitosan is usually between $70 \%$ and $95 \%$, and the molecular weight is from 10 to $1000 \mathrm{kDa}$ [37]. Physical and mechanical properties are dependent on the relative proportions of $N$-acetyl-D-glucosamine and D-glucosamine residues [38]. Chitosan molecule has amino and hydroxyl groups, which can be chemically tailored providing a high versatility and is metabolized by certain human enzymes (lysozymes). Its adhesive properties in a swollen state have been shown to persist during repeated contacts of chitosan and the substrate [37], which implicates that, in addition to the adhesion by hydration, many other mechanisms, such as hydrogen bonding and ionic interactions, might also have to be involved. It shows also a $\mathrm{pH}$-sensitive behavior as a weak polybase due to the large quantities of amino groups on its chain. This material dissolves quite fast at low $\mathrm{pH}$, whereas it is insoluble at higher $\mathrm{pH}$ values. The mechanism of $\mathrm{pH}$ sensitive swelling involves the protonation of amine groups of chitosan under low pH conditions. In addition, chitosan improves blood coagulation [39], accelerating wound healing [40,41]; thus, it can act as an ideal wound dressing as it exhibits a positive charge, film-forming capacity, mild gelation characteristics, and a strong tissue-adhesive property. As studies indicate that chitosan improves the functions of inflammatory cells such as polymorphonuclear leukocytes, macrophages, and fibroblasts promoting granulation and organization [39], it can be adopted in open and large wounds [42]. Chitosan is relatively fragile in its dry form and has to be blended with other materials or cross-linked prior to process into a nerve guide conduit (NGC). Xie et al. (2008) [43] produced conduits by blending chitosan with PLA and showed that sciatic nerve regeneration across a $10-\mathrm{mm}$ defect was superior to a silicone tube, but quite far from autografts in terms of both area and number of regenerated axons. As previously reported, formaldehyde-cross-linked chitosan conduits containing PGA filaments were implanted into a 30-mm sciatic nerve gap in dogs, obtaining interesting results.

p0175 Hyaluronic acid (HA) or hyaluronan is a linear polysaccharide that consists of alternating disaccharide units of $\alpha$-1,4-D-glucuronic acid and $\beta-1,3-N$-acetyl-Dglucosamine, linked by $\beta(1 \rightarrow 3)$ bonds [44]. HA is a naturally occurring nonsulfated glycosaminoglycan and a major macromolecular component of the intercellular matrix of most connective tissues [45]. Hyaluronan is widely distributed in vertebrates, and it is a component of the cell coat of many strains of bacteria. The role of HA is the mediation of physiological functions via interaction with binding proteins and cell surface receptors, including morphogenesis, regeneration, wound healing, and tumor invasion, as well as in the dynamic regulation of such interactions on cell signaling and behavior. Because of its viscoelastic nature, biocompatibility, and 
nonimmunogenicity, HA is widely adopted in cosmetic, medical, and pharmaceutical applications. HA is extracted from umbilical cord, rooster comb, synovial fluid, or vitreous humor. It can be also easily produced in large scales through microbial fermentation, from strains of bacteria such as streptococci [45], avoiding the risk of animal-derived pathogens. Several research works show that HA-based NGCs are completely biodegradable, noncytotoxic, and biocompatible. Concerning the healing of peripheral nervous system injury, topical application of HA gel improved axonal regrowth and reduced neural adhesion in rat sciatic nerve injury. Concerning spinal cord injury, cultured embryonic spinal cord tissue (incorporated in HA) transplanted into the gap after spinal cord transection showed a slight functional recovery. Other studies reported an HA-poly-D-lysine copolymer hydrogel introduced as a scaffold for brain defects healing in rats; further investigation may be performed to proper evaluate this potentiality in SCI repair [1].

p0180 Agarose is a purified linear galactan hydrocolloid isolated from agar or agarbearing marine algae. Structurally, it is a linear polymer consisting of alternating D-galactose and 3,6-anhydro-L-galactose units. Due to its physical properties, agarose dissolved into water forms a gel with a rigid net forming a threedimensional plastic and porous reticulum. Thus, agarose gel appears as nonpyrogenic, colorless, and transparent gel and viscous elastic at temperatures above $45^{\circ} \mathrm{C}$. It is slowly desorbed mainly by macrophage phagocytosis, and the specific enzymatic destruction of the polymer is done by means of $\alpha$-galactosidase, involved in cellular pentose cycle. Agarose is diffused as substrate in different biocompatibility tests (cytotoxicity, genotoxicity, mutagenesis, sensitivity, and subcutaneous implants) and as substrate for cell cultures [46]. Unstructured solid agarose hydrogels were implanted in rat spinal cord, avoiding immune or inflammatory response and maintaining adequate stability for more than 1-month. Neither axons nor cells penetrated such hydrogels, which suggested that agarose walls could effectively delineate pathways for regenerating axons. Freeze-drying process was performed to produce spongy agarose scaffolds, incorporated with neurotrophic factors, able to stimulate the axonal growth. Spongy agarose nerve guidance scaffolds with and without recombinant BDNF protein were tested in adult rat model for spinal cord injury. Cellular and axonal responses within and around scaffolds were evaluated 1 month after implantation. Scaffolds were found well integrated together with the host tissue, individual channels were penetrated by cells, and axons grew through scaffolds in an extremely linear manner. The further augmentation of BDNF protein displayed an increase in axons growing inside the scaffolds [47].

p0185 Alginates are block copolymers of L-guluronic and D-mannuronic acid residues connected by 1:4 glycosidic linkages. They are natural polysaccharide polymers isolated from brown seaweed (Phaeophyceae). The relative proportions of D-mannuronic and L-guluronic acids are species dependent and can be influenced by growth conditions. The ability to form hydrogels, in presence of calcium ions, together with their safety and biocompatibility, has conducted to a variety of applications in nutraceutical, cosmetic, and pharmaceutical products for more than 100 years [48]. Alginate can be processed via chemical and physical reactions to produce 
derivatives having different structures, properties, functions, and applications. They have been investigated for applications in liver, nerve, heart, and cartilage tissue engineering. Unfortunately, some drawbacks to alginate include mechanical weakness and poor cell adhesion. To overcome these limits, the strength and cell behavior of alginate have been enhanced by combination with other materials [11]. The major mechanism for degradation of alginate materials in humans is disintegration due to gradual exchange of gelling calcium ions with sodium. The relatively high concentration of calcium required to achieve high degree of cross-linking could be cytotoxic and detrimental to neural regeneration. To overcome this limit, ethylene diamine was adopted to covalently cross-link alginate that was further freeze-dried, obtaining a porous sponge. Then, it was implanted into a 7-mm sciatic nerve gap in rat model [49]. At 4.5 months after surgery, compound motor action potentials (CMAPs) were reported from the plantar foot muscles of rats, following stimulation at the sciatic notch. Subsequently, alginate foams were implanted alone or in combination with PGA mesh in the treatment of a 50-mm sciatic nerve defect in a cat model. After 8 months, regenerated nerve cables were reported in all animals, in both groups [50].

\section{s0030 Poly- $\beta$-hydroxybutyrate}

p0190 This homopolymer is a linear head-to-tail of (R)- $\beta$ hydroxybutyric acid, which forms crystalline cytoplasmic granules in the wide variety of bacteria. It is a wellknown biodegradable and biocompatible microbial polyester, which degrades slowly at body temperature and produces a nontoxic metabolite secreted by urine. PHB has been previously investigated as a wound healing device, showing capability to stimulate the cellular growth [11]. A bidimensional scaffold based on PHBcompressed fibers, coated by alginate and fibronectin, was implanted in adult rats and seeded with SCs. This solution has shown to reduce spinal cord cavitation as well as retrograde degeneration of injured spinal tract neurons [51].

\section{s0035 Poly(ether-ester)}

p0195 Poly(p-dioxanone) (PDS or PDO) is a colorless, crystalline, biodegradable synthetic polymer of multiple repeating ether-ester units. It is produced by ROP of the monomer $p$-dioxanone. The glass transition temperature is in between -10 and $0^{\circ} \mathrm{C}$, and crystallinity is around $55 \%$. The production process requires heat and an organometallic catalyst as zirconium acetylacetone or zinc L-lactate. It is commonly extruded to produce biodegradable sutures, but to avoid its spontaneous depolymerization, the lowest processing temperature is recommended. Its high flexibility is given by the ether oxygen group in the backbone of the polymer chain. It is degraded by hydrolysis, and the end products are mainly excreted in urine, the remainder being eliminated by digestive or exhaled as $\mathrm{CO}_{2}$ [52]. Rat DRGs were cultured onto PDS nanofibers for 10 days, to evaluate if the fibers alignment could influence the directional neuritic growth. Neurites showed a directional growth that led the fiber 
alignment of the scaffold. Due to degradation properties of PDS, a glia substrate was located onto the scaffold to improve the stability. Astrocytes seeded onto either aligned or random fibers showed a directional growth pattern similar to the underlying scaffold. These glia-seeded scaffolds, once cocultured with DRGs, gave the matrix alignment to and elated the intensive growth of the extending neurites. This study underlines the potential to generate an aligned scaffold that influences both the directionality and growth dynamics of DRG neurites [53].

\section{s0040 Poly(glycerol sebacic acid)}

p0200 Poly(glycerol sebacic acid) (PGS) is produced by polycondensation of sebacic acid with glycerol. This tough biocompatible polymer exhibits shape memory properties. Due to its low glass transition and melting temperatures, the temporary shape has to be kept below room temperature. PGS (known as biorubber) main features are interesting mechanical properties, rubber-like elasticity, and surface erosion biodegradation. PGS shows similar in vitro and in vivo biocompatibility to PLGA. PGS reported no harmful effect on SC metabolic activity, attachment, or proliferation and did not induce apoptosis [54].

\section{s0045 Poly(phosphoesters)}

p0205 Poly(phosphoesters) (PPEs) are a novel category, which possess some attractive properties. They contain a characteristic phosphoester linkage in the backbone, which is more easily degraded via hydrolysis in physiologically conditions than the ester linkage. Moreover, the degradation products are milder than the acids released in polyester hydrolysis. Biocompatibility of PPEs was tested adopting primary neurons and neuronal cell lines. The interesting results led [55] to test the performance of poly(bis(hydroxyethyl)terephthalate-ethyl phosphoester) as an NGC material. This scaffold, based on two different molecular weights of the polymer, was implanted into a 1-mm rat sciatic nerve gap, and regeneration was evaluated af ter 3 months. No visible inflammatory response was observed, and only a thin fibrous capsule was generated around the tubes. Of 12 rats, 11 implanted with the higher-molecular-weight NGC reported positive reflex response that it could lead to a sensory recovery. Conversely the scaffold with lower molecular weight showed poor performance. The biocompatibility of this polymer was assessed, and a second work was conducted adopting the delivery of microencapsulated nerve growth factor; the results showed that this system further improved peripheral nerve regeneration [56].

\section{s0050 Polyphosphazenes}

p0210 Poly[(organo)phosphazenes] are a class of extremely versatile polymers commonly used as shape memory polymers and as stimuli-responsive materials. They do not have carbon atoms in their backbone; their structure shows alternate sequences of 
phosphorus and nitrogen atoms, with two side groups of varied nature [57]. Polydichlorophosphazene is extremely hydrolytically unstable; however, it can be readily substituted with nucleophilic substituents to produce a wide range of stable polyphosphazenes with different properties. These characteristics are strongly dependent on the side substituents and their ratios. The synthetic flexibility of these materials can create water-soluble or high hydrophobic polymers. The biodegradation occurs via hydrolysis producing low-toxicity compounds including ammonia and phosphates. The hydrolytic stability can be managed to produce a wide spectrum of materials with differing degradation rates [58]. A novel electrically conductive and biodegradable polyphosphazene polymer containing parent aniline pentamer (PAP) and glycine ethyl ester (GEE) as side chains was synthesized through a nucleophilic substitution reaction for its potential application in nerve regeneration. Degradation studies carried out in phosphate-buffered saline at $37^{\circ} \mathrm{C}$ showed a mass loss of about $50 \%$ after 70 days. The polymer exhibited no cytotoxicity (evaluated with SCs), indicating that such scaffold exhibits interesting potential in tissue engineering for peripheral nerve regeneration [59].

\section{s0055 Polyacrylates}

p0215 Poly(2-hydroxyethyl methacrylate) (PHEMA) is one of the earliest polymers used as implantable material. Polymerized from 2-hydroxyethyl methacrylate using free radical precipitation, PHEMA forms a hydrogel biologically inert. One of the main concerns about PHEMA is its low biodegradability; their biodegradation, however, can be manipulated by modifying the porosity by photopatterning. PHEMA scaffolds show poor cell adhesion capability; to improve this characteristic, some peptides can be introduced to stimulate specific cellular response. A hydrogel based on copolymer of 2-hydroxyethyl methacrylate (HEMA) with 2-aminoethyl methacrylate (AEMA) was modified with laminin-derived peptides, thereby maintaining the geometry of the scaffold while introducing bioactive peptides that enhance cell adhesion and neurite outgrowth [60]. In addition, PCL fibers embedded in transparent cross-linked PHEMA hydrogel were produced for SC culture. Sonication of this compound in acetone produces the complete dissolution of PCL, leaving longitudinally oriented, fiber-free channels in the hydrogel. This scaffold shows the potential to enhance nerve regeneration after transection injuries of the spinal cord by increasing the available surface area and providing guidance to extending axons and invading cells [61].

\section{s0060 Polyanhydrides}

p0220 This group is characterized by anhydride bonds, which connect repeat units of the polymer backbone chain. These biodegradable materials are very reactive and hydrolytically unstable. This permits a surface erosion degradation, interesting characteristic for short-term release of drugs or bioactive agents. This material degrades in vivo into nontoxic diacid monomers. The anhydride bonds are water-labile; the 
polymer chain breaks apart at the anhydride bond. This results in two carboxylic acid groups, which are easily metabolized [62]. To promote nerve regeneration, aligned poly(lactic-co-glycolic acid)/bioactive polyanhydride fibrous substrates were produced with diameters of $600 \pm 200 \mathrm{~nm}$. SCs and dissociated rat DRGs shown elongated and proper proliferation in a direction parallel to orientated fibers with significantly longer SC process length and neurite outgrowth when compared with randomly orientated fibers. This study suggests that an aligned bioactive salicylic acid-based polyanhydride fibers could be an interesting promise as a supplement scaffold for the interior of a degradable polymer NGC [63].

\section{s0065 Poly(alkyl cyanoacrylates)}

p0225 These biodegradable and biocompatible polymers are widely used for tissueadhesive applications. Cyanoacrylates are acrylic monomers, which polymerize in the presence of water; the humidity in the air can activate the polymerization, producing long chains of poly(alkyl cyanoacrylates) (PACAs). They must be treated to avoid any spontaneous reaction [64]. The polymerization process follows free radical or anionic/zwitterionic mechanism. Stitch sutures are still the "gold standard" to hold a nerve guide in place, but they are also a cause of local inflammatory response. Glues of several kinds have been investigated as an alternative, but they are not easy to apply. In 2006, a new concept of nerve guide (known as "NeuroBox"), which is double-halved, rigid, and adopting cyanoacrylic glues, was tested onto a rat model. In the first group were implanted a NeuroBox to promote the regeneration of an experimentally produced $4 \mathrm{~mm}$ gap in the sciatic nerve. In the second one, the gap was left without repair. The last one was assembled by harvesting 10 contralateral intact nerves to document the normal anatomy. Semithin sections for visible light microscopy and ultrathin sections for TEM (transmission electron microscopy) were analyzed. Results showed that application of ethyl-2-cyanoacrylate directly to the epineurium produced no significant insult to the underlining nerve fibers nor impaired nerve regeneration. No regeneration occurred in the second group [65].

\section{s0070 Poly(propylene fumarate)}

p0230 Poly(propylene fumarate) (PPF) is an unsaturated linear polyester that can be subjected to cross-linking with a vinyl monomer, because of the presence of a double bond of the fumarate. PPF degrades by simple hydrolysis of the ester bonds into nontoxic products of propylene glycol, poly(acrylic acid-co-fumaric acid), and fumaric acid. Due to in situ characteristics (such as cross-linking, biodegradability, and injectability), it has been evaluated for fabricating preformed scaffolds [66]. They are produced by using ultraviolet (UV) laser stereolithography to photocross-link a linear, biodegradable polymer into a three-dimensional implant [67]. PPF scaffolds demonstrated to provide proper spatial maintenance and to promote repair of tissue defects. A multichannel PPF scaffold combined with collagen 
biomaterial was performed to produce a delivery system coated with neurotrophin-3 containing an engineered collagen-binding domain (CBD-NT3). The parallelaligned multichannel structure of this scaffold guides the direction of neural tissue regeneration across the lesion site and promotes reestablishment of bridge connectivity. This combination improves the inhibitory microenvironment and stimulates axonal and neuronal regeneration, survival of various types of functional neurons, and remyelination and synapse formation of regenerated axons after SCI. This innovative method effectively promotes neural tissue regeneration after transected spinal injury by providing a regrowth-supportive microenvironment and eventually induces functional improvement [68].

\section{s0075 Polyvinyl alcohol}

p0235 Poly(vinyl alcohol) (PVOH or PVA) is a water-soluble synthetic polymer. It is adopted in soft contact lenses, eye drops, embolization particles, tissue adhesion barriers, and cartilage replacement. PVA is white (colorless) and odorless. It is a linear synthetic polymer produced via partial or full hydrolysis of polyvinyl acetate to remove the acetate groups. The amount of hydroxylation can tailor the physical, chemical, and mechanical properties. Main characteristics exploited for medical applications are low protein adsorption, biocompatibility, high water solubility, and resistance to most organic solvents. PVA is normally cross-linked (obtaining hydrogels) to reduce the solubility in water. This method gives the structural stability to the hydrogel after the swelling in the presence of water. The degree of cross-linking affects the amount of fluid uptake and thus all the main properties [69]. The ability of oriented foams to support in vivo peripheral nerve regeneration was evaluated in a rat model. PLA foams were coated with PVA to improve wettability and cell adhesion. Biocompatibility of the PVA-coated foams was evaluated by measuring cell viability and neuritogenesis. A sciatic nerve gap of 5-mm length was bridged with this polymer implant showing macrotubes of $100 \mu \mathrm{m}$ diameter. Four weeks after implantation, the polymer foam was still in situ. It was well integrated and had restored an anatomic continuity. An intensive cell migration was reported at the outer surface of the polymer implant, but not within the macrotubes. This dense cellular microenvironment reported to stimulate axogenesis [70].

\section{s0080 Biopolymers technology s0085 Properties (degradability, toxicity, biocompatibility, etc.)}

p0240 The main characteristics of a bioresorbable device (such as mechanical properties and degradation time) can be designed to a specific application by tailoring the molecular weight, crystallinity, and hydrophilicity of the polymer. As for example, compositions with higher crystallinity and molecular weight improve mechanical properties and decrease resorption rates. On the contrary, higher amorphous and hydrophilic structures and a lower molecular weight resorb faster, often sacrificing 
mechanical strength. The degradation process involves polymer long chains, which are reduced into segments that can be absorbed by cells. In the human body, degradation follows two phases:

o0010 1) Segments are dissolved in extracellular fluids due to phagocytosis or metabolization.

00015 2) Fission of long chains occurs due to the hydrolysis and/or enzymatic attack.

p0255 It means that the main advantage of biodegradable polymers could be that the products of degradation are not toxic or completely eliminated from the body by natural metabolic pathway with minimal side effects [71]. The products of degradation process define the polymers biocompatibility. Because biomaterials implantation can reduce local $\mathrm{pH}$ affecting the integrity of the cells, they need to satisfy a number of prerequisites before that can be used in medical applications. To verify this feature, its components should be subjected to different tests [72], performed as recommended by various organizations and federations. These tests consist of a sequence of research protocols, described and regulated in many countries, for correct use of experimental materials under evaluation, thereby determining their safety for clinical application in human. The preliminary tests of cytotoxicity are conducting in vitro, whereas the final toxicity tests are performed in vivo (such as intracutaneous reactivity, sensitization, genotoxicity, skin irritation, allergenicity, and teratology) [73].

\section{s0090 Compounding, mechanical properties, and degradation time}

p0260 Due to an inhomogeneous molecular size, polymers are defined as "polydisperse"; this is a fundamental parameter to determine physical properties (such as strength, viscosity, transition temperature, and stiffness). In fact, an identification of an average value of molecular weight [74] became indispensable, as for example, a high value of this parameter corresponds to high mechanical resistance [75]. The degree of polymerization has intense effect on mechanical characteristics; as chain length increases, properties such as ductility, tensile strength, and hardness rise sharply and eventually level off. The Melt Flow Index (MFI) is an indirect measure of molecular weight, but it gives the ability of the material to flow under pressure at specific temperature [76]. In polymer melts, the flow viscosity at a fixed temperature rises rapidly with the increasing degree of polymerization. The use of highmolecular-weight materials (with low MFI) can bring to viscosity problems during common production processes (such as extrusion, spinning, and molding).

p0265 The twin-screw extrusion is the preferred method for compounding, but polymer processing is complicated because it is sensitive to heat and shear. The biopolymers compounding on this extruder with additives, fillers, impact modifiers, and/or other polymers promotes the functionality of the final product. This process is also affected to hydrolytic degradation if moisture is present in polymer molten. High temperatures, pressure, and moisture bring to a molecular-weight loss and decreased mechanical properties. To avoid degradation, desiccant and vacuum driers are 
required prior to melt blending. Moreover, bioresorbable compounds must be aircooled upon exiting the extruder. Pelletized strands destined for finished device processing must be thoroughly dried and properly packaged to avoid exposure to air moisture that can cause premature degradation [77].

\section{s0095 Extrusion and fiber manufacturing}

p0270 During the extrusion, a polymer melt is pumped through a shaping die and formed in a profile such as a film, a fiber, a tube, and a profile. Two main architectures for extruders are known: ram (piston) and screw. The ram adopts a positive displacement pump based on pressure, the volume is decreased, the fluid is moved, and the pressure increases. The screw one is a viscosity pump based on the gradient of friction between screw and barrel, generating the processing pressure and consequent fluid deformation. As anticipated, the twin-screw extruders are widely used as mixing and compounding devices, whereas the benefit of single-screw extruders is the precise metering capability. Plastifying extruders are normally fed with solid pellets or powder (Fig. 6.1). Feed screws can be divided in three zones: the conveying zone, the

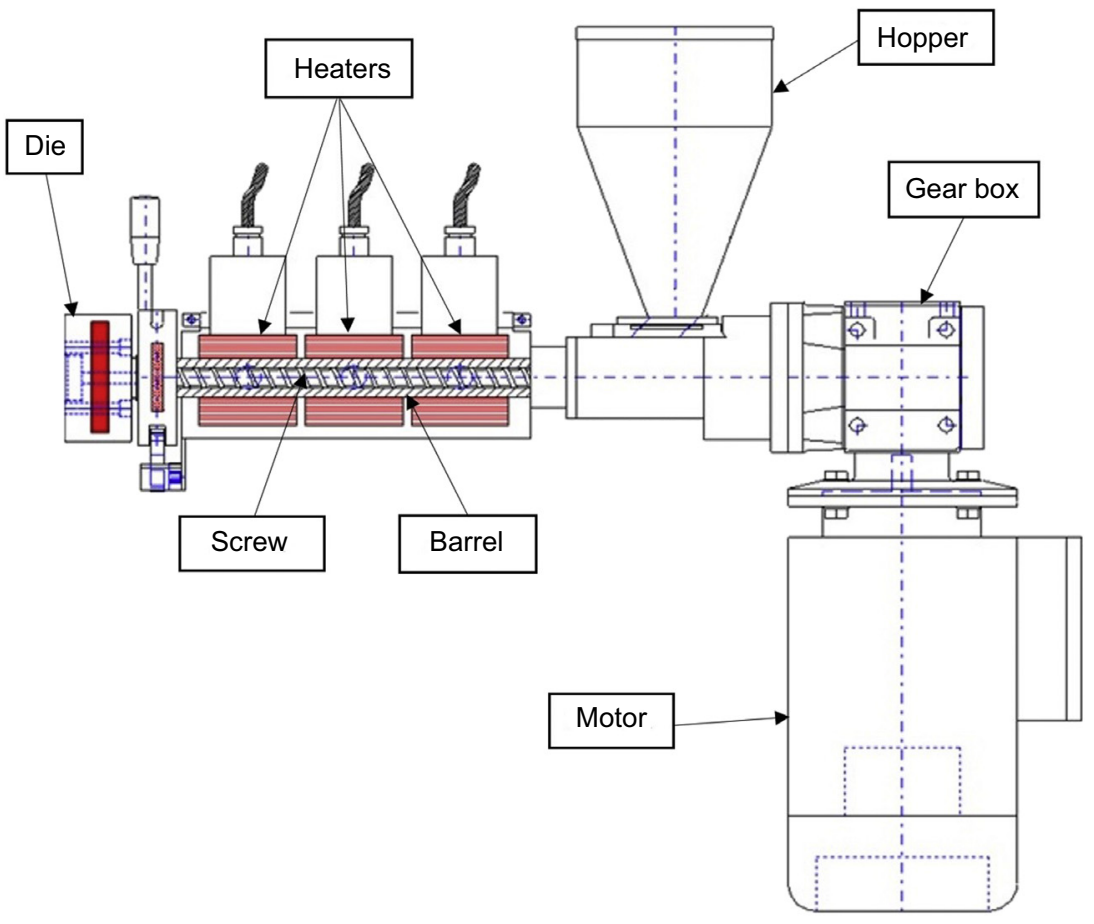

f0010 FIGURE 6.1

Schematic parts of a single-screw extruder. 
compressing zone, and the metering zone. Polymer particles fall through the hopper and then into the screw chamber. Here the conveying and compression processes start. Into the screw channel the polymer is melted and mixed to obtain the adequate isotropy, dispersion, and distribution; finally, it is pumped through the die [78]. The overall extrusion process can be divided in four steps:

u0030 - the particulate handling,

u0035 - melting,

u0040 - mixing, and

u0045 - pumping.

p0295 Because bioresorbable polymers are highly hydrophilic and tend to absorb moisture, they have to be handled with care. If these materials are not properly dry, they will not melt and recrystallize as expected, making molding and extrusion difficult. At the same time, they are heating sensible; in fact, the molecular structures can be damaged through exposure to excessive temperatures during drying. The presence of moisture makes even more severe the effect of heat on polymer degradation.

p0300 Biomaterials commonly show low melt temperature and a high crystallization temperature, and generally, they are extremely viscous, except at a tight temperature range between the two. Hence, there is a very small temperature range at which materials can be properly processed; in this specific area, the biomaterial is molten, the viscosity is at the proper level for injection molding or extrusion, and there will be no premature crystallization.

p0305 Due to their particular molecular structures, bioresorbable polymers are very limited in terms of the amount of time they can remain at molten without consequences, so cycle times must be kept within this range, which is often quite short. It is also critical to handle these kind of polymers properly before extrusion. PLA pellets should be kept sealed until the material is ready for drying/processing. Pellets stored in silos should be purged with dry air or nitrogen to minimize moisture absorption. Before the extrusion, it is generally recommended for this biomaterial to be dried in a desiccant dryer, to a moisture level lower than $250 \mathrm{ppm}$. Obviously any cross-contamination must be avoided so the extruder cleaning must be performed with accuracy. Moreover, all metal parts in this process that have stagnant flow areas (adapters, screen changers, and die) should be made of stainless steel and/or hard-chrome plated for increased corrosion resistance.

p0310 Another interesting technique able to produce nano- and microfibers is the electrospinning. It is deep investigated especially for biomedical applications (Fig. 6.2). A high electric voltage $(10-20 \mathrm{kV})$ is applied to a needle to charge the polymer (in solution or melt); the outgoing droplet is stretched due to the electrostatic repulsion that contrasts the surface tension. At a certain point (Taylor cone), a stream of liquid evaporates from the surface; if the liquid molecular cohesion is sufficient, there is no breakage of the stream (otherwise, droplets are electrosprayed) and a charged liquid jet is produced [79]. As the fiber dries in flight, the mode of current flow changes from ohmic to convective as the charge moves to the surface of the fiber. The jet is then stretched by a lashing process generated by electrostatic repulsion started 


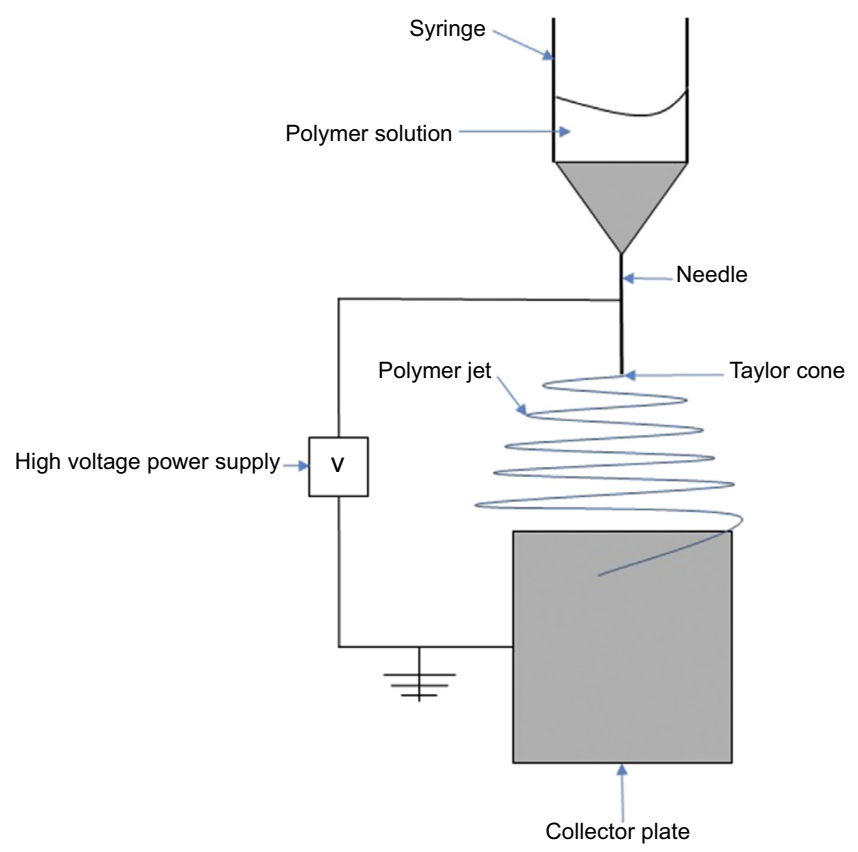

f0015 FIGURE 6.2

Schematic layout of electrospinning apparatus.

at small bends in the fiber, until it is finally deposited on the grounded collector. The elongation and thinning of the fiber resulting from this bending instability leads to the formation of uniform fibers [80].

\section{s0100 Molding}

p0315 In general, in polymer manufacturing technology, there are four molding processes adopted:

o0020 1) injection molding;

o0025 2) reactive injection molding;

o0030 3) compression molding; and

00035 4) rotational molding.

p0340 In polymeric devices manufacturing, the injection molding results absolutely in the most diffused technology [81], which permits to achieve complicated shapes with very precise dimensions. The desired shape is produced adopting a polymer melt pushed into a die; the melt is normally pressurized through a piston or a screw. As soon as the die is closed, the process starts with the injection of melt, and a holding pressure is maintained to compensate the material shrinkage. Residual moisture and/or gases are extracted by outpourings. A one-way valve is positioned at the end 


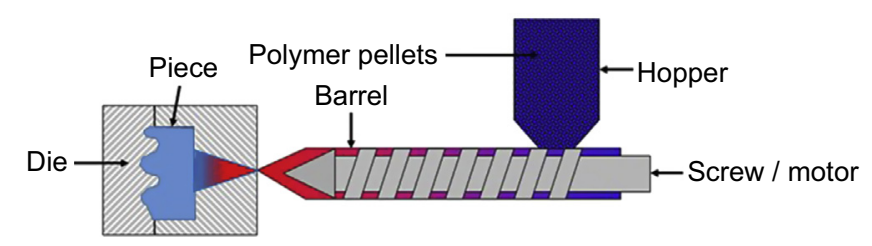

f0020 FIGURE 6.3

Schematic layout of injection molding.

of the screw to contrast the back flow. Before the ejection, the product is cooled (Fig. 6.3).

p0345 The second type of molding was developed to manage large dimension devices, where the maintenance of homogenous melt and a proper clamping pressure are critical. In this process, the polymerization is activated inside the mold, to adopt low-viscosity liquid. Not all materials can be processed; polyurethans are the most diffused.

p0350 The compression molding adopts a closed die process; it is the favorite choice for high-volume composite parts. A thermoplastic or partially polymerized thermosetting polymer is positioned in a heating chamber. The material charge is positioned into the die, the heated mold halves are closed, and up to $140 \mathrm{~atm}$ pressure is applied with a hydraulic press. During this process, the polymer undergoes complete polymerization or cross-linking. The production cycle is quite fast (maximum $5 \mathrm{~min}$ ), and it depends on size and thickness. The wastes are poor, but it is very difficult to produce objects with complicated shapes, as for example, undercuts [82] (Fig. 6.4).

p0355 The rotational molding (also known as rotomolding or rotocast) is a thermoplastic process designed for the production of hollow shapes. The powder or resin

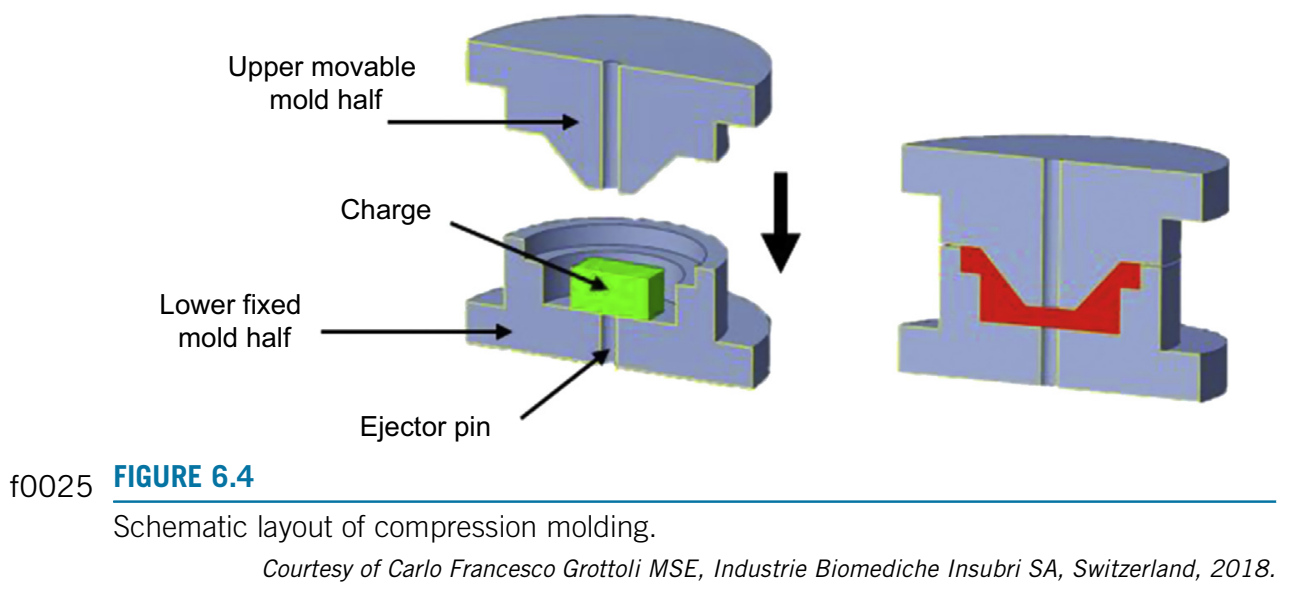


is distributed into a hollow mold, positioned inside an oven. Due to a biaxial rotation, the material melts and coats the entire surface of mold cavity. After the tool cooling, the piece is extracted. The main process is divided into six steps: induction (initial temperature rise), melting and sintering, bubbles removal and densification, precooling, polymer melt crystallization, and final cooling. The main rotocast disadvantage is the long heating and cooling time required [83].

\section{s0105 Coating}

p0360 During the past decade, the idea to improve medical devices capabilities adopting surface modification was deeply investigated. This approach is useful to manage cell adhesion, hydrophilicity, degradation, mechanical characteristics, lubricity, drugs elution, etc. It can be done adopting different techniques such as plasma treatment or substrate coating. In this case, a material in liquid status is commonly deposited onto a solid substrate. The lower the surface tension of the liquid, the easier it will be to create an adequate wet film from it. The difference between the surface tension of a coating and the surface energy of a solid substrate affects how the liquid coating flows onto the substrate. It is also responsible for the quality of the bond between the substrate and the dry film. If the surface tension of the substrate is lower than the surface tension of the coating, it will not spread out and create a film. A continuous augmentation in the coating surface tension will result in a proper wetting and a better dry film adhesion. In detail whether a liquid coating will spread across a solid substrate can be determined from the surface energies of the involved materials by using this equation:

$$
\mathrm{S}=\gamma \mathrm{SA}+(\mathrm{CA}-\mathrm{SC} \gamma)
$$

where

$\mathrm{u} 0050 \quad \mathrm{~S}$ is the coefficient of spreading.

u0055 $\gamma \mathrm{SA}$ is the surface energy of the substrate in air.

u0060 $\quad \gamma$ CA is the surface energy of the liquid coating in air.

u0065 $\gamma \mathrm{SC}$ is the interfacial energy between the coating and the substrate.

p0385 If $S$ will result positive, the liquid covers the surface and the coating adheres properly. In the negative case, the adhesion will result poor and the coating will not completely cover the substrate [84].

\section{s0110 Solvent casting}

p0390 This technique is normally used for the production of polymeric films. It starts with the polymeric dissolution in a solvent, adopting the proper parameters, for the creation of a viscous solution. Sometimes, the use of additives is required to achieve specific characteristics. Then heaters or vacuum pumps are adopted to avoid the creation of air bubbles inside the film. At this step, the solution is filtered and deposited onto a belt. The tension of this last one is managed to guarantee flatness, no 


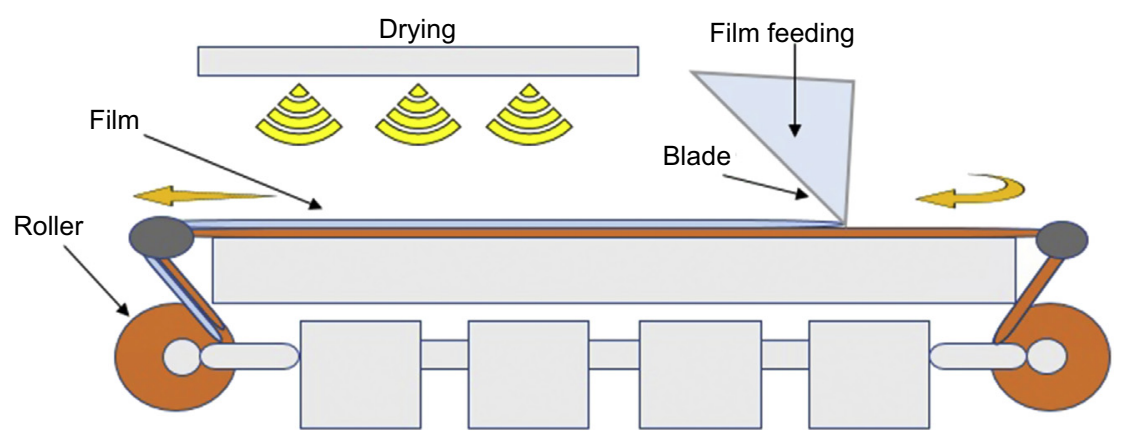

f0030 FIGURE 6.5

Schematic layout of solvent-casting apparatus.

Courtesy of Carlo Francesco Grottoli MSE, Industrie Biomediche Insubri SA, Switzerland, 2018.

vibrations, and avoid the possible belt thermal expansion. To ensure the proper thickness, a spreader is adopted for the solution deposition. The liquid film is normally dried by hot air stream [85] (Fig. 6.5).

\section{s0115 Foaming}

p0395 This technology is applied to produce polymeric porous structure; it can be divided into four steps:

00040 1. Creation of a homogeneous polymer/gas mixture

00045 2. Nucleation of cells

00050 3. Cell growth

o0055 4. Cell stabilization

p0420 During this first step, a blowing agent has to diffuse into the melt or solid bead and remain in the polymer-gas solution. To achieve the bubbles formation (cell nucleation), the gas saturation has to be reached (at a determined pressure and temperature) through the solubility reduction. Normally, some powders are introduced to promote the nucleation. During cell growth, the gas diffuses out of the melt to the nucleation sites. The supersaturation caused by the pressure drop or temperature increase is the driving force of this process. To obtain foams with a favorable cell size, distribution, and properties, the morphology must be stabilized and cell growth has to cease; otherwise, cell coalescence or coarsening takes place and damages the final foam morphology [86].

\section{s0120 Hydrogels manufacturing}

p0425 Hydrogels constitute a group of polymeric materials; their hydrophilic and swelling capabilities render them able to hold large amounts of water and exhibit different 
tissue-like properties. Hence, they have been deeply investigated for applications in soft-tissue regeneration. Some of them are an interesting solution especially for their temperature-dependent physical properties. As for example, they can be injectable at room temperature and become rigid and resistant at body temperature [87]. Hydrogels are formed by self-assembling through noncovalent interactions with ionic species, through covalent cross-linking triggered by chemical reaction or through thermal transition. The swelling properties can be managed by the surface modification to react to a specific stimulus (temperature, $\mathrm{pH}$, molecules, electric signals, ionic strength, and magnetic signals) [88]. These materials show a viscoelastic nature and water-filled interiors; these special characteristics provide an adequate microenvironment for cells encapsulation, delivery, and tissue formation [89]. Natural polymers (such as collagen, alginate, fibrin, hyaluronic acid, chitosan, and dextran) are deeply investigated due to their biological characteristics, as for example, cell recruiting, modulation of inflammatory microenvironment, and promotion of neovascularization. On the other side, the limits are represented by poor mechanical performance, hard purification, disease transmission, and immunogenic risks.

p0430 Due to these disadvantages, biocompatible and biodegradable synthetic polymers were also introduced. As for example, poly(ethylene glycol) (PEG) is adopted as an excipient in many pharmaceutical products (such as lubricating eye drops) [90].

\section{s0125 Micro- and nanoparticles manufacturing}

p0435 These kind of products are intensively exploited in the drug delivery field; in particular, they are adopted to manage the pharmacokinetic and/or pharmacodynamic properties of different types of drug molecules [91]. Different approaches are used for the production of polymeric bioresorbable micro- (in the range of $1-1000 \mu \mathrm{m}$ ) and nanoparticles (in the range of $10-1000 \mathrm{~nm}$ ), as for example, solvent evaporation, salting out, dialysis, supercritical fluid technology, micro- and miniemulsion, and interfacial polymerization. The technology adopted is directly connected to several factors, as for example, active agents released, particle size and distribution desired, and starting materials. [92]. Commonly, chitosan, gelatin, albumin, and sodium alginate are the natural materials used for the production of such particles; however, also many synthetic polymers are interested: polylactides, polyglycolides, polycaprolactone, poly(ethylene glycol), poly(vinyl alcohol), poly(lactide co-glycolides), etc.

p0440 The principal methods adopted for particles production are as follows:

00060 1) Dispersion of preformed polymer (solvent evaporation, salting out, dialysis, emulsification, supercritical fluid, and nanoprecipitation).

00065 2) Monomers polymerization (controlled/living radical polymerization, emulsion, micro and miniemulsion, and interfacial polymerization). 
p0455 There are three general physicochemical mechanisms of drug release [93]:

00070 1) Hydration and swelling of polymer particles, followed by release through diffusion.

00075 2) Enzymatic reaction and consequent polymeric disruption and/or degradation, therefore drug release from the inner cavity.

o0080 3) Dissociation of the bioactive molecule from polymer and its release from the swelled particles.

\section{s0130 Additive manufacturing}

p0475 This technology adopts the layer-by-layer manufacturing to create polymeric products. Starting from a three-dimensional model, the piece is printed slices by slices [94]. In medical applications, it is commonly used to replicate organs or tissues in a larger scale, to evaluate properly the disease, before the surgery. Moreover, cellfree scaffolds were adopted for bone and osteochondral tissue reconstruction [95]. The target still remains to create three-dimensional CAD-CAM (computer-aided design and computer-aided manufacturing) living tissues adding stem cells harvested from the patient [96].

\section{s0135 Composite materials}

p0480 To overcome the limits offered by standard products, composite materials are commonly produced combining two or more constituent material with significantly different properties. Each individual component remains separate and distinct within the finished structure; however, the result shows improvement characteristics. In medicine, in particular in tissue engineering, this technology is recently investigated to improve both biological and mechanical performance of implantable medical devices. The target is to replicate the native tissue improving the healing capabilities of standard materials [97].

\section{s0140 Sterilization}

p0485 Due to the possible degradation of medical polymers, the sterilization is a sensible and critical step. Steam autoclaving frequently used in hospital is not a good option for bioresorbable polymers because of the risk of polymer degradation caused by moisture and heating. In addition, gamma-radiation normally applied to packaged medical products could negatively affect biopolymers reducing the molecular weight due to the chemical chains breakage. Beta-radiation (high-speed electrons), is less aggressive with respect to the previous one, but low penetration power limits the choice of packaging. Ethylene oxide gas normally is well tolerated by biodegradable polymers; however, this method is highly toxic and requires long period of degassing and specific analysis to guarantee no residuals inside the device. Due to these limits, methods such as plasma and supercritical carbon dioxide are deeply investigated [10]. 


\section{s0145 Future trends}

p0490 As previously described, bioresorbable polymers are versatile biomaterials that can be engineered to achieve specific requirements. These materials were deeply investigated in spine surgery where a limited existence of an implant can provide better performance, when compared with permanent ones. Spinal cord injuries often lead to persistent neurological dysfunction due to failure in axon regeneration. Nguyen and coworkers introduced a biofunctionalized platform to provide contact guidance and sustained nonviral drug/gene delivery for nerve injury repair. This innovative scaffold is made by aligned poly( $\varepsilon$-caprolactone-co-ethyl ethylene phosphate) (PCLEEP) electrospun nanofibers distributed in a three-dimensional configuration within a collagen hydrogel. To evaluate the efficacy of this platform, in vivo experiments were performed in rat model. In particular, aligned axon regeneration was observed as early as 1 week postinjury. Moreover, no excessive inflammatory response and scar tissue formation was observed. These results demonstrate the potential of this kind of scaffold for neural tissue engineering applications. Other researchers describe an interesting animal trial of a drug therapy for chronic spinal cord injury, which led to an improvement in limb coordination during walking [98]. It was performed using dogs that had suffered naturally occurring spinal cord injuries. They vary in terms of severity and timing; these dogs offer a good opportunity to evaluate this therapy in a heterogeneous population of subjects. These kind of injuries represent similar conditions that typically occur in human trauma. The therapy is based on chondroitinase $\mathrm{ABC}$, which is a bacterial enzyme that removes sugar side chains from extracellular matrix molecules including chondroitin sulfate proteoglycans. These are found within the intact and injured nervous system and are inhibitors of axonal growth. It is already reported that the degradation of chondroitin sulfate sugar side chains with chondroitinase $A B C$ improves axon growth in vitro and in vivo. This therapy has also been shown to enhance neuroplasticity in different spinal networks, leading to functional improvements in breathing, walking, and grasping. In conclusion, this study confirms that it is feasible and effective to evaluate candidate therapies for spinal cord injury in well-powered, blinded, randomized, controlled trials using a heterogeneous and relatively large group of naturally injured large animals. These data show that this therapy is safe and effective in walking improvement. The next step will be to move on to clinical trials to achieve useful therapies for humankind.

\section{References}

[1] Nomura H, Tator $\mathrm{CH}$, Shoichet MS. Bioengineered strategies for spinal cord repair. J Neurotrauma 2006;23(3/4):496-507.

[2] Galletti PM, Boretos JW. Report on the consensus development conference on "clinical applications of biomaterials," 1-3 November 1983. J Biomed Mater Res 1983;17: $539-55$. 
[3] Perale G, Hilborn J. Bioresorbable polymers for biomedical applications. From fundamentals to translational medicine. Woodhead Publishing; 2016, ISBN 9780081002629. p. 628.

[4] Törmälä P, Laiho J, Helevirta P, Rokkanen P, Vainionpää S, Böstman O, Kilpikari J. Resorbable surgical devices. In: Proceedings of the Vth international conference on polymers in medicine surgery. The Netherlands: Leeuwenhorst Congress Center; 1986.

[5] Zhao X, Courtney JM, Qian H. Bioactive materials in medicine: design and applications. Woodhead Publishing; 2011, ISBN 9781845696245. p. 288.

[6] Hench LL, Splinter RJ, Allen WC, Greenlee TK. Bonding mechanisms at the interface of ceramic prosthetic materials. J Biomed Mater Res Symp 1971;5(6):117-41.

[7] Agrawal CM, Ray RB. Biodegradable polymeric scaffolds for musculoskeletal tissue engineering. J Biomed Mater Res 2001;55(2):141-50.

[8] Kellomäki M, Niiranen H, Puumanen K, Ashammakhi N, Waris T, Törmälä P. Bioabsorbable scaffolds for guided bone regeneration and generation. Biomaterials 2000; 21:2495-505.

[9] Nair LS, Laurencin CT. Biodegradable polymers as biomaterials. Prog Polym Sci 2007; 32:762-98.

[10] Buchanan F. Degradation rate of bioresorbable materials: prediction and evaluation. Cambridge, UK: Woodhead; 2008.

[11] Tabesh H, Amoabediny G, Salehi Nik N, Heydari M, Yosefifard M, Ranaei Siadat SO, Mottaghy K. The role of biodegradable engineered scaffolds seeded with Schwann cells for spinal cord regeneration. Neurochem Int 2009;54:73-83.

[12] Albertsson AC, Varma IK. Aliphatic polyesters. In: Biopolymers online, Part 4. Polyesters; 2005.

[13] Wang CH, Li J, Teo CS, Lee T. The delivery of BCNU to brain tumors. J Control Release 1999;61:21-41.

[14] Zinn M, Witholt B, Egli T. Occurrence, synthesis and medical application of bacterial polyhydroxyalkanoate. Adv Drug Deliv Rev 2001;53(1):5-21.

[15] Wu Q, Wang Y, Chen GQ. Medical application of microbial biopolyesters polyhydroxyalkanoates. Artif Cells Blood Substit Immobil Biotechnol 2009;37(1): $1-12$.

[16] Brigham CJ, Sinskey AJ. Applications of polyhydroxyalkanoates in the medical industry. Int J Biotech Wellness Industr 2012;1:53-60.

[17] Schneider AK. Polymers of high melting lactide. United States Patent 2703316. 1955.

[18] Patist CM, Mulder MB, Gautier SE, Maquet V, Jerome R, Oudega M. Freeze-dried pol$\mathrm{y}(\mathrm{D}, \mathrm{L}-\mathrm{lactic}$ acid $)$ macroporous guidance scaffolds impregnated with brain-derived neurotrophic factor in the transected adult rat thoracic spinal cord. Biomaterials 2004;25(9):1569-82.

[19] Majola A, Vainionpää S, Vihtonen K, Mero M, Vasenius J, Törmälä P, Rokkanen P. Absorption, biocompatibility, and fixation properties of polylactic acid in bone tissue: an experimental study in rats. Clin Orthop 1991;268:260-9.

[20] Evans GR, Brandt K, Widmer MS, et al. In vivo evaluation of poly(L-lactic acid) porous conduits for peripheral nerve regeneration. Biomaterials 1999;20:1109-15.

[21] Nakamura T, Hitomi S, Watanabe S, Shimizu Y, Jamshidi K, Hyon SH, Ikada Y. Bioabsorption of polylactides with different molecular properties. J Biomed Mater Res 1989;23(10):1115-30.

[22] Zhang X, Hua H, Shen X, Yang Q. In vitro degradation and biocompatibility of poly(Llactic acid)/Chitosan fiber composites. Polymer 2007;48:1005-11. 
[23] Wang X, Hu W, Cao Y, Yao J, Wu J, Gu X. Dog sciatic nerve regeneration across a 30$\mathrm{mm}$ defect bridged by a chitosan/PGA artificial nerve graft. Brain 2005;128:1897-910.

[24] Xu XJ, Sy JC, Shastri VP. Towards developing surface eroding poly(a-hydroxy acids). Biomaterials 2006;27:3021-30.

[25] Prabhakar RL, Brocchini S, Knowles J. Effect of glass composition on the degradation properties and ion release characteristics of phosphate glass-polycaprolactone composites. Biomaterials 2005;26:2209-18.

[26] Perez MH, Zinutti C, Lamprecht A, Ubrich N, Aster A, Hoffman M, Bodmeier R, Maincent $\mathrm{P}$. The preparation and evaluation of poly $(\mathcal{E}$-caprolactone) microparticles containing both a lipophilic and a hydrophilic drug. J Control Release 2000;65:429-38.

[27] Lowry KJ, Hamson KR, Bear L, Peng YB, Calaluce R, Evans ML, Anglen JO, Allen WC. Polycaprolactone/glass bioabsorbable implant in a rabbit humerus fracture model. J Biomed Mater Res 1997;36:536-41.

[28] Schnell E, Klinkhammer K, Balzer S, Brook G, Klee D, Dalton P. Guidance of glial cell migration and axonal growth on electrospun nanofibers of poly-e-caprolactone and a collagen/poly-e-caprolactone blend. Biomaterials 2007;28:3012-25.

[29] Nishinari K, Takahashi R. Interaction in polysaccharide solutions and gels. Curr Opin Colloid Interface Sci 2003;8:396-400.

[30] Venugopal J, Ramakrishna S. Applications of polymer nanofibers in biomedicine and biotechnology. Appl Biochem Biotechnol 2005;125:147-57.

[31] Walstra P. Physical chemistry of foods. NY, USA: Marcel Dekker Inc.; 2003. p. 137-201.

[32] Ferreira L, Gil MH, Cabrita AM, Dordick JS. Biocatalytic synthesis of highly ordered degradable dextran-based hydrogels. Biomaterials 2005;26:4707-16.

[33] Zhang Y, Chu CC. Biodegradable dextran-polylactide hydrogel networks: their swelling, morphology and the controlled release of indomethacin. J Biomed Mater Res 2002;59:318-28.

[34] Klaver CL, Caplan MR. Bioactive surface for neural electrodes: decreasing astrocyte proliferation via transforming growth factor beta-1. J Biomed Mater Res A 2007; 81(4):1011-6.

[35] Massia SP, Holecko MM, Ehteshami GR. In vitro assessment of bioactive coatings for neural implant applications. J Biomed Mater Res 2004;68A:177-86.

[36] Khor E, Lim LY. Implantable applications of chitin and chitosan. Biomaterials 2003;24: 2339-49.

[37] George M, Abraham TE. Polyionic hydrocolloids for the intestinal delivery of protein drugs: alginate and chitosan - a review. J Control Release 2006;114:1-14.

[38] Huang Y, Onyeri S, Siewe M, Moshfeghian A, Madihally SV. In vitro characterization of chitosan-gelatin scaffolds for tissue engineering. Biomaterials 2005;26:7616-27.

[39] Okamoto Y, Yano R, Miyatake K, Tomohiro I, Shigemasa Y, Minami S. Effects of chitin and chitosan on blood coagulation. Carbohydr Polym 2003;53:337-42.

[40] Kweon DK, Song SB, Park YY. Preparation of water-soluble chitosan/heparin complex and its application as wound healing accelerator. Biomaterials 2003;24:1595-601.

[41] Ueno H, Mori T, Fujinaga T. Topical formulations and wound healing applications of chitosan. Adv Drug Deliv Rev 2001;52:105-15.

[42] Alemdaroglu C, Degim Z, Celebi N, Zor F, Ozturk S, Erdogan D. An investigation on burn wound healing in rats with chitosan gel formulation containing epidermal growth factor. Burns 2006;32:319-27. 
[43] Xie F, Li QF, Gu B, Liu K, Shen GX. In vitro and in vivo evaluation of a biodegradable chitosan-PLA composite peripheral nerve guide conduit material. Microsurgery 2008; 28:471-9.

[44] Laurent TC, Laurent UBG, Fraser JRE. Functions of hyaluronan. Ann Rheum Dis 1995; $54: 429-32$.

[45] Liao YH, Jones SA, Forbes B, Martin GP, Brown MB. Hyaluronan: pharmaceutical characterization and drug delivery. Drug Deliv 2005;12:327-42.

[46] Varoni E, Tschon M, Palazzo B, Nitti P, Martini L, Rimondini L. Agarose gel as biomaterial or scaffold for implantation surgery: characterization, histological and histomorphometric study on soft tissue response. Connect Tissue Res 2012;53(6):548-54.

[47] Stokols S, Tuszynski M. Freeze-dried agarose scaffolds with uniaxial channels stimulate and guide linear axonal growth following spinal cord injury. Biomaterials 2006; 27:443-51.

[48] Donati I, Paoletti S. Material properties of alginates. Alginates: Biol Appl 2009;13: $1-53$.

[49] Suzuki K, Suzuki Y, Tanihara M, Ohnishi K, Hashimoto T, Endo K, Nishimura Y. Reconstruction of rat peripheral nerve gap without sutures using freezedried alginate gel. J Biomed Mater Res 2000;49:528-33.

[50] Sufan W, Suzuki Y, Tanihara M, Ohnishi K, Suzuki K, Endo K, Nishimura Y. Sciatic nerve regeneration through alginate with tubulation or nontubulation repair in cat J Neurotrauma 2001;18:329-38.

[51] Novikov LN, Novicova LN, Mosahebi A, Wiberg M, Terenghi G, Kellerth J. A novel biodegradable implant for neuronal rescue and regeneration after spinal cord injury. Biomaterials 2002;23:3369-76.

[52] Kumbar S, Laurencin C, Deng M. Natural and synthetic biomedical polymers. Elsevier Science; 2014

[53] Chow WN, Simpson DG, Bigbee JW, Colello RJ. Evaluating neuronal and glial growth on electrospun polarized matrices: bridging the gap in percussive spinal cord injuries. Neuron Glia Biol 2007;3(2):119-26.

[54] Sundback CA, Shyn JY, Wang Y, Faquin WC, Langer RS, Vacanti JP, Hadlock TS. Biocompatibility analysis of a poly(glycerol sebacate) as a nerve guide material. Biomaterials 2005;26:5454-64.

[55] Wang S, Wan AC, Xu X, Gao S, Mao HQ, Leong KW, Yu H. A new nerve guide conduit material composed of a biodegradable poly(phosphoester). Biomaterials 2001;22: 1157-69.

[56] Xu X, Yee W-C, Hwang PYK, Yu H, Wan ACA, Gao S, Boon K-L, Mao H-Q, Leong KW, Wang S. Peripheral nerve regeneration with sustained release of poly(phosphoester) microencapsulated nerve growth factor within nerve guide conduits. Biomaterials 2003;24:2405-12.

[57] Mark JE, Allcock HR, West R. Inorganic polymers. New York: Oxford University Press, Inc.; 2005.

[58] Teasdale I, Brüggemann O. Polyphosphazenes: multifunctional, biodegradable vehicles for drug and gene delivery. Polymers 2013;5:161-87.

[59] Zhang Q, Yan Y, Li S, Feng T. The synthesis and characterization of a novel biodegradable and electroactive polyphosphazene for nerve regeneration. Mater Sci Eng C 2010; 30(1):160-6.

[60] Yu T, Shoichet MS. Guided cell adhesion and outgrowth in peptide-modified channels for neural tissue engineering. Biomaterials 2005;26:1507-14. 
[61] Flynn L, Dalton PD, Shoichet MS. Fiber templating of poly(2hydroxyethylmethacrylate) for neural tissue engineering. Biomaterials 2003;24: 4265-72.

[62] Saltzman WM. Drug delivery: engineering principles for drug therapy. Oxford [England]; New York: Oxford University Press; 2001.

[63] Griffin J, Delgado-Rivera R, Meiners S, Uhrich KE. Salicylic acid-derived poly(anhydride-ester) electrospun fibers designed for regenerating the peripheral nervous system. J Biomed Mater Res A 2011;97(3):230-42.

[64] Dossi M, Storti G, Moscatelli D. Synthesis of poly(alkyl cyanoacrylates) as biodegradable polymers for drug delivery applications. Macromol Symp 2010;289:124-8.

[65] Merolli A, Marceddu S, Rocchi L, Catalano F. In vivo study of ethyl-2-cyanoacrylate applied in direct contact with nerves regenerating in a novel nerve-guide. J Mater Sci Mater Med 2010;21:1979-87.

[66] Lee KW, Wang S, Lu L, Jabbari E, Currier BL, Yaszemski MJ. Fabrication and characterization of poly(propylene fumarate) scaffolds with controlled pore structures using 3dimensional printing and injection molding. Tissue Eng 2006;12(10).

[67] Fisher JP, Vehof JWM, Dean D, van der Waerden JPCM, Holland TA, Mikos AG, Jansen JA. Soft and hard tissue response to photocrosslinked poly(propylene fumarate) scaffolds in a rabbit model. J Biomed Mater Res 2002;59(3):547-56.

[68] Chen X, Zhao Y, Li X, Xiao Z, Yao Y, Chu Y, Farkas B, Romano I, Brandi F, Dai J. Functional multichannel poly(propylene fumarate)-collagen scaffold with collagenbinding neurotrophic factor 3 promotes neural regeneration after transected spinal cord injury. Adv Healthc Mater 2018;7(14):1-13.

[69] Baker MI, Walsh SP, Schwartz Z, Boyan BD. A review of polyvinyl alcohol and its uses in cartilage and orthopedic applications. J Biomed Mater Res Part B 2012;100B: 1451-7.

[70] Maquet V, Martin D, Malgrange B, Franzen R, Schoenen J, Moonen G, Jérôme R. Peripheral nerve regeneration using bioresorbable macroporous polylactide scaffolds. J Biomed Mater Res 2000;52(4):639-51.

[71] Marin E, Briceño MI, Caballero-George C. Critical evaluation of biodegradable polymers used in nanodrugs. Int J Nanomed 2013;8:3071-91.

[72] Schmalz G. Material science: biological aspects. J Dent Res 2002;81(10):660-3.

[73] Pertici G, Carinci F, Carusi G, Epistatu D, Villa T, Crivelli F, Rossi F, Perale G. Composite polymer-coated mineral scaffolds for bone regeneration: from material characterization to human studies. J Biol Regul Homeost Agents 2015;29(3).

[74] Misra GS. Introductory polymer chemistry. New York: J. Wiley \& Sons; 1993.

[75] Gowariker VR, Viswanathan NV, Sreedhar J. Polymer science. New York: Wiley; 1986.

[76] Scheirs J. Compositional and failure analysis of polymers: a practical approach. Chichester; New York: Wiley; 2000.

[77] Niaounakis M. Biopolymers: processing and products. William Andrew; 2014.

[78] Osswald TA, Hernández-Ortiz JP. Polymer processing: modeling and simulation. Munich; Cincinnati: Hanser Publishers; 2006.

[79] Sill TJ, von Recum HA. Electrospinning: applications in drug delivery and tissue engineering. Biomaterials 2008;29(13):1989-2006.

[80] Li D, Xia Y. Electrospinning of nanofibers: reinventing the wheel? Adv Mater 2004; 16(14):1151-70.

[81] Han CD. Rheology and processing of polymeric materials. Oxford; New York: Oxford University Press; 2007. 
[82] Tadmor Z, Gogos CG. Principles of polymer processing. Hoboken, N.J.: WileyInterscience; 2006.

[83] Jenkins M, Stamboulis A, editors. Durability and reliability of medical polymers. Elsevier; 2012.

[84] Packham DE. Surface energy, surface topography and adhesion. Int J Adhesion Adhes 2003;23(6):437-48.

[85] Siemann U. Solvent casting technology - the most suitable tool for LCD polarizer film production. In: Proceedings of the twenty-fifth international display research conference - eurodisplay 2005; 2005. p. 240-2.

[86] Raps D, Hossieny N, Park CB, Altstädt V. Past and present developments in polymer bead foams and bead foaming technology. Polymer 2015;56:5-19.

[87] Tous E, Purcell B, Ifkovits JL, Burdick JA. Injectable acellular hydrogels for cardiac repair. J Cardiovas Trans Res 2011;4:528-42.

[88] Kopeček J. Hydrogel biomaterials: a smart future? Biomaterials 2007;28(34):5185-92.

[89] Slaughter BV, Khurshid SS, Fisher OZ, Khademhosseini A. Hydrogels in regenerative medicine. Adv Mater 2009;21:3307-29.

[90] Radhakrishnan J, Maheswari Krishnan U, Sethuraman S. Hydrogel based injectable scaffolds for cardiac tissue regeneration. Biotechnol Adv 2014;32:449-61.

[91] Badiee A, Heravi Shargh V, Khamesipour A, Jaafari MR. Micro/nanoparticle adjuvants for antileishmanial vaccines: present and future trends. Vaccine 2013;31(5):735-49.

[92] Petros RA, De Simone JM. Strategies in the design of nanoparticles for therapeutic applications. Nat Rev Drug Discov 2010;9:615-27.

[93] Nagavarma BVN, Yadav Hemant KS, Ayaz A, Vasudha LS, Shivakumar HG. Different techniques for preparation of polymeric nanoparticles - a review. Asian J Pharmaceut Clin Res 2012;5(3):16-23.

[94] Petrovic V, Vincente Haro Gonzalez J, Jordá Ferrando O, Delgado Gordillo J, Ramón Blasco Puchades J, Portolés Griñan L. Additive layered manufacturing: sectors of industrial application shown through case studies. Int J Prod Res 2011;49(4):1061-79.

[95] Melchelsa FPW, Domingosc MAN, Kleina TJ, Maldaa J, Bartoloc PJ, Hutmachera DW. Additive manufacturing of tissues and organs. Prog Polym Sci 2012;37(8):1079-104.

[96] Hockaday LA, Kang KH, Colangelo NW, Cheung PYC, Duan B, Malone E, Wu J, Girardi LN, Bonassar LJ, Lipson H, Chu CC, Butcher JT. Rapid 3D printing of anatomically accurate and mechanically heterogeneous aortic valve hydrogel scaffolds. Biofabrication 2012;4(3):1-22.

[97] Péreza RA, Wona JE, Knowlesb JC, Kim HW. Naturally and synthetic smart composite biomaterials for tissue regeneration. Adv Drug Deliv Rev 2013;65(4):471-96.

[98] $\mathrm{Hu} \mathrm{H}$, Granger N, Pai B, Bellamkonda R, Jeffery N. Therapeutic efficacy of microtubule-embedded chondroitinase $\mathrm{ABC}$ in a canine clinical model of spinal cord injury. Brain 2018;141:1017-27.

\section{Further reading}

[1] Aston SJ, Rees TD. Vicryl sutures. Aesthet Plast Surg 1977;1:289-93.

[2] Galea CF, Inkerman PA. Dextran analysis of raw sugar. Part I: a specific method for total dextran. Int Sugar J 1993;95(1136):309-13. 
104 CHAPTER 6 Elements of material science and polymeric biomaterials

[3] Lembeck B, Wülker N. Severe cartilage damage by broken poly-L-lactic acid (PLLA) interference screw after ACL reconstruction. Knee Surg Sport Traumatol Arthrosc 2005; 13(4):283-6.

[4] Nguyen LH, Gao M, Lin J, Wu W, Wang J, Chew SY. Three-dimensional aligned nanofibers-hydrogel scaffold for controlled non-viral drug/gene delivery to direct axon regeneration in spinal cord injury treatment. Sci Rep 2017;7:42212. https://doi.org/ 10.1038/srep42212.

[5] Sun J, Tan H. Alginate-based biomaterials for regenerative medicine applications. Materials 2013;6:1285-309.

[6] Williams DF. Williams dictionary of biomaterials. Liverpool University Press; 1999. 


\section{CHAPTER}

\section{c0007 Soft and rigid scaffolds for spinal cord injury regeneration}

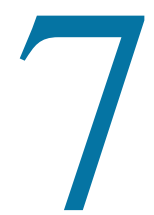

Š́rka Kubinová, PharmD, PhD

Department of Biomaterials and Biophysical Methods, Institute of Experimental Medicine of the Czech Academy of Sciences, Prague, Czech Republic

\section{s0010 Introduction}

p0015 Spinal cord injury (SCI) is a devastating trauma that results in motor and sensory disabilities with serious consequences for the lives of patients and their families. Yet, despite the extensive research and the number of ongoing clinical trials, there is still no effective clinical treatment that would regenerate the long-tract axonal damage and recover the lost neuronal function in patients.

p0020 The failure of functional recovery after SCI is mainly caused by the low regenerative capability of the adult axons and the establishment of an inhibitory environment, scar tissue, and demyelinization around the lesion that further hinders axonal repair [1].

p0025 Therapeutic strategies developed for the SCI target various aspects of the complex SCI pathology, with the aim to overcome the growth-inhibiting environment and promote endogenous repair processes [2]. These include neuroprotection, the activation of intrinsic neuronal regeneration capacity, removal of inhibition [3], the regulation of gliosis and scar formation, reducing excitotoxicity and the inflammatory response, replacing lost neurons and glia or providing cellular bridges for axon growth. Combinations of these therapies may reveal synergistic effects and represent a promising therapeutic approach in SCI treatment $[4,5]$.

p0030 Traumatic SCI, especially at its chronic stage, is frequently associated with the formation of fluid-filled pseudocystic cavities with a lack of permissive cellular substrate that together with inhibitory glial scar tissue, constitute a physical hurdle preventing cell infiltration and axonal regeneration. Therefore, the development of nontoxic, biomaterial-based scaffolds, able to bridge the lesion and provide the necessary microenvironment for the neural tissue remodeling, is among the most important issues in SCI repair strategies [6-8]. The scaffolds have been developed to simply fill the cavity to more sophisticated conduits with guidance architectures and can be combined with cells, drugs, or nucleic acid material $[8,9]$. 


\section{s0015 Scaffold properties and characterization}

p0035 A commonly used approach for the development of materials suitable for tissue repair is to mimic the biological and mechanical properties of the cell microenvironment seen in vivo. In the native tissue, the cells reside in the extracellular matrix (ECM), which is a complex fibrillary network mostly composed of collagen, elastin, proteoglycans, and glycoproteins. ECM creates not only a structural framework to maintain tissue integrity, but also provides spatial and biochemical information for the cells, which is essential for the regulation of intracellular signaling and cell behavior.

p0040 As scaffolds, various synthetic or natural-derived biomaterials have been shown to have a positive impact on the central nervous system (CNS) regeneration after implantation. The advantage of chemically synthetized biomaterials is the control over their manufacture, simple preparation of the synthesis with the possibility of modification, and the ability to tune the mechanical properties, porosity, or degradation rate (if relevant). On the other hand, synthetic materials fail to mimic the complex morphological structure and chemistry of the native ECM, and usually require additional modifications to enhance their bioactivity and cell adhesiveness. The most explored materials are aliphatic polyesters, such as polylactide, polyglycolide, and their copolymers, or polycaprolactone [9].

p0045 In contrast to synthetic materials, natural scaffolds are considered as biocompatible and nontoxic with a high ability to induce tissue remodeling, but might be variable in composition. Natural-based materials used for CNS tissue repair include single ECM molecules, such as collagen, fibronectin, hyaluronic acid, or other naturally occurring polysaccharides (alginate, agarose, chitosan, gellan gum) or their combinations. Along with this, a promising technique of how to produce a natural scaffold that resembles the structural and biochemical cues of the native tissue is tissue decellularization, in which the ECM is obtained by cell removal from the source tissue [10].

p0050 Generally, materials developed for application into the CNS must fulfill basic scaffold parameters, such as biocompatibility, controlled porosity and permeability, ease of application, and mechanical properties that match the soft neural tissue, which minimizes the possible irritation of the surrounding host tissue after the scaffold implantation. For the application of scaffolds for tissue bridging, they should provide support for cell attachment, growth, and differentiation [11]. In addition to this, multidrug delivery systems are also developed for the local controlled release of therapeutic agents into SCI [12-14].

p0055 Among the different types in the wide field of materials for soft tissue replacement, a significant interest is focused on hydrogels. They typically form a threedimensional network of hydrophilic polymers that swell in water, which can make their mechanical properties similar to those of the neural tissue and concomitantly enable ion and metabolite exchange within tissue fluids $[15,16]$.

p0060 The character of the scaffold determines the mode of application. Hydrogels in a liquid phase can be injected into a lesion site, to polymerize in situ, which clearly 
minimize tissue damage and enable the conformation of the material to the lesion irregularities, but the properties of injectable scaffolds can be more difficult to control. On the other hand, solid implantable scaffolds can provide controlled porosity enabling physical guidance for axon growth. At the same time, these materials must be flexible and mechanically stable, not to cause additional damage to the spinal cord tissue or structural collapse during implantation.

p0065 Brain and spinal cord are some of the most compliant human tissues. It is therefore essential that a hydrogel implant's stiffness is comparable to the nervous tissue and the implant is mechanically capable of withstanding the forces caused by the motions of the spinal cord without compression of the surrounding tissue. In the case of a too-stiff material, the implant fails to properly conform to the shape of the lesion and the mismatch of mechanical properties between the implant and the soft spinal cord may cause the formation of cysts or fibrotic tissue and thus further hinder tissue repair.

p0070 The mechanical properties of the material are not only critical for the successful integration of the hydrogel scaffold into the surrounding tissue, but also have an influence on cell phenotype and function, and play an important role in axonal elongation and maturation. It has been shown that growth cones generate traction force and respond to the mechanical stress caused by substrate rigidity [17]. In CNS neuronal cultures, soft substrates increased neurite outgrowth and branching, whereas astrocyte growth was enhanced on stiffer substrates and vice versa [18]. Another study demonstrated that spinal cord neurons extend more primary dendrites but shorter axons on stiffer gels $(30 \mathrm{kPa})$ [19]. It was noted that the substrate stiffness-dependent axon outgrowth and traction forces were found to be different in PNS (dorsal root ganglion (DRG)) and CNS (hippocampal) neurons. Although neurites from DRG neurons displayed maximal outgrowth on substrates with a Young's modulus of $\sim 1 \mathrm{kPa}$, hippocampal neurite outgrowth was independent of substrate stiffness [20].

p0075 A stiff substrate (shear modulus $10 \mathrm{kPa}$ ) also induced the upregulation of inflammatory genes and proteins in primary rat microglial cells and astrocytes, when compared to a substrate with a physiological stiffness value (100 Pa). Correspondingly, an in vivo, stiff implant triggered significantly enhanced levels of glial cell activation and subsequent inflammation compared to a softer implant. This suggests that adjusting the mechanical stiffness of neural implants to that of neural tissue could minimize adverse reactions and improve biocompatibility [21].

p0080 On the contrary, tissue softening was described in the scar tissue after stab injury in the cortex or crash injury in the spinal cord, which correlated with increased expression levels of glial intermediate filaments (GFAP, vimentin) and extracellular matrix components (laminin, collagen IV). Importantly, in the spinal cord, the white matter was found to be softer than the gray matter, with the gray matter being approximately twice as stiff as the white matter ( $420 \mathrm{~Pa}$ for gray and $177 \mathrm{~Pa}$ for white matter). The authors suggest that the softening of the tissue after CNS injuries in adult mammals might be one of the factors contributing to the loss of neuronal regrowth, due to the altered mechanical signals environment [22]. 
p0085 Apart from the stiffness, the key component of the microenvironmental milieu is the scaffold micro/nano topography, which may control cell migration, proliferation, and stem cell differentiation by the modulation of actin cytoskeleton [23]. The appropriate pore size distribution is then essential to provide a suitable microenvironment for the endogenous tissue infiltration and sufficient vascularization, cell migration, and proliferation. In conventional hydrogels, porosity is defined by the average distance between the cross-linked polymer chains forming the hydrogel network, and is typically on the scale of a few, to tens of nanometers. To achieve axonal guidance across the lesion site, the microarchitecture of the pores can be formed into the longitudinal oriented fibers, aligned channels or porous conduits by various techniques such as; the salt-leaching method [24], solid/liquid phase separation method [25], freeze casting method, wrapping an aligned nanofiber sheet into a tube [26], or three-dimensional (3D) printing. Nerve guidance channels fabricated from nondegradable materials include, e.g., silicone, polyacrylonitrile/polyvinylchloride, poly(tetrafluoro-ethylene), poly(2-hydroxyethyl methacrylate) (PHEMA) [8], templated agarose [27,28] or trimethylene carbonate-caprolactone conduit with poly-p-dioxanone microfilaments [29]. Generally, scaffolds with pore sizes around 50-100 $\mu \mathrm{m}$ seem to be suitable for neuronal regeneration [30,31].

p0090 Another important factor is the scaffold degradability. Our studies suggest that both nondegradable as well as degradable scaffolds are able to bridge the SCI lesion, and support axonal growth and infiltration of the host tissue elements. A considerable disadvantage of the nondegradable scaffolds is the limited space available for the tissue repair, which is given by the pore size, that does not allow complete tissue replacements. On the other hand, in the case of degradable materials, it is necessary to use long-term, absorbable biomaterials to ensure the formation of stable new tissue through the lesion site. Fast degrading hydrogels do not allow proper tissue replacement and additional cysts may be formed again after their resorption [32].

p0095 Cell adhesiveness is required for the delivery of transplanted cells, as well as for support of the host cell ingrowth into the implant. Factors affecting the cell attachment and spreading on polymer surfaces include the chemical composition or the charge of the surface, surface roughness and topography, and the balance between hydrophilic and hydrophobic microdomains. A well-known strategy to modify biomaterial surfaces is the immobilization of specific integrin-binding epitopes of ECM molecules, such as those derived from fibronectin, laminin, or collagens. The advantage of using the short peptides rather than the whole protein is their easy manufacture, high stability and the possibility of being bound to the substrate surface at a controlled density to achieve the desired receptor binding, or to generate cell-specific materials when the adhesion ligand for a certain type of cell is used. Well-characterized cell adhesion ligands are fibronectin-derived RGD [Arg-GlyAsp] or laminin-derived YIGSR [Tyr-Ile-Gly-Ser-Arg] and IKVAV [Ile-Lys-ValAla-Val] peptides [33,34].

p0100 Generally, the development of scaffolds for SCI repair includes a whole range of materials of various chemistry and structure, while a number of those either alone or in combination with cells or other factors have also been proved in vivo in animal 
models of SCI $[6,35]$. In this chapter, the results on synthetic and natural hydrogels developed by our group for neural tissue regeneration after SCI are summarized and critically discussed in relation to tissue integration and ability to support transplanted cells or promote neural tissue repair and functional outcome.

p0105 Synthetic materials developed by our group include resorbable and nonresorbable hydrogels based on synthetic polymers, such as PHEMA $[33,36]$ and poly[N(2-hydroxypropyl)methacrylamide] (PHPMA) [37]. Methacrylate hydrogels have widely been used as a biomaterial due to their good biocompatibility and the possibility to tailor their physical and chemical properties. An advantage of these materials is that monomers, such as HEMA and HPMA, can easily be copolymerized with various reactive comonomers, and these polymers can be enhanced with a variety of functional units (e.g., peptide domains) that can increase cell adhesion and allow signaling to cell surface receptors. Moreover, such variability of PHEMA modification allowed us to compare scaffolds with the same chemistry but different porosity and stiffness in the same SCI model, to find the critical value when the scaffold fails in proper tissue integration and support for the axonal ingrowth [38].

p0110 In comparison to the synthetic hydrogels, we tested natural injectable hydrogels derived from ECM, including hyaluronic acid or biological hydrogels prepared from decellularized tissues that were able to polymerize in situ and were soft and degradable.

p0115 A complex evaluation of the hydrogels then included the physicochemical characterization of the material, in vitro evaluation in cell adhesion, growth, and differentiation, and in vivo experiments using acute or chronic models of SCI, such as spinal cord hemisection and transection, or compression injury where the hydrogels were either implanted or injected into the acute, subacute, or chronic lesion in rats. The hydrogels were also combined with various types of stem cells, such as mesenchymal stem cells (MSCs) or neural progenitor cells, which were mostly beneficial for the tissue regeneration $[37,39]$.

\section{s0020 Synthetic hydrogels based on PHPMA and PHEMA s0025 Macroporous PHPMA hydrogel modified with RGD}

p0120 The very first material tested in our group in the neural tissue was the nondegradable macroporous PHPMA hydrogel (NeuroGel). PHPMA was prepared from $\mathrm{N}$-(2hydroxypropyl)-methacrylamide (HPMA) by heterophase separation using radical polymerization in a pore-forming solvent with a divinyl cross-linking agent [40] (Fig. 7.1A). PHPMA hydrogel was then functionalized by the synthetic peptide Gly-Gly-Arg-Gly-Asp, which includes the cell-adhesive sequence RGD [41]. The swollen PHPMA-RGD hydrogel displayed an elastic modulus $\sim 100 \mathrm{~Pa}$ that allowed the best match between the mechanical properties of the hydrogel and the neural parenchyma, and porosity $\sim 88 \%$ with a mean pore diameter of $\sim 20 \mu \mathrm{m}$. The hydrogel was implanted into the neonatal and adult spinal cord transections, where it revealed a good integration into the host tissue with a significant degree of cell and blood vessel infiltration into the polymer hydrogel implants [40]. 

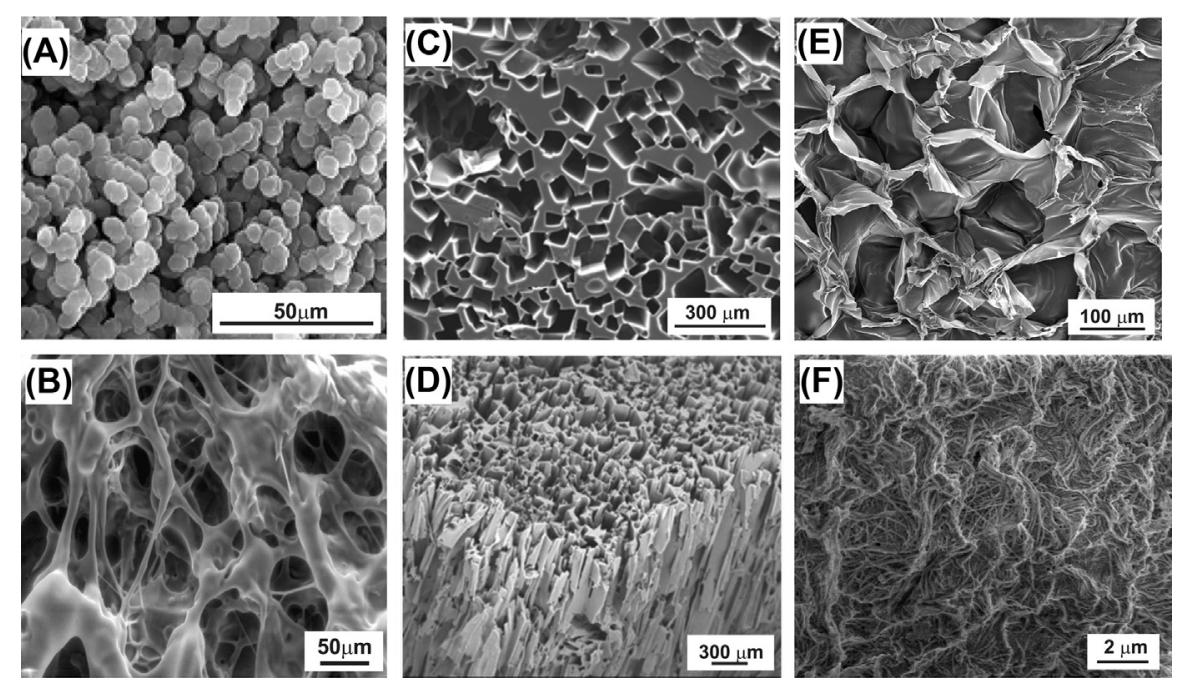

f0010

FIGURE 7.1

SEM image of the scaffolds with various porosity, used as implants in experimental SCI. (A) PHPMA-RGD hydrogel prepared by heterophase separation. The pores are formed by spaces among the building blocks of the hydrogel, microglobules. (B) PHEMA hydrogel prepared with salt porogen. (C, D) PHEMA hydrogel with parallel-oriented channels prepared by salt porogen. (E) Hyaluronic acid (HA-PH-RGD) hydrogel. (F) ECM hydrogel derived from decellularized human umbilical cord.

p0125 To bridge a chronic spinal cord lesion, PHPMA-RGD hydrogel was implanted, either alone or seeded with rat MSCs, into a balloon-induced compression lesion in rats 5 weeks after the induction of compression injury. After 6 months, a behavioral analysis (BBB score) showed a statistically significant improvement in rats with the combined treatment, hydrogel with MSCs, compared with the control group without implantation. The combined therapy prevented tissue atrophy, and the hydrogels were infiltrated with axons myelinated with Schwann cells, blood vessels, and astrocytes. Moreover, rat MSCs were preserved in the hydrogels even 5 months after implantation (the animals were immunosuppressed). These results proved that PHPMA-RGD hydrogel, seeded with MSCs, was able to successfully bridge a spinal cord cavity and provide a scaffold for tissue regeneration [37].

\section{s0030 PHEMA hydrogels with different charges}

p0130 Surface charge is considered to be one of the factors influencing cell adhesion and tissue ingrowth into an implanted hydrogel. We showed that positively charged PHEMA hydrogels promoted in vivo tissue infiltration and axonal ingrowth, in contrast to PHEMA scaffolds containing negative charges [42]. In this study, Lesny et al. [42] modified PHEMA hydrogels with differently charged polar groups on 
their surfaces: copolymers of HEMA with sodium methacrylate (MA-) carried a negative electric charge, copolymers of HEMA with [2-(methacryloyloxy)ethyl]trimethylammonium chloride (MOETA + ) carried a positive electric charge and terpolymers of HEMA, MA-, and MOETA + carried both, positive and negative electric charges. When seeded with MSC culture, the highest cellular density was found in the positively charged HEMA-MOETA + copolymers while a lower cell density was found in the negatively charged HEMA-MA- copolymers and in polyelectrolyte complexes.

p0135 Similarly, after implantation into a spinal cord hemisection cavity in rats, HEMA-based hydrogels carrying functional groups with a positive charge (HEMA+ and HEMA \pm ) improved axonal ingrowth inside the implants, compared to implants without any charge. However, only positively charged functional groups promoted connective tissue infiltration and extended axonal regeneration inside a hydrogel bridge [43]. This is in line with the preferential adhesion of neurons and axonal growth on polycationic surfaces, such as poly-lysine, that enhances electrostatic interaction between negatively charged cells or proteins.

p0140 In a later study, Hejcl et al. [44] demonstrated the synergic effect of porosity and surface modification of PHPMA hydrogels on MSC in vitro adhesiveness and in vivo survival. Rat MSCs were seeded on four different types of hydrogels: HPMA-RGD prepared by heterophase separation (HPMA-HS-RGD), and three other hydrogels polymerized in the presence of a solid porogen (SP): HPMA-SP, HPMA-SP-RGD, and positively charged HEMA-MOETA + [44]. The synthesis of hydrogels prepared in the presence of an SP resulted in a web-like architecture (Fig. 7.1B), while the HPMA hydrogel prepared by heterophase separation was composed of small globular-shaped microparticles with the spaces between them serving as pores for tissue ingrowth (Fig. 7.1A).

p0145 In vitro cell attachment was found superior for the positively charged HEMAMOETA+ and HPMA-SP-RGD hydrogels, compared with the HPMA-SP and HPMA-HS-RGD. After implantation into the SCI hemisection, the highest MSC survival was then found in the HEMA-MOETA+hydrogels; however, only a small ingrowth of blood vessels and axons was observed in this material. On the other hand, HPMA-SP and HPMA-HS-RGD hydrogels promoted a statistically significant greater ingrowth of axons and less connective tissue elements into the implant.

p0150 Importantly, the RGD sequence that attached to both types of HPMA hydrogels (SP and HS), significantly enhanced the number of blood vessels inside the implanted hydrogels but did not influence the axonal ingrowth. These results demonstrated that the combination of web-like SP porosity and presence of adhesive peptide motif of HPMA-SP-RGD hydrogel showed the best combination for bridging a spinal cord lesion, such as axonal growth and vascularization, while the positively charged HEMA-MOETACl hydrogel served as the best carrier of MSCs [44].

p0155 These results were then confirmed by the recent study, where PHPMA, PHEMA, and a PHEMA hydrogel with attached fibronectin were implanted into the spinal cord hemisection. After 3 months, the PHPMA scaffold supported the regrowth of axons far better compared to PHEMA, even when PHEMA was enriched with fibronectin [45]. 
Similarly to the previous work, a denser blood vessel network was found in the PHEMA hydrogel with fibronectin, which is rich to the RGD peptide sequence [44].

\section{s0035 Cholesterol-modified superporous PHEMA hydrogels}

p0160 In addition to the surface charge, we have shown that the incorporation of highly hydrophobic domains of naturally occurring compounds present in cell membrane lipids, such as cholesterol, in hydrophilic PHEMA gels significantly improves its bioadhesive properties and supports cell adhesion and proliferation (Fig. 7.2).

p0165 Cholesterol-modified superporous PHEMA scaffolds have been prepared by the bulk radical copolymerization of 2-hydroxyethyl methacrylate (HEMA), cholesterol methacrylate, and the cross-linking agent ethylene dimethacrylate in the presence of ammonium oxalate crystals to introduce interconnected superpores in the matrix [36]. To increase the swellability and softness of this scaffold, an additional monomer, 2-[(methoxycarbonyl)methoxy]ethyl methacrylate, was incorporated in the polymerization recipe and hydrolyzed to [2-(methacryloyloxy)ethoxy]acetic acid (MOEAA), thus introducing carboxyl groups in the polymer. Three types of PHEMA hydrogels containing $2 \mathrm{wt} \%, 4 \mathrm{wt} \%$, and $8 \mathrm{wt} \%$ of MOEAA were then prepared, which resulted in high porosity $(65 \mathrm{vol} \%)$ and the formation of interconnected pores $30-100 \mu \mathrm{m}$ and relatively rigid elastic modulus $\sim 17,19$, and $10 \mathrm{kPa}$ respectively [46]. Interestingly, when implanted into an acute spinal cord hemisection in rats, no marked differences in the tissue response to hydrogels of such elastic modulus were found. All the hydrogel implants were found to be well integrated; they closely adhered to the surrounding tissue, revealed the ingrowth of neurofilament-positive fibers and a number of newly formed blood vessels into
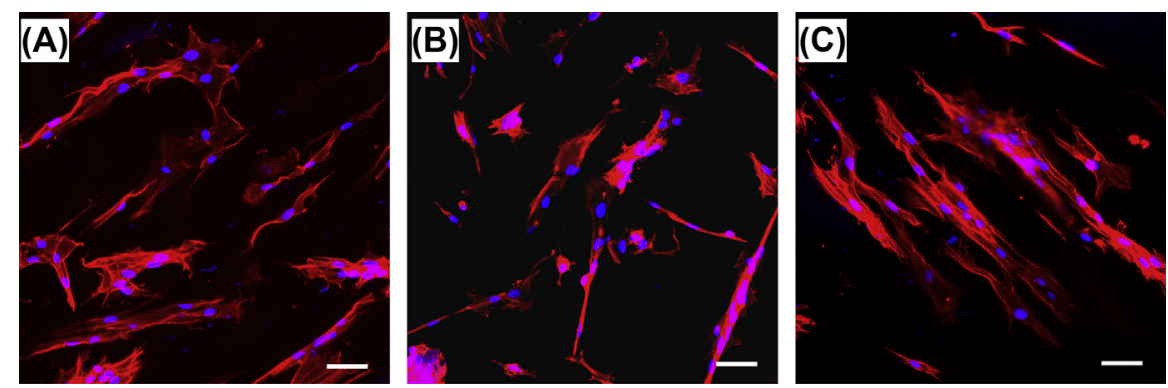

f0015

\section{FIGURE 7.2}

Confocal images of immunofluorescent staining for phalloidin (red) and cell nuclei (DAPI, blue) of rat MSCs seeded on cholesterol-modified superporous PHEMA hydrogels containing (A) 2\% MOEAA, (B) 4\% MOEAA, and (C) $8 \%$ MOEAA hydrogels scaffolds for 2 days. MSCs spread mostly into the pores of the hydrogels. Scale bar: (A-C) $50 \mu \mathrm{m}$.

Modified from Kubinova S, Horak D, Hejcl A, Plichta Z, Kotek J, Sykova E. Highly superporous cholesterolmodified poly(2-hydroxyethyl methacrylate) scaffolds for spinal cord injury repair. J Biomed Mater Res A 2011;

99:618-629. 

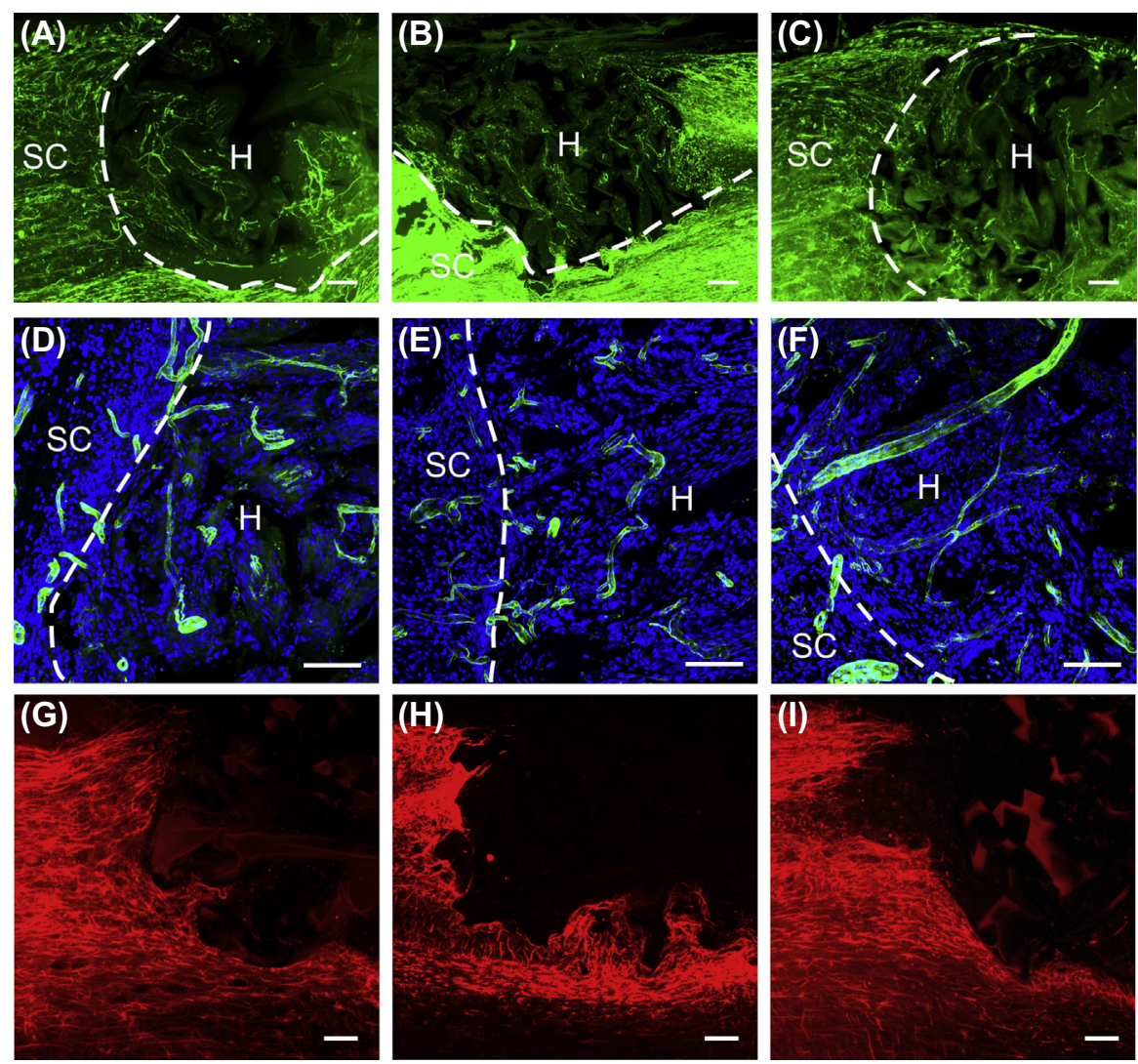

f0020 FIGURE 7.3

Longitudinal sections of the implanted cholesterol-modified superporous PHEMA hydrogels containing (A, D, G) 2\% MOEAA, (B, E, H) 4\% MOEAA, and (C, F, I) $8 \%$ MOEAA 4 weeks after the hemisection. Staining for (A-C) neurofilaments (NF160), (D-F) blood vessels (RECA staining) and cell nuclei (DAPI), and (G-I) astrocytes (GFAP). The dashed line marks the border between the implanted hydrogel $(H)$ in the spinal cord tissue (SC). Scale bar: (A-C, G-I) $100 \mu \mathrm{m}$, (D-F) $50 \mu \mathrm{m}$ [46].

the pores, throughout the whole implant 4 weeks after implantation (Fig. 7.3). Importantly, only a sparse foreign body reaction with foamy macrophages and giant cell granulomas was found locally around the implanted hydrogels; but no adverse reaction with chronic inflammation or fibrosis was detected.

\section{s0040 Superporous PHEMA hydrogels modified by cell-adhesive peptides}

p0170 To improve cell adhesion of the PHEMA hydrogel, we used a well-known surface modification with laminin-derived peptide sequence SIKVAV [Ser-Ile-Lys-ValAla-Val] or fibronectin-derived peptide RGD, and demonstrated that these 
modifications can improve rat MSC adhesion and growth, as well as differentiation of the human fetal neural stem cells $[33,47]$.

p0175 To prepare this material, we used the copolymerization of HEMA with 2aminoethyl methacrylate (AEMA) to produce a reactive polymer hydrogel, capable of modification with the laminin-derived Ac-CGGASIKVAVS-OH peptide terminated with acetylcysteine. P(HEMA-AEMA) hydrogel was then reacted with $\gamma$-thiobutyrolactone to introduce sulfhydryl groups, which after activation with 2,2'-dithiodipyridine immobilized the Ac-CGGASIKVAVS-OH sequence via the $\mathrm{SH}$ groups of cysteine.

p0180 Alternatively, the cell-adhesive peptides (SIKVAV, RGD) or fibronectin (Fn) monomer were immobilized onto the copolymer of HEMA with AEMA by the maleimide-thiol coupling reaction. The maleimide group was introduced to the P(HEMA-AEMA) hydrogels by the reaction of their amino groups with $\mathrm{N}-\gamma-$ maleimidobutyryl-oxysuccinimide ester (GMBS). All modified scaffolds supported the adhesion and growth of MSCs and maintained their multilineage potential. The cell proliferation was then far more pronounced on the Fn-modification with several biologically active domains, than on the hydrogels modified with the single SIKVAV or RGD peptide sequence $[34,47]$. When implanted in vivo, the Fn-modified HEMA scaffolds showed an increased connective tissue infiltration compared to the plain HEMA hydrogels and a higher blood vessel ingrowth [45].

\section{s0045 PHEMA hydrogels with oriented porosity}

p0185 Regarding the predominant direction of afferent and efferent spinal cord tracts in the craniocaudal or vice versa direction, the key challenge in the design of neural tissue engineered scaffolds is to develop a scaffold with parallel guiding channels or pores. Only such pores are able to promote the prospective ingrowth of axons and neurons and reconnect the proximal and distal parts of the SCI.

p0190 To achieve this goal, we optimized the previously developed SIKVAV-modified PHEMA hydrogels with parallel oriented, interconnected pores using a salt-leaching method with ammonium oxalate needle-like crystals (Fig. 7.1C and D) [33,36]. Three types of superporous SIKVAV-modified P(HEMA-AEMA) hydrogels were prepared with $8 \%, 4 \%$, and $0 \%$ (wt $\%$ ) of MOEAA that resulted in materials of $57 \%-77 \%$ porosity, average pore size $\sim 60 \mathrm{~mm}$ diameter, and the modulus of elasticity 6.7, 27.4, and $45.3 \mathrm{kPa}$ along the pore axis and 2.9, 3.6, and $11 \mathrm{kPa}$ in a perpendicular direction [38].

p0195 After implantation into the spinal cord hemisection, the best tissue response was found with the SIKVAV-modified P(HEMA-AEMA) hydrogel, possessing moderate values of the modulus of elasticity $(27 \mathrm{kPa}$ in the direction along the pores and $3.6 \mathrm{kPa}$ in a perpendicular direction) and 68\% porosity (Fig. 7.4B). This hydrogel also allowed the bridging of the lesioned spinal cord and promoted the ingrowth of axons, blood vessels, and Schwann cells into the hydrogel pores.

p0200 Sparse axonal ingrowth was then observed into the porous structure of the softest P(HEMA-8\%AEMA) hydrogel (77\% porosity, modulus elasticity $6.7 \mathrm{kPa}$ along the 

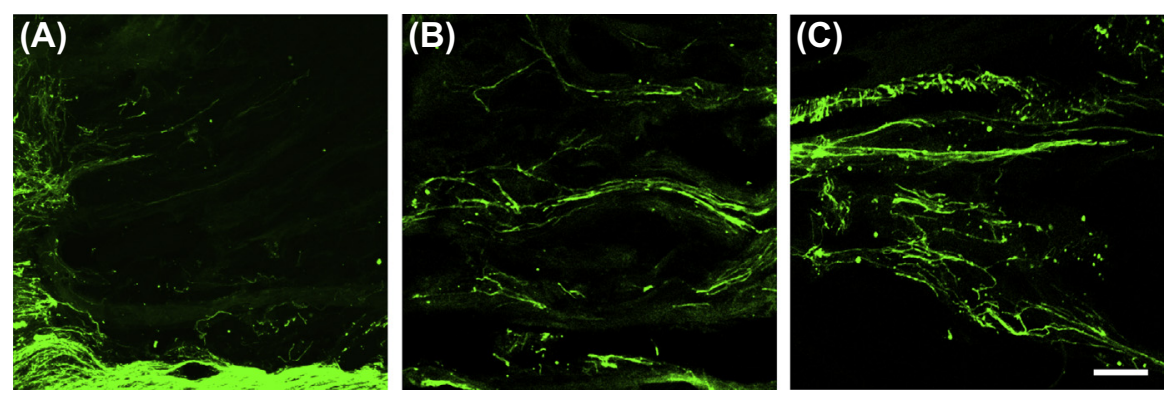

f0025

GURE 7.4

Neurofilament staining (NF-160) of longitudinal sections of spinal cord with implanted SIKVAV-modified P(HEMA-AEMA) hydrogels after 2 months: (A) 77\% porosity, modulus elasticity $6.7 \mathrm{kPa}$ along the pores and $2.9 \mathrm{kPa}$ perpendicular to the pores; (B) $68 \%$ porosity, modulus elasticity $27 \mathrm{kPa}$ along the pores and $3.6 \mathrm{kPa}$ perpendicular to the pores; (C) $57 \%$ porosity, modulus elasticity $45 \mathrm{kPa}$ along the pores and $11 \mathrm{kPa}$ perpendicular to the pores. Scale bar $=100 \mu \mathrm{m}$.

Modified from Kubinova S, Horak D, Hejcl A, Plichta Z, Kotek J, Proks V, et al. SIKVAV-modified highly superporous PHEMA scaffolds with oriented pores for spinal cord injury repair. J Tissue Eng Reg Med 2015;

9(11):1298-1309.

pores and $2.9 \mathrm{kPa}$ perpendicular to the pores) that might have collapsed due to the thinness of its pore walls, while the axons lost their guiding substrate in the mass of the infiltrating tissue (Fig. 7.4A). The third type of P(HEMA-4\%AEMA) hydrogel with an elasticity modulus of $45 \mathrm{kPa}$ and a porosity of $57 \%$ was found to be too stiff for optimal tissue integration, as large cavities were observed at the hydrogelhost tissue interface (Fig. 7.5 A and B). However, even such a stiff hydrogel promoted axonal ingrowth into its pores in spite of the cavities formed at the hydrogeltissue interface (Fig. 7.4C). These results suggest that even the overly stiff guiding substrate supports axonal growth in vivo, which is in contradiction to the preferential axonal elongation on the soft substrate found in other reports [18].

p0205 In the following study, SIKVAV-modified P(HEMA-AEMA)-oriented hydrogel with a moderate modulus of elasticity was implanted into a spinal cord transection to analyze the time-related dynamics of the tissue infiltration of this scaffold during 6 months [48]. This study showed that while the ingrowth of connective tissue into the channels of P(HEMA-AEMA) hydrogel was rather fast, as it completely infiltrated the pores within the first week, the axons infiltrated the scaffold in a slower and more gradual manner. Moreover, seeding of the hydrogel with MSCs did not show any effect on the hydrogel infiltration over time with respect to the speed or the amount of any tissue infiltration. In addition, after 6 months, only rare axons crossing the hydrogel bridge and infiltrating the caudal stump were observed [48].

p0210 The failure of the long-term promotion of axonal ingrowth suggests that using SIKVAV modification, together with the support of MSCs within the synthetic PHEMA scaffold, was insufficient for the axonal regrowth across the transection 


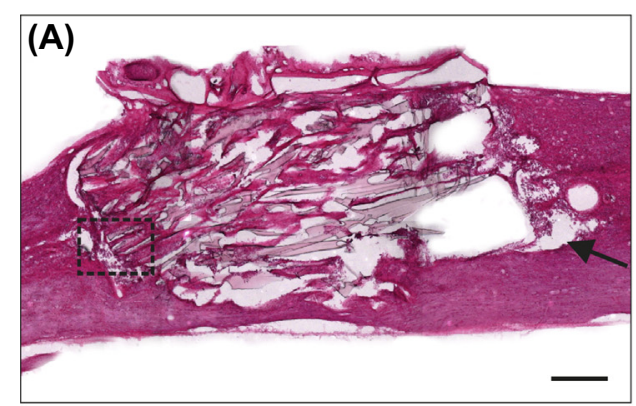

(C)

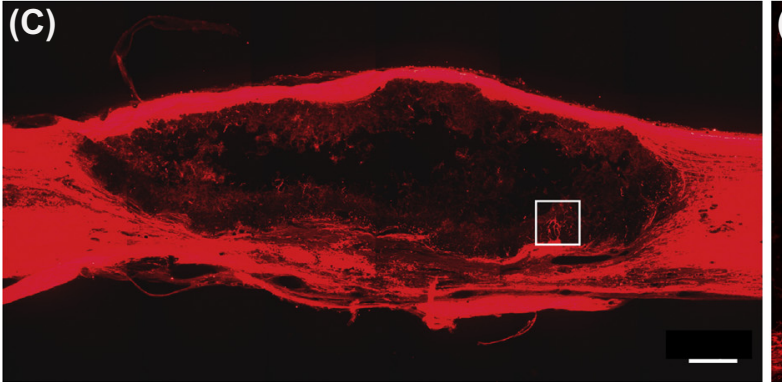

FIGURE 7.5

Rigid and soft hydrogels implanted into SCI lesion. (A, B) Hematoxylin-eosin staining of longitudinal sections of spinal cord with implanted oriented SIKVAV-modified P(HEMAAEMA) hydrogels after 2 months (57\% porosity, modulus elasticity $45 \mathrm{kPa}$ along the pores and $11 \mathrm{kPa}$ perpendicular to the pores). The arrow shows the formation of cyst between the scaffold and spinal cord tissue due to the mechanical mismatch. (B) Detailed micrographs show cyst filled with macrophages with a foamy cytoplasm (arrow). (C, D) Neurofilament staining of longitudinal section of spinal cord lesion with implanted soft PHPMA-RGD hydrogel (elastic modulus $100 \mathrm{~Pa}$ ). (D) Detailed micrographs show axonal sprouting into the hydrogel (Hejčl et al., unpublished). Scale bar: $(A, C)=500 \mu \mathrm{m}$, $(B)=10 \mu \mathrm{m}$, and $(D)=50 \mu \mathrm{m}$.

(A and B) Modified from Kubinova S, Horak D, Hejcl A, Plichta Z, Kotek J, Proks V, et al. SIKVAV-modified highly superporous PHEMA scaffolds with oriented pores for spinal cord injury repair. J Tissue Eng Reg Med 2015;

9(11):1298-1309.

lesion. In addition to the benefit of an axonal guiding scaffold, a combination with further factors that have the potential to stimulate axonal growth and regenerative response is thus necessary to achieve axonal reconnection and functional SCI repair.

p0215 For example, Zeng et al. [49] used gelatin porous sponges seeded with MSCs overexpressing of an NT-3 receptor cocultured with NT-3 overexpressing Schwann cells before the implantation, to induce MSC differentiation toward the neuronal lineage. Eight weeks after implantation into the rat spinal cord transection, the treated rats had significantly improved hindlimb function and also recovered cortical motor evoked potential, while MSC-derived neuron-like cells 
maintained their synapse-like structures and integrated into the host neural network in vivo. In a later study, the efficacy of this treatment was also confirmed using the same experimental setting with NT-3 receptor overexpression in neural stem cells [50].

p0220 In another study, Gao et al. prepared template agarose scaffolds seeded with bone marrow stromal cells secreting brain-derived neurotrophic factor (BDNF) that supported motor axon regeneration into a severe spinal cord injury model, while BDNF significantly enhances axonal growth organized into fascicles of highly linear configuration [28].

\section{S0050 PHEMA hydrogel modified with serotonin}

p0225 Serotonin is a multifunctional molecule, which serves as a neurotransmitter, but can also support the attachment of implanted or endogenous cells and positively influence and facilitate neuronal differentiation. To evaluate the effect of serotonin in SCI, PHEMA hydrogel was modified with covalently bound serotonin molecules (PHEMA-5HT) and implanted into a spinal cord hemisection in rats, alone or with a combination of the predifferentiated human neural stem cell line (SPC-01), derived from a fetal spinal cord [39].

p0230 In vitro, the PHEMA-5HT hydrogel supported the expansion and growth of spinal progenitor cells in a 3D artificial environment and serotonin molecules facilitated the neural differentiation. However, when implanted in vivo, the neural progenitor cells seeded on a PHEMA-5HT hydrogel migrated out from the polymer center and survived at the cell-hydrogel border or in the spinal cord tissue, where they expressed relevant markers of neuronal differentiation or of astrocytes. Over a period of 3 months, most of the implanted cells migrated from the implant and no long-term effect that would lead to tissue reconstruction or improvement of the locomotor and sensory functions was observed. These results suggest that the serotonin bound to PHEMA hydrogel did not provide an ideal long-term support for the continued growth and differentiation of the cells within the implant, that rather migrate out of the hydrogel without the support of tissue repair [39].

\section{s0055 Degradable PHEMA hydrogels}

p0235 In our recent work, the degradable PHEMA hydrogels were prepared from a linear copolymer $(\mathrm{Mw}=49 \mathrm{kDa}$; threshold limiting glomerular filtration) of HEMA, 2-(acethylthio) ethyl methacrylate (ATEMA), and zwitterionic 2-methacryloyloxyethyl phosphorylcholine (MPC). The deprotection of ATEMA thiol groups by triethylamine, followed by their oxidation with $2,2^{\prime}$-dithiodipyridine (DTP), resulted in the formation of reductively degradable polymers with disulfide bridges. Degradation of the hydrogels was controlled by changing the concentrations of ATEMA and DTP in the reaction mixture. The cross-linked P(HEMA-ATEMA-MPC) hydrogels were proved to be nontoxic and can be considered promising for application in tissue engineering and drug delivery systems [12]. 


\section{s0060 Natural-based materials}

p0240 Regarding limited tissue regeneration effects of synthetic hydrogels, we focused on the natural hydrogels based on ECM, such as hyaluronic acid and ECM hydrogels prepared by tissue decellularization. The advantage of these materials is their injectability, which allows in situ gelation together with cell encapsulation as well as degradability. When compared with the previously used synthetic materials, these hydrogels resulted in much better tissue ingrowth when applied into the SCI lesion; however, due to the fast degradation, complete tissue regeneration was not achieved.

\section{s0065 Hyaluronic acid hydrogel modified with RGD}

p0245 Hyaluronic acid (HA) is an important structural component of ECM, and it is widely used due to its biocompatibility, biodegradability, and nonimmunogenicity as a biomaterial in various clinical settings. Among its structural functions, HA also acts as a signaling molecule via specific HA receptors to actively modulate tissue regeneration [51,52]. Native HA does not form a gel or support cell adhesion. Therefore, it is necessary to chemically modify the functional groups of HA and tune its chemical, physical, or biological properties according to the special demands of the particular application. We used the hydroxyphenyl derivative of HA (HA-PH, $\mathrm{Mw}=400 \mathrm{kDa}$ ), which is capable of forming a covalently cross-linked hydrogel with the required mechanical properties $[53,54]$. The cross-linking reaction of this HA-PH derivative can be triggered by enzyme horseradish peroxidase and hydrogen peroxide in situ under physiological conditions, without a negative effect on encapsulated cells during cross-linking reaction and gel forming [55]. In addition, the HAPH derivative was coupled with 3-(4-hydroxyphenyl) propionic acid - L-lysine aminohexanoic acid - L-glycine - L - arginine - L-glycine - L-aspartic acid (HAPH-RGD) sequence, which allowed the attachment of 3-(4-hydroxyphenyl) propionic acid (PH moiety) and RGD cell adhesive motive to polymer backbone in one synthetic step (Fig. 7.1E) [56]. The mechanical properties of HA-PH-RGD hydrogel can be optimized to be mechanically suitable for the soft neural tissue with an optimal time of gelation, to enable a hydrogel injection into the site of the defect, while the used cross-link reaction was found noncytotoxic and was enabled to form cell-laden hydrogel under physiological conditions.

p0250 The neuroregenerative potential of HA-PH-RGD alone or combined with human Wharton's jelly-derived mesenchymal stem cells (hWJ-MSCs) was evaluated after injection into the subacute spinal cord hemisection. We demonstrated that HAPH-RGD promoted axonal ingrowth into the lesion and this effect was further enhanced when HA-PH-RGD was loaded with hWJ-MSCs (Fig. 7.6). Moreover, hWJ-MSCs delivered in HA-PH-RGD hydrogel also led to the upregulated mRNA expression of genes related to axonal growth, and both M1-like and M2like macrophage [56].

p0255 Nevertheless, in contrast to the improvement in parameters related to the tissue repair, no significant changes in locomotor functions were observed in animals 

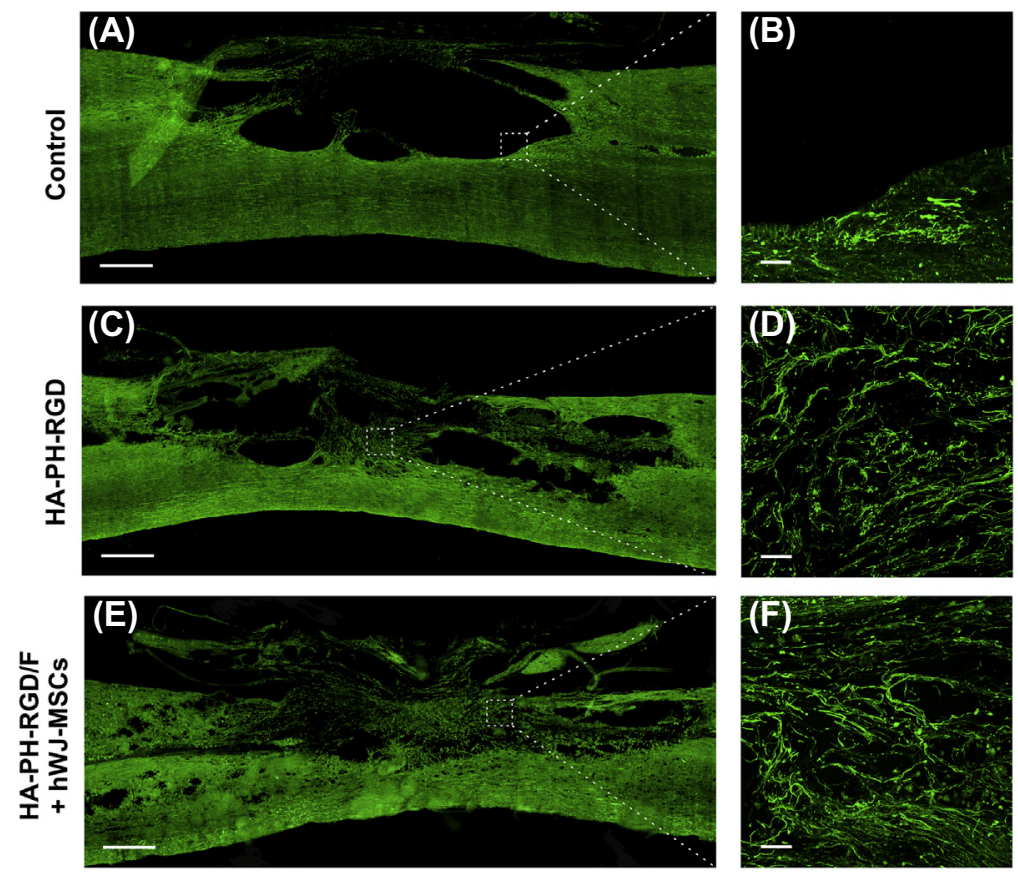

f0035 FIGURE 7.6

Neurofilament staining (NF-160) of longitudinal sections of the spinal cord lesion in (A, B) controls and at 8 weeks after the subacute injection of (C, D) HA-PH-RGD and (E, F) HAPH-RGD hydrogels combined with fibronectin (F) and hWJ-MSCs. Squares (A, C, E) are also shown under the higher magnification insets (B, D, F). Scale bar: $500 \mu \mathrm{m}(A, C, E)$, $50 \mu \mathrm{m}(\mathrm{B}, \mathrm{D}, \mathrm{F})$.

Modified from Zaviskova K, Tukmachev D, Dubisova J, Vackova I, Hejcl A, Bystronova J, et al. Injectable hydroxyphenyl derivative of hyaluronic acid hydrogel modified with RGD as scaffold for spinal cord injury repair. J

Biomed Mater Res A 2018;106(4):1129-1140.

treated with both hydrogel alone or in combination with hWJ-MSCs. This was probably caused by the insufficient number of transplanted cells, which was limited by the size of the lesion and capacity of the hydrogel to home the cells $\left(\sim 3 \times 10^{4}\right.$ cells in $\sim 5 \mu \mathrm{L}$ ), and did not exceed the level at which the functional locomotor improvement could be achieved. Therefore, the cells were able to promote axonal ingrowth, but their effect was too weak for the improvement of the motor functions, which was demonstrated on SCI with a much higher cell number [57].

\section{s0070 Extracellular matrix-based hydrogels}

p0260 Biologic scaffolds composed of native ECM represent natural materials with many advantages, such as a complex biomolecular composition, low 
immunogenicity, or the retention of growth factors and other biochemical cues. The therapeutic effect of ECM scaffolds is based on their ability to facilitate constructive tissue remodeling to the tissue-appropriate structures, by modulation of the immune response and stimulation of endogenous regenerative processes $[10,58]$. Although the degraded ECM scaffold is gradually replaced by the endogenous tissue, the degradation product called matricriptic proteins contribute to the recruitment of host cells and have a stimulatory effect on site-specific tissue remodeling [10].

p0265 Decellularized biomaterials can be created by eliminating cells from native tissues/organs using a combination of physical, chemical, and enzymatic methods. To form injectable hydrogels, ECM from various decellularized tissues can be enzymatically solubilized to a liquid form, using, for example, pepsin solubilization at $\mathrm{pH}<2$. The hydrogels have the ability to be physically crosslinked in situ at physiological $\mathrm{pH}$ and temperature that allow them to be noninvasively injected into the body [59]. Such material can be administered in a localized manner and are able to fill the defects caused by injury, conforming themselves to lesion irregularities with minimal surrounding tissue damage. The decellularization approach provides unique flexibility for choosing the most appropriate tissue source for the scaffold generation. ECM-based scaffolds, obtained from the variety of tissues, have been used for the reconstruction of, for example, myocardium, muscles, blood vessels, valves, bone, kidney, liver, and CNS tissues [10].

p0270 In our research, we have developed or optimized effective and reproducible techniques for the preparation of ECM-based hydrogels from human umbilical cord tissue (UC-ECM) (Fig. 7.1F) as well as porcine tissues-urinary bladder (UB-ECM), spinal cord (SC-ECM), and brain [59-61]. We have proved the ability of these hydrogels to promote attachment and proliferation of human MSCs, as well as the neuro-promoting capacity of these structures.

p0275 We have also analyzed the biocompatibility, immunogenicity, and capacity of ECM hydrogels to promote CNS repair. We applied acute SCI hemisection model in rats to compare the effect of ECM hydrogels, derived from CNS- (porcine SC$\mathrm{ECM}$ ) or non-CNS tissues (porcine UB-ECM) on spinal cord tissue remodeling [32]. We found that both ECM-based hydrogels bridged the lesion cavity, modulated the innate immune response, and provided the benefit of a stimulatory substrate for in vivo neural tissue regeneration (Fig. 7.7).

p0280 It is interesting that we could not find any significant differences in the chemotactic or neurotrophic properties in vitro and in vivo between CNS-derived and non-CNS-derived ECM hydrogels. In this context, due to human origin, high accessibility, and no ethical constraints, UC-ECM represents a promising tissue source for hydrogel preparation [60].

p0285 The control of ECM scaffold degradation is essential for the constructive tissue remodeling process, as the degrading biomaterial is gradually replaced by endogenous cells that build a new functional ECM equivalent, as opposed to the scar tissue. As previously shown by ourselves and others, in the inflammatory conditions of the 

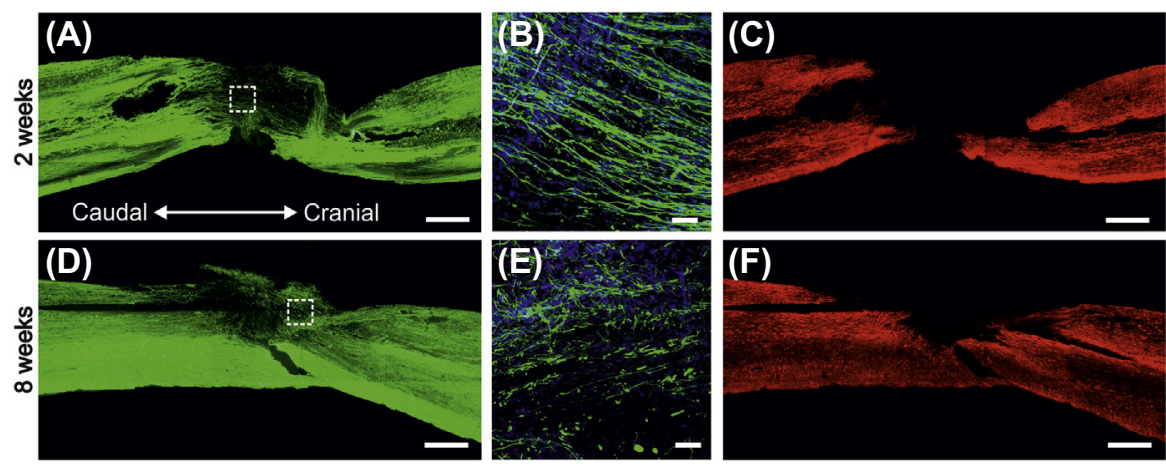

f0040

FIGURE 7.7

Representative images of the spinal cord lesion (A-C) 2 weeks and (D-F) 8 weeks after the injection of SC-ECM hydrogels. Immunofluorescent staining for (A, B, D, E) neurofilaments (NF160), (C, F) astrocytes (GFAP), and (B, E) cell nuclei (DAPI, blue). Squares $(A, D)$ are also shown under the higher magnification insets ( $B$ and $E$ ). Scale bar: (A, C, D, F) $500 \mu \mathrm{m}$; (B, E) $50 \mu \mathrm{m}$.

Modified from Tukmachev D, Forostyak S, Koci Z, Zaviskova K, Vackova I, Vyborny $K$, et al. Injectable extracellular matrix hydrogels as scaffolds for spinal cord injury repair. Tissue Eng 2016;22(3-4):306-317.

lesion the implanted ECM hydrogels are quickly populated with the resident cells, significantly accelerating the degradation rate of ECM-based scaffolds. In effect, rapid hydrogel degradation might burden the neuroregenerative potential of the ECM hydrogels and significantly influence the character of the tissue replacement. Therefore, the establishment of nontoxic, biocompatible, and reproducible techniques to prolong and control the degradability of ECM-derived hydrogels represents the challenging issue of applying such biomaterials for neural regeneration. A common method of how to reduce the degradation of various biomaterials is chemical cross-linking.

p0290 In our recent study, we used $1 \mathrm{mM}$ genipin cross-linking to improve the structural stability of the ECM hydrogel derived from a human umbilical cord, and evaluated the feasibility of the obtained materials for neural tissue repair. Genipin is a natural cross-linking low-toxic agent, derived from gardenia fruit, which can bridge free amino groups of lysine or hydroxylysine residues of different polypeptide chains, by monomeric or oligomeric cross-links in collagen as well as biological tissues $[62,63]$. To stabilize ECM scaffolds, genipin cross-linking was used in myocardial matrix hydrogel [64], decellularized spinal cord [65], or whole-liver decellularized grafts [66], where it revealed a lower cytotoxicity and in vivo immunogenicity than that of their glutaraldehyde-treated counterparts. We found $1 \mathrm{mM}$ genipin as a suitable cross-linker that enhanced in vitro as well as in vivo biostability of UC-ECM hydrogel without an adverse tissue response or enhanced inflammatory reaction [67]. 


\section{s0075 Conclusions}

p0295 The development of biomaterials, including scaffolds seeded with stem cells or serving as drug delivery systems, plays a key role in SCI repair. In this context, many types of scaffolds are able to support axonal growth and spouting after SCI, but fail to achieve a significant functional recovery, mainly due to the reduced regeneration of long-tract axons through sites of SCI [68]. We have demonstrated that both synthetic and natural hydrogels have the potential for tissue bridging, which suggests that the neural tissue is extensively flexible in regards to the implant acceptability. PHPMA hydrogels revealed a greater ability to support axonal growth when compared to PHEMA hydrogels, even when the latter one was modified with IKVAV, RGD, or fibronectin [38,45]. Correspondingly, PHPMA-RGD hydrogel, when combined with MSCs, improved motor functions in chronic SCI [37]. However, despite various modifications or seeding with different cell types, we commonly found failure of the long-term promotion of axonal ingrowth into the synthetic nondegradable scaffolds. This suggests a limited ability of synthetic nondegradable scaffolds to induce functional tissue repair that requires additional treatment approaches. Moreover, the limited pore space available for the tissue repair and no experimental data demonstrating the safety the long-term implantation (within years) weaken the potential of these materials for their further development for clinical use. Therefore, from the clinical aspect, injectable natural materials, such as HA or ECM hydrogels, have a much higher potential due to the ease of application, soft mechanical properties, and degradability.

p0300 It is generally accepted that SCI therapy requires a combined treatment approach that would comprehensively restart endogenous repair processes. The combined treatment of synthetic as well as natural materials with MSCs revealed an enhancement in tissue repair in most of the studies, but often failed in the induction of behavioral improvement. This indicates that providing a substrate for the tissue bridging, either synthetic or natural, is not sufficient for functional recovery and needs to be potentiated by an effective number of transplanted cells or other active factors, and also proper rehabilitation.

p0305 Finally, to develop scaffolds for SCI, cost effectiveness and reproducibility of the manufacture, which is required to be performed under GMP (Good Manufacture Practice) conditions, must be taken into consideration to allow the transfer of the scaffold production into clinical practice. In this regard, it must be mentioned that several clinical trials, to assess the safety and functional improvements after the implantation of a PLGA/poly(lysine) scaffold and a collagen scaffold loaded with MSCs in SCI patients, are already ongoing in the USA and China, respectively (http://www.clinicaltrials.gov/). 


\section{Acknowledgment}

This work was supported by project CZ.02.1.01/0.0./0.0/15_003/0000419 and EATRIS LM2015064 from the Ministry of Education, Youth and Sports of the Czech Republic.

\section{References}

[1] Rowland JW, Hawryluk GW, Kwon B, Fehlings MG. Current status of acute spinal cord injury pathophysiology and emerging therapies: promise on the horizon. Neurosurg Focus 2008;25(5):E2.

[2] Varma AK, Das A, Wallace G, Barry J, Vertegel AA, Ray SK, et al. Spinal cord injury: a review of current therapy, future treatments, and basic science frontiers. Neurochem Res 2013;38(5):895-905.

[3] Fawcett JW. The extracellular matrix in plasticity and regeneration after CNS injury and neurodegenerative disease. Progress Brain Res 2015;218:213-26.

[4] Fuhrmann T, Anandakumaran PN, Shoichet MS. Combinatorial therapies after spinal cord injury: how can biomaterials help? Adv Healthc Mater 2017;6(10).

[5] Liu SW, Schackel T, Weidner N, Puttagunta R. Biomaterial-supported cell transplantation treatments for spinal cord injury: challenges and perspectives. Front Cell Neurosci $2018 ; 11$.

[6] Kubinova S, Sykova E. Biomaterials combined with cell therapy for treatment of spinal cord injury. Regen Med 2012;7(2):207-24.

[7] Pego AP, Kubinova S, Cizkova D, Vanicky I, Mar FM, Sousa MM, et al. Regenerative medicine for the treatment of spinal cord injury: more than just promises? J Cell Mol Med 2012;16(11):2564-82.

[8] Straley KS, Foo CW, Heilshorn SC. Biomaterial design strategies for the treatment of spinal cord injuries. J Neurotrauma 2010;27(1):1-19.

[9] Pires LR, Pego AP. Bridging the lesion-engineering a permissive substrate for nerve regeneration. Regen Biomater 2015;2(3):203-14.

[10] Badylak SF, Freytes DO, Gilbert TW. Extracellular matrix as a biological scaffold material: structure and function. Acta Biomater 2009;5(1):1-13.

[11] Haggerty AE, Oudega M. Biomaterials for spinal cord repair. Neurosci Bull 2013;29(4): 445-59.

[12] Rossi F, Perale G, Papa S, Forloni G, Veglianese P. Current options for drug delivery to the spinal cord. Expert Opin Drug Deliv 2013;10(3):385-96.

[13] Ziemba AM, Gilbert RJ. Biomaterials for local, controlled drug delivery to the injured spinal cord. Front Pharmacol 2017;8.

[14] Perale G, Rossi F, Santoro M, Peviani M, Papa S, Llupi D, et al. Multiple drug delivery hydrogel system for spinal cord injury repair strategies. J Control Release 2012;159(2): 271-80.

[15] Perale G, Rossi F, Sundstrom E, Bacchiega S, Masi M, Forloni G, et al. Hydrogels in spinal cord injury repair strategies. ACS Chem Neurosci 2011;2(7):336-45. 
[16] Hejcl A, Lesny P, Pradny M, Michalek J, Jendelova P, Stulik J, et al. Biocompatible hydrogels in spinal cord injury repair. Physiol Res 2008;57(Suppl. 3):S121-32.

[17] Franze K, Gerdelmann J, Weick M, Betz T, Pawlizak S, Lakadamyali M, et al. Neurite branch retraction is caused by a threshold-dependent mechanical impact. Biophys $\mathrm{J}$ 2009;97(7):1883-90.

[18] Georges PC, Miller WJ, Meaney DF, Sawyer ES, Janmey PA. Matrices with compliance comparable to that of brain tissue select neuronal over glial growth in mixed cortical cultures. Biophys J 2006;90(8):3012-8.

[19] Jiang FX, Yurke B, Firestein BL, Langrana NA. Neurite outgrowth on a DNA crosslinked hydrogel with tunable stiffnesses. Ann Biomed Eng 2008;36(9):1565-79.

[20] Koch D, Rosoff WJ, Jiang JJ, Geller HM, Urbach JS. Strength in the periphery: growth cone biomechanics and substrate rigidity response in peripheral and central nervous system neurons. Biophys J 2012;102(3):452-60.

[21] Moshayedi P, Ng G, Kwok JCF, Yeo GSH, Bryant CE, Fawcett JW, et al. The relationship between glial cell mechanosensitivity and foreign body reactions in the central nervous system. Biomaterials 2014;35(13):3919-25.

[22] Moeendarbary E, Weber IP, Sheridan GK, Koser DE, Soleman S, Haenzi B, et al. The soft mechanical signature of glial scars in the central nervous system. Nat Commun 2017;8:14787.

[23] Miyoshi H, Adachi T. Topography design concept of a tissue engineering scaffold for controlling cell function and fate through actin cytoskeletal modulation. Tissue Eng Part B 2014;20(6):609-27.

[24] Guo BL, Sun Y, Finne-Wistrand A, Mustafa K, Albertsson AC. Electroactive porous tubular scaffolds with degradability and non-cytotoxicity for neural tissue regeneration. Acta Biomater 2012;8(1):144-53.

[25] Khorasani MT, Mirmohammadi SA, Irani S. Polyhydroxybutyrate (PHB) scaffolds as a model for nerve tissue engineering application: fabrication and in vitro assay. Int $\mathrm{J}$ Polym Mater 2011;60(8):562-75.

[26] Xie JW, MacEwan MR, Liu WY, Jesuraj N, Li XR, Hunter D, et al. Nerve guidance conduits based on double-layered scaffolds of electrospun nanofibers for repairing the peripheral nervous system. Acs Appl Mater Inter 2014;6(12):9472-80.

[27] Gros T, Sakamoto JS, Blesch A, Havton LA, Tuszynski MH. Regeneration of long-tract axons through sites of spinal cord injury using templated agarose scaffolds. Biomaterials 2010;31(26):6719-29.

[28] Gao MY, Lu P, Bednark B, Lynam D, Conner JM, Sakamoto J, et al. Templated agarose scaffolds for the support of motor axon regeneration into sites of complete spinal cord transection. Biomaterials 2013;34(5):1529-36.

[29] Novikova LN, Kolar MK, Kingham PJ, Ullrich A, Oberhoffner S, Renardy M, et al. Trimethylene carbonate-caprolactone conduit with poly-p-dioxanone microfilaments to promote regeneration after spinal cord injury. Acta Biomater 2018;66:177-91.

[30] Yuan N, Tian W, Sun L, Yuan RY, Tao JF, Chen DF. Neural stem cell transplantation in a double-layer collagen membrane with unequal pore sizes for spinal cord injury repair. Neural Regen Res 2014;9(10):1014-9.

[31] Jurga M, Dainiak MB, Sarnowska A, Jablonska A, Tripathi A, Plieva FM, et al. The performance of laminin-containing cryogel scaffolds in neural tissue regeneration. Biomaterials 2011;32(13):3423-34. 
[32] Tukmachev D, Forostyak S, Koci Z, Zaviskova K, Vackova I, Vyborny K, et al. Injectable extracellular matrix hydrogels as scaffolds for spinal cord injury repair. Tissue Eng 2016;22(3-4):306-17.

[33] Kubinova S, Horak D, Kozubenko N, Vanecek V, Proks V, Price J, et al. The use of superporous Ac-CGGASIKVAVS-OH-modified PHEMA scaffolds to promote cell adhesion and the differentiation of human fetal neural precursors. Biomaterials 2010; 31(23):5966-75.

[34] Kubinova S, Horak D, Vanecek V, Plichta Z, Proks V, Sykova E. The use of new surfacemodified poly(2-hydroxyethyl methacrylate) hydrogels in tissue engineering: treatment of the surface with fibronectin subunits versus Ac-CGGASIKVAVS-OH, cysteine, and 2-mercaptoethanol modification. J Biomed Mater Res A 2014;102(7):2315-23.

[35] Assuncao-Silva RC, Gomes ED, Sousa N, Silva NA, Salgado AJ. Hydrogels and cell based therapies in spinal cord injury regeneration. Stem Cell Int 2015;2015:948040.

[36] Kubinova S, Horak D, Sykova E. Cholesterol-modified superporous poly(2hydroxyethyl methacrylate) scaffolds for tissue engineering. Biomaterials 2009; 30(27):4601-9.

[37] Hejcl A, Sedy J, Kapcalova M, Toro DA, Amemori T, Lesny P, et al. HPMA-RGD hydrogels seeded with mesenchymal stem cells improve functional outcome in chronic spinal cord injury. Stem Cell Dev 2010;19(10):1535-46.

[38] Kubinova S, Horak D, Hejcl A, Plichta Z, Kotek J, Proks V, et al. SIKVAV-modified highly superporous PHEMA scaffolds with oriented pores for spinal cord injury repair. J Tissue Eng Regenerat Med 2015;9(11):1298-309.

[39] Ruzicka J, Romanyuk N, Hejcl A, Vetrik M, Hruby M, Cocks G, et al. Treating spinal cord injury in rats with a combination of human fetal neural stem cells and hydrogels modified with serotonin. Acta Neurobiol Exp 2013;73(1):102-15.

[40] Woerly S, Petrov P, Sykova E, Roitbak T, Simonova Z, Harvey AR. Neural tissue formation within porous hydrogels implanted in brain and spinal cord lesions: ultrastructural, immunohistochemical, and diffusion studies. Tissue Eng 1999;5(5):467-88.

[41] Woerly S, Pinet E, de Robertis L, Van Diep D, Bousmina M. Spinal cord repair with PHPMA hydrogel containing RGD peptides (NeuroGel). Biomaterials 2001;22(10): 1095-111.

[42] Lesny P, Pradny M, Jendelova P, Michalek J, Vacik J, Sykova E. Macroporous hydrogels based on 2-hydroxyethyl methacrylate. Part 4: growth of rat bone marrow stromal cells in three-dimensional hydrogels with positive and negative surface charges and in polyelectrolyte complexes. J Mater Sci Mater Med 2006;17(9):829-33.

[43] Hejcl A, Lesny P, Pradny M, Sedy J, Zamecnik J, Jendelova P, et al. Macroporous hydrogels based on 2-hydroxyethyl methacrylate. Part 6: 3D hydrogels with positive and negative surface charges and polyelectrolyte complexes in spinal cord injury repair. J Mater Sci Mater Med 2009;20(7):1571-7.

[44] Hejcl A, Ruzicka J, Kapcalova M, Turnovcova K, Krumbholcova E, Pradny M, et al. Adjusting the chemical and physical properties of hydrogels leads to improved stem cell survival and tissue ingrowth in spinal cord injury reconstruction: a comparative study of four methacrylate hydrogels. Stem Cell Dev 2013;22(20):2794-805.

[45] Hejcl A, Ruzicka J, Kekulova K, Svobodova B, Proks V, Mackova H, et al. Modified methacrylate hydrogels improve tissue repair after spinal cord injury. Int J Mol Sci $2018 ; 19(9)$. 
[46] Kubinova S, Horak D, Hejcl A, Plichta Z, Kotek J, Sykova E. Highly superporous cholesterol-modified poly(2-hydroxyethyl methacrylate) scaffolds for spinal cord injury repair. J Biomed Mater Res A 2011;99:618-29.

[47] Mackova H, Plichta Z, Proks V, Kotelnikov I, Kucka J, Hlidkova H, et al. RGDS- and SIKVAVS-modified superporous poly(2-hydroxyethyl methacrylate) scaffolds for tissue engineering applications. Macromol Biosci 2016;16:1621-31.

[48] Hejcl A, Ruzicka J, Proks V, Mackova H, Kubinova S, Tukmachev D, et al. Dynamics of tissue ingrowth in SIKVAV-modified highly superporous PHEMA scaffolds with oriented pores after bridging a spinal cord transection. J Mater Sci Mater Med 2018;29(7).

[49] Zeng X, Qiu XC, Ma YH, Duan JJ, Chen YF, Gu HY, et al. Integration of donor mesenchymal stem cell-derived neuron-like cells into host neural network after rat spinal cord transection. Biomaterials 2015;53:184-201.

[50] Lai BQ, Che MT, Du BL, Zeng X, Ma YH, Feng B, et al. Transplantation of tissue engineering neural network and formation of neuronal relay into the transected rat spinal cord. Biomaterials 2016;109:40-54.

[51] Litwiniuk M, Krejner A, Speyrer MS, Gauto AR, Grzela T. Hyaluronic acid in inflammation and tissue regeneration. Wounds 2016;28(3):78-88.

[52] Knopf-Marques H, Pravda M, Wolfova L, Velebny V, Schaaf P, Vrana NE, et al. Hyaluronic acid and its derivatives in coating and delivery systems: applications in tissue engineering, regenerative medicine and immunomodulation. Adv Healthc Mater 2016; 5(22):2841-55.

[53] Darr A, Calabro A. Synthesis and characterization of tyramine-based hyaluronan hydrogels. J Mater Sci Mater Med 2009;20(1):33-44.

[54] Wolfova L, Pravda M, Foglarova M, Memcova M, Niedoba K, Velebny V. Derivates based on hyaluronic acid, capable of forming hydrogels, method of preparation thereof, hydrogels based on said derivatives, method of preparation thereof and use. WO2013127374 A1. 2013.

[55] Kučera L, Weinfurterová R, Dvořákova J, Kučera J, Pravda M, Foglarová M, et al. Chondrocyte cultivation in hyaluronan-tyramine cross-linked hydrogel. Int J Polymer Mater Polymer Biomaterials 2015;64(13):661-74.

[56] Zaviskova K, Tukmachev D, Dubisova J, Vackova I, Hejcl A, Bystronova J, et al. Injectable hydroxyphenyl derivative of hyaluronic acid hydrogel modified with RGD as scaffold for spinal cord injury repair. J Biomed Mater Res A 2018;106(4):1129-40.

[57] Krupa P, Vackova I, Ruzicka J, Zaviskova K, Dubisova J, Koci Z, et al. The effect of human mesenchymal stem cells derived from Wharton's jelly in spinal cord injury treatment is dose-dependent and can be facilitated by repeated application. Int J Mol Sci 2018;19(5).

[58] Kubinova S. Extracellular matrix based biomaterials for central nervous system tissue repair: the benefits and drawbacks. Neural Regen Res 2017;12(9):1430-2.

[59] Medberry CJ, Crapo PM, Siu BF, Carruthers CA, Wolf MT, Nagarkar SP, et al. Hydrogels derived from central nervous system extracellular matrix. Biomaterials 2013;34(4): $1033-40$.

[60] Koci Z, Vyborny K, Dubisova J, Vackova I, Jager A, Lunov O, et al. Extracellular matrix hydrogel derived from human umbilical cord as a scaffold for neural tissue repair and its comparison with extracellular matrix from porcine tissues. Tissue Eng $\mathrm{C}$ Methods 2017;23(6):333-45. 
[61] Crapo PM, Medberry CJ, Reing JE, Tottey S, van der Merwe Y, Jones KE, et al. Biologic scaffolds composed of central nervous system extracellular matrix. Biomaterials 2012;33(13):3539-47.

[62] Sung HW, Huang RN, Huang LL, Tsai CC, Chiu CT. Feasibility study of a natural crosslinking reagent for biological tissue fixation. J Biomed Mater Res 1998;42(4): 560-7.

[63] Daniel M, NK K, Myron S. Injectable collagen-genipin gel for the treatment of spinal cord injury: in vitro studies. Adv Funct Mater 2011;21(24):4788-97.

[64] Wassenaar JW, Braden RL, Osborn KG, Christman KL. Modulating in vivo degradation rate of injectable extracellular matrix hydrogels. J Mater Chem B 2016;4(16): 2794-802.

[65] Jiang T, Ren XJ, Tang JL, Yin H, Wang KJ, Zhou CL. Preparation and characterization of genipin-crosslinked rat acellular spinal cord scaffolds. Mater Sci Eng C Mater Biol Appl 2013;33(6):3514-21.

[66] Wang Y, Bao J, Wu X, Wu Q, Li Y, Zhou Y, et al. Genipin crosslinking reduced the immunogenicity of xenogeneic decellularized porcine whole-liver matrices through regulation of immune cell proliferation and polarization. Sci Rep 2016;6:24779.

[67] Vyborny K, Vallova J, Koci Z, Kekulova K, Jirakova K, Jendelova P, Hodan J, Kubinova S. Genipin and EDC crosslinking of extracellular matrix hydrogel derived from human umbilical cord for neural tissue repair. SciRep 2019;9(1):10674.

[68] Eva R, Andrews MR, Franssen EH, Fawcett JW. Intrinsic mechanisms regulating axon regeneration: an integrin perspective. Int Rev Neurobiol 2012;106:75-104. 

Non-Print Items

\begin{abstract}
Spinal cord injury often results in a permanent loss of motor and sensory functions, due to the low ability of adult axons to regenerate. One of the treatment strategies is the development of scaffolding materials that bridge the damaged tissue or cavity and promote endogenous axonal regrowth. In this chapter, the results from the implantation or injection of various synthetic or natural polymers with various modification, stiffness, and porosity are summarized and critically discussed.
\end{abstract}

\title{
Keywords:
}

Extracellular matrix, Hyaluronic acid, Hydrogel, Hydroxyethyl methacrylate, Hydroxypropyl methacrylamide, Regeneration, Spinal cord injury, Stem cells. 


\title{
CHAPTER
}

\section{c0008 Nanomaterials for spinal cord injury (SCl) regeneration}

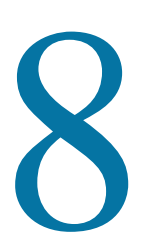

\author{
Emanuele Mauri, PhD ${ }^{\mathrm{a}}$, Maurizio Masi, PhD ${ }^{\mathrm{b}}$ \\ ${ }^{a}$ Department of Engineering, Tissue Engineering and Chemistry for Engineering Unit, Università \\ Campus Bio-Medico di Roma, Rome, Italy; ${ }^{\mathrm{b}}$ Department of Materials, Chemistry and Chemical \\ Engineering "G. Natta", Politecnico di Milano, Milan, Italy
}

\section{s0010 Nanotechnology and nanomedicine: an overview}

p0010 During the last decades, nanotechnology has become a widespread scientific approach in many different biological and medical fields, due to its unique benefits of improved performances and its potential toward the clinical translation [1-3]. It is defined by the US National Nanotechnology Initiative as "it is concerned with materials and systems whose structures and components exhibit novel and significantly improved physical, chemical and biological properties, phenomena and processes due to their nanoscale size" [4]. It means that qualitatively new and advantageous properties emerge from the materials at the nanoscale: with their tiny dimensions, these materials possess unique physiochemical properties such as conductivity, strength, durability, and chemical reactivity. Generally characterized by a size range between 10 and $1000 \mathrm{~nm}$, nanomaterials guarantee a higher surface area per unit volume resulting in higher surface activity and subsequent higher interaction with the external stimuli. Their use in medical applications is generally recognized as "nanomedicine" and characterized by therapeutic aims to improve diseases diagnosis and curative modalities, reduce healthcare costs, and meet the societal and ethical challenges $[5,6]$.

p0015 In particular, the advent of nanomedicine may provide the innovative tools to overcome the current constraints about disorders that have not an efficient cure and whose therapeutic protocol study is still under investigation. In this scenario, the neurological diseases have a pivotal role: they affect many people with permanent deficits, and in particular spinal cord injury (SCI) involves also the youngest and most active segment of our society. Multifactorial damages occur as primary and secondary SCI, regarding the interruption of ascending and descending nerves pathway and the resulting paraplegia or tetraplegia. Nowadays, the research has investigated the direct administration of protective drugs or antibodies, the neural implantation, the electrical stimulation, and the environmental modification [7-9], recording some promising results in recovering specific spinal cord

Spinal Cord Injury (SCI) Repair Strategies. https://doi.org/10.1016/B978-0-08-102807-0.00008-9 Copyright $\odot 2020$ Elsevier Ltd. All rights reserved. 
functionalities [10-13], but the major problems regarding the oriented axonal growth, remyelination, and reknitting tissue remain unsolved.

p0020 For these reasons, the nanoscale approach could develop innovative methods for tackling the SCI pathophysiological challenges. In detail, nanomaterials offer the opportunity to combine the neuroprotective and neuroregenerative pathways that represent the milestones for treating SCI. Nanocarriers have the capacity to increase the bioavailability of neuroprotective drugs through delivery in targeted sites and extended circulation times [14,15], Moreover, they provide large specific surface, which allows the surface binding of targeting moieties, receptors, and therapeutic biomolecules, increases the mutual interactions with the physiological compartments and enhances the stability of loaded drugs or proteins. On the other hand, the neuroregeneration aims could be implemented using nanomatrices that mimic the extracellular environment and influence the cellular proliferation, growth, and differentiation [16-18].

p0025 Other benefits are related to the ability of self-assembling, scavenger of reactive oxygen species, or imaging and detection tool that could be addressed through functionalization strategies, exploiting the physicochemical features of the raw materials. The smart adjustment of these features can overcome the conventional practices of therapeutic administration and design a nanomedicine context that opens new horizons for prevention, diagnosis, and treatment of severe diseases. Research has proposed different nanomaterial configurations: nanoparticles, nanogels, nanotubes, nanowires, nanofibers, nanorods, quantum dots, and hollow spheres. In the SCI therapy, particular focus is related to the first three tools, due to their more attractive compliance with the neuronal scenario.

\section{s0015 Design of nanomaterials}

p0030 The spinal cord environment is segregated by the blood spinal cord barrier (BSCB) that allows the transition of few molecules from the circulating blood to the nervous extracellular fluid. BSCB performs the spinal cord pathological protection from immunological and biochemical insults and foreign substances not recognizable by specific markers. As a result, drug permeability is limited using oral, intravenous, and intraarterial chemical routes: the clinic administration exploits the principle of higher blood osmotic pressure and the permeability of endothelial cells to promote the delivery of mannitol hypertonic solution and bradykinin and outline some curative guidelines [19,20]. Moreover, the surgical approach regarding the use of catheters and implanted mini-pumps represents an alternative but accompanied by risks and potential side effects $[21,22]$.

p0035 Innovative non-conventional methods suggest the design of biomaterials that can be placed into desired areas and able to release the therapeutic compounds in situ. Together with the basic criteria of biocompatibility, another important aspect is the matching of the biomaterial mechanical properties to those of the host tissue, resembling the stiffness of the spinal cord components that could overcome the 
drawbacks related to the risks of further inflammations within the treated site. Macrophages, microglia, astrocytes, oligodendrocytes, and even neurons to an extent can all respond and release inflammatory cytokines: the biomaterial has to interact with the resident cells' immune response in a proactive manner to tune the injured condition [23]. The synthesis of a nanotool able of specific cellular targeting implements the chance to selectively interact with a cell population and gives the opportunity to modulate its response to the external injury, preventing or reducing the harmful progression of the lesion. In particular, one major goal is the option to preserve the microglia curative and protective effect, avoiding deleterious proinflammatory events during the neurodegeneration $[24,25]$. The nanomaterial design can also be considered as a strategic choice to address a multitarget strategy based on the neuroprotective and neuroregenerative joint action; about this, the use of bioinert or biointeractive tools is distinctive. The first ones are generally presented as nondegradable materials, avoiding the issues of degradation rate control and toxicity of degradation products; however, these systems generate higher risks of activation of proinflammatory responses or unconventional spatial rearrangement of the biological compartments due to their hindrance as foreign matter. Otherwise, the second ones are composed by degradable components that are often more inherently adhesive to neurons or glial cells.

p0040 Generally, the nanomaterials synthesis is performed using top-down, bottom-up, or hybrid methods [26]. The top-down approach involves the conversion of a bulk material to a nanosized configuration through controlled etching, elimination, or layering of the initial substance; optical, soft and nanoimprint, block copolymer, and scanning probe lithographic protocols belong to this category. The bottom-up strategies are based on the arrangement of atoms, molecules, macromolecules, or supramolecules exploiting their peculiar and reciprocal interactions to produce a stable nanoassembling, without waste or by-products that need to be eliminated; however, some technological vigilance is required to solve the questionable points about the surface preparation and conditioning for the controlled deposition of the atoms, control of impurities, and uniformity of the nanostructure. Atomic layer deposition, sol-gel nanofabrication, molecular self-assembly, physical and chemical vapor phase deposition, and DNA scaffolding are the guidelines to design nanotools following this method. Instead, the hybrid tactic is based on the integration of the two previously discussed techniques to produce a more flexible design of nanostructures [27].

\section{s0020 Key properties}

p0045 The fundamental parameters to promote the use of nanocarriers in the SCI progress are as follows: size, shape, and surface charge, as shown in Fig. 8.1. The ability to control them allows affecting the nanosystem pathway through biological fluids, the cellular internalization, and the therapeutic delivery kinetics. In detail:

u0010 - diameter values represent a critical factor to the cellular uptake kinetics, discriminating between nanocarriers that are ingested by cell to achieve the 


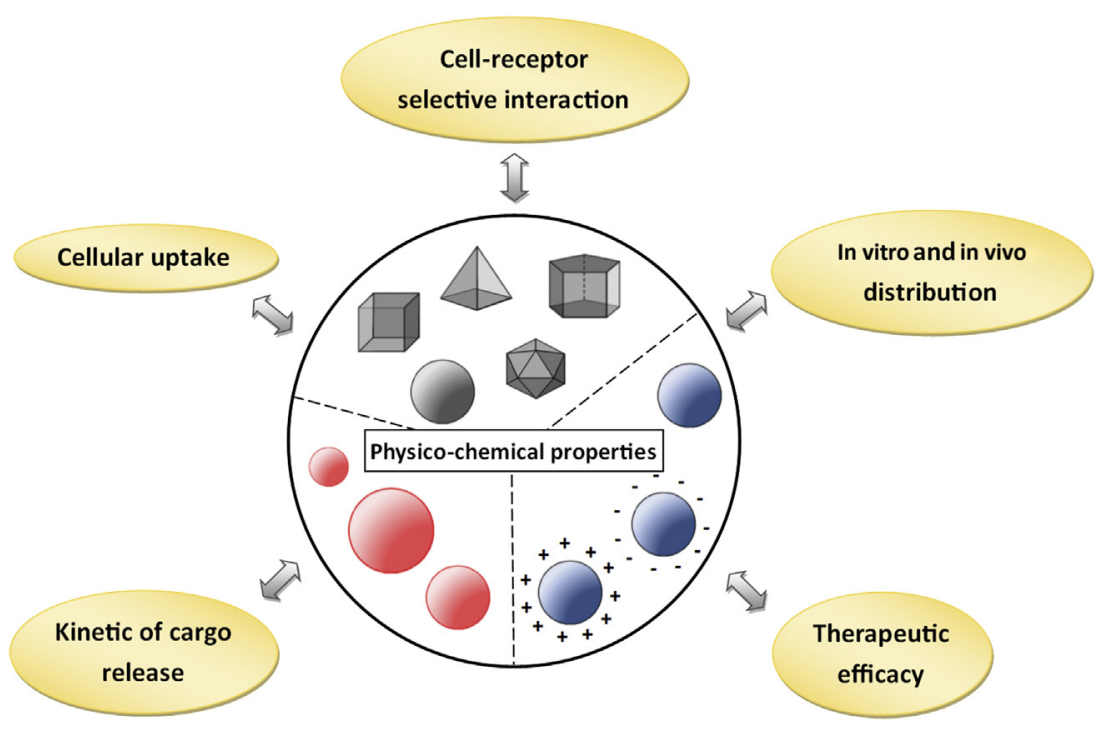

f0010 FIGURE 8.1

Scheme of the main parameters affecting the nanomaterial fate and efficacy in SCl.

desired effect and those are intended to remain in the extracellular environment over time. The SCI transport kinetics are strongly influenced by size; smaller particles could show higher distribution in tissues and across the biological barrier in less time than larger ones [28,29], and the ligand conjugation produces a carrier design with improved biological signals to interact with specific receptors/transporters expressed by neural cells, also enhancing the BSCB penetration efficiency and evading the reticuloendothelial system; however, limited ability for controlled modification in a batch-to-batch synthesis approach is an undesired effect [30];

u0015 - shape is another aspect addressing the nanoparticles development, due to its influence in body distribution and cell uptake [31]: nanoscale can be designed as spherical, cubic, tubular, rod-like, and irregular configuration. The evaluation of the optimal shape and physical properties of the nanovectors can be performed according to the therapeutic needs with respect to the studied model of disease or clinical context: in nervous system, nanorods with targeting peptides are characterized by higher specific and lower nonspecific accumulation in the desired vascular environment compared to nanoparticles, avoiding the immune clearance [32], whereas bioconcave nanoparticles were able to improve the release of the therapeutic cargo than spherical, tubular and spindle geometries [33]. The shape of nanoparticles in the circulation is of particular interest because it has a significant impact on hydrodynamics and biological polyvalent interactions;

u0020 - charge and surface properties are the third crucial feature for SCI application [34]; generally, positively charged nanoparticles are more easily internalized by 
cell populations than system with a neutral or negative surface charge $[35,36]$. However, quantum dots and surface functionalization can modulate this scenario; in particular, the first ones are internalized proportionally to the absolute value of surface charge (higher the modulus, higher the uptake) whereas the modification with specific chemical groups, proteins, or growth factors could create a charge shift, altering the distribution of positive and negative groups, with the aim to target specific area [37]. In addition, this parameter affects the nanotool fate because it can improve the interactions with the immune system or the transportation in blood stream by reducing the selective efficacy to SCI through electrostatic modulation. Common approaches to increase the nanoparticles penetration in central nervous system are related to the PEGylation: PEG is able to reduce the charge-based protein contacts and the aggregation creating a superficial shield and increase the nanoparticle solubility [33].

\section{s0025 Materials}

p0065 The design of nanomaterials to support medical drugs or biomolecules performing the desired pharmacokinetics, reduced toxicity, therapeutic efficacy, and water inertness and to promote the selective interactions with the cell compartments involved in SCI is generally based on the use of polymers. They are categorized as naturally or synthetically derived materials. The natural polymers provide structures similar to living tissues, stimulating cell responses, promoting excellent cell adhesion and growth, or reducing immunological reactions and toxicity; however, the production of nanomatrices from biological materials with homogeneous and reproducible structures is a challenge and these tools generally suffer of poor mechanical properties, which limit their use. Otherwise, synthetic materials offer the chance to tailor the scaffold architecture, in molecular weight, composition, availability, degradation, and mechanical properties, but with the main drawback of the risk of rejection due to lack of bioactivity or biocompatibility. The therapeutic aim determines that polymer type is chosen and combining natural and synthetic polymers is a solution to enhance the biological suitability. The former ones are generally silicone, lipids, protein polymers, involving collagen, albumin, and fibrin, and polysaccharide polymers, such as chitosan, hyaluronic acid, chondroitin sulfate, agarose, alginate, cellulose, and heparin, whereas the synthetic polymers are polylactic acid (PLA), poly(lactic-co-glycolic acid) (PLGA), polyethyleneimine (PEI), polyethylene glycol (PEG), polyglycolic derivatives, polyacrylates, polymethacrylates, polycyanoacrylates, and poly( $\varepsilon$-caprolactone) (PCL). The chemical structures of the main polymers used in SCI treatments are reported in Fig. 8.2. The presence of specific chemical groups and the peculiar physicochemical properties guarantee the synthesis of carriers having specific capabilities with respect to drug pharmacokinetics and adjustable with further functionalizations to penetrate the BSCB. In particular, PLGA present a significant advantage justifying its consistent use: among the synthetic polymers, it is approved by the FDA and EMA in many drug delivery systems, leading PLGA-based nanomatrices in a promising position for clinical trials [38]. 

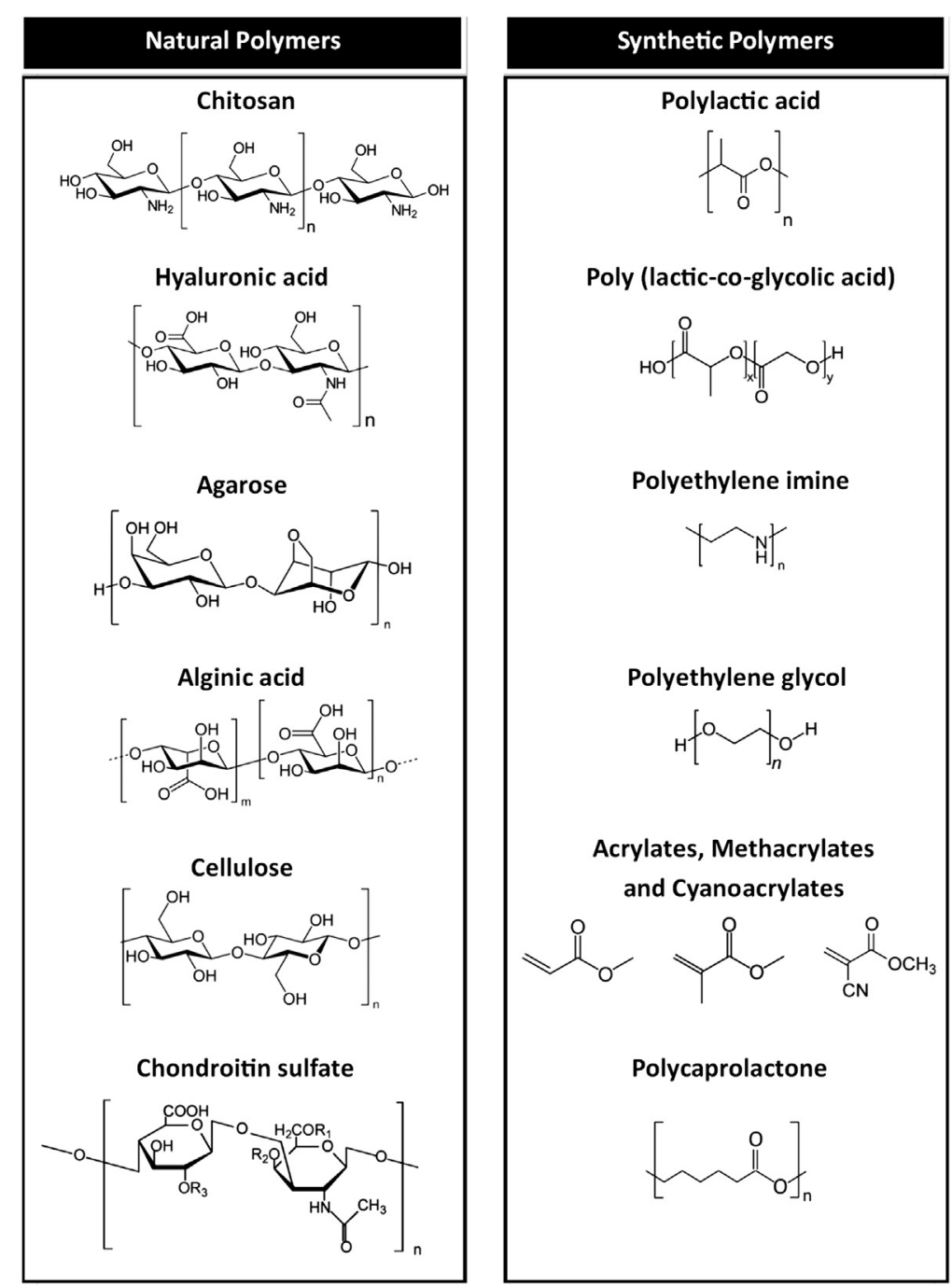

f0015

FIGURE 8.2

Chemical structures of the natural and synthetic polymers used in $\mathrm{SCl} . \mathrm{R}_{1}=\mathrm{H}$, $\mathrm{R}_{2}=\mathrm{SO}_{3} \mathrm{H}, \mathrm{R}_{3}=\mathrm{H}$

Biodegradable polymers similar to PLGA can be used for sustained therapeutic release from delivery systems and concurrently supply a suitable tissue scaffold [13]. They can protect their cargo from degradation and enhance their stability.

p0070 Depending on the purpose of nanocarriers, specific characteristics are essential to create unique interactions with the SCI biological compartment. Nanoparticles, nanogels, and nanotubes are the main tools developed to lead to desired outcomes. 


\section{s0030 Nanoparticles in SCI}

p0075 Nanoparticles (NPs) are classified as colloidal systems obtained by polymers physically or chemically linked. According to the synthesis procedure, they are generally recognized as nanospheres or nanocapsules (Fig. 8.3). The first ones are solid matrix-like systems where drug is dissolved, entrapped, encapsulated, chemically bound, or adsorbed to the constituent polymeric chains; they are characterized by diameter values within the range of $100-200 \mathrm{~nm}$, with a considerable polydispersity parameter [39]. Their size improves the biological circulation, but they are also predominantly subjected to the reticuloendothelial phagocytic activity. For these reasons, the synthesis and the modification of the nanostructures play a pivotal role to address the nanospheres fate in the complex SCI environment. For example, commonly developed configurations involve the use of different surfactants (such as poloxamine, poloxamer, and Brij) adsorbed to the NPs surface or the coating with amphiphilic layer, to confer a "water-look" to the nanocarriers and minimize the immune internalization process [40]. However, promising results have been obtained over a short period of time; whereas, over a long time, the surfactant trend to desorb from the NPs surface have showed no differences in cell uptake between the coated and uncoated systems.

p0080 On the other side, nanocapsules are identified as vesicular structures composed by a single external layer of polymeric membrane and a lipophilic therapeutic core that generate a "drug storage" for in situ release [41]. Their utility is focused on the hydrophobic drug delivery and generally are synthetized using polyester homopolymers such as PLA, PLGA, PCL, or PEG. The latter is used to avoid the opsonization and to reduce the cytotoxicity of the final nanocapsules. Main advantages are related to the ability to incorporate hydrophobic drugs at concentrations greater than their intrinsic water solubility. Alternatively, when the vesicle core is an aqueous phase and the surrounding coating is a polymer bilayer, the particles are indicated as polymersomes $[42,43]$. The use of amphiphilic copolymers guarantees the formation of a

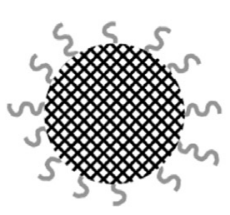

\section{Nanosphere}

Size: $100-200 \mathrm{~nm}$

Phase-separated

solid matrix core

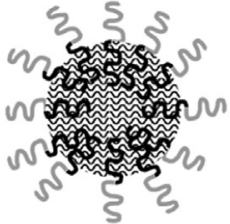

Nanocapsule

Size: $100-300 \mathrm{~nm}$

Polymeric membrane surrounding a drug reservoir or oily core

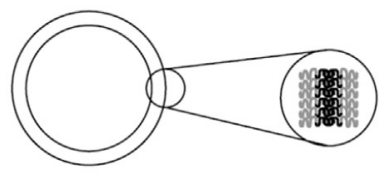

\section{Polymersome}

Size: $5 \mathrm{~nm}-5 \mu \mathrm{m}$

Polymers assembled

in a bilayer around an

hydrophilic reservoir

fO020 FIGURE 8.3

Putative structures of nanospheres, nanocapsules, and polymersomes. 
nanostructure able to preserve the therapeutic water-soluble cargo and release it over time. Indeed, these materials are bioinert but not biodegradable. Latest researchers have developed the synthesis of polymersomes composed of PEGylated polyesters or polycarbonates to improve the block copolymers biodegradability, addressing a leading aspect for in vivo applications. Polymersomes can be promising as artificial cells, vehicles for drug delivery or for mimicking cell membranes $[44,45]$. NPs have been demonstrated to aid particularly in drug delivery to spinal cord, and the conjugations with autocatalytic components (such as ceria) have shown the preliminary neuroprotective role in vitro mouse models $[46,47]$.

\section{s0035 Synthesis techniques}

p0085 The choice of the preparation method for NPs mainly depends on the employed polymeric materials. Acrylates derivatives are commonly used, and the formation of nanostructures requires polymerization techniques; in particular, nanospheres could be synthetized through emulsion polymerization (e.g., referring to the use of poly(methylmethacrylate)s and poly(ethylcyanoacrylate)s) or interfacial polymerization (when poly(alkylcyanoacrylate)s are involved). Emulsion polymerization considers the emulsification process of relatively hydrophobic monomer molecules in water by an oil-in-water emulsifier, followed by the initiation reaction using either a water-insoluble or an oil-soluble initiator; the propagation reaction of free radicals provides a high number of polymeric particles, dispersed in an aqueous continuous phase. The nucleation and the growth steps control the colloidal and physical properties of latex products [48]. To prevent the NPs aggregation, ionic and nonionic surfactants and protective colloids are used as stabilizers and physically adsorbed or chemically incorporated onto the particle surface. In this context, the therapeutic cargo is incorporated by dissolving it in the polymerization medium or through adsorption onto the nanospheres. The interfacial polymerization is a polycondensation reaction between two highly reactive monomers dissolved in two immiscible liquids or between a reactive phase containing an initiator or a catalyst and a monomer. The polymer formation occur at the interface region, and the reactants will more likely encounter the growing polymer chains instead of other monomers, resulting in high molecular weight structures. The preservation of molecular weight and NPs size range is performed through precipitation methods. In addition, in this case, the internalization of therapeutic compound can be achieved via adsorption or dispersion in one phase during the polymerization process [49].

p0090 Other nanosphere preparations regard nanoprecipitation, solvent evaporation, salting out, controlled gelification, desolvation technique, and coacervation methods. They are briefly discussed later.

u0025 - Nanoprecipitation: it is based on the interfacial deposition of a polymer following the displacement of a semipolar solvent miscible with water from an organic solution. The addition to the aqueous phase in the presence or absence 
of a surfactant can induce the precipitation of the polymer forming the nanostructures [50,51].

u0030 - Solvent evaporation: polymers and drugs are dissolved in an organic phase; then the addition of a suitable emulsifying agent, such as gelatin or polyvinyl alcohol, dispersed in water allows the formation of an o/w emulsion and the latter is subjected to solvent evaporation via continuous mixing or increasing temperature or by reducing pressure, obtaining the final NPs [52].

u0035 - Salting out: the polymer is dissolved in an organic solvent and the resulting solution is added to an aqueous phase, containing suitable emulsifier and high concentration of salts, in particular magnesium chloride hexahydrate or magnesium acetate tetrahydrate; the mechanical shear stress induces the emulsification. Pure water is then added to finalize the o/w emulsion formation under mild stirring to enhance diffusion of organic solvent into aqueous phase and form the nanospheres. These are recovered by centrifugation or cross-flow filtration, removing salting out reagents [53].

u0040 - Controlled gelification: this method is strictly related to the use of sodium alginate and calcium chloride to form gel state, and the addition of poly-L-lysine induces the design of polyelectrolyte complex. The obtained nanospheres are collected through centrifugation [54,55].

u0045 - Desolvation technique: this method is applicable when the NPs are composed of natural polymers. The active molecules are dissolved in ethanol or acetone (desolvating agent) dropwise to the polymeric aqueous solution under stirring, and a cross-linking agent is added to perform the physicochemical formation of NPs. The resulting suspension is centrifuged and lyophilized [56].

u0050 - Coacervation: it is an ionic gelation approach based on the electrostatic interactions between the polymer and therapeutic cargo aqueous phases, resulting in a coacervate having the particle size in the nanometer range [57].

p0125 On the other hand, the synthesis of nanocapsules is preferentially performed through interfacial deposition of preformed polymers [58]. The self-assembling of these block polymers is driven by the different affinity of the solvents to each used polymer, and thermodynamic forces affect the size and morphology [59]. In this procedure, the polymeric chains and the biomolecules that need to be encapsulated are dissolved in a water-miscible organic solvent (such as acetone); then a lipophilic surfactant and an oil, which is miscible with the solvent but immiscible with the polymeric mixture, are added and the resulting system is dispersed into an aqueous solution usually containing a hydrophilic stabilizing agent. Under stirring, the solvent diffuses into the aqueous phase and the polymer aggregates around the oil droplet. In the last decades, improved process are developed making use of emulsificationdiffusion techniques that through the subsequent addition of water to the system polymer biomolecules, omitting the oil in the formulation, induces solvent diffusion into the external phase, resulting in the formation of colloidal particles [60-62].

p0130 The polymersomes syntheses occur through film rehydration, similarly to liposome preparation. The copolymer is dissolved in organic solvent, such as 
chloroform, that evaporates under nitrogen stream leaving a thin film of polymer, which is rehydrated with the aqueous phase and via sonication and extrusion submicron vesicles are obtained with a narrow size distribution [63]. Alternatively, the use of amphiphilic polymers can promote the application of a $\mathrm{pH}$-switch method, that is, a bottom-up self-assembly process that can be precisely manipulated [64]: here, the aqueous phase can be acid or base, showing the reversible spontaneous formation of biocompatible vesicles [65].

p0135 NPs design could involve the employment of protein, due to their therapeutic effects promoting the functional axon regeneration and the potential recovery of some motor abilities [66]. In this case, the use of organic solvents causes toxicity and denaturation, whereas the sonication could lead to protein aggregation and consequent damage of its bioactivity [67]. According to these considerations, the protein-based NPs are synthetized using coacervation in aqueous solution and subsequent stabilization with a cross-linking agent. The fundamental step in this approach is the progressive addition of a desolvation factor (generally natural salts or alcohols) to the aqueous protein solution: the protein ternary structure is altered and in correspondence of a certain degree of desolvation, protein and polymer aggregates are generated. These nanostructures are then stabilized with glutaraldehyde or glyoxal. Finally, the system turbidity has to be finely tuned to obtain well-dispersed protein-based nanoparticles, without formation of macroscopic clusters [33,68].

\section{s0040 Nanoparticle functionalization guidelines}

p0140 Generally, the smart combination of different polymers represents a partial solution to the main needs of SCI treatment. Crossing the BSCB, improving the delivery efficacy, ensuring specific cell interactions, and releasing the controlled NPs cargo could be aimed through the polymer conjugation with specific moieties [69]. Indeed, used polymers possess peculiar chemical groups that can be exploited to link desired molecules or functionalities: alkoxyl, amino, carboxyl, hydroxyl, and alkene groups are involved in the physical or chemical modification. The encapsulation of the curative cargo can be performed exploiting the recognition groups based on the electrostatic or hydrophobic/hydrophilic interactions or via covalent bonds formation that can be stable or cleavable under specific stimuli, such as temperature, $\mathrm{pH}$, salts, and solvents. In detail, alkene groups can be involved in polymerization processes obtaining nanosystems editable through physical bonds. For example, the first polymer-based NPs applied in the central nervous system scenario were formed by poly(butylcyanoacrylate), obtained by polymerization between butylcyanocrylate monomers and dextran, and then functionalized with polysorbate-80 (Tween 80 ) that due to its chemical structure with hydrophilic and hydrophobic groups generates a superficial NPs coating. This modification has exhibited capacity of penetrating the neural barrier and of delivering in the central nervous system [70,71]. Moreover, the NPs coating with the antioxidant enzyme superoxide dismutase has showed neuroprotective effects [72]. Polyesters, such as PLA, PGA, and PLGA, are linked to peptides, enzymes, dyes, or surfactants to overcome the BSCB constraints. PLA carboxyl groups are activated 
using epoxide moieties to perform the grafting of transactivating transcriptor peptide through ring opening reaction [73] and allows the drug distribution in the parenchyma. Carboxyl and hydroxyl PLGA terminal groups can be functionalized with thiol chemistry, linking glutathione-modified PEG to target delivery [74]; otherwise, the PLGA hydrogen-bond acceptors (the carbonyl) and hydrogen-bond donors (the hydroxyl) guarantee the synthesis of NPs able to delivery growth factors (for example, fibroblast growth factor-2 [75]) that can reduce the vessels vasoconstriction in the tissue surrounding the SCI primary damages and promote the angiogenesis. Similar approach is used to internalized bioactive neurotrophin within the PLGA nanomatrices, exploiting the emulsion-solvent evaporation method, commonly used in the reactions concerning proteins [76]; in this case, the final aim is the axonal regeneration and the functional improvements after the lesion. NPs delivery of neurotrophic agents is currently being developed for SCI use focusing on their effect to support the survival of damage neurons and stimulate the axonal sprouting along a specific path though the spinal cord [77]. The release of enzymes represents another strategy widely investigated to counteract the SCI contusive effects; in particular, the chondroitinase ABC $(\mathrm{ch} A B C)$ shows promise in the axonal regeneration cleaving the proteoglycans residues that support the glial scar formation. The needs to preserve the nature and the bioactivity of the enzyme require the design of NPs able to incorporate the biomolecule without affecting its chemical composition: for this reason, physical interactions are preferred, synthetizing PLGA or PLA nanostructures via emulsion fabrication methods $[33,78]$.

p0145 Further suitable polymers for functionalization strategies are cationic polymers. In particular, the presence of amine groups along the chains or as terminal moieties allows the development of different chemistry to link biomolecules. PEI can be functionalized with thiol or maleimide groups to form disulfide cross-linking with rabies virus glycoprotein peptide design a nanomaterial capable of neuron-specific delivery in vivo, carrying also neurogenic microRNA into the brain [79]. Chitosan amine moieties can react with carboxyl groups of PEG grafting biotin to design NPs where the biotin acts as affinity site for monoclonal antibody OX26, which is able to reduce the neuron vulnerability to death, once delivered to the spinal cord environment [80]. This suggests that chitosan NPs could be surface modified displaying a variety of ligands, including transferrin receptor antibodies.

p0150 PEG is another polymer suitable for NPs functionalization: the reactivity of its hydroxyl groups and the opportunity to link different chemical groups to its terminal moieties encourage the use as a coating layer of preformed NPs. The approach shows that the protective coating affects the phagocytic activity depending on the PEG density and molecular weight, through covalent linkage [41]. The development of silica NPs represents another application of PEG nanosystem decoration: in this case, the goals are two: one is related to increase the PEG bioavailability that, in SCI, presents well-documented neuroprotective effects and seals damaged cell membranes $[81,82]$; the second one is the modulation of release of hydralazine-loaded silica NPs due to the polymer steric hindrance and the reduction of free radicalmediated injury [83]. 


\section{2}

\section{CHAPTER 8 Nanomaterials for spinal cord injury (SCI) regeneration}

p0155 According to the NPs internalization and release of therapeutic molecules, the nanostructure traceability represents an important aspect to monitor and control the SCI treatment. Usually, magnetic resonance imaging (MRI) agents or dyes are used and chemically linked to the polymer chains. Specific and nonspecific cellular imaging techniques are developed: the first one requires NPs carrying some peculiar recognition elements such as peptide, sugar, fluorescent protein, or antibodies, whereas the second one is based on the design of functional chemical bonds for dye to ensure the nanostructure tracking in vitro and in vivo environment. Polymers are functionalized with reactive biotin, carboxyl groups, azido or alkyne bearing artificial amino acids, or monosaccharides; whereas, the MRI is performed using iron particles or quantum dots [84-86].

\section{s0045 Case study 1: design of PLGA nanoparticles for sustained release of bioactive neurotrophin}

p0160 The potential of nanoparticles as carriers of specific proteins interfering the degenerative scenario of SCI is clearly showed by Elliott Donaghue and colleagues [76]. Neurotrophin has been demonstrated to induce the axonal regeneration pathway in animal models, and the critical point is related to the local administration to ensure the sustained and minimally invasive release in situ. Due to the nature of the polymer, PLGA was capable to entrap the neutrophin-3 (NT-3) in nanostructures with diameter around $220 \mathrm{~nm}$, using a double emulsion-solvent evaporation approach that did not affect the peculiar properties of the cargo. Moreover, their dispersion in a hyaluronan/methyl cellulose matrix allowed injecting the NPs into the desired intrathecal space to achieve the local NT-3 release for up to 50 days in vitro and 28 days in vivo, by simple diffusion and noncovalent interactions between NT-3 and the polymeric chains. During the administration, the immunohistochemistry studies showed significant growth of axons and recovery of locomotor ability, and no effects on the astroglial response occurred, as illustrated in Fig. 8.4. The benefits in the use of these NPs also concern the possibility to limit the number of administrations, reducing the risks of causing further tissue lesions. These results demonstrate the ability of PLGA-based NPs dispersed in suitable polymeric matrix as bioactive protein delivery in a clinically relevant SCI model.

\section{s0050 Nanogels in SCI}

p0165 Tuning the atomic and molecular scale of matter allows the design of biomaterials with innovative or improved physical, chemical and biological features. In this scenario, nanogels (NGs) are in the spotlight due to their potential in medicine, bioengineering, and diagnostic applications [87-89]. The key advantages related to the use of nanogels in SCI are their hydrophilic nature, their improved colloidal stability compared to surfactant micelles, longer retention of loaded cargo, and their swelling behavior. In particular, this last property points out the unique nature of the 

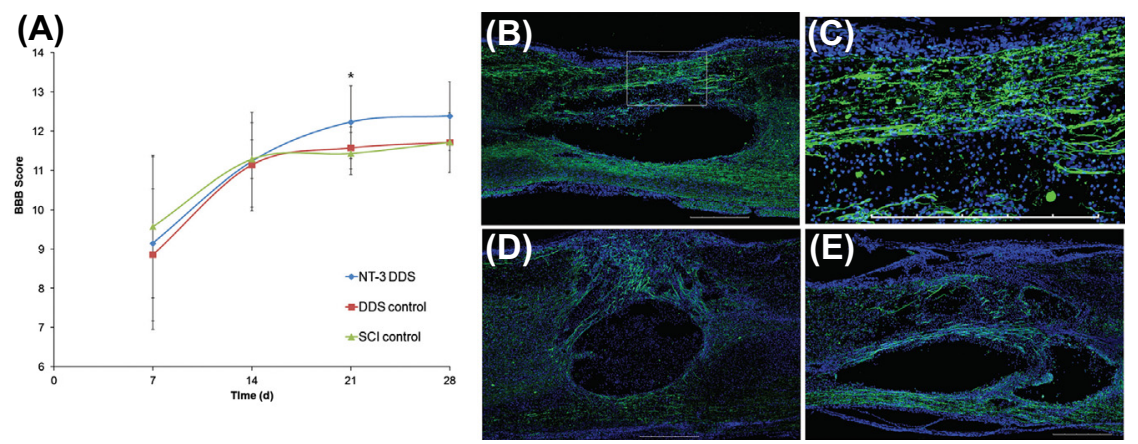

f0025 FIGURE 8.4

(A) Comparison of locomotor recovery ability in mice after injection of PGLA NPs loaded with NT-3 (NT-3 DDS, blue), after injection of only NPs (DDS control, red), and without treatment ( $\mathrm{SCl}$ control, green). (B-E) Immunohistochemistry imagines showing the local sustained release of NT-3 promotes axonal growth (in green); (B) and (C) are representatives of treatment with PGLA NPs loaded with NT-3, (D) of DDS control, and (E) of $\mathrm{SCl}$ control.

Adapted from Elliott Donaghue I, Tator CH, Shoichet MS. Sustained delivery of bioactive neurotrophin-3 to the injured spinal cord. Biomater Sci 2015;3(1):65-72; with permission of The Royal Society of Chemistry.

Copyright 2015.

nanostructures that create synergy with the biological environment: NGs are capable to incorporate a great amount of water or biological fluids, preserving the structural integrity. In this manner, they introduce the swelling feature, representative of macroscopic hydrogel systems, at the nanoscale. NGs final configuration is the result of an established equilibrium between the elongation of the polymer chains in contact with solvent molecules and the elastic retroactive forces that counterbalance the polymer deformation; this ensures the exchange of ions and metabolites with tissue fluids to maintain the biological chemical balance with the surrounding environment and the diffusion of biomolecules throughout the nanotool $[90,91]$. NGs present the peculiar properties of nanoparticles and hydrogels and for this reason can be categorized as soft materials, which combine the characteristics of solid and fluids. In addition, the physical and chemical nature of the polymers used in NGs synthesis tunes the parameters of surface tension, biodistribution, and circulation time in targeted cells or organs because the resulting intrinsic deformability permits them to cross the physiological barriers more easily than other stiffer nanoparticles, which would be locked or deported during the biological pathways. Last works even propose the application of external driving forces, such as limited pressure, to guide NGs into channels smaller than their size, exploiting their deformability [92,93]. Similar to NPs, the development and engineering of NGs for repair damaged spinal cord require the satisfaction of biological criteria, such as the nonactivation of immune response, nontoxicity, and the formation of degradation products completely 
compatible with the surrounding tissue. Secondary, any nanocarriers should be easily introduced into the spinal cord without producing further damage. This can be a real challenge, considering that the natural response to spinal injury is the glial scar generation. Finally, the device must be able to remain placed in situ over long periods of time, to allow for release of therapeutic cargo, reduction of inflammatory response, or stimulation of nerves growth [94].

\section{s0055 Synthesis techniques}

p0170 The presence of specific chemical groups in the starting materials is the guideline to promote the NGs formation through covalent links or physical interconnections: amide, alkyne, alkene, hydroxyl, carboxamide, and sulfo groups are commonly involved in this design of three-dimensional nanostructures, and the corresponding residual moieties arranged along the macromolecular chains are mainly responsible for the NGs capacity to absorb water [95]. Chemical strategy utilizes heterogeneous polymerization of low molecular weight monomers or the cross-linking of polymer precursors. The first method is referred to the controlled/living radical polymerization, where specific initiators give rise to propagation reaction that confines the cross-linking to intraparticle rather than interparticle level. In this manner, particles are characterized by an internal structure comparable to that of macroscopic hydrogel scaffolds [96]. It includes inverse emulsion, miniemulsion, precipitation, and dispersion polymerization processes that allow incorporating functional groups inside the network or on the surface to facilitate the attractiveness toward the conjugation with growth factors, peptides, or cell receptors [97,98]. On the other side, the use of polymer precursors generally regards the self-assembling of amphiphilic or triblock copolymers or polymers with reactive sites that directly form covalent linkages. Reaction principles belonging to this method are about click chemistry, thiol-disulfide exchange, Schiff base reaction, photo- or thermally induced crosslinking, amide bond formation, catalyzed coupling, and the chemistry of ketones, aldehydes, epoxides, or other groups [87].

p0175 Alternatively, physical cross-linking produces polymer interconnections formed by hydrogen bonding, Van der Waals, electrostatic, or hydrophilic/hydrophobic interactions, which make NGs more fragile and degradable than the chemical counterparts due to the weak nature of the linkage, more sensitive to the sol-gel transition caused by environmental stimuli changes $[99,100]$. However, two last innovative methods are currently investigated and are placed alongside the traditional approaches: the particle replication in nonwetting templates (PRINT) technology and the molecular imprinting. PRINT fabricates monodisperse nanoparticles from a wide range of matrix materials and uses nonwetting elastomeric molds of fluoropolymers that enable high-resolution imprint lithography and eliminate the formation of an interconnecting film between the molded objects. In this manner, tunability and precise control are permitted over NGs size, shape, composition, physicochemical properties, surface properties, and loading cargo [101]. Molecular imprinted polymers are materials capable of molecular recognition that could be 
exploited to selective cell targeting in drug treatment and to avoid the immunological and inflammatory response [102]. The principles of this method consider the covalent or noncovalent conjugation between a chosen target protein and a functional monomer to form the so-called template molecule; then, polymerization occurs using cross-linker agents and finally, the template protein is removed, leaving an imprinted cavity complementary to itself in size and shape. This synthesis of NGs is very complex, but could open new options in the drug delivery and recognition of the inflamed cell responses in SCI.

\section{s0060 Nanogels functionalization principles}

p0180 As previously discussed, nanosystems are employed to carry drugs, proteins, peptides, enzymes, or oligonucleotides in desired areas and control their fate. The NGs functionalization is addressed to introduce chemical moieties supporting the physical or chemical bonds formation not only with the cargo but also according to external stimuli such as temperature, $\mathrm{pH}$, pressure, ions, and specific molecular recognition, to ensure release of bioactive molecules as diffusion-controlled, swelling-controlled, or chemical-controlled.

p0185 Different pathways can be followed to prepare a modified substrate, as shown in Fig. 8.5, that can be grouped as follows [103]:

u0055 - Amide bonds formation through ester activation, that produces a very stable linkage toward extreme chemical environments.

u0060 - Esterification, that represents one of the most adjustable moieties for polymer functionalization.

u0065 - Click chemistry, that ensures high yields and purity, regiospecificity, versatility, fast kinetics, working in biological-compatible solvents, and facile tuning of structural and mechanical properties using stoichiometry, to generate biohybrid materials with a diverse selection of new and improved properties. This approach regards the following: copper-catalyzed or copper-free strainpromoted azide-alkyne cycloaddition, Michael addition, Diels-Alder reaction, thiol-ene reaction, and oxime reaction.

u0070 • Ring-opening reactions, that represent very versatile methods for polymer transformations. The nucleophilic ring opening of strained heterocycles, such as epoxides, aziridines, and azlactones, enables the introduction of desired heteroatoms on the polymer backbone.

p0210 These biorthogonal strategies create an affinity bond between the biomolecules and the polymeric nanonetwork, covalently linking receptors, peptides, and drugs. For example, carbazone coupling can be used to decrease the drug release rate of doxorubicin linked to PVA-based nanosystems [104]; whereas, pH-sensitive bonds, such as ester and hydrazone, could be encouraged for the controlled release under variation of $\mathrm{pH}$ values according to the inflammatory conditions. External stimuli are also exploited in the preparation of innovative redox-responsive NGs to modulate their biodistribution, enhance the permeability and retention effect, and finally, 


\section{6}

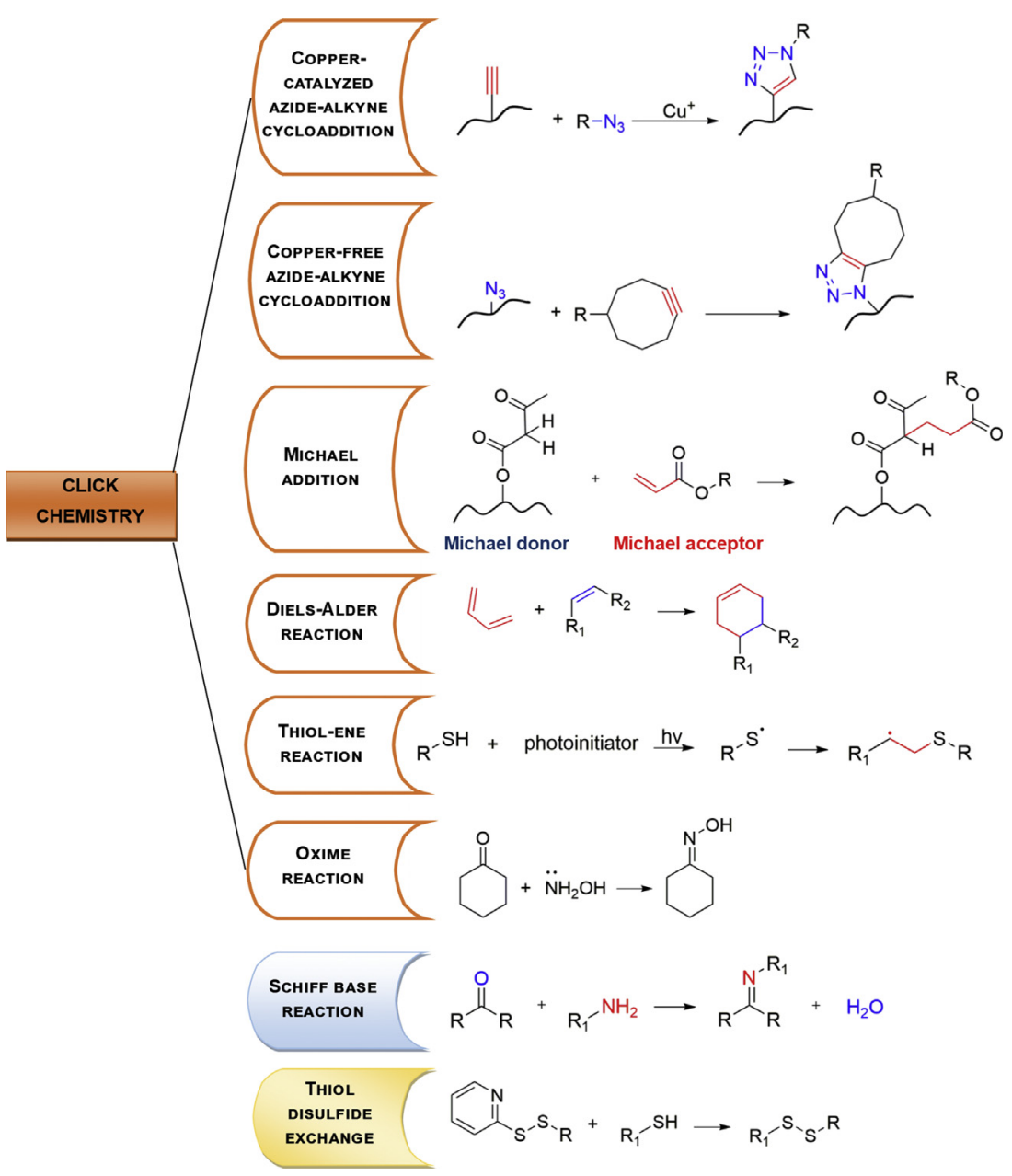

Functionalization techniques involved in nanogel design for SCl.

rapidly release the loaded drug at the site of action when the redox stimulus is applied [88]. NGs functionalization could also be used to enhance noncovalent interactions between the active molecules and polymer moieties: cationic nanocarriers are synthetized through the smart radical polymerization of different methacrylate derivatives to encapsulate genes to emphasize the pathway for bone formation and regeneration after a heterotopic ossification that may occur after the SCI [105]. Another well-established approach is related to the use of PEI: properly functionalizing through the chemical strategies mentioned previously and preserving its amine groups, this polymer is attractive for encapsulation of genes, peptides, and growth 
factors; indeed, the amines protonation allows for the entrapment of biomolecules without distorting the corresponding chemical structure or spatial organization and, as a result, the cargo preserves its activity during and after the aimed cell internalization [87]. Moreover, great interest is focused on the thermos-responsive materials, in particular acrylates; through free radical polymerization, controlled radical polymerization, or ring opening polymerization, it is possible to conjugate polymers to different hydrophobic or hydrophilic moieties modulating the critical solution temperature and the amount of the entrapped bioactive component. These nanocarriers could cross the biological barriers and show their curative effects under external stimuli related to irradiation sources or hyperthermia [106].

\section{s0065 Case study 2: nanogels as thiol-sensitive intracellular drug delivery systems}

p0215 In nanomedicine, loading of nanocarriers with hydrophilic therapeutic molecules presents the main drawback of their undesired rapid diffusion toward the external biological environment, according to the high clearance of body fluids, which in turn reduces pharmacological activity at the target site. To overcome this aspect, chemical functionalization strategies can be pursued. Mauri and coworkers [107] have designed functionalized nanogels through the smart combination of two strategies: thiol chemistry and click chemistry. The former has been used to form a disulfide bond between rhodamine (used as drug mimetic) and PEG, and the latter to label PEI with Cy5 dye for nanogel traceability in vitro, exploiting the copper-catalyzed alkyne-azide cycloaddition. The resulting modified polymers produced the final nanoscaffold via carbamate bonds formation. The selectivity of drug delivery was related to the disulfide bond that can be disrupted intracellularly by glutathione or cysteines present in the cytosol. Given the key role of microglia in modulating SCI inflammation, nanogels were tested in combination with microglia cultures. Fluorescence colocalization studies showed that nanogels were internalized by target cells, and that the drug mimetic was selectively released in the cytosol after 4 days, without any external stimuli (Fig. 8.6). In this case, the introduction of a sensitive cleavable bond (disulfide) avoids losing the drugs content in body fluids and ensures the drug uptake and release in the microglia potentially promoting the target therapeutic effect.

\section{s0070 Nanochannels in SCI}

p0220 Together with nanoparticles and nanogels, neuroprotective and neuroregenerative treatments are addressed using nanoscaled tubular structures. Nanotubes (NTs), nanowires (NWs), nanofibers (NFs), and conduits are widely synthetized to limit the SCI immune response, the cell oxidative stress, the loss of cell interconnection in the lesion site, and the permanent demyelination. 
(A)

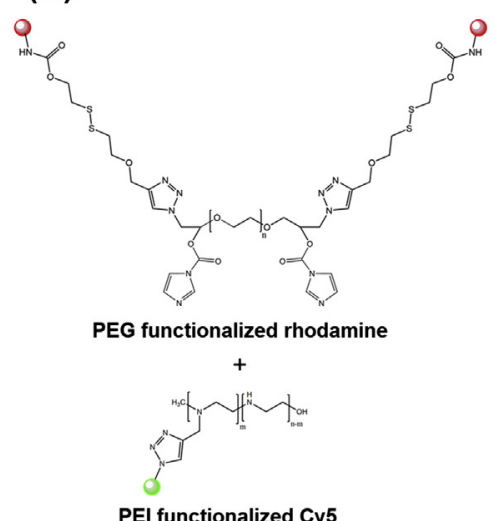

PEI functionalized Cy5
(B)

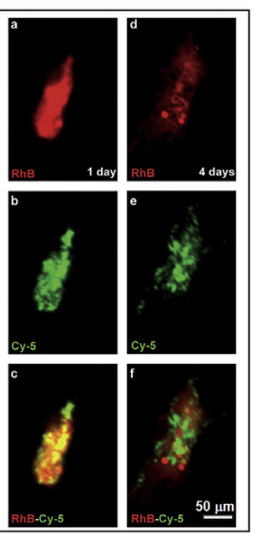

f0035 FIGURE 8.6

(A) Scheme of functionalized nanogels, with rhodamine used as drug mimetic (red dots) and Cy5 (green dot). (B) Nanogel internalization in microglia and signal delocalization of rhodamine (red) and Cy5 (green) after 4 days.

Adapted from Mauri E, et al. Double conjugated nanogels for selective intracellular drug delivery. RSC Adv 2017; 7(48):30345-56; with permission of The Royal Society of Chemistry. Copyright 2017.

\section{s0075 Nanotubes}

p0225 Nanotubes are carbon-based materials generally made of a graphene thick sheet rolled up into a seamless cylinder with diameter of the order of nanometer [108]. They have attracted great attention in the SCI panorama due to their electrical conductivity, flexibility, strength, durability, and the presence of active sites for easy chemical functionalization. In detail, their use as scaffold and drug carrier in neurological compartments is promoted by Refs. [109,110]:

u0075 - the compliance with the neural elements, NTs structure and size resemble cytoskeletal elements in neurons, ion channels, and signaling proteins;

u0080 - the versatile electrophysical properties, NTs can be proportionally modulated to the charge transport features of the neural interface and support the electrophysiological activity in nerve cells;

u0085 - the conductivity, that is not altered by oxidation in aqueous solutions;

u0090 - the mechanical and chemical properties, that allow for prolonged implantation in spinal cord tissues.

p0250 NTs design shows higher potential through the conjugation of smart biomolecules to create a hospitable and suitable niche for neuron growth. One of the early strategies regards the NTs functionalization with 4-hydroxynonenal to improve the neurons optimal spatial orientation and reciprocal interconnections [111]; whereas, in the last years, PEG is used as coating layer and tested in in vivo SCI 
f0040 FIGURE 8.7
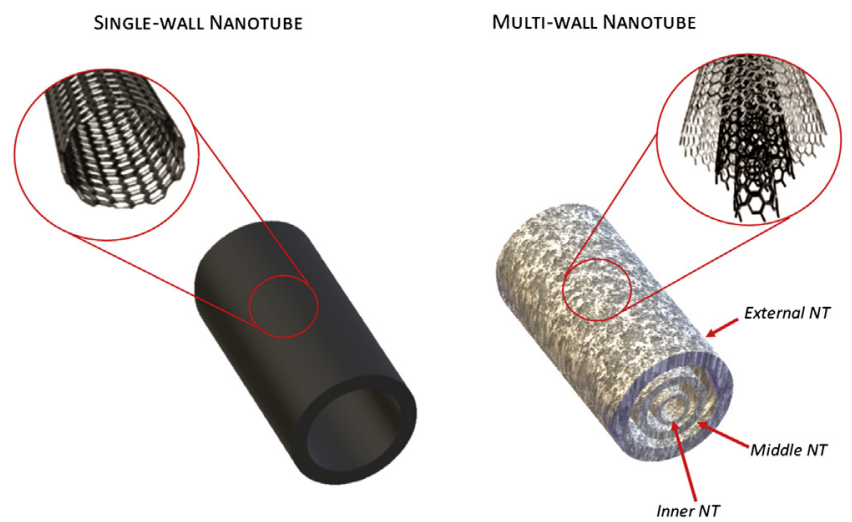

Nanotube structure: single-wall and multi-wall configurations.

Adapted from Choudhary V, et al. Polymer/Carbon Nanotube Nanocomposites, Carbon Nanotubes-Polymer Nanocomposites, Siva Yellampalli, IntechOpen (2011) https://doi.org/10.5772/18423.

rat model. As discussed by Roman and coworkers [112], the NTs injection into the lesion site during the secondary injury phase allows a modest improved locomotor recovery, decrease of the injured volume, and increase of the neurofilament-positive fibers and the corticospinal tract fibers in the lesion. Although these nanocarriers are not completely biodegradable, their presence in biological environment does not cause neither toxicity nor reactive gliosis improvement. Ding and colleagues [113] have proposed multiwall NTs (Fig. 8.7) functionalized with neurotrophic factors, brain receptors, or genes that show neuroprotective role and functional recovery in injured mice. NTs can be superficially charged with specific chemical groups or using anisotropic electrical conductive materials to control the neurite outgrowth. These nanocarriers are also appropriate to the formation of composite nanomatrices: Nafion and chitosan are the innovative proposal [108]. In summary, NTs are mainly focused on the neurons curative and protective effects.

\section{s0080 Nanowires}

p0255 Nanowires are slender structures characterized by a length extremely longer than the diameter. Their application is intended to increase the bioavailability of neuroprotective compound to which NWs are conjugated [114]. This material presents distinctive electrical and optical properties due to its composition based on the use of metals, semiconductors, insulators, or organic compounds, which offer the opportunity to design heterostructures with tuned electronic properties. Sharma and coworkers have tested $\mathrm{TiO}_{2}$-based $\mathrm{NWs}$ capable to improve the efficacy of neuroprotective Acure compounds tagged to the nanocarriers [115]. The axonal regeneration and alignment are explored synthetizing Ga- or magnetic Ni- 
modified NWs, as proposed, respectively, by Hallstrom [116] and Johansson [117]. In addition, electroconductive polymers are linked to the NWs to perform neuronal cell adhesion and proliferation: polypyrrole is used by Bechara and coworkers on PCL NWs through polymerization [118] providing physiological levels of electrical stimulation to cells. NWs are also applicable in electronics, optics, chemical sensors, and biological systems [119-121].

\section{s0085 Nanofibers}

p0260 Neural regeneration could be supported using nanofibers. Their porous networks resemble the extracellular matrix environment, and their configuration has been shown to promote the adhesion, proliferation, and differentiation of various cells [108]. Notably, NFs diameter values and spatial orientation represent the main parameters that influence the cell spreading and differentiation: decreasing diameter, higher degree of cell proliferation, and lower degree of cell aggregation has been observed; whereas, aligned NFs enable to increase the rate of neural stem cells differentiation than randomly oriented fibers, as discussed by Yang, Xie, and colleagues [122,123]. Moreover, the NFs surface modification is a potential strategy to improve the neuron growth and neurite extension. Polymers converted to NFs present changes in their properties, including antibacterial ability, mechanical features, biodegradability, cell adhesion, and proliferation, and need to be regulated by controlling the structure and properties of the composite scaffold with other synthetic polymers. Proposed surface functionalizations regard the grafting of specific chemical moieties (such as carboxylic groups in collagen-modified NFs) to improve the cell attachment, laminin (in PCL NFs) to control the neurite outgrowth [124], or the physical entrapment of antiinflammatory molecules (such as Rolipram in PLGA NFs) to promote axon growth and reduce the population of astrocytes and chondroitin sulfate proteoglycans in the lesion site [125]. Promising results are also obtained through the design of electrospun polyamide NFs and their functionalization with peptides [7].

\section{s0090 Conduits}

p0265 Conduits are tubes able to cover the nerves gap forming a "bridge" between their proximal and distal ends. In this manner, conduits provide a physical guidance toward the restoration of neural interconnections. These nanofibrous systems can be obtained via electrospinning and are already successfully used in peripheral nerve regeneration [126,127]. Recent studies propose the application of PLGA-chitosan or PCL conduits to close gap within the range of $15-80 \mathrm{~mm}$ in nerve model obtaining partial remyelination of axons with a promising myelin sheaths thickness. However, the main limitation is that these nanofibrous systems must be invasively implanted in the desired area and this could cause further damages, infections, or surgical complications [128]. 


\section{s0095 Conclusions}

p0270 To summarize, nanomaterials are great potential tools for treating various causes of SCI. In particular, a neuroregenerative approach requires the smart combination with a neuroprotective strategy to be efficient: suitable biomaterials need to be designed using physical and chemical methods to obtain desired dimensions, shape, surface charge, and mechanical properties. In addition, nanomaterial functionalization allows to introduce specific moieties that, otherwise, it is not possible to record on the nanomatrices, overcoming the main drawbacks of bioavailability of active principles, prolonged circulation time, and cross of BSCB. Important progress has been made in choosing the right therapeutic molecules and developing nanosystems with the capacity to mitigate SCI effects. Different types of cells are involved, and a selective action requires the use of specific grafting between the cargo and the polymeric scaffolds. The first steps have been made, and they provide the avenue through which the cure to SCI may be attained.

\section{References}

[1] Svenson S. Clinical translation of nanomedicines. Curr Opin Solid State Mater Sci 2012;16(6):287-94.

[2] Hua S, et al. Current trends and challenges in the clinical translation of nanoparticulate nanomedicines: pathways for translational development and commercialization. Front Pharmacol 2018;9.

[3] Singh T, et al. Application of nanotechnology in food science: perception and overview. Front Microbiol 2017;8.

[4] Riehemann K, et al. Nanomedicine-challenge and perspectives. Angew Chem Int Ed 2009;48(5):872-97.

[5] Satalkar P, Elger BS, Shaw DM. Defining nano, nanotechnology and nanomedicine: why should it matter? Sci Eng Ethics 2016;22(5):1255-76.

[6] Jeevanandam J, et al. Review on nanoparticles and nanostructured materials: history, sources, toxicity and regulations. Beilstein J Nanotechnol 2018;9:1050-74.

[7] Tyler JY, Xu XM, Cheng JX. Nanomedicine for treating spinal cord injury. Nanoscale 2013;5(19):8821-36.

[8] White-Schenk D, Shi R, Leary JF. Nanomedicine strategies for treatment of secondary spinal cord injury. Int J Nanomed 2015;10:923-38.

[9] Hamid S, Hayek R. Role of electrical stimulation for rehabilitation and regeneration after spinal cord injury: an overview. Eur Spine J 2008;17(9):1256-69.

[10] Nas K, et al. Rehabilitation of spinal cord injuries. World J Orthoped 2015;6(1):8-16.

[11] Ievins A, Moritz CT. Therapeutic stimulation for restoration of function after spinal cord injury. Physiology 2017;32(5):391-8.

[12] Rosenzweig ES, et al. Restorative effects of human neural stem cell grafts on the primate spinal cord. Nat Med 2018;24(4):484.

[13] Nejati-Koshki K, et al. An update on application of nanotechnology and stem cells in spinal cord injury regeneration. Biomed Pharmacother 2017;90:85-92. 
[14] Anselmo AC, et al. Elasticity of nanoparticles influences their blood circulation, phagocytosis, endocytosis, and targeting. ACS Nano 2015;9(3):3169-77.

[15] Banik BL, Fattahi P, Brown JL. Polymeric nanoparticles: the future of nanomedicine. Wiley Interdiscip Rev Nanomed Nanobiotechnol 2016;8(2):271-99.

[16] Liu XQ, Tang RZ. Biological responses to nanomaterials: understanding nano-bio effects on cell behaviors. Drug Deliv 2017;24(2):1-15.

[17] Przybylski S, et al. Influence of nanoparticle-mediated transfection on proliferation of primary immune cells in vitro and in vivo. PLoS One 2017;12(5).

[18] Mahmoudi M, et al. Effect of nanoparticles on the cell life cycle. Chem Rev 2011; 111(5):3407-32.

[19] Wang JX, et al. Magnetic nanoparticles for MRI of brain tumors. Curr Pharmaceut Biotechnol 2012;13(12):2403-16.

[20] Huang LJ, et al. Nanomaterial applications for neurological diseases and central nervous system injury. Prog Neurobiol 2017;157:29-48.

[21] Oladosu FA, et al. Novel intrathecal and subcutaneous catheter delivery systems in the mouse. J Neurosci Methods 2016;264:119-28.

[22] Chen B, et al. Repair of spinal cord injury by implantation of bFGF-incorporated HEMA-MOETACL hydrogel in rats. Sci Rep 2015;5.

[23] Khaing ZZ, et al. Advanced biomaterials for repairing the nervous system: what can hydrogels do for the brain? Mater Today 2014;17(7):332-40.

[24] Su F, et al. Fluoxetine and S-citalopram inhibit M1 activation and promote M2 activation of microglia in vitro. Neuroscience 2015;294:60-8.

[25] Papa S, et al. Modulators of microglia: a patent review. Expert Opin Ther Pat 2016; 26(4):427-37.

[26] Biswas A, et al. Advances in top-down and bottom-up surface nanofabrication: techniques, applications \& future prospects. Adv Colloid Interface Sci 2012;170(1-2): $2-27$.

[27] Schobel J, et al. Bottom-up meets top-down: patchy hybrid nonwovens as an efficient catalysis platform. Angew Chem Int Ed 2017;56(1):405-8.

[28] Sonavane G, Tomoda K, Makino K. Biodistribution of colloidal gold nanoparticles after intravenous administration: effect of particle size. Colloids Surfaces B Biointerfaces 2008;66(2):274-80.

[29] Etame AB, et al. Design and potential application of PEGylated gold nanoparticles with size-dependent permeation through brain microvasculature. Nanomed Nanotechnol Biol Med 2011;7(6):992-1000.

[30] Saraiva C, et al. Nanoparticle-mediated brain drug delivery: overcoming blood-brain barrier to treat neurodegenerative diseases. J Control Release 2016;235:34-47.

[31] Decuzzi P, et al. Size and shape effects in the biodistribution of intravascularly injected particles. J Control Release 2010;141(3):320-7.

[32] Kolhar P, et al. Using shape effects to target antibody-coated nanoparticles to lung and brain endothelium. Proc Natl Acad Sci USA 2013;110(26):10753-8.

[33] Zuidema JM, Gilbert RJ, Osterhout DJ. Nanoparticle technologies in the spinal cord. Cells Tissues Organs 2015;202(1-2):102-15.

[34] Jo DH, et al. Size, surface charge, and shape determine therapeutic effects of nanoparticles on brain and retinal diseases. Nanomed Nanotechnol Biol Med 2015;11(7): 1603-11.

[35] Yue ZG, et al. Surface charge affects cellular uptake and intracellular trafficking of chitosan-based nanoparticles. Biomacromolecules 2011;12(7):2440-6. 
[36] Xiao K, et al. The effect of surface charge on in vivo biodistribution of PEGoligocholic acid based micellar nanoparticles. Biomaterials 2011;32(13):3435-46.

[37] Tenzer S, et al. Rapid formation of plasma protein corona critically affects nanoparticle pathophysiology. Nat Nanotechnol 2013;8(10):772-U1000.

[38] Nejati-Koshki K, et al. Synthesis and in vitro study of cisplatin-loaded Fe3O4 nanoparticles modified with PLGA-PEG(6000) copolymers in treatment of lung cancer. J Microencapsul 2014;31(8):815-23.

[39] Liu JB, Xiao YH, Allen C. Polymer-drug compatibility: a guide to the development of delivery systems for the anticancer agent, ellipticine. J Pharm Sci 2004;93(1): $132-43$.

[40] Allen TM. The use of glycolipids and hydrophilic polymers in avoiding rapid uptake of liposomes by the mononuclear phagocyte system. Adv Drug Deliv Rev 1994;13(3): 285-309.

[41] Soppimath KS, et al. Biodegradable polymeric nanoparticles as drug delivery devices. J Control Release 2001;70(1-2):1-20.

[42] Discher DE, Eisenberg A. Polymer vesicles. Science 2002;297(5583):967-73.

[43] Chidanguro T, O'Dowd F, Simon Y. Design and fabrication of mechanically stable polymer vesicles from crosslinkable linear amphiphilic triblock copolymers. Abstr Pap Am Chem Soc 2018:255.

[44] Roy S, Nallani M. Surface functionalized biodegradable polymersome for targeted drug delivery. Abstr Pap Am Chem Soc 2017:254.

[45] Meng FH, et al. Biodegradable polymersomes. Macromolecules 2003;36(9):3004-6.

[46] Kim YT, Caldwell JM, Bellamkonda RV. Nanoparticle-mediated local delivery of methylprednisolone after spinal cord injury. Biomaterials 2009;30(13):2582-90.

[47] Das M, et al. Auto-catalytic ceria nanoparticles offer neuroprotection to adult rat spinal cord neurons. Biomaterials 2007;28(10):1918-25.

[48] Chern CS. Emulsion polymerization mechanisms and kinetics. Prog Polym Sci 2006; 31(5):443-86.

[49] Raaijmakers MJT, Benes NE. Current trends in interfacial polymerization chemistry. Prog Polym Sci 2016;63:86-142.

[50] Salatin S, et al. Development of a nanoprecipitation method for the entrapment of a very water soluble drug into Eudragit RL nanoparticles. Res Pharmaceut Sci 2017; 12(1):1-14

[51] Qi L, et al. Synthesis of methylprednisolone loaded ibuprofen modified dextran based nanoparticles and their application for drug delivery in acute spinal cord injury. Oncotarget 2017;8(59):99666-80.

[52] Deshmukh R, Wagh P, Naik J. Solvent evaporation and spray drying technique for micro- and nanospheres/particles preparation: a review. Dry Technol 2016;34(15): $1758-72$.

[53] Nestor M-M, David Q-G, Eric A. The impact of the salting-out technique on the preparation of colloidal particulate systems for pharmaceutical applications. Recent Pat Drug Deliv Formul 2012;6(3):236-49.

[54] Pant A, Negi JS. Novel controlled ionic gelation strategy for chitosan nanoparticles preparation using TPP- $\beta$-CD inclusion complex. Eur J Pharm Sci 2018;112:180-5.

[55] Gwak S-J, et al. Chitosan/TPP-hyaluronic acid nanoparticles: a new vehicle for gene delivery to the spinal cord. J Biomater Sci Polym Ed 2012;23(11):1437-50.

[56] Irache, J.M.aEspuelas, S., Albumin nanoparticles, in Nanotechnologies for the life sciences. 
[57] Gwak S-J, et al. Multifunctional nanoparticles for gene delivery and spinal cord injury: multifunctional nanoparticles for gene delivery and SCI 2015;103.

[58] Letchford K, Burt H. A review of the formation and classification of amphiphilic block copolymer nanoparticulate structures: micelles, nanospheres, nanocapsules and polymersomes. Eur J Pharm Biopharm 2007;65(3):259-69.

[59] Lodge TP, Pudil B, Hanley KJ. The full phase behavior for block copolymers in solvents of varying selectivity. Macromolecules 2002;35(12):4707-17.

[60] Quintanar-Guerrero D, et al. Preparation and characterization of nanocapsules from preformed polymers by a new process based on emulsification-diffusion technique. Pharmaceut Res 1998;15(7):1056-62.

[61] Guterres SS, Alves MP, Pohlmann AR. Polymeric nanoparticles, nanospheres and nanocapsules, for cutaneous applications. Drug Target Insights 2007;2:147-57.

[62] Moinard-Checot D, et al. Nanoparticles for drug delivery: review of the formulation and process difficulties illustrated by the emulsion-diffusion process. J Nanosci Nanotechnol 2006;6(9-10):2664-81.

[63] Ahmed F, Discher DE. Self-porating polymersomes of PEG-PLA and PEG-PCL: hydrolysis-triggered controlled release vesicles. J Control Release 2004;96(1):37-53.

[64] Tian XH, et al. LRP-1-mediated intracellular antibody delivery to the central nervous system. Sci Rep 2015;5.

[65] LoPresti C, et al. Polymersomes: nature inspired nanometer sized compartments. J Mater Chem 2009;19(22):3576-90.

[66] Infante JC. Nanoparticle-based systems for delivery of protein therapeutics to the spinal cord. Front Neurosci 2018;12.

[67] Vulic K, Shoichet MS. Affinity-based drug delivery systems for tissue repair and regeneration. Biomacromolecules 2014;15(11):3867-80.

[68] Nicolas J, et al. Design, functionalization strategies and biomedical applications of targeted biodegradable/biocompatible polymer-based nanocarriers for drug delivery. Chem Soc Rev 2013;42(3):1147-235.

[69] Orive G, et al. Biomaterials for promoting brain protection, repair and regeneration. Nat Rev Neurosci 2009;10(9). 682-U47.

[70] Kreuter J, et al. Direct evidence that polysorbate-80-coated poly( butylcyanoacrylate) nanoparticles deliver drugs to the CNS via specific mechanisms requiring prior binding of drug to the nanoparticles. Pharmaceut Res 2003;20(3):409-16.

[71] Patel T, et al. Polymeric nanoparticles for drug delivery to the central nervous system. Adv Drug Deliv Rev 2012;64(7):701-5.

[72] Varma AK, et al. Spinal cord injury: a review of current therapy, future treatments, and basic science frontiers. Neurochem Res 2013;38(5):895-905.

[73] Rao KS, et al. TAT-conjugated nanoparticles for the CNS delivery of anti-HIV drugs. Biomaterials 2008;29(33):4429-38.

[74] Geldenhuys W, et al. Brain-targeted delivery of paclitaxel using glutathione-coated nanoparticles for brain cancers. J Drug Target 2011;19(9):837-45.

[75] Kang CE, et al. Localized and sustained delivery of fibroblast growth factor- 2 from a nanoparticle-hydrogel composite for treatment of spinal cord injury. Cells Tissues Organs 2013;197(1):55-63.

[76] Elliott Donaghue I, Tator CH, Shoichet MS. Sustained delivery of bioactive neurotrophin-3 to the injured spinal cord. Biomater Sci 2015;3(1):65-72.

[77] Donaghue IE, et al. Cell and biomolecule delivery for tissue repair and regeneration in the central nervous system. J Control Release 2014;190:219-27. 
[78] Zuidema JM, et al. Magnetic NGF-releasing PLLA/iron oxide nanoparticles direct extending neurites and preferentially guide neurites along aligned electrospun microfibers. ACS Chem Neurosci 2015;6(11):1781-8.

[79] Hwang DW, et al. A brain-targeted rabies virus glycoprotein-disulfide linked PEI nanocarrier for delivery of neurogenic microRNA. Biomaterials 2011;32(21): 4968-75.

[80] Aktas Y, et al. Development and brain delivery of chitosan-PEG nanoparticles functionalized with the monoclonal antibody OX26. Bioconjug Chem 2005;16(6): 1503-11.

[81] Chen BJ, et al. Affinity for, and localization of, PEG-functionalized silica nanoparticles to sites of damage in an ex vivo spinal cord injury model. J Biol Eng 2012;6(1).

[82] Luo J, Shi RY. Polyethylene glycol inhibits apoptotic cell death following traumatic spinal cord injury. Brain Res 2007;1155:10-6.

[83] Cho Y, et al. Repairing the damaged spinal cord and brain with nanomedicine. Small 2008;4(10):1676-81.

[84] Provenzale JM, Silva GA. Uses of nanoparticles for central nervous system imaging and therapy. Am J Neuroradiol 2009;30(7):1293-301.

[85] Feng LH, et al. Conjugated polymer nanoparticles: preparation, properties, functionalization and biological applications. Chem Soc Rev 2013;42(16):6620-33.

[86] Papa S, et al. Polymeric nanoparticle system to target activated microglia/macrophages in spinal cord injury. J Control Release 2014;174:15-26.

[87] Mauri E, Perale G, Rossi F. Nanogel functionalization: a versatile approach to meet the challenges of drug and gene delivery. ACS Appl Nano Mater 2018;1(12):6525-41.

[88] Ghorbani M, Hamishehkar H. Redox-responsive smart nanogels for intracellular targeting of therapeutic agents: applications and recent advances. J Drug Target 2019; 27(4):408-22.

[89] Bin S, et al. Nano-carrier mediated co-delivery of methyl prednisolone and minocycline for improved post-traumatic spinal cord injury conditions in rats. Drug Dev Ind Pharm 2017;43(6):1033-41.

[90] Wu HQ, Wang CC. Biodegradable smart nanogels: a new platform for targeting drug delivery and biomedical diagnostics. Langmuir 2016;32(25):6211-25.

[91] Nayak S, Lyon LA. Soft nanotechnology with soft nanoparticles. Angew Chem Int Ed 2005;44(47):7686-708.

[92] Zhang L, et al. Softer zwitterionic nanogels for longer circulation and lower splenic accumulation. ACS Nano 2012;6(8):6681-6.

[93] Hendrickson GR, Lyon LA. Microgel translocation through pores under confinement. Angew Chem Int Ed 2010;49(12):2193-7.

[94] Siebert JR, Eade AM, Osterhout DJ. Biomaterial approaches to enhancing neurorestoration after spinal cord injury: strategies for overcoming inherent biological obstacles. BioMed Res Int 2015;2015:20.

[95] Hamidi M, Azadi A, Rafiei P. Hydrogel nanoparticles in drug delivery. Adv Drug Deliv Rev 2008;60(15):1638-49.

[96] Antonietti M. Microgels-polymers with a special molecular architecture. Angew Chem Int Ed Engl 1988;27(12):1743-7.

[97] Sumerlin BS. Proteins as initiators of controlled radical polymerization: grafting-from via ATRP and RAFT. ACS Macro Lett 2012;1(1):141-5.

[98] Sanson N, Rieger J. Synthesis of nanogels/microgels by conventional and controlled radical crosslinking copolymerization. Polym Chem 2010;1(7):965-77. 
[99] Lalitha K, et al. Stimuli responsive hydrogels derived from a renewable resource: synthesis, self-assembly in water and application in drug delivery. J Mater Chem B 2015; 3(27):5560-8.

[100] Sasaki Y, Akiyoshi K. Nanogel engineering for new nanobiomaterials: from chaperoning engineering to biomedical applications. Chem Rec 2010;10(6):366-76.

[101] $\mathrm{Xu} \mathrm{J}$, et al. Future of the particle replication in nonwetting templates (PRINT) technology. Angew Chem Int Ed 2013;52(26):6580-9.

[102] Takeuchi T, et al. Molecularly imprinted nanogels acquire stealth in situ by cloaking themselves with native dysopsonic proteins. Angew Chem Int Ed 2017;56(25): 7088-92.

[103] Blasco E, et al. 50th anniversary perspective: polymer functionalization. Macromolecules 2017;50(14):5215-52.

[104] Ossipov D, et al. Orthogonal chemoselective assembly of hyaluronic acid networks and nanogels for drug delivery. Macromolecules 2013;46(10):4105-13.

[105] Shrivats AR, et al. Cationic nanogel-mediated Runx 2 and osterix siRNA delivery decreases mineralization in MC3T3 cells. Clin Orthop Relat Res 2015;473(6):2139-49.

[106] Bergueiro J, Calderón M. Thermoresponsive nanodevices in biomedical applications. Macromol Biosci 2015;15(2):183-99.

[107] Mauri E, et al. Double conjugated nanogels for selective intracellular drug delivery. RSC Adv 2017;7(48):30345-56.

[108] Ramezani F, Nasirinezhad F, Abotaleb N. A review of nanotechnology strategies for neuron regeneration after spinal cord injury. J Med Physiol 2016;1(2):13.

[109] Nunes A, et al. Application of carbon nanotubes in neurology: clinical perspectives and toxicological risks. Arch Toxicol 2012;86(7):1009-20.

[110] Kabiri M, et al. Neuroregenerative effects of olfactory ensheathing cells transplanted in a multi-layered conductive nanofibrous conduit in peripheral nerve repair in rats. J Biomed Sci 2015;22(1):35.

[111] Mattson MP, Haddon RC, Rao AM. Molecular functionalization of carbon nanotubes and use as substrates for neuronal growth. J Mol Neurosci 2000;14(3):175-82.

[112] Roman JA, et al. Single-Walled carbon nanotubes chemically functionalized with polyethylene glycol promote tissue repair in a rat model of spinal cord injury. J Neurotrauma 2011;28(11):2349-62.

[113] Ding SH, et al. Neuroprotective effect of functionalized multi-walled carbon nanotubes on spinal cord injury in rats. Int J Clin Exp Pathol 2015;8(12):15769-77.

[114] Sharma HS. Nano neuro science: emerging concepts on nanoneurotoxicity and nanoneuroprotection. Nanomedicine 2007;2(6):753-8.

[115] Sharma HS, et al. Nanowired-drug delivery enhances neuroprotective efficacy of compounds and reduces spinal cord edema formation and improves functional outcome following spinal cord injury in the rat. Brain Edema Xiv 2010;106:343.

[116] Hallstrom W, et al. Rectifying and sorting of regenerating axons by free-standing nanowire patterns: a highway for nerve fibers. Langmuir 2009;25(8):4343-6.

[117] Johansson F, et al. Cell guidance by magnetic nanowires. Exp Cell Res 2010;316(5): 688-94.

[118] Bechara S, Wadman L, Popat KC. Electroconductive polymeric nanowire templates facilitates in vitro C17.2 neural stem cell line adhesion, proliferation and differentiation. Acta Biomater 2011;7(7):2892-901.

[119] Wang N, Cai Y, Zhang RQ. Growth of nanowires. Mater Sci Eng R Rep 2008;60(1-6): $1-51$. 
[120] Kubinova S, Sykova E. Nanotechnology for treatment of stroke and spinal cord injury. Nanomedicine 2010;5(1):99-108.

[121] Rahong S, et al. Recent developments in nanowires for bio-applications from molecular to cellular levels. Lab Chip 2016;16(7):1126-38.

[122] Yang F, et al. Characterization of neural stem cells on electrospun poly(L-lactic acid) nanofibrous scaffold. J Biomater Sci Polym Ed 2004;15(12):1483-97.

[123] Xie JW, et al. Conductive core-sheath nanofibers and their potential application in neural tissue engineering. Adv Funct Mater 2009;19(14):2312-8.

[124] Li WS, et al. Electrospun nanofibers immobilized with collagen for neural stem cells culture. J Mater Sci Mater Med 2008;19(2):847-54.

[125] Zhu YQ, et al. Nanofibrous patches for spinal cord regeneration. Adv Funct Mater 2010;20(9):1433-40.

[126] Matsumoto K, et al. Peripheral nerve regeneration across an 80-mm gap bridged by a polyglycolic acid (PGA)-collagen tube filled with laminin-coated collagen fibers: a histological and electrophysiological evaluation of regenerated nerves. Brain Res 2000;868(2):315-28.

[127] Jiang T, et al. Electrospinning of polymer nanofibers for tissue regeneration. Prog Polym Sci 2015;46:1-24.

[128] Tuszynski MH, et al. Guidelines for the conduct of clinical trials for spinal cord injury as developed by the ICCP Panel: clinical trial inclusion/exclusion criteria and ethics. Spinal Cord 2006;45:222. 



\title{
Non-Print Items
}

\begin{abstract}
Materials at the nanoscale represent the innovative tools to overcome the main constraints of the classical administration routes in the spinal card injury (SCI) treatment. The well choice of starting polymers and their physical or chemical functionalization with specific moieties allow designing nanostructures able to selectively interact with cells, control the release of their therapeutic cargo, and promote the regeneration of neuronal pathways. Here, a wide discussion about the main nanostructures applied in SCI is proposed, describing the main parameters and bioconjugation strategies that affect their efficacy in in vitro and in vivo studies.
\end{abstract}

\section{Keywords:}

Functionalization strategies, Nanogels, Nanoparticles, Nanotubes, Polymers, Therapeutic delivery. 


\title{
CHAPTER
}

\section{c0009 Cell therapies for spinal cord injury regeneration}

\author{
Eduardo D. Gomes, MSc, PhD ${ }^{1,2}$, Luís A. Rocha, MSc ${ }^{1,2}$, \\ Rita C. Assunção-Silva, MSc ${ }^{1,2}$, Rui Lima, MSc ${ }^{1,2}$, N.A. Silva, BSc, PhD ${ }^{1,2}$, \\ António J. Salgado, BSc, PhD, DSc ${ }^{1,2,3}$ \\ ${ }^{1}$ Life and Health Sciences Research Institute (ICVS), School of Medicine, University of Minho, \\ Campus de Gualtar, Braga, Portugal; ${ }^{2} I C V S / 3 B$ 's - PT Government Associate Laboratory, Bragal \\ Guimarães, Portugal; ${ }^{3} \mathrm{Dr}$, ICVS, School of Medicine, University of Minho, Braga, Portugal
}

p0015 The transplantation of cells to treat a pathology, either isolated from the own individual (autologous) or from another person (allogeneic) is a reality for some diseases, mostly leukemias and lymphomas [1]. For many years, the rationale behind such approach has been to replace the damaged cell population or injured tissues. In central nervous system (CNS) applications, one of the first experiments was done by Jorge Francisco Tello from Ramon y Cajal's lab, attempting to transplant predegenerated peripheral nerve segments onto proximal stumps of severed optic nerves [2]. Amazingly, retinal axons could grow into these transplanted nerves. Similarly, in the early 1980 s, this observation was again put to the test, but this time in a spinal cord injury (SCI) context. Richardson et al. demonstrated that severed spinal cord axons could regrow onto autologous peripheral nerve grafts (from sciatic nerve), and in some rats even further to the caudal stumps of $10 \mathrm{~mm}$ gap injuries [3]. In addition, after performing a peripheral graft bridge between the medulla oblongata and the spinal cord, some CNS axons could grow more than $30 \mathrm{~mm}$ into the grafts [4]. These experiments were made to understand the reason why peripheral nervous system (PNS) neurons have a regenerative capacity, different from CNS neurons. One of the main hypotheses was that the environment influenced neuronal regrowth, explaining why CNS neurons could easily grow into peripheral nerve grafts. Another, and a quite relevant one, was the presence of Schwann cells (SCs) in the PNS. Thus, even before Richardson, David, and Aguayo's experiments, Richard Bunge and others have explored the properties of SCs in vitro, with the ultimate goal of using these cells as a potential therapeutic strategy to CNS repair [5,6]. In this case, the transplanted SCs would not serve as a replacement for neuronal populations, but rather as a supportive substrate for inducing endogenous neuronal repair.

p0020 Based on this dichotomy of purposes (cell replacement vs. cell supportive), different cell populations have been proposed as candidates for SCI repair. Neural stem/progenitor cells (NSCs/NPCs), embryonic stem cells (ESCs), and more recently induced pluripotent stem cells (iPSCs) have been applied with the main

Spinal Cord Injury (SCI) Repair Strategies. https://doi.org/10.1016/B978-0-08-102807-0.00009-0 Copyright $\odot 2020$ Elsevier Ltd. All rights reserved. 
objective of replacing lost neural cells; whereas, SCs, olfactory ensheathing cells (OECs), or mesenchymal stem cells (MSCs) have been mostly used as a strategy to support endogenous neuronal regrowth. This chapter intends to describe the use of each cell population for SCI treatment, discussing their major achievements and the path that lies ahead for the successful integration of cell transplantation in SCI therapies. A summary with the most relevant studies for the type of cells herein explored will also be presented in Table 9.1.

\section{s0010 ESCs, iPSCs, and NSCs}

p0025 The concept of stem cells can be traced back to the end of the 19th century [7]. At the time, it started as a theoretical postulate, trying to explain why some tissues had the capacity to self-renew, such as blood or skin for example.

\section{s0015 ESCs}

p0030 In 1981, Martin Evans and Kaufman were the firsts to successfully culture embryonic pluripotent cells from mouse blastocysts [8]. This breakthrough experiment opened a huge door to gene-targeted therapies but also for the potential application of these cells to numerous degenerative diseases. Later in 1998, the first human ESCs were successfully cultured, maintaining proliferation and differentiation potential in vitro for several months [9].

p0035 The ability of ESCs to differentiate into neural and glial cells in in vitro culture systems has been extensively explored using different strategies. Retinoic acid- and embryoid body-based protocols have been used to induce neural differentiation of ESCs in culture, resulting in the activation of a complex system of neuronal gene expression provided by neuronal-like cells [10] and in the production of oligodendrocytes, capable of producing myelin for the myelination of neurons in culture [11]. Another approach consisting in the use of specific factors in mouse ESCs culture was found to efficiently direct cell differentiation into dopaminergic and serotonergic neurons $[12,13]$. The use of cell culture media specifically defined for ESCs commitment to the neural fate is also an alternative method [14]. Of particular interest is the possibility to genetically modify the ESCs, to obtain neuronal precursors-enriched cultures [15].

p0040 The suitability of ESCs-based approaches for SCI treatment has also been investigated in a number of SCI models. Keirstead et al. transplanted NSCs obtained from mouse ESCs into a rat spinal cord, after an induced thoracic SCI. Most transplanted cells survived, migrated away from the injury site, and were shown to preferentially differentiate into oligodendrocytes and astrocytes [16]. Still, induced mouse ESCderived oligodendrocytes transplanted into demyelinated rat spinal cords were found to contribute to the remyelination of host axons [11]. Later on, Kakinohana et al. showed that the injection of human ESC-derived neural cell precursors (hNPCs) into ischemia-injured lumbar spinal cord of rats and lumbar spinal cord of naïve immunosuppressed minipigs resulted in successful engraftment of hNPCs and their 
t0010 Table 9.1 Some of the most relevant studies using cell therapy in $\mathrm{SCl}$ research.

\begin{tabular}{|c|c|c|c|c|}
\hline $\begin{array}{l}\text { Cellular } \\
\text { population }\end{array}$ & $\begin{array}{l}\text { Study } \\
\text { setting }\end{array}$ & SCI model & Major outcomes & References \\
\hline \multirow[t]{2}{*}{ ESCs } & In vivo & Rat; contusion & $\begin{array}{l}\text { Neuronal, oligodendrocytic and astrocytic differentiation; hindlimb weight } \\
\text { support and partial coordination }\end{array}$ & [18] \\
\hline & Clinical & Patients; chronic & $\begin{array}{l}\text { Regeneration in some axonal tracts; motor gains; improved bladder and } \\
\text { bowel sensation; No teratoma formation or immune response }\end{array}$ & {$[20,21]$} \\
\hline \multirow[t]{2}{*}{ iPSCs } & In vivo & Rat; hemisection & $\begin{array}{l}\text { Increased axonal outgrowth; establishment of synapses between host } \\
\text { neurons and grafts and vice versa }\end{array}$ & [43] \\
\hline & In vivo & $\begin{array}{l}\text { Common marmoset; } \\
\text { contusion }\end{array}$ & $\begin{array}{l}\text { Functional gains on upper and lower limbs; increased axonal outgrowth } \\
\text { and angiogenesis; reduced demyelination }\end{array}$ & [45] \\
\hline \multirow[t]{2}{*}{ NSCs/NPCs } & In vivo & $\begin{array}{l}\text { Rhesus monkey; } \\
\text { hemisection }\end{array}$ & $\begin{array}{l}\text { Synaptic formation between NPC-derived axons and host motor neurons; } \\
\text { motor improvements on object manipulation and locomotion }\end{array}$ & [54] \\
\hline & Clinical & Patients; chronic & $\begin{array}{l}\text { Three out of four patients responded to the treatment; one of the subjects } \\
\text { improved from complete to incomplete SCI; no serious adverse events } \\
\text { reported }\end{array}$ & [57] \\
\hline \multirow[t]{2}{*}{ BM-MSCs } & In vivo & Rat; contusion & $\begin{array}{l}\text { Functional recovery; axonal sprouting; tissue preservation; reduced } \\
\text { cavities }\end{array}$ & {$[83,85,87]$} \\
\hline & Clinical & $\begin{array}{l}\text { Patients; subacute } \\
\text { and chronic }\end{array}$ & $\begin{array}{l}\text { Improved ASIA, Barthel Index, Ashworth scoring; improved bladder } \\
\text { function; safe and feasible }\end{array}$ & [89-92] \\
\hline \multirow[t]{3}{*}{ UC-MSCs } & In vivo & $\begin{array}{l}\text { Rat; complete } \\
\text { transection }\end{array}$ & $\begin{array}{l}\text { Axonal growth and regeneration; locomotor improvement; production of } \\
\text { neurotrophic factors }\end{array}$ & [99] \\
\hline & In vivo & $\begin{array}{l}\text { Dogs; balloon } \\
\text { compression }\end{array}$ & Axonal remyelination; hindlimb motor recovery & {$[100,101]$} \\
\hline & Clinical & $\begin{array}{l}\text { Patients; chronic } \\
\text { thoracolumbar }\end{array}$ & $\begin{array}{l}\text { Improvements in algesia, tactile sensation, motion; improved bowel and } \\
\text { bladder function; safety }\end{array}$ & [103] \\
\hline \multirow[t]{3}{*}{ ASCs } & In vivo & $\begin{array}{l}\text { Rat; balloon } \\
\text { compression }\end{array}$ & Functional recovery; axonal regeneration; tissue preservation & [118] \\
\hline & In vivo & Dogs & Functional recovery; neural differentiation & {$[119,121]$} \\
\hline & Clinical & $\begin{array}{l}\text { Patients; acute and } \\
\text { chronic }\end{array}$ & $\begin{array}{l}\text { Improved ASIA score; median nerve improvement; adverse effects in some } \\
\text { patients }\end{array}$ & [126] \\
\hline \multirow[t]{2}{*}{ SCs } & In vivo & Rat; contusion & Axonal growth; improvement in motor function & [137] \\
\hline & Clinical & Patients; subacute & No additional spinal cord damage; no adverse events; safe & [144] \\
\hline \multirow[t]{2}{*}{ OECs } & In vivo & Rat; hemisection & $\begin{array}{l}\text { Improved regenerative capacity of CST neurons; improved forepaw } \\
\text { reaching skills }\end{array}$ & [152] \\
\hline & Clinical & Patients; chronic & $\begin{array}{l}\text { The procedure is feasible and safe; no significant alterations in motor and } \\
\text { sensorial scores }\end{array}$ & [168-170] \\
\hline
\end{tabular}


maturation into neurons [17]. Moreover, mouse ESCs transplanted 9 days following a rat spinal cord contusion injury led to functional improvements [18].

p0045 Finally, ESCs clinical applications in SCI patients started through a Phase I clinical trial provided by Geron's company in 2011. A cohort of patients with complete subacute thoracic SCI was transplanted with predifferentiated oligodendrocyte precursor cells (OPCs) derived from human ESCs for safety studies. Unfortunately, Geron's program was aborted later in that year due to financial constraints. Nevertheless, to date no safety issues were reported in five patients submitted to those ESC transplants [19]. Later in 2016, Shroff published a retrospective study of a cohort of 226 SCI patients treated with human ESCs in India [20]. The ESCs treatment in paraplegic and quadriplegic patients promoted gains in voluntary movement of the areas below injury level, improvement in bladder sensation and control, bowel sensation and control as well as in the gait and handgrip [21]. The improvements in Shroff's patients were reflected in magnetic resonance imaging and tractography reports that show regeneration of the lost axonal connections. Moreover, none of the patients in this study developed teratomas or an immune response, without administration of steroids or immunosuppressants, being the adverse effects observed mild and resolved without any sequel [20].

p0050 Nevertheless, there are several concerns regarding the safety of transplanting ESCs in humans, including the possible formation of teratomas following ESCderived cells engraftment [22]. Therefore, the application of prolonged differentiation protocols, fluorescence or magnetic activated cell sorting, and inhibition of proliferation signaling pathways by genetic manipulation, collectively decrease the incidence of tumor formation and efficiently increase the conversion of ESCs into neural cells [22-25].

p0055 Besides these technical issues related with ESCs therapy, there is a controversial discussion concerning human ESCs research, due to the use of human oocytes and human embryos, which usually die in the experimental process, and also how they are acquired [26]. In 2001, following a temporary prohibition for ESCs research in the United States, indignation resulted among scientists and liberal bioethicists. The fact that ESCs are derived from early human embryos present a moral dilemma for many people. Although Geron's Ethics Advisory Commission [27], the National Bioethics Advisory Commission (1999), and the American Association for the Advancement of Science [28] published guidelines for ethical development of therapies based on these cells, the debate continues. Therefore, both sides have the right to present their arguments, to consciously inform the society and legislators, to finally establish the boundaries for the use of ESCs. Despite this controversy, ESCs represent an interesting source of cells to study developmental biology and to find mechanisms that occur during this phase.

\section{s0020 iPSCs}

p0060 Recently, another type of pluripotent stem cells, known as induced pluripotent stem cells (iPS cells or iPSCs), emerged as a possible alternative to obtain stem 
cells directly from adult tissues for autologous transplantation. The iPSCs technology resulted from a pioneer work developed by Yamanaka's lab in Japan in 2006, which showed that the introduction of four transcription factors reverted the phenotype of differentiated adult cells into pluripotent stem cells [29]. Takahashi and Yamanaka originally generated iPSCs from embryonic and adult murine fibroblasts using retroviral transfection with the transcription factors Oct4, Sox2, Klf2, and c-Myc genes [29]. The expression of these genes was sufficient to reprogram somatic cells to a pluripotent state, making possible their use as autologous source material tissues from mice [29], rats [30], rhesus monkeys [31], and humans [32-35].

p0065 iPSCs are often compared to ESCs, as they share similar characteristics, such as pluripotency, self-renewal capacity, and gene expression [36]. Moreover, the potential to acquire abnormal karyotypes and genetic amplification associated with teratoma formation is also a common feature between the two cell types [37]. Reprogramming transcription factors that induce the pluripotency and selfrenewing state in somatic cells has well-known oncogenic properties. Considering this limitation of traditional reprogramming techniques, researchers have been focused on the replacement of the transcription factors required for reprogramming. For instance, previous studies have shown that c-Myc is a potent oncogene and can spontaneously reactivate following the induction of pluripotency, resulting in tumor formation [38]. In the reprogramming process, c-Myc was found to be mostly involved in speed and efficiency, being dispensable for the successful reprogramming of fibroblasts [39]. Moreover, Kim and colleagues demonstrated that Oct4 together with either Klf4 or c-Myc can reprogram NSCs into pluripotent stem cells, proposing that endogenous expression of Sox 2 plus exogenous expression of two factors, including Oct4, is sufficient to induce the generation of iPSCs from adult NSCs [40]. Besides the reprogramming process, Miura et al. have revealed that the responsiveness of mouse iPSCs to induction of neural differentiation and their safety after transplantation vary greatly depending on the somatic cells from which the iPSCs are derived [41].

p0070 The fact that iPSCs can be derived directly from adult tissues offers an unlimited supply of autologous cells, which could be used to generate transplants without the risk of immune rejection. However, safety issues such as those related to tumor formation should be determined before their clinical application. Therefore, it is crucial to carefully test iPSCs for tumorigenicity [42].

p0075 Tuszynski and colleagues explored the potential of iPSCs engraftment, differentiation, and axonal outgrowth when driven to NSC fate. For that, human iPSCs were differentiated into NSCs and implanted into immunodeficient rats 2 weeks after C5 lateral hemisection lesions. Three months later, they found an abnormal axonal outgrowth, where axons extended nearly the entire rostral-caudal axis of the adult rat nervous system and formed synapses with the host neurons, while these axons penetrated human iPSC grafts and also formed synapses [43]. The same laboratory reported that NSCs extended over very long distances when grafted to the injured spinal cord [44], despite the inhibitory milieu of adult CNS. Nevertheless, NSCs 
derived from human iPSCs promoted higher axonal growth, extending in both greater number and over virtually the entire length of the rat CNS [43].

p0080 A preclinical study investigated the therapeutic potential of transplanting preevaluated neural stem/progenitor cell (NSPC) clones derived from murine and human iPSCs into a nonhuman primate model of contusive SCI (common adult marmoset). Similarly to previous studies, the grafted cells were found to survive and differentiate into neurons, astrocytes, and oligodendrocytes, without evidence of tumor formation. In addition, there was an enhancement in axonal sparing/regrowth and angiogenesis at the lesion site, and the prevention of the lesion epicenter demyelination. At the end of the treatment, a functional recovery of the animals after SCI was observed [45]. Following this, Okano's group has seen, very recently, the first clinical trial using iPSCs to treat SCI to be approved by the Japanese health ministry. The study will consist of four patients that will receive the transplantation of patient-specific iPSC-derived NPCs to test the safety and efficacy of using this approach, before considering the expansion to a larger clinical trial [46]. Therefore, the results of this study will be extremely important to understand the iPSC technology, and for sure will serve as a barometer for future therapeutic applications on SCI and other CNS disorders.

\section{s0025 NSCs/NSPCs}

p0085 Another cell population with a possible interest for SCI research is adult multipotent NSCs, which are particularly appealing due to their CNS origin. These cells have been shown to generate the three main neural cell lineages of the mammalian CNS in culture [47]. Thus, they can hypothetically allow the replacement of spinal neurons lost after injury and differentiate toward astrocytes, to restore the nonneuronal milieu of the preinjured spinal cord, or toward oligodendroglia, to allow remyelination [48]. In line with this observation, Piltti and colleagues showed that the timing for the transplantation of these cells following SCI, as well as its location, is crucial to determine their fate. Therefore, NSPCs transplanted into the lesion site predominantly differentiated into astrocytes, in contrast to their transplantation to host parenchyma where they majorly differentiated into oligodendrocytes [49]. Several other studies, however, have proved the capacity of these cells to differentiate in situ into neurons, which functionally integrated the host tissue, following transplantation in SCI models [44,50,51].

p0090 In a cervical contusion-induced SCI in primates, in vitro-expanded NSPCs were grafted 9 days after injury and were shown to survive and differentiate into the neural lineages. In addition, there was a decrease in the injury cavities extent, as well as a significant increase of the spontaneous motor activity of the transplanted animals [52]. Furthermore, demyelinated axons in immunodeficient mice with traumatic SCI were remyelinated after transplantation of fetal brain-derived human CNS stem cells, grown in neurospheres. These cells also differentiated into neurons that exhibited the ability of synapse formation with host neurons [53]. 
p0095 Regarding the exploration of NSCs/NSPCs as cell therapeutic agents for SCI, Tuszynski's lab has been in the forefront of understanding the mechanisms that these cells trigger in vivo upon transplantation, as well as their behavior regarding the integration of functional neuronal networks. Therefore, Lu et al. transplanted GFP (green fluorescent protein)-expressing NSCs into T3 completely transected rats, 2 weeks after injury. The authors showed that GFP-positive cells were able to entirely fill the lesion cavity 7 weeks after transplantation and to evenly differentiate into neurons and oligodendrocytes, as well as astrocytes. Grafted NSCs did not migrate following implantation and showed the expression of both mature neuronal and spinal motor neuron markers. Importantly, this study demonstrated that not only transplanted NSCs were able to project their axons rostrally and caudally over long distances (reaching C4 and L1) but also to differentiate into neurons that interacted with host neurons by forming functional synapses. This long-distance axonal growth was correlated with mTOR signaling as its inhibition led to a 50\% decrease in graft axonal outgrowth. In addition, rats receiving NSCs were able to recover the movement of all hindlimb joints, in contrast to lesioned animals that presented minimal movement and partially regained electrophysiological response caudal to transection. The retransection of NSC-grafted animals rostrally to the initial lesion, 6 weeks postgraft, abolished motor recovery of these animals, as well as the recovered evoked response, proving that functional gains were due to host-graft relay interactions [44].

p0100 Focusing on these findings, Kadoya et al. explored the use of GFP-labeled NPCs in regenerating corticospinal tract (CST) projections after SCI due to its extreme importance in motor behavior and previous failed attempts in restoring CST function. Initially, the authors found that 6 weeks after transplantation corticospinal axons regenerated into the grafts being able to form contacts with their dendrites, and establishing synapses, in a T3 transection rat model. To go beyond this observation and understand the molecular mechanisms associated with CST regeneration, the researchers performed C4-CST lesions immediately followed by NPC grafting and used optogenetics to understand whether synaptic contacts between endogenous CST and grafted cells were functional in three of the animals. Photic stimulus created excitatory postsynaptic currents in two of the rats; whereas, using the glutamatergic blocker 6,7-dinitroquinoxaline-2,3-dione blocked the passage of signal showing that it resulted from glutamatergic synaptic activation and not from some other experimental artifact. This study also demonstrated that lesion to CST acts as a sort of priming for CST regeneration as in a subset of animals the authors proved that invasion of the graft by host CST only occurred in animals where a C5 injury was made, and not in animals that underwent sham surgery following NPC implantation. Interestingly, this study also showed that the site where NPCs were obtained impacts the degree of CST regeneration into grafts, proving that cells from spinal cord origin promoted significantly higher CST axonal infiltration than NPCs from telencephalon or hindbrain. Statistically significant improvements in fine motor behavior were also found [51]. 
p0105 Going into a more relevant animal model (rhesus monkeys), Rosenzweig and coworkers transplanted human spinal cord-derived NPCs into C7 spinal segment, 2 weeks after its hemisection. Grafts were shown to survive following a period of 9 months, expressing both glial and neuronal markers. Differently from rodent studies, the researchers encountered difficulties in graft survival and had to optimize the method, highlighting the importance of this type of studies in developing more robust and safer therapeutic hypotheses before performing clinical trials in humans. Interestingly, and contrarily to previous data, differentiation of NPCs mostly yielded neurons and no mature myelinating oligodendrocytes differentiated. Correlating with previous data, a large number of GFP-positive axons appeared from the graft, reaching up to $50 \mathrm{~mm}$ both rostral and caudal do the graft, extending and growing over white matter tracts. However, these axons did not present features of maturation 9 months posttransplantation. Notably, NPC-derived human axons commonly directed onto host motor neurons, forming synaptic contacts with an excitatory phenotype, restoring the necessary communication between motor cortex and the rostral part of the injured spinal cord. Nevertheless, any of these axons were found to be myelinated, even 9 months postimplantation, suggesting that a longer followup period would be necessary to attain fully matured NPC-derived cells and to possibly understand the full potential of this approach before translating it into humans. The study also showed that host axons could invade the graft, more concretely from neuronal populations fundamental for motor behavior (CST- and serotoninergic-derived axons). Using a scoring system that evaluated different motor parameters, the researchers found statistical differences in both the manipulation of objects and locomotion between animals that had/had not surviving grafts [54].

p0110 Kumamaro and colleagues, building on all these observations questioned whether the projections from host axons, most importantly CST prolongations, contacted with phenotypically relevant neurons within the grafts and if the differentiative fate of transplanted NPCs resembled native spinal cord neuronal population. Two weeks after grafting into a bilateral C4 dorsal spinal lesion rat model, NPCs expressed markers for immature and mature neurons, together with populations that showed markers for general motor neurons and premotor interneurons. It is worth noting that most grafted cells (73.6\%) expressed sensory interneuron markers, similarly to donor E14 rat spinal cord cells. Six months following implantation, NPCs differentiated into totally mature neurons, being the majority excitatory neurons and some other populations showed markers for cholinergic motor interneurons and inhibitory neurons. The authors then showed that at both timepoints ( 2 and 6 months) grafted cells presented discrete domains of motor and sensory neurons, once again resembling the organization of the native spinal cord. Remarkably, host CST projections connected with grafted neuronal domains expressing motor interneuron markers, avoiding sensory parts, 2 weeks and 6 months after grafting. This study also showed that CST projections were able to form active synapses with motor neuron populations within the graft 2 weeks after receiving the graft. These results were corroborated 6 months following grafting showing that the synaptic connections established in early timepoints between CST and grafted cells 
have long-term stability, keeping the specificity toward motor interneuron populations. As with the previous study, Tuszynski's group transplanted human spinalderived NSCs into nonhuman primates and corroborated the findings attained in rodents, thus showing that these grafts differentiate to resemble the native spinal cord cellular population and are permeable to CST axonal projections. Moreover, these projections establish functional synaptic contacts, specifically, with motor neuron populations present in the grafts, independently of specie that opens up an opportunity to use this therapeutic approach in human clinical trials [55]. In line with this, Stem Cells Inc. Company (Switzerland) established the world's first clinical trial in spinal cord-injured humans using these cells. In 2011, a Phase I/II clinical trial was designed to assess both safety and preliminary efficacy of a single transplantation of purified fetal human NSCs, as a treatment for chronic thoracic SCI, for both complete and incomplete injuries. The study enrolled seven patients with complete injuries [American Spinal Injury Association (ASIA) Impairment Scale A-AIS A] and five patients with incomplete injuries (AIS B). The cells were directly injected into their spinal cords, and they were temporarily immunosuppressed. Clinical updates were reported on a total of 8 of the 12 patients enrolled in the clinical trial. With regards to AIS A patients, there was significant posttransplant gain in sensory function in four patients up to date. Concerning AIS B subjects, two of three patients had significant gain in sensory perception, the third remaining unaltered [56]. Interestingly, most of these improvements were lost once the immunosuppression regiments were interrupted (nonpublished data). This highlights the relevance of autologous transplantation in SCI context. Another Phase I trial was carried out in patients suffering from chronic SCI using NSI-566 NPC cell line [57] that previously proved to be safe in Phase I and II trials in patients with amyotrophic lateral sclerosis [58]. The long-term safety of the cells had been previously tested in rats lesioned by compression in T10 by Curtis et al. The authors showed that the cells were able to differentiate into the three neural lineages, as well as to sprout their axons across the lesioned area and to establish synaptic contacts with host interneurons and motor neurons both below and above lesion site, setting up a promising platform to proceed to human assays. The clinical study comprised four individuals that received NSI-566 spinal cord implantation, with a follow-up period of 18-27 months. None of the patients showed serious adverse events during this interval, therefore tolerating the procedure. Three of the patients responded to the treatment and showed some positive secondary outcomes up to 27 months. More concretely, one of the subjects showed ameliorations in muscle responsiveness in lower limbs. Remarkably, these improvements reverted the SCI from complete to incomplete. Another of the individuals saw new voluntary activity in abdominal muscle and paraspinal muscles, 6 months following transplantation, together with ability to sense needle insertion, 18 months after grafting. Finally, other subjects presented sensorial and motor improvements at 6-, 12-, and 18-month checkpoints. This was translated in gain in the control of voluntary motor of the abdominal wall muscles and some superficial paraspinal muscles at the 12 months posttransplantation. However, the researchers noted that this patient showed anti-HLA antibodies 
98 days following transplantation but that was further observed not to be related to the treatment [58].

p0115 Even though the clinical study of NSCs/NPCs to treat SCI is still in its early phase, preclinical evidences are encouraging and clearly demonstrate that these cells have potential to serve as cellular therapies against the condition. Their capacity to differentiate into the three neural lineages (restoring native spinal cord cell phenotype), together with the ability to interact with host CST axons and motor neurons, establishing new neuronal circuits are probably the biggest advantages that NSCs/ NPCs present. Nevertheless, it is worth underlining that more mechanistic studies are needed to understand how the environment dictates the direction these cells differentiate, to better control the mature cellular populations obtained in situ, and how they can help modulating injured tissue apart from recreating new motor circuitry, just a small part of the complexity of SCI. Due to this, combinatorial studies using approaches targeting specific SCI secondary events and NSCs/NPCs will probably get us closer to finding a feasible way to restore spinal cord function beyond motor improvements.

\section{s0030 Mesenchymal stem cells}

p0120 The term MSC was used for the first time by Caplan et al. in 1991 [59] but these cells had been already well described, particularly by the work of Friedenstein and colleagues in the 1960s and 1970s [60,61]. However, before the recognition of the MSCs' existence, some experiments dating back to the 19th century showed that transplants of bone marrow could induce ectopic bone formation in other regions of the body, suggesting an important role for bone marrow in osteogenesis. That role was later confirmed by Tavassoli and Crosby in 1968 [62]. Friedenstein's work helped to clarify that a subset of cells from the bone marrow stroma was responsible for the osteogenic potential [60]. Moreover, he and his colleagues showed that these cells could be distinguished from hematopoietic cells due to their adherence to surfaces, fibroblast-like morphology, and capacity to form colonies from a single cell [61]. Later, Friedenstein named these cells as osteogenic stem cells [63] or bone marrow stromal stem cells [64]. As mentioned earlier, Caplan suggested the name mesenchymal stem cell, a term that is still today under debate [7]. The essential characteristics of these cells were later defined by Dominici et al. in 2006. In a statement of the International Society for Cellular Therapy, where the word "stem" was replaced for "stromal" to highlight the origin of these cells, it was defined that MSCs should (1) be plastic-adherent; (2) express the clusters of differentiation (CD) CD105, CD73, and CD90, lacking the expression of CD45, CD34, CD14 or CD11b, CD79- $\alpha$ or CD19, and human leukocyte antigen DR isotype (HLA-DR) surface markers; and (3) differentiate in vitro into osteoblasts, adipocytes, and chondroblasts [65]. Based on these criteria, MSC-like cells have been identified in numerous other tissue sources besides bone marrow, including the adipose tissue [66], the umbilical cord [67], skeletal muscles [68], dental tissues 
[69], synovial fluid [70], palatine tonsil [71], parathyroid gland [72], fallopian tube [73], brain [74], placenta [75], endometrial tissues [76], and uterine cervix [77]. Despite all these sources, bone marrow-derived MSCs (BM-MSCs), adipose tissue-derived stem/stromal cells (ASCs), and MSCs derived from the umbilical cord (UC-MSCs) are the most studied and their potential application to SCI has been addressed for many years. The main logic behind MSCs transplantation into SCI was, at the beginning, to find an alternative stem cell population that could replace damaged neurons and glial cells. However, as seen in other disease models, the main mechanism of action of MSCs does not seem to be related with neural differentiation, but rather with a general support and protection of endogenous cells. An interesting study by Zhang and colleagues, pointed in that direction. Even though different neural differentiation protocols were implemented to ASCs before transplantation, undifferentiated cells induced similar recovery of locomotor functions and identical numbers of neurofilament (NF)-positive cells in comparison to ASCs submitted to differentiation protocols, following a thoracic contusion injury in rats [78]. Similar findings were reported by Arboleda et al. in 2011. In this study, undifferentiated or ASCs submitted to sphere-differentiation protocols were transplanted into a rat model of SCI compression, 1-week postinjury. Functional improvements were observed in both groups, indicating a possible paracrine mechanism for the effects observed, rather than differentiation [79].

\section{s0035 BM-MSCs}

p0125 One of the first studies using BM-MSCs was performed by Chopp et al. in 2000, demonstrating that the transplantation of rat-derived BM-MSCs into the lesion epicenter led to functional improvements of injured rats 1 week after contusion of the spinal cord, as assessed by the Basso, Beattie, and Bresnahan (BBB) locomotor test [80]. Others had similar results, attributing this improvement to the remyelination of the injury site by both oligodendrocytes and SCs, with increased conduction velocity of the host axons $[81,82]$. Functional recovery accompanied by guided axonal growth and sprouting was also reported after grafting of BM-MSCs into contused rat spinal cords $[83,84]$. Importantly, host tissue preservation [84] and reduced lesion cavities $[85,86]$ were also observed, which seems to be related to neuroprotective effects of the transplanted cells. Although BM-MSCs are usually able to migrate to the injury site, the number of surviving cells is considerable low [87]. Therefore, some studies investigated the synergistic effect of BM-MSCs transplantation with other therapies, such as biomolecules. Lu et al. used BM-MSCs transduced to overexpress the brain-derived neurotrophic factor (BDNF) in a cervical spinal cord injury rat model, which resulted in a significant increase of host axonal growth. Additionally, increased levels of BDNF were detected in the rat spinal cord after treatment with BDNF-transduced BM-MSCs, as well as nerve growth factor (NGF) and neurotrophin-3 (NT-3) [88]. Based on the promising data of BMMSCs transplantation-based therapies in animal models, their safety and clinical effects were investigated in SCI patients. BM-MSCs administration to SCI patients 
demonstrated improvements in ASIA impairment scale, Barthel Index (quality of life), and Ashworth scoring (spasticity), in both subacute [89,90] and chronic injuries [89-92]. Bladder function was also found to improve upon BM-MSCs transplantation [90]. All the reported clinical studies differed in the route and frequency of administration of BM-MSCs, as well as on the number of the cohorts used, but all defend BM-MSCs administration to be feasible and safe and to improve patients' overall quality of life. However, successful application of BM-MSCs in the clinical practice requires more detailed examinations on the effects of these cells on SCI patients. Besides, BM-MSCs isolation is still a painful, invasive procedure, accompanied by a risk of infection [93], so alternative alternatives sources to the bone marrow are still needed.

\section{s0040 UC-MSCs}

p0130 UC-MSCs represent a potential alternative to BM-MSCs for allogeneic mesenchymal cell-based therapies. This nonhematopoietic MSC population was well described by Sarugaser et al. to present a rapid doubling time, high frequencies of colony-forming unit-fibroblast frequency and high major histocompatibility complexes $(\mathrm{MHC}+/+$ ) phenotype [94]. In contrast to BM-MSCs, UC-MSCs are immune naive and so present less graft rejection and posttransplant infections [95,96]. Saporta et al. was one of the first transplanting human UC-MSCs (HUC-MSCs) in rats after SCI. Cells obtained from the cord blood were systemically injected 1 and 5 days after injury, to test the therapeutic effect of HUC-MSCs at acute and subacute stage, respectively. In the subacute stage (5 days postinjury), HUC-MSCs were found to significantly improve animals' locomotor function, not observed for the acute stage (1-day postinjury). In addition, transplanted HUC-MSCs were able to migrate to the damaged tissue [97]. Similar results were found by Kao et al. in 2008. Interestingly, after transplantation of these cells, neurotrophic factors such as glial cell line-derived neurotrophic factor (GDNF) and vascular endothelial growth factor (VEGF) were detected in the injured spinal cord, possibly contributing to the observed improvement of animals' neurological functions [98]. The production of neurotrophic factors in the host spinal cord after HUC-MSCs transplantation was also reported by Yang et al. in the same year. In this study, cells obtained from the umbilical cord Wharton's Jelly were transplanted into the injury site of rats after complete spinal cord transection. Transplanted cells survived for 16 weeks within the lesion site, and produced human neutrophil-activating protein-2, NT-3, basic fibroblast growth factor (bFGF), glucocorticoid-induced tumor necrosis factor receptor, and VEGF. This was accompanied by significant locomotor improvements of the treated animals, and axonal growth and regeneration in the CST and in the lesion site [99]. The therapeutic potential of UC-MSCs has also been studied in larger SCI models such as dogs. Lim et al. [100] and, later on, Lee at al [101]. have reported the ability of blood-derived UC-MSCs to promote axonal remyelination of dog's spinal cord after injury, accompanied by a significant improvement in nerve conduction. In addition, hindlimb significant recovery was found in four of 
five HUC-MSCs transplanted dogs, which was sustained for at least 3 years following transplantation [101]. More recently, the clinical application of HUCMSCs from the Wharton's Jelly was found to be effective in 13 out of 22 patients with SCI after cells' intrathecal injection, inferred by significant improvements on algesia, tactile sensation, motion, and activity, as well as in bowel and bladder function. Additionally, no treatment-related adverse events occurred from 3 months to 3 years after transplantation, proving the safety of this therapy [102]. Overall neurological functional recovery of patients after thoracolumbar spinal cord injury was also reported by others, after transplantation of Wharton's Jelly-derived HUCMSCs. In this study, the observed improvements in the transplanted patients were superior to that of rehabilitation therapy and self-healing patients [103]. Besides these promising data, clinical studies with HUC-MSCs are still rare because of the concerns about safety and efficiency. Therefore, important issues on this approach require to be addressed in future clinical trials for treating SCI [104].

\section{s0045 ASCs}

p0135 ASCs emerged as a potential candidate for SCI therapy, as they can be obtained in larger quantities in comparison to BM-MSCs and UC-MSCs and are equally suited for biomedical applications [105].

p0140 The existence of cells with multipotent differentiation potential in the adipose tissue was suspected following some pathological observations such as osseous heteroplasia, where ectopic bone formation occurs within adipose tissue layers [106]. The histological analysis of these tissues revealed the presence of chondrocytes and osteoblasts, besides adipocytes [107]. In this sense, many authors started to explore the potentialities of this cell population, which later confirmed to fulfill the criteria defined for MSCs [65]. ASCs are responsible for replacing approximately $10 \%$ of mature adipocytes that turn over each year [108]. However, they have many other properties that can be useful when applied to neurodegenerative diseases. The main advantages of ASCs over other sources are that while maintaining most of the characteristics of an MSC population, they can be obtained from an accessible source, in a relatively simple procedure that can be repeated over time. Besides their multipotent potential, ASCs are known for their paracrine activity, secreting several growth factors, cytokines, extracellular matrix (ECM) molecules, among others [109]. Additionally, these cells have immunomodulatory properties and tend to migrate into injured tissues (homing ability). Finally, the reported capacity of ASCs to modulate the environment, rather than their differentiation ability, has been associated to the positive results observed in several medical conditions, such as neurological disorders $[110,111]$.

p0145 One of the first works describing the transplantation of ASCs into an SCI model was performed by Kang and colleagues, in 2006. In this study, the authors tried to differentiate rat ASCs toward OPCs, before transplantation. In fact, ASCs increased the expression of platelet-derived growth factor receptor $\alpha$, an OPCs-associated marker. Then, 3 days after a complete CST transection at T9-T10 level, ASCs- 
OPCs were autologously injected through the tail vein. At 4 weeks postinjury, injected cells survived and migrated preferentially to the injured spinal cord, although they could also be found in other organs such as the brain, liver, kidneys, or lungs. More importantly, transplanted rats demonstrated improved locomotor functions, as assessed by the BBB test [112]. Using a different approach, the same authors isolated cytoplasmic extracts of rat ASCs and transplanted them directly into the spinal cord, immediately after injury. This led to a significant suppression of apoptotic death and less astrogliosis, together with improved hindlimb function. The content of the extracts was not determined; however, it seemed to influence apoptotic mediators, by reducing caspase- 3 and Bcl2-associated X protein (Bax), while increasing B-cell lymphoma 2 (Bcl2) expression [113].

p0150 So far, both rat and human-derived ASCs have been addressed in different rodent models, from transection to contusion injuries, mostly at the thoracic region but some also at cervical level [114]. ASCs have been mostly administered intraspinally, but also intrathecally and intravenously with positive results $[115,116]$, which in the last case highlights the homing capacity of these cells to injured areas. Cells are usually transplanted 1 week after injury to avoid the aggressive environment and cell death often associated with immediate transplants. However, some works opted for treatments right after lesion [114,117], demonstrating not only significant cell survival, but also positive functional and histological effects of this approach. This unveils an important neuroprotective character of ASCs. In another interesting study, Menezes et al. showed that the transplantation of human ASCs $30 \mathrm{~min}$ after a T8-T9 balloon compression injury in rats improved animal's functional recovery, tissue preservation, and axonal regeneration. These results seemed to be associated with laminin deposition [118]. ASCs have also already been applied to larger animal models such as dogs. Dr. Kweon's group in South Korea has published several studies where canine-derived ASCs are transplanted into dogs with lumbar compression injuries [119-121]. Either alone [119] or combined with other strategies $[120,122]$, transplanted ASCs have demonstrated positive results in this animal model. At a veterinary setting, the transplantation of these cells proved to be a safe procedure, with no major complications, and showed some preliminary effects on locomotion and electromyogram activity [123,124]. Considering such data, at least two SCI clinical trials have been put forward using ASCs $[125,126]$. No serious adverse events were observed, and some preliminary improvements were seen in some patients.

p0155 As mentioned earlier, the factors and cytokines secreted by ASCs, also known as secretome, are mainly responsible for the effects observed until now. The cell secretome can be in fact used as a novel tool for the treatment of different conditions. Our group and others have shown that ASCs' secretome has a neuroprotective effect on cortical neurons subjected to inflammation in vitro [127]. It also favors the metabolic viability and cell density of hippocampal neuronal cultures, which seems to be related with the presence of NGF, VEGF, hepatocyte growth factor, and stem cell factor in the secretome [128]. ASC secretome was also reported to have an antiinflammatory effect over human monocytes, reducing the production of tumor 
necrosis factor alpha, nitric oxide, or prostaglandin E2 and inducing the production of transforming growth factor $\beta 1$ and interleukin 10 by M2 macrophages [129]. More recently, ASCs secretome demonstrated to promote neurite outgrowth from dorsal root ganglia explants, when compared to that mediated by MSCs isolated from the bone marrow and the umbilical cord [130]. In this sense, the future application of factors secreted by ASCs to an SCI context is a promising strategy, avoiding phenomena such as cell rejection or low cell survival.

\section{s0050 Glial cells (supportive cells) s0055 Schwann cells}

p0160 As mentioned earlier, SCs have been studied in SCI research for decades [131-133]. SCs are glial cells with a myelinating function, only present in the peripheral nervous system, where spontaneous regeneration occurs. There, SCs are known to surround peripheral axons with a spirally wrapped myelin sheath, leading to increased signal conduction [134].

p0165 Applying the myelinating SCs to treat the damaged spinal cord appeared to be logic, therefore the use of dissociated SCs in SCI preclinical models became frequent and often with promising results $[131,133]$. One of the first studies using SC cultures to transplant to the injured CNS was performed by Kromer and Cornbrooks in 1985 [135], moving away from experiments using complete peripheral nerve grafts [4].

p0170 Mary Bartlet Bunge's group from The Miami Project to Cure Paralysis has been working with SCs for SCI repair for several years now. The first preliminary data came in 1991, with Paino and Bunge (1991) showing that SCs enclosed in collagen rolls led to axonal ingrowth in SCI rats, similar to what was seen before with peripheral nerve grafts [136]. In another study, two million SCs were transplanted at the injury site 1 week after a moderate midthoracic contusion (T9), and led to increased spared tissue, higher numbers of myelinated axons, and also more brainstem and propriospinal axons reaching longer distances beyond the graft. This resulted in modest but significant improvements in motor behavior [137]. Later, Barakat et al. transplanted SC cellular grafts into chronically injured rats, 8 weeks after injury. The survival of SC grafts was $17.1 \%$ and they were shown to support the ingrowth of numerous NF-positive axons within the injury site, besides promoting the growth of several ascending and descending fibers. Once again, SCs led to significant improvements in motor function [138].

p0175 There have been works differentiating other cells into SCs, broadening the availability of SC sources. In a very interesting work, Biernaskie and Sparling et al. used a thoracic rat SCI model to demonstrate that mouse skin-derived precursors (SKPs) differentiated toward SCs were more effective in bridging the lesion site, myelinating spared axons, or enhancing locomotor recovery than the same nondifferentiated SKPs or neurospheres from the forebrain subventricular zone [139]. More recently, the same authors demonstrated that both rat SKP-derived or nerve-derived SCs 
promoted neural repair and functional recovery after incomplete cervical spinal cord crush injury [140]. SCs have also been genetically modified to express and secrete neurotrophic factors. Menei et al. showed that BDNF-secreting SCs enhanced axonal regrowth following SCI transection in the rat [141].

p0180 Similar to other cell-based therapies, SC transplantation has also been coupled with other therapeutic strategies. Pearse et al. again from Mary Bartlet Bunges's group, combined SC transplantation with cyclic adenosine monophosphate (cAMP) modulation treatment in SCI rats. Using rolipram to inhibit cAMP hydrolysis, in combination with SC grafts, the decrease of cAMP levels after SCI was successfully obtained, promoting at both axonal sparing and myelination of the treated animals. By further adding an injection of dibutyryl-cAMP near the graft, they could significantly enhance axonal sparing and myelination and also improve locomotion [142]. A recent study combining SC transplantation with macrophage depletion following contusive SCI in rats observed a reduced cystic cavity size and lesion volume in comparison to SC transplants alone [143].

p0185 Overall, these studies demonstrated interesting positive effects of SC transplants. The proposed mechanisms of action include neuroprotection, axon regeneration, and myelination. All these positive preclinical results recently led to the design of clinical trial from the Miami Project to Cure Paralysis team [144]. In this study, autologous transplants of SCs from the sural nerve were applied to subacute SCI patients (4-7 weeks after injury). After transplantation into the injury epicenter in a total of six subjects, there were no adverse events and no evidence of additional spinal cord damage, mass lesion, or syrinx formation, up to 1 year posttransplantation. These results show that SCs transplantation can be safe. However, further studies must be pursued to completely support the use of SC into SCI patients in the future.

\section{s0060 Olfactory ensheathing cells}

p0190 OECs are a special type of cells present in the olfactory system, known for ensheathing and surrounding axons from olfactory neurons. They are specifically located in the lamina propria of the olfactory mucosa at the PNS and in the external layer of the olfactory bulb-the olfactory nerve layer (ONL) at the CNS [145]. They are glial cells that support the growth of olfactory receptor neurons in the olfactory bulb [146] and participate in the continuous renewal of neurons in that system [145]. More specifically, OECs communicate with olfactory axons by evoking calcium signals via glutamatergic and purinergic pathways [147]. OECs' cytoplasmic projections envelop packages of axons, acting as insulators, avoiding contact with CNS inhibitory molecules [145].

p0195 Based on cellular morphology and expression of different markers, it is believed that different OEC subpopulations exist. For example, all OECs in the ONL express S100 $\beta$ and glial fibrillary acidic protein (GFAP) markers, but the ones sitting in the inner layer express neuropeptide $\mathrm{Y}$, while those in the outer layer express the low-affinity nerve growth factor receptor (p75) and neural cell adhesion molecule (E-NCAM) [145]. However, it is difficult to draw a clear separation of OEC 
populations, because the same cell can change the expression of its markers [145]. Moreover, OECs are migratory cells and their morphology and gene expression might differ in response to the environment [145]. In vitro cultures can be used as a model to understand this phenomena, although the conclusions are limited due to the different stimulus that OECs are subjected to, in comparison to those from in vivo scenario. Isolated OECs in vitro may assume predominantly two phenotypes: (i) astrocyte-like in shape, with low or no expression of p75; (ii) SCs-like morphology, with high expression of p75.

p0200 Work from Doucette was fundamental to identify OECs as a distinct type of glial cell present in the olfactory system, and also to characterize their importance in that environment $[148,149]$. The discovery of their role on neuronal growth led researchers to hypothesize their potential beneficial application to other CNS lesions.

p0205 Ramón-Cueto and colleagues were pioneers in the use of these cells for CNS applications. They started to characterize these cells in 1992, using cultures of rat olfactory bulbs and olfactory nerves [150]. At the time, the term olfactory ensheathing glia was often applied. Two years later, the same authors transplanted OECs into rhizotomized spinal cords at lower thoracic level (T10 dorsal root). Three weeks after transplantation, the growth of dorsal root axons into the spinal cord, which did not happen in nontransplanted rats, was observed [151]. Regarding SCI applications, Li et al. (1997) published one of the first works describing the transplantation of OECs that led to an improved regenerating capacity of CST fibers following a unilateral cervical injury in rats. The transplant also led to improved forepaw reaching skills in treated rats [152]. Later, Ramón-Cueto et al. (1998) tested OECs transplantation coupled with channels made of synthetic polymers filled with SCs, after a T9 transection injury in rats. Six weeks after injury, numerous NF-, growthassociated protein 43 (GAP43)-, calcitonin gene-related peptide (CGRP)-, and serotonin-positive fibers traversed the glial scars previously formed. Interestingly, the growth of serotonin fibers avoided the interior of the channels, growing instead supported by connective tissue bridges formed on the exterior of the channels. Importantly, they verified that OECs migrated after transplantation, overcoming the traditionally inhibitory factors such as the glial scar, and were present in the same locations of regenerated axons [153]. In the following years, a great volume of studies using OECs as a tool for SCI repair emerged. Either transplanted immediately following injury [154] or at a chronic stage [155], applied into transection or contusion injuries at different spinal levels [156,157], transplants of OECs have shown a regenerative potential and a capacity to induce functional recovery in several cases. Moreover, OECs isolated from both adult and young animals have been used [158,159], and obtained from two distinct regions: the lamina propria from the olfactory mucosa and the outer layers of the olfactory bulb. Overall, both types of OECs were capable of inducing beneficial effects in SCI models $[160,161]$. From a translational point of view, important studies have used OECs from species other than rodents, including dogs [162], pigs [163] or human cells [164]. In this last work, the authors demonstrated that OECs transplanted 1-week 
after a T10 contusion injury reduced lesion volume and GFAP intensity, while improving hindlimb function [164].

p0210 There are several mechanisms associated with OECs' effects. For instance, OECs were suggested to interact with astrocytes and meningeal cells, being able to grow with astrocytes in culture and in the glial scar, which is an advantage in comparison to SCs [165]. Some other studies even reported that OECs reduced astrocytic reactivity and CSPGs (chondroitin sulfate proteoglycans) expression [166]. OECs were also shown to produce ECM proteases, which might help in their migratory capacity within the nervous tissue [167].

p0215 The relevance of preclinical data led several groups to attempt OECs transplantation into patients. Lima et al. transplanted olfactory mucosa autografts into seven chronic SCI patients, demonstrating that the procedure was feasible and relatively safe [168]. Later, Mackay-Sim et al. also demonstrated that autologous transplantation of mucosal OECs was safe, free of serious adverse effects after a 3-year followup, even though no significant improvements were seen in transplanted patients [169]. More recently, Tabakow et al. (2013) obtained similar results [170] and very interestingly, published a clinical case of OECs transplantation from the olfactory bulbs, which resulted in significant recovery of patients' function [171].

\section{s0065 Conclusions}

p0220 The use of cell transplantation for SCI purposes mainly aims to replace lost cell populations or to provide suitable substrate for neuronal regeneration. All the referred types of cells have the potential to be further studied in the context of this condition (Table 9.1), even though perhaps the most promising application lies on using iPSCs. With these cells, it will be possible to obtain virtually endless amounts of autologous pluripotent cells, without the risk of patient rejection, and they could be differentiated to specific cells of interest according to the objective of the study. The use of MSCs also holds great promise, as they can be obtained from various sources, and very importantly from adult individuals. Understanding the MSCs immunomodulatory effects, together with the possibility of designing cell-free therapies based on the MSCs' secretome, will be of the utmost importance for SCI and other neurodegenerative diseases. Nevertheless, due to the complexity of SCI, the most exciting possibilities rely on combining cellular transplantation, of one or more distinct cell types, with approaches that target precise secondary events, helping to build more robust and broad therapeutic options.

\section{Acknowledgements}

Funding from Prémios Santa Casa Neurociências - Prize Melo e Castro for Spinal Cord Injury Research (MC-04/17). This work is funded by national funds through the Portuguese Foundation for Science and Technology (FCT) under the scope of grant reference TUBITAK/ 
0007/2014 and predoctoral fellowships reference numbers PDE/BDE/113596/2015, SFRH/ $\mathrm{BD} / 103075 / 2014, \mathrm{PD} / \mathrm{BDE} / 127835 / 2016, \mathrm{PD} / \mathrm{BDE} / 127836 / 2016$. This article has been developed under the scope of the projects NORTE-01-0145-FEDER-000013 supported by the Northern Portugal Regional Operational Programme (NORTE 2020), under the Portugal 2020 Partnership Agreement, through the European Regional Development Fund (FEDER). This work has been funded by FEDER funds, through the Competitiveness Factors Operational Programme (COMPETE), and by National funds, through the Foundation for Science and Technology (FCT), under the scope of the project POCI-01-0145-FEDER-007038 and POCI-01-0145-FEDER-029206.

\section{References}

[1] Ballen KK, Gluckman E, Broxmeyer HE. Umbilical cord blood transplantation: the first 25 years and beyond. Blood 2013;122(4):491-8.

[2] Cajal SR, DeFelipe J, Jones EG. Cajal's degeneration and regeneration of the nervous system. Oxford University Press; 1991.

[3] Richardson PM, McGuinness UM, Aguayo AJ. Axons from CNS neurons regenerate into PNS grafts. Nature 1980;284(5753):264-5.

[4] David S, Aguayo AJ. Axonal elongation into peripheral nervous system "bridges" after central nervous system injury in adult rats. Science 1981;214(4523):931-3.

[5] Bunge MB. Efficacy of Schwann cell transplantation for spinal cord repair is improved with combinatorial strategies. J Physiol 2016;594(13):3533-8.

[6] Bunge MB, Williams AK, Wood PM, Uitto J, Jeffrey JJ. Comparison of nerve cell and nerve cell plus Schwann cell cultures, with particular emphasis on basal lamina and collagen formation. J Cell Biol 1980;84(1):184-202.

[7] Bianco P, Robey PG, Simmons PJ. Mesenchymal stem cells: revisiting history, concepts, and assays. Cell Stem Cell 2008;2(4):313-9.

[8] Evans MJ, Kaufman MH. Establishment in culture of pluripotential cells from mouse embryos. Nature 1981;292(5819):154-6.

[9] Thomson JA, Itskovitz-Eldor J, Shapiro SS, Waknitz MA, Swiergiel JJ, Marshall VS, Jones JM. Embryonic stem cell lines derived from human blastocysts. Science 1998; 282(5391):1145-7.

[10] Bain G, Kitchens D, Yao M, Huettner JE, Gottlieb DI. Embryonic stem cells express neuronal properties in vitro. Dev Biol 1995;168(2):342-57.

[11] Liu S, Qu Y, Stewart TJ, Howard MJ, Chakrabortty S, Holekamp TF, McDonald JW. Embryonic stem cells differentiate into oligodendrocytes and myelinate in culture and after spinal cord transplantation. Proc Natl Acad Sci USA 2000;97(11):6126-31.

[12] Kawasaki H, Mizuseki K, Nishikawa S, Kaneko S, Kuwana Y, Nakanishi S, Nishikawa SI, Sasai Y. Induction of midbrain dopaminergic neurons from ES cells by stromal cell-derived inducing activity. Neuron 2000;28(1):31-40.

[13] Lee SH, Lumelsky N, Studer L, Auerbach JM, McKay RD. Efficient generation of midbrain and hindbrain neurons from mouse embryonic stem cells. Nat Biotechnol 2000;18(6):675-9.

[14] Ying QL, Stavridis M, Griffiths D, Li M, Smith A. Conversion of embryonic stem cells into neuroectodermal precursors in adherent monoculture. Nat Biotechnol 2003;21(2): $183-6$. 
[15] Abranches E, Bekman E, Henrique D, Cabral JM. Expansion and neural differentiation of embryonic stem cells in adherent and suspension cultures. Biotechnol Lett 2003; 25(9):725-30.

[16] Keirstead HS, Nistor G, Bernal G, Totoiu M, Cloutier F, Sharp K, Steward O. Human embryonic stem cell-derived oligodendrocyte progenitor cell transplants remyelinate and restore locomotion after spinal cord injury. J Neurosci 2005;25(19):4694-705.

[17] Kakinohana O, Juhasova J, Juhas S, Motlik J, Platoshyn O, Galik J, Hefferan M, Yuan S, Vidal J, Carson C. Survival and differentiation of human embryonic stem cell-derived neural precursors grafted spinally in spinal ischemia-injured rats or in naive immunosuppressed minipigs: a qualitative and quantitative study. Cell Transplantation 2012;21(12):2603-19.

[18] McDonald JW, Liu XZ, Qu Y, Liu S, Mickey SK, Turetsky D, Gottlieb DI, Choi DW. Transplanted embryonic stem cells survive, differentiate and promote recovery in injured rat spinal cord. Nat Med 1999;5(12):1410-2.

[19] Pollack A. Geron is shutting down its stem cell clinical trial. N Y Times, November 14, 2011.

[20] Shroff G. Human embryonic stem cell therapy in chronic spinal cord injury: a retrospective study. Clin Ttransl Sci 2016;9(3):168-75.

[21] Shroff G, Barthakur K. Bowel/bladder sensation and control in patients with spinal cord injury treated with human embryonic stem cell therapy. EC Neurol 2015;2: $47-54$.

[22] Li J-Y, Christophersen NS, Hall V, Soulet D, Brundin P. Critical issues of clinical human embryonic stem cell therapy for brain repair. Trends Neurosci 2008;31(3): $146-53$.

[23] Chambers SM, Fasano CA, Papapetrou EP, Tomishima M, Sadelain M, Studer L. Highly efficient neural conversion of human ES and iPS cells by dual inhibition of SMAD signaling. Nat Biotechnol 2009;27(3):275.

[24] Brederlau A, Correia AS, Anisimov SV, Elmi M, Paul G, Roybon L, Morizane A, Bergquist F, Riebe I, Nannmark U. Transplantation of human embryonic stem cellderived cells to a rat model of Parkinson's disease: effect of in vitro differentiation on graft survival and teratoma formation. Stem Cell 2006;24(6):1433-40.

[25] Tate CC, Shear DA, Tate MC, Archer DR, Stein DG, LaPlaca MC. Laminin and fibronectin scaffolds enhance neural stem cell transplantation into the injured brain. J Tissue Eng Regenerat Med 2009;3(3):208-17.

[26] Sleeboom-Faulkner M. Debates on human embryonic stem cell research in Japan: minority voices and their political amplifiers. Sci Cult 2008;17(1):85-97.

[27] Gea B, Lebacqz K, Mendiola MM, Peters T, Young EW, Zoloth-Dorfman L. Research with human embryonic stem cells: ethical considerations. Hastings Cent Rep 1999; 29(2):31-6.

[28] Chapman A, Frankel M, Garfinkel M, Science AAftAo. Stem cell research and applications: monitoring the frontiers of biomedical research.

[29] Takahashi K, Yamanaka S. Induction of pluripotent stem cells from mouse embryonic and adult fibroblast cultures by defined factors. Cell 2006;126(4):663-76.

[30] Liao J, Cui C, Chen S, Ren J, Chen J, Gao Y, Li H, Jia N, Cheng L, Xiao H. Generation of induced pluripotent stem cell lines from adult rat cells. Cell Stem Cell 2009;4(1): $11-5$. 
[31] Liu H, Zhu F, Yong J, Zhang P, Hou P, Li H, Jiang W, Cai J, Liu M, Cui K. Generation of induced pluripotent stem cells from adult rhesus monkey fibroblasts. Cell Stem Cell 2008;3(6):587-90.

[32] Takahashi K, Tanabe K, Ohnuki M, Narita M, Ichisaka T, Tomoda K, Yamanaka S. Induction of pluripotent stem cells from adult human fibroblasts by defined factors. Cell 2007;131(5):861-72.

[33] Yu J, Vodyanik MA, Smuga-Otto K, Antosiewicz-Bourget J, Frane JL, Tian S, Nie J, Jonsdottir GA, Ruotti V, Stewart R. Induced pluripotent stem cell lines derived from human somatic cells. Science 2007;318(5858):1917-20.

[34] Lowry W, Richter L, Yachechko R, Pyle A, Tchieu J, Sridharan R, Clark A, Plath K. Generation of human induced pluripotent stem cells from dermal fibroblasts. Proc Natl Acad Sci USA 2008;105(8):2883-8.

[35] Park I-H, Zhao R, West JA, Yabuuchi A, Huo H, Ince TA, Lerou PH, Lensch MW, Daley GQ. Reprogramming of human somatic cells to pluripotency with defined factors. Nature 2008;451(7175): 141 .

[36] Puri MC, Nagy A. Concise review: embryonic stem cells versus induced pluripotent stem cells: the game is on. Stem Cell 2012;30(1):10-4.

[37] Gutierrez-Aranda I, Ramos-Mejia V, Bueno C, Munoz-Lopez M, Real PJ, Macia A, Sanchez L, Ligero G, Garcia-Parez JL, Menendez P. Human induced pluripotent stem cells develop teratoma more efficiently and faster than human embryonic stem cells regardless the site of injection. Stem Cell 2010;28(9):1568-70.

[38] Okita K, Ichisaka T, Yamanaka S. Generation of germline-competent induced pluripotent stem cells. Nature 2007;448(7151):313.

[39] Nakagawa M, Koyanagi M, Tanabe K, Takahashi K, Ichisaka T, Aoi T, Okita K, Mochiduki Y, Takizawa N, Yamanaka S. Generation of induced pluripotent stem cells without Myc from mouse and human fibroblasts. Nat Biotechnol 2008;26(1):101.

[40] Kim JB, Zaehres H, Wu G, Gentile L, Ko K, Sebastiano V, Araúzo-Bravo MJ, Ruau D, Han DW, Zenke M. Pluripotent stem cells induced from adult neural stem cells by reprogramming with two factors. Nature 2008;454(7204):646.

[41] Miura K, Okada Y, Aoi T, Okada A, Takahashi K, Okita K, Nakagawa M, Koyanagi M, Tanabe K, Ohnuki M. Variation in the safety of induced pluripotent stem cell lines. Nat Biotechnol 2009;27(8):743.

[42] Tsuji O, Miura K, Okada Y, Fujiyoshi K, Mukaino M, Nagoshi N, Kitamura K, Kumagai G, Nishino M, Tomisato S, et al. Therapeutic potential of appropriately evaluated safe-induced pluripotent stem cells for spinal cord injury. Proc Natl Acad Sci USA 2010;107(28):12704-9.

[43] Lu P, Woodruff G, Wang Y, Graham L, Hunt M, Wu D, Boehle E, Ahmad R, Poplawski G, Brock J. Long-distance axonal growth from human induced pluripotent stem cells after spinal cord injury. Neuron 2014;83(4):789-96.

[44] Lu P, Wang Y, Graham L, McHale K, Gao M, Wu D, Brock J, Blesch A, Rosenzweig ES, Havton LA. Long-distance growth and connectivity of neural stem cells after severe spinal cord injury. Cell 2012;150(6):1264-73.

[45] Kobayashi Y, Okada Y, Itakura G, Iwai H, Nishimura S, Yasuda A, Nori S, Hikishima K, Konomi T, Fujiyoshi K, et al. Pre-evaluated safe human iPSC-derived neural stem cells promote functional recovery after spinal cord injury in common marmoset without tumorigenicity. PLoS One 2012;7(12):e52787. 
[46] Cyranoski D. 'Reprogrammed' stem cells to treat spinal-cord injuries for the first time. Nature February 22, 2019.

[47] Weiss S, Dunne C, Hewson J, Wohl C, Wheatley M, Peterson AC, Reynolds BA. Multipotent CNS stem cells are present in the adult mammalian spinal cord and ventricular neuroaxis. J Neurosci 1996;16(23):7599-609.

[48] Lu P, Jones LL, Snyder EY, Tuszynski MH. Neural stem cells constitutively secrete neurotrophic factors and promote extensive host axonal growth after spinal cord injury. Exp Neurol 2003;181(2):115-29.

[49] Piltti KM, Salazar DL, Uchida N, Cummings BJ, Anderson AJ. Safety of epicenter versus intact parenchyma as a transplantation site for human neural stem cells for spinal cord injury therapy. Stem Cells Transl Med 2013;2(3):204-16.

[50] Bonner JF, Connors TM, Silverman WF, Kowalski DP, Lemay MA, Fischer I. Grafted neural progenitors integrate and restore synaptic connectivity across the injured spinal cord. J Neurosci 2011;31(12):4675-86.

[51] Kadoya K, Lu P, Nguyen K, Lee-Kubli C, Kumamaru H, Yao L, Knackert J, Poplawski G, Dulin JN, Strobl H, et al. Spinal cord reconstitution with homologous neural grafts enables robust corticospinal regeneration. Nat Med 2016;22(5):479-87.

[52] Iwanami A, Yamane J, Katoh H, Nakamura M, Momoshima S, Ishii H, Tanioka Y, Tamaoki N, Nomura T, Toyama Y, et al. Establishment of graded spinal cord injury model in a nonhuman primate: the common marmoset. J Neurosci Res 2005;80(2): $172-81$.

[53] Cummings BJ, Uchida N, Tamaki SJ, Salazar DL, Hooshmand M, Summers R, Gage FH, Anderson AJ. Human neural stem cells differentiate and promote locomotor recovery in spinal cord-injured mice. Proc Natl Acad Sci USA 2005;102(39): 14069-74.

[54] Rosenzweig ES, Brock JH, Lu P, Kumamaru H, Salegio EA, Kadoya K, Weber JL, Liang JJ, Moseanko R, Hawbecker S, et al. Restorative effects of human neural stem cell grafts on the primate spinal cord. Nat Med 2018;24(4):484-90.

[55] Kumamaru H, Lu P, Rosenzweig ES, Kadoya K, Tuszynski MH. Regenerating corticospinal axons innervate phenotypically appropriate neurons within neural stem cell grafts. Cell Reports 2019;26(9):2329-39. e2324.

[56] Levi AD, Okonkwo DO, Park P, Jenkins 3rd AL, Kurpad SN, Parr AM, Ganju A, Aarabi B, Kim D, Casha S, et al. Emerging safety of intramedullary transplantation of human neural stem cells in chronic cervical and thoracic spinal cord injury. Neurosurgery 2018;82(4):562-75.

[57] Curtis E, Martin JR, Gabel B, Sidhu N, Rzesiewicz TK, Mandeville R, Van Gorp S, Leerink M, Tadokoro T, Marsala S. A first-in-human, phase I study of neural stem cell transplantation for chronic spinal cord injury. Cell Stem Cell 2018;22(6): 941-50. e946.

[58] Glass JD, Hertzberg VS, Boulis NM, Riley J, Federici T, Polak M, Bordeau J, Fournier C, Johe K, Hazel T, et al. Transplantation of spinal cord-derived neural stem cells for ALS: analysis of phase 1 and 2 trials. Neurology 2016;87(4):392-400.

[59] Caplan AI. Mesenchymal stem cells. J Orthop Res 1991;9(5):641-50.

[60] Friedenstein AJ, Deriglasova UF, Kulagina NN, Panasuk AF, Rudakowa SF, Luria EA, Ruadkow IA. Precursors for fibroblasts in different populations of hematopoietic cells as detected by the in vitro colony assay method. Exp Hematol 1974;2(2): 83-92. 
[61] Friedenstein AJ, Chailakhjan RK, Lalykina KS. The development of fibroblast colonies in monolayer cultures of Guinea-pig bone marrow and spleen cells. Cell Tissue Kinet 1970;3(4):393-403.

[62] Tavassoli M, Crosby WH. Transplantation of marrow to extramedullary sites. Science 1968;161(3836):54-6.

[63] Friedenstein AJ, Chailakhyan RK, Gerasimov UV. Bone marrow osteogenic stem cells: in vitro cultivation and transplantation in diffusion chambers. Cell Tissue Kinet 1987;20(3):263-72.

[64] Owen M, Friedenstein AJ. Stromal stem cells: marrow-derived osteogenic precursors. Ciba Foundat Sympos 1988;136:42-60.

[65] Dominici M, Le Blanc K, Mueller I, Slaper-Cortenbach I, Marini F, Krause D, Deans R, Keating A, Prockop D, Horwitz E. Minimal criteria for defining multipotent mesenchymal stromal cells. The International Society for Cellular Therapy position statement. Cytotherapy 2006;8(4):315-7.

[66] Zuk PA, Zhu M, Ashjian P, De Ugarte DA, Huang JI, Mizuno H, Alfonso ZC, Fraser JK, Benhaim P, Hedrick MH. Human adipose tissue is a source of multipotent stem cells. Mol Biol Cell 2002;13(12):4279-95.

[67] Lee OK, Kuo TK, Chen WM, Lee KD, Hsieh SL, Chen TH. Isolation of multipotent mesenchymal stem cells from umbilical cord blood. Blood 2004;103(5):1669-75.

[68] Jackson WM, Nesti LJ, Tuan RS. Potential therapeutic applications of muscle-derived mesenchymal stem and progenitor cells. Expert Opinion on Biological Therapy 2010; 10(4):505-17.

[69] Huang GT, Gronthos S, Shi S. Mesenchymal stem cells derived from dental tissues vs. those from other sources: their biology and role in regenerative medicine. J Dent Res 2009;88(9):792-806.

[70] Jones EA, English A, Henshaw K, Kinsey SE, Markham AF, Emery P, McGonagle D. Enumeration and phenotypic characterization of synovial fluid multipotential mesenchymal progenitor cells in inflammatory and degenerative arthritis. Arthritis Rheum 2004;50(3):817-27.

[71] Janjanin S, Djouad F, Shanti RM, Baksh D, Gollapudi K, Prgomet D, Rackwitz L, Joshi AS, Tuan RS. Human palatine tonsil: a new potential tissue source of multipotent mesenchymal progenitor cells. Arthritis Research \& Therapy 2008;10(4):R83.

[72] Shih YR, Kuo TK, Yang AH, Lee OK, Lee CH. Isolation and characterization of stem cells from the human parathyroid gland. Cell Proliferation 2009;42(4):461-70.

[73] Jazedje T, Perin PM, Czeresnia CE, Maluf M, Halpern S, Secco M, Bueno DF, Vieira NM, Zucconi E, Zatz M. Human fallopian tube: a new source of multipotent adult mesenchymal stem cells discarded in surgical procedures. J Transl Med 2009; 7:46.

[74] Paul G, Ozen I, Christophersen NS, Reinbothe T, Bengzon J, Visse E, Jansson K, Dannaeus K, Henriques-Oliveira C, Roybon L, et al. The adult human brain harbors multipotent perivascular mesenchymal stem cells. PLoS One 2012;7(4):e35577.

[75] Fukuchi Y, Nakajima H, Sugiyama D, Hirose I, Kitamura T, Tsuji K. Human placentaderived cells have mesenchymal stem/progenitor cell potential. Stem Cell 2004;22(5): 649-58.

[76] Schwab KE, Hutchinson P, Gargett CE. Identification of surface markers for prospective isolation of human endometrial stromal colony-forming cells. Hum Reprod 2008; 23(4):934-43. 
[77] Eiro N, Sendon-Lago J, Seoane S, Bermudez MA, Lamelas ML, Garcia-Caballero T, Schneider J, Perez-Fernandez R, Vizoso FJ. Potential therapeutic effect of the secretome from human uterine cervical stem cells against both cancer and stromal cells compared with adipose tissue stem cells. Oncotarget 2014;5(21):10692-708.

[78] Zhang HT, Luo J, Sui LS, Ma X, Yan ZJ, Lin JH, Wang YS, Chen YZ, Jiang XD, $\mathrm{Xu}$ RX. Effects of differentiated versus undifferentiated adipose tissue-derived stromal cell grafts on functional recovery after spinal cord contusion. Cell Mol Neurobiol 2009;29(8):1283-92.

[79] Arboleda D, Forostyak S, Jendelova P, Marekova D, Amemori T, Pivonkova H, Masinova K, Sykova E. Transplantation of predifferentiated adipose-derived stromal cells for the treatment of spinal cord injury. Cell Mol Neurobiol 2011;31(7):1113-22.

[80] Chopp M, Zhang XH, Li Y, Wang L, Chen J, Lu D, Lu M, Rosenblum M. Spinal cord injury in rat: treatment with bone marrow stromal cell transplantation. Neuroreport 2000;11(13):3001-5.

[81] Akiyama Y, Radtke C, Honmou O, Kocsis JD. Remyelination of the spinal cord following intravenous delivery of bone marrow cells. Glia 2002;39(3):229-36.

[82] Akiyama Y, Radtke C, Kocsis JD. Remyelination of the rat spinal cord by transplantation of identified bone marrow stromal cells. J Neurosci 2002;22(15):6623-30.

[83] Himes BT, Neuhuber B, Coleman C, Kushner R, Swanger SA, Kopen GC, Wagner J, Shumsky JS, Fischer I. Recovery of function following grafting of human bone marrow-derived stromal cells into the injured spinal cord. Neurorehabilitation Neural Repair 2006;20(2):278-96.

[84] Ankeny DP, McTigue DM, Jakeman LB. Bone marrow transplants provide tissue protection and directional guidance for axons after contusive spinal cord injury in rats. Exp Neurol 2004;190(1):17-31.

[85] Ohta M, Suzuki Y, Noda T, Ejiri Y, Dezawa M, Kataoka K, Chou H, Ishikawa N, Matsumoto N, Iwashita Y, et al. Bone marrow stromal cells infused into the cerebrospinal fluid promote functional recovery of the injured rat spinal cord with reduced cavity formation. Exp Neurol 2004;187(2):266-78.

[86] Sheth RN, Manzano G, Li X, Levi AD. Transplantation of human bone marrowderived stromal cells into the contused spinal cord of nude rats. J Neurosurg Spine 2008;8(2):153.

[87] Č́ižková D, Rosocha J, Vanický I, Jergová S, Č́žžek M. Transplants of human mesenchymal stem cells improve functional recovery after spinal cord injury in the rat. Cell Mol Neurobiol 2006;26(7):1165-78.

[88] Lu P, Jones LL, Tuszynski MH. BDNF-expressing marrow stromal cells support extensive axonal growth at sites of spinal cord injury. Exp Neurol 2005;191(2):344-60.

[89] Syková E, Homola A, Mazanec R, Lachmann H, Konrádová ŠL, Kobylka P, Pádr R, Neuwirth J, Komrska V, Vávra V, et al. Autologous bone marrow transplantation in patients with subacute and chronic spinal cord injury. Cell Transplantation 2006; 15(8-9):675-87.

[90] Geffner LF, Santacruz P, Izurieta M, Flor L, Maldonado B, Auad AH, Montenegro X, Gonzalez R, Silva F. Administration of autologous bone marrow stem cells into spinal cord injury patients via multiple routes is safe and improves their quality of life: comprehensive case studies. Cell Transplantation 2008;17(12):1277-93.

[91] Kishk NA, Gabr H, Hamdy S, Afifi L, Abokresha N, Mahmoud H, Wafaie A, Bilal D. Case control series of intrathecal autologous bone marrow mesenchymal stem cell 
therapy for chronic spinal cord injury. Neurorehabilitation Neural Repair 2010;24(8): $702-8$.

[92] Kakabadze Z, Kipshidze N, Mardaleishvili K, Chutkerashvili G, Chelishvili I, Harders A, Loladze G, Shatirishvili G, Kipshidze N, Chakhunashvili D, et al. Phase 1 trial of autologous bone marrow stem cell transplantation in patients with spinal cord injury. Stem Cell Int 2016;2016:8.

[93] Hass R, Kasper C, Böhm S, Jacobs R. Different populations and sources of human mesenchymal stem cells (MSC): a comparison of adult and neonatal tissue-derived MSC. Cell Commun Signal 2011;9(1):12.

[94] Sarugaser R, Lickorish D, Baksh D, Hosseini MM, Davies JE. Human umbilical cord perivascular (HUCPV) cells: a source of mesenchymal progenitors. Stem Cell 2005; 23(2):220-9.

[95] Tse W, Laughlin MJ. Umbilical cord blood transplantation: a new alternative option. ASH Educ Program B 2005;2005(1):377-83.

[96] Knutsen AP, Wall DA. Kinetics of T-cell development of umbilical cord blood transplantation in severe T-cell immunodeficiency disorders. J Allergy Clin Immunol 1999; 103(5):823-32.

[97] Saporta S, Kim J-J, Willing AE, Fu ES, Davis CD, Sanberg PR. Human umbilical cord blood stem cells infusion in spinal cord injury: engraftment and beneficial influence on behavior. J Hematother Stem Cell Res 2003;12(3):271-8.

[98] Kao C-H, Chen S-H, Chio C-C, Lin M-T. Human umbilical cord blood-derived CD34+ cells may attenuate spinal cord injury by stimulating vascular endothelial and neurotrophic factors. Shock 2008;29(1):49-55.

[99] Yang C-C, Shih Y-H, Ko M-H, Hsu S-Y, Cheng H, Fu Y-S. Transplantation of human umbilical mesenchymal stem cells from Wharton's jelly after complete transection of the rat spinal cord. PLoS One 2008;3(10):e3336.

[100] Lim J-H, Byeon Y-E, Ryu H-H, Jeong Y-H, Lee Y-W, Kim WH, Kang K-S, Kweon O-K. Transplantation of canine umbilical cord blood-derived mesenchymal stem cells in experimentally induced spinal cord injured dogs. J Vet Sci 2007;8(3): $275-82$.

[101] Lee J-H, Chung W-H, Kang E-H, Chung D-J, Choi C-B, Chang H-S, Lee J-H, Hwang S-H, Han H, Choe B-Y, et al. Schwann cell-like remyelination following transplantation of human umbilical cord blood (hUCB)-derived mesenchymal stem cells in dogs with acute spinal cord injury. J Neurol Sci 2011;300(1):86-96.

[102] Liu J, Han D, Wang Z, Xue M, Zhu L, Yan H, Zheng X, Guo Z, Wang H. Clinical analysis of the treatment of spinal cord injury with umbilical cord mesenchymal stem cells. Cytotherapy 2013;15(2):185-91.

[103] Cheng H, Liu X, Hua R, Dai G, Wang X, Gao J, An Y. Clinical observation of umbilical cord mesenchymal stem cell transplantation in treatment for sequelae of thoracolumbar spinal cord injury. J Transl Med 2014;12(1):253.

[104] Park D-H, Lee J-H, Borlongan CV, Sanberg PR, Chung Y-G, Cho T-H. Transplantation of umbilical cord blood stem cells for treating spinal cord injury. Stem Cell Rev Rep 2011;7(1):181-94.

[105] Strioga M, Viswanathan S, Darinskas A, Slaby O, Michalek J. Same or not the same? Comparison of adipose tissue-derived versus bone marrow-derived mesenchymal stem and stromal cells. Stem Cell Dev 2012;21(14):2724-52.

[106] Gimble JM, Katz AJ, Bunnell BA. Adipose-derived stem cells for regenerative medicine. Circ Res 2007;100(9):1249-60. 
[107] Kaplan FS, Hahn GV, Zasloff MA. Heterotopic ossification: two rare forms and what they can teach us. J Am Acad Orthopaed Surg 1994;2(5):288-96.

[108] Salgado A J, Reis R L, Sousa N, Gimble J M. Adipose tissue derived stem cells secretome: soluble factors and their roles in regenerative medicine. Curr Stem Cell Res Ther 2010;5(2):103-10.

[109] Kokai LE, Marra K, Rubin JP. Adipose stem cells: biology and clinical applications for tissue repair and regeneration. Transl Res 2014;163(4):399-408.

[110] McIntosh KR, Frazier T, Rowan BG, Gimble JM. Evolution and future prospects of adipose-derived immunomodulatory cell therapeutics. Expert Rev Clin Immunol 2013;9(2):175-84.

[111] Chang KA, Kim HJ, Joo Y, Ha S, Suh YH. The therapeutic effects of human adiposederived stem cells in Alzheimer's disease mouse models. Neurodegener Dis 2014; 13(2-3):99-102.

[112] Kang SK, Shin MJ, Jung JS, Kim YG, Kim CH. Autologous adipose tissue-derived stromal cells for treatment of spinal cord injury. Stem Cells and Development 2006; 15(4):583-94.

[113] Kang SK, Yeo JE, Kang KS, Phinney DG. Cytoplasmic extracts from adipose tissue stromal cells alleviates secondary damage by modulating apoptosis and promotes functional recovery following spinal cord injury. Brain Pathol 2007;17(3):263-75.

[114] Kolar MK, Kingham PJ, Novikova LN, Wiberg M, Novikov LN. The therapeutic effects of human adipose-derived stem cells in a rat cervical spinal cord injury model. Stem Cells and Development 2014;23(14):1659-74.

[115] Min J, Kim JH, Choi KH, Yoon HH, Jeon SR. Is there additive therapeutic effect when GCSF combined with adipose-derived stem cell in a rat model of acute spinal cord injury? J Korean Neurosurg Soc 2017;60(4):404-16.

[116] Ohta Y, Hamaguchi A, Ootaki M, Watanabe M, Takeba Y, Iiri T, Matsumoto N, Takenaga M. Intravenous infusion of adipose-derived stem/stromal cells improves functional recovery of rats with spinal cord injury. Cytotherapy 2017;19(7):839-48.

[117] Zhou Z, Chen Y, Zhang H, Min S, Yu B, He B, Jin A. Comparison of mesenchymal stromal cells from human bone marrow and adipose tissue for the treatment of spinal cord injury. Cytotherapy 2013;15(4):434-48.

[118] Menezes K, Nascimento MA, Goncalves JP, Cruz AS, Lopes DV, Curzio B, Bonamino M, de Menezes JR, Borojevic R, Rossi MI, et al. Human mesenchymal cells from adipose tissue deposit laminin and promote regeneration of injured spinal cord in rats. PLoS One 2014;9(5):e96020.

[119] Ryu H-H, Lim J-H, Byeon Y-E, Park J-R, Seo M-S, Lee Y-W, Kim WH, Kang K-S, Kweon O-K. Functional recovery and neural differentiation after transplantation of allogenic adipose-derived stem cells in a canine model of acute spinal cord injury. J Vet Sci 2009;10(4):273-84.

[120] Kim Y, Jo S-h, Kim WH, Kweon O-K. Antioxidant and anti-inflammatory effects of intravenously injected adipose derived mesenchymal stem cells in dogs with acute spinal cord injury. Stem Cell Res Ther 2015;6(1):229.

[121] Lee SH, Kim Y, Rhew D, Kim A, Jo KR, Yoon Y, Choi KU, Jung T, Kim WH, Kweon O-K. Effect of canine mesenchymal stromal cells overexpressing heme oxygenase-1 in spinal cord injury. J Vet Sci 2017;18(3):377-86.

[122] Lee SH, Kim Y, Rhew D, Kuk M, Kim M, Kim WH, Kweon O-K. Effect of the combination of mesenchymal stromal cells and chondroitinase $\mathrm{ABC}$ on chronic spinal cord injury. Cytotherapy 2015;17(10):1374-83. 
[123] Escalhão CCM, Ramos IP, Hochman-Mendez C, Brunswick THK, Souza SAL, Gutfilen B, et al. Safety of allogeneic canine adipose tissue-derived mesenchymal stem cell intraspinal transplantation in dogs with chronic spinal cord injury. Stem Cell Int 2017;2017:11.

[124] Krueger E, Magri LMS, Botelho AS, Bach FS, Rebellato CLK, Fracaro L, Fragoso FYI, Villanova JA, Brofman PRS, Popović-Maneski L. Effects of lowintensity electrical stimulation and adipose derived stem cells transplantation on the time-domain analysis-based electromyographic signals in dogs with SCI. Neurosci Lett 2019;696:38-45.

[125] Ra JC, Shin IS, Kim SH, Kang SK, Kang BC, Lee HY, Kim YJ, Jo JY, Yoon EJ, Choi HJ, et al. Safety of intravenous infusion of human adipose tissue-derived mesenchymal stem cells in animals and humans. Stem Cell Dev 2011;20(8):1297-308.

[126] Hur JW, Cho T-H, Park D-H, Lee J-B, Park J-Y, Chung Y-G. Intrathecal transplantation of autologous adipose-derived mesenchymal stem cells for treating spinal cord injury: a human trial. J Spinal Cord Med 2016;39(6):655-64.

[127] Szekiova E, Slovinska L, Blasko J, Plsikova J, Cizkova D. The neuroprotective effect of rat adipose tissue-derived mesenchymal stem cell-conditioned medium on cortical neurons using an in vitro model of SCI inflammation. Neurol Res 2018;40(4):258-67.

[128] Ribeiro CA, Fraga JS, Grãos M, Neves NM, Reis RL, Gimble JM, Sousa N, Salgado AJ. The secretome of stem cells isolated from the adipose tissue and Wharton jelly acts differently on central nervous system derived cell populations. Stem Cell Res Ther 2012;3(3): 18 .

[129] Guillén MI, Platas J, Pérez del Caz MD, Mirabet V, Alcaraz MJ. Paracrine antiinflammatory effects of adipose tissue-derived mesenchymal stem cells in human monocytes. Front Physiol 2018;9(661).

[130] Assunção-Silva RC, Mendes-Pinheiro B, Patrício P, Behie LA, Teixeira FG, Pinto L, Salgado AJ. Exploiting the impact of the secretome of MSCs isolated from different tissue sources on neuronal differentiation and axonal growth. Biochimie 2018;155: $83-91$.

[131] Xu XM, Guenard V, Kleitman N, Bunge MB. Axonal regeneration into Schwann cellseeded guidance channels grafted into transected adult rat spinal cord. J Comp Neurol 1995;351(1):145-60.

[132] Xu XM, Guenard V, Kleitman N, Aebischer P, Bunge MB. A combination of BDNF and NT-3 promotes supraspinal axonal regeneration into Schwann cell grafts in adult rat thoracic spinal cord. Exp Neurol 1995;134(2):261-72.

[133] Xu XM, Chen A, Guenard V, Kleitman N, Bunge MB. Bridging Schwann cell transplants promote axonal regeneration from both the rostral and caudal stumps of transected adult rat spinal cord. J Neurocytol 1997;26(1):1-16.

[134] Oudega M, Xu XM. Schwann cell transplantation for repair of the adult spinal cord. J Neurotrauma 2006;23(3-4):453-67.

[135] Kromer LF, Cornbrooks CJ. Transplants of Schwann cell cultures promote axonal regeneration in the adult mammalian brain. Proc Natl Acad Sci USA 1985;82(18):6330-4.

[136] Paino CL, Bunge MB. Induction of axon growth into Schwann cell implants grafted into lesioned adult rat spinal cord. Exp Neurol 1991;114(2):254-7.

[137] Takami T, Oudega M, Bates ML, Wood PM, Kleitman N, Bunge MB. Schwann cell but not olfactory ensheathing glia transplants improve hindlimb locomotor performance in the moderately contused adult rat thoracic spinal cord. J Neurosci 2002;22(15): $6670-81$. 
[138] Barakat DJ, Gaglani SM, Neravetla SR, Sanchez AR, Andrade CM, Pressman Y, Puzis R, Garg MS, Bunge MB, Pearse DD. Survival, integration, and axon growth support of glia transplanted into the chronically contused spinal cord. Cell Transplantation 2005;14(4):225-40.

[139] Biernaskie J, Sparling JS, Liu J, Shannon CP, Plemel JR, Xie Y, Miller FD, Tetzlaff W. Skin-derived precursors generate myelinating Schwann cells that promote remyelination and functional recovery after contusion spinal cord injury. J Neurosci 2007; 27(36):9545-59.

[140] Sparling JS, Bretzner F, Biernaskie J, Assinck P, Jiang Y, Arisato H, Plunet WT, Borisoff J, Liu J, Miller FD, et al. Schwann cells generated from neonatal skinderived precursors or neonatal peripheral nerve improve functional recovery after acute transplantation into the partially injured cervical spinal cord of the rat. J Neurosci 2015;35(17):6714-30.

[141] Menei P, Montero-Menei C, Whittemore SR, Bunge RP, Bunge MB. Schwann cells genetically modified to secrete human BDNF promote enhanced axonal regrowth across transected adult rat spinal cord. Eur J Neurosci 1998;10(2):607-21.

[142] Pearse DD, Pereira FC, Marcillo AE, Bates ML, Berrocal YA, Filbin MT, Bunge MB. cAMP and Schwann cells promote axonal growth and functional recovery after spinal cord injury. Nat Med 2004;10(6):610-6.

[143] Lee YS, Funk LH, Lee JK, Bunge MB. Macrophage depletion and Schwann cell transplantation reduce cyst size after rat contusive spinal cord injury. Neural Regen Res 2018;13(4):684-91.

[144] Anderson KD, Guest JD, Dietrich WD, Bartlett Bunge M, Curiel R, Dididze M, Green BA, Khan A, Pearse DD, Saraf-Lavi E, et al. Safety of autologous human schwann cell transplantation in subacute thoracic spinal cord injury. J Neurotrauma 2017;34(21):2950-63.

[145] Gomez RM, Sanchez MY, Portela-Lomba M, Ghotme K, Barreto GE, Sierra J, Moreno-Flores MT. Cell therapy for spinal cord injury with olfactory ensheathing glia cells (OECs). Glia 2018;66(7):1267-301.

[146] Assinck P, Duncan GJ, Hilton BJ, Plemel JR, Tetzlaff W. Cell transplantation therapy for spinal cord injury. Nat Neurosci 2017;20(5):637-47.

[147] Rieger A, Deitmer JW, Lohr C. Axon-glia communication evokes calcium signaling in olfactory ensheathing cells of the developing olfactory bulb. Glia 2007;55(4):352-9.

[148] Doucette JR. The glial cells in the nerve fiber layer of the rat olfactory bulb. Anat Rec 1984;210(2):385-91.

[149] Doucette R. Glial influences on axonal growth in the primary olfactory system. Glia 1990;3(6):433-49.

[150] Ramon-Cueto A, Nieto-Sampedro M. Glial cells from adult rat olfactory bulb: immunocytochemical properties of pure cultures of ensheathing cells. Neuroscience 1992; 47(1):213-20.

[151] Ramon-Cueto A, Nieto-Sampedro M. Regeneration into the spinal cord of transected dorsal root axons is promoted by ensheathing glia transplants. Exp Neurol 1994; 127(2):232-44.

[152] Li Y, Field PM, Raisman G. Repair of adult rat corticospinal tract by transplants of olfactory ensheathing cells. Science 1997;277(5334):2000-2.

[153] Ramon-Cueto A, Plant GW, Avila J, Bunge MB. Long-distance axonal regeneration in the transected adult rat spinal cord is promoted by olfactory ensheathing glia transplants. J Neurosci 1998;18(10):3803-15. 
[154] Thornton MA, Mehta MD, Morad TT, Ingraham KL, Khankan RR, Griffis KG, Yeung AK, Zhong H, Roy RR, Edgerton VR, et al. Evidence of axon connectivity across a spinal cord transection in rats treated with epidural stimulation and motor training combined with olfactory ensheathing cell transplantation. Exp Neurol 2018; 309:119-33.

[155] Keyvan-Fouladi N, Raisman G, Li Y. Functional repair of the corticospinal tract by delayed transplantation of olfactory ensheathing cells in adult rats. J Neurosci 2003; 23(28):9428-34.

[156] Stamegna JC, Felix MS, Roux-Peyronnet J, Rossi V, Feron F, Gauthier P, Matarazzo V. Nasal OEC transplantation promotes respiratory recovery in a subchronic rat model of cervical spinal cord contusion. Exp Neurol 2011;229(1):120-31.

[157] Cao L, Liu L, Chen ZY, Wang LM, Ye JL, Qiu HY, Lu CL, He C. Olfactory ensheathing cells genetically modified to secrete GDNF to promote spinal cord repair. Brain 2004;127(Pt 3):535-49.

[158] Ramon-Cueto A, Cordero MI, Santos-Benito FF, Avila J. Functional recovery of paraplegic rats and motor axon regeneration in their spinal cords by olfactory ensheathing glia. Neuron 2000;25(2):425-35.

[159] Lopez-Vales R, Fores J, Verdu E, Navarro X. Acute and delayed transplantation of olfactory ensheathing cells promote partial recovery after complete transection of the spinal cord. Neurobiol Dis 2006;21(1):57-68.

[160] Lu J, Feron F, Mackay-Sim A, Waite PM. Olfactory ensheathing cells promote locomotor recovery after delayed transplantation into transected spinal cord. Brain 2002; 125(Pt 1):14-21.

[161] Richter MW, Fletcher PA, Liu J, Tetzlaff W, Roskams AJ. Lamina propria and olfactory bulb ensheathing cells exhibit differential integration and migration and promote differential axon sprouting in the lesioned spinal cord. J Neurosci 2005;25(46): 10700-11.

[162] Carwardine D, Prager J, Neeves J, Muir EM, Uney J, Granger N, Wong LF. Transplantation of canine olfactory ensheathing cells producing chondroitinase $\mathrm{ABC}$ promotes chondroitin sulphate proteoglycan digestion and axonal sprouting following spinal cord injury. PLoS One 2017;12(12).

[163] Imaizumi T, Lankford KL, Burton WV, Fodor WL, Kocsis JD. Xenotransplantation of transgenic pig olfactory ensheathing cells promotes axonal regeneration in rat spinal cord. Nat Biotechnol 2000;18(9):949-53.

[164] Gorrie CA, Hayward I, Cameron N, Kailainathan G, Nandapalan N, Sutharsan R, Wang J, Mackay-Sim A, Waite PME. Effects of human OEC-derived cell transplants in rodent spinal cord contusion injury. Brain Research 2010;1337:8-20.

[165] Andrews MR, Stelzner DJ. Evaluation of olfactory ensheathing and schwann cells after implantation into a dorsal injury of adult rat spinal cord. J Neurotrauma 2007; 24(11):1773-92.

[166] Lakatos A, Barnett SC, Franklin RJ. Olfactory ensheathing cells induce less host astrocyte response and chondroitin sulphate proteoglycan expression than Schwann cells following transplantation into adult CNS white matter. Exp Neurol 2003;184(1): 237-46.

[167] Pastrana E, Moreno-Flores MT, Gurzov EN, Avila J, Wandosell F, Diaz-Nido J. Genes associated with adult axon regeneration promoted by olfactory ensheathing cells: a new role for matrix metalloproteinase 2. J Neurosci 2006;26(20):5347-59. 
[168] Lima C, Pratas-Vital J, Escada P, Hasse-Ferreira A, Capucho C, Peduzzi JD. Olfactory mucosa autografts in human spinal cord injury: a pilot clinical study. J Spinal Cord Med 2006;29(3):191-203. discussion 204-196.

[169] Mackay-Sim A, Feron F, Cochrane J, Bassingthwaighte L, Bayliss C, Davies W, Fronek P, Gray C, Kerr G, Licina P, et al. Autologous olfactory ensheathing cell transplantation in human paraplegia: a 3-year clinical trial. Brain 2008;131(Pt 9):2376-86.

[170] Tabakow P, Jarmundowicz W, Czapiga B, Fortuna W, Miedzybrodzki R, Czyz M, Huber J, Szarek D, Okurowski S, Szewczyk P, et al. Transplantation of autologous olfactory ensheathing cells in complete human spinal cord injury. Cell Transplantation 2013;22(9):1591-612.

[171] Tabakow P, Raisman G, Fortuna W, Czyz M, Huber J, Li D, Szewczyk P, Okurowski S, Miedzybrodzki R, Czapiga B, et al. Functional regeneration of supraspinal connections in a patient with transected spinal cord following transplantation of bulbar olfactory ensheathing cells with peripheral nerve bridging. Cell Transplantation 2014; 23(12):1631-55. 


\title{
Non-Print Items
}

\begin{abstract}
The transplantation of cells to promote spinal cord injury regeneration presents as an interesting approach to address the condition. The idea behind the concept is to either provide the lesion site with relevant CNS populations that will substitute dying neurons, oligodendrocytes or astrocytes and help reestablishing lost connections and tissue homeostasis or to use cells from other sources that guarantee trophic support and enhance regeneration. In this way, we review the use of the most relevant cell types in spinal cord injury ranging from embryonic stem cells, neural stem cells and induced pluripotent stem cells to mesenchymal stem cells and glial cells (focusing on Schwann Cells and olfactory ensheating cells).
\end{abstract}

\section{Keywords:}

Cell transplantation, IPSCs, MSCs, Neuronal regeneration, NSCs/NPCs, Spinal cord injury. 


\section{CHAPTER}

\section{c0010 Functional properties of progenitor-like cells in the spinal cord: implications for self-repair}

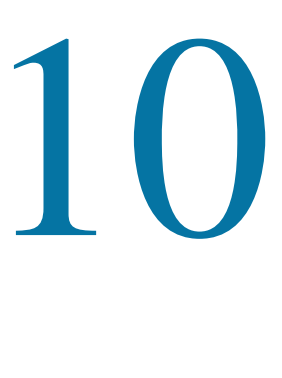

Raúl E. Russo, PhD

Professor, Departamento de Neurofisiología Celular y Molecular, Instituto de Investigaciones Clemente Estable, Montevideo, Uruguay

\section{s0010 Introduction}

p0010 The first progenitors in the neural tube are neuroepithelial cells that at the onset of neurogenesis become radial glia $(\mathrm{RG})$, the founders of most neurogenic lineages during development [1]. Both neuroepithelial cells and RG have a pronounced polarity with an apical pole bearing a single primary cilium protruding into the ventricular lumen and a distal process in contact with the pia [1,2]. Ependymal cells in the adult spinal cord have been proposed to arise from the ventral neuroepithelium [3], some originating from Olig2 progenitors from the $\mathrm{pMN}$ domain that also generates motoneurons and oligodendrocytes [4]. However, a recent study using genetic lineage tracing based on a Wnt target gene showed that a small population of ependymal cells originate from progenitors in the dorsal neural tube [5]. Although the cells lining the central canal (CC) keep the pronounced apicobasal polarity of their progenitors, they are morphologically heterogeneous with some having a cuboidal shape and few (2-4) cilia whereas others mostly located in the dorsal and ventral poles of the CC (called radial ependymocytes or tanycytes) have a long basal process that contacts the basal lamina of blood vessels or the pia matter [6-8]. Several markers related to neural stem/progenitor cells such as Sox2, CD15, CD133, nestin, vimentin, BLBP, and glial fibrillary acidic protein (GFAP) are expressed by subpopulations of cells lining the $\mathrm{CC}[7,9,10]$. It has been suggested that the true neural stem cells able to sustain several passages of neurospheres and generate astrocytes, oligodendrocytes, and neurons are GFAP RG-like cells located on the dorsal pole of the ependyma [11]. In line with this, dorsal ependymal cells derived from Wnt responsive RG yield the larger clones and may have a higher proliferative potential compared to ependymal cells in ventral domains [5].

p0015 Although ependymal cells in the mouse stop dividing about 9 weeks after birth [11], some cells in the adult CC still react to injury by proliferating and migrating toward the lesion [12-14]. Most ependyma-derived cells differentiate into astrocyte-like cells, whereas a few become oligodendrocytes that establish a close

Spinal Cord Injury (SCI) Repair Strategies. https://doi.org/10.1016/B978-0-08-102807-0.00010-7 
association with axonal sprouts $[10,15]$. Ependyma-derived cells seem to play a central role in the formation of the scar thereby limiting the extension of damage [15a] . Astrocyte-like and oligodendrocyte-like cells derived from the ependyma concentrate in the core of the scar and release growth factors that improve the survival of neurons around the lesion (Sabelström et al., 2013). Thus, the ependyma appears to generate a "good" component of the scar that should be optimized to increase functional recovery after injury. However, the contribution of progenitor-like cells in the $\mathrm{CC}$ to scarring may be related to the type of injury as demyelinating lesions do not reactivate dormant ependymal cells [16] and some compression lesions induce a minimal contribution of ependymal cells to the glial scar [17].

p0020 Despite the ependyma latent stem cell niche seems a potential resource for selfrepair, the mechanisms by which injury re-activates "dormant" progenitor-like cells in the $\mathrm{CC}$ remain virtually unknown. The cells lining the $\mathrm{CC}$ are heterogeneous from the morphological, molecular, and functional point of view. I review here different functional features that represent potential targets for the regulation of ependymal cell biology to improve the endogenous repair mechanisms after injury.

\section{s0015 Membrane properties of cells in the CC stem cell niche}

p0025 The membrane properties of the cells lining the CC have been characterized both in the spinal cord of turtles and rats $[8,18-20]$. In turtles, the electrophysiological properties of cells lying on the lateral aspects of the $\mathrm{CC}$ were similar to glial cells and progenitor cells in the developing brain with hyperpolarized resting membrane potentials, low input resistances, and dominated by leak conductances [20]. However, leak subtraction revealed a delayed a rectifier $\mathrm{K}^{+}$current $\left(\mathrm{I}_{\mathrm{KD}}\right)$ in a subpopulation of ependymal cells on the lateral CC of turtles [20]. Whereas cells in the dorsal and ventral aspects of the turtle $\mathrm{CC}$ display similar electrophysiological phenotypes than those in lateral domains, a subpopulation with morphological features of RG have a conductance that is active at resting membrane potentials and deactivates slowly when the membrane is hyperpolarized [21]. The mechanisms and functional relevance of this voltage-gated conductance remain to be explored.

p0030 The membrane properties of ependymal cells in the spinal cord of neonatal rats shared common features with those in nonmammalian vertebrates (Fig. 10.1). In the lateral domains of the $\mathrm{CC}$ of neonatal rats, most cells had passive membrane properties, low input resistances, and hyperpolarized resting membrane potentials [8]; Fig. 10.1C). However, most single ependymal cells lying on the lateral domains and having a basal process projecting away from the $\mathrm{CC}$ display a delayed rectifier current. Different from neurogenic RG in the developing mammalian cortex [22], ependymal cells with the morphological phenotype of RG located in the midline domains of the neonatal rat $\mathrm{CC}$ have complex electrophysiological properties displaying various combinations of a $\mathrm{I}_{\mathrm{KD}}$, an inactivating A-type current $\left(\mathrm{I}_{\mathrm{A}}\right)$ and/or calcium currents (Fig. 10.1A and B). The presence of $\mathrm{I}_{\mathrm{KD}}$ is a common feature among adult progenitors, as it is functionally expressed in hippocampal nestin + type 2 cells [23] and 


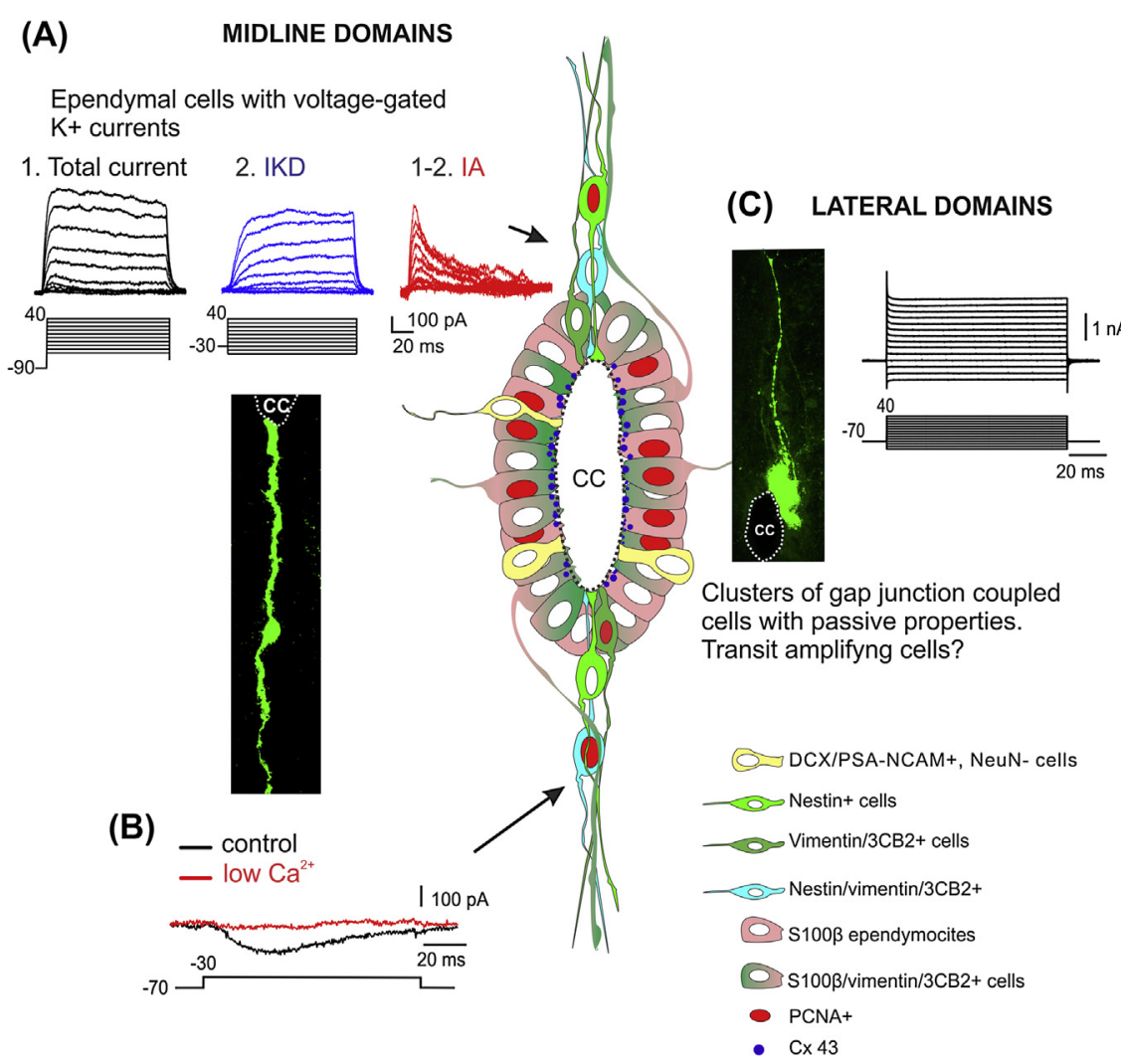

f0010 FIGURE 10.1 Cartoon Showing the Functional Organization of the Ependyma Stem Cell Niche in the Spinal Cord.

In midline domains, the membrane properties of ependymal cells are complex, with different types of voltage-gated $\mathrm{K}^{+}$currents (A). A small subpopulation also displayed a $\mathrm{Ca}^{2+}$ inward current (B). In the neonatal rat, ependymal cells on the dorsal and ventral poles of the central canal have the morphological phenotype of radial glia as shown in the image of a biocytin-filled cell on the left. In lateral domains, cells are functionally coupled via gap junctions (image on the right) and display passive membrane properties (C). Modified from Marichal N, García G, Radmilovich M, Trujillo-Cenóz O, Russo RE. Spatial domains of progenitorlike cells and functional complexity of a stem cell niche in the neonatal rat spinal cord. Stem Cells 2012;30:

2020-31.

GFAP + cells in the subventricular zone (SVZ) stem cell niche [24]. Although $\mathrm{I}_{\mathrm{A}}$ has not been reported in stem cells of the adult SVZ [24], progenitors from the embryonic [25] and neonatal [26] SVZ as well as cultured human stem cells [27] display $\mathrm{I}_{\mathrm{A}}$. Interestingly, the electrophysiological phenotype of CC-contacting cells in midline domains with conspicuous $\mathrm{I}_{\mathrm{KD}}$ and $\mathrm{I}_{\mathrm{A}}$ is remarkably similar to that of oligodendrocyte progenitors [28], raising the possibility that they are bipolar precursors committed 
to the oligodendrocyte lineage [29] during a postnatal developmental stage in which active myelination occurs. It remains to be explored whether these cells can differentiate in oligodendrocyte progenitor cells with a multipolar morphology and expression of NG2 or PDGFR $\alpha$. Finally, in a minor subpopulation of RG-like cells in midline domains of the $\mathrm{CC}$ of neonatal rats, depolarizing current pulses induced an inward calcium current $\left(\mathrm{I}_{\mathrm{Ca}}\right)$ strong enough to generate a low threshold spike [8]; (Fig. 10.1B). $\mathrm{I}_{\mathrm{Ca}}$ has been described in some floor plate cells [30], a fact that may reflect a possible origin of this type of ependymal cell in the ventral neuroepithelium. $\mathrm{Ca}^{2+}$ electrogenesis plays an important part during development by regulating events such as neural induction [31] and neuronal differentiation for the proper maturation of spinal circuits [32]. Another possibility is that RG-like ependymal cells displaying $\mathrm{I}_{\mathrm{Ca}}$ could be precursors showing the first signs of differentiation into cerebrospinal fluid-contacting neurons (CSFcNs), [18]. Because the regenerative potential of ependymal cells decline over time [33], it is mandatory in future studies to explore the membrane properties of these cells in the adult spinal cord.

p0035 The complex repertoire of $\mathrm{K}^{+}$currents in ependymal cells of the spinal cord of nonmammalian vertebrates and rodents may regulate fundamental properties of these progenitor-like cells. $\mathrm{I}_{\mathrm{KD}}$ channels are important as regulators of cell proliferation [34-36] whereas $\mathrm{I}_{\mathrm{A}}$ channels appear essential for the proliferation of multipotent neural stem cells obtained from humans [27]. It is then possible that $\mathrm{K}^{+}$ channels in ependymal cells are part of epigenetic mechanisms regulating proliferation. Furthermore, $\mathrm{I}_{\mathrm{A}}$ has been implied in the differentiation of oligodendrocyte progenitor cells [37] and rat spinal cord astrocytes [38]. It is tempting to speculate that voltage-gated $\mathrm{K}^{+}$channels will also participate in the transformation of RG to postmitotic ependymal cells in the spinal cord.

\section{s0020 Signaling via connexins (CX) in the CC stem cell niche}

p0040 Gap junction coupling among progenitor-like cells in the $\mathrm{CC}$ is a phylogenetic preserved feature $[8,20]$. In turtles - a low vertebrate with an active CC stem cell niche and remarkable self-repair capabilities - ependymal cells on the lateral aspects of the $\mathrm{CC}$ are electrically and metabolically coupled via gap junctions [20]. In contrast, CC-contacting cells in the dorsal and ventral poles of the turtle $\mathrm{CC}$ are isolated or coupled with one or two cells at the most [20]. The large cluster of cells coupled via gap junctions in the turtle match the expression of brain lipid binding protein (BLBP) and the transcription factor Pax6 and Nkx6.1 [20], a molecular signature of multipotent progenitors [39]. Both, Cx43 and Cx26, support Cx signaling in the $\mathrm{CC}$ of the turtle, resembling the $\mathrm{Cx}$ expression profile in progenitors of the developing cortex $[40,41]$. Cx43 is spatially segregated to the apical poles of progenitorlike cells on the lateral aspects of the CC suggesting that gap junction coupling relies mostly on this type of Cx [20]. In contrast, Cx26 is expressed at some distance of the $\mathrm{CC}$ lumen and both in the lateral and midline domains. The fact that the proliferation rate on the lateral aspects of the CC of the intact [20] and injured [42] turtle spinal 
cord is higher than in midline domains suggests that gap junction coupling is an important functional feature to maintain an active progenitor cell phenotype.

p0045 Ependymal cells in the neonatal spinal cord are-like their nonmammalian counterparts - coupled via gap junctions in the lateral domains of the CC whereas RGlike cells with complex electrophysiological phenotypes are uncoupled in the poles of the ependymal [8]; (Fig. 10.1C). Cx43 is also expressed in the rat CC with a spatial profile similar to that of the reptilian spinal cord. Whether Cx26 is expressed in ependymal cells of neonatal rodents is not clear and remains to be explored.

p0050 Communication of neural stem cells via $\mathrm{Cx}$ is critical for the regulation of many functions [43]. Cx play an important role in the regulation of proliferation, migration and differentiation [44], with both $\mathrm{Cx} 43$ and Cx26 expressing differentially during development $[41,45]$. Indeed, in the ventricular zone of the developing cortex gap junction blockers reduced the proliferation of progenitors suggesting $\mathrm{Cx}$ are needed for cells to enter the $\mathrm{S}$ phase [46]. The importance of $\mathrm{Cx}$ as regulators of proliferation is retained in adult neurogenic niches as the knockout of $\mathrm{Cx} 30$ and $\mathrm{Cx} 43$ in progenitors of the adult hippocampus reduce their proliferation rate leading to a decreased number of new granule cells [47]. Whether these effects are due to interference with communication via gap junction or the blockade of hemichannels is not clear. Hemichannels seem to play a central role in the generation of coordinated $\mathrm{Ca}^{2+}$ waves that synchronize the cell cycle of a group of neighboring radial glia [48]. The fact that progenitors contacting the lateral aspects of the CC both in turtles [20] and in neonatal rodents [8] have a higher proliferative rate suggests that $\mathrm{Cx}$ are also critical to support an active $\mathrm{CC}$ stem cell niche. The mechanisms by which $\mathrm{Cx}$ may regulate the behavior of progenitor cells and their progeny are not clear. Strong metabolic coupling of spinal precursors at their apical poles may allow an efficient communication of molecules that regulate key aspects of their biology, such as mitotic activity or differentiation [44]. Gap junctions may also provide genetic communication among neighboring progenitor cells as they allow the passage of microRNA (miRNA), being Cx43 more efficient than $\mathrm{Cx} 26$ or $\mathrm{Cx} 30$ [49]. Finally, $\mathrm{Cx} 43$ and $\mathrm{Cx} 26$ may also affect the proliferation, migration, and differentiation of spinal precursors by nonconventional mechanisms via molecular interactions with a variety of proteins [50]. Interestingly, the role of $\mathrm{Cx}$ in neuronal migration during development in the cortical plate is actually mediated by facilitation of cell-cell adhesion and not by their functions as channels [51]. Although gap junctions are not needed for the tangential migration of inhibitory interneurons, $\mathrm{Cx} 43$ plays a role in the switch from tangential to radial migration, an action mediated by the $\mathrm{C}$ terminus of $\mathrm{Cx} 43$ [52].

p0055 The available information raises the possibility that gap junction communication and $\mathrm{Cx}$ hemichannels may be main regulators of the biology of ependymal cells in the intact and injured spinal cord. Interestingly, Cx43 mRNA increases as early as $4 \mathrm{~h}$ after complete transection of the rat spinal cord and remains elevated at least 4 weeks after injury [53]. The role played by $\mathrm{Cx} 43$ in response to injury of the nervous system is controversial, with some reports suggesting that increased levels are detrimental $[54,55]$ whereas others claim that $\mathrm{Cx} 43$ downregulation results in an expansion in the lesion size [56,57]. The interference with $\mathrm{Cx} 43$ function after spinal cord injury 
(SCI) reduces the reactive astrogliosis and neuronal death [58] and improves functional recovery [59]. However, whether $\mathrm{Cx}$ hemichannels and gap junction coupling in the $\mathrm{CC}$ stem cell niche are regulated by injury remains to be experimentally tested. Interestingly, both $\mathrm{Cx} 43$ and $\mathrm{Cx} 26$ express in the skin and have a major role in wound healing [60]. In epithelia, the wound-induced changes of Cx26 and Cx43 expression occur in a complex spatiotemporal manner. Indeed, in the skin of mice and humans, Cx43 and Cx26 are initially downregulated, to later increase in epidermal cells close to the wound while remaining in a downregulated state at the edges of the lesion [60-62]. Cx26 remains highly expressed in later stages of skin wound healing [60]. The use of antisense technology to interfere with Cx43 function shows that inhibition of Cx43 speeds up wound closure [63] probably by facilitating keratinocyte proliferation and transformation into a migratory phenotype [60]. Interestingly, blockade of Cx43 hemichannels regulates the expression of several wound healingassociated genes in human gingival fibroblasts that may promote faster and scarless wound healing [64]. It is tempting to speculate that similar to wound healing in other tissues, Cx play a major part in the "awakening" of dormant progenitor-like cells in the ependyma, potentially regulating their proliferation and migration toward the injury site. Understanding the role of gap junction coupling and $\mathrm{Cx}$ hemichannels in the normal and injury-activated CC may provide valuable clues to improve the contribution of this stem cell niche to self-repair.

\section{s0025 Regulation of the CC stem cell niche by neurotransmitters}

p0060 Stem cells niches are homeostatically regulated by the environment. In neurogenic niches of the adult brain, progenitors are controlled by factors such as age and activity [65], hormones [66], and neurotransmitters [67]. The decline in neurogenesis with age in the mammalian brain seems related with a reduction in the activity of progenitor cells by different mechanisms $[68,69]$. Similarly, the mitotic activity of spinal ependymal cells gradually declines as postnatal development proceeds to become halted in the adult spinal cord $[11,70]$. The molecular and cellular mechanisms by which proliferation stops during early postnatal life in the $\mathrm{CC}$ stem cell niche remain unknown.

p0065 In the embryo, the behavior of progenitors and newborn cells is regulated by the activity of developing neural circuits via the influence of different neurotransmitter systems [71,72]. For example, in adult stem cell niches of the brain progenitor cells have been shown to be regulated by $\gamma$-amino butyric acid (GABA), glutamate, acetylcholine, dopamine, serotonin, and nitric oxide [67]. GABA released from newborn neurons in the SVZ inhibits the proliferation of neighboring progenitors thereby implementing a feedback control system to adjust neurogenesis to functional demands $[24,73,74]$. Less is known about the regulation by neurotransmitter signaling in spinal stem cell niches but neurotransmitters produced by cellular components of the niche or by surrounding axonal fibers may also modulate the behavior of CC-contacting progenitors [21,75-77]. Both in nonmammalian vertebrates and mammals, ependymal cells are surrounded by CSFcNs that have the molecular 
signature of immature neurons (expression of DCX and PSA-NCAM) and synthetize GABA $[21,78,79]$. In turtles, abundant GABAergic terminals spread over the CC $[21,80]$ making functional synaptic contacts with CSFcNs [19] thereby suggesting that there is an active GABAergic signaling system in this stem cell niche. Indeed, functional studies in turtles [21] showed that GABA generates currents with components mediated by GABA transporters (GAT) and $\mathrm{GABA}_{\mathrm{A}}$ receptors in clusters of gap junction coupled BLBP + progenitors on the lateral domains of the CC. Uncoupling $\mathrm{BLBP}+$ progenitors with carbenoxolone suggests that individual progenitors react differently to GABA with various combinations of GABA transporter- and ionic-induced currents. GABA also depolarizes ependymal cells of the spinal cord of juvenile rats [76], suggesting that GABAergic signaling on CC-contacting progenitors is a phylogenetic conserved trait.

p0070 CSFcNs intermingled with ependymal cells also have functional $\mathrm{GABA}_{\mathrm{A}}$ receptors $[18,19,21]$. $\mathrm{GABA}_{\mathrm{A}}$ receptor activation generates responses ranging from depolarizations that may in some cases be strong enough to elicit spike firing to hyperpolarizations from rest [18,21]. GABA-induced depolarization in neurons is a distinctive functional feature in the developing brain [71,81] and migrating neuroblasts of the SVZ and rostral migratory stream [82]. Both in the SVZ and the dentate gyrus, GABA provides an excitatory drive to newborn neurons because of high intracellular concentration of $\mathrm{Cl}^{-}$resulting from a dominant activity of NKCC1 [83]. When neurons complete their differentiation, the action of GABA switches from excitation to inhibition because of the downregulation of NKCC1 and/or increase of $\mathrm{KCC} 2$ activity that leads to a reduction of intracellular $\mathrm{Cl}^{-}[84,85]$. Similarly, the excitatory action of GABA in CSFcNs stems from a depolarized equilibrium potential for $\mathrm{Cl}^{-}$because of a predominance of NKCC1 over KCC2 [21]. Cells hyperpolarized by GABA generally spiked repetitively-an electrophysiological phenotype of more differentiated neurons [86,87] — and have measurable KCC2 activity, which is in line with the idea that the turtle $\mathrm{CC}$ is a neurogenic stem cell niche similar to those of the adult mammalian brain. $\mathrm{GABA}_{\mathrm{A}}$ receptor activation induces an increase in intracellular $\mathrm{Ca}^{2+}$ that requires extracellular $\mathrm{Ca}^{2+}$, in both progenitors and immature neurons of the turtle CC [21]. These studies show that GABAergic signaling around the $\mathrm{CC}$ shares fundamental properties with those in the embryo and adult neurogenic niches, suggesting that GABA may be part of the mechanisms regulating the $\mathrm{CC}$ stem cell niche. Future research should be directed to unveil the source of ambient GABA around the $\mathrm{CC}$ and the functional role of GABAergic signaling on CC-contacting progenitors and CSFcNs.

p0075 Receptors gated by ATP may be important to regulate the properties of spinal progenitors, particularly in the context of SCI as ATP levels around the lesion epicenter are increased for several hours after a lesion [88]. Purinergic signaling plays a central role during development by regulating processes such as progenitor cell proliferation, migration, differentiation, and synapse formation [89]. During cortical development, ATP released through $\mathrm{Cx}$ hemichannels in RG activates P2Y1 receptors generating $\mathrm{Ca}^{2+}$ waves that propagate among neighboring $\mathrm{RG}$ [48]. These $\mathrm{Ca}^{2+}$ waves regulate the mitotic activity of $\mathrm{RG}$ and may allow the 
synchronization of the cell cycle of a cohort of progenitors [48]. Purinergic signaling participates in the expansion of the ventricular zone stem cell niche [90] and initiates important events as transient release of ATP triggers the development of the eye by inducing the expression of Pax6 via P2Y1 receptor activation [91]. Other nucleotides may also regulate adult neurogenesis, as the ATP hydrolyzing ectonucleotidase NTPDase2 is a characteristic of adult neurogenic niches [92].

p0080 In the context of SCI, ATP may act as a diffusible "danger signal" alerting about damage [93]. Purinergic signaling has been also implied in the secondary expansion of tissue damage after injury $[88,94]$. The P2X7 receptor merits particular attention in relation to injury because they have a rather low sensitivity (activation at $100 \mu \mathrm{M}$ to $3 \mathrm{mM}$ of ATP) [95], and may activate significantly only after a lesion. The other peculiar feature of $\mathrm{P} 2 \mathrm{X} 7$ receptors is that they are highly permeable to $\mathrm{Ca}^{2+}$ and when activated for several seconds become permeable to the large cation $\mathrm{N}$ methyl-D-glucamine $[96,97]$. Ependymal cells on both the medial and lateral domains of the $\mathrm{CC}$ of neonatal rats have functional $\mathrm{P} 2 \mathrm{X} 7$ receptors [77]. The activation of P2X7 receptors by ATP or its selective agonist BzATP generates a slow inward current and $\mathrm{a} \mathrm{Ca}^{2+}$ wave that propagates bidirectionally from the site of ATP application (Fig. 10.2A and B). It is possible that P2X7 receptors may be a key component of the response of the ependymal stem cell niche to SCI. For some ependymal cells with distal processes surrounding injured tissue, $\mathrm{P} 2 \mathrm{X} 7$ receptors activated by increased extracellular ATP will generate a $\mathrm{Ca}^{2+}$ wave propagating toward the $\mathrm{CC}$, generating a local $\mathrm{Ca}^{2+}$ increase in key cellular compartments such as the nucleus and the apical process of $\mathrm{CC}$-contacting progenitors. $\mathrm{Ca}^{2+}$ transients may modulate nuclear gene expression, activating or repressing function-specific transcription factors that may affect events such as proliferation, differentiation, and migration [98,99] of ependymal cells (Fig. 10.2C). For example, interference of $\mathrm{Ca}^{2+}$ signaling by blockade or genetic knockdown of purinergic receptors impairs the migration of intermediate neuronal progenitors to the SVZ [100]. $\mathrm{Ca}^{2+}$ waves propagated to or generated at the apical pole of CC-contacting RG may generate changes in this cellular compartment with major functional consequences (Fig. 10.2C). Whether the increase in $\mathrm{Ca}^{2+}$ induced by $\mathrm{P} 2 \mathrm{X} 7$ receptors results beneficial for repair or is detrimental by activating caspases inducing apoptosis of ependymal cells [101] is a key problem to tackle in future research.

p0085 Acetylcholine (Ach) is an additional transmitter that may have a functional role in the ependymal stem cell niche. The $\mathrm{CC}$ of rats is surrounded by choline acetyltransferase (ChaT) fibers and Ach depolarizes ependymal cells and CSFcNs via activation of $\alpha 7$ - and non- $\alpha 7$ nicotinic Ach receptors [75]. Furthermore, activation of nicotinic receptors increases proliferation of ependymal cells both in vitro and in vivo [75]. This study suggests that cholinergic receptors may be part of the mechanisms by which the microenvironment of the injured cord signals upon ependymal cells to leave the normal quiescent state. In line with this possibility, inflammation in a model of autoimmune encephalomyelitis decreases the rate of proliferation of ependymal cells, an effect that can be counteracted by administration of nicotine [102]. 

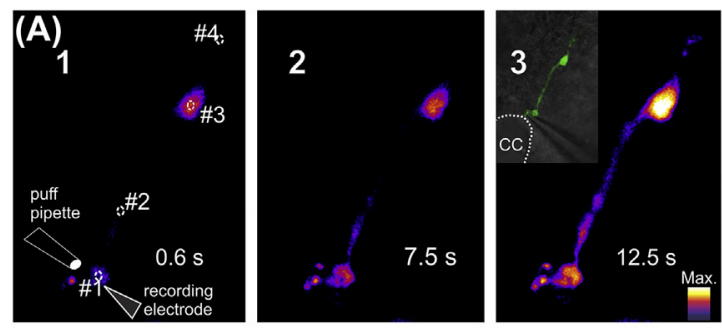

(B)

(C)

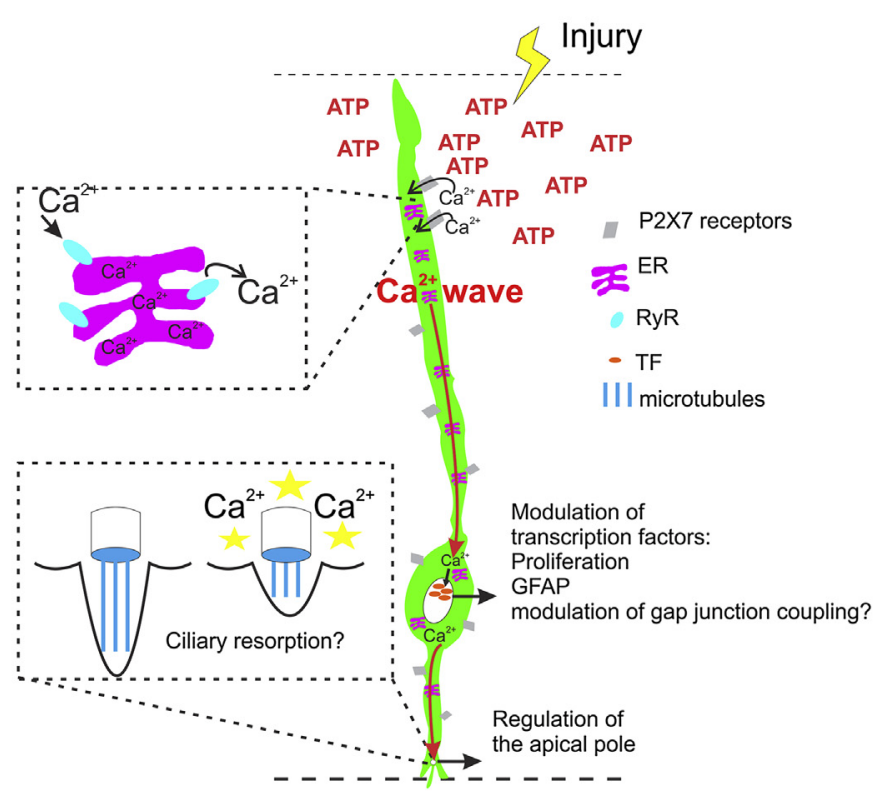

f0015 FIGURE 10.2 Purinergic Signaling in the Ependyma Stem Cell Niche.

(A) The application of BzATP (a selective P2X7 receptor agonist) on the end foot of a progenitor-like cell contacting the central canal (CC) induced a $\mathrm{Ca}^{2+}$ wave that started in the end foot $(1,2)$ propagated away from the CC lumen (3). \#1-4 in panel A1 indicate regions of interest (ROI) for the analysis of fluorescence. (B) Time course of the $\mathrm{Ca}^{2+}$ wave in different ROIs ( $\Delta \mathrm{F} / \mathrm{Fo}$ ) after transient application of BzATP. (C) Cartoon showing the possible role of purinergic signaling in the reactivation of the ependyma stem cell niche after spinal cord injury.

Modified from Marichal N, Fabbiani G, Trujillo-Cenóz O, Russo RE. Purinergic signalling in a latent stem cell niche of the rat spinal cord. Purinergic Signal 2016;12:331-41.

\section{s0030 Concluding remarks}

p0090 It is now clear that the CC is a complex structure composed of cells with heterogeneous properties. Some of these cells display characteristics of progenitors and may have retained some of the programs of the primitive neural stem cells to generate 
the new cells needed for repair. In nonmammalian vertebrates, the $\mathrm{CC}$ reacts to injury and orchestrates self-repair mechanisms that lead to functional recovery [103,104]. Whereas it is clear now that ependymal cells in the spinal cord of mammals also react to injury and contribute to limit the extension of the lesion, it is obvious that this reaction is not sufficient to restore function satisfactorily. To unleash the full power of the CC stem cell niche for repair, we need to better comprehend the biology of ependymal cells and how they are regulated by intrinsic mechanisms and microenvironmental signals. As described here, the complexity and heterogeneity of various functional properties of ependymal cells (e.g.,: voltage-gated $\mathrm{K}^{+}$channels, gap junction coupling and $\mathrm{Cx}$ hemichannels, neurotransmitter signaling) are just starting to emerge and represent potential opportunities to manipulate the CC stem cell niche for increased regeneration and meaningful functional recovery.

\section{References}

[1] Kriegstein A, Alvarez-Buylla A. The glial nature of embryonic and adult neural stem cells. Annu Rev Neurosci 2009;32:149-84.

[2] Alvarez-Buylla A, García-Verdugo JM, Tramontin AD. A unified hypothesis on the lineage of neural stem cells. Nat Rev Neurosci 2001;2:287-93.

[3] Fu HUI, Qi Y, Tan MIN, Cai JUN, Hu X, Liu Z, Qiu M. Molecular mapping of the origin of postnatal spinal cord ependymal cells: evidence that adult ependymal cells are derived from Nkx6.1 + ventral neural progenitor cells. J Comp Neurol 2003; 456:237-44.

[4] Masahira N, Takebayashi H, Ono K, Watanabe K, Ding L, Furusho M, Ogawa Y, Nabeshima Y, Alvarez-Buylla A, Shimizu K, Ikenaka K. Olig2-positive progenitors in the embryonic spinal cord give rise not only to motoneurons and oligodendrocytes, but also to a subset of astrocytes and ependymal cells. Dev Biol 2006;293:358-69.

[5] Xing L, Anbarchian T, Tsai JM, Plant GW, Nusse R. Wnt/ $\beta$-catenin signaling regulates ependymal cell development and adult homeostasis. Proc Natl Acad Sci U S A 2018; 115:E5954-62.

[6] Alfaro-Cervello C, Soriano-Navarro M, Mirzadeh Z, Alvarez-Buylla A, GarciaVerdugo JM. Biciliated ependymal cell proliferation contributes to spinal cord growth. J Comp Neurol 2012;520:3528-52.

[7] Hugnot JP, Franzen R. The spinal cord ependymal region: a stem cell niche in the caudal central nervous system. Front Biosci 2011;16:1044-59.

[8] Marichal N, García G, Radmilovich M, Trujillo-Cenóz O, Russo RE. Spatial domains of progenitor-like cells and functional complexity of a stem cell niche in the neonatal rat spinal cord. Stem Cells 2012;30:2020-31.

[9] Hamilton LK, Truong MK, Bednarczyk MR, Aumont A, Fernandes KJ. Cellular organization of the central canal ependymal zone, a niche of latent neural stem cells in the adult mammalian spinal cord. Neuroscience 2009;164:1044-56.

[10] Meletis K, Barnabé-Heider F, Carlén M, Evergren E, Tomilin N, Shupliakov O, Frisén J. Spinal cord injury reveals multilineage differentiation of ependymal cells. PLoS Biol 2008;6:1494-507.

[11] Sabourin JC, Ackema KB, Ohayon D, Guichet PO, Perrin FE, Garces A, Ripoll C, Charite J, Simonneu L, Ketenmann H, Zine A, Pivat A, Valmier J, Pattyn A, 
Hugnot JP. A mesenchymal-like ZEB1+ niche harbors dorsal radial glial fibrillary acidic protein-positive stem cells in the spinal cord. Stem Cells 2009;27:2722-33.

[12] Beattie MS, Bresnahan JC, Komon J, Tovar CA, Van Meter M, Anderson DK, Faden AI, Hsu CY, Noble LJ, Salzman S, Young W. Endogenous repair after spinal cord contusion injuries in the rat. Exp Neurol 1997;148:453-63.

[13] Johansson CB, Momma S, Clarke DL, Risling M, Lendahl U, Frisén J. Identification of a neural stem cell in the adult mammalian central nervous system. Cell 1999;96: $25-34$.

[14] Mothe AJ, Tator CH. Proliferation, migration, and differentiation of endogenous ependymal region stem/progenitor cells following minimal spinal cord injury in the adult rat. Neuroscience 2005;131:177-87.

[15] Barnabé-Heider F, Göritz C, Sabelström H, Takebayashi H, Pfrieger FW, Meletis K, Frisén J. Origin of new glial cells in intact and injured adult spinal cord. Cell Stem Cell 2010;7:470-82.

[15a] Sabelström H, Stenudd M, Réu P, Dias DO, Elfineh M, Zdunek S, Damberg P, Göritz C, Frisén J. Resident neural stem cells restrict tissue damage and neuronal loss after spinal cord injury in mice. Science 2013;342:637-40.

[16] Lacroix S, Hamilton LK, Vaugeois A, Beaudoin S, Breault-Dugas C, Pineau I, Lévesque SA, Grégoire CA, Fernandes KJ. Central canal ependymal cells proliferate extensively in response to traumatic spinal cord injury but not demyelinating lesions. PLoS One 2014;9:e85916.

[17] Ren Y, Ao Y, O'Shea TM, Burda JE, Bernstein AM, Brumm AJ, Muthusamy N, Ghashghaei HT, Carmichael ST, Cheng L, Sofroniew MV. Ependymal cell contribution to scar formation after spinal cord injury is minimal, local and dependent on direct ependymal injury. Sci Rep 2017;7:41122.

[18] Marichal N, García G, Radmilovich M, Trujillo-Cenóz O, Russo RE. Enigmatic central canal contacting cells: immature neurons in "standby mode"? J Neurosci 2009;29: 10010-24.

[19] Russo RE, Fernández A, Reali C, Radmilovich M, Trujillo-Cenóz O. Functional and molecular clues reveal precursor-like cells and immature neurones in the turtle spinal cord. J Physiol 2004;3:831-8.

[20] Russo RE, Reali C, Radmilovich M, Fernández A, Trujillo-Cenóz O. Connexin 43 define functional domains of neurogenic precursors in the spinal cord. J Neurosci 2008;28:3298-309.

[21] Reali C, Fernández A, Radmilovich M, Trujillo-Cenóz O, Russo RE. GABAergic signalling in a neurogenic niche of the turtle spinal cord. J Physiol 2011;589:5633-47.

[22] Noctor SC, Flint AC, Weissman TA, Wong WS, Clinton BK, Kriegstein AR. Dividing precursor cells of the embryonic cortical ventricular zone have morphological and molecular characteristics of radial glia. J Neurosci 2002;22:3161-73.

[23] Filippov V, Kronenberg G, Pivneva T, et al. Subpopulation of nestin-expressing progenitor cells in the adult murine hippocampus shows electrophysiological and morphological characteristics of astrocytes. Mol Cell Neurosci 2003;23:373-82.

[24] Liu X, Bolteus AJ, Balkin DM, Henschel O, Bordey A. GFAP-expressing cells in the postnatal subventricular zone display a unique glial phenotype intermediate between radial glia and astrocytes. Glia 2006;54:394-410.

[25] Smith DO, Rosenheimer JL, Kalil RE. Delayed rectifier and A-type potassium channels associated with Kv 2.1 and $\mathrm{Kv} 4.3$ expression in embryonic rat neural progenitor cells. PLoS One 2008;3:e1604. 
[26] Stewart RR, Zigova T, Luskin MB. Potassium currents in precursor cells isolated from the anterior subventricular zone of the neonatal rat forebrain. J Neurophysiol 1999;81: 95-102.

[27] Schaarschmidt G, Wegner F, Schwarz SC, Schmidt H, Schwarz J. Characterization of voltage-gated potassium channels in human neural progenitor cells. PLoS One 2009;4: e6168. https://doi.org/10.1371/journal.pone.0006168.

[28] Chittajallu R, Aguirre A, Gallo V. NG2-positive cells in the mouse white and grey matter display distinct physiological properties. J Physiol 2004;561:109-22.

[29] Levine JM, Reynolds R, Fawcett JW. The oligodendrocyte precursor cell in health and disease. Trends Neurosci 2001;24:39-47.

[30] Frischknecht F, Randall AD. Voltage- and ligand-gated ion channels in floor plate neuroepithelia of the rat. Neuroscience 1998;85:1135-49.

[31] Webb SE, Moreau M, Leclerc C, Miller AL. Calcium transients and neural induction in vertebrates. Cell Calcium 2005;37:375-85.

[32] Spitzer NC, Root CM, Borodinsky LN. Orchestrating neuronal differentiation: patterns of Ca2+ spikes specify transmitter choice. Trends Neurosci 2004;27:415-21.

[33] Li X, Floriddia EM, Toskas K, Fernandes KJL, Guérout N, Barnabé-Heider F. Regenerative potential of ependymal cells for spinal cord injuries over time. EBioMedicine 2016;13:55-65.

[34] Chittajallu R, Chen Y, Wang H, Yuan X, Ghiani CA, Heckman T, McBain CJ, Gallo V. Regulation of Kv1 subunit expression in oligodendrocyte progenitor cells and their role in G1/S phase progression of the cell cycle. Proc Natl Acad Sci 2002;99:2350-5.

[35] Ghiani CA, Yuan X, Eisen AM, Knutson PL, DePinho RA, McBain CJ, Gallo V. Voltage-activated $\mathrm{K}+$ channels and membrane depolarization regulate accumulation of the cyclin-dependent kinase inhibitors p27(Kip1) and p21(CIP1) in glial progenitor cells. J Neurosci 1999;19:5380-92.

[36] MacFarlane SN, Sontheimer H. Changes in ion channel expression accompany cell cycle progression of spinal cord astrocytes. Glia 2000a;30:39-48.

[37] Sontheimer H, Trotter J, Schachner M, Kettenmann H. Channel expression correlates with differentiation stage during the development of oligodendrocytes from their precursor cells in culture. Neuron 1989;2:1135-45.

[38] MacFarlane SN, Sontheimer H. Modulation of Kv1.5 currents by Src tyrosine phosphorylation: potential role in the differentiation of astrocytes. J Neurosci 2000b;20:5245-53.

[39] Pinto L, Götz M. Radial glial cell heterogeneity-the source of diverse progeny in the CNS. Prog Neurobiol 2007;83:2-23.

[40] Dermietzel R, Traub O, Hwang TK, Beyer E, Bennett MV, Spray DC, Willecke K. Differential expression of three gap junction proteins in developing and mature brain tissues. Proc Natl Acad Sci 1989;86:10148-52.

[41] Nadarajah B, Jones AM, Evans WH, Parnavelas JG, et al. Differential expression of connexins during neocortical development and neuronal circuit formation. J Neurosci 1997;17:3096-111.

[42] Rehermann MI, Marichal N, Russo R, Trujillo-Cenoz O. Neural Reconnection in the transected spinal cord of the freshwater turtle Trachemys dorbignyi. J Comp Neurol 2009;515:197-214.

[43] Elias LA, Kriegstein AR. Gap junctions: multifaceted regulators of embryonic cortical development. Trends Neurosci 2008;31:243-50.

[44] Bruzzone R, Dermietzel R. Structure and function of gap junctions in the developing brain. Cell Tissue Res 2006;326:239-48. 
[45] Bittman KS, LoTurco JJ. Differential regulation of connexin 26 and 43 in murine neocortical precursors. Cerebr Cortex 1999;9:188-95.

[46] Bittman K, Owens DF, Kriegstein AR, LoTurco JJ. Cell coupling and uncoupling in the ventricular zone of developing neocortex. J Neurosci 1997;17:7037-44.

[47] Kunze A, Congreso MR, Hartmann C, Wallraff-Beck A, Hüttmann K, Bedner P, Requardt R, Seifert G, Redecker C, Willecke K, Hofmann A, Pfeifer A, Theis M, Steinhäuser C. Connexin expression by radial glia-like cells is required for neurogenesis in the adult dentate gyrus. Proc Natl Acad Sci U S A 2009;106:11336-41.

[48] Weissman TA, Riquelme PA, Ivic L, Flint AC, Kriegstein AR. Calcium waves propagate through radial glial cells and modulate proliferation in the developing neocortex. Neuron 2004;43:647-61.

[49] Zong L, Zhu Y, Liang R, Zhao HB. Gap junction mediated miRNA intercellular transfer and gene regulation: a novel mechanism for intercellular genetic communication. Sci Rep 2016;6:19884.

[50] Kardami E, Dang X, Iacobas DA, Nickel BE, Jeyaraman M, Srisakuldee W, Makazan J, Tanguy S, Spray DC. The role of connexins in controlling cell growth and gene expression. Prog Biophys Mol Biol 2007;94:245-64.

[51] Elias LA, Wang DD, Kriegstein AR. Gap junction adhesion is necessary for radial migration in the neocortex. Nature 2007;448:901-7.

[52] Elias LA, Turmaine M, Parnavelas JG, Kriegstein AR. Connexin 43 mediates the tangential to radial migratory switch in ventrally derived cortical interneurons. J Neurosci 2010;30:7072-7.

[53] Lee IH, Lindqvist E, Kiehn O, Widenfalk J, Olson L. Glial and neuronal connexin expression patterns in the rat spinal cord during development and following injury. J Comp Neurol 2005;489:1-10.

[54] Frantseva MV, Kokarovtseva L, Naus CG, Carlen PL, MacFabe D, Perez Velazquez JL. Specific gap junctions enhance the neuronal vulnerability to brain traumatic injury. J Neurosci 2002a;22:644-53.

[55] Frantseva MV, Kokarovtseva L, Perez Velazquez JL. Ischemia-induced brain damage depends on specific gap-junctional coupling. J Cereb Blood Flow Metab 2002b;22: 453-62.

[56] Nakase T, Söhl G, Theis M, Willecke K, Naus CC. Increased apoptosis and inflammation after focal brain ischemia in mice lacking connexin43 in astrocytes. Am J Pathol 2004; 164:2067-75.

[57] Siushansian R, Bechberger JF, Cechetto DF, Hachinski VC, Naus CC. Connexin43 null mutation increases infarct size after stroke. J Comp Neurol 2001;440:387-94.

[58] O'Carroll SJ, Alkadhi M, Nicholson LF, Green CR. Connexin 43 mimetic peptides reduce swelling, astrogliosis, and neuronal cell death after spinal cord injury. Cell Commun Adhes 2008;15:27-42.

[59] Cronin M, Anderson PN, Cook JE, Green CR, Becker DL. Blocking connexin43 expression reduces inflammation and improves functional recovery after spinal cord injury. Mol Cell Neurosci 2008;39:152-60.

[60] Wong P, Tan T, Chan C, Laxton V, Chan YWF, Liu T, Wong WT, Tse G. The role of connexins in wound healing and repair: novel therapeutic approaches. Front Physiol 2016;7:596. https://doi.org/10.3389/fphys.2016.00596.

[61] Becker DL, Thrasivoulou C, Phillips AR. Connexins in wound healing; perspectives in diabetic patients. Biochim Biophys Acta 2012;1818:2068-75. 
[62] Coutinho P, Qiu C, Frank S, Wang CM, Brown T, Green CR, Becker DL. Limiting burn extension by transient inhibition of Connexin 43 expression at the site of injury. Br J Plast Surg 2005;58:658-67.

[63] Kretz M, Euwens C, Hombach S, Eckardt D, Teubner B, Traub O, Willecke K, Ott T. Altered connexin expression and wound healing in the epidermis of connexin-deficient mice. J Cell Sci 2003;116:3443-52.

[64] Tarzemany R, Jiang G, Jiang JX, Larjava H, Häkkinen L. Connexin 43 hemichannels regulate the expression of wound healing-associated genes in human gingival fibroblasts. Sci Rep 2017;7:14157.

[65] Kempermann G. Activity dependency and aging in the regulation of adult neurogenesis. In: Gage FH, Kempermann G, Song H, editors. Adult neurogenesis cold spring. New York: Harbor Laboratory Press; 2008. p. 341-62.

[66] Lucassen PJ, Oomen CA, van Dam AM, Czéh B. Regulation of hippocampal neurogenesis by systemic factors including stress, glucocorticoids, sleep and inflammation. In: Gage FH, Kempermann G, Song H, editors. Adult neurogenesis. New York: Cold Spring Harbor Laboratory Press; 2008. p. 363-96.

[67] Jang MH, Song H, Ming GL. Regulation of adult neurogenesis by neurotransmitters. In: Gage FH, Kempermann G, Song H, editors. Adult neurogenesis. New York: Cold Spring Harbor Laboratory Press; 2008. p. 397-421.

[68] Hattiangady B, Shetty AK. Aging does not alter the number or phenotype of putative stem/progenitor cells in the neurogenic region of the hippocampus. Neurobiol Aging 2008;29:129-47.

[69] Molofsky AV, Slutsky SG, Joseph NM, He S, Pardal R, Krishnamurthy J, Sharpless NE, Morrison SJ. Increasing p16INK4a expression decreases forebrain progenitors and neurogenesis during ageing. Nature 2006;443:448-52.

[70] Alexovič Matiašová A, Ševc J, Tomori Z, Gombalová Z, Gedrová Š, Daxnerová Z. Quantitative analyses of cellularity and proliferative activity reveals the dynamics of the central canal lining during postnatal development of the rat. J Comp Neurol 2017;525:693-707.

[71] Ben-Ari Y, Spitzer NC. Phenotypic checkpoints regulate neuronal development. Trends Neurosci 2010;33:485-92.

[72] Wang DD, Kriegstein AR. Defining the role of GABA in cortical development. J Physiol 2009;587:1873-9.

[73] Haydar TF, Wang F, Schwartz ML, Rakic P. Differential modulation of proliferation in the neocortical ventricular and subventricular zones. J Neurosci 2000;20:5764-74.

[74] LoTurco JJ, Owens DF, Heath MJ, Davis MB, Kriegstein AR. GABA and glutamate depolarize cortical progenitor cells and inhibit DNA synthesis. Neuron 1995;15: 1287-98.

[75] Corns LF, Atkinson L, Daniel J, et al. Cholinergic enhancement of cell proliferation in the postnatal neurogenic niche of the mammalian spinal cord. Stem Cells 2015;33: 2864-76.

[76] Corns LF, Deuchars J, Deuchars SA. GABAergic responses of mammalian ependymal cells in the central canal neurogenic niche of the postnatal spinal cord. Neurosci Lett 2013;553:57-62.

[77] Marichal N, Fabbiani G, Trujillo-Cenóz O, Russo RE. Purinergic signalling in a latent stem cell niche of the rat spinal cord. Purinergic Signal 2016;12:331-41. 
[78] Roberts BL, Maslam S, Scholten G, Smit W. Dopaminergic and GABAergic cerebrospinal fluid-contacting neurons along the central canal of the spinal cord of the eel and trout. J Comp Neurol 1995;354:423-37.

[79] Stoeckel ME, Uhl-Bronner S, Hugel S, Hugel S, Veinante P, Klein MJ, Mutterer J, Freund-Mercier MJ, Schlichter R. Cerebrospinal fluid-contacting neurons in the rat spinal cord, a gamma-aminobutyric acidergic system expressing the P2X2 subunit of purinergic receptors, PSA-NCAM, GAP-43 immunoreactivities: light and electron microscopic study. J Comp Neurol 2003;457:159-74.

[80] Trujillo-Cenóz O, Fernández A, Radmilovich M, Reali C, Russo RE. Cytological organization of the central gelatinosa in the turtle spinal cord. J Comp Neurol 2007;502: 291-308.

[81] Ben-Ari Y. Excitatory actions of GABA during development: the nature of the nurture. Nat Rev Neurosci 2002;3:728-39.

[82] Wang DD, Krueger DD, Bordey A. GABA depolarizes neuronal progenitors of the postnatal subventricular zone via GABAA receptor activation. J Physiol 2003;550: 785-800.

[83] Bordey A. Enigmatic GABAergic networks in adult neurogenic zones. Brain Res Brain Res Rev 2007;53:124-34.

[84] Ganguly K, Schinder AF, Wong ST, Poo M. GABA itself promotes the developmental switch of neuronal GABAergic responses from excitation to inhibition. Cell 2001;105: 521-32.

[85] Rivera C, Voipio J, Payne JA, Ruusuvuori E, Lahtinen H, Lamsa K, Pirvola U, Saarma M, Kaila K. The K+/Cl- co-transporter KCC2 renders GABA hyperpolarizing during neuronal maturation. Nature 1999;397:251-5.

[86] Russo RE, Hounsgaard J. Dynamics of intrinsic electrophysiological properties in spinal cord neurones. Prog Biophys Mol Biol 1999;72:329-65.

[87] Spitzer NC, Vincent A, Lautermilch NJ. Differentiation of electrical excitability in motoneurons. Brain Res Bull 2000;53:547-52.

[88] Wang X, Arcuino G, Takano T, Lin J, Peng WG, Wan P, Li P, Xu Q, Liu QS, Goldman SA, Nedergaard M. P2X7 receptor inhibition improves recovery after spinal cord injury. Nat Med 2004;10:821-7.

[89] Zimmermann H. Nucleotide signaling in nervous system development. Pflógers Arch 2006;452:573-88.

[90] Lin JH, Takano T, Arcuino G, Wang X, Hu F, Darzynkiewicz Z, Nunes M, Goldman SA, Nedergaard M. Purinergic signaling regulates neural progenitor cell expansion and neurogenesis. Dev Biol 2007;302:356-66.

[91] Massé K, Bhamra S, Eason R, Dale N, Jones EA. Purine-mediated signalling triggers eye development. Nature 2007;449:1058-62.

[92] Abbracchio MP, Burnstock G, Boeynaems JM, Barnard EA, Boyer JL, Kennedy C, Knight GE, Fumagalli M, Gachet C, Jacobson KA, Weisman GA. International Union of Pharmacology. Update and subclassification of the P2Y G protein-coupled nucleotide receptors: from molecular mechanisms and pathophysiology to therapy. Pharmacol Rev 2006;58:281-341.

[93] Abbracchio MP, Burnstock G, Verkhratsky A, Zimmermann H. Purinergic signalling in the nervous system: an overview. Trends Neurosci 2009;32:19-29. 
[94] Peng W, Cotrina ML, Han X, Yu H, Bekar L, Blum L, Takano T, Tian GF, Goldman SA, Nedergaard M. Systemic administration of an antagonist of the ATPsensitive receptor P2X7 improves recovery after spinal cord injury. Proc Natl Acad Sci USA 2009;106:12489-93.

[95] Khakh BS, North RA. P2X receptors as cell-surface ATP sensors in health and disease. Nature 2006;442:527-32.

[96] Surprenant A, North RA. Signaling at purinergic P2X receptors. Annu Rev Physiol 2009;71:333-59.

[97] Surprenant A, Rassendren F, Kawashima E, North RA, Buell G. The cytolytic P2Z receptor for extracellular ATP identified as a P2X receptor (P2X7). Science 1996;272: $735-8$.

[98] Glaser T, Resende RR, Ulrich H. Implications of purinergic receptor-mediated intracellular calcium transients in neural differentiation. Cell Commun Signal 2013;11: 12. https://doi.org/10.1186/1478-811X-11-12.

[99] Miras-Portugal MT, Gomez-Villafuertes R, Gualix J, Diaz-Hernandez JI, Artalejo AR, Ortega F, Delicado EG, Perez-Sen R. Nucleotides in neuroregeneration and neuroprotection. Neuropharmacology 2015;104:243-54.

[100] Liu X, Hashimoto-Torii K, Torii M, Haydar TF, Rakic P. The role of ATP signalling in the migration of intermediate neuronal progenitors to the neocortical subventricular zone. Proc Natl Acad Sci U S A 2008;105:11802-7.

[101] Gandelman M, Peluffo H, Beckman JS, Cassina P, Barbeito L. Extracellular ATP and the P2X7 receptor in astrocyte-mediated motor neuron death: implications for amyotrophic lateral sclerosis. J Neuroinflammation 2010;7:33.

[102] Gao Z, Nissen JC, Legakis L, Tsirka SE. Nicotine modulates neurogenesis in the central canal during experimental autoimmune encephalomyelitis. Neuroscience 2015; 297:11-21.

[103] Diaz Queiroz JP, Echeverri K. Spinal cord regeneration: where fish, frogs and salamanders lead the way, can we follow? Biochem J 2013;451:353-64.

[104] Tanaka EM, Ferretti P. Considering the evolution of regeneration in the central nervous system. Nat Rev Neurosci 2009;10:713-23. 
Non-Print Items

\begin{abstract}
The ependyma of the spinal cord is a stem cell niche that reacts to spinal cord injury and contributes new cells to the glial scar limiting the extension of the lesion. The intrinsic and extrinsic mechanisms that regulate the biology of progenitor-like cells in the ependyma remain little understood. Both in nonmammalian vertebrates and mammals, the ependyma is a complex structure formed by cells with heterogeneous functional properties. The cells lining the lateral aspects of the central canal (CC) - where most of the proliferative potential seem to rest - have membrane properties dominated by leak conductances and are functionally coupled via gap junctions. In contrast, cells mostly located in the dorsal and ventral poles of the $\mathrm{CC}$ with the morphological phenotype of radial glia are functionally isolated and display voltage-gated $\mathrm{K}^{+}$ currents. In addition to these intrinsic properties, the $\mathrm{CC}$ stem cell niche is surrounded by active spinal circuits that release neurotransmitters that may influence ependymal cells. In this chapter, I review the diversity of the functional properties of ependymal cells, the possible neurotransmitter systems that may convey signals from the microenvironment, and how these functional features may regulate the behavior of the $\mathrm{CC}$ stem cell niche in the normal and injured spinal cord.
\end{abstract}

\title{
Keywords:
}

Endogenous progenitor cells, Central canal, Spinal cord injury, Self-repair, Neural plasticity, P2X7 receptors, Connexins. 


\title{
CHAPTER
}

\section{c0011 Paracrine effects for spinal cord injury regeneration

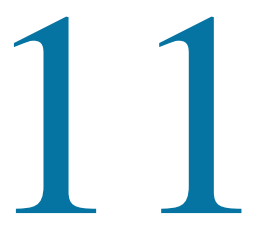

\author{
Simonetta Papa, PhD ${ }^{1}$, Irma Vismara, PhD ${ }^{1}$, Pietro Veglianese, PhD ${ }^{1,2}$ \\ ${ }^{1}$ Dipartimento di Neuroscienze, Istituto di Ricerche Farmacologiche Mario Negri IRCCS, Milan, \\ Italy, ${ }^{2}$ Head of Unit, Neuroscience, Istituto di Ricerche Farmacologiche Mario Negri, Milan, Italy
}

\section{s0010 Introduction}

p0010 Spinal cord injury (SCI) arises from mechanical trauma to the spine and leads to permanent disability. Its pathophysiology can be summarized in two complex phases. The primary event - the primary injury - is the consequence of compression, contusion, or transection of the spinal cord below the lesioned vertebral tract. The injured spinal cord suffers "imminent" cell damage followed by neuronal death, vascular ruptures, and breakages of the blood-spinal cord barrier. The primary injury is followed by sustained posttraumatic degeneration of the cord-the secondary injury-that leads to further damage and neurological dysfunction [1]. In the acute stage of the secondary injury, an inflammatory phenotype promotes further local inflammation [2] and the recruitment of peripheral monocyte macrophages in the site of injury $[3,4]$. With time, this inflammatory response triggers a self-propelling detrimental mechanism, impairing and limiting any recovery attempts $[3,5]$. In addition, other inflammatory cells such as neutrophils and lymphocytes are involved in the spread of the secondary injury, acting on oligodendrocytes and neuronal cells [3,5]. After the spinal cord damage, activated microglia, macrophages, and astrocytes deposit a barrier of extracellular matrix composed of proteoglycans, generating a glial scar that inhibits spontaneous axonal regeneration [6]. The current procedure in case of SCI is surgical stabilization and decompression of the spinal cord, combined with high-dose methylprednisolone [7]. This approach, however, is still controversial as it gives only limited improvements in outcome, often with severe side effects [8].

p0015 Despite major progress in pharmacological and surgical approaches, aimed at finding definitive therapies for this condition, there is still no treatment able to restore complete motor activity. The most probable reason for these disappointing results lies in SCI progression itself. SCI is a very complex process with different temporal development of degenerative biochemical pathways, and a single-target therapy is probably not enough to counteract the spread of secondary injury. The multitherapeutic ability of stem cells, able to release potential beneficial factors in the damaged site, has been evaluated as one of the most promising strategies for SCI [9]. Stem cell therapy seems promising because a single cell type acts on various pathological pathways, avoiding the use of different drugs whose interactions are often hard to control.

Spinal Cord Injury (SCI) Repair Strategies. https://doi.org/10.1016/B978-0-08-102807-0.00011-9 Copyright $\odot 2020$ Elsevier Ltd. All rights reserved. 
p0020 It was widely assumed that the mechanism of protection of stem cells depend on their differentiation into central nervous system(CNS) cells, replacing dead cells after the primary lesion. Although promising results have been reported on the basis of this theory, today it is increasingly accepted that the paracrine release of antiinflammatory and proregenerative mediators by stem cells plays a key role in the utility of this treatment.

\section{s0015 Stem cell-based therapies after SCI}

p0025 With developments in cell and molecular biology, the clinical use of stem cells holds out promise as a therapeutic option [10]. After SCI, the spinal cord attempts to repair itself by endogenous regenerative mechanisms, such as (i) the migration of Schwann cells, the myelinating and regeneration-promoting cell in the peripheral nervous system, into the damaged tissue to myelinate spinal cord axons [11]; (ii) the increased expression of regeneration-associated genes in damaged neurons [12,13]; (iii) the proliferation of local adult stem cells and progenitor cells [14]. However, axonal growth is hampered by myelin debris growth inhibitors and by the scar tissue [15]. Moreover, the newborn stem cells and progenitor cells do not integrate functionally into the injured spinal cord tissue. Thus, the overall result is a failure of endogenous regenerative events to repair on the injured spinal cord. Their characteristic abilities to self-renew and differentiate into any cell type in the body means the promise of stem cells is also justified in SCI, offering a potential therapy for SCI patients. Different cell therapies have been widely investigated preclinically in SCI models and are considered potentially multiprotective. These approaches are amply discussed in the review by Vismara [9].

\section{s0020 Stem cells can act in different ways}

p0030 Stem cells promote neural regeneration and rescue impaired neural function after SCI, providing antiinflammatory, immunomodulatory, trophic, and antiapoptotic effects. Hypothetically, the stem cell protection after SCI is based on two main mechanisms: (i) cell differentiation and replacement, (ii) neuroprotection and neuroregeneration through the paracrine release of beneficial factors that preserves other cells.

o0010 (i) Cell differentiation and replacement: The CNS is a promising target for cell replacement therapy, because while there is a large amount of information on the ontogenesis of neurons and glia that can be applied to generate clinically relevant cell types, on the other hand there is a lack of effective therapeutic approaches for most CNS diseases. The multi/pluripotent nature of stem cells makes them able to differentiate in different cell lines according to different specific stimuli, including neurons and glial cells $[16,17]$. One major strategy for treating the injured spinal cord is to induce stem cells to differentiate into specific phenotypes to replace the desired cells (neurons or glia) and sustain regeneration of the tissue. 
Several cell-based therapeutic approaches have been proposed and evaluated for treating SCI [18], and different groups have reported in motor and sensory function after transplantation of neuron or glia predifferentiated mouse stem cells in rat or mouse SCI animal models $[19,20]$. Bone marrow mesenchymal stem cells (BM-MSCs) differentiate into cells of the mesodermal lineage but also under certain experimental condition, into cells of the neuronal and glial lineage. A very recent work demonstrated that engrafted BM-MSCs could differentiate into neuronal-like and glial-like cells by Tanshinone IIA. The authors suggested that neuronal-like cells could presumably replace missing or damaged neurons, modulate immune responses, and facilitate tissue regeneration, thus improving the neurological deficits following SCI [21].

Another recent approach exploits the capacity of olfactory ectomesenchymal stem cells (OE-MSCs) by neural crest to differentiate into motor neuron-like cells after treatment with a differentiation cocktail containing retinoic acid and Sonic Hedgehog, suggesting their therapeutic use for motor neuron-related disorders of the CNS such as SCI [22]. In addition, different strategies have been used for treating SCI such as inducing embryonic stem cells (ESCs) to differentiate and replace the desired cells, or to secrete factors that could promote tissue sparing and neuronal regeneration [9]. Despite these encouraging results, it is now clear that for many stem cells the protective mechanism does not mainly depend on the differentiation $[23,24]$. The phenotypic heterogeneity and myriad connections of its neuronal elements, the four-dimensional complexity of its synaptic architecture, and the regionally variable and poorly understood nature of neuronal interactions with astrocytes, oligodendrocytes, and glial progenitor cells make it difficult to establish a precise cell reconstruction mechanism. It is therefore legitimate to think that the mechanisms of stem cell efficacy do indeed differ from or go beyond cell differentiation.

00015 (ii) Neuroprotection and neuroregeneration: It has been demonstrated that many biomolecules are produced by adult multipotent stem cells (mesenchymal stem cells) or pluripotent stem cells (embryonic/neuronal stem cells). Stem cells act by parasecreting neurotrophic factors at the lesion site to enhance the regenerative capacity of damaged tissue and limit the spread of spinal damage and the chronicization of inflammation. Different data suggest the existence of a tissue microenvironment where stem cell factors influence cell survival, inflammation, angiogenesis, repair, and regeneration in a temporal and spatial manner. The mechanisms of paracrine factor release are discussed in the next few sections.

\section{s0025 Stem cells protect by paracrine release of therapeutic mediators}

p0055 Evidence is emerging that the protective effect of stem cells depends on a paracrine effect rather than cell differentiation after transplant. Therapeutic benefit through a paracrine mechanism would not require the cells to be present in the damaged tissue. 
It was recently demonstrated that the use of conditioned medium from adult stem cells is sufficient to recapitulate the beneficial effects of the cells in vitro and in vivo [25]. This and similar reports from other laboratories have led to the proposal that adult stem cells exert their therapeutic benefits through the release of biologically active proteins, or paracrine factors, acting on resident cells. Indeed, there is now a large body of data supporting the hypothesis that paracrine factors are essential for the reparative effects of adult stem cells after delivery into the injured tissue. This suggests that the stem cells themselves are not strictly necessary in the injured tissue for their neuroprotective functions.

p0060 Among the different stem cells that can be used, mesenchymal stem cells (MSCs) are particularly appealing for SCI repair and currently offer the most promise stem cells in preclinical and clinical research [9] on account of their relative ease of access and efficient in vitro expansion [26]. In addition, ESCs, neural stem cells (NSCs), and induced pluripotent stem cells (iPSCs) have a therapeutic value after transplantation into the injured spinal cord (Fig. 11.1). Different studies on stem cell trophic factor production, and the implications for tissue regeneration, are summarized below.

\section{s0030 Mesenchymal stem cells}

p0065 MSCs are the most studied cells for SCI medicine [27]. Compared to other stem cells their use raises no ethical concerns, they can be used in autologous transplants, and they are safe when inserted into the CNS [26]. They can be isolated from different sources: BM-MSCs, umbilical cord MSCs (UC-MSCs), amniotic fluid (AF-MSCs),

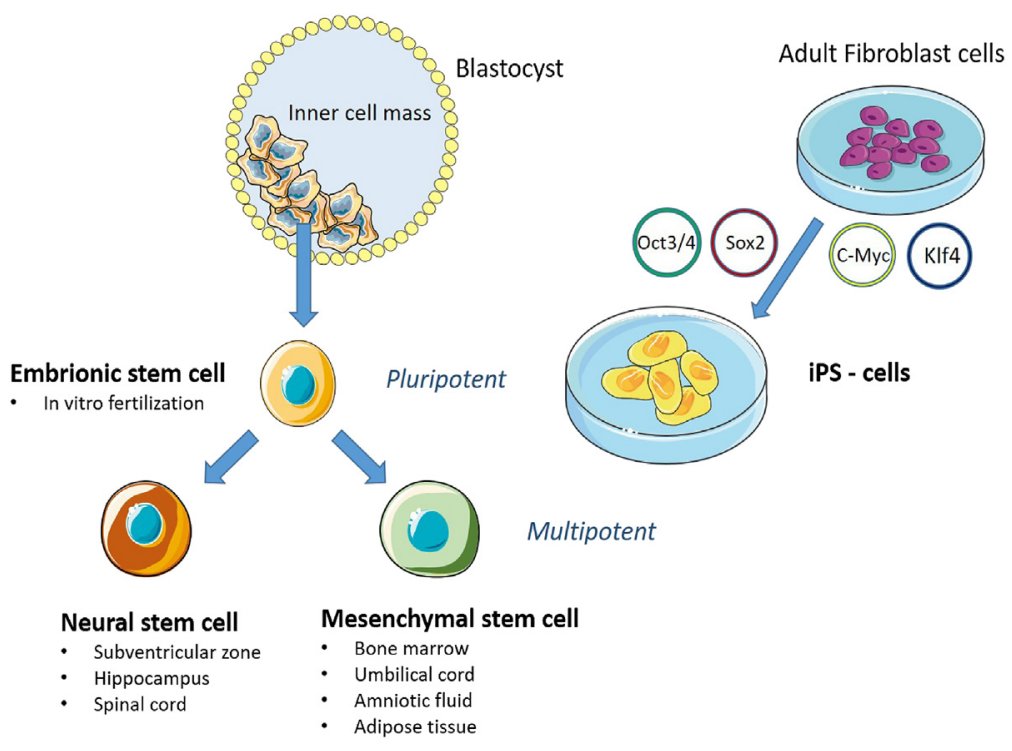

fO010 FIGURE 11.1

Origin and sources of stem cells for SCl therapy. 
and adipose MSCs (A-MSCs). Experimental studies employing MSCs have shown improvements in motor activity ([28,28a]). MSCs were originally considered to be pluripotent with the ability to turn into neurons or glia cells, but these findings have been challenged, suggesting an alternative paracrine effect [29]. BM-MSCs release many trophic factors into their environment to support tissue protection and regeneration, such as brain-derived neurotrophic factor (BDNF), nerve growth factor (NGF), vascular endothelial growth factor (VEGF), and glial cell-derived neurotrophic factor (GDNF) [30,31]. BM-MSCs can abolish lymphocyte proliferation and differentiation, or promote the conversion of macrophages to an antiinflammatory and regenerative phenotype [32], showing immunosuppressive properties [31,33]. MSCs isolated from umbilical cord or cord blood of newborns (UC-MSCs) showed encouraging therapeutic effects in many preclinical SCI studies [34,35] including antiinflammatory [36-38], neurotrophic [37], antiapoptotic [27], and angiogenic action [39]. AFMSCs contributed to improved functional recovery in preclinical SCI models in different studies $[40,41]$. These authors reported more preserved myelin, an angiogenic effect and fever inflammatory cells in the injured site, likely mediated by hepatocyte growth factor [40], BDNF and VEGF [42]. Axon growth is also supported by AF-MSCs transplanted into the transection cavity of the spinal cord of primates [43].

p0070 A-MSCs can be obtained from adipose tissue after lipoplasty or liposuction. When transplanted in situ preclinically, they show functional effects associated with angiogenesis, antiinflammatory, and antiapoptotic activity [44] together with neuroregeneration [45-47]. These regenerative mechanisms are promoted by a mix of factors such as trophic growth factors (BDNF and GDNF) [48], extracellular matrix proteins, proteases, cytokines, and several immunomodulatory molecules [49]. In addition, transplanted A-MSCs promote regeneration and preservation of tissue, limiting glial scar formation, stimulating axonal regrowth [46], depositing laminin [47], and preventing oxidative and inflammatory mechanisms in the injury site [49].

\section{s0035 Embryonic stem cells}

p0075 ESCs are pluripotent cells with the ability to differentiate into nearly all cell types, including neuronal and glial cells [50]. Therefore, they are a promising source of differentiated oligodendrocytes and motoneurons and could be used to treat neurological disorders and traumas, including SCI [51]. They produce protective factors after neural differentiation, such as insulin-like growth factor, fibroblast growth factor, platelet-derived growth factor, and VEGF that promote functional recovery after transplantation into the injured spinal cord of nonhuman primates [52].

\section{s0040 Neural stem cells}

p0080 NSCs are multipotent, self-renewing cells that generate the neurons and glia of the nervous system of all animals during embryonic development. Some NSCs persist in 
specific zones of the adult vertebrate brain (subventricular zone, hippocampus, and central canal of the spinal cord) and continue to produce neurons throughout life $[53,54]$. These can differentiate into neurons and glial cells when appropriately stimulated $[53,54]$. NSCs transplanted into the damaged spinal cord lead to functional recovery replacing neuronal cells and acting by trophic support (BDNF, CNTF, GDNF, NGF, and IGF-1) [55,56], mediating remyelination [57] and preserving axons [58].

\section{s0045 Induced pluripotent stem cells}

p0085 Through the introduction of four different transcription factors, Oct3/4 (octamer-4), Sox2 (sex-determining region Y-box2), Klf4 (Kruppel-like factor), and C-Myc pluripotent cells can be obtained by reprogramming differentiated cells such as fibroblasts [59]. This technique was first described by Takahashi and Yamanaka $[60,61]$ for mouse fibroblasts and has now been applied for other mouse cells [62] and for human somatic cells [63]. These cells offer a valid alternative to ESCs. They avoid the ethical concerns about the use of embryos and reduce the risk of rejection because autologous transplantation is possible. Expression of neurotrophic factors (hepatocyte growth factor HGF, NGF, and BDNF) was demonstrated after transplantation of human iPSC-derived NSCs in an SCI mouse model, able to preserve neuronal cells and promote axonal regeneration [64]. NGF, BDNF, FGF8, and GDNF have also been reported [65] as paracrine effects underlying the functional improvement in vivo. However, further investigation is needed to clarify their paracrine action and to allow their clinical use. The transcription factors are introduced using retroviral vectors, and selection markers are needed to identify the reprogrammed cells. The use of the oncogene c-Myc and the integration of retroviral vectors into the genome are other critical aspects for their clinical use. Nevertheless, iPS cell technology is promising and has opened up exciting avenues for the clinical application of pluripotent cells without the ethical obstacles involved in the use of ESCs.

p0090 Protective mediators are released by two different mechanisms: (i) free delivery or (ii) exosome-mediated delivery (Fig. 11.2).

00020 (i) Free delivery of protective mediators. The wide variety of growth factors and chemokines produced by stem cells that can promote tissue repair are freely delivered in the damaged tissue. Elevated levels of proteins such as BDNF, NGF, VEGF, basic fibroblast growth factor (bFGF), hepatocyte growth factor (HGF), and insulin-like growth factor 1 (IGF1) might underlie the functional benefits with stem cells.

o0025 (ii) Exosome-mediated delivery of protective mediators. Stem cells can release a variety of extracellular vesicles, which can be classified as exosomes (30-100 nm), microvesicles (MVs, 100-1000 nm), and apoptotic bodies. These membrane-bound vesicles are secreted naturally by many types of cells. In general, MSCs are the most promising source of exosomes for 
f0015 FIGURE 11.2

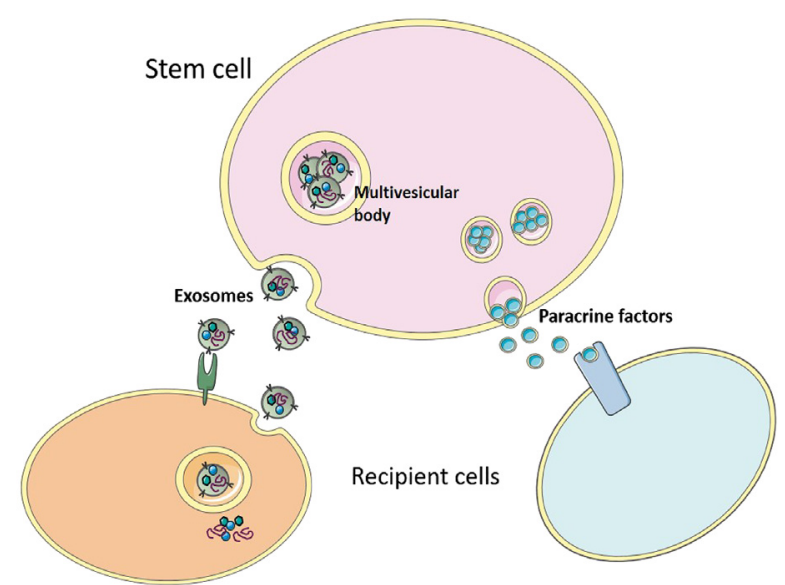

Exosomes-mediated delivery of factors: $\quad$ Free delivery of paracrine factors:

- Exosomes are secreted by multivesicular bodies - Soluble proteins are secreted by fusion of through fusion with cell membrane secretory granules with cell membrane

Their potential effect can be local and remote

Communication via cell receptors or intracellular uptake by endocytosis

- Their effect is related to cells in close proximity

- Communication via cell receptors

Mechanisms of release of paracrine factors.

neurotherapeutic applications [43]. Exosomes contain proteins, lipids, and various nucleic acids, including mRNAs, miRNAs, and long noncoding RNAs (lncRNAs). These exosomal RNAs can be taken up by distant cells and lead to the protein translation in the target cells. Thus, exosomes are natural carriers of signal molecules that act as physiological regulators of cell-to-cell communication [66].

p0105 MSC exosomes carry exosome-related markers such as Alix, tetraspanins (CD9, CD63, and CD81), and heat-shock proteins including Hsp60, Hsp70, and Hsp90. Besides these, the composition of MSC exosomes depends on the cell sources (from which tissue the MSCs were isolated) and their physiological states [67].

p0110 It is increasingly evident that exosomes derived from MSCs may have comparable therapeutic potential to the cells themselves. Exosomes derived from MSCs have shown therapeutic potential for many diseases [43]. Zhang et al. reported that exosomes derived from MSCs can promote axonal growth of cortical neurons, indicating a potential therapeutic strategy to enhance axonal growth after CNS injury [68]. MSC exosomes also contribute to the improvement of impaired neurological functions, implying potential clinical applications [69]. These results raise the possibility that exosomes from MSCs might be a promising therapeutic tool for SCI and could serve as the source for producing commercially exosomes. Exosomes are less immunogenic, more biocompatible, and stable than other viral or liposome-based gene delivery systems. 
p0115 It suggested that exosomes cross the blood-brain barrier and enter the CNS through intercellular junctions of endothelial cells. In addition, exosomes can be genetically engineered, to boost their therapeutic efficiency. These features suggest that exosomes could be developed as an ideal vehicle for therapeutic delivery. Therefore, future studies will have to engineer exosome delivery systems containing a high density of the therapeutic molecules, to target specific cells in specific situations.

\section{s0050 The paracrine release of therapeutic mediators}

p0120 The mechanism of the therapeutic success of stem cells involves several trophic factors released after transplantation. This evidence indicates that it is more important to act on the secretion and focus on the paracrine mechanism of the different factors rather than on the differentiation. Therefore, the approaches mainly reported in the literature to boost the release of protective factors are genetic engineering and in vitro preconditioning.

p0125 Genetic engineering aims to manipulate the genome of an organism to modify the genetic composition of cells. This strategy is now applied to stem cells and there is already evidence that it can further enhance the intrinsic potential of releasing protective factors and confer new functions on cells [9]. Different transgenes have been used so as to secrete large enough amounts of trophic factors to support degenerated cells and regeneration.

p0130 Wang and his group used viral and nonviral plasmids to genetically modify BMMSCs to overexpress neurotrophin 3. Treating rodents with SCI, they found greater regeneration of the axons and better motor activity [70]. Overexpressing BDNF, the same cells improve neurological function in vivo [71]. In addition, increasing the expression not only of BDNF but also of chABC in BM-MSCs both functional recovery and axonal regeneration have been obtained in SCI animal models [72].

p0135 Other investigations involving genetically modified UC-MSC with VEGF and GNDF genes suggest a protective role when these cells are administered to rats after spinal cord contusion. There was preservation of cytoarchitecture and an improvement in motor performance [73]. Moreover, prolonged presence in the site of lesion of the factors VEGF and FGF2 overexpressed by genetically modified UC-MSCs led to reduction of cavitation and sustain the number of myelinated fibers in the injured animals [73a]. Finally, A-MSCs overexpressing both neurogenin-2, a proneural gene, and BDNF and VEGF, promoted functional recovery and inhibit glial scar formation [74].

p0140 Several in vivo treatments have been proposed using preconditioned cells in vitro, improving the pathological outcome. These modified cells can preserve axons, reduce apoptosis and the number of macrophages at the site of the lesion, and improve motor function in vivo [75]. For instance, the reduction of oxygen (hypoxia) in hostile grafting environments results in a better therapeutic effect of stem cells [76]. Hypoxia increased the therapeutic potential of BM-MSCs as it inhibited apoptosis and preserved the spinal cord barrier in a rat model of spinal cord ischemia/reperfusion injury 
[77]. Preconditioning with lower oxygen also acts on UC-MSCs, which became able to boost the expression of HGF, BDNF, and VEGF [75].

p0145 Finally, in recent years, other innovative strategies involving the use of 3D scaffolds have been examined to raise cell viability after transplantation or to create niches in which cells can grow and produce protective factors without being influenced by the hostile environment typical of the damaged spinal cord [75].

\section{s0055 Engineered biomaterial for stem cell delivery in SCI}

p0150 The limited viability of transplanted stem cells in the injury site is a critical issue. Several studies have suggested adding biopolymeric support for cell delivery [78], providing localized targeted therapy to maximize the efficacy. Loaded hydrogels remain temporally localized in the spinal cord and act as reservoirs, delivering factors secreted by implanted stem cells [36] or sustaining them structurally to fill the gap in the damaged site. In addition, the hydrogel preserves the cells from the hostile environment generated by the damaged spinal cord [36]. For instance, to maximize the efficacy of BM-MSCs after transplantation in vivo, smart three-dimensional support has been proposed to mimic the stem cell niche, creating a more sustainable and permissive environment for cell viability and axonal regeneration [79,80,80a]. Several studies have aimed to increase the survival and efficacy of UC-MSCs by supporting the graft with polymeric scaffolds that mimic a physiological niche in situ and can preserve them from the hostile environment while at the same time permitting paracrine release of factors in situ [36,36a].

p0155 To facilitate connection of the damaged spinal cord segment, several biodegradable scaffolds have been transplanted with A-MSCs to address the regenerative processes promoted by these stem cells $[34,81]$.

p0160 To sustain ESC viability in the damaged spinal cord, polymeric scaffolds have been developed for therapeutic intervention, offering advantages in supporting stem cell survival after transplantation [82]. A natural or synthetic polymers scaffold has also been tested to support Schwann cell survival boosting the efficacy of the treatment [83-85].

\section{s0060 Conclusions}

p0165 Immediately after the primary injury, SCI presents a progressive multifaceted neuropathology that makes the situation worse, becoming chronic after some months. This complexity has led new medical strategies to focus to therapies to counteract more than one neuropathological event in SCI, and multifunctional therapies may be a new promising medical approach. MSCs are considered the most promising sources for cellular therapies after SCI. The mechanisms of the biological behavior of MSCs and their complicated function in vivo are not fully understood, which is a very important obstacle to improving the therapeutic effects and designing better therapeutic strategies. 
p0170 Stem cell therapies were conventionally associated with cell differentiation for the replacement of damaged tissues, but they also secrete soluble factors in response to external stimuli [86]. The regulatory mechanisms mediated by paracrine release of protective factors will undoubtedly provide valuable insights for improving SCmediated therapy. Various growth factors, cytokines, chemokines, miRNA, extracellular matrix constituents, and immunomodulatory molecules have been identified and characterized from different types of stem cell, and many of them have some protective and regenerative activity when released in the injury site.

p0175 Cell therapies using stem cells to treat SCI have yielded some important results but a number of impediment remain for their clinical application. One limitation to using MSCs as a therapeutic mediator is that there is no standard protocol on how these cells should be used. Definitions of the key factors useful to obtain therapeutic action are fundamental to improve the reproducibility and effectiveness of the cell therapies. Protocols used in preclinical experiments have not been standardized; the nonhomogeneous origins of the stem cells used create variability, and different routes of administration have been tested but only a few studies have compared them [9]. To overcome this limitation, a clinical-grade protocol must also be adopted in preclinical experiments, in accordance with current good manufacturing practice, to ensure reproducibility of the protocols.

p0180 There is an urgent need for more well-characterized studies to identify the potential mechanisms of the paracrine action. These could offer several advantages such a more controlled effect of stem cells, or using potential identified biomolecules for a free cell-based approach. The variability of cell survival must be overcome, so it can be varied depending on the precise therapeutic needs. Some groups found that using only the secretome produced by neural stem cells ameliorated the motor function after SCI $[87,88]$. It was recently demonstrated that stem cells can release extracellular vesicles (EVs): exosomes and microvesicles. EVs are considered mediators in cell communication in many cell types. EVs can recapitulate the therapeutic effects of stem cell transplantation in some pathology [89] by transferring biologically active molecules as cargo, such as proteins, lipids, and mRNA from the stem cells to the damaged cells. Exosome therapies also offer the exciting prospect of developing cell-free stem cell therapies and ultimately it may be possible to engineer synthetic exosomes with controlled therapeutic payloads [90,91] providing easy controlled therapies. However, the amounts of secreted factors or EVs and the dosage necessary to obtain a functional effect in vivo remain to be clarified.

p0185 In conclusion, there is much evidence that stem cells act through a paracrine effect in SCI, but further effort is still needed to identify the types and amounts of biomolecules delivered and their mechanisms of action to control the stem cell effects for a reparative and regenerative intervention in SCI.

p0190 Cell-free therapy is an appealing alternative to cell-based therapy. To act only with promising identified molecules in situ gives a more controlled effect that can be modified according to therapeutic needs. This could overcome the existing limitations of cell-based therapy, giving advantages for more controlled translation of preclinical research into clinical practice by ensuring a reproducible and optimized 
cell-derived medical application in SCI. In addition, to facilitate and optimize bioactive molecule delivery biodegradable scaffolds could be developed for controlled microfluidic release of neuroprotective/neuroregenerative factors for tailored, timed therapeutic intervention in situ.

\section{References}

[1] Silva NA, Sousa N, Reis RL, Salgado AJ. From basics to clinical: a comprehensive review on spinal cord injury. Prog Neurobiol 2014;114:25-57. https://doi.org/10. 1016/j.pneurobio.2013.11.002.

[2] David S, López-Vales R, Wee Yong V. Harmful and beneficial effects of inflammation after spinal cord injury: potential therapeutic implications. Handb Clin Neurol 2012; 109:485-502. https://doi.org/10.1016/B978-0-444-52137-8.00030-9.

[3] Kigerl KA, Gensel JC, Ankeny DP, Alexander JK, Donnelly DJ, Popovich PG. Identification of two distinct macrophage subsets with divergent effects causing either neurotoxicity or regeneration in the injured mouse spinal cord. J Neurosci 2009;29: 13435-44. https://doi.org/10.1523/JNEUROSCI.3257-09.2009.

[4] Shechter R, Schwartz M. Harnessing monocyte-derived macrophages to control central nervous system pathologies: no longer "if" but "how." J Pathol 2013;229: 332-46. https://doi.org/10.1002/path.4106.

[5] Papa S, Caron I, Erba E, Panini N, De Paola M, Mariani A, Colombo C, Ferrari R, Pozzer D, Zanier ER, Pischiutta F, Lucchetti J, Bassi A, Valentini G, Simonutti G, Rossi F, Moscatelli D, Forloni G, Veglianese P. Early modulation of proinflammatory microglia by minocycline loaded nanoparticles confers long lasting protection after spinal cord injury. Biomaterials 2016;75:13-24. https://doi.org/10.1016/j. biomaterials.2015.10.015.

[6] Orr MB, Gensel JC. Spinal cord injury scarring and inflammation: therapies targeting glial and inflammatory responses. Neurotherapeutics 2018;15:541-53. https://doi.org/ 10.1007/s13311-018-0631-6.

[7] Bracken MB, Holford TR. Neurological and functional status 1 year after acute spinal cord injury: estimates of functional recovery in national acute spinal cord injury study II from results modeled in national acute spinal cord injury study III. J Neurosurg 2002;96:259-66.

[8] Breslin K, Agrawal D. The use of methylprednisolone in acute spinal cord injury: a review of the evidence, controversies, and recommendations. Pediatr Emerg Care 2012;28: 1238-45. quiz 1246-1248, https://doi.org/10.1097/PEC.0b013e3182724434.

[9] Vismara I, Papa S, Rossi F, Forloni G, Veglianese P. Current options for cell therapy in spinal cord injury. Trends Mol Med 2017;23:831-49. https://doi.org/10.1016/j. molmed.2017.07.005.

[10] Murray PE, Garcia-Godoy F. Stem cell responses in tooth regeneration. Stem Cell Dev 2004;13:255-62. https://doi.org/10.1089/154732804323099181.

[11] Takami T, Oudega M, Bates ML, Wood PM, Kleitman N, Bunge MB. Schwann cell but not olfactory ensheathing glia transplants improve hindlimb locomotor performance in the moderately contused adult rat thoracic spinal cord. J Neurosci 2002;22:6670-81. https://doi.org/20026636. 
[12] Filbin MT. Recapitulate development to promote axonal regeneration: good or bad approach? Philos Trans R Soc Lond, B, Biol Sci 2006;361:1565-74. https://doi.org/ 10.1098/rstb.2006.1885.

[13] Gardiner NJ. Integrins and the extracellular matrix: key mediators of development and regeneration of the sensory nervous system. Dev Neurobiol 2011;71:1054-72. https:// doi.org/10.1002/dneu.20950.

[14] Mothe AJ, Tator CH. Review of transplantation of neural stem/progenitor cells for spinal cord injury. Int J Dev Neurosci 2013;31:701-13. https://doi.org/10.1016/j. ijdevneu.2013.07.004.

[15] Silver J, Miller JH. Regeneration beyond the glial scar. Nat Rev Neurosci 2004;5: 146-56. https://doi.org/10.1038/nrn1326.

[16] Bhaskar B, Mekala NK, Baadhe RR, Rao PS. Role of signaling pathways in mesenchymal stem cell differentiation. Curr Stem Cell Res Ther 2014;9:508-12.

[17] Wu J, Izpisua Belmonte JC. Stem cells: a renaissance in human biology research. Cell 2016;165:1572-85. https://doi.org/10.1016/j.cell.2016.05.043.

[18] Buzhor E, Leshansky L, Blumenthal J, Barash H, Warshawsky D, Mazor Y, Shtrichman R. Cell-based therapy approaches: the hope for incurable diseases. Regen Med 2014;9:649-72. https://doi.org/10.2217/rme.14.35.

[19] Deep A, Adeeb N, Hose N, Rezaei M, Fard SA, Tubbs RS, Yashar P, Liker MA, Kateb B, Mortazavi MM. Mouse models of spinal cord injury and stem cell transplantation. Transl Res Anat 2015;1:2-10. https://doi.org/10.1016/j.tria.2015.10. 001.

[20] van Gorp S, Leerink M, Kakinohana O, Platoshyn O, Santucci C, Galik J, Joosten EA, Hruska-Plochan M, Goldberg D, Marsala S, Johe K, Ciacci JD, Marsala M. Amelioration of motor/sensory dysfunction and spasticity in a rat model of acute lumbar spinal cord injury by human neural stem cell transplantation. Stem Cell Res Ther 2013;4: 57. https://doi.org/10.1186/scrt209.

[21] Zhang X-M, Ma J, Sun Y, Yu B-Q, Jiao Z-M, Wang D, Yu M-Y, Li J-Y, Fu J. Tanshinone IIA promotes the differentiation of bone marrow mesenchymal stem cells into neuronal-like cells in a spinal cord injury model. J Transl Med 2018;16:193. https:// doi.org/10.1186/s12967-018-1571-y.

[22] Bagher Z, Kamrava SK, Alizadeh R, Farhadi M, Absalan M, Falah M, Faghihi F, ZareSadeghi A, Komeili A. Differentiation of neural crest stem cells from nasal mucosa into motor neuron-like cells. J Chem Neuroanat 2018;92:35-40. https://doi.org/10. 1016/j.jchemneu.2018.05.003.

[23] Kusuma GD, Carthew J, Lim R, Frith JE. Effect of the microenvironment on mesenchymal stem cell paracrine signaling: opportunities to engineer the therapeutic effect. Stem Cell Dev 2017;26:617-31. https://doi.org/10.1089/scd.2016.0349.

[24] Liang X, Ding Y, Zhang Y, Tse H-F, Lian Q. Paracrine mechanisms of mesenchymal stem cell-based therapy: current status and perspectives. Cell Transplant 2014;23: 1045-59. https://doi.org/10.3727/096368913X667709.

[25] Bollini S, Gentili C, Tasso R, Cancedda R. The regenerative role of the fetal and adult stem cell secretome. J Clin Med 2013;2:302-27. https://doi.org/10.3390/jcm2040302.

[26] Qu J, Zhang H. Roles of mesenchymal stem cells in spinal cord injury. Stem Cell Int 2017;2017:1-12. https://doi.org/10.1155/2017/5251313.

[27] Dasari VR, Veeravalli KK, Dinh DH. Mesenchymal stem cells in the treatment of spinal cord injuries: a review. World J Stem Cell 2014;6:120-33. https://doi.org/10.4252/ wjsc.v6.i2.120. 
[28] Lu L-L, Liu Y-J, Yang S-G, Zhao Q-J, Wang X, Gong W, Han Z-B, Xu Z-S, Lu Y-X, Liu D, Chen Z-Z, Han Z-C. Isolation and characterization of human umbilical cord mesenchymal stem cells with hematopoiesis-supportive function and other potentials. Haematologica 2006;91:1017-26.

[28a] Volkman R, Offen D. Concise review: mesenchymal stem cells in neurodegenerative diseases. Stem Cell 2017;35:1867-80. https://doi.org/10.1002/stem.2651.

[29] Tian L, Prabhakaran MP, Ramakrishna S. Strategies for regeneration of components of nervous system: scaffolds, cells and biomolecules. Regen Biomater 2015;2:31-45. https://doi.org/10.1093/rb/rbu017.

[30] Kanekiyo K, Nakano N, Homma T, Yamada Y, Tamachi M, Suzuki Y, Fukushima M, Saito F, Ide C. Effects of multiple injection of bone marrow mononuclear cells on spinal cord injury of rats. J Neurotrauma 2017;34:3003-11. https://doi.org/10.1089/neu. 2016.4841.

[31] Neirinckx V, Cantinieaux D, Coste C, Rogister B, Franzen R, Wislet-Gendebien S. Concise review: spinal cord injuries: how could adult mesenchymal and neural crest stem cells take up the challenge? Stem Cell 2014;32:829-43. https://doi.org/10. 1002/stem.1579.

[32] Nakajima H, Uchida K, Guerrero AR, Watanabe S, Sugita D, Takeura N, Yoshida A, Long G, Wright KT, Johnson WEB, Baba H. Transplantation of mesenchymal stem cells promotes an alternative pathway of macrophage activation and functional recovery after spinal cord injury. J Neurotrauma 2012;29:1614-25. https://doi.org/10.1089/ neu.2011.2109.

[33] Corcione A, Benvenuto F, Ferretti E, Giunti D, Cappiello V, Cazzanti F, Risso M, Gualandi F, Mancardi GL, Pistoia V, Uccelli A. Human mesenchymal stem cells modulate B-cell functions. Blood 2006;107:367-72. https://doi.org/10.1182/blood2005-07-2657.

[34] Park D-H, Lee J-H, Borlongan CV, Sanberg PR, Chung Y-G, Cho T-H. Transplantation of umbilical cord blood stem cells for treating spinal cord injury. Stem Cell Rev 2011; 7:181-94. https://doi.org/10.1007/s12015-010-9163-0.

[35] Schira J, Gasis M, Estrada V, Hendricks M, Schmitz C, Trapp T, Kruse F, Kögler G, Wernet P, Hartung H-P, Müller HW. Significant clinical, neuropathological and behavioural recovery from acute spinal cord trauma by transplantation of a well-defined somatic stem cell from human umbilical cord blood. Brain 2012;135:431-46. https:// doi.org/10.1093/brain/awr222.

[36] Caron I, Rossi F, Papa S, Aloe R, Sculco M, Mauri E, Sacchetti A, Erba E, Panini N, Parazzi V, Barilani M, Forloni G, Perale G, Lazzari L, Veglianese P. A new three dimensional biomimetic hydrogel to deliver factors secreted by human mesenchymal stem cells in spinal cord injury. Biomaterials 2016;75:135-47. https://doi.org/10. 1016/j.biomaterials.2015.10.024.

[36a] Liu S, Li C, Xing Y, Tao F. Effect of microenvironment modulation on stem cell therapy for spinal cord injury pain. Neural Regen Res 2014;9(5):458-9. https://doi.org/ 10.4103/1673-5374.130057.

[37] Chua SJ, Bielecki R, Yamanaka N, Fehlings MG, Rogers IM, Casper RF. The effect of umbilical cord blood cells on outcomes after experimental traumatic spinal cord injury. Spine 2010;35:1520-6. https://doi.org/10.1097/BRS.0b013e3181c3e963.

[38] Papa S, Vismara I, Mariani A, Barilani M, Rimondo S, De Paola M, Panini N, Erba E, Mauri E, Rossi F, Forloni G, Lazzari L, Veglianese P. Mesenchymal stem cells encapsulated into biomimetic hydrogel scaffold gradually release CCL2 chemokine in situ 
preserving cytoarchitecture and promoting functional recovery in spinal cord injury. J Control Release 2018;278:49-56. https://doi.org/10.1016/j.jconrel.2018.03.034.

[39] Kao C-H, Chen S-H, Chio C-C, Lin M-T. Human umbilical cord blood-derived CD34+ cells may attenuate spinal cord injury by stimulating vascular endothelial and neurotrophic factors. Shock 2008;29:49-55. https://doi.org/10.1097/shk. 0b013e31805cddce.

[40] Bottai D, Scesa G, Cigognini D, Adami R, Nicora E, Abrignani S, Di Giulio AM, Gorio A. Third trimester NG2-positive amniotic fluid cells are effective in improving repair in spinal cord injury. Exp Neurol 2014;254:121-33. https://doi.org/10.1016/j. expneurol.2014.01.015.

[41] Gao S, Ding J, Xiao H-J, Li Z-Q, Chen Y, Zhou X-S, Wang J-E, Wu J, Shi W-Z. Antiinflammatory and anti-apoptotic effect of combined treatment with methylprednisolone and amniotic membrane mesenchymal stem cells after spinal cord injury in rats. Neurochem Res 2014;39:1544-52. https://doi.org/10.1007/s11064-014-1344-9.

[42] Zhou H-L, Zhang X-J, Zhang M-Y, Yan Z-J, Xu Z-M, Xu R-X. Transplantation of human amniotic mesenchymal stem cells promotes functional recovery in a rat model of traumatic spinal cord injury. Neurochem Res 2016;41:2708-18. https://doi.org/10. 1007/s11064-016-1987-9.

[43] Sankar V, Muthusamy R. Role of human amniotic epithelial cell transplantation in spinal cord injury repair research. Neuroscience 2003;118:11-7.

[44] Kang S-K, Shin M-J, Jung JS, Kim YG, Kim C-H. Autologous adipose tissue-derived stromal cells for treatment of spinal cord injury. Stem Cell Dev 2006;15:583-94. https://doi.org/10.1089/scd.2006.15.583.

[45] Kokai LE, Marra K, Rubin JP. Adipose stem cells: biology and clinical applications for tissue repair and regeneration. Transl Res 2014;163:399-408. https://doi.org/10.1016/ j.trs1.2013.11.009.

[46] Kolar MK, Kingham PJ, Novikova LN, Wiberg M, Novikov LN. The therapeutic effects of human adipose-derived stem cells in a rat cervical spinal cord injury model. Stem Cell Dev 2014;23:1659-74. https://doi.org/10.1089/scd.2013.0416.

[47] Menezes K, Nascimento MA, Gonçalves JP, Cruz AS, Lopes DV, Curzio B, Bonamino M, de Menezes JRL, Borojevic R, Rossi MID, Coelho-Sampaio T. Human mesenchymal cells from adipose tissue deposit laminin and promote regeneration of injured spinal cord in rats. PLoS One 2014;9:e96020. https://doi.org/10.1371/ journal.pone.0096020.

[48] Ohta Y, Takenaga M, Tokura Y, Hamaguchi A, Matsumoto T, Kano K, Mugishima H, Okano H, Igarashi R. Mature adipocyte-derived cells, dedifferentiated fat cells (DFAT), promoted functional recovery from spinal cord injury-induced motor dysfunction in rats. Cell Transplant 2008;17:877-86.

[49] Kim N, Cho S-G. New strategies for overcoming limitations of mesenchymal stem cell-based immune modulation. Int J Stem Cells 2015;8:54-68. https://doi.org/10. 15283/ijsc.2015.8.1.54.

[50] Erceg S, Ronaghi M, Stojković M. Human embryonic stem cell differentiation toward regional specific neural precursors. Stem Cell 2009;27:78-87. https://doi.org/10.1634/ stemcells.2008-0543.

[51] Damdimopoulou P, Rodin S, Stenfelt S, Antonsson L, Tryggvason K, Hovatta O. Human embryonic stem cells. Best Pract Res Clin Obstet Gynaecol 2016;31:2-12. https://doi.org/10.1016/j.bpobgyn.2015.08.010. 
[52] Iwai H, Shimada H, Nishimura S, Kobayashi Y, Itakura G, Hori K, Hikishima K, Ebise H, Negishi N, Shibata S, Habu S, Toyama Y, Nakamura M, Okano H. Allogeneic neural stem/progenitor cells derived from embryonic stem cells promote functional recovery after transplantation into injured spinal cord of nonhuman primates. Stem Cells Transl Med 2015;4:708-19. https://doi.org/10.5966/sctm.2014-0215.

[53] Emgård M, Piao J, Aineskog H, Liu J, Calzarossa C, Odeberg J, Holmberg L, Samuelsson E-B, Bezubik B, Vincent PH, Falci SP, Seiger A, Åkesson E, Sundström E. Neuroprotective effects of human spinal cord-derived neural precursor cells after transplantation to the injured spinal cord. Exp Neurol 2014;253:138-45. https://doi.org/10.1016/j.expneurol.2013.12.022.

[54] Parr AM, Kulbatski I, Zahir T, Wang X, Yue C, Keating A, Tator CH. Transplanted adult spinal cord-derived neural stem/progenitor cells promote early functional recovery after rat spinal cord injury. Neuroscience 2008;155:760-70. https://doi.org/10. 1016/j.neuroscience.2008.05.042.

[55] Hawryluk GWJ, Mothe A, Wang J, Wang S, Tator C, Fehlings MG. An in vivo characterization of trophic factor production following neural precursor cell or bone marrow stromal cell transplantation for spinal cord injury. Stem Cell Dev 2012;21: 2222-38. https://doi.org/10.1089/scd.2011.0596.

[56] Lu P, Jones LL, Snyder EY, Tuszynski MH. Neural stem cells constitutively secrete neurotrophic factors and promote extensive host axonal growth after spinal cord injury. Exp Neurol 2003;181:115-29.

[57] Hawryluk GWJ, Spano S, Chew D, Wang S, Erwin M, Chamankhah M, Forgione N, Fehlings MG. An examination of the mechanisms by which neural precursors augment recovery following spinal cord injury: a key role for remyelination. Cell Transplant 2014;23:365-80. https://doi.org/10.3727/096368912X662408.

[58] Kadoya K, Lu P, Nguyen K, Lee-Kubli C, Kumamaru H, Yao L, Knackert J, Poplawski G, Dulin JN, Strobl H, Takashima Y, Biane J, Conner J, Zhang S-C, Tuszynski MH. Spinal cord reconstitution with homologous neural grafts enables robust corticospinal regeneration. Nat Med 2016;22:479-87. https://doi.org/10. 1038/nm.4066.

[59] Khazaei M, Ahuja CS, Fehlings MG. Induced pluripotent stem cells for traumatic spinal cord injury. Front Cell Dev Biol 2016;4:152. https://doi.org/10.3389/fcell.2016. 00152.

[60] Takahashi K, Yamanaka S. Induction of pluripotent stem cells from mouse embryonic and adult fibroblast cultures by defined factors. Cell 2006a;126:663-76. https://doi. org/10.1016/j.cell.2006.07.024.

[61] Takahashi K, Yamanaka S. Induction of pluripotent stem cells from mouse embryonic and adult fibroblast cultures by defined factors. Cell 2006b;126:663-76. https://doi. org/10.1016/j.cell.2006.07.024.

[62] Okada S. The pathophysiological role of acute inflammation after spinal cord injury. Inflamm Regen 2016;36:20. https://doi.org/10.1186/s41232-016-0026-1.

[63] Yu B, Gu X. Combination of biomaterial transplantation and genetic enhancement of intrinsic growth capacities to promote CNS axon regeneration after spinal cord injury. Front Med 2018. https://doi.org/10.1007/s11684-018-0642-z.

[64] Nori S, Okada Y, Yasuda A, Tsuji O, Takahashi Y, Kobayashi Y, Fujiyoshi K, Koike M, Uchiyama Y, Ikeda E, Toyama Y, Yamanaka S, Nakamura M, Okano H. Grafted human-induced pluripotent stem-cell-derived neurospheres promote motor functional 
recovery after spinal cord injury in mice. Proc Natl Acad Sci USA 2011;108: 16825-30. https://doi.org/10.1073/pnas.1108077108.

[65] Romanyuk N, Amemori T, Turnovcova K, Prochazka P, Onteniente B, Sykova E, Jendelova P. Beneficial effect of human induced pluripotent stem cell-derived neural precursors in spinal cord injury repair. Cell Transplant 2015;24:1781-97. https:// doi.org/10.3727/096368914X684042.

[66] Sun D, Zhuang X, Zhang S, Deng Z-B, Grizzle W, Miller D, Zhang H-G. Exosomes are endogenous nanoparticles that can deliver biological information between cells. Adv Drug Deliv Rev 2013;65:342-7. https://doi.org/10.1016/j.addr.2012.07.002.

[67] Assunção-Silva RC, Gomes ED, Sousa N, Silva NA, Salgado AJ. Hydrogels and cell based therapies in spinal cord injury regeneration. Stem Cell Int 2015;2015. 948040, https://doi.org/10.1155/2015/948040.

[68] Zhang Y, Chopp M, Meng Y, Katakowski M, Xin H, Mahmood A, Xiong Y. Effect of exosomes derived from multipluripotent mesenchymal stromal cells on functional recovery and neurovascular plasticity in rats after traumatic brain injury. J Neurosurg 2015;122:856-67. https://doi.org/10.3171/2014.11.JNS14770.

[69] Zhang Y, Chopp M, Liu XS, Katakowski M, Wang X, Tian X, Wu D, Zhang ZG. Exosomes derived from mesenchymal stromal cells promote axonal growth of cortical neurons. Mol Neurobiol 2017;54:2659-73. https://doi.org/10.1007/s12035-0169851-0.

[70] Wang L-J, Zhang R-P, Li J-D. Transplantation of neurotrophin-3-expressing bone mesenchymal stem cells improves recovery in a rat model of spinal cord injury. Acta Neurochir 2014;156:1409-18. https://doi.org/10.1007/s00701-014-2089-6.

[71] Lu P, Jones LL, Tuszynski MH. BDNF-expressing marrow stromal cells support extensive axonal growth at sites of spinal cord injury. Exp Neurol 2005;191:344-60. https:// doi.org/10.1016/j.expneurol.2004.09.018.

[72] Xiong L-L, Li Y, Shang F-F, Chen S-W, Chen H, Ju S-M, Zou Y, Tian H-L, Wang T-H, Luo C-Z, Wang X-Y. Chondroitinase administration and pcDNA3.1-BDNF-BMSC transplantation promote motor functional recovery associated with NGF expression in spinal cord-transected rat. Spinal Cord 2016;54:1088-95. https://doi.org/10.1038/ sc.2016.55.

[73] Mukhamedshina YO, Garanina EE, Masgutova GA, Galieva LR, Sanatova ER, Chelyshev YA, Rizvanov AA. Assessment of glial scar, tissue sparing, behavioral recovery and axonal regeneration following acute transplantation of genetically modified human umbilical cord blood cells in a rat model of spinal cord contusion. PLoS One 2016;11:e0151745. https://doi.org/10.1371/journal.pone.0151745.

[73a] Shămardanova GF, Mukhamedshina IO, rizvanov AA, Salafutdinov II, Chelyshev IA. [Effects of transplantation of human umbilical cord blood mononuclear cells, expressing VEGF and FGF2 genes, into the area of spinal cord traumatic lesion]. Morfologiia 2012;142:31-6.

[74] Tang L, Lu X, Zhu R, Qian T, Tao Y, Li K, Zheng J, Zhao P, Li S, Wang X, Li L. Adipose-derived stem cells expressing the neurogenin-2 promote functional recovery after spinal cord injury in rat. Cell Mol Neurobiol 2016;36:657-67. https://doi.org/10. 1007/s10571-015-0246-y.

[75] Zhilai Z, Biling M, Sujun Q, Chao D, Benchao S, Shuai H, Shun Y, Hui Z. Preconditioning in lowered oxygen enhances the therapeutic potential of human umbilical mesenchymal stem cells in a rat model of spinal cord injury. Brain Res 2016;1642: 426-35. https://doi.org/10.1016/j.brainres.2016.04.025. 
[76] Sart S, Ma T, Li Y. Preconditioning stem cells for in vivo delivery. Biores Open Access 2014;3:137-49. https://doi.org/10.1089/biores.2014.0012.

[77] Wang Z, Fang B, Tan Z, Zhang D, Ma H. Hypoxic preconditioning increases the protective effect of bone marrow mesenchymal stem cells on spinal cord ischemia/reperfusion injury. Mol Med Rep 2016;13:1953-60. https://doi.org/10.3892/mmr.2016. 4753.

[78] Agbay A, Edgar JM, Robinson M, Styan T, Wilson K, Schroll J, Ko J, Khadem Mohtaram N, Jun MB-G, Willerth SM. Biomaterial strategies for delivering stem cells as a treatment for spinal cord injury. Cells Tissues Organs 2016;202:42-51. https:// doi.org/10.1159/000446474.

[79] Han WM, Jang YC, García AJ. Engineered matrices for skeletal muscle satellite cell engraftment and function. Matrix Biol 2017;60-61:96-109. https://doi.org/10.1016/j. matbio.2016.06.001.

[80] Onuma-Ukegawa M, Bhatt K, Hirai T, Kaburagi H, Sotome S, Wakabayashi Y, Ichinose S, Shinomiya K, Okawa A, Enomoto M. Bone marrow stromal cells combined with a honeycomb collagen sponge facilitate neurite elongation in vitro and neural restoration in the hemisected rat spinal cord. Cell Transplant 2015;24:1283-97. https://doi.org/10.3727/096368914X682134.

[80a] Ritfeld G, Rauck B, Novosata TL, Parkb D, Patela P, AC Roosg R, Wangc Y, Oudega M. The effect of a polyurethane-based reverse thermal gel on bone marrow stromal cell transplant survival and spinal cord repair. Biomaterials 2014;35(6): 1924-31. https://doi:10.1016/j.biomaterials.2013.11.062.

[81] Ferrero-Gutierrez A, Menendez-Menendez Y, Alvarez-Viejo M, Meana A, Otero J. New serum-derived albumin scaffold seeded with adipose-derived stem cells and olfactory ensheathing cells used to treat spinal cord injured rats. Histol Histopathol 2013;28:89-100. https://doi.org/10.14670/HH-28.89.

[82] Johnson PJ, Tatara A, McCreedy DA, Shiu A, Sakiyama-Elbert SE. Tissue-engineered fibrin scaffolds containing neural progenitors enhance functional recovery in a subacute model of SCI. Soft Matter 2010;6:5127-37. https://doi.org/10.1039/ c0sm00173b.

[83] Lin X-Y, Lai B-Q, Zeng X, Che M-T, Ling E-A, Wu W, Zeng Y-S. Cell transplantation and neuroengineering approach for spinal cord injury treatment: a summary of current laboratory findings and review of literature. Cell Transplant 2016;25:1425-38. https:// doi.org/10.3727/096368916X690836.

[84] Patel V, Joseph G, Patel A, Patel S, Bustin D, Mawson D, Tuesta LM, Puentes R, Ghosh M, Pearse DD. Suspension matrices for improved Schwann-cell survival after implantation into the injured rat spinal cord. J Neurotrauma 2010;27:789-801. https:// doi.org/10.1089/neu.2008.0809.

[85] Williams RR, Henao M, Pearse DD, Bunge MB. Permissive Schwann cell graft/spinal cord interfaces for axon regeneration. Cell Transplant 2015;24:115-31. https://doi. org/10.3727/096368913X674657.

[86] Das M, Sundell IB, Koka PS. Adult mesenchymal stem cells and their potency in the cell-based therapy. J Stem Cells 2013;8:1-16.

[87] Cheng I, Park DY, Mayle RE, Githens M, Smith RL, Park HY, Hu SS, Alamin TF, Wood KB, Kharazi AI. Does timing of transplantation of neural stem cells following spinal cord injury affect outcomes in an animal model? J Spine Surg 2017;3:567-71. https://doi.org/10.21037/jss.2017.10.06. 
[88] Teixeira FG, Carvalho MM, Sousa N, Salgado AJ. Mesenchymal stem cells secretome: a new paradigm for central nervous system regeneration? Cell. Mol Life Sci 2013;70: 3871-82. https://doi.org/10.1007/s00018-013-1290-8.

[89] Zhang B, Yeo RWY, Tan KH, Lim SK. Focus on extracellular vesicles: therapeutic potential of stem cell-derived extracellular vesicles. Int J Mol Sci 2016;17:174. https:// doi.org/10.3390/ijms17020174.

[90] Luan X, Sansanaphongpricha K, Myers I, Chen H, Yuan H, Sun D. Engineering exosomes as refined biological nanoplatforms for drug delivery. Acta Pharmacol Sin 2017a;38:754-63. https://doi.org/10.1038/aps.2017.12.

[91] Luan X, Sansanaphongpricha K, Myers I, Chen H, Yuan H, Sun D. Engineering exosomes as refined biological nanoplatforms for drug delivery. Acta Pharmacol Sin 2017b;38:754-63. https://doi.org/10.1038/aps.2017.12.

\section{Further reading}

[1] Ahuja CS, Wilson JR, Nori S, Kotter MRN, Druschel C, Curt A, Fehlings MG. Traumatic spinal cord injury. Nat Rev Dis Primers 2017;3:17018. https://doi.org/10.1038/nrdp. 2017.18.

[2] Anthony DF, Shiels PG. Exploiting paracrine mechanisms of tissue regeneration to repair damaged organs. Transplant Res 2013;2:10. https://doi.org/10.1186/2047-1440-2-10.

[3] Baraniak PR, McDevitt TC. Stem cell paracrine actions and tissue regeneration. Regen Med 2010;5:121-43. https://doi.org/10.2217/rme.09.74.

[4] Batista CEM, Mariano ED, Marie SKN, Teixeira MJ, Morgalla M, Tatagiba M, Li J, Lepski G. Stem cells in neurology-current perspectives. Arq Neuropsiquiatr 2014;72: 457-65.

[5] Caplan AI, Bruder SP. Mesenchymal stem cells: building blocks for molecular medicine in the 21st century. Trends Mol Med 2001;7:259-64.

[6] Donnelly EM, Lamanna J, Boulis NM. Stem cell therapy for the spinal cord. Stem Cell Res Ther 2012;3:24. https://doi.org/10.1186/scrt115.

[7] Katsuda T, Kosaka N, Takeshita F, Ochiya T. The therapeutic potential of mesenchymal stem cell-derived extracellular vesicles. Proteomics 2013;13:1637-53. https://doi.org/ 10.1002/pmic.201200373.

[8] Kyurkchiev D, Bochev I, Ivanova-Todorova E, Mourdjeva M, Oreshkova T, Belemezova K, Kyurkchiev S. Secretion of immunoregulatory cytokines by mesenchymal stem cells. World J Stem Cell 2014;6:552-70. https://doi.org/10.4252/wjsc.v6. i5.552.

[9] Linero I, Chaparro O. Paracrine effect of mesenchymal stem cells derived from human adipose tissue in bone regeneration. PLoS One 2014;9:e107001. https://doi.org/10. 1371/journal.pone.0107001.

[10] Mendonça MVP, Larocca TF, de Freitas Souza BS, Villarreal CF, Silva LFM, Matos AC, Novaes MA, Bahia CMP, de Oliveira Melo Martinez AC, Kaneto CM, Furtado SBC, Sampaio GP, Soares MBP, dos Santos RR. Safety and neurological assessments after autologous transplantation of bone marrow mesenchymal stem cells in subjects with chronic spinal cord injury. Stem Cell Res Ther 2014;5:126. https://doi.org/10.1186/ scrt516. 
[11] Mentkowski KI, Snitzer JD, Rusnak S, Lang JK. Therapeutic potential of engineered extracellular vesicles. AAPS J 2018;20:50. https://doi.org/10.1208/s12248-018-0211-z.

[12] Nakano M, Nagaishi K, Konari N, Saito Y, Chikenji T, Mizue Y, Fujimiya M. Bone marrow-derived mesenchymal stem cells improve diabetes-induced cognitive impairment by exosome transfer into damaged neurons and astrocytes. Sci Rep 2016;6: 24805. https://doi.org/10.1038/srep24805.

[13] Nandoe Tewarie RS, Hurtado A, Bartels RH, Grotenhuis A, Oudega M. Stem cell-based therapies for spinal cord injury. J Spinal Cord Med 2009;32:105-14.

[14] Ngangan AV, Waring JC, Cooke MT, Mandrycky CJ, McDevitt TC. Soluble factors secreted by differentiating embryonic stem cells stimulate exogenous cell proliferation and migration. Stem Cell Res Ther 2014;5:26. https://doi.org/10.1186/scrt415.

[15] Oliveri RS, Bello S, Biering-Sørensen F. Mesenchymal stem cells improve locomotor recovery in traumatic spinal cord injury: systematic review with meta-analyses of rat models. Neurobiol Dis 2014;62:338-53. https://doi.org/10.1016/j.nbd.2013.10.014.

[16] Pashoutan Sarvar D, Shamsasenjan K, Akbarzadehlaleh P. Mesenchymal stem cellderived exosomes: new opportunity in cell-free therapy. Adv Pharmaceut Bull 2016; 6:293-9. https://doi.org/10.15171/apb.2016.041.

[17] Phillips MI, Tang YL. Genetic modification of stem cells for transplantation. Adv Drug Deliv Rev 2008;60:160-72. https://doi.org/10.1016/j.addr.2007.08.035.

[18] Shămardanova GF, Mukhamedshina IO, Arkhipova SS, Salafutdinov II, Rizvanov AA, Chelyshev IA. Posttraumatic changes of rat spinal cord after transplantation of human umbilical cord blood mononuclear cells transfected with VEGF and FGF2 genes. Morfologiia 2011;140:36-42.

[19] Shende P, Subedi M. Pathophysiology, mechanisms and applications of mesenchymal stem cells for the treatment of spinal cord injury. Biomed Pharmacother 2017;91: 693-706. https://doi.org/10.1016/j.biopha.2017.04.126.

[20] Watanabe K, Nakamura M, Iwanami A, Fujita Y, Kanemura Y, Toyama Y, Okano H. Comparison between fetal spinal-cord- and forebrain-derived neural stem/progenitor cells as a source of transplantation for spinal cord injury. Dev Neurosci 2004;26: 275-87. https://doi.org/10.1159/000082144. 



\title{
Non-Print Items
}

\begin{abstract}
Despite major progresses in pharmacological and surgical approaches, there is still no treatment able to restore complete motor activity after spinal cord injury (SCI). Stem cell therapy represents a promising strategy to repair the damaged spinal cord. Even if a lot of studies looked at stem cells differentiation to replace neuronal and glial cells for a better functional outcome, is becoming clear that different functional improvements after stem cells therapy are due to a paracrine activity of transplanted cells rather than cell replacement. In this section we will discuss the paracrine mechanisms for tissue repair and regeneration after stem cell transplantation in SCI. It focuses on stem cell factors production, effect in tissue restoration and novel delivery strategies to boost their protective effects.
\end{abstract}

\section{Keywords:}

Biofactors, Paracrine activity, Secreted factors, Stem cells, Spinal cord injury. 


\section{CHAPTER}

\section{c0012 Pharmacological therapies and factors delivery for spinal cord injury regeneration}

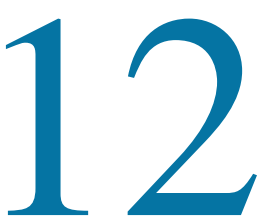

Silviya Petrova Zustiak, PhD, Saahil Sheth, Mozhdeh Imaninezhad, PhD

Biomedical Engineering, Parks College of Engineering, Aviation and Technology, Saint Louis

University, Saint Louis, MO, United States

\section{s0010 Introduction}

p0010 Spinal cord injury (SCI) is one of the major causes of irreversible nerve injury, affecting both sensory and motor neurons [1]. Injuries to the spinal cord pose a significant health problem; in the United States alone, about 12,000 people per year join the population of approximately 270,000 current victims of SCI [2]. Furthermore, SCI has a high rate of prevalence in the younger population, characteristically, young men between 16 and 30 years old, creating physical, emotional, and economic burdens on both the individual and society [2-5]. It is estimated that the annual economic impact of SCI is more than four billion dollars [6].

p0015 The spinal cord functions mainly in transmitting signals between the brain and the body, thus, damage to the spinal cord leads to a complete or partial loss of function in the nervous system below the lesion level [7]. Consequently, strategies to promote functional repair of the spinal cord after injury have been a major focus of translational neuroscience research [8]. Current treatment strategies mainly revolve around eliminating further damage to the spinal cord through surgery to decompress and stabilize any unstable lesions followed by a rehabilitation program [9]. Although such approaches may improve certain clinical outcomes for SCI patients, there are no therapeutic approaches that fully restore the loss of functionality caused by the injury. To restore functionality, SCI therapies would need to first reduce the rate of cell death and minimize the extent of injury, and then facilitate regeneration to repair the damaged nervous tissue [10]. Progress in neuroscience research and the growing availability of advanced interventions, including novel materials and pharmaceutical delivery approaches, make the idea of SCI repair possible [11]. It is now well established that mature central nerves system (CNS) axons maintain an intrinsic ability to regenerate when provided with a permissive environment at the injury site [12]. Several strategies including molecular therapies and hydrogel biomaterial scaffolds have been developed to engineer such permissive environments to promote 
regeneration and functional recovery after SCI [13]. Here, we describe recent developments in such strategies, with focus on hydrogels in particular, as the most commonly used approach to provide a permissive environment as well as a depot for neurotrophins, growth factors, and other pharmaceuticals delivery in SCI repair.

\section{s0015 Newer developments in spinal cord injury repair}

p0020 A combination of factors is responsible for the lack of axonal regeneration and minimal functional recovery usually observed after SCI: a lack of permissive scaffolds within lesion sites, the formation of fluid-filled cysts and scar tissue, the presence of inhibitory molecules in scar tissue and myelin that impair axonal regeneration, diminished neurotrophic support for severed neurons, and intrinsic neuronal changes such as axon demyelination, cell atrophy, and cell death [14-17] (Fig. 12.1). Novel pharmacological therapies include a combination of techniques, which are meant to facilitate functional recovery upon SCI: biomaterial scaffolds, cell transplantation, and delivery of bioactive molecules.

p0025 Biomaterial scaffolds are usually meant to provide structural support for axonal regeneration and could also serve as depots for sustained release of bioactive molecules. Biomaterials have been investigated for their ability to reconstruct tissue architecture, to provide targeted guidance for regenerating axons, and to limit the formation of scar tissue $[18,19]$. It is important to note that tissue loss at the injury site is progressive [20], implying that therapeutic strategies would limit but not fully prevent tissue loss even when applied shortly following injury. To successfully design a scaffold that can be used as a treatment for SCI, many considerations must be taken into account. The scaffold should lessen glial scar formation, yet contain cell adhesive sites to allow regenerating neurons to extend axons into the

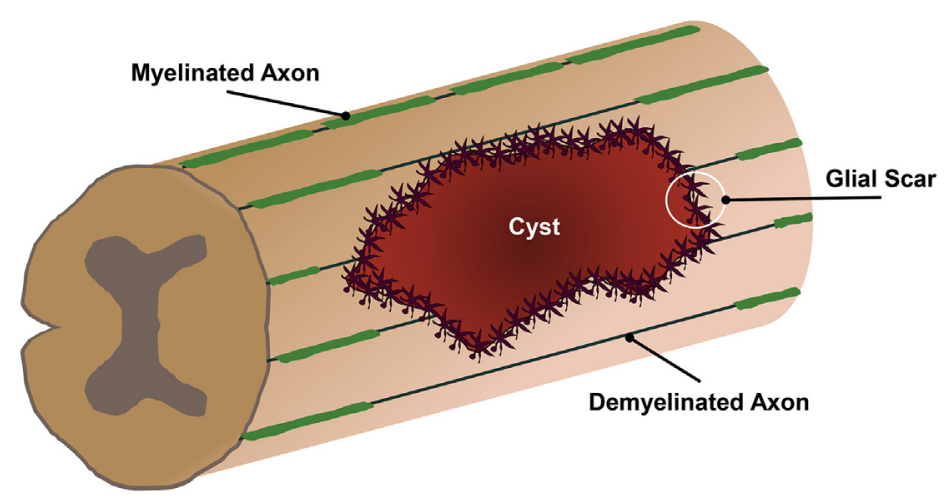

f0010 FIGURE 12.1

Schematic representing a fluid-filled cyst, a glial scar and localized axonal demyelination at a spinal cord injury site. 
injury site, and to reach the distal site [20]. Hydrogels, which act as mimics of the extracellular matrix (ECM) and can support cell attachment, proliferation, and differentiation, have been typically used for promoting tissue regeneration at the lesion site following SCI [21]. Common hydrogels used in SCI will be briefly discussed in this chapter.

p0030 Cells have also been delivered to act as support cells, secrete bioactive molecules, or even replenish damaged neural cells (neurons, oligodendrocytes) [20,22]. Up until now, the implantation of a variety of cell types, including fibroblasts [23], stem cells [24], olfactory ensheathing [25], Schwann cells [26,27], and bone marrow stem cells [28] into a lesion site, has resulted in axon regeneration and moderate sensory and motor improvements in SCI animal models. The properties, advantages, and disadvantages of these cells are comprehensively reviewed elsewhere [29-31] and will not be a major focus of this book chapter.

p0035 Finally, multiple strategies have been developed to deliver bioactive molecules, such as neurotrophins, growth factors, and small molecule pharmaceuticals, in a manner that maximizes therapeutic benefits while minimizing side effects. The remarkable advances in molecular biology in the past decades have led to a better understanding of the mechanisms implicated in the pathophysiology of SCI as well as mechanisms that control axonal growth and regeneration [29], paving the way for the development of targeted therapies. Molecular therapies usually focus on the modulation of inflammatory response, administration of growth factors to promote axonal growth, and molecules that prevent inhibition of regeneration [32,33]. For therapeutic delivery, localized or targeted delivery methods are preferred over systemic delivery, as they result in improved efficacy and reduction in side effects. Localized delivery is typically accomplished via hydrogels, microparticles, and nanoparticles or a combination thereof, which could be directly injected in the intrathecal space following SCI. Nanoparticles in particular could also be delivered systemically in some targeted approaches. However, systemic administration of nanoparticles has a limited therapeutic window; it has been demonstrated that the blood-spinal cord barrier is permeable to nanoparticles as large as $200 \mathrm{~nm}$ for only up to $96 \mathrm{~h}$ post-SCI [34]. Interestingly, nanomaterials themselves have been shown to have neuroprotective properties and have been explored in the treatment of SCI; these have been reviewed elsewhere [35]. In this chapter, we discuss technological developments in materials for neurotrophins, growth factors, and other therapeutics delivery in detail (Fig. 12.2).

\section{s0020 Implantable and injectable hydrogels as permissive scaffolds and depots for therapeutic delivery}

p0040 In the field of CNS regeneration, injectable hydrogel delivery has been extensively explored to stimulate the native repair mechanisms, promote regeneration, and target inhibitory factors [36]. Hydrogels are viscoelastic materials formed through 

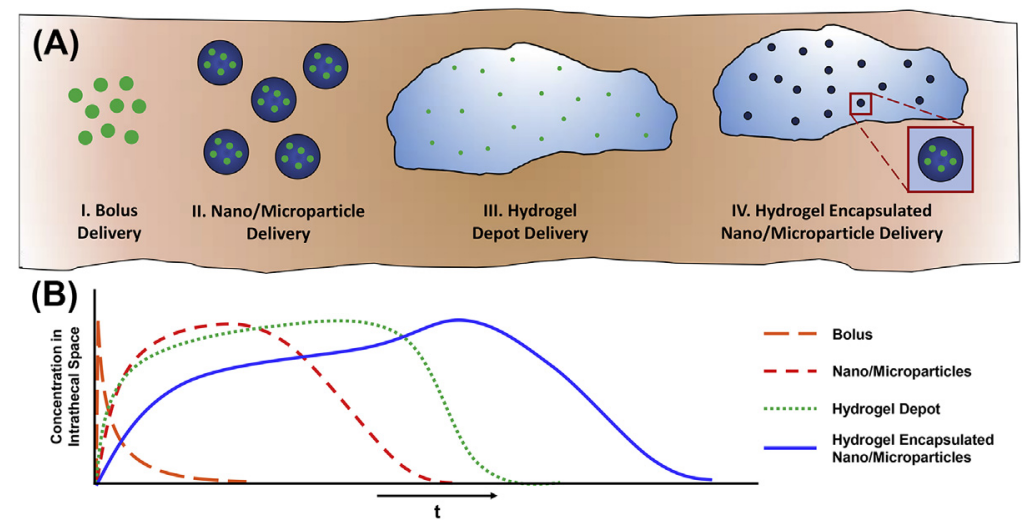

f0015 FIGURE 12.2

(A) Schematic depicting four methods of localized delivery in the intrathecal space near the spinal cord injury site: (I) a bolus dose of therapeutic; (II) therapeutic-loaded nano/ microparticles; (III) therapeutic-loaded hydrogel depot; (IV) therapeutic-loaded nano/ microparticles encapsulated in a hydrogel depot. (B) Theoretical plots depicting the concentration of drug found in the intrathecal space over time when delivered using the four methods described earlier. It is anticipated that free therapeutics will be cleared quickly, while therapeutics released from drug delivery devices will have improved bioavailability.

chemical or physical cross-linking of polymers leading to a three-dimensional structure with an interconnected mesh network [37]. Hydrogels can act both as permissive scaffolds that support cell infiltration and guide axonal growth and as depots for therapeutic delivery to promote axonal regeneration and suppress inflammation [38]. Although various hydrogels have been proposed for SCI treatment, the design of these biomaterials should satisfy several essential criteria: biocompatibility, tailored mechanical, physical, and biochemical properties, biodegradability, porosity, and permeability [29,39,40]. For example, poly 2-hydroxyethyl methacrylate (HEMA) hydrogels containing Schwann cells were implanted into a rat lesioned optic tract for the first time in the 1990s, acting as prosthetic bridges and promoting axonal regeneration [41]. Further work by Plant et al. [42] examined the effect of adhesive Arginine-Glycine-Aspartic Acid (RGD) peptides and amino sugars chemically incorporated in hydrogels, when implanted in the lesioned optic tract or cerebral cortex of rats. The results showed that the highest density of axons for both lesions was observed in hydrogels functionalized with RGD compared to nonmodified or amino sugar-modified hydrogels [42], underscoring the importance of the material's biochemical properties and cell adhesion on cellular infiltration and axon regeneration.

p0045 In particular, injectable hydrogels, which are injected as a solution and form a solid gel in vivo, would be advantageous as they enable the filling of small or irregular defects such as cavities, cysts, or the space between severed areas of the spinal 
cord [43]. These gels can serve as a scaffold, filling any void because of SCI to form a regenerative template to direct cellular infiltration and matrix deposition [44]. For example, agarose hydrogels that have the ability to polymerize in situ have been shown to fill different types of neurological defects and adapt to the shape of the lesion [45]. In addition, injectable biomaterials can be mixed with cells or therapeutics before delivery at the injury site and release of their payload can be tailored by tuning the hydrogels' physical and chemical properties [46]. Last but not least, injectable hydrogels require less-invasive surgeries, shortening the surgical operation time and reducing the postoperative loss of function, pain, and scar size [21]. Injectable hydrogel materials for spinal cord regeneration have been reviewed recently [44].

p0050 Hydrogels can be classified into three main categories: natural, synthetic, and composite hydrogels [37]. Natural hydrogels are based on naturally derived polymers such as agarose, alginate, collagen, fibrin, and chitosan [43]. Natural hydrogels have been implemented in nerve regeneration applications because of their inherent biocompatibility and ability to interact with native tissue [47]. For example, agarose hydrogels loaded with neurotrophic factors and antiscar agents have been shown to enhance neural regeneration [45]. In another study, a templated agarose scaffold seeded with bone marrow stromal cells secreting brain-derived neurotrophic factor (BDNF) proved to support motor axon regeneration after a complete transection SCI model [48]. Another naturally derived hydrogel material is alginate, which has hydrophobic domains that provide good retention of proteins that can be released upon the dissociation of the hydrophobic junction [49]. Alginate hydrogels have been studied for the delivery of growth factors including vascular endothelial growth factor (VEGF) [50]. For example, alginate hydrogels can respond to mechanical signaling and upregulate the release of VEGF to promote blood vessel formation, which has applications in neural regeneration [50]. Hydrogels based on collagen have also been extensively explored for SCI treatment. Collagen is one of the most abundant proteins found in the ECM of mammals. Therefore, collagenderived biomaterials are highly biocompatible, biodegradable, and noncytotoxic [51]. However, the main drawback of collagen is the variability of its mechanical properties and enzymatic degradation rate in vivo [51,52]. Fibrin-based hydrogels are another alternative for SCI treatment. Fibrin is produced during the coagulation cascade following an injury. A fibrin monomer is produced when fibrinogen is cleaved by thrombin and, therefore, these monomers spontaneously polymerize and create a 3D matrix [53]. Importantly for neural tissue engineering applications, fibrin can be injected in its liquid state and form a solid scaffold in vivo [54]. A few disadvantages of fibrin-based materials are their fast degradation [55,56] and easy contamination by blood-borne pathogens or prion proteins [57]. Furthermore, fibrin can contain traces of autologous mammalian fibrinogen, which inhibits neurite growth [58] as well as activates resident astrocyte scar formation [59].

p0055 Synthetic hydrogels typically offer better batch-to-batch variability compared to natural materials, and their properties can be more robustly tailored to fit a specific application [60]. However, a limitation for most synthetic hydrogels is the lack of 
cell-binding sites [61]. Recent studies have overcome this limitation by incorporating bioactive molecules, such as peptides and growth factors, into synthetic materials to mediate specific cell functions [62-64]. Some of the most commonly used synthetic hydrogels in neural tissue engineering are poly(lactic acid) (PLA) [65,66], poly(lactic-co-glycolic acid) (PLGA) [67,68], poly(ethylene glycol) (PEG) [69-71], poly(2-hydroxyethyl methacrylate) (pHEMA) [72-74], and poly(hydroxypropyl methacrylate) (pHPMA) [75,76]. PLA hydrogels are used in tissue engineering due to their biodegradability, biocompatibility, thermal plasticity, and a wide range of mechanical properties, which can match the properties of the targeted tissue [77]. However, the low degradation rate of PLA and the high crystallinity of its fragments can cause an inflammatory reaction in the body. This disadvantage can be overcome by using a combination of L-lactic and D, L-lactic acid monomers to make PLGA hydrogels to allow degradation without the formation of crystalline fragments [78]. Compared to PLA, PLGA hydrogels offer superior control over degradation properties by varying the ratio of PLA:PGA monomers. Both PLA and PLGA hydrogels degrade hydrolytically, and the degradation products are removed through natural pathways [79]. Despite being used for clinical applications, pure PLGA is limited due to the lack of adequate mechanical properties for implantation into the chosen target tissue $[35,80,81]$. PEG polymers are bioinert, biocompatible, and approved by the US Food and Drug Administration for clinical use. However, PEG polymers are nondegradable, but degradability could be imparted by adding hydrolytically degradable cross-linkers $[82,83]$ or enzymatically degradable peptide sequences [84] into the three-dimensional gel network. pHEMA hydrogels have been mainly explored as nerve guidance channels due to their viscoelastic properties similar to those of nervous tissue [85].

\section{s0025 Delivery of neurotrophic factors in SCI repair s0030 Neurotrophic factors in SCI repair}

p0060 Neurotrophic factors (NTFs) are a class of growth factors secreted by cells specifically to aid neuronal growth [86-88]. NTFs are responsible for axonal growth, synaptic plasticity, and general function of neurons in the nervous system. In particular, NTFs have been shown to promote axonal growth and synaptic formation following SCI [88]. Along with the natural regenerative benefits, NTFs have also been used to promote the differentiation of progenitor cells, such as astrocytes [89], neurons [90], and neural stem cells (NSCs) [88] in cell transplantation approaches. There is a plethora of NTFs that have the potential to contribute to functional recovery following SCI, including nerve growth factor (NGF), neurotrophin-3 (NT-3), and BDNF. NGF is perhaps the most recognized and well-studied NTF to date. The growth factor dates as far back as 1954 when it was used to augment the growth and differentiation of nerve cells in mice [91]. NGF, although termed a "growth factor," is slightly different than the traditional growth factors, because it regulates the 
function of differentiated neurons by promoting the growth rather than the proliferation of these cells [92]. NGF has also proven to be a critical component in SCI treatment, promoting neural regeneration and functional recovery in rat models [93,94]. NT-3 is another NTF that provides substantial benefits for repair following SCI. It has been shown to prevent atrophy in neurons, promote axonal regeneration, and restore functionality in the hindlimb of an SCI animal model $[88,95,96]$. BDNF is the most commonly found NTF in the mammalian CNS [97]; it has been shown to play an important role in the survival, differentiation, and plasticity of neurons in the CNS by impeding apoptosis and promoting axonal growth and reorganization [97]. All of these NTFs are excellent candidates for delivery alone or in combination after SCI to prevent further damage while initiating and enhancing neuronal growth.

p0065 Other neurotrophic factors, such as glial-derived neurotrophic factor (GDNF) [98], ciliary neurotrophic factor (CNTF) [99], VEGF [21], and fibroblast growth factor (FGF) [100], have also been considered for SCI repair. For example, long-term GDNF intrathecal infusion has been shown to have a strong neuroprotective effect on white matter and certain axonal sparing following injury in adult rats [98]. CNTF plays a role in motor neuron survival and outgrowth [101]. Transplantation of CNTF-expressing oligodendrocyte precursor cells has been shown to promote remyelination and functional recovery after SCI [99]. However, studies suggest that CNTF contributes to glial scar formation after SCI, making it undesirable as a therapeutic in the CNS [102]. Angiogenic factors, such as VEGF and FGF-2, have also been studied for their potential to promote nerve regeneration after SCI as vascularization helps overcome an ischemic environment that limits regeneration [21,103]. VEGF, in particular, is one of the most widely researched growth factors for any tissue regeneration. VEGF is capable of inducing angiogenesis and endothelial cellular activity including the synthesis of several cytokines and other growth factors. In the context of SCI, Widenfalk et al. [104] showed that VEGF can lead to increased blood vessel density in the wounded areas of a spinal contusion and can improve tissue sparing. FGF-2 can reduce immediate vasoconstriction of vessels in the tissue surrounding the primary injury, promote angiogenesis, and prevent neuronal cell death $[105,106]$. In SCI, FGF-2 has been shown to significantly enhance functional recovery of hind limb movements in the absence of significant tissue sparing after SCI in rats [107]. Lastly, sonic hedgehog (Shh) is a multifunctional growth factor that acts on several aspects of spinal cord development and has been shown to contribute to motor neuron regeneration in a zebra fish SCI model [108].

\section{s0035 Strategies for sustained delivery of neurotrophic factors to improve efficacy}

p0070 However, the widespread use of NTFs in SCI treatment is hampered by their short half-life, low bioavailability, poor ability to pass biological barriers, and generally unfavorable pharmacokinetic properties [109]. For example, NTFs cannot be delivered systemically via an oral route [110], which would have been noninvasive and 
would have enabled easy patient self-administration. Systemic delivery via an intravenous injection is also not feasible, due to the NTFs' short half-life and inability to pass the blood-spinal cord biological barrier [111,112]. Hence, local delivery methods such as bolus intrathecal injections or prolonged pump-driven infusions through an indwelling catheter have been developed. Such interventions not only bypass the blood-spinal cord barrier, but also deliver the therapeutic at the site of injury to maximize efficacy and reduce side effects [112]. However, external mini-pumps are invasive and can cause scarring, compression of the spinal cord, and infections at the skin port of entry [36]. Bolus injections, on the other hand, fail to maximize NTFs efficacy, as the upregulated proteolytic enzymatic activity after SCI can quickly degrade the administered NTFs and repeated injections cause tissue damage at the injection site, which further hinders recovery.

p0075 For ultimate clinical translation, a drug delivery system is required for localized and sustained release to improve efficacy and reduce side effects [112]. Ideally, such a sustained delivery system would protect NTFs from degradation and even successfully target them to localize them at the injured site of the spinal cord [113]. As mentioned earlier, hydrogels have most commonly been studied as sustained delivery vehicles for NTFs to treat SCI [114]. One disadvantage of hydrogels is that release is typically controlled by simple diffusion or noncovalent interactions, which could lead to high burst release [115]. One way to overcome this limitation is to provide further barriers to NTF release, such as for example encapsulating the neurotrophic factors in microparticles or tethering them to nanoparticles, which could then be delivered as is [116] or encapsulated in a hydrogel scaffold [44]. Overall, such materials developed for NTFs delivery should also be injectable for minimally invasive delivery and degradable to avoid a removal surgery [117,118]. The direct injection of micro- or nanoparticles is a good option, but one concern is that the unconfined particles are free to leave the zone of injection and can migrate to the liver or the spleen, where they can be cleared from the body [119]. When particles are incorporated in a support matrix such as a hydrogel, they offer sustained release, while localized at the site of injection [112]. The support matrix also provides a buffering zone for counteracting the surface burst release from the particles [120]. Furthermore, the hydrogel matrix creates a suitable environment for inducing tissue regeneration at the lesion by providing an artificial ECM [21].

\section{s0040 Examples of delivery approaches for neurotrophic factors in SCI repair}

p0080 One of the most frequently used polymers in drug delivery is PLGA, and its degradation rate and consequently drug release are readily controlled by the ratio of PLA to PGA, as mentioned earlier. For example, an 85:15 ratio of PLA to PGA degrades significantly slower than a 50:50 ratio [114]. Encapsulating neurotrophins in PLGA has been shown to not only enable localized sustained delivery, but also to protect the growth factors from enzymatic degradation. Benoit et al. [121] developed biocompatible degradable PLGA microspheres for local delivery of several 
molecules, including NGF. Their work illustrated the ability to encapsulate NGF that was subsequently released over several months and, importantly, remained active upon release. Burdick and coworkers [122] also utilized PLGA microspheres $(1-10 \mu \mathrm{m})$, but encapsulated them in an injectable hydrogel to simultaneously deliver multiple neurotrophins, where each growth factor exhibited an individual release profile. To do so, NT-3 was encapsulated in the PLGA microspheres and CNTF in the bulk hydrogel, leading to a rapid release of CNTF from the hydrogel phase and a slow continuous release of NT-3 from the degradable microspheres. In another study, Wang et al. [113] showed that intraspinal injection of GDNFloaded PLGA nanoparticles had no effect on gliosis, but effectively preserved neuronal fibers and led to the hindlimb locomotor recovery in rats with SCI. Lowry et al. [123] showed that administration of biodegradable PLGA microspheres that provide sustained, controlled delivery of Shh resulted in significant functional improvement in two different mouse models of SCI-contusion and dorsal hemioversection. The authors attributed the positive results to increased proliferation of oligodendrocyte cells, decreased astrocytic scar formation, and increased nerve fiber sprouting and growth.

p0085 Another biocompatible and injectable hydrogel developed for SCI applications is one composed of hyaluronan and methylcellulose (HAMC) [124]. It is a fast-gelling, noncell adhesive, and degradable material, which is already at the gelation point before injection, but is injectable due to its shear-thinning property. In vivo rat studies (1 month duration) show that HAMC is biocompatible within the intrathecal space and may even provide therapeutic benefit in locomotor behavior and inflammation, positioning it as a promising candidate for localized therapeutic delivery to the injured spinal cord [124]. HAMC hydrogel has been used to deliver erythropoietin [125] and FGF-2 [126]. More commonly, HAMC has been used as a depot for small molecule or protein release, such as the neuroprotective molecules NBQX and FGF-2 or neuroregenerative molecules dbcAMP and EGF, where the therapeutic molecules were loaded in particles, which were then suspended in the gel [106]. Although HAMC gels have inferior mechanical properties, PLGA particles can be embedded into the hydrogels to improve biostability compared to HAMC alone [106]. The improved rheological properties and biostability of HAMC upon PLGA particle loading have been attributed to hydrophobic interactions between the methylcellulose methyl groups and PLGA nanoparticles [112]. Importantly, the hydrogel provides a buffering zone that helps attenuate the rate of drug release and also localizes the particles at the injection site [127].

p0090 Similarly to HAMC alone, the composite HAMC/PLGA system have been shown well-tolerated in the intrathecal space of spinal cord injured rats, showing no increase in inflammation, scarring, or cavity volume relative to controls, and no effect on locomotor function up to 28 days [112]. The PLGA/HAMC composite materials have been used by multiple groups for neurotrophic factor delivery in SCI. For example, Donaghue and coworkers [115] showed that when NT-3 was loaded in PLGA nanoparticles dispersed in an HAMC hydrogel, NT-3 exhibited a prolonged release of over 50 days in vitro and over 28 days in vivo when injected in the 
intrathecal space. The release profile was triphasic in vitro, with a minimal burst and slow release initially attributed to either hydrophobic or electrostatic interactions between the NT-3 and the PLGA nanoparticles, followed by a period of faster and then again a slower release. In vivo, delivery of NT-3 resulted in significant axon growth improvements and locomotor function [115]. In another study, to enhance the endogenous angiogenic response after spinal cord injury, FGF-2-loaded PLGA nanoparticles embedded in HAMC were injected into the intrathecal space leading to higher blood vessel density in the dorsal horns 28 days post-injury, compared to FGF-2-free controls [105]. To prolong neurotrophin release even further and improve the stability of the HAMC/PLGA delivery system, various excipients, such as PEG 400, trehalose, and $\mathrm{MgCO}_{3}$, have also been tested [128]. Trehalose showed no significant improvement over the excipient-free formulation, while coencapsulation of PEG 400 significantly improved NT-3 detection immediately after encapsulation, but lead to decrease in release duration from 28 to $7 \mathrm{~d}$ making it unsuitable for long-term release formulations [128]. Coencapsulated $\mathrm{MgCO}_{3}$, on the other hand, dramatically enhanced the bioactivity of NT-3 up to $28 \mathrm{~d}$, which was attributed to the ability of $\mathrm{MgCO}_{3}$ to neutralize the low $\mathrm{pH}$ inside PLGA particles, an environment in which NT-3 has been shown to be particularly vulnerable to structural damage [128].

p0095 Hydrogels other than HAMC have been used to encapsulate neurotrophin-loaded PLGA nanoparticles. Ansorena et al. [129] illustrated that GDNF release kinetics was much slower with minimal burst release when the PLGA microspheres were incorporated into an alginate:fibrinogen hydrogel compared to GDNF incorporated directly into the gel (free GDNF). This difference could be explained by a combination of factors, such as the hindered diffusion of GDNF across the PLGA-hydrogel boundary, by interactions between the alginate and GDNF, and by slower microsphere degradation due to reduced water accessibility upon confinement in the hydrogel [129]. Interestingly, animals treated with free GDNF-experienced superior functional recovery compared with the animals treated with GDNF microsphereloaded hydrogels, possibly due to the differences in release profiles [129]. GDNFloaded PLGA microsphere have also been embedded in fibrin gels, where gel encapsulation again led to prolonged release in vitro and delayed degradation in vivo (compared to fibrin gels alone) [130]. When implanted into a delayed nerve repair site, the GDNF-loaded nanoparticle/gels system led to improved early nerve regeneration, as measured by histomorphometry and motor neuron regeneration [130]. In another study, BDNF and VEGF-loaded PLGA microspheres were embedded in a hyaluronic acid (HA) hydrogel, leading to $~ 20 \%-30 \%$ growth factor release within $150 \mathrm{~h}$ with excellent preservation of protein bioactivity [131]. Biocompatibility was demonstrated by seeding NSCs on the hydrogels, where the cells were able to adhere and proliferate on the composite materials [131].

p0100 Delivery systems other than PLGA particles have been used for neurotrophins release. For example, hollow collagen microspheres were loaded with NGF postfabrication with a high loading efficiency due to the hollow structure and release was tailored for up to 13 days by varying collagen cross-linking [116]. In another 
example, NT-3 released from chitosan particles promoted the differentiation of neural stem cells into neurons, while reducing the overall dose required for differentiation and preventing NT-3 denaturation and hydrolization [132]. Localized and sustained release of CNTF from degradable sodium hyaluronate gelatinous particles have been shown to enhance locomotion, increase axonal regrowth as well as replenish $\beta$-tubulin-III-positive neuron-like cells in rats following SCI [133]. Another group incorporated a combination of free VEGF and VEGF-loaded chitosan-dextran sulfate nanoparticles in an injectable alginate/fibrinogen-based hydrogel [21]. Their results showed that this delivery system induced angiogenesis and neurite growth at the injury site [21]. Hamann et al. [100] delivered epidermal growth factor (EGF) and FGF-2 via a collagen-based injectable intrathecal delivery device. The rationale for choosing EGF and FGF-2 was their ability to stimulate the proliferation and differentiation of ependymal cells and functional recovery in the spinal cord [100].

\section{s0045 Neurotrophic factor delivery in combination therapies for SCI repair}

p0105 In some cases, protein delivery has been combined with other therapeutic approaches such as nerve guidance channels (NGCs), which are designer scaffolds that bridge the distal and proximal ends of a severed nerve to support and guide axon regeneration [134]. Various therapeutic molecules, including NTFs, have been used in sustained release formulations with varying levels of success to improve regeneration and functional recovery after injury when combined with NGCs. In one study, NGF was incorporated into NGCs made of poly(2hydroxyethyl methacrylate-co-methyl methacrylate), P(HEMA-co-MMA), either directly or by first encapsulating the NGF in PLGA microspheres [135]. Sustained release of NGF was achieved from NGCs with both NGF-loaded microspheres and NGF alone incorporated into the inner layer. However, the release of NGF from NGCs with microspheres was limited by a slow-degrading microsphere formulation and by the maximum amount of microspheres that could be incorporated into the NGCs structure [135]. In another study, Yang and colleagues [136] fabricated porous nerve conduits of poly(lactide-co-glycolide) (PLG) for the sustained release of NGF for up to 42 days, which stimulated neurite outgrowth from primary dorsal root ganglion. EGF has also been incorporated in PLGA microspheres within chitosan/chitin NGCs, where the released EGF was able to successfully promote the formation of neurospheres in culture during the first 14-21 days of release [134]. In another study, De Laporte et al. [103] developed bridges by fusing growth factorloaded PLGA microspheres, allowing localized and sustained release of VEGF and FGF-2 to promote angiogenesis and nerve growth in an SCI model. Implanted bridges releasing VEGF and FGF-2 increased the infiltration of endothelial cells and the vascularization, but did not significantly impact neurofilament staining [103].

p0110 In another combination therapy approach, Tobias et al. [137] have shown that alginate-encapsulated BDNF-producing fibroblasts (Fb/BDNF) survived 1 month in culture, made bioactive neurotrophins, survived transplantation into the injured 
spinal cord in the absence of immune suppression, and provided a permissive environment for host axonal growth. Tobias et al. [138] extended these studies by examining the effects of grafting encapsulated $\mathrm{Fb} / \mathrm{BDNF}$ into a subtotal cervical hemisection on recovery of forelimb and hindlimb function and axonal growth in the absence of immune suppression. Grafting of encapsulated Fb/BDNF resulted in partial functional recovery, where recovery was significantly greater when compared to animals that received unencapsulated $\mathrm{Fb} / \mathrm{BDNF}$ without immune suppression, but similar to that of immune suppressed animals receiving unencapsulated Fb/BDNF [138]. In another approach, Kokai et al. [139] created a double-walled microsphere delivery system for GDNF with a sustained release profile $>50$ days in vitro, by coating the PLGA microspheres with an additional polymer layer (e.g., PLA), and then incorporating them into porous PCL nerve guides [139]. The nerve guides were then implanted in a $1.5 \mathrm{~cm}$ defect in a rat sciatic nerve gap, resulting in an increase in tissue integration and presence of Schwann cells after 6 weeks [139].

p0115 A summary of delivery approaches for neurotrophic factors, either as single or combination therapy, is provided in Table 12.1 .

\section{s0050 Delivery strategies for other therapeutic molecules in SCI}

p0120 Systemic administration of a high dose of methylprednisolone (MP), a synthetic corticosteroid, is the only recommended neuroprotective route to prevent further secondary injury damage following acute SCI [7]. It has been demonstrated that MP given within $8 \mathrm{~h}$ of SCI can improve neurologic recovery in humans [141]. Systemic MP administration leads to only modest improvements in neurological recovery, but is associated with multiple adverse side effects, including wound infections, pneumonia, and acute myopathy [142]. Hence, a localized and sustained delivery of MP by means of using a controlled drug delivery system could be advantageous in enhancing its efficiency as an antiinflammatory and neuroprotective agent, while alleviating side effects [5]. In one such approach, MP was encapsulated in PLGA nanoparticles and compared to systemic and local delivery of free MP in a rat model of SCI [143]. Relative to systemic or local delivery of free MP, MP-nanoparticle therapy significantly reduced lesion volume and improved behavioral outcomes due to improved MP pharmacokinetic properties as well as the targeted and prolonged administration [143]. Carboxymethylchitosan/polyamidoamine (CMCht/ PAMAM) dendrimer nanoparticles of $109 \mathrm{~nm}$ have also been shown to sustain MP release over 14 days and to be successfully internalized by microglial cells without impacting their viability in vitro [5]. Following lateral hemisection lesions in Wistar rats, nanoparticle uptake by the spinal tissue was observed $3 \mathrm{~h}$ post-administration and led to significantly improved locomotor function [5]. Similar to growth factor-loaded nanoparticles, MP-loaded particles could be encapsulated in a hydrogel to prevent the particles from leaving the injection zone and migrating toward distal tissues and organs, such as liver and spleen. For example, MP-loaded 
t0010 Table 12.1 Hydrogels and hydrogel particles for the delivery of neurotrophic factors.

\begin{tabular}{|c|c|c|c|c|}
\hline Matrix & Particles & Neurotrophins & $\begin{array}{l}\text { In vitro/ } \\
\text { in vivo }\end{array}$ & References \\
\hline $\begin{array}{l}\text { PLGA } \\
\text { nanoparticles }\end{array}$ & - & NGF & In vivo & {$[121]$} \\
\hline- & PLGA nanoparticles & GDNF & $\begin{array}{l}\text { In vitro, } \\
\text { in vivo }\end{array}$ & {$[113]$} \\
\hline- & Chitosan carriers & NT-3 & In vitro & [132] \\
\hline- & $\begin{array}{l}\text { Hollow collagen } \\
\text { microspheres }\end{array}$ & NGF & In vitro & [116] \\
\hline HAMC & PLGA nanoparticles & NT-3 & $\begin{array}{l}\text { In vitro, } \\
\text { in vivo }\end{array}$ & {$[115,128]$} \\
\hline HAMC & PLGA nanoparticles & FGF-2 & $\begin{array}{l}\text { In vitro, } \\
\text { in vivo }\end{array}$ & {$[105]$} \\
\hline $\begin{array}{l}\text { Hyaluronic } \\
\text { acid }\end{array}$ & PLGA microspheres & BDNF, VEGF & In vitro & {$[140]$} \\
\hline Fibrin & PLGA microspheres & GDNF & $\begin{array}{l}\text { In vitro, } \\
\text { in vivo }\end{array}$ & {$[130]$} \\
\hline $\begin{array}{l}\text { Alginate: } \\
\text { Fibrinogen }\end{array}$ & PLGA microspheres & GDNF & In vivo & {$[129]$} \\
\hline $\begin{array}{l}\text { Alginate: } \\
\text { Fibrinogen }\end{array}$ & $\begin{array}{l}\text { CS/DS nanoparticles } \\
\text { and }\end{array}$ & & & \\
\hline $\begin{array}{l}\text { PLGA } \\
\text { microspheres }\end{array}$ & - & VEGF & $\begin{array}{l}\text { In vitro, } \\
\text { in vivo }\end{array}$ & {$[21]$} \\
\hline $\begin{array}{l}\text { PEG } \\
\text { hydrogels }\end{array}$ & PLGA microspheres & CNTF, NT-3 & In vitro & {$[122]$} \\
\hline $\begin{array}{l}\text { PCL nerve } \\
\text { guides }\end{array}$ & $\begin{array}{l}\text { Double-walled PLGA } \\
\text { microspheres }\end{array}$ & GDNF & In vivo & {$[139]$} \\
\hline- & $\begin{array}{l}\text { Sodium hyaluronate } \\
\text { gelatinous particles }\end{array}$ & CNTF & In vivo & {$[133]$} \\
\hline- & PLGA microspheres & Shh & $\begin{array}{l}\text { In vitro, } \\
\text { in vivo }\end{array}$ & {$[123]$} \\
\hline $\begin{array}{l}\text { Poly(2- } \\
\text { hydroxyethyl } \\
\text { methacrylate- } \\
\text { co-methyl } \\
\text { methacrylate }\end{array}$ & PLGA microspheres & NGF & & {$[135]$} \\
\hline- & PLG microspheres & NGF & $\begin{array}{l}\text { In vitro, } \\
\text { in vivo }\end{array}$ & [136] \\
\hline- & Alginate microspheres & $\begin{array}{l}\text { Fibroblast } \\
\text { secreted BDNF }\end{array}$ & $\begin{array}{l}\text { In vitro, } \\
\text { in vivo }\end{array}$ & {$[137,138]$} \\
\hline Collagen & - & EGF, FGF-2 & In vivo & [100] \\
\hline $\begin{array}{l}\text { Chitosan/ } \\
\text { chitin }\end{array}$ & PLGA microspheres & EGF & In vitro & {$[134]$} \\
\hline- & PLG microspheres & VEGF, FGF-2 & $\begin{array}{l}\text { In vitro, } \\
\text { in vivo }\end{array}$ & {$[103]$} \\
\hline
\end{tabular}


PLGA nanoparticles embedded in agarose hydrogels have been shown to reduce inflammation and lesion volume following SCI [142]. Lastly, MP co-delivery with other therapeutic molecules, such as minocycline, has been proposed as an effective therapy [144]. The two drugs were delivered in a hemisectioned SCI in rats by means of albumin-coupled, chitosan-stabilized, PLGA nanoparticles and resulted in reduced lesion volume and improved behavioral outcomes compared to direct administration of the same drug combination [144].

p0125 Minocycline is an antibiotic and antiinflammatory drug that has been shown to target secondary injury mechanisms in SCI, such as inflammation, free radicals and oxidative stress, glutamate excitotoxicity, calcium influx, mitochondrial dysfunction, ischemia, hemorrhage, and edema [145]. However, minocycline when administered systemically has shown neuroprotective efficacy only at high doses, which are associated with liver toxicity and even death after prolonged treatment [146]. To reduce off-target effects, minocycline has been targeted directly at activated microglia and macrophages by means of both nonbiodegradable poly(methylmethacrylate) (a mimetic drug compound To-Pro3 was used) [147] and biodegradable PCL-based [148] nanoparticles. Sustained release of minocycline led to reduced cell proliferation and phenotypically altered the cells from a round-shape phagocytic-like to a more arborized resting phenotype; improved outcomes were directly attributed to increased drug uptake by cells compared to free drug [148]. This work underscores the importance of targeting the therapeutic to the desired cell type for increased efficacy. Another group developed an agarose hydrogel loaded with dextran sulfate-minocycline complexes self-assembled by metal ions, which were injectable directly into the intrathecal space for local minocycline delivery [149]. Sustained release of minocycline from this system was shown more effective in reducing secondary injury and promoting locomotor functional recovery than systemic minocycline injection of a 30-40-fold higher dose [149]. Another group showed that administration of minocycline-loaded PCL nanoparticles in an SCI mouse model was able to modulate the resident microglial cells, reducing the proinflammatory response and improving behavioral outcome up to 63 days postinjury [150]. This work also underscored the importance of attenuating microglia activation and M1 macrophages recruitment to the injured site shortly after SCI to limit secondary injury.

p0130 Another small molecule drug considered for SCI recovery is flavopiridol, which is a synthetic cell-cycle inhibitor. Flavopiridol is suggested to act by inhibiting astrocyte proliferation and migration, the synthesis of inflammatory factors and the formation of a glial scar at the site of injury and by promoting neuronal survival $[151,152]$. Although direct intrathecal administration has shown good efficacy in experimental SCI models [151], the drug has significant side effects, such as diarrhea [153]. To alleviate side effects, localized sustained delivery has been proposed by means of PLGA nanoparticles [154]. Localized delivery in a right-hemisection SCI animal model significantly improved motor recovery, increased neuronal preservation and decreased glial scarring and cavitation, and consequently improved the motor recovery of injured animals [154]. Another drug suggested for SCI treatment, 
Chicago sky blue (CSB), is a small molecule inhibitor of macrophage migration inhibitory factor. CSB is structurally related to glutamate, which is a major excitatory CNS neurotransmitter and has been implicated in many pain states. Liposomal delivery of CSB has been shown to protect axons from demyelination and to modulate inflammation [34]. Ferulic acid is another small neuroprotective molecule due to its antiinflammatory and antioxidant properties. When ferulic acid was packed into chitosan nanoparticles, it prolonged their circulation time upon systemic administration and led to significant preservation of axons and myelin and also reduced cavity volume, astrogliosis, and inflammatory response at the lesion site, which resulted in functional recovery following SCI [155].

p0135 Anti-NogoA antibody is another promising therapeutic that acts by blocking the axonal growth inhibitor NogoA and thus, has recently garnered interest in promoting axonal regeneration following SCI. Anti-NogoA has been successfully embedded in PLGA nanoparticles dispersed in a HAMC hydrogel for sustained release, where coencapsulation with additives such as trehalose, significantly improved anti-NogoA bioactivity and co-encapsulation with $\mathrm{MgCO}_{3}$ and $\mathrm{CaCO}_{3}$ prolonged release [127]. This delivery system was further adapted for localized co-delivery of NT-3 and anti-NogoA in an impact/compression model of SCI and led to increased axon density and improved locomotor function [156]. Furthermore, Prostaglandin $\mathrm{E}_{1}\left(\mathrm{PGE}_{1}\right)$ is a naturally occurring small molecule that has multiple therapeutic benefits, such as vasodilation, inhibition of platelet aggregation, cytoprotection, and angiogenesis, which could be beneficial in the treatment of SCI. Takenaga et al. [157] developed Nano $\mathrm{PGE}_{1}$ (nanoparticles containing prostaglandin $\mathrm{E}_{1}$ ) composed of poly(L-lactic acid) and poly(ethylene glycol) (PEG)-poly(D,L-lactide) (PEGPDLLA) block copolymers with a mean diameter of around $100 \mathrm{~nm}$. Their results demonstrated that Nano $\mathrm{PGE}_{1}$ significantly and dose-dependently promoted functional recovery from SCI-induced motor dysfunction animal model, due to the accumulation of nanoparticles in the injured site, where $\mathrm{PGE}_{1}$ release prevented cell death, induced angiogenesis, and improved blood flow [157].

p0140 Dibutyryl cyclic adenosine monophosphate (dbcAMP) is a membranepermeable analogue of cyclic-AMP, which is a downstream molecule in neurotrophin signaling and has been shown to enable neurite outgrowth in the presence of myelin inhibitors both in vitro and in vivo. In one study, sustained release of dbcAMP encapsulated in PLGA microspheres, which were then dispersed in an oligo[(polyethylene glycol)fumarate] (OPF) hydrogel and injected in a transected thoracic spinal cord, rescued mesenchymal stem cells (MSCs)-induced inhibition of axonal regeneration [158]. dbcAMP was also shown to reduce capillary formation in the presence of MSCs, which was coupled with significant functional improvements [158]. Pearse et al. [159] locally injected bolus dbcAMP in conjunction with Schwann cells transplant to improve sparing of injured tissue and behavioral outcomes in injured mice. In another study, Kim et al. [160] showed that controlled release of dbcAMP from PLGA microspheres embedded within chitosan guidance channels, enhanced survival, and guided differentiation of neural stem progenitor cells (NSPCs) in vitro and in vivo. The authors used untreated NSPCs, NSPCs 
transplanted with dbcAMP-releasing microspheres, and NSPCs pre-differentiated with dbcAMP for 4 days in vitro, which were then transplanted after rat spinal cord transection and assessed 2 and 6 weeks later. Interestingly, NSPC survival was highest in the dbcAMP-pre-treated group, having $\sim 80 \%$ survival, which is highly significant given that stem-cell transplantation usually results in $\sim 1 \%$ survival [160]. At 6 weeks, the animals implanted with dbcAMP-pre-differentiated NSPCs also saw extensive axonal regeneration and improved functional recovery [160].

p0145 Other molecules that have been implicated in reducing neuronal damage and hence improving functional recovery after nerve injuries are thrombin inhibitors. Thrombin has been suggested to play an important role in inflammatory responses to nerve injury, leading to scar formation and reactive gliosis [161]. For example, a recent small clinical study suggested that antithrombin therapy with intravenously administered argatroban may be useful in the treatment of intracerebral hemorrhage; intracerebral hemorrhage typically leads to high morbidity of neurons and even mortality following injury [162]. Another thrombin inhibitor, hirudin, released in a controlled manner from PLGA microspheres embedded in Pluronic F-127 hydrogels and implanted in a lateral demyelination lesion animal model, led to a reduction in activated macrophages, improved motor function recovery and increase in oligodendrocytes [163]. Reukov et al. [164] found that superoxide dismutase (SOD) and antiglutamate N-methyl D-aspartate receptor 1 (NR1) can be attached simultaneously to poly(butyl cyanoacrylate) (PBCA) nanoparticles, showing neuroprotective efficacy in vitro with rat cerebellar cell cultures challenged by superoxide. Degradable and biocompatible free radical scavengers, such as SOD, can lead to a reduction in cell damage during secondary injury.

p0150 Nanomaterials themselves can be neuroprotective and can penetrate the bloodbrain barrier if they are less than $200 \mathrm{~nm}$. For example, intravenously injected micelles composed of self-assembled monomethoxy poly(ethylene glycol)-poly(D,Llactic acid) (mPEG-PDLLA) diblock copolymers were shown to recover locomotor function and reduce the lesion volume and the inflammatory reaction in SCI rats [165]. Furthermore, chitosan, which is a natural, nontoxic, biodegradable, polycationic polymer, has been shown to serve as a suppressor of reactive oxygen species and lipid peroxidation of cell membranes, hence preserving neural cells following acute SCI [166]. It has been demonstrated that chitosan nanoparticles restored nerve impulse transmission after severe compression injury in guinea pigs [167]. A summary of the delivery approaches for small molecule delivery in SCI repair is presented in Table 12.2.

\section{s0055 Summary}

p0155 A number of studies involving various therapeutic molecules and delivery approaches for SCI repair were reviewed in this chapter. In particular, we highlighted the importance of the delivery method to maximize therapeutic efficacy while 
t0015 Table 12.2 Hydrogels and hydrogel particles for the delivery of other therapeutics in SCl repair.

\begin{tabular}{|c|c|c|c|c|}
\hline Matrix & Particles & Therapeutics & In vitro/in vivo & References \\
\hline- & PLGA nanoparticles & MP & In vitro, in vivo & [143] \\
\hline Agarose & PLGA nanoparticles & MP & In vivo & [142] \\
\hline- & CMCht/PAMAM dendrimer nanoparticles & MP & In vitro, in vivo & [5] \\
\hline Chitosan & PLGA nanoparticles & MP, minocycline & In vivo & [144] \\
\hline- & PLGA nanoparticles & Flavopiridol & In vitro, in vivo & [154] \\
\hline HAMC & PLGA nanoparticles & Anti-NogoA & In vitro & [127] \\
\hline HAMC & PLGA nanoparticles & Anti-NogoA, NT-3 & In vivo & [156] \\
\hline OPF hydrogel & PLGA microspheres & dbcAMP & In vitro, in vivo & [158] \\
\hline Chitosan NGCs & PLGA microspheres & dbcAMP & In vitro, in vivo & [160] \\
\hline- & - & dbcAMP + Schwann cells & In vivo & [159] \\
\hline- & Liposomal nanocarrier & CSB & In vitro, in vivo & [34] \\
\hline- & PEG-PDLLA nanoparticles & PGE1 & In vitro, in vivo & [118] \\
\hline Agarose & PMMA nanoparticles & To-Pro3 & In vitro, in vivo & [147] \\
\hline- & PCL nanoparticles & Minocycline & In vitro, in vivo & {$[148,150]$} \\
\hline Agarose & $\begin{array}{l}\text { Self-assembled dextran sulfate-minocycline } \\
\text { complexes }\end{array}$ & Minocycline & In vivo & [149] \\
\hline- & mPEG-PDLLA diblock copolymer micelles & - & In vivo & [165] \\
\hline- & Chitosan nanoparticles & - & In vivo & [167] \\
\hline- & PBCA nanoparticles & SOD and NR1 & In vitro, in vivo & [164] \\
\hline $\begin{array}{l}\text { Pluronic F-127 } \\
\text { hydrogels }\end{array}$ & PLGA microspheres & Hirudin & In vitro, in vivo & [163] \\
\hline- & Chitosan nanoparticles & Ferulic acid & In vitro, in vivo & [155] \\
\hline
\end{tabular}


reducing unwanted side effects. Traditional methods of delivery, including oral, topical, and intravenous, are simple to administer, but have multiple disadvantages. For example, neurotrophic factors have poor pharmacokinetic properties: they are rapidly cleared from the body and cannot penetrate biological barriers. They also have broad biological activity, which implies that they will have multiple site effects when not targeted to the site of injury. Small molecule drugs, such as MP, which have multiple dangerous side effects, are also excellent candidates for localized delivery to reduce drug concentration at off-target sites. Here, we describe some of the techniques currently being developed to enable sustained and localized delivery of neurotrophic factors: drug-loaded injectable hydrogel/matrix, drug-loaded micro- or nanoparticles, or a combination of the two. Localizing the drug delivery provides two main benefits: a higher concentration at the target site and a significant reduction in potential adverse effects. Sustained and localized delivery of therapeutic molecules has been shown to lead to improved functional recovery in animal models and has a tremendous translational potential.

\section{References}

[1] Park J, et al. Nerve regeneration following spinal cord injury using matrix metalloproteinase-sensitive, hyaluronic acid-based biomimetic hydrogel scaffold containing brain-derived neurotrophic factor. J Biomed Mater Res A 2009;93A(3): 1091-9.

[2] Spinal cord injury facts and figures at a glance. J Spinal Cord Med 2012;35(6):480-1.

[3] Carlson GD, Gorden C. Current developments in spinal cord injury research. Spine J 2002;2(2):116-28.

[4] Sharma HS. A select combination of neurotrophins enhances neuroprotection and functional recovery following spinal cord injury. Ann N Y Acad Sci 2007;1122(1): 95-111.

[5] Cerqueira SR, et al. Microglia response and in vivo therapeutic potential of methylprednisolone-loaded dendrimer nanoparticles in spinal cord injury. Small 2013;9(5):738-49.

[6] Varma A, et al. Spinal cord injury: a review of current therapy, future treatments, and basic science frontiers. Neurochem Res 2013;38(5):895-905.

[7] Kan M, Ling EA, Lu J. Stem cell therapy for spinal cord injury. Curr Med Chem 2010; 17(36):4492-510.

[8] Srikanth M, Kessler JA. Nanotechnology-novel therapeutics for CNS disorders. Nat Rev Neurol 2012;8(6):307-18.

[9] Wilson JR, Forgione N, Fehlings MG. Emerging therapies for acute traumatic spinal cord injury. CMAJ 2013;185(6):485-92.

[10] Wilcox JT, Cadotte D, Fehlings MG. Spinal cord clinical trials and the role for bioengineering. Neurosci Lett 2012;519(2):93-102.

[11] McDonald JW, Sadowsky C. Spinal-cord injury. The Lancet 2002;359(9304):417-25.

[12] Coumans JV, et al. Axonal regeneration and functional recovery after complete spinal cord transection in rats by delayed treatment with transplants and neurotrophins. J Neurosci 2001;21(23):9334-44. 
[13] McCreedy DA, Sakiyama-Elbert SE. Combination therapies in the CNS: engineering the environment. Neurosci Lett 2012;519(2):115-21.

[14] Profyris C, et al. Degenerative and regenerative mechanisms governing spinal cord injury. Neurobiol Dis 2004;15(3):415-36.

[15] Piantino J, et al. An injectable, biodegradable hydrogel for trophic factor delivery enhances axonal rewiring and improves performance after spinal cord injury. Exp Neurol 2006;201(2):359-67.

[16] Taylor L, et al. Neurotrophin-3 gradients established by lentiviral gene delivery promote short-distance axonal bridging beyond cellular grafts in the injured spinal cord. J Neurosci 2006;26(38):9713-21.

[17] Novikova LN, Novikov LN, Kellerth J-O. Differential effects of neurotrophins on neuronal survival and axonal regeneration after spinal cord injury in adult rats. J Comp Neurol 2002;452(3):255-63.

[18] Nomura H, Tator CH, Shoichet MS. Bioengineered strategies for spinal cord repair. J Neurotrauma 2006;23(3-4):496-507.

[19] Katz JS, Burdick JA. Hydrogel mediated delivery of trophic factors for neural repair. Wiley Interdiscip Rev 2009;1(1):128-39.

[20] Tabesh $\mathrm{H}$, et al. The role of biodegradable engineered scaffolds seeded with Schwann cells for spinal cord regeneration. Neurochem Int 2009;54(2):73-83.

[21] des Rieux A, et al. Vascular endothelial growth factor-loaded injectable hydrogel enhances plasticity in the injured spinal cord. J Biomed Mater Res A 2014;102(7): 2345-55.

[22] Wu M-c, et al. Cellular transplantation-based evolving treatment options in spinal cord injury. Cell Biochem Biophys 2015;71(1):1-8.

[23] Jin Y, et al. Transplants of fibroblasts genetically modified to express BDNF promote axonal regeneration from supraspinal neurons following chronic spinal cord injury. Exp Neurol 2002;177(1):265-75.

[24] Shrestha B, et al. Repair of injured spinal cord using biomaterial scaffolds and stem cells. Stem Cell Res Ther 2014;5(4):91.

[25] Shen Y, et al. Guidance of olfactory ensheathing cell growth and migration on electrospun silk fibroin scaffolds. Cell Transplant 2010;19(2):147-57.

[26] Pearse DD, et al. cAMP and Schwann cells promote axonal growth and functional recovery after spinal cord injury. Nat Med 2004;10:610-6.

[27] Biernaskie J, et al. Skin-derived precursors generate myelinating Schwann cells that promote remyelination and functional recovery after contusion spinal cord injury. J Neurosci 2007;27(36):9545-59.

[28] Wright KT, et al. Concise review: bone marrow for the treatment of spinal cord injury: mechanisms and clinical applications. Stem Cell 2011;29(2):169-78.

[29] Silva NA, et al. From basics to clinical: a comprehensive review on spinal cord injury. Prog Neurobiol 2014;114(0):25-57.

[30] Thuret S, Moon LDF, Gage FH. Therapeutic interventions after spinal cord injury. Nat Rev Neurosci 2006;7(8):628-43.

[31] Willerth SM, Sakiyama-Elbert SE. Cell therapy for spinal cord regeneration. Adv Drug Deliv Rev 2008;60(2):263-76.

[32] Bregman BS, et al. Neurotrophic factors increase axonal growth after spinal cord injury and transplantation in the adult rat. Exp Neurol 1997;148(2):475-94.

[33] Li X-L, et al. Temporal changes in the expression of some neurotrophins in spinal cord transected adult rats. Neuropeptides 2007;41(3):135-43. 
[34] Saxena T, et al. Nanocarrier-Mediated inhibition of macrophage migration inhibitory factor Attenuates secondary injury after spinal cord injury. ACS Nano 2015;9(2): 1492-505.

[35] Kubinová Š, Syková E. Nanotechnology for treatment of stroke and spinal cord injury. Nanomedicine 2010;5(1):99-108.

[36] Elliott Donaghue I, et al. Cell and biomolecule delivery for tissue repair and regeneration in the central nervous system. J Control Release 2014;190(0):219-27.

[37] Hoffman AS. Hydrogels for biomedical applications. Adv Drug Deliv Rev 2012;64: $18-23$.

[38] Ordikhani F, Sheth S, Zustiak SP. Polymeric particle-mediated molecular therapies to treat spinal cord injury. Int J Pharm 2017;516(1-2):71-81.

[39] Zhang N, Yan H, Wen X. Tissue-engineering approaches for axonal guidance. Brain Res Rev 2005;49(1):48-64.

[40] Little L, Healy KE, Schaffer D. Engineering biomaterials for synthetic neural stem cell microenvironments. Chem Rev 2008;108(5):1787-96.

[41] Plant GW, Harvey AR, Chirila TV. Axonal growth within poly (2-hydroxyethyl methacrylate) sponges infiltrated with Schwann cells and implanted into the lesioned rat optic tract. Brain Res 1995;671(1):119-30.

[42] Plant GW, Woerly S, Harvey AR. Hydrogels containing peptide or aminosugar sequences implanted into the rat brain: influence on cellular migration and axonal growth. Exp Neurol 1997;143(2):287-99.

[43] Assuncao-Silva RC, et al. Hydrogels and cell based therapies in spinal cord injury regeneration. Stem Cell Int 2015;2015:24.

[44] Macaya D, Spector M. Injectable hydrogel materials for spinal cord regeneration: a review. Biomed Mater 2012;7(1):012001.

[45] Jain A, et al. In situ gelling hydrogels for conformal repair of spinal cord defects, and local delivery of BDNF after spinal cord injury. Biomaterials 2006;27(3):497-504.

[46] Ziemba AM, Gilbert RJ. Biomaterials for local, controlled drug delivery to the injured spinal cord. Front Pharmacol 2017;8(245).

[47] Perale G, et al. Hydrogels in spinal cord injury repair strategies. ACS Chem Neurosci 2011;2(7):336-45.

[48] Gao M, et al. Templated agarose scaffolds for the support of motor axon regeneration into sites of complete spinal cord transection. Biomaterials 2013;34(5):1529-36.

[49] Leonard M, et al. Hydrophobically modified alginate hydrogels as protein carriers with specific controlled release properties. J Control Release 2004;98(3):395-405.

[50] Lee KY, et al. Controlled growth factor release from synthetic extracellular matrices. Nature 2000;408(6815):998.

[51] Gorgieva S, Kokol V. Collagen-vs. gelatine-based biomaterials and their biocompatibility: review and perspectives. In: Biomaterials applications for nanomedicine. InTech; 2011.

[52] Lynn A, Yannas I, Bonfield W. Antigenicity and immunogenicity of collagen. J Biomed Mater Res B Appl Biomater 2004;71(2):343-54.

[53] Mosesson M. Fibrinogen and fibrin structure and functions. J Thromb Haemost 2005; 3(8):1894-904.

[54] Sharp KG, et al. Salmon fibrin treatment of spinal cord injury promotes functional recovery and density of serotonergic innervation. Exp Neurol 2012;235(1):345-56.

[55] Bensald W, et al. A biodegradable fibrin scaffold for mesenchymal stem cell transplantation. Biomaterials 2003;24(14):2497-502. 
[56] Sieminski AL, Gooch KJ. Salmon fibrin supports an more sprouts and decreased degradation while maintaining sprout length relative to human fibrin in an in vitro angiogenesis model. J Biomater Sci Polym Ed 2004;15(2):237-42.

[57] Fischer MB, et al. Binding of disease-associated prion protein to plasminogen. Nature 2000;408(6811):479-83.

[58] Schachtrup C, et al. Fibrinogen inhibits neurite outgrowth via $\beta 3$ integrin-mediated phosphorylation of the EGF receptor. Proc Natl Acad Sci USA 2007;104(28): 11814-9.

[59] Schachtrup C, et al. Fibrinogen triggers astrocyte scar formation by promoting the availability of active TGF-beta after vascular damage. J Neurosci 2010;30(17): $5843-54$

[60] Straley KS, Po Foo CW, Heilshorn SC. Biomaterial design strategies for the treatment of spinal cord injuries. J Neurotrauma 2010;27(1):1-19.

[61] Zhu J, Marchant RE. Design properties of hydrogel tissue-engineering scaffolds. Expert Rev Med Devices 2011;8(5):607-26.

[62] Freudenberg U, et al. A star-PEG-heparin hydrogel platform to aid cell replacement therapies for neurodegenerative diseases. Biomaterials 2009;30(28):5049-60.

[63] Deiber JA, et al. Characterization of cross-linked polyampholytic gelatin hydrogels through the rubber elasticity and thermodynamic swelling theories. Polymer 2009; 50(25):6065-75.

[64] Lutolf MP. Biomaterials: spotlight on hydrogels. Nat Mater 2009;8(6):451-3.

[65] Wang HB, et al. Creation of highly aligned electrospun poly-L-lactic acid fibers for nerve regeneration applications. J Neural Eng 2008;6(1):016001.

[66] Hurtado A, et al. Robust CNS regeneration after complete spinal cord transection using aligned poly-L-lactic acid microfibers. Biomaterials 2011;32(26):6068-79.

[67] Olson HE, et al. Neural stem cell- and Schwann cell-loaded biodegradable polymer scaffolds support axonal regeneration in the transected spinal cord. Tissue Eng 2009;15(7):1797-805.

[68] Fan J, et al. Neural regrowth induced by PLGA nerve conduits and neurotrophin-3 in rats with complete spinal cord transection. J Biomed Mater Res B Appl Biomater 2011;97B(2):271-7.

[69] Luo J, Borgens R, Shi R. Polyethylene glycol immediately repairs neuronal membranes and inhibits free radical production after acute spinal cord injury. J Neurochem 2002;83(2):471-80.

[70] Shi R, Borgens RB. Acute repair of crushed Guinea pig spinal cord by polyethylene glycol. J Neurophysiol 1999;81(5):2406-14.

[71] Zustiak SP, et al. Hydrolytically degradable poly (ethylene glycol) hydrogel scaffolds as a cell delivery vehicle: characterization of PC12 cell response. Biotechnol Prog 2013;29(5):1255-64.

[72] Tsai EC, et al. Synthetic hydrogel guidance channels facilitate regeneration of adult rat brainstem motor axons after complete spinal cord transection. J Neurotrauma 2004; 21(6):789-804.

[73] Tsai EC, et al. Matrix inclusion within synthetic hydrogel guidance channels improves specific supraspinal and local axonal regeneration after complete spinal cord transection. Biomaterials 2006;27(3):519-33.

[74] Patist CM, et al. Freeze-dried poly(D,L-lactic acid) macroporous guidance scaffolds impregnated with brain-derived neurotrophic factor in the transected adult rat thoracic spinal cord. Biomaterials 2004;25(9):1569-82. 
[75] Woerly S, et al. Spinal cord repair with PHPMA hydrogel containing RGD peptides (NeuroGel). Biomaterials 2001;22(10):1095-111.

[76] Woerly S, et al. Heterogeneous PHPMA hydrogels for tissue repair and axonal regeneration in the injured spinal cord. J Biomater Sci Polym Ed 1998;9(7):681-711.

[77] Lopes MS, Jardini A, Maciel Filho R. Poly (lactic acid) production for tissue engineering applications. Proc Eng 2012;42:1402-13.

[78] Fukushima K, Kimura Y. An efficient solid-state polycondensation method for synthesizing stereocomplexed poly(lactic acid)s with high molecular weight. J Polym Sci A Polym Chem 2008;46(11):3714-22.

[79] Gentile P, et al. An overview of poly(lactic-co-glycolic) acid (PLGA)-Based biomaterials for bone tissue engineering. Int J Mol Sci 2014;15(3):3640-59.

[80] Kwon BK, Sekhon LH, Fehlings MG. Emerging repair, regeneration, and translational research advances for spinal cord injury. Spine 2010;35(21S):S263-70.

[81] Lee JY, et al. Polypyrrole-coated electrospun PLGA nanofibers for neural tissue applications. Biomaterials 2009;30(26):4325-35.

[82] Zustiak SP, Leach JB. Hydrolytically degradable poly (ethylene glycol) hydrogel scaffolds with tunable degradation and mechanical properties. Biomacromolecules 2010; 11(5): $1348-57$.

[83] Jain E, et al. Control of gelation, degradation and physical properties of polyethylene glycol hydrogels through the chemical and physical identity of the crosslinker. J Mater Chem B 2017;5(14):2679-91.

[84] Lutolf M, Hubbell J. Synthesis and physicochemical characterization of end-linked poly (ethylene glycol)-co-peptide hydrogels formed by Michael-type addition. Biomacromolecules 2003;4(3):713-22.

[85] Dalton PD, Flynn L, Shoichet MS. Manufacture of poly (2-hydroxyethyl methacrylateco-methyl methacrylate) hydrogel tubes for use as nerve guidance channels. Biomaterials 2002;23(18):3843-51.

[86] Skaper SD. The neurotrophin family of neurotrophic factors: an overview. In: Neurotrophic factors. Springer; 2012. p. 1-12.

[87] Lindsay RM, et al. Neurotrophic factors: from molecule to man. Trends Neurosci 1994;17(5):182-90.

[88] Lu P, et al. Neural stem cells constitutively secrete neurotrophic factors and promote extensive host axonal growth after spinal cord injury. Exp Neurol 2003;181(2): 115-29.

[89] Hughes SM, et al. Ciliary neurotrophic factor induces type-2 astrocyte differentiation in culture. Nature 1988;335(6185):70.

[90] Knüsel B, et al. Promotion of central cholinergic and dopaminergic neuron differentiation by brain-derived neurotrophic factor but not neurotrophin 3. Proc Natl Acad Sci USA 1991;88(3):961-5.

[91] Cohen S, Levi-Montalcini R, Hamburger V. A nerve growth-stimulating factor isolated from sarcom as 37 and 180. Proc Natl Acad Sci USA 1954;40(10):1014-8.

[92] Skaper SD. Nerve growth factor. Mol Neurobiol 2001;24(1-3):183-99.

[93] Chen G, et al. Combined treatment with FK506 and nerve growth factor for spinal cord injury in rats. Exp Therap Med 2013;6(4):868-72.

[94] Tuszynski MH, et al. Nerve growth factor delivery by gene transfer induces differential outgrowth of sensory, motor, and noradrenergic neurites after adult spinal cord injury. Exp Neurol 1996;137(1):157-73. 
[95] Guo J, et al. Cotransplant of neural stem cells and NT-3 gene modified Schwann cells promote the recovery of transected spinal cord injury. Spinal Cord 2007;45(1):15.

[96] Hapner SJ, et al. Neural differentiation promoted by truncated trkC receptors in collaboration with p75NTR. Dev Biol 1998;201(1):90-100.

[97] Balaratnasingam S, Janca A. Brain derived neurotrophic factor: a novel neurotrophin involved in psychiatric and neurological disorders. Pharmacol Ther 2012;134(1): $116-24$.

[98] Iannotti C, et al. A neuroprotective role of glial cell line-derived neurotrophic factor following moderate spinal cord contusion injury. Exp Neurol 2004;189(2):317-32.

[99] Cao Q, et al. Transplantation of CNTF-expressing adult oligodendrocyte precursor cells promotes remyelination and functional recovery after spinal cord injury. J Neurosci 2010;30(8):2989-3001.

[100] Jimenez Hamann MC, Tator CH, Shoichet MS. Injectable intrathecal delivery system for localized administration of EGF and FGF-2 to the injured rat spinal cord. Exp Neurol 2005;194(1):106-19.

[101] Vergara C, Ramirez B. CNTF, a pleiotropic cytokine: emphasis on its myotrophic role. Brain Res Rev 2004;47(1-3):161-73.

[102] Ishii K, et al. Neutralization of ciliary neurotrophic factor reduces astrocyte production from transplanted neural stem cells and promotes regeneration of corticospinal tract fibers in spinal cord injury. J Neurosci Res 2006;84(8):1669-81.

[103] De Laporte L, et al. VEGF and FGF-2 delivery from spinal cord bridges to enhance angiogenesis following injury. J Biomed Mater Res A 2011;98(3):372-82.

[104] Widenfalk J, et al. Vascular endothelial growth factor improves functional outcome and decreases secondary degeneration in experimental spinal cord contusion injury. Neuroscience 2003;120(4):951-60.

[105] Kang CE, et al. Localized and sustained delivery of fibroblast growth factor-2 from a nanoparticle-hydrogel composite for treatment of spinal cord injury. Cells Tissues Organs 2012;197(1):55-63.

[106] Baumann MD, et al. An injectable drug delivery platform for sustained combination therapy. J Control Release 2009;138(3):205-13.

[107] Rabchevsky AG, et al. Basic fibroblast growth factor (bFGF) enhances functional recovery following severe spinal cord injury to the rat. Exp Neurol 2000;164(2):280-91.

[108] Reimer MM, et al. Sonic hedgehog is a polarized signal for motor neuron regeneration in adult zebrafish. J Neurosci 2009;29(48):15073-82.

[109] Geral C, Angelova A, Lesieur S. From molecular to nanotechnology strategies for delivery of neurotrophins: emphasis on brain-derived neurotrophic factor (BDNF). Pharmaceutics 2013;5(1):127-67.

[110] Thorne RG, et al. Delivery of insulin-such as growth factor-I to the rat brain and spinal cord along olfactory and trigeminal pathways following intranasal administration. Neuroscience 2004;127(2):481-96.

[111] Hendriks WTJ, et al. Viral vector-mediated gene transfer of neurotrophins to promote regeneration of the injured spinal cord. In: Progress in brain research. Elsevier; 2004. p. 451-76.

[112] Baumann MD, et al. Intrathecal delivery of a polymeric nanocomposite hydrogel after spinal cord injury. Biomaterials 2010;31(30):7631-9.

[113] Wang Y-C, et al. Sustained intraspinal delivery of neurotrophic factor encapsulated in biodegradable nanoparticles following contusive spinal cord injury. Biomaterials 2008;29(34):4546-53. 
[114] Madigan NN, et al. Current tissue engineering and novel therapeutic approaches to axonal regeneration following spinal cord injury using polymer scaffolds. Respir Physiol Neurobiol 2009;169(2):183-99.

[115] Elliott Donaghue I, Tator CH, Shoichet MS. Sustained delivery of bioactive neurotrophin-3 to the injured spinal cord. Biomater Sci 2015;3(1):65-72.

[116] Kraskiewicz H, et al. Assembly of protein-based hollow spheres encapsulating a therapeutic factor. ACS Chem Neurosci 2013;4(9):1297-304.

[117] Gu H, Yue Z. Controlled release strategy based on biodegradable microspheres for neurodegenerative disease therapy. INTECH Open Access Publisher; 2012.

[118] Kokai LE, et al. Protein bioactivity and polymer orientation is affected by stabilizer incorporation for double-walled microspheres. J Control Release 2010;141(2): $168-76$.

[119] Filippo R, et al. Polymer hydrogel functionalized with biodegradable nanoparticles as composite system for controlled drug delivery. Nanotechnology 2015;26(1):015602.

[120] Kumar P, et al. Nanoparticulate strategies for the five R's of traumatic spinal cord injury intervention: restriction, repair, regeneration, restoration and reorganization. Nanomedicine 2014;9(2):331-48.

[121] Benoit J-P, et al. Development of microspheres for neurological disorders: from basics to clinical applications. J Control Release 2000;65(1-2):285-96.

[122] Burdick JA, et al. Stimulation of neurite outgrowth by neurotrophins delivered from degradable hydrogels. Biomaterials 2006;27(3):452-9.

[123] Lowry N, et al. The effect of long-term release of Shh from implanted biodegradable microspheres on recovery from spinal cord injury in mice. Biomaterials 2012;33(10): 2892-901.

[124] Gupta D, Tator CH, Shoichet MS. Fast-gelling injectable blend of hyaluronan and methylcellulose for intrathecal, localized delivery to the injured spinal cord. Biomaterials 2006;27(11):2370-9.

[125] Kang CE, et al. A new paradigm for local and sustained release of therapeutic molecules to the injured spinal cord for neuroprotection and tissue repair. Tissue Eng 2008; 15(3):595-604.

[126] Kang CE, et al. Spinal cord blood flow and blood vessel permeability measured by dynamic computed tomography imaging in rats after localized delivery of fibroblast growth factor. J Neurotrauma 2010;27(11):2041-53.

[127] Stanwick JC, Baumann MD, Shoichet MS. In vitro sustained release of bioactive antiNogoA, a molecule in clinical development for treatment of spinal cord injury. Int J Pharm 2012;426(1-2):284-90.

[128] Stanwick JC, Baumann MD, Shoichet MS. Enhanced neurotrophin-3 bioactivity and release from a nanoparticle-loaded composite hydrogel. J Control Release 2012; 160(3):666-75.

[129] Ansorena E, et al. Injectable alginate hydrogel loaded with GDNF promotes functional recovery in a hemisection model of spinal cord injury. Int J Pharm 2013;455(1-2): $148-58$.

[130] Wood MD, et al. Fibrin gels containing GDNF microspheres increase axonal regeneration after delayed peripheral nerve repair. Regen Med 2013;8(1):27-37.

[131] Wang Y, et al. Combination of hyaluronic acid hydrogel scaffold and PLGA microspheres for supporting survival of neural stem cells. Pharmaceut Res 2011;28(6): $1406-14$. 
[132] Yang Z, et al. The effect of the dosage of NT-3/chitosan carriers on the proliferation and differentiation of neural stem cells. Biomaterials 2010;31(18):4846-54.

[133] Wang N, et al. Sodium hyaluronate-CNTF gelatinous particles promote axonal growth, neurogenesis and functional recovery after spinal cord injury. Spinal Cord 2014;52(7): 517-23.

[134] Goraltchouk A, et al. Incorporation of protein-eluting microspheres into biodegradable nerve guidance channels for controlled release. J Control Release 2006;110(2):400-7.

[135] Piotrowicz A, Shoichet MS. Nerve guidance channels as drug delivery vehicles. Biomaterials 2006;27(9):2018-27.

[136] Yang Y, et al. Neurotrophin releasing single and multiple lumen nerve conduits. J Control Release 2005;104(3):433-46.

[137] Tobias CA, et al. Grafting of encapsulated BDNF-producing fibroblasts into the injured spinal cord without immune suppression in adult rats. J Neurotrauma 2001; 18(3):287-301.

[138] Tobias CA, et al. Alginate encapsulated BDNF-producing fibroblast grafts permit recovery of function after spinal cord injury in the absence of immune suppression. J Neurotrauma 2005;22(1):138-56.

[139] Kokai LE, Ghaznavi AM, Marra KG. Incorporation of double-walled microspheres into polymer nerve guides for the sustained delivery of glial cell line-derived neurotrophic factor. Biomaterials 2010;31(8):2313-22.

[140] Wang Y, et al. Combination of hyaluronic acid hydrogel scaffold and PLGA microspheres for supporting survival of neural stem cells. Pharmaceut Res 2011;28(6):1406.

[141] Bracken MB, et al. A randomized, controlled trial of methylprednisolone or naloxone in the treatment of acute spinal-cord injury. N Engl J Med 1990;322(20):1405-11.

[142] Chvatal SA, et al. Spatial distribution and acute anti-inflammatory effects of Methylprednisolone after sustained local delivery to the contused spinal cord. Biomaterials 2008;29(12):1967-75.

[143] Kim Y-t, Caldwell J-M, Bellamkonda RV. Nanoparticle-mediated local delivery of methylprednisolone after spinal cord injury. Biomaterials 2009;30(13):2582-90.

[144] Bin S, et al. Nano-carrier mediated co-delivery of methyl prednisolone and minocycline for improved post-traumatic spinal cord injury conditions in rats. Drug Dev Industr Pharm 2017;43(6):1033-41.

[145] Shultz RB, Zhong Y. Minocycline targets multiple secondary injury mechanisms in traumatic spinal cord injury. Neural Regen Res 2017;12(5):702.

[146] Casha S, et al. Results of a phase II placebo-controlled randomized trial of minocycline in acute spinal cord injury. Brain 2012;135(4):1224-36.

[147] Papa S, et al. Polymeric nanoparticle system to target activated microglia/macrophages in spinal cord injury. J Control Release 2014;174(0):15-26.

[148] Papa S, et al. Selective nanovector mediated treatment of activated proinflammatory microglia/macrophages in spinal cord injury. ACS Nano 2013;7(11):9881-95.

[149] Wang Z, et al. Local delivery of minocycline from metal ion-assisted self-assembled complexes promotes neuroprotection and functional recovery after spinal cord injury. Biomaterials 2017;112:62-71.

[150] Papa S, et al. Early modulation of pro-inflammatory microglia by minocycline loaded nanoparticles confers long lasting protection after spinal cord injury. Biomaterials 2016;75:13-24.

[151] Li C, et al. Intrathecal administration of flavopiridol promotes regeneration in experimental model of spinal cord injury. Turk Neurosurg 2016;26(6):922-9. 
[152] Wu J, et al. Delayed cell cycle pathway modulation facilitates recovery after spinal cord injury. Cell Cycle 2012;11(9):1782-95.

[153] Rudek MA, et al. Clinical pharmacology of flavopiridol following a 72-hour continuous infusion. Ann Pharmacother 2003;37(10):1369-74.

[154] Ren H, et al. Repair of spinal cord injury by inhibition of astrocyte growth and inflammatory factor synthesis through local delivery of flavopiridol in PLGA nanoparticles. Biomaterials 2014;35(24):6585-94.

[155] Wu W, et al. Neuroprotective ferulic acid (FA)-glycol chitosan (GC) nanoparticles for functional restoration of traumatically injured spinal cord. Biomaterials 2014;35(7): 2355-64.

[156] Elliott Donaghue I, Tator CH, Shoichet MS. Local delivery of neurotrophin-3 and antiNogoA promotes repair after spinal cord injury. Tissue Eng 2016;22(9-10):733-41.

[157] Takenaga M, et al. Nano PGE1 promoted the recovery from spinal cord injury-induced motor dysfunction through its accumulation and sustained release. J Control Release 2010;148(2):249-54.

[158] Rooney GE, et al. Sustained delivery of dibutyryl cyclic adenosine monophosphate to the transected spinal cord via oligo [(Polyethylene glycol) fumarate] hydrogels. Tissue Eng 2011;17(9-10):1287-302.

[159] Pearse DD, et al. cAMP and Schwann cells promote axonal growth and functional recovery after spinal cord injury. Nat Med 2004;10(6):610.

[160] Kim H, et al. Effects of dibutyryl cyclic-AMP on survival and neuronal differentiation of neural stem/progenitor cells transplanted into spinal cord injured rats. PLoS One 2011;6(6):e21744.

[161] NISHINO A, et al. Thrombin may contribute to the pathophysiology of central nervous system injury. J Neurotrauma 1993;10(2):167-79.

[162] Matsuoka H, Hamada R. Role of thrombin in CNS damage associated with intracerebral haemorrhage. CNS Drugs 2002;16(8):509-16.

[163] Sellers DL, et al. Poly(lactic-co-glycolic) acid microspheres encapsulated in Pluronic F-127 prolong hirudin delivery and improve functional recovery from a demyelination lesion. Biomaterials 2014;35(31):8895-902.

[164] Reukov V, Maximov V, Vertegel A. Proteins conjugated to poly (butyl cyanoacrylate) nanoparticles as potential neuroprotective agents. Biotechnol Bioeng 2011;108(2): $243-52$.

[165] Shi Y, et al. Effective repair of traumatically injured spinal cord by nanoscale block copolymer micelles. Nat Nanotechnol 2010;5(1):80-7.

[166] Cho Y, Shi R, Borgens RB. Chitosan produces potent neuroprotection and physiological recovery following traumatic spinal cord injury. J Exp Biol 2010;213(9):1513-20.

[167] Chen B, et al. Pushing the science forward: chitosan nanoparticles and functional repair of CNS tissue after spinal cord injury. J Biol Eng 2013;7(1):15. 


\title{
Non-Print Items
}

\begin{abstract}
Spinal cord injury (SCI) is one of the major causes of irreversible nerve injury, affecting both sensory and motor neurons, which poses a significant physical, emotional, and economic burden on both the individual and society. Current treatments are aimed at symptomatic alleviation and do not lead to complete functional recovery. In this chapter, we describe emerging pharmacological therapies being developed to facilitate functional recovery upon SCI, with primary focus on the delivery of small molecule pharmaceuticals, growth factors, and neurotrophins. We also discuss biomaterial scaffolds that provide a permissive environment for neural regeneration, while serving as deports for pharmaceuticals release. Current research suggests that sustained and localized delivery of therapeutic molecules leads to improved functional recovery in animal models and has a tremendous translational potential.
\end{abstract}

\section{Keywords:}

Drug delivery, Growth factors, Hydrogels, Microparticles, Nanoparticles, Neurotrophins, Pharmaceuticals, Spinal cord injury. 


\title{
CHAPTER
}

\section{c0013 Reliability on animal models}

\author{
María Permuy, DVM, PhD, Mónica López-Peña, PhD, \\ Antonio González-Cantalapiedra, DVM, PhD, \\ Fernando María Muñoz Guzón, DVM, PhD
}

Department of Anatomy, Animal Production and Veterinary Clinical Sciences, Universidade de Santiago de Compostela, Lugo, Spain

\section{s0010 Introduction}

p0010 Animal models of spinal cord injury (SCI) are necessary not only for evaluating new therapies and surgical techniques but also to improve understanding of the molecular pathways involved [1] and predict clinical outcomes and prognosis.

p0015 The ideal animal models should mimic the pathological features of human SCI, be reproducible and available, and have the capacity to create different degrees of SCI severity and functional outcomes [2]. However, before choosing an appropriate animal model of SCI, it is necessary to identify the main aims and purpose of the study.

p0020 This chapter describes the main species and models available for use in SCI preclinical research. The authors highlight the regulations, ethical aspects, and publications related to using animals in research. Finally, we provide advice that we consider useful for researchers to design a protocol.

\section{s0015 Animal species used in experimental spinal cord injury repair}

p0025 In relation to animal species used as SCI experimental models, one review published in 2017 on over 2209 SCI animal studies concluded that the most commonly used animal species in SCI research was the rat (72.4\%) followed by mice (16\%) and rabbits $(2.4 \%)$. For large animal models in that review, the dog was the most common model used $(2.3 \%)$, followed by cats $(2.2 \%)$, pigs $(1.5 \%)$, and nonhuman primates $(1.5 \%)$; the minority of the models included sheep and goats [3]. Advantages and disadvantages of each species are summarized in Table 13.1. 
t0010 Table 13.1 Animal species used in spinal cord injury research.

\begin{tabular}{|c|c|c|c|}
\hline Species & Advantages & Disadvantages & Main models \\
\hline Rat & $\begin{array}{l}\text { Available, and easy to } \\
\text { handle. } \\
\text { Well-established model. } \\
\text { Similar outcomes } \\
\text { (humans). } \\
\text { Allow monitorization } \\
\text { (physiologic and } \\
\text { pathologic parameters). }\end{array}$ & $\begin{array}{l}\text { Differences in } \\
\text { neuroanatomy, } \\
\text { neurophysiology and } \\
\text { immunology. } \\
\text { Faster and higher } \\
\text { spontaneous recovery. } \\
\text { Quadrupedal. } \\
\text { Small size. } \\
\text { Low cerebrospinal fluid. }\end{array}$ & $\begin{array}{l}\text { Contusion } \\
\text { Compression } \\
\text { Transection } \\
\text { Secondary } \\
\text { Dislocation } \\
\text { Distraction }\end{array}$ \\
\hline Mouse & Transgenic models. & $\begin{array}{l}\text { Similar disadvantages with } \\
\text { rats. }\end{array}$ & $\begin{array}{l}\text { Contusion } \\
\text { Compression }\end{array}$ \\
\hline Dog & $\begin{array}{l}\text { Similarities in lesion } \\
\text { development. } \\
\text { Similar neuroanatomy and } \\
\text { tissue size. } \\
\text { Easy neurological } \\
\text { examination. } \\
\text { Spontaneous SCI models. }\end{array}$ & $\begin{array}{l}\text { Ethical issues. } \\
\text { Cost. } \\
\text { Quadrupedal. }\end{array}$ & $\begin{array}{l}\text { Contusion } \\
\text { Compression } \\
\text { Distraction }\end{array}$ \\
\hline $\begin{array}{l}\text { Nonhuman } \\
\text { primate }\end{array}$ & $\begin{array}{l}\text { Best approximate human } \\
\text { SCl. } \\
\text { Bipedal. } \\
\text { Hand movements and } \\
\text { coordination. }\end{array}$ & $\begin{array}{l}\text { Ethical issues. } \\
\text { Cost. }\end{array}$ & $\begin{array}{l}\text { Contusion } \\
\text { Compression } \\
\text { Transection } \\
\text { Distraction }\end{array}$ \\
\hline Swine & $\begin{array}{l}\text { Neuroanatomical } \\
\text { similarities (white and gray } \\
\text { matter distribution, } \\
\text { vasculature). } \\
\text { Similar quantity of } \\
\text { cerebrospinal fluid. } \\
\text { Surgery, analytics, and } \\
\text { implantable devices (same } \\
\text { as humans). }\end{array}$ & $\begin{array}{l}\text { Some differences in } \\
\text { functional organization of } \\
\text { motor system. } \\
\text { Cost. } \\
\text { Does not detect discrete } \\
\text { therapeutic effects (motor). } \\
\text { Quadrupedal. }\end{array}$ & $\begin{array}{l}\text { Contusion } \\
\text { Compression } \\
\text { Transection } \\
\text { Secondary } \\
\text { Distraction }\end{array}$ \\
\hline $\begin{array}{l}\text { Small } \\
\text { ruminant }\end{array}$ & $\begin{array}{l}\text { Size and anatomical } \\
\text { similarities with humans. } \\
\text { Easily trained. }\end{array}$ & $\begin{array}{l}\text { Not well-established } \\
\text { model (few studies). } \\
\text { Quadrupedal. }\end{array}$ & \\
\hline Marsupial & $\begin{array}{l}\text { Bipedal. } \\
\text { Born at early stage. }\end{array}$ & $\begin{array}{l}\text { Not well-established } \\
\text { model (few studies). }\end{array}$ & \\
\hline Zebrafish & $\begin{array}{l}\text { Inexpensive, easily bred, } \\
\text { high number of eggs and } \\
\text { larvae. } \\
\text { Adult spinal cord } \\
\text { regeneration. } \\
\text { Transparent embryo. }\end{array}$ & $\begin{array}{l}\text { Differences with mammals } \\
\text { (anatomical, neurological, } \\
\text { physiological, behavioral, } \\
\text { etc.). }\end{array}$ & \\
\hline Amphibian & $\begin{array}{l}\text { Stage-dependent spinal } \\
\text { cord regeneration. } \\
\text { External development } \\
\text { High number of eggs or } \\
\text { froglets. }\end{array}$ & $\begin{array}{l}\text { Differences with mammals } \\
\text { (anatomical, neurological, } \\
\text { physiological, behavioral, } \\
\text { etc.). }\end{array}$ & \\
\hline
\end{tabular}


Table 13.1 Animal species used in spinal cord injury research.-cont'd

\begin{tabular}{l|l|l|l} 
Species & Advantages & Disadvantages & Main models \\
Lamprey & $\begin{array}{l}\text { Studies in axon } \\
\text { regeneration. } \\
\text { Transparent brain. }\end{array}$ & $\begin{array}{l}\text { Differences with mammals } \\
\text { (anatomical, neurological, } \\
\text { physiological, behavioral, } \\
\text { etc.). }\end{array}$ & \\
Turtle & Partial SCl regeneration. & $\begin{array}{l}\text { Differences with mammals } \\
\text { (anatomical, neurological, } \\
\text { physiological, behavioral, } \\
\text { etc.). }\end{array}$ & \\
\hline SCl, spinal cord injury. &
\end{tabular}

\section{s0020 Rodents}

p0030 Rodent models were widely used in SCI repair research owing to their availability, easy maintenance, and cost. However, rat and mouse models differ in several key points, including the presence of fibroblast-like cells in mouse chronic SCI [4] and the temporal difference in inflammatory infiltration between the species [5]. Also, evidence suggests that in mice, a modest degree of regeneration occurs in the lesion site; they have an atypical cyst-like structure that has axonal regeneration different from that seen in rats and primates.

p0035 On the other hand, rat models enable the monitorization of physiological and pathological parameters in SCI and demonstrate functional, electrophysiological, and morphological outcomes similar to those of humans [6]. For those reasons, rats are commonly used in preliminary studies, whereas mice are particularly useful for genetic studies owing to the availability of their transgenic models [4].

p0040 Comparing the functional and anatomical outcomes of a contusion model in rats with human chronic SCI, Metz et al. [6] identified that spinal cord atrophy and the size of the lesion significantly correlated with electrophysiological and functional outcomes in both rats and humans. That study provides evidence that rat contusive SCI could be a valid model to evaluate the structural and functional benefits of some therapies. However, many experimental treatments that proved their efficacy in rats were difficult to translate to human clinical trials. This discrepancy may result from differences in neuroanatomy, neurophysiology, and immune systems.

p0045 Rodent models have several disadvantages for translating results to human clinical trials. Rodents exhibit a high degree of spontaneous recovery from SCI [2]; this recovery is faster than those observed in humans. This fact has to be considered when evaluating the effects of potential therapies. Also, rodents and humans have several neuroanatomical differences such as the mainly dorsal corticospinal tract in rats [3], their small size, and that they are quadrupeds. This difference in size makes these animals unavailable for studying long-distance axon regeneration, 
which is important in humans for SCI repair (1 mm of growth in rodents may be significant but not enough in larger animals and humans). The model may be not acceptable for studying surgical therapies or implantable devices. In addition, the thickness of the spinal cord and the amount of cerebrospinal fluid have to be considered factors that can influence results in drug-based therapies [7].

p0050 Contusion, compression, transection, and secondary models are well-established for use in rats, as well as in outcome tests for evaluating SCI induction and recovery.

\section{s0025 Large animals}

p0055 There has been increasing interest in the use of nonhuman primates or large animal models, such as dogs and pigs. These models may allow more effective translation of potential therapies in human clinical trials. However, the high cost compared with rodents, the need for special facilities, and the small available sample size have limited their use in SCI research [8].

\section{s0030 Canines}

p0060 Canine models can be considered intermediate between rodents and human clinical trials; they are the large animal model most used in the SCI literature [3]. However, there are increasing ethical issues relating to using dogs in medical research owing to their status as companion animals. Several different models were described for the use of dogs since the first one described in 1911 by Allen.

p0065 The adult dog is considered a superior translational model to human clinical trials compared with rodents. Canine SCI have similarities to human SCI in the mechanism of lesion development, tissue size, and neuroanatomy; the spinal cord length is similar to that of humans [9]. It has also the advantage of an easy neurological examination [2]; it is feasible to train dogs on functional testing.

p0070 Moreover, preclinical trials in dogs with spontaneous acute SCI, as in spontaneous intervertebral disc herniation, can be performed. That gives the opportunity to screen potential human treatments in a natural model with many similarities to human acute SCI $[9,10]$.

\section{s0035 Primates}

p0075 Nonhuman primate SCI models are the ones that best approximate human SCI [11] because they have genetic, biological, and physiological similarities. Those reasons made these animals essential models for evaluating potential therapies. Another advantage is that some motor functions, including hand movements, hand-to-eye coordination, posture, and locomotion (bipedal) are not present in other animals. The number of studies using this species is rising [12], as is the need to create reliable models in nonhuman primates, because most data obtained in rodent models lack in translation to human clinical trials.

p0080 Main limitations of using nonhuman primates are ethical concerns and the high cost [13]. From an ethical point of view, the use of nonhuman primates should be permitted only in biomedical areas that are essential for the benefit of humans, 
such potentially life-threatening or debilitating conditions. In addition, researchers should provide an accurate description of the benefits, harm to animals, and limitations, and be realistic about the potential outputs and impacts [14]. Therefore, modeling severe SCI with impairment of bladder and bowel functions is not possible in nonhuman primates for both ethical and economic reasons.

p0085 SCI models most used in primates are contusion lesions. Although these models are well-documented, the individual neuroanatomy induces variability and it is not always possible to predict the neurological deficit. Also, transection models are used in these animals, as well as surgical resection of spinal cord sections [12].

\section{s0040 Swine}

p0090 In many aspects of SCI research, swine models are considered a good alternative to using nonhuman primates and meet criteria for model validity. As SCI models, these species have been used in several techniques, including compression, ischemic injuries, root avulsion with ventral myelotomy, vertebral distraction, and transection models, among others [7].

p0095 These animals have an anatomy, physiology, and pathophysiology similar to those of humans and are considered to provide a stable neurological deficit $[15,16]$. Similarities between pigs and humans are the anatomical position of the corticospinal tract, gray and white matter distribution, the position of spinal cord blood vessels, and neural development [15]. Also, surgical and analytical instruments as well as implantable devices used in these animals are similar or even the same as those used in humans. Finally, research in swine does not raise the same ethical concerns as those in nonhuman primates and dogs.

p0100 A clear limitation of the swine as a translational model for human SCI is that pigs lack neuroanatomical or functional organization of the motor system found in humans and primates. Thus, the pig and minipig models are unsuitable for detecting the discrete therapeutic effects associated with distinct motor axons populations $[15,16]$. Finally, these models are expensive compared with rodents and need special facilities.

\section{s0045 Other mammals}

s0050 Small ruminants

p0105 The advantages of using these animals is their similarity in size, anatomical features of the spinal cord, and the possibility of training them more easily than other large animals for behavioral tasks such as treadmill ambulation of cervical flexion to evaluate SCI repair [17]. Despite these possible advantages, the use of small ruminant models is anecdotical in the literature [3].

\section{s0055 Macropodid marsupials}

p0110 In an attempt to establish a natural bipedal model for SCI repair different from nonhuman primates, macropodid marsupials as the Tammar wallaby were studied. Along with primates, these marsupials are a major group of bipedal animals. It has been proposed that the spinal cord circuits used in bipedal gait are similar 
between macropodids and humans. The other advantage of using marsupials to study spinal cord regeneration is that in those animals are born at an early stage of central nervous system development (equivalent to embryonic stages in rodents), when the capability of natural repair of the spinal cord, which mammals exhibit in an immature stage, can be studied [18].

\section{s0060 Nonmammals \\ s0065 Zebrafish}

p0115 Studies of spinal cord regeneration in those animals have shown how animal species lose the ability of neuroregeneration with evolution. Zebrafish (Danio rerio) have the advantages of being cheap and easily bred, with the possibility of producing large number of eggs or larvae adequate for genetic manipulation and with an incredible capacity for spinal cord regeneration in adults. For instance, in zebrafish with complete SCI transection under the influence of the overexpression of connective tissue growth factor, a glial bridge is formed between dissected sections with the restoration of spinal functions [19]. Also, it is important to highlight the transparency of its embryo, which allows in vivo analysis of functional neural integration.

p0120 Zebrafish could serve as an invaluable model to target functional regeneration of SCI in humans and to complement SCI research based on other mammal models [20].

\section{s0070 Amphibians}

p0125 Anuran amphibians such as Xenopus laevis represent a case of stage-dependent spinal cord regeneration. These animals are capable of regenerating the spinal cord before metamorphosis, but not after. The presence of both regenerative and nonregenerative stages, its external development (which allows easy access to examine and manipulate spinal cord), its robustness, the easy generation of hundreds of larvae or froglets, and advances in genetic manipulations make this species a good model for studying regenerative biology including SCI regeneration [21].

\section{s0075 Lampreys}

p0130 Some studies used lampreys (Petromyzon marinus) as animal models to study axon regeneration. In contrast to mammals, these animals recover normal locomotion spontaneously several weeks after complete SCI owing to plastic changes and regenerative processes [22]. Other advantages seen in marine lampreys are that, because of the transparency of the lamprey brain, descending neurons and axons can be visualized in vivo. Lampreys are considered a good vertebrate model for the in vivo study of molecular mechanisms underlying the death or regeneration of descending neurons after SCI.

\section{s0080 Turtles}

p0135 The only known amniotes with partial spinal cord regenerating capacity are slider turtles, a group of American freshwater turtles such as Traquemys spp. They are 
considered the animal closest phylogenetically to mammals that have this ability. Therefore, this group of animals represents an intermediate stage between non-regenerating species as mammals and fully regenerating animals such as most anamniote vertebrates [23]. Cellular and functional processes that take place after traumatic spinal injury in turtles were characterized by Reherman et al. [24].

\section{s0085 Ethical}

p0140 The use of animals is a relatively recent concept. For centuries, new surgical procedures or medical treatments were tried immediately in patients, who therefore became a sacrifice to the goal of medical progress. One cause was modern warfare, which created the need to replace or repair major body parts. From the 18th century onward, the use of animals was rapidly standardized, mainly to study anatomy and physiology. However, human experimentation followed until the midtwentieth century $[24 \mathrm{a}]$.

p0145 After World War II, the Nuremberg Code (1947), and later the Declaration of Helsinki (1964), were redacted to protect humans from the incorrect use of medical research. These regulations established the bases for using animals and humans as experimental subjects, making voluntary consent mandatory in the case of humans. At the same time, they considered that all experiments should be designed and based on the results of animal experimentation.

p0150 Many advances have been made in medicine (medical devices, human and animal drugs, and biological products), improving the quality of life for numerous patients. Professional efficacy and general safety rules generated scientific and governmental efforts to formalize bench trials and animal testing as prerequisites for using these advanced devices in patients. This created the development of regulations and reports to protect animals and humans used as subjects of research.

p0155 At the same time, over the last quarter of the 20th century, the perception of society changed regarding animals and their place in research, owing to the efforts of people involved in the cause of animal rights. The first steps were given in the scientific community, because it considered that it had a moral obligation to provide animals with a degree of human treatment, while improving the reproducibility of research results. These were the principles of replacement, refinement, and reduction (3Rs) that were published in 1959 by Russell and Burch [25]. The consequence was that in 1963, guidelines were published to comply with current standards for the treatment of animals in research by the National Research Council. This has been updated eight times, including in 2011 [26].

p0160 Later, a movement emerged among people headed from philosophy departments in universities in the 1970s. Their argument was that the boundary between humans and animals is completely arbitrary and that animals have to be treated with respect. The most renowned authors of the movement were Peter Singer (Animal Liberation, [67]) and Tom Regan (The Case for Animal Rights, [27]). The movement considers that animals have rights and value equal to those of humans. Essentially, they stated 
that the human needs for health and survival provided no moral justification for inflicting harm or risks to animals [26], and that it was not ethically acceptable to conduct scientific investigations using animals in most cases.

p0165 These ideas gained widespread acceptance in society and the scientific community. Supporters encouraged governments to change regulations regarding the use of animals in research and to follow the principles of 3Rs. However, published polls indicated that $60-80 \%$ of the population accepts the use of animals in biomedical research if the pain and suffering of animals is reduced or eliminated [28].

p0170 Unfortunately, regulations will not eliminate the need for animal experimentation. Nevertheless, it has led to increased interest in alternative models. Many organizations have improved and reduced the use of animals. One of the most important is the National Center for the Replacement, Refinement and Reduction of Animals in Research (NC3Rs). It is a United Kingdom-based scientific organization dedicated to replacing, refining, and reducing the use of animals in research and testing. They provide a 3Rs peer review and advice service to the United Kingdom's major funding bodies and have an extensive library of 3 Rs resources. These resources include guidelines, practical information, and themed hubs. Links to publications, other online resources, and video and training materials are also available.

\section{s0090 Regulatory affairs and design of procedures}

p0175 The use of laboratory animals is governed by an interrelated, dynamic system of regulations, policies, guidelines, and procedures. In the European Union (EU), all procedures mandate following Directive 2010/63/EU on protecting animals used for scientific purposes. This provides detailed rules and minimum standards for animal protection, although a more restrictive protection may apply in some EU States in terms of space available per animal.

p0180 In the United States, the use is covered by a Federal Animal Protection; researchers should use the Guide for the Care and Use of Laboratory Animals (Eighth Edition, 2011. National Academies Press, Washington, DC). This guide covers US regulatory requirements and may be useful for non-US entities because principles are applicable to other locations.

p0185 In general, regulations must keep to the following principles:

$\mathrm{u} 0010$ - Replacement and reduction of the use of animals in procedures and refinement of breeding. Researchers should consider using alternatives such as in vitro systems, computer simulations, and/or mathematical models before performing an animal procedure.

u0015 - In any case, procedures should avoid or minimize discomfort, distress, and pain using appropriate sedation, analgesia, and anesthesia. Procedures should clearly include humane end points. More information about euthanasia may be obtained from Web resources from the American Veterinary Medical Association [29]. The publication includes information about species-specific euthanasia methods and confirmation of death for vertebrates, invertebrates, and other 
lower-order species. The guide gives additional information about humane handling of animals before and during euthanasia.

u0020 - The origin, breeding, transportation, marking, care and accommodation of animals, with a special point in minimal requirements in terms of housing dimensions, lighting, flooring, etc.

u0025 - Operations of breeders, suppliers, and users and specific requirements for the information of personnel, including those in veterinary care.

$\mathrm{u} 0030$ - The evaluation and authorization of projects involving the use of animals in procedures.

p0215 In addition to local regulations, several associations have Web or published resources supplying information about related topics. We recommend the lecture of specific manuals of the Laboratory Animal Science Association, Animal Research Advisory Committee and NC3Rs. In many instances, these guides are Web accessible. The Experimental Design Assistant is a free online tool from the NC3Rs, designed to guide researchers in designing their experiments, ensuring that they use the minimum number of animals consistent with their scientific objectives, methods to reduce subjective bias, and appropriate statistical analysis.

p0220 In some cases, preclinical assays may involve using implanted medical devices or tissue-engineered products. US government agencies such as the Food and Drug Administration (FDA) and regulatory bodies such as the American Society for Testing and Materials, International Organization for Standardization (ISO), and US Pharmacopeial Convention have made great efforts to provide procedures, protocols, guidelines, and standards that may be used in the in vivo assessment of the safety of medical devices or cell therapies. In general (for regulatory or experimental models), all protocols should adhere to ISO 10993-1 and to 3R international protocols. The ISO 10993 standard presents a structured program for evaluating biocompatibility in which matrices indicate required tests according to specific types of tissue contact and the duration of contact [30].

\section{s0095 Main experimental models}

p0225 Animal models in SCI repair can be classified based on:

u0035 - spinal cord level affected,

u0040 - injury mechanism used to induce the lesion, or

u0045 - time from lesion to treatment.

p0245 Based on the spinal level injured, most studies have induced a thoracic-level lesion $(81 \%)$ [3], but because of clinical relevance in humans, research has also focused on cervical-level models $(12 \%)$ [3,31]. The lumbar spine was used in $5.1 \%$ of studies; in a few cases $(0.7 \%)$, use of the sacral spine was reported [3].

p0250 Based on the mechanism of injury, SCI models in animals can be classified according to mechanical traumatic injury: contusion, compression, transection, 
and nontraumatic or secondary SCI [1,3]. Each can be used in different animal species. In the review by Sharif-Alhoseini, $94.5 \%$ of studies in SCI research were of mechanical injuries, whereas $5.5 \%$ were nontraumatic. In the same study, contusion models were the most common pattern of injury used in animal SCI models, followed by compression. The review was found that $1.1 \%$ of studies evaluated used more than one pattern. Types are summarized in Table 13.2.

p0255 Based on the time from lesion to treatment, SCI models can be chronic studies, in which the SCI is chronically established, and acute studies, in which the treatment

t0015 Table 13.2 Spinal cord injury models based on injury mechanisms.

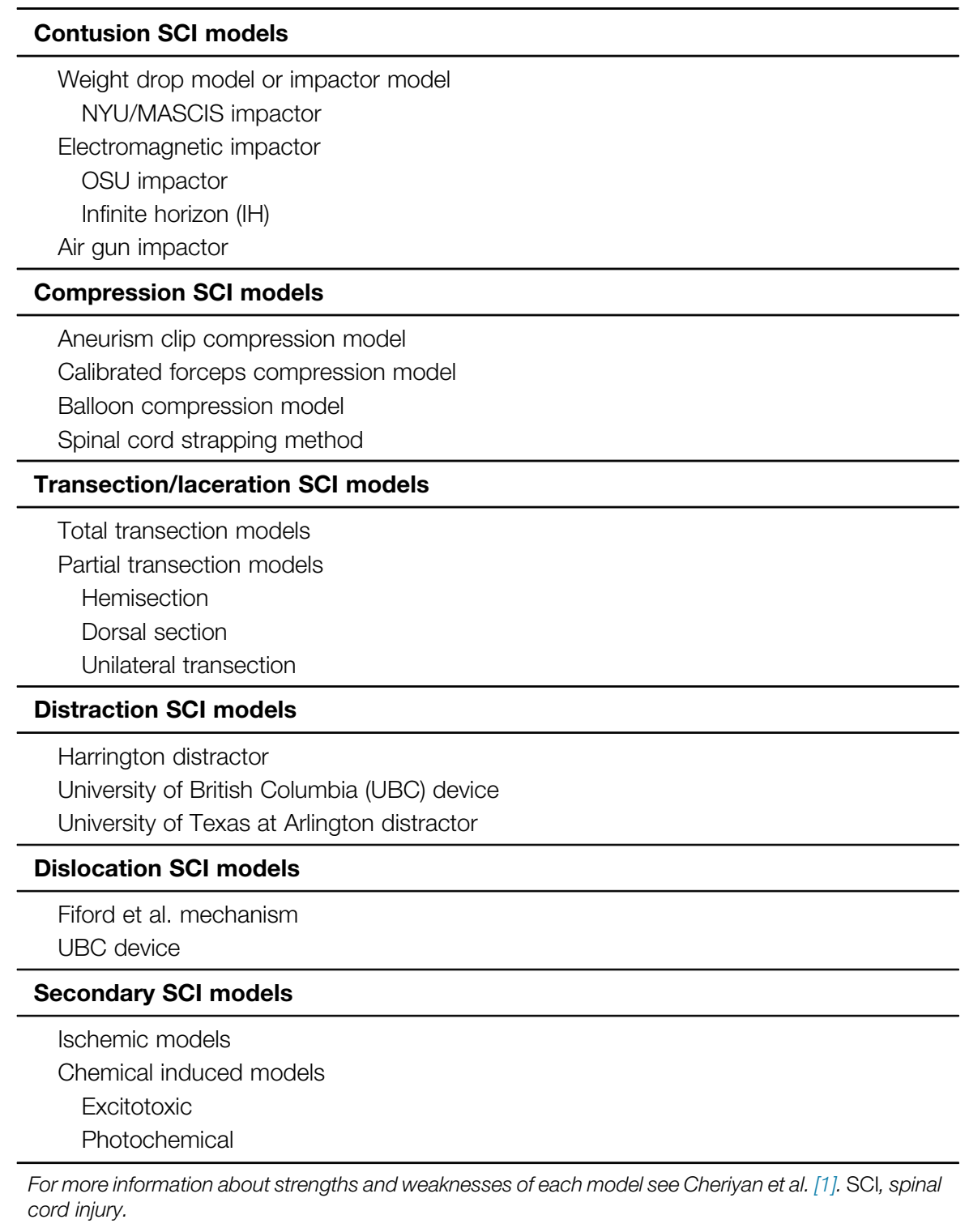


began within hours or days. In chronic studies, it is important to achieve the longterm survival of animals. It is considered more appropriate for studies of effective axonal regeneration. However, chronic studies are rarely used because it is difficult to maintain paraplegic animals to develop a chronic lesion owing to complications and the special care they need [32]. It is important to consider that with short-term studies, the absence of the effect of therapies may simply result from a lack of time.

\section{s0100 Models based on spinal injury level}

p0260 Although the most common region involved in SCI in humans is cervical, cervical models of SCI can result in respiratory compromise and are associated with high mortality because of interruption of the bulbospinal respiratory drive or impairment of the diaphragm [33]. To solve this problem, most studies used the thoracic region for SCI models, which are also apparently reliable and easily reproducible [3].

p0265 Cervical and thoracic levels differ in several aspects such as spinal diameter, the distance between neuronal bodies and the injured site, specific ascending and descending pathways, vascularization, the proportion of white and gray matter, and the size of the different (sensory and motor) neuron populations [34]. For these reasons, results of thoracic and cervical models may not be easily translatable.

\section{s0105 Cervical}

p0270 Because of differences in the thoracic region, cervical SCI models were developed [31]. At the cervical level, ethical and medical arguments prevent the use of bilateral injuries, because these lesions would paralyze both forelimbs and hind limbs, as well as result in respiratory compromise. Cervical models are important because regaining arm and hand function is considered a priority for improving the quality of life in humans with tetraplegia, and because cervical lesions are predominant in human pathology.

\section{s0110 Thoracic}

p0275 This level of lesions permits the isolation and study of white matter deficits [33]. Complete SCI at low thoracic levels causes permanent paralysis of the hind limbs with impairment in the sensory and motor functions. In those animals, it is mandatory to provide correct bladder emptying, as well as treatment of possible ulcers.

\section{s0115 Lumbar and sacral}

p0280 Sacral injuries are restricted to tail symptoms and can be used to monitor recovery from several treatments [35].

\section{s0120 Models based on injury mechanism \\ s0125 Contusion}

p0285 Lesions are caused by a transient physical impact applied to the spinal cord that displaces and damages tissues. It is the oldest and most commonly used method for SCI 
models and is available in different animal species such as rodents and large animals $[7,36]$.

p0290 Contusion models can produce different SCI severities. The lesions are characterized by inflammation, ischemia, hemorrhagic necrosis, and central cavitation. This type of model may be used in all spinal cord areas. In cervical SCI models, unilateral contusion (or hemicontusion) is the most used owing to the life-threatening effects that can occur after complete cervical lesions [3].

p0295 Contusion models need to employ a laminectomy to expose the spinal cord. There is the possibility of several complications because of the surgical technique. In addition, tissues such as muscles and tendons surrounding the spinal cord have an important role in regeneration [37].

p0300 Different techniques should be included, with variations depending on animal species, such as the weight drop model, electromagnetic impactor, or air pressure devices.

\section{s0130 Weight drop model or impactor model}

p0305 In this model, initially a laminectomy is performed on an anesthetized animal to expose the dorsal surface of the spinal cord. Then, a given weight is dropped from a known height, using a perpendicular guide tube. When the weight impacts the cord, the underlying tissue is subjected to dynamic compression. Depending on both the weight and height, the force applied to the spinal cord is different, resulting in varying degrees of SCI [2].

p0310 In 1911, Allen described the first animal experiment using this method in dogs, in which a known mass was dropped onto the dorsal surface of the dura from a prescribed height. Later, the technique was modified for use in rats [38]. Since then, several different weight drop models have been described. The New York University (NYU) impactor device is one of the most popular used models in rats [39] (later renamed the Multicenter Animal Spinal Cord Injury Study or MASCIS). This method drops a 10-g weight from different heights directly onto the spinal dura at T9-T10. In this model, a severe loss of hind limb locomotor movement was found in all animals, except at a height of $6.25 \mathrm{~mm}$ (the shortest one). The spinous processes of vertebrae on both sides of the lesion are secured with clamps to avoid motion of the column during the impact.

p0315 The NYU/MASCIS impactor measures several biomechanical outcomes. Disadvantages are that it can be used only in a rat strain (Long Evans rats) of a concrete age (77 \pm 1 days), with a fixed anesthetic protocol and an impact exactly $60 \pm 1 \mathrm{~min}$ after the induction of anesthesia. The most recent version of the MASCIS device was introduced in 2012 and includes electromagnetic control and digital recording of the impact parameters [1].

\section{s0135 Electromagnetic impactor}

p0320 One electromechanical device developed for contusion SCI models was the Ohio State University (OSU) impactor. In this case, after laminectomy and securing of proximal and distal vertebra with clamps, the impactor probe was carefully screwed 
to the dura surface to displace the spinal cord $30 \mu \mathrm{m}$ (with 3000 dyne force) to provide a consistent starting point. Then, the system was triggered and the device impacted the cord. Is possible to measure the force transmitted and the total displacement $[4 a, b]$. The OSU impactor has an advantage in that it was adapted for use with mice [4]. Although this device is not available commercially, it provides excellent control of all biomechanical aspects of the injury; it is possible to modify the severity of the lesion [2].

p0325 Another type of electromagnetic impactor used in rats is the Infinite Horizon (IH) impactor. In this case, the force is generated by a stepping motor to produce a controlled impact after a laminectomy [1]. The IH impactor can be set at different force levels to provide mild to severe lesions. The main limitation of this impactor is the unreliability of the clamps in holding the spinal column firmly, which might cause a parenchymal injury. Impactor mechanisms have been developed for its use in large animal models $[7,36]$.

s0140 Air gun impactor

p0330 The air gun impactor was developed in the early 2010s for use in rats; it is based on an air pressure-mediated contusion with the advantage of no direct contact with the spinal cord. In this model, the T10 is stabilized with clamps to the adjacent vertebrae, and the vertebral arch is drilled with a dental drill $2 \mathrm{~mm}$ in diameter through which the injector is inserted and positioned onto the dura, and the air shot is delivered. Problems with this device are that resulting lesions are inconsistent, lesions that are gradable in severity cannot be produced, and it is not yet a validated method [40].

\section{s0145 Compression spinal cord injury models}

p0335 The lesion is based on compression of the spinal cord over time. These models are helpful in simulating spinal canal occlusion in humans. They enable the study of both the effects and time of decompression. Some of these models are in fact contusion-compression models that involve an acute impact followed by persisting compression.

\section{s0150 Aneurism clip compression}

p0340 Rivlin and Tator first described this model in 1978. It is the model that best mimics the ventral contusive compression normally seen in humans. It is the most commonly used compression model in rats and mice, is easy to use, and is inexpensive. In this model, the injury is obtained through dorsoventral compression using modified Kerr-Lougheed aneurism clips [41] calibrated to apply a known compression force for a predetermined time. Adjusting the clip force and the duration of the compression can modify the severity of injury. This model is relatively inexpensive, it can be used to produce a focal SCI in different parts of the spinal cord [42], and it mimics common clinical injuries in human SCI. Also, it can be used to inflict ischemia-reperfusion SCI models that may occur during surgeries. Disadvantages are that the clip compression model could create variations owing to the velocity 


\section{CHAPTER 13 Reliability on animal models}

of the clips closing and the delivered force at the time of application [1], a surgical laminectomy is necessary.

p0345 Aneurism clips were initially designed for use in rats; however, there are smaller and larger clips for in mice [43] and large animals [44]. Also, it can be used for cervical, thoracic, and lumbar spine injuries.

\section{s0155 Calibrated forceps compression}

p0350 Calibrated forceps compression can be used to produce a lateral compression injury, inserting calibrated forceps in either side of the spinal cord after a laminectomy. This method was designed initially for use in T13 lesions in guinea pigs [42], but it was adapted to other species such as rats or mice. Although it is a simple technique, this model lacks the initial impact injury normally seen in human SCI, so it is not a clinically relevant model for therapeutic development [1]. Nevertheless, it is the best choice when precision in compression is desired for a specific segment of the spinal cord [45].

\section{s0160 Balloon compression}

p0355 The application of balloon compression was first described by Tarlov in 1953 $[46,47]$. Since then, several different techniques have been developed in rats [48], dogs [37,49], and nonhuman primates [50]. This is a simple technique that uses a balloon catheter introduced in the epidural or subdural space, filled with air or fluid, to produce a closed injury similar to those seen in human clinical cases [51]. The degree of damage is produced by the volume of the balloon inflation and the time of compression [48], but it is difficult to standardize the initial impact. The oldest surgical techniques included a laminectomy to introduce the catheter cranially one or two spinal levels high, but there are techniques that do not suggest it, so the method does not cause damage to surrounding structures [49]. Balloon compression models typically lack the acute component of human traumatic SCI [1].

\section{s0165 Spinal cord strapping method}

p0360 The spinal cord strapping method is a technique used in rats; it was developed in 2008 [52]. In this method, a curved surgical needle is inserted through one intervertebral foramen (on one side of the column) to pass anterior to the spinal cord and back to the skin through the contralateral foramen so that the suture wraps around the spinal cord. The suture is fixed to the experimental device (called the SCStrapper) in one end and to a suspended weight in the other; compression is induced by a weight drop. This method has the advantage of not requiring laminectomy (a $100 \%$ survival rate was reported) and force can be computed based on the weight of the mass dropped. Another advantage of this technique is that it provides compression on all sides of the cord instead of only laterally or dorsoventrally [1]. A disadvantage of the method is that the researcher is blinded to when the needle passes through the spinal canal, so the procedure can injure the spinal cord rather than pass anterior to it. 


\section{s0170 Transection/laceration spinal cord injury models}

p0365 The models include partial or total section of the spinal cord at a particular level and focal myelotomy (incision), with resection and aspiration methods that damage the spinal cord axons [2]. The most used are the transection ones.

p0370 Transection models are performed by interrupting the spinal cord (cutting it with surgical scissors) after a laminectomy and may be complete or incomplete. Partial transection models include hemisection, unilateral transection, and dorsal lesions. A complete transection model is relatively simple to reproduce [1]. In contrast, the reproducibility of partial lacerations is inconsistent [2] and the ratio of spontaneous recovery is higher.

p0375 Complete transection models are useful for studying the possible effects of scaffolds, biomaterials, or neurotrophic factors. These are appropriate models for investigating degeneration and regeneration of the spinal cord, tissue engineering, or neuroplasticity [53]. Partial transection models are valuable for nerve grafting research, plasticity, and comparisons of injured and noninjured pathways in the same animal [1]. This type of injury of the spinal cord is uncommon in clinical practice, and thus transection/laceration models do not truly reflect the pathophysiology of human SCI and are inappropriate for studying neuroprotective treatments [54].

\section{s0175 Distraction spinal cord injury models}

p0380 Distraction spinal cord injury models involve controlled stretching of the cord with the objective of simulating tension forces experienced by the spinal cord during SCI [1].

\section{s0180 Harrington distractor}

p0385 This model was designed in 2004 for use in rats [55]. The procedure involves a laminectomy in the site of the lesion. Then, for one vertebra caudal to this site and one rostral, modified Harrington hooks are placed under the laminae. The hooks are connected to a computer-controlled motor that controls the length, strength, and duration of the distraction, producing from mild to severe SCI. A major limitation of the model is variability within the injury grade. Also, this model is not yet validated.

\section{s0185 University of British Columbia device}

p0390 This device was designed for use in cervical SCI models in rats and can cause contusions, distractions, and dislocations. The severity of the lesion can be varied depending on the displacement of the spinal cord. This device is capable of inflicting high-speed distraction, creating an injury similar to human vertebral dislocation, but it is not yet validated [56].

\section{s0190 University of Texas at Arlington distractor}

p0395 The objective of this device is to inflict a bidirectional distraction (compared with the University of British Columbia [UBC], which is unidirectional). This technique 
does not need a laminectomy, so it is less invasive. It needs only the use of clamps secured to vertebral bodies and attached to linear actuators that simultaneously distract T9 and T11. It is possible to create different degrees of SCI. Limitations of the device are that there is variability in behavioral scoring, the instrument is not commercially available, and the lesions are produced at a lower speed than the UBC, which may affect the capacity to mimic human injury [57].

\section{s0195 Dislocation spinal cord injury models}

p0400 Fiford et al. [58] developed a mechanism to replicate column dislocation using vertebral displacement. The device has two horizontal beams attached to the lateral surfaces of adjacent vertebrae; one is rigidly fixed and the other is used to displace the lower vertebrae laterally at a specific distance for $1 \mathrm{~s}$ at a specific speed. A longer displacement means great SCI.

p0405 The UBC device also can produce an anterior dislocation model in which the vertebrae are dislocated dorsally. This model is not validated, but it closely reproduces anterior fracture-dislocation injuries seen in humans [56].

\section{s0200 Secondary mechanism spinal cord injury models}

p0410 Secondary injuries of the spinal cord begin within minutes after the initial trauma and continue for weeks or months, causing progressive damage. Secondary injury can be divided into acute, subacute, and chronic phases. The acute phase includes vascular damage, ionic imbalance, excitotoxicity, free radical accumulation, lipid peroxidation, inflammation, edema, and cell death. The subacute phase involves, among others, apoptosis, demyelination of axons, matrix remodeling, and glial scar formation. Finally, in chronic phases, the cystic cavities are formed [59].

p0415 Secondary SCI models are based on changes that occur after the initial lesion; they are divided into ischemic and chemically mediated models.

\section{s0205 Ischemic}

p0420 Ischemic models are established by temporal aortic occlusion using aneurism clips. These types of models are performed mostly to test the effect of drugs and growth factors after SCI [3] and to resemble ischemic events after spinal cord trauma.

\section{s0210 Chemically mediated}

p0425 Chemically mediated models focus on the secondary spinal cord damage sequence caused by the rapid increase in excitatory amino acid concentrations after an SCI injury. This rapid increase has a crucial role in the pathology of both gray and white matter [60]. These models are useful for investigating molecular mechanisms involved in SCI and the effect of therapies on specific pathways, but they are less useful in terms of overall approximation to SCI pathology. Other limitations of these models are related to the difficulty in exactly reproducing the lesion in terms of location, extent, and characteristics of the injury [1]. 
s0215 Excitotoxic Excitotoxic models involve administering different molecules (e.g., glutamate, $N$-methyl-D-aspartate, kainate) into the spinal cord to promote degeneration in gray and white matter, as well as neuron and oligodendrocyte death [2].

s0220 Photochemically induced models This method was first developed in 1986 [61] and is widely used in neurotrauma. It consists of inducing a photochemical reaction by activating an argon laser to induce the production of oxygen molecules on the endothelial surface of spinal blood vessels, causing damage to the tissues. Previous to irradiation of the area, this model involves the injection or direct application of a nontoxic photosensitive dye (such as rose bengal). Is a reliable and reproducible model and does not require mechanical trauma.

\section{s0225 Spinal cord injury evaluation tests [62]}

p0440 Behavior examinations are fundamental tests after therapeutic interventions in SCI to estimate the pretreatment and posttreatment function of neurological tracts. Those tests are well-defined for use in rodents, although some are being adapted for use in other animal species. Available tests are summarized in Table 13.3.

\section{s0230 Motor tests}

p0445 Motor tests were developed to assess the motor recovery of descending tracts after SCI. Among these tests, the Irvine, Beatties, and Bresnahan (IBB) scale and, the skilled forelimb reaching task, and the limb grip strength test are the most commonly used.

\section{s0235 Irvine, Beatties, and Bresnahan scale}

p0450 The IBB scale is a 10-point scale used to identify the recovery of forelimb functions after unilateral cervical SCI. It is based on the evaluation of joint positions, digit movements, and grasping method recorded on a video.

\section{s0240 Skilled forelimb reaching task}

p0455 This task is time consuming and needs several weeks to train rats to forelimb pellet retrieval. For qualitative assessment, 10 components of movements (e.g., digital flexion, movements of the elbow, grasp, supination are rated from $0=$ normal to 2 = absent). For quantitative assessment, the success rate of each animal is calculated, including the total number of pellets retrieved and eaten and the time required. A major limitation of this test is that a partial lesion may not impair reaching success and qualitative scores.

\section{s0245 Limb grip strength test}

p0460 This test is used to evaluate both forelimb and hind limb functions measuring the strength of the flexor muscles. Grip strength measurements are important because they can predict motor deficits after SCI. Acclimatization of rats to the measuring apparatus is necessary before the test begins. In the test, the animal is allowed to 
t0020 Table 13.3 Spinal cord injury evaluation tests in rats.

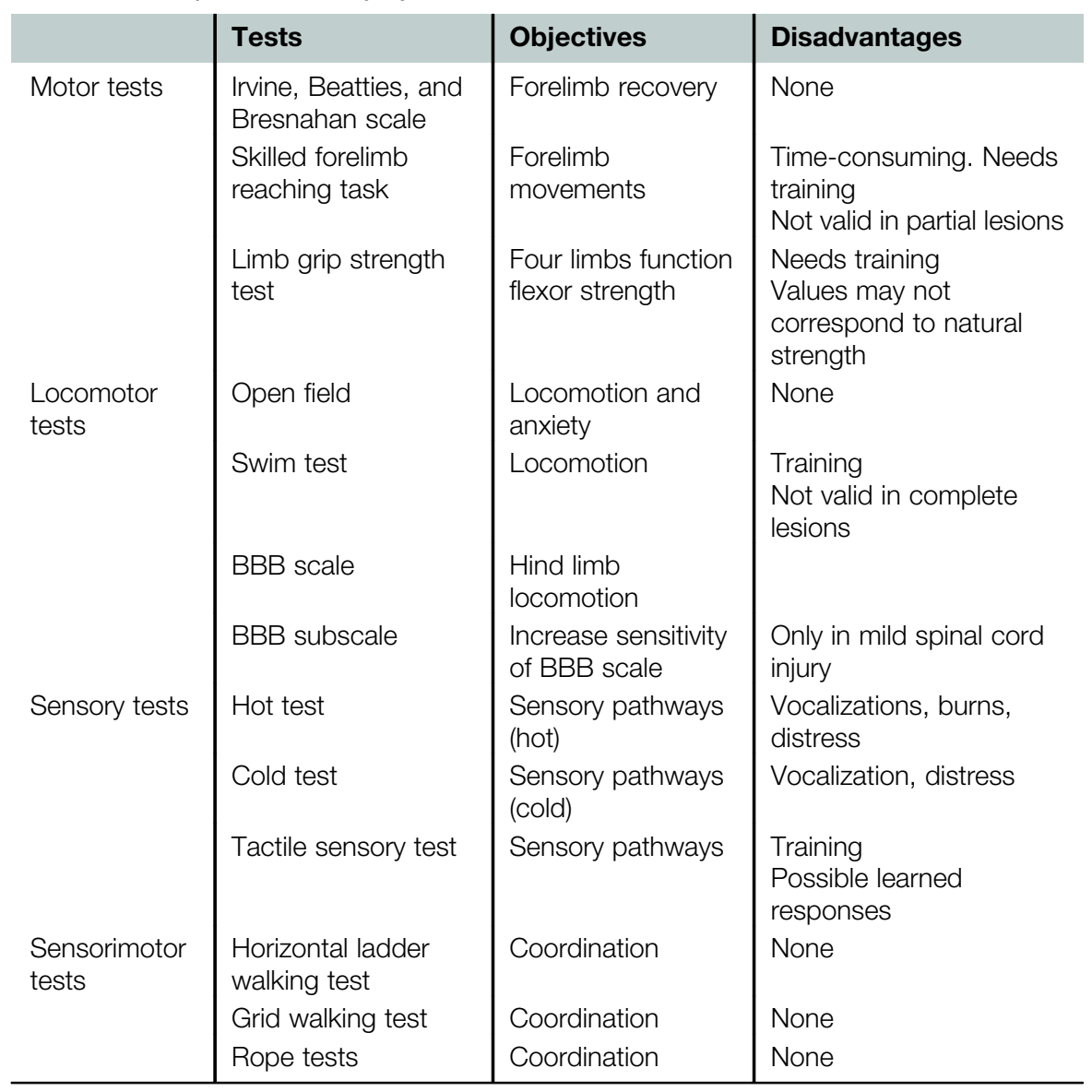

BBB, Basso, Beattie, and Bresnahan.

grab a metal grid or pull bar with any of the paws, and then is pulled away (horizontally), measuring the pull strength until the rat releases the bar. For each limb, various tests are performed to evaluate the maximum grid strength. It is important to consider that animals may hesitate to grasp the bar if they feel unpleasant when they are pulled away, so the measured value may differ from the natural grip strength value.

\section{s0250 Locomotor tests}

p0465 To evaluate recovery from SCI, locomotor tests are commonly used in rats. The most used are the open-field test, swim test, Basso, Beattie, and Bresnahan (BBB) scale, and BBB subscoring scale. 
s0255 Open field test

p0470 This is the simplest method to evaluate locomotor activity and anxiety in rats after an SCI. The method uses a $60 \times 60-\mathrm{cm}$ open field arena with the bottom subdivided into $12 \times 12-\mathrm{cm}$ squares. The animal is placed in the center and the number of squares crossed during a predetermined time is recorded.

s0260 Swim test

p0475 Typically, a plexiglass swim tank $(150 \times 15 \times 30 \mathrm{~cm})$ is filled with water to a depth of $20 \mathrm{~cm}$, and the animal is observed during swimming in a total of five trials. Movements of the limbs and the distance between the hind limb and the tail are evaluated on an eight-point scale. An important limitation of the test is that it cannot be performed in complete lesions and animals should be trained before SCI.

s0265 Basso, Beattie, and Bresnahan scale and Basso, Beattie, and Bresnahan subscoring scale

p0480 The BBB scale is a 21-point qualitative scale that measures hind limb locomotion function. It evaluate joint movements, coordination, stability, toe clearance, and tail position. This test has the advantages of needing no training and not requiring special apparatus [63].

p0485 To solve the sensitivity of the BBB scale, a 7-point subscoring BBB scale was developed; its limitation is that it is valid only in mild SCI [64].

\section{s0270 Sensory test}

p0490 The sensory test is used to evaluate sensory pathways after SCI, analyzing the reactions of rats in the presence of heat, cold, or pain.

\section{s0275 Test for hot sensation}

p0495 To test the sensory stimulation of hind limbs after thoracic SCI, a $50^{\circ} \mathrm{C}$ preheated plate is used. The animal is placed on this plate for a maximum of $60 \mathrm{~s}$; the test is considered positive if the animal shows signs of pain or discomfort. A major limitation is that the animal may vocalize or have burn injuries during the test. It is important to take animals away from the heat after a maximum of $60 \mathrm{~s}$ to avoid burn injuries in desensitized animals.

s0280 Test for cold sensation

p0500 This test it is useful for sensory nociceptor testing and usually applies ethyl chloride on a shaved skin area. The response is recorded from 0 (no response) to 3 (sustained vocalization).

s0285 Tactile sensory test or neuropathic pain test

p0505 Before the test, animals have to be habituated to the cage where the microfilaments are placed. Two validated methods (up/down plantar Von Frey test and Von Frey hair monofilaments) are used. In both, withdrawal of the paws after stimuli indicates that the spinal reflex is active. A disadvantage of these tests is that repetitive measurements needed may lead to learned responses. 


\section{s0290 Sensorimotor tests}

p0510 Sensorimotor tests are used to evaluate sensory and motor integration after SCI.

s0295 Horizontal ladder walking test

p0515 This test it is useful to evaluate stepping and coordination of forelimbs and hind limbs. In this case, a horizontal ladder with rungs at 2-cm intervals is used. Animals are allowed to cross it from the original cage to a new one, with a reward. After several days of training, irregular rung patterns are used to normalize difficulty and enhance comparability during learning. Animal movements across the ladder are recorded and evaluated for seven categories.

s0300 Grid walking test

p0520 The grid walking test is useful for predicting and evaluating afferent/efferent sensorimotor integration. There are several versions, such as horizontal grid walking and ladder beam or inclined grid climbing, among others.

s0305 Rope climbing and horizontal walking test

p0525 After training, animals are required to climb a $160-\mathrm{cm}$ vertical rope four times, and the climbing is recorded on a video. The video records the number of foot slips for 42 days after the injury. In the horizontal rope walking test, rats are trained to cross an 83-cm rope five consecutive times. They are also recorded to assess steps and falls quantitatively.

\section{s0310 Quality control (Good Laboratory Practice, Good Clinical Practice, and Good Clinical Laboratory Practice)}

p0530 With new knowledge about the causes and mechanisms of diseases obtained from basic research, there is an increased demand for new drugs and principles of treatment. Basic biomedical research refers to the use of fundamental scientific principles in medical and biological research directed toward developing tools to detect, prevent, or treat human disease. Such research involves the discovery and exploratory studies that precede the regulated phases of drug development or programs to develop other methods of disease control.

p0535 Because regulatory research is time-consuming and requires vast financial, human, and technical resources, the data obtained in basic research activities need to be reliable to ensure a solid basis for deciding whether to invest in the further development of a strategy or product. When the working hypothesis is not well-conceived or the experiment is incorrectly conducted, the experiments will not lead to a true advance in knowledge acceptable to the scientific community. In addition, unregulated procedures will not be accepted outside the country of origin. The objective of regulations is to provide a unified standard to facilitate the mutual acceptance of 
laboratory and clinical data by regulatory authorities in these jurisdictions. Thus, these regulations cover the following aspects:

u0050 - the nonclinical stage to establish drug or procedure safety,

u0055 - the clinical stage to establish safety and efficacy in humans, and

$\mathrm{u} 0060$ - the postapproval stage, in which the drug is monitored for safety and its production is carefully controlled.

p0555 All of these aspects are regulated by quality practices in basic biomedical research. Good Laboratory Practice (GLP) covers the nonclinical stage and clinical and postapproval are ruled by Good Clinical Laboratory Practice (GCLP). All of these guidelines were written after discussion with organizations engaged in nonclinical safety studies and clinical trials and published by the World Health Organization for wide diffusion. If we apply them to preclinical studies, the results will be cleared as Stage 2 procedures implementing the use of the 3Rs principles.

p0560 The formal concept of GLP originated in the United States in the 1970s because of concerns about the validity of nonclinical safety data submitted to the FDA in the context of New Drug Applications. They were a reaction to cases of malpractice and fraud in the nonclinical testing of drugs performed by some pharmaceutical companies and contract research organizations. The inspection of studies and test facilities revealed instances of inadequate planning and incompetent execution of studies, insufficient documentation of methods and results, and even cases of fraud.

p0565 A little later, in 1981, the Organization for Economic Cooperation and Development (OECD) assembled an expert group to formulate the first OECD Principles of GLP. Compliance with these principles is a prerequisite for the mutual acceptance of data (OECD GLP Guideline), reducing costs. A number of OECD member countries have incorporated these principles into their national legislation. With the amendment of the EU in Commission Directive 1999/11/EC to Council Directive 87/18/ EEC, the OECD GLP was formally introduced into European legislation.

p0570 The GLP principles set out requirements for the appropriate management of nonclinical safety studies. This helps researchers to perform their work in compliance with their own preestablished scientific design. The principles may be considered a set of standards for ensuring the quality, reliability, and integrity of studies, the reporting of verifiable conclusions, and the traceability of data. These rules are applied to studies planned and conducted in a manufacturer's laboratory, a contract or subcontract facility, or a university or public sector laboratory.

p0575 Whatever the industry targeted, GLP stresses the importance of these main points:

u0065 - Resources: organization, personnel, facilities, and equipment;

u0070 - Characterization: substances or methods and test systems;

u0075 - Rules: protocols and standard operating procedures;

u0080 - Results: raw data, final report, and archives; and

u0085 - Quality assurance: independent monitoring of research processes. 
p0605 Depending on national legal situations, GLP requirements for nonclinical laboratory studies conducted to evaluate drug safety, including medical devices for human use, cover the following classes of studies:

u0090 - Single dose toxicity;

u0095 - Repeated dose toxicity (subacute and chronic);

u0100 - Reproductive toxicity (fertility, embryo-fetal toxicity and teratogenicity, and peri/postnatal toxicity);

u0105 - Mutagenic potential;

u0110 - Carcinogenic potential;

u0115 - Toxicokinetics (pharmacokinetic studies that provide systemic exposure data for these studies);

u0120 - Pharmacodynamic studies designed to test the potential for adverse effects (safety pharmacology); and

u0125 - Local tolerance studies, including phototoxicity, irritation and sensitization studies, or testing for suspected addictive and/or withdrawal effects of drugs.

p0650 Good Clinical Practice (GCP) is an international ethical and scientific quality standard for designing, conducting, recording, and reporting trials that involve the participation of human subjects. Compliance with this standard provides public assurance that the rights, safety, and well-being of trial subjects are protected, consistent with the principles that have their origin in the Declaration of Helsinki.

p0655 GCLP applies the principles established under GLP for data generation used in regulatory submissions relevant to the analysis of samples from a clinical trial. At the same time, it ensures that the objectives of the GCP principles are carried out. This ensures the reliability and integrity of data generated by analytical laboratories.

\section{s0315 Economic aspects}

p0660 Preclinical models performed with animals have a high cost, and not only economic. This happens mainly for three reasons. First, all procedures usually ended with dead animals or seriously hurt ones; second, the installation and handling of animals is expensive because of the strict conditions of humidity, temperature, isolation, and safety mechanisms. In addition, tests performed on samples may be as expensive those on experimental animals. The degree of neurological deficit during the progress of lesions may be assessed using an examination score, in vivo magnetic resonance imaging, and neurophysiological examination using specific and costly equipment.

p0665 Laboratory evaluation of these models has unique characteristics, such as the need for heavy-duty microtomes, saws for bone sectioning, microgrinders, and special equipment to improve the final result. In some cases, the existence of implants adds complexity to the process.

p0670 Histological, quantification, and immunohistochemical analyses are usually methods selected to evaluate specimens after the procedure ends. 
p0675 Other methods that may be used to evaluate samples involved in animal studies are microbiological (cultures and polymerase chain reaction assays) and the detection of proteins and another substances involved in mechanisms related to tissue degeneration and inflammation.

\section{s0320 Publications}

p0680 Scientists who use animals in research have the responsibility of ensuring that the studies are appropriately reported in terms of reproducibility and the translation of findings into potential clinical benefits. The problem is that in many cases, the reporting of research is inadequate and has incomplete information. To avoid it, Animal Research: Reporting of In Vivo Experiments (ARRIVE) guidelines have been published simultaneously in several bioscience research journals and have been endorsed in the journal instructions of most indexed publications for inclusions as supplementary information together with the main text and figures. These guides maximize output from research and should be enough information to allow the experiment to be repeated.

p0685 The ARRIVE guidelines, originally published in PLOS Biology, were developed in consensus with the scientific community (journal editors, research funders, and scientists) as part of an NC3Rs initiative to improve the standard of reporting of research using animals [65]. It consists of a checklist of 20 items describing the minimum information that all scientific publications reporting research using animals should include. Among the aspects reported are the number and specific characteristics of the animals used; details of housing and husbandry; and the experimental, statistical, and analytical methods. The results of the publication should reflect relevant characteristics of the experimental group and the health status of the animals, the number of animals included in each analysis, statistical facts with a measure of precision (standard error or confidence interval), and details about adverse effects.

\section{s0325 Remarks and recommendations}

p0690 Selecting a correct injury model, as well as the animal species, is essential for the reliability of results and possible translation to humans. Every model has advantages and disadvantages. It is necessary to employ selection criteria before starting the experimental procedure. It is also important to choose the best animal model and SCI pattern to answer each specific question.

p0695 Animal models of SCI have several limitations, such as the different neuroanatomy between animal species and humans [1]. Rodents have a spinal cord anatomy different from that of humans and subhuman primates; there are also differences between humans and large animal models [66].

p0700 Another thing to consider when translating the results of an animal model to humans is that, in contrast to clinical cases in humans, experimental animals injured 
under controlled conditions have more reproducible and less complex lesions. Normally, in SCI models, lesions are simple and concrete in a spinal region, whereas in human traumatic SCI, often lesions are multiple or complex [13]. Also, two other factors have a crucial role in experimental SCI and differ from human trauma: laminectomy and anesthesia. In most models, a laminectomy (which causes spinal instability and the trauma of surrounding tissues) is necessary to induce the SCI and may have an impact on the physiological responses to injury. On the other hand, animals are anesthetized during the SCI induction, and this anesthesia affects recovery after injury, including blood pressure and metabolism, among others [53].

p0705 To date, several preclinical discoveries with proved efficacy in animal models have been transferred to clinical trials. However, the most have failed to reproduce the same efficacy in humans as in animals. It is important to choose the animal species and study design carefully to reflect the clinical features of human SCI as much as possible.

\section{References}

[1] Cheriyan T, Ryan DJ, Weinreb JH, Cheriyan J, Paul JC, Lafage V, et al. Spinal cord injury models: a review. Spinal Cord 2014;52(8):588-95.

[2] Nardone R, Florea C, Höller Y, Brigo F, Versace V, Lochner P, et al. Rodent, large animal and non-human primate models of spinal cord injury. Zoology (Jena) 2017; 123:101-14.

[3] Sharif-Alhoseini M, Khormali M, Rezaei M, Safdarian M, Hajighadery A, Khalatbari MM, et al. Animal models of spinal cord injury: a systematic review. Spinal Cord 2017;55(8):714-21.

[4] Jakeman LB, Guan Z, Wei P, Ponnappan R, Dzwonczyk R, Popovich PG, et al. Traumatic spinal cord injury produced by controlled contusion in mouse. J Neurotrauma 2000;17(4):299-319.

[4a] Bresnahan JC, Beattie MS, Todd FD, Noyes DH. A behavioral and anatomical analysis of spinal cord injury produced by a feedback-controlled impaction device. Exp Neurol 1987;95(3):548-70.

[4b] Stokes BT. Experimental spinal cord injury: A dynamic and verifiable injury device. J Neurotrauma 1992;9(2):129-34.

[5] Sroga JM, Jones TB, Kigerl KA, McGaughy VM, Popovich PG. Rats and mice exhibit distinct inflammatory reactions after spinal cord injury. J Comp Neurol 2003;462(2): 223-40.

[6] Metz GA, Curt A, van de Meent H, Klusman I, Schwab ME, Dietz V. Validation of the weight-drop contusion model in rats: a comparative study of human spinal cord injury. J Neurotrauma 2000;17(1):1-17.

[7] Zuchner M, Lervik A, Kondratskaya E, Bettembourg V, Zhang L, Haga HA, et al. Development of a multimodal apparatus to generate biomechanically reproducible spinal cord injuries in large animals. Front Neurol 2019;10:223.

[8] Kwon BK, Streijger F, Hill CE, Anderson AJ, Bacon M, Beattie MS, et al. Large animal and primate models of spinal cord injury for the testing of novel therapies. Exp Neurol 2015;269:154-68. 
[9] Jeffery ND, Smith PM, Lakatos A, Ibanez C, Ito D, Franklin RJ. Clinical canine spinal cord injury provides an opportunity to examine the issues in translating laboratory techniques into practical therapy. Spinal Cord 2006;44(10):584-93.

[10] Prado C, Fratini P, de Sá Schiavo Matias G, Bocabello RZ, Monteiro J, Dos Santos CJ, et al. Combination of stem cells from deciduous teeth and electroacupuncture for therapy in dogs with chronic spinal cord injury: a pilot study. Res Vet Sci 2019;123: $247-51$.

[11] Iwanami A, Yamane J, Katoh H, Nakamura M, Momoshima S, Ishii H, et al. Establishment of graded spinal cord injury model in a nonhuman primate: the common marmoset. J Neurosci Res 2005;80(2):172-81.

[12] Baklaushev VP, Durov OV, Kim SV, Gulaev EV, Gubskiy IL, Konoplyannikov MA, et al. Development of a motor and somatosensory evoked potentials-guided spinal cord Injury model in non-human primates. J Neurosci Methods 2019;311: 200-14.

[13] Cizkova D, Murgoci AN, Cubinkova V, Humenik F, Mojzisova Z, Maloveska M, et al. Spinal cord injury: animal models, imaging tools and the treatment strategies. Neurochem Res 2019. https://doi.org/10.1007/s11064-019-02800-w.

[14] SCHEER (Scientific Committee on Health Environmental and Emerging Risks). Final opinion on the need for non-human primates in biomedical research, production and testing of products and devices (Internet). 2017. Available from: http://ec.europa.eu/ environment/chemicals/lab_animals/pdf/Scheer_may2017.pdf.

[15] Foditsch EE, Miclaus G, Patras I, Hutu I, Roider K, Bauer S, et al. A new technique for minimal invasive complete spinal cord injury in minipigs. Acta Neurochir 2018; 160(3):459-65.

[16] Navarro R, Juhas S, Keshavarzi S, Juhasova J, Motlik J, Johe K, et al. Chronic spinal compression model in minipigs: a systematic behavioral, qualitative, and quantitative neuropathological study. J Neurotrauma 2012;29(3):499-513.

[17] Wilson S, Abode-Iyamah KO, Miller JW, Reddy CG, Safayi S, Fredericks DC, et al. An ovine model of spinal cord injury. J Spinal Cord Med 2017;40(3):346-60.

[18] Saunders NR, Dziegielewska KM, Whish SC, Hinds LA, Wheaton BJ, Huang Y, et al. A bipedal mammalian model for spinal cord injury research: The tammar wallaby F1000Res 2017;6:921.

[19] Mokalled MH, Patra C, Dickson AL, Endo T, Stainier DY, Poss KD. Injury-induced ctgfa directs glial bridging and spinal cord regeneration in zebrafish. Science 2016; 354(6312):630-4.

[20] Ghosh S, Hui SP. Regeneration of Zebrafish CNS: adult neurogenesis. Neural Plast 2016;2016:5815439.

[21] Muñoz R, Edwards-Faret G, Moreno M, Zuñiga N, Cline H, Larraín J. Regeneration of Xenopus laevis spinal cord requires Sox2/3 expressing cells. Dev Biol 2015;408(2): 229-43.

[22] Barreiro-Iglesias A, Sobrido-Cameán D, Shifman MI. Retrograde Activation of the extrinsic apoptotic pathway in spinal-projecting neurons after a complete spinal cord injury in lampreys. BioMed Res Int 2017;2017:5953674.

[23] Valentin-Kahan A, García-Tejedor GB, Robello C, Trujillo-Cenóz O, Russo RE, Alvarez-Valin F. Gene expression profiling in the injured spinal cord of Trachemys scripta elegans: an amniote with self-repair capabilities. Front Mol Neurosci 2017; 10:17. 
[24] Rehermann MI, Marichal N, Russo RE, Trujillo-Cenóz O. Neural reconnection in the transected spinal cord of the freshwater turtle Trachemys dorbignyi. J Comp Neurol 2009;515(2):197-214.

[24a] Dennis Jr MB. Protection of research animals. In: von Recum AF, editor. Handbook of biomaterials evaluation. 2nd ed. Philadelphia, USA: Taylor \& Francis; 1999. p. $465-74$.

[25] Tannenbaum J, Bennett BT. Russell and Burch's then and now: the need for clarify in definition and purpose. J Am Assoc Lab Anim Sci 2015;54(2):120-32.

[26] Duncan E, Lemons JE, Mayesh JP, et al. New products and standards. In: Ratner BD, Hoffman AS, Schoen FJ, Lemons JE, editors. Biomaterials science. An introduction to materials in medicine. San Diego, CA, USA: Elsevier Academic Press; 2004. p. 783-804.

[27] Regan T. The case for animal rights. USA: University of California Press; 1983.

[28] Smith AC, Fosse RT, Ramp WK, Swindle M. Ethics and regulations for the care and use of laboratory animals. In: An YH, Friedman RJ, editors. Animal models in orthopaedic research. Boca Raton, FL, USA: CRC Press; 1999. p. 3-14.

[29] AVMA. Guidelines for the euthanasia of animals: 2013 edition. Schaumburg, IL, USA: American Veterinary Medical Association; 2013. Available from: avma.org.

[30] Anderson JM, Bianco RW, et al. Biological testing of biomaterials. In: Ratner BD, Hoffman AS, Schoen FJ, Lemons JE, editors. Biomaterials science. An introduction to materials in medicine. San Diego, CA, USA: Elsevier Academic Press; 2004. p. $355-409$.

[31] Dunham KA, Siriphorn A, Chompoopong S, Floyd CL. Characterization of a graded cervical hemicontusion spinal cord injury model in adult male rats. J Neurotrauma 2010;27(11):2091-106.

[32] Zurita M, Aguayo C, Bonilla C, Otero L, Rico M, RodrÍguez A, et al. The pig model of chronic paraplegia: a challenge for experimental studies in spinal cord injury. Prog Neurobiol 2012;97(3):288-303.

[33] Lane MA, Fuller DD, White TE, Reier PJ. Respiratory neuroplasticity and cervical spinal cord injury: translational perspectives. Trends Neurosci 2008;31(10):538-47.

[34] Pearse DD, Lo TP, Cho KS, Lynch MP, Garg MS, Marcillo AE, et al. Histopathological and behavioral characterization of a novel cervical spinal cord displacement contusion injury in the rat. J Neurotrauma 2005;22(6):680-702.

[35] Bennett DJ, Gorassini M, Fouad K, Sanelli L, Han Y, Cheng J. Spasticity in rats with sacral spinal cord injury. J Neurotrauma 1999;16(1):69-84.

[36] Petteys RJ, Spitz SM, Syed H, Rice RA, Sarabia-Estrada R, Goodwin CR, et al. Design and testing of a controlled electromagnetic spinal cord impactor for use in large animal models of acute traumatic spinal cord injury. J Clin Neurosci 2017;43:229-34.

[37] Fukuda S, Nakamura T, Kishigami Y, Endo K, Azuma T, Fujikawa T, et al. New canine spinal cord injury model free from laminectomy. Brain Res Brain Res Protoc 2005; 14(3):171-80.

[38] Wrathall JR, Pettegrew RK, Harvey F. Spinal cord contusion in the rat: production of graded, reproducible, injury groups. Exp Neurol 1985;88(1):108-22.

[39] Gruner JA. A monitored contusion model of spinal cord injury in the rat. J Neurotrauma 1992 Summer;9(2):123-6. discussion 126-8.

[40] Marcol W, Slusarczyk W, Gzik M, Larysz-Brysz M, Bobrowski M, GrynkiewiczBylina B, et al. Air gun impactor-a novel model of graded white matter spinal cord injury in rodents. J Reconstr Microsurg 2012;28(8):561-8. 
[41] Rivlin AS, Tator CH. Effect of duration of acute spinal cord compression in a new acute cord injury model in the rat. Surg Neurol July 1978;10(1):38-43.

[42] Blight AR. Morphometric analysis of a model of spinal cord injury in Guinea pigs, with behavioral evidence of delayed secondary pathology. J Neurol Sci 1991; 103(2):156-71

[43] Joshi M, Fehlings MG. Development and characterization of a novel, graded model of clip compressive spinal cord injury in the mouse: Part 1. Clip design, behavioral outcomes, and histopathology. J Neurotrauma 2002;19(2):175-90.

[44] Lee JH, Jones CF, Okon EB, Anderson L, Tigchelaar S, Kooner P, et al. A novel porcine model of traumatic thoracic spinal cord injury. J Neurotrauma 2013;30(3): $142-59$.

[45] Abdullahi D, Annuar AA, Mohamad M, Aziz I, Sanusi J. Experimental spinal cord trauma: a review of mechanically induced spinal cord injury in rat models. Rev Neurosci 2017;28(1):15-20.

[46] Tarlov IM. Spinal cord compression studies: II. Time limits for recovery after acute compression in dogs. Arch Neurol Phychiatry 1954a;70:813-9.

[47] Tarlov IM. Spinal cord compression studies: III. Time limits for recovery after gradual compression in dogs. Arch Neurol Phychiatry 1954b;71:271-90.

[48] Vanicky I, Urdzíková L, Saganová K, Cízková D, Gálik J. A simple and reproducible model of spinal cord injury induced by epidural balloon inflation in the rat. J Neurotrauma 2001;18(12):1399-407.

[49] Lim JH, Jung CS, Byeon YE, Kim WH, Yoon JH, Kang KS, et al. Establishment of a canine spinal cord injury model induced by epidural balloon compression. J Vet Sci 2007;8(1):89-94.

[50] Nesathurai S, Graham WA, Mansfield K, Magill D, Sehgal P, Westmoreland SV, et al. Model of traumatic spinal cord injury in Macaca fascicularis: similarity of experimental lesions created by epidural catheter to human spinal cord injury. J Med Primatol 2006;35(6):401-4.

[51] Khan M, Griebel R. Acute spinal cord injury in the rat: comparison of three experimental techniques. Can J Neurol Sci 1983;10(3):161-5.

[52] da Costa ES, Carvalho AL, Martinez AM, De-Ary-Pires B, Pires-Neto MA, de AryPires R. Strapping the spinal cord: an innovative experimental model of CNS injury in rats. J Neurosci Methods 2008;170(1):130-9.

[53] Kundi S, Bicknell R, Ahmed Z. Spinal cord injury: current mammalian models. Am J Neurosci 2013;4(1):1-12.

[54] Schwab ME, Bartholdi D. Degeneration and regeneration of axons in the lesioned spinal cord. Physiol Rev 1996;76(2):319-70.

[55] Dabney KW, Ehrenshteyn M, Agresta CA, Twiss JL, Stern G, Tice L, et al. A model of experimental spinal cord trauma based on computer-controlled intervertebral distraction: characterization of graded injury. Spine 2004;29(21):2357-64.

[56] Choo AM, Liu J, Liu Z, Dvorak M, Tetzlaff W, Oxland TR. Modeling spinal cord contusion, dislocation, and distraction: characterization of vertebral clamps, injury severities, and node of Ranvier deformations. J Neurosci Methods 2009;181(1):6-17.

[57] Seifert JL, Bell JE, Elmer BB, Sucato DJ, Romero MI. Characterization of a novel bidirectional distraction spinal cord injury animal model. J Neurosci Methods 2011; 197(1):97-103.

[58] Fiford RJ, Bilston LE, Waite P, Lu J. A vertebral dislocation model of spinal cord injury in rats. J Neurotrauma April 2004;21(4):451-8. 
[59] Oyinbo CA. Secondary injury mechanisms in traumatic spinal cord injury: a nugget of this multiply cascade. Acta Neurobiol Exp 2011;71(2):281-99.

[60] Park E, Velumian AA, Fehlings MG. The role of excitotoxicity in secondary mechanisms of spinal cord injury: a review with an emphasis on the implications for white matter degeneration. J Neurotrauma 2004;21(6):754-74.

[61] Watson BD, Prado R, Dietrich WD, Ginsberg MD, Green BA. Photochemically induced spinal cord injury in the rat. Brain Res 1986;367(1-2):296-300.

[62] Ahmed RU, Alam M, Zheng YP. Experimental spinal cord injury and behavioral tests in laboratory rats. Heliyon 2019;5(3):e01324.

[63] Basso DM, Beattie MS, Bresnahan JC. Graded histological and locomotor outcomes after spinal cord contusion using the NYU weight-drop device versus transection. Exp Neurol 1996;139(2):244-56.

[64] Lankhorst AJ, Duis SE, ter Laak MP, Joosten EA, Hamers FP, Gispen WH. Functional recovery after central infusion of alpha-melanocyte-stimulating hormone in rats with spinal cord contusion injury. J Neurotrauma 1999;16(4):323-31.

[65] Kilkenny C, Browne WJ, Cuthill IC, Emerson M, Altman DG. Improving bioscience research reporting: the ARRIVE guidelines for reporting animal research. PLoS Biol 2010;8(6): 1000412.

[66] Plemel JR, Duncan G, Chen KW, Shannon C, Park S, Sparling JS, et al. A graded forceps crush spinal cord injury model in mice. J Neurotrauma 2008;25(4):350-70.

[67] Singer P. Animal liberation: a new ethics for our treatment of animals. USA: Harper Collins; 1975.

\section{Further reading}

[1] Allen AR. Surgery of experimental lesion of spinal cord equivalent to crush injury of fracture dislocation of spinal column. A preliminary report. J Am Med Assoc 1911;57: 878-80.

[2] Dennis Jr MB. Protection of research animals. In: von Recum AF, editor. Handbook of biomaterials evaluation. 2nd ed. Philadelphia, USA: Taylor \& Francis; 1999. p. 465-74.

[3] European Union: Directive 2010/63/EU of the European Parliament and of the Council on 22 September 2010 on the protection of animals used for scientific purposes. Available in: eur-lex.europa.eu.

[4] LASA. Guiding principles for preparing for and undertaking aseptic surgery. In: Lilley E, Berdoy M, editors. A report by the LASA education, training and ethics section; 2017. http://www.lasa.co.uk/publications/.

[5] National Research Council. Guide for the care and use of laboratory animals. 8th ed. Washington DC, USA: The National Academies Press; 2011 Availabe in: https:// grants.nih.gov/grants/olaw/guide-for-the-care-and-use-of-laboratory-animals.pdf.

[6] Trials of war criminals before the Nuremberg military tribunals under control Council law No. 10U.S. Nuremberg Code. (Internet). Washington, DC, USA: U.S. Government Printing Office; 1949. Available from: https://history.nih.gov/research/downloads/ nuremberg.pdf.

[7] World Health Organization. Good clinical laboratory practice (GCLP). 2009. Available from: https://www.who.int/tdr/publications/documents/gclp-web.pdf. 
[8] World Health Organization. Handbook: good laboratory practice (GLP): quality practices for regulated non-clinical research and development. 2nd ed. 2009 Available from: $\quad$ https://www.who.int/tdr/publications/training-guideline-publications/goodlaboratory-practice-handbook/en/.

[9] World Health Organization. Handbook of quality practices in basic biomedical research. 2006. Available from: https://www.who.int/tdr/publications/training-guideline-publications/handbook-quality-practices-biomedical-research/en/. 



\title{
Non-Print Items
}

\begin{abstract}
Animal models of spinal cord injury (SCI) are necessary not only for evaluating new therapies and surgical techniques but also to improve understanding of the molecular pathways involved and predict clinical outcomes and prognosis. The ideal animal models should mimic the pathological features of human SCI, be reproducible and available, and have the capacity to create different degrees of SCI severity and functional outcomes. However, before choosing an appropriate animal model of SCI, it is necessary to identify the main aims and purpose of the study.

This chapter describes the main species and models available for use in SCI preclinical research. The authors highlight the regulations, ethical aspects, and publications related to using animals in research. Finally, we provide advice that we consider useful for researchers to design a protocol.
\end{abstract}

\section{Keywords:}

Animal models, Ethics, Injury models, Regulatory affairs, SCI repair. 


\section{CHAPTER}

\section{c0014 Fundamentals and application of modeling in support of spinal cord injury repair strategies}

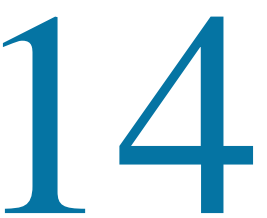

Tommaso Casalini, PhD ${ }^{1,2}$

${ }^{1}$ Institute for Chemical and Bioengineering, Department of Chemistry and Applied Bioscience, ETH Zurich, Zurich, Switzerland; ${ }^{2}$ Polymer Engineering Laboratory, Institute for Mechanical Engineering and Materials Technology, SUPSI, Manno, Switzerland

\section{s0010 Introduction}

p0010 The concept of engineering the design of biomedical devices with the purpose to obtain a target release profile, that is, the current drug delivery paradigm, dates back to 1960s [1]. The first modeling attempts took the first steps in the same period, when Prof. Takeru Higuchi instilled the principles of physical chemistry in his wellknown and still employed Higuchi equation, which constituted the fundamental pillar for the design of drug delivery systems in the 1960-85 period [2]. After more than 50 years, mathematical modeling reinforced even more his role in the field, emerging as a tool that allows, on the one side, rationalizing the experimental observations, and on the other side obtaining a fundamental understanding of the most important phenomena. Indeed, the system of interest does not appear as a "black box" anymore, but its behavior can be described through a suitable system of equations that highlights the constitutive laws. The acquired knowledge is not an end in itself, but it can be used to optimize the design of the device and the experimental activity, by means of simulations that can suggest $a$ priori, at least in trends, the impact of a change in formulation on system behavior. The evolution of the mathematical modeling from Higuchi equation is the net result of several synergistic factors, such as an increased availability of computational power, the formulation of new algorithms for a robust numerical solution of complex problems (e.g., moving boundaries) and the introduction of new approaches "borrowed" from different fields, such as molecular dynamics or dissipative particle dynamics. The first part of this chapter aims at providing the fundamental principles behind the most commonly used modeling approaches in the literature, underlining advantages and limitations, and the potential range of applicability. The second part is devoted to the discussion of specific examples of modeling related to systems of interest for spinal cord injury (SCI) repair. 


\section{s0015 Modeling approaches}

p0015 The discussed modeling approaches are here classified according to their intrinsic accessible time and length scales. On one hand, molecular dynamics allow performing simulations at atomic resolution, with time and length scale in the order of ns (up to $\mu \mathrm{s}$ ) and $\mathrm{nm}$, respectively; coarse-grained models extend time and length scales depending on the level of coarse-graining of the system. On the other hand, macroscale models (i.e., based on fundamental mass, energy and momentum conservation equations) have the largest accessible time and length scales, in the order of $s$ and $m$, respectively, but the molecular detail is lost. Of course, each approach has its own strong points, limitations and range of applicability but, above all, provides different insights about the system of interest because of the different involved time and length scales. This constitutes the strength of the multiscale modeling, where the descriptions at different time and length scales are synergistically combined and provide and all-around overview of system behavior.

\section{s0020 Molecular dynamics}

p0020 Molecular dynamics (MD) simulations represent atoms as spheres that mutually interact by means of a potential energy function called force field (FF). Molecular coordinates and velocities as a function of time are obtained integrating Newton's equation of motion:

$$
m_{i} \frac{d^{2} r_{i}}{d t^{2}}=F_{i}=-\nabla U(r(t))
$$

where $m_{i}$ is the mass of the $i$ th atom, $r_{i}$ are the spatial coordinates of the $i$ th atom, $t$ is time, $F_{i}$ is the force acting on the $i$ th atom, and $U(r(t))$ is the potential energy (i.e., the force field) as a function of atomic coordinates $r$, which are, in turn, function of time. The molecular system is thus modeled as a many-body system that obeys the laws of classical mechanics. This is a good approximation that decays when quantum effects are relevant, for example, when dealing with the motion of light species $\left(\mathrm{H}_{2}, \mathrm{D}_{2}, \mathrm{He}\right)$ or vibrational motions with a frequency $\nu$, so that $h \nu$ is higher than the thermal energy $k_{B} T$ (where $h$ is the Planck constant, $k_{B}$ is the Boltzmann constant, and $T$ is the absolute temperature) [3]. A force field accounts for both intramolecular and intermolecular interactions, and it is parameterized to best reproduce structural properties or conformational energies obtained through high-level quantum chemistry calculations or experimental data, such as hydration enthalpies or structural parameters obtained from NMR experiments [4]. The literature offers general-purpose force fields, such as GAFF (General Amber Force Field), GROMOS (GROningen MOlecular Simulations), OPLS (Optimized Potential for Liquid Simulations), and CHARMM (Chemistry at Harvard Macromolecular Mechanics), as well as FF tailored for a specific category of molecules, such as proteins, carbohydrates, nucleic acids, and lipids [4]. The reliability of a molecular simulation strongly depends on the accuracy of the employed force field. MD simulations do not take into account 
electrons; therefore, chemical reactions or excited states cannot be studied; they are the ideal tool for those systems whose behavior is mainly determined by noncovalent interactions, such as electrostatics and van der Waals. Moreover, MD simulations allow accounting for environmental effects by means of explicit solvent molecules, ions, and other solute molecules. Typical MD applications are the study of binding poses of drug molecules with target proteins or their permeation in lipid bilayers (that mimic cellular membranes or liposomal carriers), interactions of nucleic acids with drugs or carriers, self-assembling of amphiphilic compounds, conformation of polymeric systems (such as dendrimers), and crystallization [5-7]. The main simulation outputs are molecular trajectories, whose subsequent postprocessing can provide structural information (binding poses, ion and solvent distribution around a solute, protein conformations, etc.) as well as energetic information, such as interaction free energies. As a matter of fact, chemical reactions can be studied by means of $a b$ initio molecular dynamics (where potential energy is computed no more with FF but through quantum chemistry, and electrons are thus accounted for) or quantum mechanics/molecular mechanics (QM/MM) methods (where a small portion of the system is described through QM, while the remaining one is described with classical FF). Although ab initio MD is suitable only for small systems (hundredth of atoms), QM/MM methods can be employed to investigate, for example, enzyme inhibition through the covalent binding of small ligands. A detailed description of these approaches is beyond the purpose of this chapter, and the interested reader is referred to exhaustive reviews [8-10]. As mentioned, the characteristic time scale of MD simulations is limited to hundredth of ns, up to few $\mu$ s for small systems, while many phenomena of interest (protein folding/unfolding, drug unbinding, etc.) occur at higher time scales (from microseconds to minutes, or more); this is due to free energy barriers that separate metastable states. This issue can be overcome by means of enhanced sampling methods, which allow the system enhancing the transitions between different metastable states separated by an energy barrier higher than thermal energy $k_{B} T$. There are three main strategies: increasing the temperature $T$, modifying the potential $U(r)$, or adding an external bias potential $V(r)$ [11]. Each approach has its own methods, such as replica exchange (first strategy), Hamiltonian replica exchange and thermodynamic integration (second strategy), umbrella sampling, metadynamics, and accelerated molecular dynamics (third strategy). Highlighting the theoretical basis and the formulation of each method is well beyond the purpose of this chapter; the interested reader is referred to ad hoc reviews [11,12]. Among the wide range of available methods, metadynamics attracted a lot of interest in different fields, including the simulation of biological systems [13]. Briefly, metadynamics allows recovering the free energy of the system of interest as a function of few relevant conformational degrees of freedom (usually referred as collective variables $[\mathrm{CVs}]$ ) through a time-dependent bias, keeping track of the sampled conformations and discouraging the sampling of conformations that have already been visited. CVs are continuous and differentiable functions of atomic coordinates, such as distances and dihedral angles, but also more complex quantities like the number of hydrogen bonds between two groups of atoms, the electrostatic 
interaction energy or the alpha helix content in a protein. The main assumption (which can be verified a posteriori) is that enhancing the sampling of few degrees of freedom should be enough to achieve the convergence of the remaining ones during the simulation. The choice of suitable $\mathrm{CV}$ is fundamental to obtain meaningful and converged results; they must not only be able to discriminate different metastable states but they should also be representative of the transition mechanism. Metadynamics is widely employed for the study of protein conformations (including folding and unfolding in the presence of denaturants), drug binding to target proteins, conformations, and assembling of polymer systems. Currently, thanks to new advancements in the field, metadynamics can be used also to study the kinetics of the system, as showed for the unbinding rate of small ligands or protein folding/ unfolding $[14,15]$.

\section{s0025 Coarse-grained models}

p0025 Coarse-grained models are aimed at building simplified representations of complex systems that allow keeping the main chemical/physical peculiarities (such as the interplay between hydrophilic and hydrophobic effects) and performing affordable and meaningful simulations, which could not be feasible with, for example, molecular dynamics because of time and length scales limitations. Groups of atoms are enclosed in beads, which interact each other according to a suitable potential energy function that accounts for both bonded (if present) and nonbonded interactions, parameterized to reproduce experimental data (such as thermodynamic properties) or the behavior of a more detailed simulation at full-atomistic level. The coarsegraining procedure can embrace a wide range of length scales; indeed, a bead (also referred as interaction site) can include few atoms (e.g., a functional group), an entire protein or a polymeric microparticle, according to the system of interest and the property under investigation. Focusing at molecular level, the coarsegrained force field MARTINI [16] (where a bead includes a group of three or four heavy atoms) attracted a lot of interest for its flexibility and reliability. Analogously to molecular dynamics, trajectories are computed by integrating Newton's equation of motion, adopting a suitable potential energy function. Although many coarsegrained models are based on ad hoc parameterizations specific for the system of interest, MARTINI force field offers a straightforward strategy for system coarsegraining, thanks to a library of interaction sites mainly divided in four categories: polar, nonpolar, apolar, and charged; each group includes subgroups according to the polarity or the hydrogen-bonding capability. Although parameters for bonded interactions must be determined from detailed simulations at atomic level, nonbonded interactions are taken into account through a simple Lennard-Jones potential, whose parameters are available and obtained to best reproduce thermodynamic properties such as free energy of hydration, free energy of vaporization, and partitioning free energies between water and different solvents. Coarse-graining involves also solvent molecules that can be explicitly included in the system; MARTINI water beads, for example, are representatives of four water molecules. MARTINI force field proved 


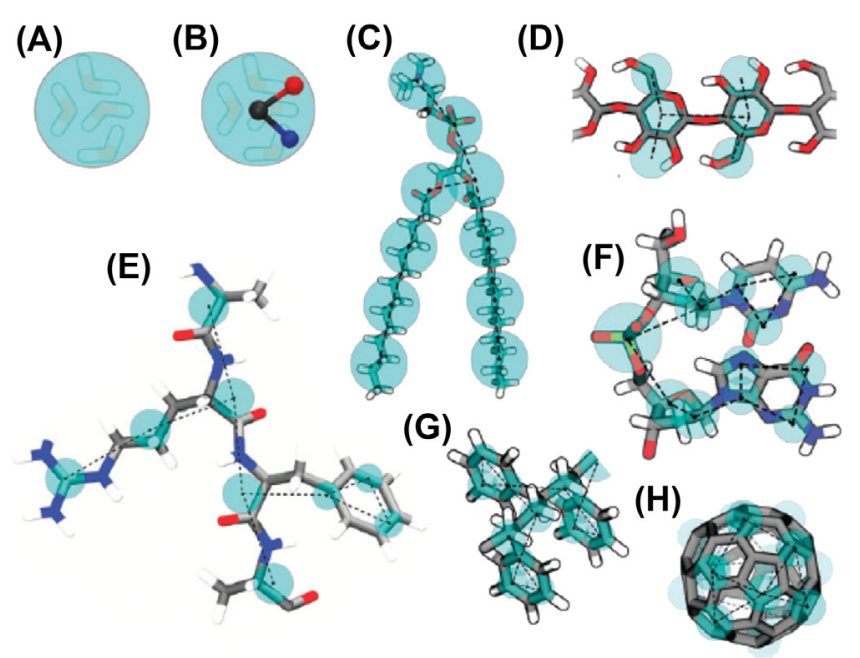

f0010 FIGURE 14.1

Example of MARTINI mapping of selected molecules. Standard water bead embedding four water molecules (A). Polarizable water bead with embedded charges (B). DMPC lipid (C). Polysaccharide fragment (D). Peptide (E). DNA fragment (F). Polystyrene fragment (G). Fullerene (H).

Reproduced with permission from Marrink SJ, Tieleman DP. Perspective on the martini model. Chem Soc Rev 2013;42:6801-22. Copyright The Royal Society of Chemistry, 2013.

to provide satisfactory results in a wide range of applications, such as peptide self-assembling, lipid membranes, emulsions, and membrane proteins [16,17]; in addition, parameters for nonbonded interactions can be further tuned to improve the agreement with detailed full-atomistic simulations. A graphical example of MARTINI mapping is provided in Fig. 14.1.

p0030 When coarse-graining involves bigger entities, such as protein or particles, commonly used approaches are, for example, Brownian dynamics (BD), dissipative particle dynamics (DPD), or Monte Carlo (MC) simulations [18,19], which are here only briefly summarized. BD and DPD are still based on Newton's equation of motion: in addition to the interparticle interaction potential (which leads to a conservative force), they also include a dissipative force (that accounts for the effect viscosity) and a random force (representing the effects of thermal fluctuations) that acts as a white noise. In MC simulations, particles still experience an interaction potential, but the dynamics are usually propagated by means of Metropolis algorithm.

\section{s0030 Macroscale models}

p0035 In the drug delivery field, macroscale models are usually employed to describe the release of active compounds from suitable dosage forms or devices, underlining the 
fundamental mechanisms behind the observed release rate. When bioresorbable polymers are employed (such as aliphatic polyesters, vide infra), macroscale models can be fruitfully employed to account for degradation kinetics. Such models can be divided into three main categories, according to the underlying approach that also determines the descriptive power [20]:

u0010 - Empirical models: they are simply constituted by an equation, which contains no theoretical insights but is able to account for the trend under investigation by properly tuning input parameters. Such models are not adequate to predict system behavior for different operative conditions due to the lack of physical meaning of involved parameters, and their importance is quite limited.

u0015 - Semiempirical models: they are largely based on experimental data such as empirical models, but contain a theoretical basis in their formulation. Involved parameters have physical meaning and can help understanding experimental data. Semiempirical models can be employed when only trends are necessary, but the descriptive power is not enough to allow performing device design.

u0020 - Mechanistic models: they are based on fundamental mass, energy, and momentum conservation principles. Input parameters have a specific physical meaning (diffusion coefficients, kinetic constants, mass transfer coefficients, etc.) and, in principle, can be independently estimated. However, empirical or semiempirical correlation can be included (such as a drug diffusivity as a function of polymer molecular weight). As they contain the fundamental description of the system through its constitutive laws, they can be used to investigate device behavior in different conditions and thus to optimize its design. Mechanistic models can be further divided into two main groups:

u0025 - Deterministic models: they use deterministic equations to account for, for example, device degradation or drug diffusion. They are extensively employed in the field, and this chapter is focused on this approach (vide infra).

u0030 - Stochastic models: they consider the phenomenon of interest, for example, chain scission, as a random event.

p0065 As mentioned, mechanistic deterministic models are based on fundamental mass, energy, and momentum conservation principles. In particular, mass balances play a key role, as they are extensively employed to characterize device degradation (when bioresorbable matrices are used) and, above all, the release of active molecules and their fate in tissues, which determine the efficacy of the proposed drug-eluting device. Energy balance is usually not essential, as the systems can be reasonably considered as isothermal. Momentum conservation equations are employed, for example, when the performances of stents are investigated: blood flow as well as the stress fields for blood vessels and the stent must be taken into account for a comprehensive analysis. Given the mare magnum of possible applications, this chapter focuses on the fruitful use of mass balances for characterizing the behavior of hydrogels and devices made of bioresorbable aliphatic polyesters, as they attracted a lot of interest also in the field of SCI repair. In this framework, macroscale models 
represent a useful tool to characterize the degradation of bioresorbable matrices and the transport phenomena, so that release rate of the loaded active molecules can be properly tuned according to the specific exigencies. The description of the underlying physics is indeed essential, because of the different involved mechanisms that determine release rate; as pointed out by Siepmann [21], several factors can influence drug release in vitro:

u0035 - Wetting of system surface with water and water penetration in the device;

u0040 - Phase transition of polymeric excipients (e.g., glassy-to-rubbery phase transition); u0045 - Drug and excipient dissolution;

u0050 • Drug and/or excipient degradation, dissolution and/or precipitation of degradation products;

u0055 - Creation of water-filled pores;

u0060 - Pore closing due to polymer swelling;

u0065 - Significant hydrostatic pressure in the device (e.g., coated dosage forms) and consequent convection-driven release;

u0070 - Creation of cracks;

u0075 - Creation of acidic or basic microenvironment in the device bulk due to degradation products;

u0080 - Changes in the rate of drug and/or excipient degradation and/or dissolution due to microenvironmental $\mathrm{pH}$ variation;

u0085 - Physical drug/excipient interactions (electrostatic, Van der Waals), which can also depend on the microenvironmental $\mathrm{pH}$;

u0090 • Time- and/or position-dependent diffusion through the device;

u0095 - Mass transport resistance at the device/environment interface;

u0100 - Penetration of acids, bases, or salts from the environment into the device.

p0140 The description is even more complicated in vivo where additional mechanisms occur, such as enzymatic degradation, protein binding on device surface, drug uptake into cells, and drainage into lymphatic system. Certainly, it is impossible to include all these phenomena in a mathematical model, which must contain only the real rate-determining processes. This aims at reducing not only model complexity, but also the number of input parameters, especially if they must be estimated from experimental data. Complex theories need many system-specific inputs; fitting many parameters at the same time can be challenging and can also lead to overfitting, which improves the agreement between experiments and theory even if the formulaically description is not appropriate. In other words, the good agreement does not imply the validity of the theoretical framework, which can be assessed by means of purely predictive simulations that can be compared with independent experimental datasets (i.e., not employed for parameters fitting). Some models have a broad range of applications; other models can be used in a more limited number of cases, but there is not a general model suitable for all possible situations; the approach depends on the specific case. Available models can be mainly classified according, for example, to the principal rate-limiting mechanism that governs drug release, as discussed in the following paragraphs. 


\section{s0035 Models based on diffusion}

p0145 In this framework, drug diffusion through the device is the rate-determining step behind release rate [22]. Fick's law (here presented in one dimension) usually constitutes the starting point:

$$
J=-D \frac{\partial C}{\partial x}
$$

where $J$ is the diffusive flux, $D$ is the diffusion coefficient, $C$ is concentration, and $x$ is the spatial coordinate along which diffusion takes place. By taking advantage of Eq. (14.2), drug concentration inside the device can be accounted for through a simple mass balance:

$$
\frac{\partial C}{\partial t}=D\left(\frac{\partial^{2} C}{\partial x^{2}}+\frac{\partial^{2} C}{\partial y^{2}}+\frac{\partial^{2} C}{\partial z^{2}}\right)
$$

where $t$ is time and $x, y, z$ are Cartesian coordinates; Eq. (14.3) can be solved by assigning proper initial conditions (concerning drug distribution in the device before release onset) and boundary conditions (usually profile symmetry at device center, and concentration value or mass flux continuity at the device/environment interface). Analytical solutions can be achieved for different geometries (slab, cylinder, sphere) if the following assumptions are satisfied:

u0105 - Diffusion coefficient is constant in time and space;

u0110 - Perfect sink conditions in the release medium (i.e., negligible drug concentration);

u0115 - Device swelling negligible or very rapid attainment of swelling equilibrium;

u0120 • Device degradation/erosion absent or negligible;

u0125 - Mass transfer resistance at device/release medium interface is negligible;

u0130 - Homogeneous initial drug distribution.

p0180 Numerical solutions become unavoidable, for example, if diffusion coefficient changes in time and space (e.g., because of device degradation and swelling) and/ or if erosion or swelling decreases or increases, respectively, device volume (moving boundary problem). In primis, the aspect that discriminates the optimal modeling approach is the dosage form, which can be classified into two categories: reservoir systems (the drug is located at the center and coated with a membrane) or monolithic systems (the drug is dispersed in a matrix). For each dosage form, two limiting situations can be identified depending on initial drug concentration, which can be above or below solubilization limit. In the first case, after water penetration, not the entire amount of drug is dissolved, but solubilized drug and nonsolubilized drug coexist in the device; this is important, as only dissolved compounds are available for diffusion. Assuming that water penetration and drug dissolution are much faster than drug diffusion, a saturated drug solution is rapidly obtained into the device and drug concentration remains constant (a released molecule is rapidly replaced by a dissolved one) and equal to the saturation value until a drug excess is present. On the other hand, and under the same assumptions, if initial drug 
concentration is below its solubilization limit, complete dissolution occurs upon water penetration; the concentration of the solubilized active compound inside the device decreases in time and space. For simple cases (i.e., if all the previously mentioned assumptions hold), analytical solutions are available for all four cases (reservoir/monolithic system, initial drug concentration above/below saturation limit) for three different geometries (slab, cylinder, sphere) as summarized by Siepmann [21,22]. Higuchi equation is the most famous example, as it describes drug release from a monolithic slab, when initial concentration is higher than solubilization limit. In general, a key point is a proper estimation of the diffusion coefficient, which can be challenging in polymeric systems, as illustrated in the "Transport Phenomena" section (vide infra).

\section{s0040 Models based on polymer swelling and polymer dissolution}

p0185 In this type of systems, a water-soluble active compound is loaded in a matrix made of a glassy polymer. Focusing on swelling, when the device is placed in water (or buffer), solvent diffusion leads to polymer swelling and volume increase. Indeed, water content into the system increases and, when a certain system-specific solvent concentration value is reached, the mobility of polymer chains is steeply enhanced. This phenomenon is also referred as polymer chain relaxation or glassy-to-rubber phase transition. This has two potential opposing effects; on the one side, volume increase implies longer diffusive paths, slowing down the active compound release. On the other side, the enhanced chain mobility increases also drug mobility, accelerating the release rate. From a modeling point of view, the system can be rationalized by identifying different zones separated by moving boundaries, as shown in Fig. 14.2. On the left side, there is the surrounding release medium, while on the right side there is the inner core of the device. The solvent diffuses through the

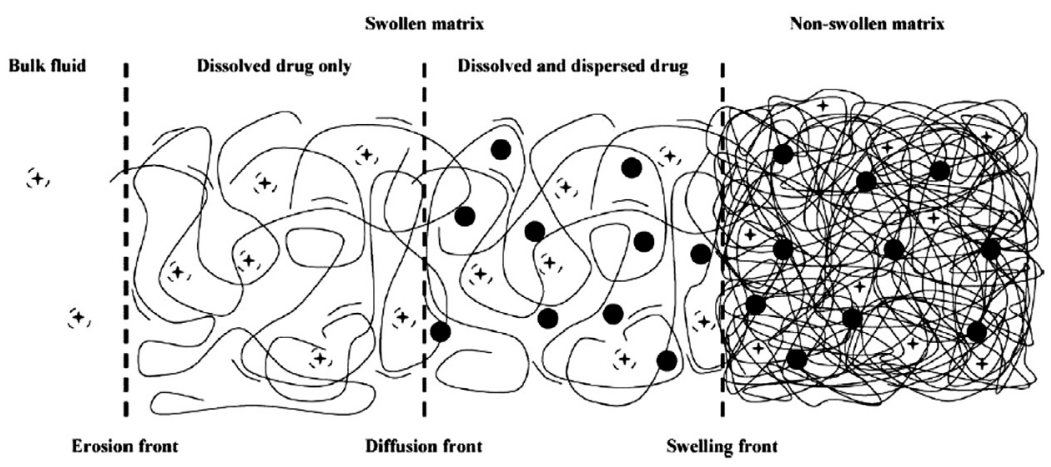

fO015 FIGURE 14.2

Schematization of a swelling-controlled drug delivery system, which contains both dissolved (black crosses) and undissolved (black filled circles) drugs.

Reproduced with permission from Siepmann J, Siepmann F. Mathematical modeling of drug delivery. Int J Pharm 2008;364:328-43. Copyright Elsevier, 2008. 
matrix and chain relaxation takes place; the boundary where this process occurs is called swelling front, and it separates the swollen portion of the matrix from the nonswollen one (located in the bulk of the device), where the polymer is still in glassy state. As mentioned, the swelling front is not stationary, but moves inward because of solvent diffusion toward the core of the device. In the swollen portion of the polymer matrix, dissolved (black crosses) and undissolved (black filled circles) drugs coexist, if the initial concentration of the active compound is higher than its solubilization limit. In this case, the concentration of dissolved drug is constant, as a released molecule is rapidly replaced by dissolution (in other words, a saturated drug solution is present in the solvent-filled pores of the matrix). When all excess drug is dissolved, drug concentration inside the device decreases. The front that separates the swollen matrix that contains only dissolved drug from the one that contains both dissolved/undissolved active molecules is called diffusion front. When water-soluble matrices are employed, it is possible to identify a third boundary located at the matrix/release medium interface, referred as the erosion front.

p0190 If polymer dissolution plays a key role (i.e., when a water-soluble matrix is employed), in addition to the solvent diffusion and solvent-induced plasticization (responsible for chain relaxation) also chain disentanglement should be taken into account. As explained for polymer swelling, a system rationalization based on sequential layers has been proposed and shown in Fig. 14.3 for an amorphous glassy polymer [23].

p0195 Available models use this layer-based description as a starting point, and can be classified according to the lumping of such layers and the assumptions concerning rate-determining transport phenomena and/or dissolution kinetics. Modeling approaches can be divided into four main categories [23]:

u0135 - Models based on phenomenology and Fickian equations;

u0140 - Models in which the rate-determining transport phenomenon is external mass transfer;

u0145 - Models based on stress relaxation;

u0150 - Anomalous transport models and scaling laws for chain disentanglement.

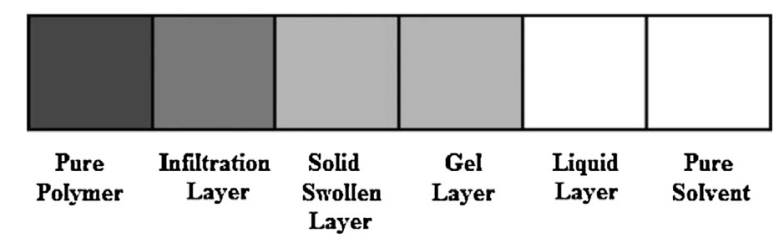

fO020 FIGURE 14.3

Schematic representation of a layer-based model for the dissolution of an amorphous glassy polymer.

Reproduced with permission from Kaunisto E, Marucci M, Borgquist P, Axelsson A. Mechanistic modelling of drug release from polymer-coated and swelling and dissolving polymer matrix systems. Int J Pharm 2011;418: 
p0220 Specific examples are reported and discussed in Applications section; a proper estimation of diffusion coefficients is still an important point, because of the role of the solvent penetration dynamics, and is discussed in a following paragraph (vide infra).

\section{s0045 Models based on polymer erosion and degradation}

p0225 Aliphatic polyesters, resulting from the homo- or copolymerization of monomers such as lactic acid, glycolic acid, and caprolactone, are widely used as raw materials for biomedical devices because they naturally degrade in vivo through hydrolysis mechanism (water molecules break ester bonds that constitute polymer backbone) and the body itself metabolizes degradation products. As expected, this has a non-negligible impact on the release rate, as chain scission creates new and wider diffusive paths. In this framework, as pointed out by Siepmann [21], it is useful to distinguish properly between degradation and erosion. Degradation identifies the chain scission process, where monomers and oligomers are obtained from the cleavage of long chains. Erosion is the mass loss from polymer bulk and can involve monomers, oligomers, and parts of polymer bulk. According to these definitions, the degradation of water-insoluble polymer is part of its erosion process. Two types of erosion can be highlighted, depending on the relative rate of water penetration and water consumption due to chain scission. If polymer degradation is much faster than water diffusion, heterogeneous or surface erosion takes place: chain cleavage involves only a small layer across the surface, while the bulk remains intact. On the other hand, if water diffusion dynamics are much faster than degradation kinetics, homogeneous or bulk erosion occurs; in this case, the system is rapidly wetted and polymer degradation involves the entire device. This concept is illustrated in Fig. 14.4.
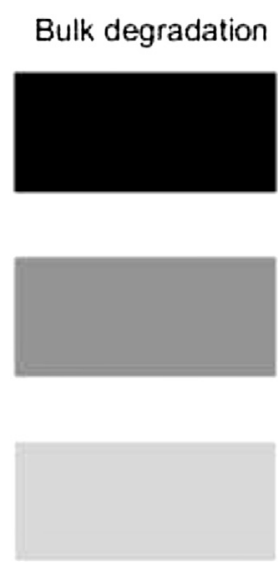

fO025 FIGURE 14.4

Schematization of bulk and surface degradation.

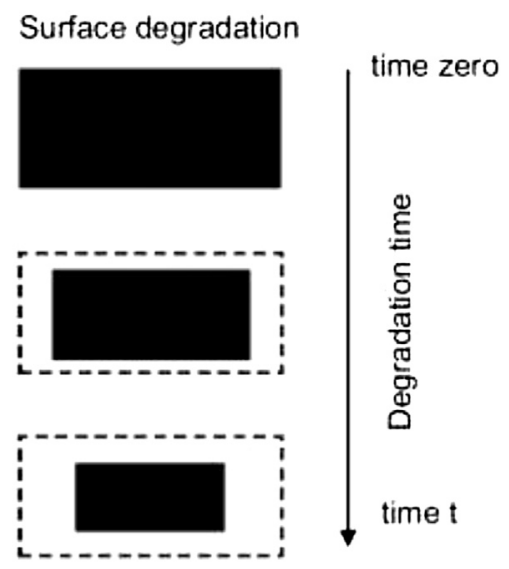

time $\mathrm{t}$

\footnotetext{
Reproduced with permission from Lao LL, Peppas NA, Boey FYC, Venkatraman SS. Modeling of drug release from

Reproduced with permission from Lao LL, Peppas NA, Boey FYC, Venkatraman SS. Modeling of drug release from
bulk-degrading polymers. Int J Pharm 2011;418:28-41. Copyright Elsevier, 2010.
} 
p0230 Aliphatic polyesters usually exhibit bulk erosion, while polyanydrides are subjected to surface erosion, as material hydrophobicity prevents a relevant water penetration within polymer matrix. The attainment of heterogeneous or homogeneous erosion depends not only on the chosen material but also on the device size. In this regard, Von Burkersroda and coworkers [25] introduced the concept of critical thickness. If the characteristic size of the device is bigger than critical thickness, surface erosion occurs, otherwise homogeneous erosion takes place. In their work, Von Burkersroda et al. provide critical thickness values for different polymers, which range from $75 \mu \mathrm{m}$ for polyanydrides to $7.4 \mathrm{~cm}$ for aliphatic polyesters, with the upper bound of $13.4 \mathrm{~m}$ for polyamides. In this framework, the description of drug release (initial drug amount can be above or below solubilization limit, vide supra) must be coupled with constitutive laws that account for both erosion mechanism and a suitable degradation kinetics, because of their relevant influence on release rate. Literature offers a wide range of examples for both surface and bulk erodible matrices, with different approaches to account for degradation kinetics, as illustrated in Applications section.

\section{s0050 Transport phenomena}

p0235 A crucial aspect of the vast majority of models is a proper description of transport phenomena. Although the diffusion in liquids and gases can be successfully characterized by existing theories [26], the use of theoretical models for diffusion in polymers and gels is still challenging because of system complexity, due to the dependence on polymer concentration and swelling [27,28]. In polymers, Fickian diffusion occurs when the temperature is above the glass transition temperature $T_{g}$ : as already pointed out, polymer chains are highly mobile and allow an easy solvent (or solute) diffusion. In other words, polymer relaxation is faster than solvent diffusion. If temperature value is below $T_{g}$, the polymer is in glassy state and the chains are not mobile enough to allow a fast solvent penetration; this represents the situation where solvent diffusion rate is faster than polymer relaxation rate, and it is usually referred as Case II diffusion. This implies the presence of a swollen and a nonswollen region separated by a sharp front, as already discussed in Models based on polymer swelling and polymer dissolution section. If the order of magnitude of the rates of the processes is similar, anomalous diffusion takes place. As extensively reviewed [27], there are several approaches that allow estimating diffusivity values in polymers and gels. They can be classified according to the physical concept they are based on:

u0155 - Theories based on obstruction effects: polymer chains are modeled as motionless with respect to solvent and solute molecules. The main assumption behind such theories is that polymer self-diffusion coefficient is much smaller than the one of diffusing molecules. Therefore, polymer matrix is represented as a fixed and impenetrable network, which leads to an increase of the mean path length experienced by diffusants. Such theories are suitable for estimating the diffusion 
coefficients of small molecules at low polymer concentrations, while they fail at high polymer concentration because solute/matrix interactions, which are not accounted for, are no more negligible.

u0160 - Theories based on hydrodynamic interactions: hydrodynamic theories account for the mutual interactions between the polymer, the solvent, and the solute. Some models can be employed also for determining the diffusivities of large molecules and/or in concentrated polymer solutions.

u0165 - Theories based on free volume: free volume is defined as the volume not occupied by the matter or, in other words, the volume of a given system at the temperature of interest minus the volume of the same system at $0 \mathrm{~K}$. The main assumption is that diffusion is mainly controlled by free volume rearrangements, which create paths where solute and solvent molecules can diffuse through.

p0255 As already mentioned, each approach has its own strengths and range of applicability. Besides such three main categories, literature offers other modeling frameworks. A relevant example is Amsden's model [27,29], which combines obstruction and hydrodynamic theories, accounting for polymer structural properties (chain stiffness, chain radius), polymer volume fraction, and size of diffusants. In addition, specific models have been developed for the diffusion of charged compounds in polyelectrolyte matrices, which also account for electrostatic interactions [30].

\section{s0055 Applications}

p0260 In the following sections, a brief overview concerning the applications of the discussed modeling approaches is provided. Because of the extent of the subject, this summary cannot be inevitably exhaustive and extensively cover all the scientific literature. The aim is to illustrate, through selected examples, the potentiality of modeling in the drug delivery field. The overview is focused on devices based on hydrogels and aliphatic polyesters, as they are the most diffuse and promising systems in the SCI repair field [31-33].

\section{s0060 Hydrogels}

p0265 Hydrogels are cross-linked hydrophilic polymer matrices that form an insoluble three-dimensional network; they are able to retain a high amount of water, up to 99\% in weight terms [34]. Their structure is usually characterized by means of three parameters: polymer volume fraction in the swollen state $v_{2, s}$, average molecular weight between cross-links $M_{c}$ and average mesh size $\xi$ (i.e., the distance between two adjacent cross-links). The starting point for uncharged networks is FloryRehner theory [35], which states that an amorphous dry polymer network immersed 
in a fluid is subjected only to two opposite forces, namely the thermodynamic force of solvent/polymer mixing and the retractive force of polymer chains. Equilibrium is reached when the forces balance each other; in Gibbs free energy:

$$
\Delta G_{\text {total }}=\Delta G_{\text {mixing }}+\Delta G_{\text {elastic }}
$$

where $\Delta G_{\text {total }}$ is Gibbs free energy of the system, $\Delta G_{\text {mixing }}$ is the contribution due to the solvent/polymer mixing (thus representative of the affinity of the matrix with the surrounding fluid), and $\Delta G_{\text {elastic }}$ accounts for the elastic retractive force. Eq. (14.4) can be expressed in chemical potentials, by differentiating with respect to the number of moles of solvent molecules at constant temperature and pressure:

$$
\mu_{1}-\mu_{1,0}=\Delta \mu_{\text {mixing }}+\Delta \mu_{\text {elastic }}
$$

where $\mu_{1}$ is solvent chemical potential inside the gel, $\mu_{1,0}$ is solvent chemical potential in the surrounding fluid. At equilibrium, the difference in chemical potential is zero. Expressing the change in chemical potential due to the mixing $\Delta \mu_{\text {mixing }}$ with heat and entropy of mixing, and the one due to elastic forces $\Delta \mu_{\text {elastic }}$ with through rubber elasticity theory, $M_{c}$ can be obtained [36,37]:

$$
\frac{1}{M_{c}}=\frac{2}{M_{n}}-\frac{\frac{v}{V_{1}}\left[\ln \left(1-v_{2, s}\right)+v_{2, s}+\chi_{1} v_{2, s}^{2}\right]}{v_{2, s}^{1 / 3}-\frac{v_{2, s}}{2}}
$$

where $M_{n}$ is the polymer average molecular weight before cross-linking, $v$ is the polymer-specific volume, $V_{1}$ is the water molar volume, and $\chi_{1}$ is the polymersolvent interaction parameter. Peppas and coworkers have extended the theory for hydrogels prepared in the presence of solvent, accounting for the changes in elastic potential due to the solvent [37]. The presence of polyelectrolytes in the polymer network complicates the description of the swelling, as an additional term must be accounted for the following:

$$
\begin{gathered}
\Delta G_{\text {total }}=\Delta G_{\text {mixing }}+\Delta G_{\text {elastic }}+\Delta G_{\text {ionic }} \\
\mu_{1}-\mu_{1,0}=\Delta \mu_{\text {mixing }}+\Delta \mu_{\text {elastic }}+\Delta \mu_{\text {ionic }}
\end{gathered}
$$

where $\Delta G_{\text {ionic }}$ and $\Delta \mu_{\text {ionic }}$ are the change in free energy and chemical potential, respectively, due to the presence of ionic moieties in the network; Eq. (14.8) is obtained analogously to Eq. (14.5). Peppas and coworkers have developed suitable expressions for the ionic contribution to the chemical potential, which depends on the ionic strength of the surrounding media and the nature of ions present in the solvent [37]. Average mesh size can be computed as follows:

$$
\xi=v_{2, s}^{-1 / 3}\left(\frac{2 C_{n} M_{c}}{M_{r}}\right)^{1 / 2} l
$$

where $C_{n}$ is the Flory characteristic ratio, $M_{r}$ is the molecular weight of the repeating unit of polymer chains, and $l$ is the bond length along polymer backbone. As 
expected, the knowledge of hydrogel structure is essential, as the intrinsic porosity determines the size of the solutes that can diffuse. In this regard, molecular dynamics simulations have been employed to study both structural/mechanical properties of the network and the diffusion of small molecules. In particular, self-diffusion coefficients can be computed from molecular trajectories using Einstein equation:

$$
D=\lim _{t \rightarrow \infty} \frac{1}{2 d t}(r(t)-r(t=0))^{2}
$$

where $D$ is the self-diffusion coefficient, $t$ is time, $d$ is the dimensionality of the system, and $r(t)$ are the molecular coordinates at time $t$. The term inside angle brackets represents the squared distance traveled by the molecule at time $t$, also referred as mean square displacement (MSD); angle brackets indicate that MSD is averaged over all solute molecules and/or multiple time origins. The limit indicates that Eq. (14.10) is valid for simulations that are long enough so that Brownian motion regime is reached. According to Eq. (14.10), the $t$ versus MSD plot is a straight line, whose slope is equal to $2 d D$. A commonly used approach to model hydrogels at molecular scale is the creation of a fundamental building block that, repeated in space by means of periodic boundary conditions, allows reproducing a regular network that constitutes a reasonable statistical description of the real system, as shown in Fig. 14.5.

p0270 Jang et al. [39] adopted the method depicted in Fig. 14.5 to study mechanical properties of single and double networks made of polyacrylic acid and polyethylene

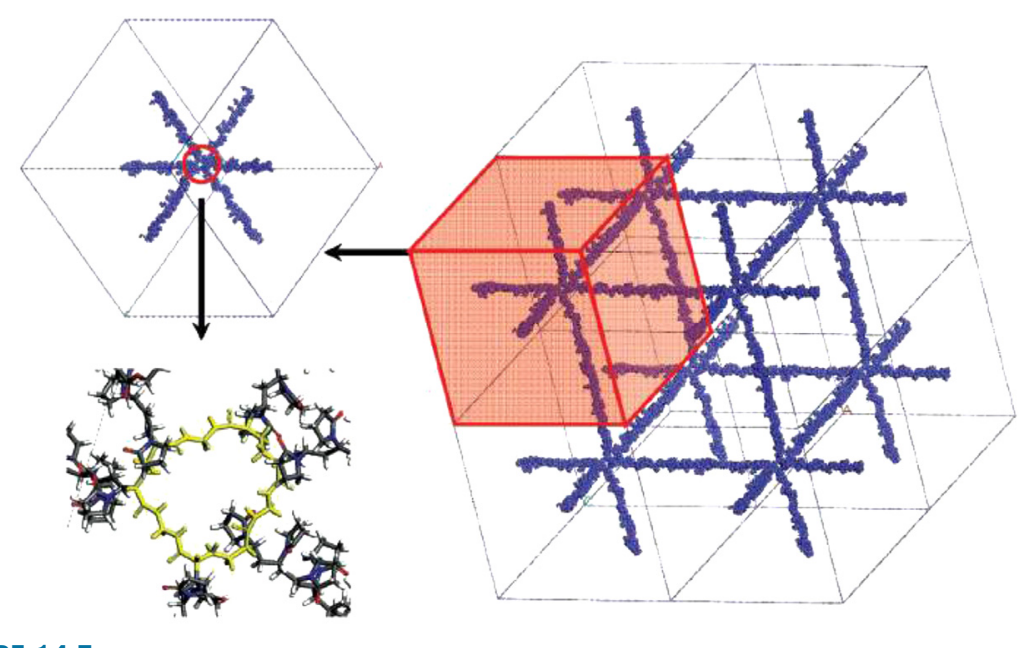

f0030 FIGURE 14.5

Building of a three-dimensional network through the repetition of a fundamental building block with periodic boundary conditions.

Reproduced with permission from Lee SG, Brunello GF, Jang SS, Lee JH, Bucknall DG. Effect of monomeric sequence on mechanical properties of P(VP-co-HEMA) hydrogels at low hydration. J Phys Chem B 2009;113: 6604-12. Copyright American Chemical Society, 2009. 
oxide, obtaining stress/strain curves from MD simulations, and the diffusion of D-glucose and ascorbic acid in the gels. In a series of papers, Lee et al. [40,41] investigated the effect of water content and polymer composition on mechanical and transport properties of hydrogels made of poly(N-vinyl-2-pyrrolidone-co-2hydroxyethyl methacrylate). Wu et al. [42] characterized the diffusion of water, ions, and rhodamine in PEO hydrogels at different cross-linking density values; diffusion coefficients showed a reasonable agreement with Amsden model. Edgecombe and coworkers [43-46] extensively used coarse-grained MC simulations to study the structure and swelling of polyelectrolyte hydrogels, assessing in detail the effects of the presence of salts, size, and charge of counterions and topological defects. Chen and Yong [47] employed dissipative particle dynamics for the simulation of swelling, obtaining a good agreement with Flory-Rehner theory. Another approach for the simulation of hydrogels is the investigation of the structure of single or multiple long chains in solution. Walter et al. $[48,49]$ employed MD to study the conformation of poly(N-isopropylacrylamide) (PNIPAM) chains in water and water/ methanol solutions at different temperatures. The same system has been studied by Deshmukh et al. [50], who focused on the effect of cross-linker type on swelling, and Alaghemandi et al. [51], who investigated the structure of single and multiple PNIPAM chains in water solution in a wide range of temperature that also included the lower critical solution temperature. Zhang and coworkers [52] studied the swelling behavior of a polyvinyl alcohol (PVA) gel in water/ethanol solution, as well as solvent diffusion within the matrix. Gautieri et al. [53] parameterized a MARTINI model of benzene diffusing in PVA hydrogels from full atomistic simulations (Fig. 14.6); the self-diffusion coefficient obtained from CG simulations is in good agreement with experimental data.

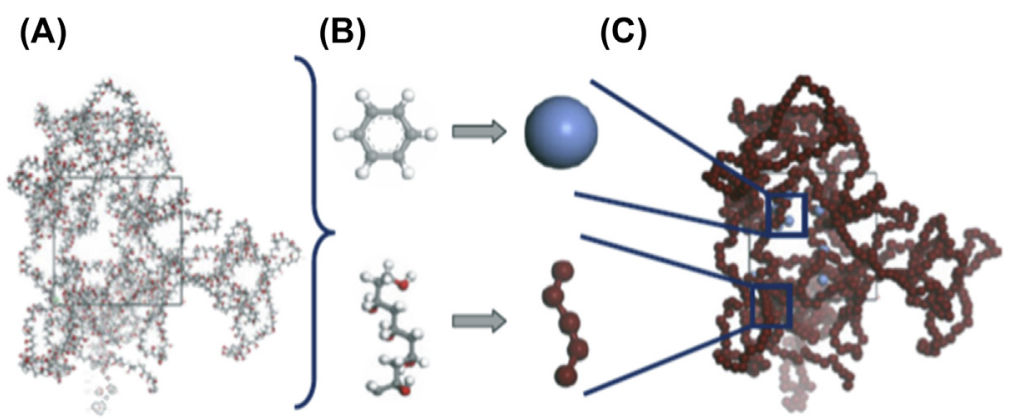

f0035 FIGURE 14.6

Atomistic model of PVA chains (A). Mapping scheme for benzene and PVA (B). CG model for benzene diffusion in PVA gels (C).

Reproduced with permission from Gautieri A, Vesentini S, Redaelli A. How to predict diffusion of medium-sized molecules in polymer matrices. From atomistic to coarse grain simulations. J Mol Model 2010;16:1845-51. Copyright Springer-Verlag, 2010. 
p0275 Molecular simulations are also a useful tool to characterize in detail the specific interactions that lead to the formation of supramolecular gels in selfassembling systems [5]. The computational analysis through MD and CG is usually limited to the early events that lead to the formation of supramolecular assemblies, as time and length scales do not allow investigating properly gelation phenomena; for this purpose, Brownian dynamics, dissipative particle dynamics, and MC simulations are the methods of choice [54]. Macroscale models are employed to describe the release rate of active molecules from the matrix. It is here useful to introduce a dimensionless number commonly referred as Deborah number:

$$
D e=\frac{\lambda}{\tau_{D}}=\frac{\lambda D}{\delta(t)^{2}}
$$

where $\lambda$ is the time scale for chain relaxation, $\tau_{D}$ is the time scale for drug diffusion, and $\delta(t)$ is the time-dependent thickness of swollen region. If $D e<<1$, drug release is dominated by diffusion and can be safely described with Fick's law and a suitable expression for diffusion coefficient (vide supra). On the other hand, if $D e>>1$, release rate is mainly determined by polymer swelling, which must be explicitly included in the model, as presented in the following examples. Ju and coworkers [55] developed a model for drug release from cylindrical devices made of hydroxypropyl methylcellulose (HPMC), accounting for swelling and polymer dissolution. The model is based on the following equation (only radial coordinate $r$ was considered):

$$
\frac{\partial \rho_{i}}{\partial t}=-\frac{\partial \rho_{i}}{\partial r} \frac{d r}{d t}+\frac{1}{r} \frac{\partial}{\partial r}\left(r D_{i} \rho_{t} \frac{\partial w_{i}}{\partial r}\right)-\rho_{i} \frac{1}{V} \frac{d V}{d t}
$$

where $\rho_{i}$ is mass concentration of the $i$ th component, $t$ is time, $D_{i}$ is the diffusion coefficient of the $i$ th component, $w_{i}$ is the mass fraction of the $i$ th component, $\rho_{t}$ is the overall mass concentration of the matrix, and $V$ is volume. The first term on the right side accounts for convection (that arises from moving boundary due to swelling), the second one is related to diffusion, and the third term is the source term takes into account concentration changes due to volume changes. Drug dissolution is not included, therefore Eq. (14.12) is valid only for water-soluble molecules. Diffusion coefficients are expressed according to the following expressions, based on NMR measurements:

$$
\begin{gathered}
\frac{D_{s, w}}{D_{w, 0}}=k_{w}^{\prime} \exp \left(k_{w} w_{w}\right) \\
\frac{D_{d}}{D_{d, 0}}=\exp \left(-k_{d, p} w_{p}-k_{d, 1} w_{1}-k_{d, d} w_{d}\right)
\end{gathered}
$$

where $D_{s, w}$ is the water self-diffusion coefficient, $D_{d}$ is the drug mutual diffusion coefficient, $k_{w}{ }^{\prime}$ is a prefactor, $k_{w}$ and $k_{d, i}$ are weighting factors, $D_{i, 0}$ is the diffusion 
coefficient of the $i$ th component, and $w_{i}$ is the mass fraction of the $i$ th component. Water diffusion coefficient $D_{w}$ is related to polymer volume fraction $\varphi_{p}$ :

$$
D_{w}=D_{s, w} \varphi_{p}
$$

p0280 Matrix dissolution is considered similar to the dissolution of an object immersed in a fluid and subjected to forced convection; it is related to the polymer disentanglement concentration (that depends on molecular weight), defined as the polymer concentration below which polymer chains detach off the matrix. Siepmann and Peppas [56] developed a comprehensive model for HPMC devices that accounts for drug and water diffusion, polymer and drug dissolution, nonconstant diffusivities, swelling, and moving boundary conditions. Polymer dissolution is described through reptation theory; above a certain critical water concentration value $c_{1 \text { crit }}$, polymer chains at device/medium interface start to disentangle and diffuse into bulk fluid. Mass loss is computed as follows:

$$
M_{p t}=M_{p 0}-k_{\text {diss }} A_{t} t
$$

where $M_{p t}$ is the polymer mass at time $t, M_{p 0}$ is the initial polymer mass, $k_{d i s s}$ is the dissolution constant, and $A_{t}$ is the surface area at time $t$. Diffusion of the $i$ th compound is described through second Fick's law in cylindrical coordinates:

$$
\frac{\partial c_{i}}{\partial t}=\frac{1}{r}\left\{\frac{\partial}{\partial r}\left(r D_{i} \frac{\partial c_{i}}{\partial r}\right)+\frac{\partial}{\partial \theta}\left(\frac{D_{i}}{r} \frac{\partial c_{i}}{\partial \theta}\right)+\frac{\partial}{\partial z}\left(r D_{i} \frac{\partial c_{i}}{\partial z}\right)\right\}
$$

where $c_{i}$ is the concentration of the $i$ th compound (water, drug) and $r, z$, and $\theta$ are radial, axial, and angular coordinates, respectively. Diffusion coefficients are expressed according free volume theory through a Fujita - type exponential dependence:

$$
D_{i}=D_{i, c r i t} \exp \left\{-\beta_{i}\left(1-\frac{c_{1}}{c_{1 c r i t}}\right)\right\}
$$

where $D_{i, c r i t}$ is the diffusion coefficient at matrix/medium interface, $\beta_{i}$ is a dimensionless constant related to concentration effects, $c_{1}$ is water concentration and $\mathrm{c}_{1 \text { crit }}$ is water concentration at matrix/medium interface. This model was successfully tested and validated against the release of theophylline at different initial drug concentrations, as shown in Fig. 14.7.

p0285 In addition to diffusion and swelling, other mechanisms can affect the release of active molecules. For example, the drug can be covalently bound to the matrix through a cleavable spacer (the release is thus determined by cleavage rate [57]) or can interact with the polymer through long-range interactions (electrostatic and van der Waals). Sakiyama-Elbert and Hubbel [58] developed a reaction-diffusion model to describe the release of basic fibroblast growth factor (bFGF) from fibrinbased hydrogels, where bFGF was noncovalently bound to the matrix. Yan, Casalini and coworkers [59] employed the same model to explain the $\mathrm{pH}$-dependent release of bone morphogenetic factor 2 protein (BMP2) from hyaluronic acid-based 


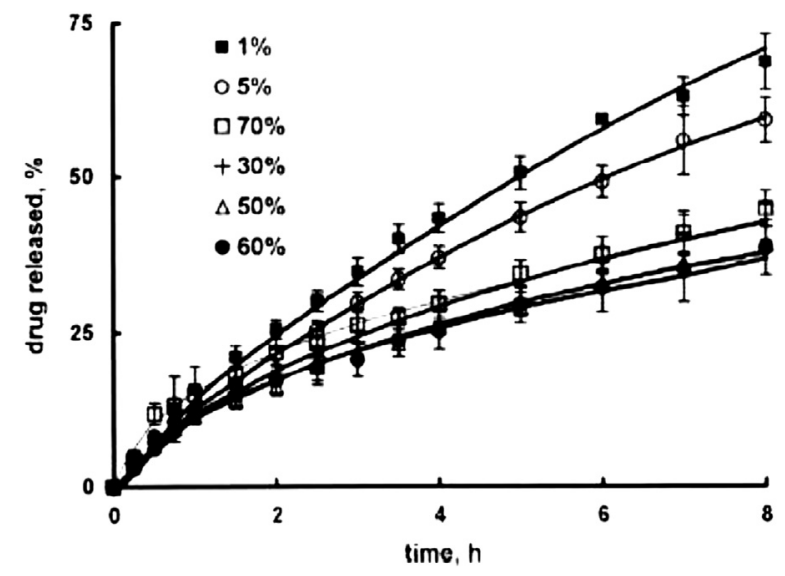

Theophylline release from HPMC tablets as a function of time for different values of initial drug loading.

Reproduced with permission from Siepmann J, Peppas NA. Modeling of drug release from delivery systems based on hydroxypropyl methylcellulose (HPMC). Adv Drug Deliv Rev 2012;64:163-74. Copyright Elsevier, 2012.

hydrogels; in this framework, a reaction-diffusion model was coupled with molecular dynamics simulations, which highlighted that the BMP2 binding to the matrix is mainly due to electrostatic interactions that depend on protein protonation state.

\section{s0065 Aliphatic polyesters}

p0290 Polylactic acid, polyglycolic acid, poly- $\varepsilon$-caprolactone, and their copolymers belong to the family of aliphatic polyesters, the most used and studied polymers in biomedical field. Their chemical structure is depicted in Fig. 14.8.

p0295 Aliphatic polyesters naturally degrade in situ because of hydrolysis mechanism, through the scission of ester bond that constitutes chain backbone. Degradation is favored in acidic environment and can be represented through the following kinetic scheme:

$$
\mathrm{P}_{n+m}+\mathrm{H}_{2} \mathrm{O}+\mathrm{H}^{+} \rightarrow \mathrm{P}_{n}+\mathrm{P}_{m}+\mathrm{H}^{+}
$$

where $P_{n+m}, P_{n}$, and $P_{m}$ are polymer chains with $n+m, n$, and $m$ monomer units, respectively, $\mathrm{H}_{2} \mathrm{O}$ is water and $\mathrm{H}^{+}$indicates that hydrolysis is favored at low pH. The main phenomena behind aliphatic polyesters degradation are currently well rationalized and can be summarized as follows:

u0170 - Water diffuses in the polymer matrix, causing the matrix to swell. Only short oligomers are water-soluble; therefore, polymer dissolution is absent at this stage.

u0175 - Water breaks ester bonds as diffusion takes place; 
<smiles>CC(O)C(=O)O</smiles>

(C)<smiles>CCC(C)C(=O)OC(C)C(=O)OC(C)C(=O)O</smiles>

(B)<smiles>CCC(=O)O</smiles>

(D)<smiles>CCCOC(=O)CO</smiles>

f0045

FIGURE 14.8

Chemical structures of lactic acid (A), glycolic acid (B) and their related polymers: polylactic acid (C) and polyglycolic acid (D).

Reproduced with permission from Casalini T. Bioresorbability of polymers: chemistry, mechanisms, and modeling. Woodh Publ Ser Biom 2017;120:65-83. Copyright Elsevier, 2017.

u0180 - The resulting small oligomers have terminal carboxylic group, whose dissociation lowers microenvironmental $\mathrm{pH}$ and thus catalyzes hydrolysis, leading to an autocatalytic mechanism;

u0185 - Small oligomers are mobile enough to diffuse out of the polymer matrix;

u0190 • If oligomers accumulate in the core because of mass transport resistances, degradation is heterogeneous because the $\mathrm{pH}$ is locally lower and thus hydrolysis is faster;

u0195 - Diffusivities increases as molecular weight decreases, as degradation opens new diffusive paths.

p0330 Degradation rate depends on many factors [60], such as composition (that determines hydrophobicity), molecular weight, crystallinity, addition of drugs and/or plasticizers, device size, and geometry. Some of them can be tuned by the user, which means that degradation rate can be in principle tailored according to the specific exigencies. In this framework, modeling activity essentially aims at describing molecular weight decrease over time and its influence on drug release, whereas only few examples of modeling at molecular scale are reported in the literature [61]. In this regard, available models differ in the description of degradation kinetics and their impact on diffusion coefficients. A very simple model can be formulated for polylactic-co-glycolic acid (PLGA) degradation, for which molecular weight decrease follows a pseudo-first-order kinetics, as experimentally assessed:

$$
\begin{gathered}
\frac{d M W_{w}}{d t}=-k_{d} M W_{w} \\
M W_{w}(t)=M W_{w}(t=0) \exp \left(-k_{d} t\right)
\end{gathered}
$$

where $M W_{w}$ is weight average molecular weight, and $k_{d}$ is the pseudo-first-order degradation kinetic constant. Eq. (14.20) can be solved analytically with a suitable 
initial condition (i.e., molecular weight before degradation onset) and Eq. (14.21) can be obtained. Siepmann and coworkers [62] used this model to describe the degradation of PLGA microparticles of different sizes, obtaining a good agreement with experimental data. They found that degradation kinetic constant increases as size increases, which is consistent with autocatalysis mechanism. Indeed, longer diffusive paths promote oligomers accumulation within the device, lowering the microenvironmental $\mathrm{pH}$ and speeding up chain scission. Such model is too simplistic to allow an optimal device design; a comprehensive modeling framework must include also transport phenomena and autocatalysis. Rothstein et al. [63] developed a model that included transport phenomena but not autocatalysis; diffusivity increase is accounted for through a position and time-dependent porosity:

$$
\begin{gathered}
\frac{\partial C_{w}}{\partial t}=\nabla\left(D_{w} \nabla C_{w}\right)-k_{d} C_{w} M W_{w} \\
\frac{\partial M W_{w}}{\partial t}=-k_{d} C_{w} M W_{w} \\
D_{e f f}=D_{A} \varepsilon(r, t)
\end{gathered}
$$

where $C_{w}$ is water concentration, $D_{w}$ is water diffusion coefficient, $D_{e f f}$ is the effective diffusivity, $D_{A}$ is the diffusion coefficient in the matrix, and $\varepsilon(r, t)$ is matrix porosity at time $t$ and position $r$. A more detailed description of degradation kinetics can be achieved by means of population balances, that is, by writing a mass balance for each chain with a generic number $n$ of repeating units, as discussed by several authors [64-68]. Nishida and coworkers [68] solved population balances with the method of statistical moments and developed simple expressions for the time evolution of number and weight average molecular weights and polydispersity; they accounted for autocatalysis but neglected transport phenomena, which implies that mass loss cannot be taken into account. A similar approach was adopted by Antheunis et al. [69,70]. Casalini et al. [66], starting from population balances and subsequently applying the method of the statistical moments, formulated a comprehensive model that includes autocatalysis and oligomers diffusion, where diffusivities depend on number average molecular weight. A third-order kinetics is assumed: degradation rate depends on polymer, water , and carboxyl terminals concentrations. The model is constituted by a system of partial differential equations that describe time and spatial evolution of monomer, oligomers (up to nonamers), water and drug concentrations, and the statistical moments of the first three orders. Indeed, average properties of interest can be easily obtained from zeroth-, first-, and second-order moments:

$$
\begin{aligned}
M W_{n} & =\frac{\mu_{1}}{\mu_{0}} M W_{m o n} \\
M W_{w} & =\frac{\mu_{2}}{\mu_{1}} M W_{m o n}
\end{aligned}
$$




$$
P D=\frac{\mu_{2} \mu_{0}}{\mu_{1}^{2}}
$$

where $\mu_{j}$ is the $j$ th order statistical moment, $M W_{n}$ is number average molecular weight, $M W_{\text {mon }}$ is the molecular weight of the repeating unit, and $P D$ is polydispersity. The model proved to catch the interplay between autocatalysis and transport phenomena thanks to the constitutive laws: indeed, only one kinetic constant value could reproduce molecular weight decay for PLGA microparticles with different sizes, while different values were needed with the pseudo-first-order model (vide supra). In addition, also the release of different loaded active compounds was satisfactorily described. Siepmann et al. [71] developed a stochastic model for drug release from PLGA microparticles, treating hydrolysis as a random event. The microsphere (only a quarter was considered, for symmetry reasons) was divided in pixels that represent polymer, drug, or water. Each polymer pixel has a characteristic lifetime that starts decreasing as soon as it comes in contact with the water, which diffuses from the release medium through the matrix. An eroded polymer pixel becomes a pore, which allows computing a time and position-dependent porosity that is in turn employed to evaluate diffusivities. A schematic representation of the system is reported in Fig. 14.9.

p0335 Such model cannot be used to quantitatively describe the molecular weight decrease over time, but provides a very good agreement with experimental data in release rate of loaded compounds. Chen et al. [72] adopted a stochastic model that also quantitatively account for degradation and mass loss. The authors assumed that the probability that as ester bond is hydrolyzed $P_{A}$ is given by the sum of two contributions: regular hydrolysis and autocatalysis:

$$
P_{A}=\frac{\lambda_{0} \exp \left(-\lambda_{0} t\right)\left(1+\beta\left(\exp \left(C_{M}-1\right)\right)\right)}{V_{0} V(t)}
$$

where $\lambda_{0}$ is the first-order degradation kinetic constant, $C_{M}$ is the monomer concentration, $V_{0}$ is the initial polymer volume, $V(t)$ is the polymer volume at time $t$, and $\beta$ is an adjustable parameter that quantifies the importance of autocatalysis. For each time step, a random number between 0 and 1 is generated; if $P_{A}$ is higher than this number, hydrolysis occurs, otherwise polymer can experience chain scission in the following step. Diffusion coefficient depends on degradation according to the following expression:

$$
D_{M}=D_{M}^{0} \exp \left(R_{M} \frac{x_{H}-x}{x_{H}}\right)
$$

where $D_{M}$ is monomer diffusion coefficient, $D_{M}^{0}$ is diffusion coefficient before degradation onset, $R_{M}$ is an empirical constant, $x$ is the status variable for the matrix (equal to 1 for polymer, 0.001 for hydrolyzed polymer, 0 for pores), and $x_{H}$ is the status variable for polymer (equal to 1 ). 
fO050 FIGURE 14.9

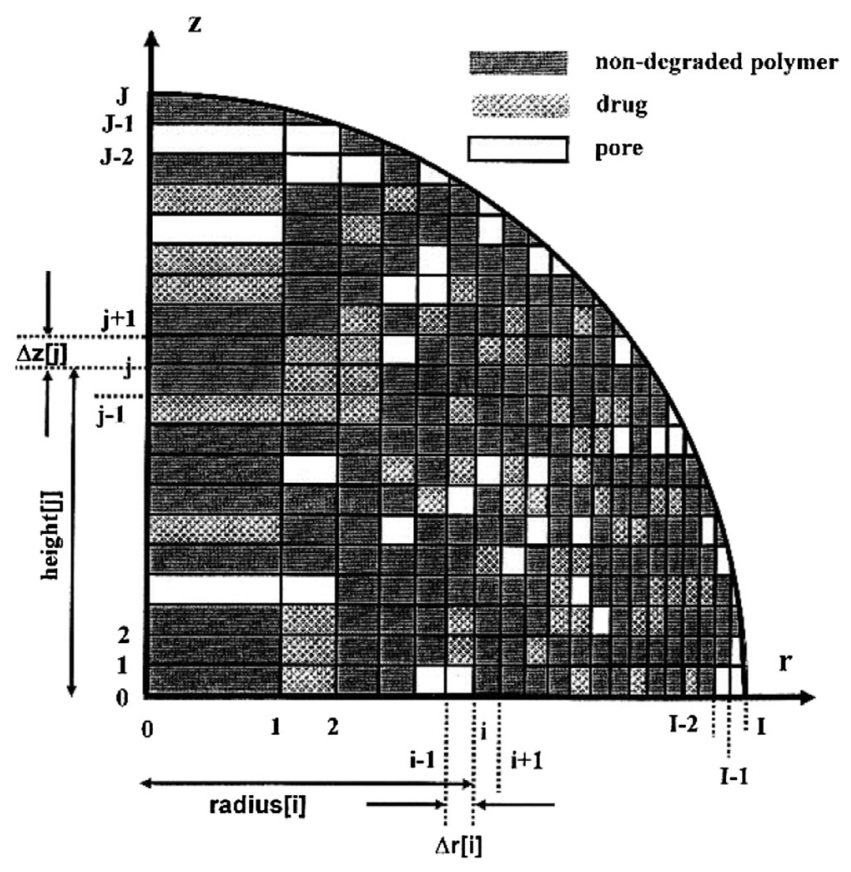

Schematic representation of a Monte Carlo simulation performed by Siepmann and coworkers. Gray pixels represent polymer, dotted pixels represent loaded drug while white pixels are pores, previously occupied by polymer that has been degraded.

Reproduced with permission from Siepmann J, Faisant N, Benoit JP. A new mathematical model quantifying drug release from bioerodible microparticles using Monte Carlo simulations. Pharmaceut Res 2002;19: 1885-93. Copyright Plenum Publishing Corporation, 2002.

\section{s0070 In vivo modeling}

p0340 The discussed modeling approaches constitute a fundamental tool to characterize the release rate and to understand that parameters can be tuned to obtain the desired performances. From a modeling point of view, the real goal is to model device behavior in vivo, to assess whether drug concentration at delivery site is maintained in a suitable range of therapeutic efficacy for a prolonged period, reducing side effects and avoiding the delivery to nontarget tissues. It is thus essential to understand the main phenomena behind drug transport in the tissue and to highlight their constitutive laws, which must be coupled with the formulaically description of the device [73]. A multiphase approach for diffusion in tissues was presented by Nicholson [74]; in this framework, the tissue is composed by three phases: intracellular space (ICS), cellular membrane (CM), and extracellular space (ECS). The molar concentration of the drug per unit volume of tissue $C$ is computed as follows:

$$
C=\alpha C_{E C S}+\beta C_{I C S}+(1-\alpha-\beta) C_{C M}
$$


where $\alpha$ is the ECS volume fraction, $\beta$ is the ICS volume fraction, and $C_{E C S}, C_{I C S}$, and $C_{C M}$ are drug moles per unit volume of each phase. Drug is subjected to diffusion through ECS and convection due to interstitial fluid in ECS, elimination, and reversible binding to tissue components. The amount of bound drug $B$ can be expressed as follows:

$$
B=\alpha B_{E C S}+\beta B_{I C S}+(1-\alpha-\beta) B_{C M}
$$

where $B_{E C S}, B_{I C S}$, and $B_{C M}$ are the moles of bound drug in each phase. Starting from a general mass balance, which accounts for convection, diffusion, and elimination, and assuming that the tissue is isotropic, the following expression for drug dispersion in the region surrounding the delivery device can be obtained:

$$
\begin{gathered}
\frac{\partial C_{E C S}}{\partial t}+\frac{\alpha}{\alpha^{*}} v_{r} \cdot \nabla C_{E C S}=\frac{\alpha}{\alpha^{*}} D_{E C S} \nabla^{2} C_{E C S}-\frac{\alpha k_{E C S}+\beta k_{I C S} P_{I C S: E C S}}{\alpha^{*}} C_{E C S} \\
\alpha^{*}=\alpha\left(1+K_{E C S}\right)+\beta P_{I C S: E C S}\left(1+K_{I C S}\right)+(1-\alpha-\beta) P_{C M: E C S} \\
K_{E C S}=\frac{B_{E C S}}{C_{E C S}} \\
K_{I C S}=\frac{B_{I C S}}{C_{I C S}} \\
P_{I C S: E C S}=\frac{C_{I C S}}{C_{E C S}} \\
P_{C M: E C S}=\frac{C_{C M}}{C_{E C S}}
\end{gathered}
$$

where $v_{r}$ is the vector containing the components of interstitial fluid velocity, $D_{E C S}$ is the diffusion coefficient in the ECS phase, $k_{E C S}$ and $k_{I C S}$ are first-order drug elimination kinetic constants, $K_{E C S}$ and $K_{I C S}$ are binding equilibrium constants, and $P_{I C S}$ : $E C S$ and $P_{C M \text { :ECS }}$ are partition coefficients. Eq. (14.30) was presented for drug diffusion in the brain, but can be applied to any tissue microenvironment, as long as the peculiar characteristics of each tissue are taken into account (different phase composition, nonisotropic diffusion, and so on).

\section{s0075 Conclusions}

p0345 This chapter aimed at providing a general overview concerning the mathematical modeling of hydrogels and aliphatic polyesters, promising candidates in the SCI repair field. Because of the extent of the topic, this summary cannot be fully exhaustive: the fundamental purpose is to illustrate the new modeling approaches at molecular scale and the established ones based on fundamental mass conservation equations. Microscale models elucidate the impact of formulation at molecular level, while macroscale models are a fundamental tool for an optimal device design. Their 
synergistic use, commonly referred as multiscale modeling, provides an all-around understanding of the system of interest, increasing even more the potential of mathematical modeling in drug delivery and SCI repair field.

\section{References}

[1] Peppas NA. Historical perspective on advanced drug delivery: how engineering design and mathematical modeling helped the field mature. Adv Drug Deliv Rev 2013;65:5-9.

[2] Siepmann J, Peppas NA. Higuchi equation: derivation, applications, use and misuse. Int J Pharm 2011;418:6-12.

[3] Frenkel D, Smit B. Understanding molecular simulation: from algorithms to applications. San Diego: Academic Press; 2002.

[4] Riniker S. Fixed-charge atomistic force fields for molecular dynamics simulations in the condensed phase: an overview. J Chem Inf Model 2018;58:565-78.

[5] Bochicchio D, Pavan GM. Molecular modelling of supramolecular polymers. Adv Phys-X 2018;3:315-37.

[6] Freddolino PL, Harrison CB, Liu YX, Schulten K. Challenges in protein-folding simulations. Nat Phys 2010;6:751-8.

[7] Katiyar RS, Jha PK. Molecular simulations in drug delivery: opportunities and challenges. Wires Comput Mol Sci 2018;8.

[8] Ryde U. QM/MM calculations on proteins. Methods Enzymol 2016;577:119-58.

[9] Duster AW, Wang CH, Garza CM, Miller DE, Lin H. Adaptive quantum/molecular mechanics: what have we learned, where are we, and where do we go from here? Wires Comput Mol Sci 2017;7.

[10] Kirchner B, di Dio PJ, Hutter J. Real-world predictions from ab initio molecular dynamics simulations. Top Curr Chem 2012;307:109-53.

[11] Camilloni C, Pietrucci F. Advanced simulation techniques for the thermodynamic and kinetic characterization of biological systems. Adv Phys-X 2018;3.

[12] Miao YL, McCammon JA. Unconstrained enhanced sampling for free energy calculations of biomolecules: a review. Mol Simul 2016;42:1046-55.

[13] Valsson O, Tiwary P, Parrinello M. Enhancing important fluctuations: rare events and metadynamics from a conceptual viewpoint. Annu Rev Phys Chem 2016;67:159-84.

[14] Cavalli A, Spitaleri A, Saladino G, Gervasio FL. Investigating drug-target association and dissociation mechanisms using metadynamics-based algorithms. Acc Chem Res 2015;48:277-85.

[15] Marinelli F, Pietrucci F, Laio A, Piana S. A kinetic model of Trp-cage folding from multiple biased molecular dynamics simulations. PLoS Comput Biol 2009;5.

[16] Marrink SJ, Tieleman DP. Perspective on the martini model. Chem Soc Rev 2013;42: 6801-22.

[17] Frederix PWJM, Patmanidis I, Marrink SJ. Molecular simulations of self-assembling bio-inspired supramolecular systems and their connection to experiments. Chem Soc Rev 2018;47:3470-89.

[18] Chen JC, Kim AS. Brownian dynamics, molecular dynamics, and Monte Carlo modeling of colloidal systems. Adv Colloid Interfac 2004;112:159-73.

[19] Liu MB, Liu GR, Zhou LW, Chang JZ. Dissipative particle dynamics (DPD): an overview and recent developments. Arch Comput Method E 2015;22:529-56. 
[20] Casalini T. Bioresorbability of polymers: chemistry, mechanisms, and modeling. Woodh Publ Ser Biom 2017;120:65-83.

[21] Siepmann J, Siepmann F. Mathematical modeling of drug delivery. Int J Pharm 2008; 364:328-43.

[22] Siepmann J, Siepmann F. Modeling of diffusion controlled drug delivery. J Control Release 2012;161:351-62.

[23] Kaunisto E, Marucci M, Borgquist P, Axelsson A. Mechanistic modelling of drug release from polymer-coated and swelling and dissolving polymer matrix systems. Int J Pharm 2011;418:54-77.

[24] Lao LL, Peppas NA, Boey FYC, Venkatraman SS. Modeling of drug release from bulkdegrading polymers. Int J Pharm 2011;418:28-41.

[25] von Burkersroda F, Schedl L, Gopferich A. Why degradable polymers undergo surface erosion or bulk erosion. Biomaterials 2002;23:4221-31.

[26] Cussler EL. Diffusion, mass transfer in fluid systems. Cambridge Cambridgeshire, New York: Cambridge University Press; 1984.

[27] Masaro L, Zhu XX. Physical models of diffusion for polymer solutions, gels and solids. Prog Polym Sci 1999;24:731-75.

[28] George SC, Thomas S. Transport phenomena through polymeric systems. Prog Polym Sci 2001;26:985-1017.

[29] Amsden B. Solute diffusion in hydrogels. An examination of the retardation effect. Polym Gels Netw 1998;6:13-43.

[30] Fatin-Rouge N, Milon A, Buffle J, Goulet RR, Tessier A. Diffusion and partitioning of solutes in agarose hydrogels: the relative influence of electrostatic and specific interactions. J Phys Chem B 2003;107:12126-37.

[31] Ziemba AM, Gilbert RJ. Biomaterials for local, controlled drug delivery to the injured spinal cord. Front Pharmacol 2017;8.

[32] Ordikhani F, Sheth S, Zustiak SP. Polymeric particle-mediated molecular therapies to treat spinal cord injury. Int J Pharm 2017;516:71-81.

[33] Perale G, et al. Hydrogels in spinal cord injury repair strategies. ACS Chem Neurosci 2011;2:336-45.

[34] Peppas NA. Hydrogels in medicine and pharmacy. Boca Raton, Fla: CRC Press; 1986.

[35] Flory PJ. Principles of polymer chemistry. Ithaca: Cornell University Press; 1953.

[36] Peppas NA, Hilt JZ, Khademhosseini A, Langer R. Hydrogels in biology and medicine: from molecular principles to bionanotechnology. Adv Mater 2006;18:1345-60.

[37] Slaughter BV, Khurshid SS, Fisher OZ, Khademhosseini A, Peppas NA. Hydrogels in regenerative medicine. Adv Mater 2009;21:3307-29.

[38] Lee SG, Brunello GF, Jang SS, Lee JH, Bucknall DG. Effect of monomeric sequence on mechanical properties of P(VP-co-HEMA) hydrogels at low hydration. J Phys Chem B 2009;113:6604-12.

[39] Jang SS, Goddard WA, Kalani MYS. Mechanical and transport properties of the poly(ethylene oxide)-poly(acrylic acid) double network hydrogel from molecular dynamic simulations. J Phys Chem B 2007;111:1729-37.

[40] Lee SG, Brunello GF, Jang SS, Bucknall DG. Molecular dynamics simulation study of P (VP-co-HEMA) hydrogels: effect of water content on equilibrium structures and mechanical properties. Biomaterials 2009;30:6130-41.

[41] Lee SG, et al. Effect of monomeric sequence on transport properties of D-glucose and ascorbic acid in poly(VP-co-HEMA) hydrogels with various water contents: molecular dynamics simulation approach. Theor Chem Acc 2012;131. 
[42] Wu YB, Joseph S, Aluru NR. Effect of cross-linking on the diffusion of water, ions, and small molecules in hydrogels. J Phys Chem B 2009;113:3512-20.

[43] Edgecombe S, Schneider S, Linse P. Monte Carlo simulations of defect-free crosslinked gels in the presence of salt. Macromolecules 2004;37:10089-100.

[44] Edgecombe S, Linse P. Monte Carlo simulations of cross-linked polyelectrolyte gels with oppositely charged macroions. Langmuir 2006;22:3836-43.

[45] Edgecombe S, Linse P. Monte Carlo simulation of polyelectrolyte gels: effects of polydispersity and topological defects. Macromolecules 2007;40:3868-75.

[46] Edgecombe S, Linse P. Monte Carlo simulation of two interpenetrating polymer networks: structure, swelling, and mechanical properties. Polymer 2008;49:1981-92.

[47] Chen SS, Yong X. Dissipative particle dynamics modeling of hydrogel swelling by osmotic ensemble method. J Chem Phys 2018;149.

[48] Walter J, Ermatchkov V, Vrabec J, Hasse H. Molecular dynamics and experimental study of conformation change of poly(N-isopropylacrylamide) hydrogels in water. Fluid Phase Equilib 2010;296:164-72.

[49] Walter J, Sehrt J, Vrabec J, Hasse H. Molecular dynamics and experimental study of conformation change of poly( $\mathrm{N}$-isopropylacrylamide) hydrogels in mixtures of water and methanol. J Phys Chem B 2012;116:5251-9.

[50] Deshmukh S, Mooney DA, MacElroy JMD. Molecular simulation study of the effect of cross-linker on the properties of poly(N-isopropyl acrylamide) hydrogel. Mol Simul 2011;37:846-54.

[51] Alaghemandi M, Spohr E. Molecular dynamics investigation of the thermo-responsive polymer poly(N-isopropylacrylamide). Macromol Theory Simul 2012;21:106-12.

[52] Zhang QG, Liu QL, Chen Y, Wu JY, Zhu AM. Microstructure dependent diffusion of water-ethanol in swollen poly(vinyl alcohol): a molecular dynamics simulation study. Chem Eng Sci 2009;64:334-40.

[53] Gautieri A, Vesentini S, Redaelli A. How to predict diffusion of medium-sized molecules in polymer matrices. From atomistic to coarse grain simulations. J Mol Model 2010;16:1845-51.

[54] Dickinson E. Structure and rheology of colloidal particle gels: insight from computer simulation. Adv Colloid Interfac 2013;199:114-27.

[55] Ju RTC, Nixon PR, Patel MV, Tong DM. Drug-release from hydrophilic matrices .2. A mathematical-model based on the polymer disentanglement concentration and the diffusion layer. J Pharm Sci 1995;84:1464-77.

[56] Siepmann J, Peppas NA. Modeling of drug release from delivery systems based on hydroxypropyl methylcellulose (HPMC). Adv Drug Deliv Rev 2012;64:163-74.

[57] Lin CC, Metters AT. Hydrogels in controlled release formulations: network design and mathematical modeling. Adv Drug Deliv Rev 2006;58:1379-408.

[58] Sakiyama-Elbert SE, Hubbell JA. Development of fibrin derivatives for controlled release of heparin-binding growth factors. J Control Release 2000;65:389-402.

[59] Yan HJ, et al. Synthetic design of growth factor sequestering extracellular matrix mimetic hydrogel for promoting in vivo bone formation. Biomaterials 2018;161: 190-202.

[60] Alexis F. Factors affecting the degradation and drug-release mechanism of poly(lactic acid) and poly[(lactic acid)-co-(glycolic acid)]. Polym Int 2005;54:36-46.

[61] Gautieri A, Mezzanzanica A, Motta A, Redealli A, Vesentini S. Atomistic modeling of water diffusion in hydrolytic biomaterials. J Mol Model 2012;18:1495-502. 


\section{8}

\section{CHAPTER 14 Fundamentals and application of modeling}

[62] Siepmann J, Elkharraz K, Siepmann F, Klose D. How autocatalysis accelerates drug release from PLGA-based microparticles: a quantitative treatment. Biomacromolecules 2005;6:2312-9.

[63] Rothstein SN, Federspiel WJ, Little SR. A unified mathematical model for the prediction of controlled release from surface and bulk eroding polymer matrices. Biomaterials 2009;30:1657-64.

[64] Arosio P, Busini V, Perale G, Moscatelli D, Masi M. A new model of resorbable device degradation and drug release - part I: zero order model. Polym Int 2008;57:912-20.

[65] Batycky RP, Hanes J, Langer R, Edwards DA. A theoretical model of erosion and macromolecular drug release from biodegrading microspheres. J Pharm Sci 1997;86: 1464-77.

[66] Casalini T, Rossi F, Lazzari S, Perale G, Masi M. Mathematical modeling of PLGA microparticles: from polymer degradation to drug release. Mol Pharm 2014;11:4036-48.

[67] Perale G, et al. A new model of resorbable device degradation and drug release: transient 1-dimension diffusional model. J Control Release 2009;136:196-205.

[68] Nishida $\mathrm{H}$, et al. Theoretical prediction of molecular weight on autocatalytic random hydrolysis of aliphatic polyesters. Macromolecules 2000;33:6595-601.

[69] Antheunis H, van der Meer JC, de Geus M, Kingma W, Koning CE. Improved mathematical model for the hydrolytic degradation of aliphatic polyesters. Macromolecules 2009; 42:2462-71.

[70] Antheunis H, van der Meer JC, de Geus M, Heise A, Koning CE. Autocatalytic equation describing the change in molecular weight during hydrolytic degradation of aliphatic polyesters. Biomacromolecules 2010;11:1118-24.

[71] Siepmann J, Faisant N, Benoit JP. A new mathematical model quantifying drug release from bioerodible microparticles using Monte Carlo simulations. Pharmaceut Res 2002; 19:1885-93.

[72] Chen YH, Zhou SW, Li Q. Mathematical modeling of degradation for bulk-erosive polymers: applications in tissue engineering scaffolds and drug delivery systems. Acta Biomater 2011;7:1140-9.

[73] Weiser JR, Saltzman WM. Controlled release for local delivery of drugs: barriers and models. J Control Release 2014;190:664-73.

[74] Nicholson C. Interaction between diffusion and Michaelis-Menten uptake of dopamine after iontophoresis in striatum. Biophys J 1995;68:1699-715. 


\title{
Non-Print Items
}

\begin{abstract}
Computational techniques are now a complementary tool to experimental activity for a comprehensive characterization of biomedical devices for drug delivery. They allow achieving a mechanistic and fundamental understanding of the systems of interest and rationalizing experimental data. The acquired knowledge can be fruitfully employed to optimize the design of the device, which does not behave as a "black box" anymore, to obtain the desired performances. Starting from the seminal contribution of Prof. Takeru Higuchi with the well-known Higuchi equation in 1961, modeling of drug delivery devices has now embraced not only a wide range of systems but a likewise variety of computational techniques. This chapter aims at providing to the interested reader a brief overview of the available approaches employed in the literature, along with specific examples of applications on system of potential interest for spinal cord injury repair.
\end{abstract}

\section{Keywords:}

Coarse, Drug delivery, Grained models, Mathematical modeling, Molecular dynamics. 


\title{
CONCLUSIONS
}

\section{c0015 Trends in regenerative therapies, combination approaches, and clinical highlights for spinal cord injury (SCl) regeneration}

\author{
Filippo Rossi, PhD ${ }^{1}$, Mario Gimona, PhD ${ }^{2}$, Giuseppe Perale, PhD ${ }^{3,4}$ \\ ${ }^{1}$ Department of Chemistry, Materials and Chemical Engineering "Giulio Natta”, Milan, Italy; \\ ${ }^{2}$ GMP Laboratory, Spinal Cord Injury and Tissue Regeneration Center Salzburg (SCI-TReCS), \\ Paracelsus Medical University (PMU), Salzburg, Austria; ${ }^{3}$ Faculty of Biomedical Sciences, \\ University of Southern Switzerland (USI), Lugano, Switzerland; ${ }^{4}$ Ludwig Boltzmann Institute for \\ Experimental and Clinical Traumatology in Vienna, Austria
}

p0010 As anticipated in the forewords, this book aims at describing the current scenario in spinal cord injury (SCI) regeneration strategies, pointing toward most promising research directions in this wide and complex field. Recent results and clinical achievements from Prof. Courtine's team opened tremendous new directions for restoring patients' functionality, but not by directly regenerating injured tissue, rather by electronically bypassing the lesion gap.

p0015 Indeed, even if many preclinical studies and promising approaches have been proposed for SCI treatment basing on regenerative purposes, unfortunately nowadays none of them showed relevant efficacy when translated to human patients $[1,2]$. One of the main reasons is that many cell-based and/or pharmacological approaches are directed only to one specific and single pathway and did not consider combination-based therapies that simultaneously work on neuroprotection (against primary injury) and neuroregeneration of the damaged tissue [3]. Moreover, other problems are strictly related to conventional pharmacological treatments and potential side effects associated. Indeed the most commonly used strategy is represented by continuous infusion by minipumps [4,5]: advantages are associated with immediate drug efficacy and limited side effects. Unfortunately, various drawbacks restrict the applicability of this route of administration, such as limited drug diffusion into the spinal cord segment and cerebrospinal fluid clearance of about 5 h. Higher doses and repeated injections are hence required. Furthermore, problems due to surgery and catheter placement are also frequently reported together with infections [4].

p0020 Recently, novel engineered biocompatible scaffolds obtained high interest in SCI treatment because they tackle some of the key features of this disease such as the localized trauma and the consequent presence of cyst that create a proper space

Spinal Cord Injury (SCI) Repair Strategies. https://doi.org/10.1016/B978-0-08-102807-0.00015-6 Copyright $\odot 2020$ Elsevier Ltd. All rights reserved. 
for scaffold implantation or injection. Once in situ, such scaffolds can release drugs, cells, or biomolecules directly into the target area avoiding problems such as immune system clearance, systemic drug overdosage, or cells leakage into circulatory torrent.

p0025 In this field, medicine and engineering should work together to design suitable systems able to maintain local drug levels within a desired functional range, satisfying the need for fewer administrations and optimizing drug concentration and patient compliance. Promising results have been achieved with hydrogels, soft injectable polymeric matrices that can jellify in situ and then release multifunctional therapies directed to counteract multiple degenerative mechanisms of SCI, trying to release not only neuroprotective but also neuroregenerative agents as schematized in Figs. 15.1 and $15.2[6]$.

p0030 Moreover, hydrogels can protect biomolecules reducing their deactivation and biodistribution of chondroitinase ABC and NT-3: respectively, a bacterial enzyme that is able to digest chondroitin sulfate glycosaminoglycans and a neurothrophine, both of which can promote axonal regeneration and sprouting supporting functional recovery in various preclinical models [7]. In general, if injected systemically, it is extremely difficult to retain them at the injury site because of their rapid diffusion into extracellular fluids: a single injection of neurotrophic factors has a limited half-life only of about $30 \mathrm{~min}$, and multiinjection rather than a single injection would hence be needed to provide a neuroprotective effect.

p0035 Following the same strategy, other factors, such as brain-derived neurotrophic factor [8], ciliary neurotrophic [9], epidermal [10], and fibroblast growth factors [11] are important promoting factors for neural regeneration and can hence be smartly delivered using hydrogels. Another interesting and very promising strategy
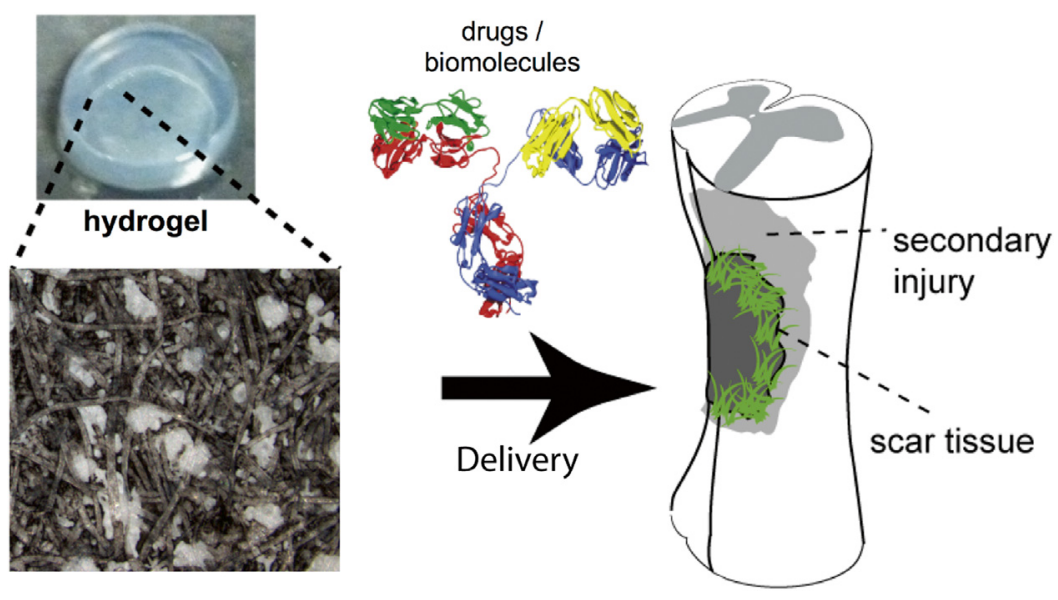

\section{f0010 FIGURE 15.1}

A cartoon represents delivery of drugs or biomolecules from the hydrogel in the damaged spinal cord. 


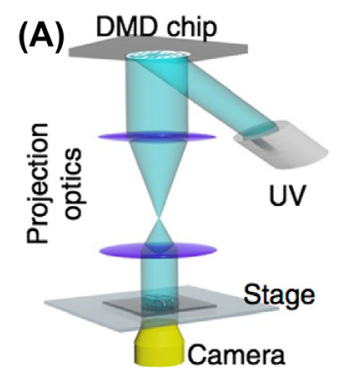

(B)
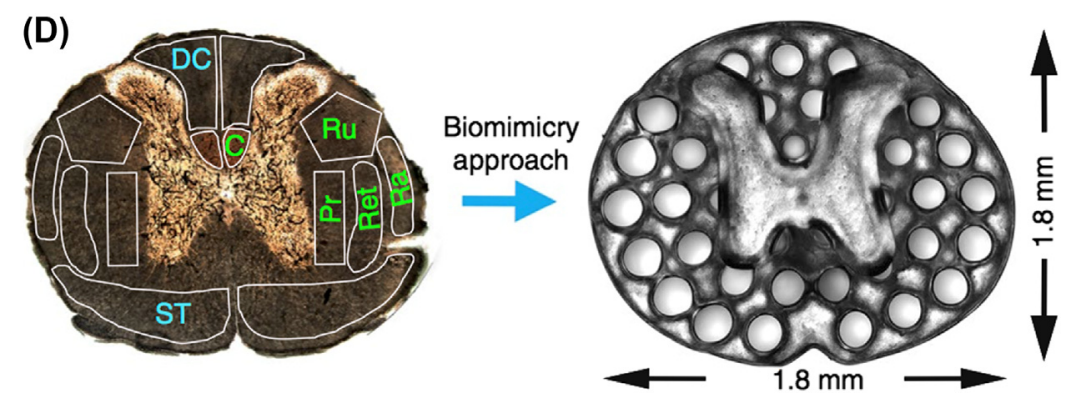

fO015 FIGURE 15.2

3D printer setup: An ultraviolet light source (365 nm wavelength); a computer for sliced image-flow generation and system synchronization;

a digital micromirror device (DMD) for optical pattern generation; a set of projection optics; a stage for sample position control; and a CCD (charge-coupled device) imaging system for online monitoring of the fabrication process.

Reprinted with permission from Koffler J, Zhu W, Qu X, Platoshyn O, Dulin JN, Brock J, et al. Biomimetic 3D-printed scaffolds for spinal cord injury repair. Nat Med 2019;25:263-269.

is represented by biofabrication of scaffolds able to mimic spinal cord tissue, as depicted in Fig. 15.2. In this direction, Koffler and coworkers used microscale continuous projection printing method to create the complexity of CNS structure [12].

p0040 In parallel, there has been growing interests in developing nanoscaled delivery tools able to enter within specific cells delivering selectively their contents, working so as Trojan horses $[13,14]$. Following this strategy, a new frontier is opened by smart combination of biomaterials to address multiple approach using both hydrogels and nanoparticles (NPs).

p0045 Naturally occurring nanoparticles, such as exosomes and other therapeutically active extracellular vesicles (EVs), are liberated by a plethora of cells, including mesenchymal stromal cells into their complex secretome, and hold great promise for future application in the acute to subacute phase of SCI. EV-enriched secretomes preserve and harness the immunomodulatory potential of mesenchymal-derived cells (MSCs) [15] and display neuroprotective activity in vivo and in vitro via multiple pathways. These allogeneic biological therapeutics present a much-improved 


\section{0}

CONCLUSIONS Trends in regenerative therapies

safety profile compared with stem cells and, albeit little is known about the potential modes of action the impact on tissue mechanotransduction and compliance is evident. EV-rich allogeneic secretomes will also enable new approaches that consider the different steps of body reactions following traumatic SCI in a timedependent fashion and thus move SCI therapy into a 4D situation. The combination of regeneration-promoting (nanoporous) materials and structure-inducing scaffolds together with vesicular secretomes may be employed to augment the body's intrinsic abilities to repair damaged tissue and eventually prepare the required regenerative environment for functional recovery and subsequent therapeutic strategies, including cell-based approaches [16] (Fig. 15.3).

p0050 Indeed, the ability to remain localized in situ, together with the possibility of controlling the delivery of hydrophilic high steric hindrance molecules, typical of hydrogels, could be combined with the cell selectivity and with the possibility of tuning the release of hydrophobic drugs, typical role of nanoparticles. Several recent studies have investigated these aspects, trying to combine the advantages of both systems: minocycline [17], methylprednisolone [7], antibodies [18], and growth factors [19] were incorporated in NPs and then loaded into hydrogels to provide a sustained release into the final target tissue, aiming at increasing medical recovery chances. An ideal biomaterial-based delivery platform must achieve localized and sustained release and a favorable risk/benefit ratio to be adopted clinically for SCI treatment. In particular, all the following characteristics in developing delivery tools have to be considered:

o0010 1. biocompatibility;

00015 2. controlled biodegradability;

o0020 3. easy injectability;
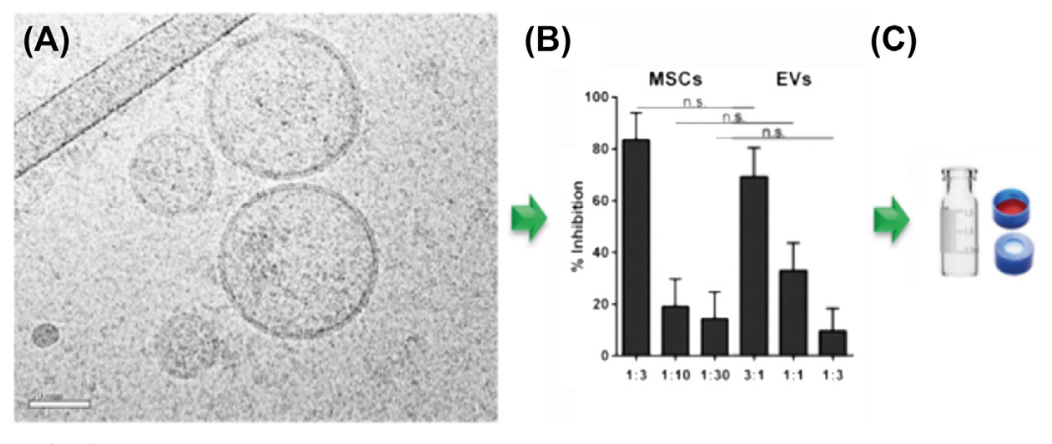

f0020 FIGURE 15.3

Mesenchymal-derived cell (MSC)-derived secretomes enriched in 50-130 nm sized membrane surrounded extracellular vesicles (EVs) (A) preserve the immunomodulatory function ( $B$; inhibition of T cell proliferation) of the parental cells and can be manufactured as ready-to-use injectable allogeneic therapeutics $(\mathrm{C})$ for immediate application in the acute phase of spinal cord injury (SCl) (scale bar $=50 \mathrm{~nm}$ ). 
00025 4. complete control over material formulation and hence complete rationalization in terms of physicochemical properties;

00030 5. ability to sustain multiple drugs delivery, at the same time and with different and independent kinetic profiles;

00035 6. total absence of any chemical interactions between moieties of materials and drugs.

p0085 These findings indicate that material science, in conjunction with bio- and nanotechnologies, can develop novel specifically designed devices able to maintain drug levels within a desired range, satisfying the need for fewer administrations, optimal use of the drug, and increased patient compliance.

p0090 Overall, the hope is that one of these therapies, or most likely a combination of some of them, will soon provide sufficiently robust animal data to withstand the regulatory and ethical hurdles to enter into clinical phase and in the end allow the achievement of effective results in patients. Even just partial recovery and preliminary clinical results will for sure open new directions in research that will finally lead to find a spectrum of solutions for this complex and high invalidating pathology.

\section{References}

[1] Morren JA, Galvez-Jimenez N. Current and prospective disease-modifying therapies for amyotrophic lateral sclerosis. Expert Opin Investig Drugs 2012;21:297-320.

[2] Rabchevsky AG, Patel SP, Springer JE. Pharmacological interventions for spinal cord injury: where do we stand? How might we step forward? Pharmacol Ther 2011;132: $15-29$.

[3] Kwon BK, Okon EB, Plunet W, Baptiste D, Fouad K, Hillyer J, et al. A systematic review of directly applied biologic therapies for acute spinal cord injury. J Neurotrauma 2011;28:1589-610.

[4] Belverud S, Mogilner A, Schulder M. Intrathecal pumps. Neurotherapeutics 2008;5: $114-22$.

[5] Cohen SP, Dragovich A. Intrathecal analgesia. Anesthesiol Clin 2007;25:863-82.

[6] Perale G, Rossi F, Santoro M, Peviani M, Papa S, Llupi D, et al. Multiple drug delivery hydrogel system for spinal cord injury repair strategies. J Control Release 2012;159: 271-80.

[7] Lee H, McKeon RJ, Bellamkonda RV. Sustained delivery of thermostabilized chABC enhances axonal sprouting and functional recovery after spinal cord injury. Proc Natl Acad Sci USA 2010;107:3340-5.

[8] Liang W, Han Q, Jin W, Xiao Z, Huang J, Ni H, et al. The promotion of neurological recovery in the rat spinal cord crushed injury model by collagen-binding BDNF. Biomaterials 2010;31:8634-41.

[9] Burdick JA, Ward M, Liang E, Young MJ, Langer R. Stimulation of neurite outgrowth by neurotrophins delivered from degradable hydrogels. Biomaterials 2006;27:452-9.

[10] Hamann MCJ, Tator CH, Shoichet MS. Injectable intrathecal delivery system for localized administration of EGF and FGF-2 to the injured rat spinal cord. Exp Neurol 2005; 194:106-19. 


\section{CONCLUSIONS Trends in regenerative therapies}

[11] Vulic K, Shoichet MS. Tunable growth factor delivery from injectable hydrogels for tissue engineering. J Am Chem Soc 2012;134:882-5.

[12] Koffler J, Zhu W, Qu X, Platoshyn O, Dulin JN, Brock J, et al. Biomimetic 3D-printed scaffolds for spinal cord injury repair. Nat Med 2019;25:263-9.

[13] Papa S, Rossi F, Vismara I, Forloni G, Veglianese P. Nanovector-mediated drug delivery in spinal cord injury: a multitarget approach. ACS Chem Neurosci 2019;10:1173-82.

[14] Mauri E, Chincarini GMF, Rigamonti R, Magagnin L, Sacchetti A, Rossi F. Modulation of electrostatic interactions to improve controlled drug delivery from nanogels. Mater Sci Eng C 2017;72:308-15.

[15] Pachler K, Ketterl N, Desgeorges A, Dunai ZA, Laner-Plamberger S, Streif D, et al. An in vitro potency assay for monitoring the immunomodulatory potential of stromal cellderived extracellular vesicles. Int J Mol Sci 2017;18:E1413.

[16] Gomes ED, Silva NA, Salgado AJ. Combinatorial therapies for spinal cord injury: strategies to induce regeneration. Neural Regen Res 2019;14:69-71.

[17] Papa S, Rossi F, Ferrari R, Mariani A, De Paola M, Caron I, et al. Selective nanovector mediated treatment of activated proinflammatory microglia/macrophages in spinal cord injury. ACS Nano 2013;7:9881-95.

[18] Stanwick JC, Baumann MD, Shoichet MS. In vitro sustained release of bioactive anti-NogoA, a molecule in clinical development for treatment of spinal cord injury. Int J Pharm 2012;426:284-90.

[19] Piotrowicz A, Shoichet MS. Nerve guidance channels as drug delivery vehicles. Biomaterials 2006;27:2018-27. 


\section{Index}

'Note: Page numbers followed by " $\mathrm{t}$ " indicate tables and "f" indicate figures.'

A

2-(Acethylthio) ethyl methacrylate (ATEMA), 117

Acetylcholine (Ach), 194

Acrylates derivatives, 136

Additive manufacturing, 97

Adipose mesenchymal stem cells (A-MSCs), 206-207

Adipose tissue-derived stem/stromal cells (ASCs) advantages, 169

lumbar compression injuries, 170

multipotent differentiation potential, 169

neural differentiation protocols, 166-167

oligodendrocyte precursor cells (OPCs), 169-170

paracrine activity, 169

secretome, 170-171

transplantation, 169-170

Agarose hydrogels, 83, 226-227

Air gun impactor, 261

Air pressure-mediated contusion, 261

Alginate hydrogels, 227

Alginates, 83-84

Aliphatic polyesters

chemical structure, 298f

classification, 79

degradation, 297-300

diffusion coefficient, 300

microsphere, 298-300

Monte Carlo simulation, 301f

poly (glycolic acid) (PGA), 80

polycaprolactone (PCL), 80-81

polyhydroxyalkanoates (PHAs), 80

poly(lactic acid) (PLA) scaffolds

macroporous, 80

poly-D-lactide (PDLA), 80

poly-L-lactide (PLLA), 80

Shellac resin, 79

statistical moments, 298-300

transport phenomena and autocatalysis, 298-300

American Spinal Injury Association (ASIA) Impairment Scale A (AIS A), 164-166

American Spinal Injury Association (ASIA) scale, 13-14, 44f, 54

2-Aminoethyl methacrylate (AEMA), 114

Amphibians, 254

Amphiphilic copolymers, 135-136

Amphiphilic polymers, 137-138
Aneurism clip compression, 261-262

Animal models

canine models, 252

chronic studies, 258-259

economic aspects, 270-271

ethical issues, 255-256

vs. human trauma, 271-272

injury mechanism, 257-258, 258t

compression spinal cord injury models, 261-262

contusion, 259-261

dislocation spinal cord injury models, 264

distraction spinal cord injury models, 263-264

secondary mechanism spinal cord injury models, 264-265

transection/laceration spinal cord injury models, 263

limitations, 271

macropodid marsupials, 253-254

nonmammals

amphibians, 254

turtles, 254-255

zebrafish, 254

primates, 252-253

publications, 271-272

quality control, 268-270

regulatory affairs and procedure design, 256-257

rodent models. See Rat models

small ruminants, 253

spinal injury level, 257-259

swine, 253

Animal Research: Reporting of In Vivo Experiments (ARRIVE) guidelines, 271

Anterior arthrodesis, 46

Anterior cord syndrome, 42, 54

Anterior spinal artery syndrome, 42

Anticoagulation prophylaxis, 45

Anti-NogoA antibody, 237

Antithrombin therapy, 238

Arachnoid mater, 1

Argatroban, 238

Astrocyte-like cells, 187-188

Astrocytes, 9

Autonomic nervous system, 16

Autonomy

executional autonomy, 70

negative obligation, 70 


\section{4}

Autonomy (Continued)

noninterference, 71

principle, 70

qualitative investigation, 70

repairing process, $70-71$

self-determination, 70-71

B

Balloon compression model, 262

Basic fibroblast growth factor (bFGF), 296-297

Basso, Beattie, and Bresnahan (BBB) scale, 267

Basso, Beattie, and Bresnahan (BBB) subscoring scale, 267

Beneficence

functional recovery priorities, $71-72$

moral obligation, 71

quality of life (QoL), 71-72

Binaural bipolar galvanic currents, 20

Bioabsorbable polymers, 78

Bioconcave nanoparticles, 132

Biodegradable biomaterials, 78-79

Biologically active (bioactive) materials, 78

Biomaterials

advantages, 77

bioinertness, 77

ceramic and glasses, 78

definition, 77

metallic implants, 77

scaffolds, 224-225

Biopolymers

additive manufacturing, 97

bioabsorbable polymers, 78

biodegradable biomaterials, 78-79

biologically active (bioactive) materials, 78

biostable materials, 78

coating, 94

composites, 78, 97

degradation process, $88-89$

extrusion process, $90-92$

foaming, 95

hydrogel manufacturing, 95-96

micro- and nanoparticles manufacturing, 96-97

molding processes, 92-94

natural biopolymers. See Natural biopolymers

poly(alkyl cyanoacrylates), 87

poly(ether-ester), 84-85

poly(glycerol sebacic acid) (PGS), 85

poly(phosphoesters) (PPEs), 85

poly(propylene fumarate) (PPF), 87-88

poly(vinyl alcohol) (PVA), 88

polyacrylates, 86

polyanhydrides, $86-87$ polyphosphazenes, $85-86$

poly- $\beta$-hydroxybutyrate (PHB), 84

properties, 89-90

single-phase materials, 78

solvent casting, 94-95, 95f

sterilization, 97

Biostable materials, 78

Blood oxygenation level-dependent (BOLD) image, 52

Blood pressure augmentation, 45

Blood spinal cord barrier (BSCB), 130

Bone marrow-derived mesenchymal stem cells (BM-MSCs), 167-168, 204-207

Bone morphogenetic factor 2 protein (BMP2), 296-297

Brain blood barrier (BBB), 7-8

Brain-derived neurotrophic factor (BDNF), 80, 114-115, 206-207

Brain lipid binding protein (BLBP), 188

Brownian dynamics (BD), 283

Brownian motion, 51

Brown-Séquard syndrome, 41-42

Bulbocavernosus (BC) reflex, 20-21

Bulk erosion, 289

C

$\mathrm{Ca}^{2+}$ electrogenesis, 188-190

Calibrated forceps compression, 262

Canine models

ethical issues, 252

and human clinical trials, 252

preclinical trials, 252

Carbazone coupling, 143-145

Carboxymethylchitosan/polyamidoamine (CMCht/PAMAM) dendrimer nanoparticles, 234-236

Cationic polymers, 139

Cell adhesiveness, 108

Cell-free therapy, 212-213

Cell therapies, 159t, 204-205. See also Stem cellbased therapies

embryonic stem cells (ESCs)

adipose tissue-derived stem/stromal cells (ASCs), 169-171

clinical trials, 160

ethical development, 160

gene-targeted therapies, 158

human embryonic stem cells-neural cell

precursors (hNPCs), 158-160

mouse embryonic stem cells-derived oligo-

dendrocytes, 158-160

neural differentiation, 158 
paraplegic and quadriplegic patients, 160 predifferentiated oligodendrocyte precursor cells (OPCs), 160

technical issues, 160

teratomas, 160

glial cells

olfactory ensheathing cells, 172-174

Schwann cells, 171-172

induced pluripotent stem cells (iPSCs)

abnormal axonal outgrowth, 161-162

neural stem/progenitor cell (NSPC) clones, 162

reprogramming process, 161

transcription factors, 160-161

tumorigenicity, 161

mesenchymal stem cells (MSCs)

adipose tissue-derived stem/stromal cells (ASCs), 166-167

bone marrow-derived MSCs (BM-MSCs),

$$
\text { 167-168 }
$$

bone marrow transplants, 166-167

characteristics, 166-167

vs. hematopoietic cells, 166-167

tissue sources, 166-167

umbilical cord-derived MSCs (UC-MSCs), 168-169

neural stem/progenitor cells (NSCs/NPCs) animal model, 164

bilateral C4 dorsal spinal lesion rat model, 164-166

cervical contusion-induced SCI, 162

clinical trials, 164-166

corticospinal tract (CST) projections, 163-166

graft axonal outgrowth, 163

graft survival, 164

transplantation, 162

peripheral nervous system (PNS) neurons, 157

transplantation, 157

Central canal (CC) stem cell niche

ependyma stem cells. See Ependyma stem cell niche

markers, 187

neural stem cells, 187

regulation, 192-194

Central cord syndrome, 41, 54

Central motor conduction time (CMCT), 21-22, 28

Cerebrospinal fluid-contacting neurons (CSFcNs), 188-190

Cervical level injury, animal model, 259

Chemically mediated models, 264-265

Chicago sky blue (CSB), 236-237

Chitin, 82
Chitosan, 82, 238

Cholesterol-modified superporous poly (2-hydroxyethyl methacrylate) (PHEMA) hydrogels

bulk radical copolymerization, 112-113

hydrophobic domains, 112

implanted hydrogels, $113 \mathrm{f}$

2-(methacryloyloxy)ethoxy]acetic acid (MOEAA), 112-113

Ciliary neurotrophic factor (CNTF), 229

c-Myc oncogene, 161

Coacervation, 137

Coarse-grained models

bonded interactions, 282-283

force field MARTINI, 282-283

MARTINI mapping, 282-283, 283f

nonbonded interactions, 282-283

procedure, 282-283

protein, 283

solvent molecules, 282-283

Coating, 94

Cold sensation test, 267

Collagen, 227, 232-233

Collective variables (CVs), 280-282

Complete cord injury, 4, 40, 41f, 54

Complete transection model, 263

Composite materials, 97

Compound muscle action potential (CMAP), 17-18

Compression molding, 93, 93f

Compression spinal cord injury models aneurism clip compression, 261-262

balloon compression, 262

calibrated forceps compression, 262

spinal canal occlusion, 261

Conduits, 148

Contact heat evoked potentials (CHEPs), 25, 33

Contralateral findings, Brown-Séquard syndrome, 41-42

Controlled gelification, 137

Contusion models

air gun impactor, 261

electromagnetic impactor, 260-261

impactor model, 260

laminectomy, 260

unilateral contusion, 260

weight drop model, 260

Corticospinal motor system, 14-15

Corticospinal tract (CST) projections, 163

Cyanoacrylates, 87

Cyclic adenosine monophosphate (cAMP) modulation, 172 


\section{6}

D

Danio rerio, 254

Deborah number, 295-296

Decellularization approach, 120

Deep venous thrombosis (DVT), 45

Dermatomal sensory evoked potentials (dSEPs), 24-25, 25f, 32-33

Desolvation technique, 137

Deterministic models, 284

Dextran, 81

Dibutyryl cyclic adenosine monophosphate (dbcAMP), 237-238

Diffusion tensor imaging (DTI), 51-52, 62

Diffusion-weighted imaging (DWI) sequences, 51

Dislocation spinal cord injury models, 264

Dissipative particle dynamics (DPD), 283

Distraction spinal cord injury models, 263-264

Dorsal ramus, 14-15

Double emulsion-solvent evaporation approach, 140

Dura mater, 1

\section{E}

Echo planar imaging (EPI), 51

Electrical perception threshold (EPT), 26

Electrical supramaximal stimulus, 22

Electromagnetic impactor, 260-261

Infinite Horizon (IH) impactor, 261

Ohio State University (OSU) impactor, 260-261

Electromyography (EMG)

motor unit action potentials (MUAPs), 19

peripheral pathways recording, 26-28

spontaneous activity, 19

Electrospinning, 91-92, 92f

Electrospun fibers, 80-81

Embryonic stem cells (ESCs), 207

adipose tissue-derived stem/stromal cells (ASCs), 169-171

clinical trials, 160

ethical development, 160

gene-targeted therapies, 158

human embryonic stem cells-neural cell precursors (hNPCs), 158-160

mouse embryonic stem cells-derived oligodendrocytes, 158-160

neural differentiation, 158

paraplegic and quadriplegic patients, 160

predifferentiated oligodendrocyte precursor cells (OPCs), 160

technical issues, 160

teratomas, 160

Empirical models, 284

Emulsion polymerization, 136
Endogenous regenerative mechanisms, 204

Energy balance, 284-285

Enhanced sampling methods, 280-282

Ependyma stem cell niche

astrocyte-like cells, 187-188

cells membrane properties

neonatal rats, 188-190

turtles, 188

connexins $(\mathrm{Cx})$ signaling

Cx43 and Cx26, 190-192

gap junction coupling, 190-192

gap junctions, 191

hemichannels, 191

metabolic coupling, 191

neuronal migration, 191

turtles, 190-191

dorsal ependymal cells, 187

functional organization, $189 \mathrm{f}$

genetic lineage tracing, 187

membrane properties

$\mathrm{Ca}^{2+}$ electrogenesis, 188-190

electrophysiological properties, 188-190

$\mathrm{I}_{\mathrm{A}}$ channels, 190

$\mathrm{I}_{\mathrm{KD}}$ channels, 190

neonatal rats, 188-190

subventricular zone (SVZ) stem cell niche,

188-190

turtles, 188

oligodendrocyte-like cells, 187-188

regenerative potential, 188-190

regulation

acetylcholine (Ach), 194

ATP, 193-194

$\gamma$-amino butyric acid (GABA), 192-193

mitotic activity, 192

purinergic signaling, 193-194, 195f

P2X7 receptor, 194

P2Y1 receptor activation, 193-194

Erb's point potential, 23f

Ethical issues

animal models, 255-256

autonomy, 70-71

beneficence, $71-72$

justice, $68-70$

management direct and indirect costs, 68

Evoked potentials (EPs)

advantages, $22-23$

contact heat evoked potentials (CHEPs), 25

dermatomal sensory evoked potentials (dSEPs), $24-25,25 f$

disadvantages, $22-23$

laser evoked potentials (LEPs), 25-26 
pudendal sensory evoked potential, 25

somatosensory evoked potentials (SEPs), 23-24, $23 f$

Excitotoxic models, 265

Executional autonomy, 70

Exosome-mediated delivery, protective mediators,

$$
\text { 208-209 }
$$

Exosomes, 208-209

Extracellular matrix (ECM)-based hydrogels

acute SCI hemisection model, 120

advantages, 119-120

cellular component free, 120

constructive tissue remodeling process, 120-12

decellularized biomaterials, 120

genipin cross-linking, 121

therapeutic effect, 119-120

Extracellular vesicles (EVs), 208-209, 212, 309-310

Extrusion process

cross-contamination, 91

drying/processing, 91

electrospinning, 91-92, $92 \mathrm{f}$

extruders, 90-91

moisture, 91

nano- and microfiber production, 91-92

viscosity, 91

\section{F}

Far-field potentials, volume-conducted, 16

Feed screws, 90-91

Ferulic acid, 236-237

Fibrin, 227

Fick's law, 286

Flaccid paralysis, 13

Flavopiridol, 236-237

Flory-Rehner theory, 291-293

Fluorescence colocalization studies, 145

Foaming, 95

Force field (FF), 280-282

Fractional anisotropy, 62

Free volume, 291

F response, 18

Functional magnetic resonance imaging (fMRI), 63-64

blood oxygenation level-dependent (BOLD) image, 52

tracers, 52

T2* sequences, $52-53$

G

Galvanic vestibular stimulation (GVS), 20

Genipin cross-linking, 121
Glassy-to-rubber phase transition, 287-288

Glial cells

olfactory ensheathing cells, 172-174

Schwann cells, 171-172

Glial-derived neurotrophic factor (GDNF), 229,

$$
233-234
$$

Glial scar, 7-8

Gliosis, 10

Glycine ethyl ester (GEE), 85-86

Glycolic acid, $298 \mathrm{f}$

Good Clinical Laboratory Practice (GCLP), 269

Good Clinical Practice (GCP), 270

Good Laboratory Practice (GLP), 269

Gradient echo (GRE), 50

Gray matter, 1-2, 3f, 14-16

Grid walking test, 268

Grip strength measurements, 265-266

H

Harrington distractor, 263

Heat-shock proteins, 209

Hirudin, 238

H/M amplitude ratio, 26-28

Horizontal ladder walking test, 268

Horizontal walking test, 268

Hospital management, 43, 44f

Hot sensation test, 267

H reflex, 18

Human embryonic stem cells-neural cell precursors (hNPCs), 158-160

Human umbilical cord-derived MSCs (UC-MSCs), 168-169

Hyaluronan and methylcellulose (HAMC), 231

Hyaluronan and methylcellulose

(HAMC)/poly(lactic-co-glycolic acid)

(PLGA) system, 231-232

Hyaluronic acid (HA) hydrogel, 82-83

cross-linking reaction, 118

HA-PH-RGD hydrogel, 118

hydroxyphenyl derivative, 118

locomotor functions, 118-119

neurofilament staining, $119 \mathrm{f}$

structural functions, 118

Hydrodynamic theories, 291

Hydrogels, 224-225. See also Scaffolds

injectable hydrogel delivery

agarose hydrogels, 226-227

biomaterial design, 225-226

natural hydrogels, 227

permissive scaffolds, 225-226

poly 2-hydroxyethyl methacrylate (HEMA), 225-226 


\section{8}

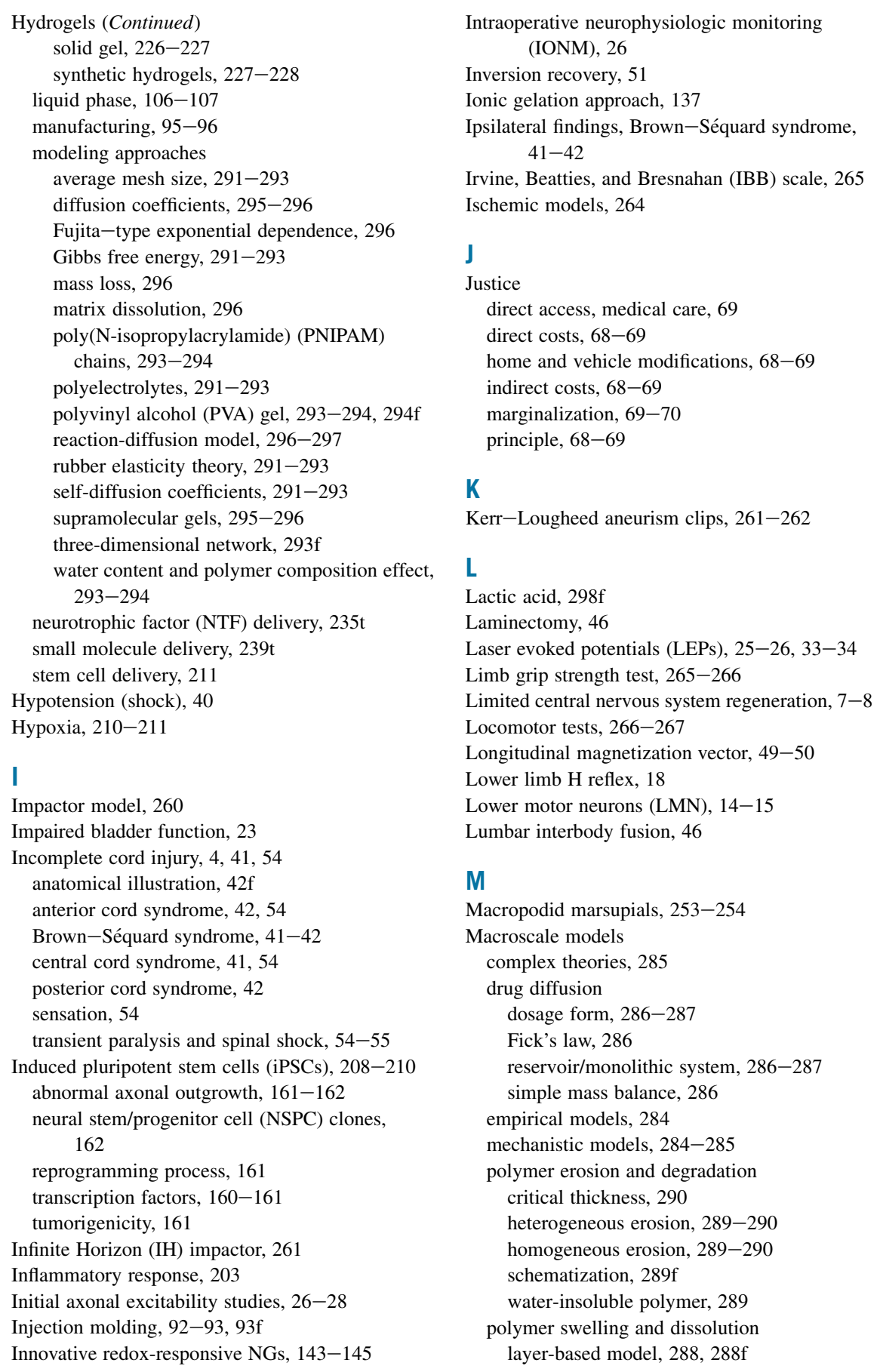

ersion recovery,

Ionic gelation approach, 137$$
41-42
$$

Irvine, Beatties, and Bresnahan (IBB) scale, 265

J

direct costs, 68-69

home and vehicle modifications, 68-69

costs, 68-69

Lactic acid, 298f

Laminectomy, 46

Locomotor tests, 266-267

Lower limb H reflex, 18

Lower motor neurons (LMN), 14-15

interbody fusion, 46

Macropodid marsupials, 253-254

dosage form, 286-287

Fick's law, 286

simple mass balance, 286

heterogeneous erosion, 289-290

, $289-290$

layer-based model, 288, 288f 
polymer chain relaxation, $287-288$ swelling-controlled drug delivery system, $287 \mathrm{f}$ swollen matrix, 287-288

water-soluble active compound, 287-288

semiempirical models, 284

transport phenomena

Fickian diffusion, 290-291

free volume, 291

hydrodynamic interactions, 291

obstruction effects, 290-291

polymer relaxation, 290-291

Magnetic resonance imaging (MRI), 43-44

acute imaging

axial T2 sequences, 55

geometric measurements, 55-58

hemorrhage, 55-58

sagittal GRE sequences, 55

sagittal T2 images, 55, 56f

short $\mathrm{T}$ inversion recovery images, $57 \mathrm{f}$

spinal cord injury without radiographic

abnormality (SCIWORA), 58-60

spinal cord transection, 59f

diffusion tensor imaging, 51-52, 62-63

diffusion-weighted imaging (DWI) sequences, 51

drawback, 62

dynamic and short duration magnetic field, 49-50

fractional anisotropy, 62

functional magnetic resonance imaging (fMRI), $52-53,63-64$

gradient echo (GRE), 50

inversion recovery, 51

magnetization vector, 49-50

mathematical transformation, 50

maximum spinal cord compression (MSCC), 61

postacute imaging

edema changes, 61

hematoma, 61

pain, 60

progressive posttraumatic cystic

syringomyelia, 61

subacute progressive ascending myelopathy, 61

short $\mathrm{T}$ inversion recovery (STIR) images, 53 spatial encoding, 49-50

spin echo (SE), 50

static magnetic field, 49-50

T2* GRE images, 53

T1 turbo SE (TSE) images, 53

T2 turbo SE (TSE) images, 53

Marginalization, 69-70

Matrix dissolution, 296
Maximum spinal cord compression (MSCC), 61

Mean square displacement (MSD), 291-293

Mechanistic models, 284

Medial lemniscus, 15-16

Melt Flow Index (MFI), 89

Membrane axonal excitability, 19-20

Meninges, 1

Mesenchymal-derived cell (MSC)-derived secretomes, $310 \mathrm{f}$

Mesenchymal stem cells (MSCs)

adipose tissue-derived stem/stromal cells (ASCs), 166-167

bone marrow-derived MSCs (BM-MSCs),

$$
\text { 167-168 }
$$

bone marrow transplants, 166-167

characteristics, 166-167

vs. hematopoietic cells, 166-167

limitation, 212

tissue sources, 166-167

umbilical cord-derived MSCs (UC-MSCs),

$$
\text { 168-169 }
$$

Metadynamics, 280-282

Metallic implants, 77

Methacrylate hydrogels, 109

2-(Methacryloyloxy)ethoxy]acetic acid (MOEAA), 112-113

Methylprednisolone (MP) delivery, 234-236

Methylprednisolone sodium succinate (MPSS), 44

Microglia, 9-10, $10 \mathrm{f}$

Microvesicles, 208-209

Minocycline delivery, 236

Molding process

compression molding, 93, 93f

injection molding, 92-93, 93f

reactive injection molding, 93

rotational molding, 93-94

Molecular dynamics (MD) simulations, 280-282

Momentum conservation principle, 284-285

Monte Carlo (MC) simulations, 283

Motor evoked potential (MEP), 21-22, 28, $29 \mathrm{f}-30 \mathrm{f}$

Motor unit action potentials (MUAPs), 19

Multicenter Animal Spinal Cord Injury Study (MASCIS), 260

Multimodal neurophysiological assessments, 34

N

Nanocapsules

structure, 135-136, $135 \mathrm{f}$

synthesis, 137

Nanocarriers, 130

charge and surface properties, 132-133 


\section{0}

\section{Index}

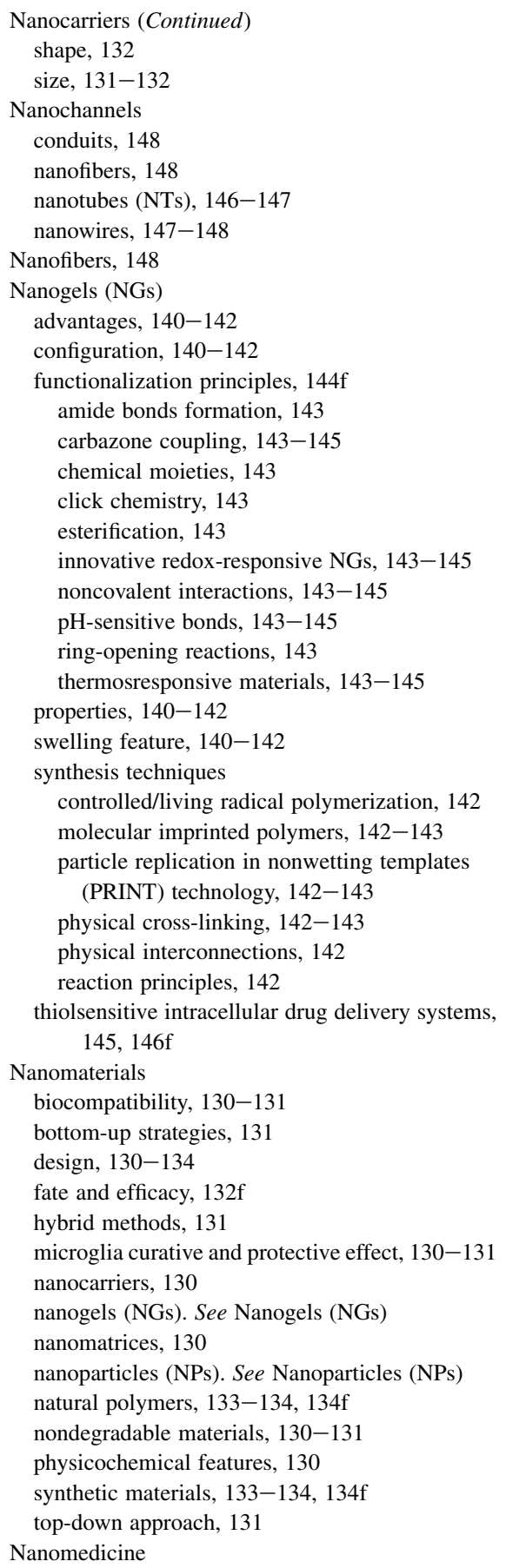

medical applications, 129

protective drugs, 129-130

Nanoparticles (NPs)

functionalization guidelines

cationic polymers, 139

encapsulation, 138-139

PEG, 139

polyesters, 138-139

polymerization, 138-139

nanocapsules, 135-136

nanostructures, 135

neuroprotective properties, 225

polymersomes, 135-136

solid matrix-like systems, 135

synthesis techniques

nanocapsules synthesis, 137

nanosphere preparations, 136-137

polymeric materials, 136

polymersomes syntheses, 137-138

protein-based NPs, 138

systemic delivery, 225

Nanoprecipitation, 136-137

Nano prostaglandin E1 (PGE1), 237

Nanorods, 132

Nanospheres, $135 \mathrm{f}$

Nanotechnology

advantageous properties, 129

definition, 129

size range, 129

Nanotubes (NTs)

functionalization, 146-147

scaffold and drug carrier, 146

single-wall and multi-wall configurations, 146-147, $147 \mathrm{f}$

Nanowires, 147-148

National Acute Spinal Cord Injury Study (NASCIS II), 44

National Center for the Replacement, Refinement and Reduction of Animals in Research (NC3Rs), 256

National Spinal Cord Injury Statistical Center,

$$
\text { 68-69 }
$$

Natural biopolymers

agarose, 83

alginates, $83-84$

chitosan, 82

dextran, 81

hyaluronic acid (HA), 82-83

polysaccharides, 81

Natural hydrogels, 227

advantage, 118

extracellular matrix-based hydrogels, 119-121 
hyaluronic acid hydrogel, 118-119

Near-field potentials, volume-conducted, 16

Nerve conduction studies

electrical response, 17-18

F response, 18

H reflex, 18

peripheral pathways recording, 26-28

Nerve excitability studies, 19-20

Nerve growth factor (NGF), 228-229

Nerve guidance channels, 108

Nerve guidance channels (NGCs), 233

Nerve "threshold, 19-20

Neural stem/progenitor cells (NSCs/NPCs), 207-208, 237-238

animal model, 164

bilateral C4 dorsal spinal lesion rat model, 164-166

cervical contusion-induced SCI, 162

clinical trials, 164-166

corticospinal tract (CST) projections, 163-166

graft axonal outgrowth, 163

graft survival, 164

transplantation, 162

NeuroBox, 87

Neuroepithelial cells, 187

Neuropathic pain (NP), 33-34

Neuropathic pain test, 267

Neurophysiological techniques, $14,17 \mathrm{f}$

bulbocavernosus (BC) reflex, 20-21

electromyography, 19

Evoked potentials (EPs). See Evoked potentials (EPs)

extracellular potentials, 16

galvanic vestibular stimulation (GVS), 20

intracellular potentials, 16

intraoperative neurophysiologic monitoring (IONM), 26

nerve conduction studies, $17-18$

nerve excitability studies, $19-20$

peripheral pathways recording, 26-28

quantitative sensory test (QST) methods, 26

sensory evoked potentials. See Evoked potentials (EPs)

spinal pathway recording, 28-34

sympathetic skin response (SSR), 20

transcranial magnetic stimulation (TMS), 21-22,

$$
21 f
$$

triple-stimulation technique, 22

volume-conducted potentials, 16

Neuroprotection, 205

Neuroregeneration, 205

Neurotrophic factor (NTF) delivery alginate encapsulated BDNF-producing fibroblasts (Fb/BDNF), 233-234

brain-derived neurotrophic factor (BDNF), 228-229

brain-derived neurotrophic factor (BDNF) and vascular endothelial growth factor

(VEGF)-loaded PLGA microspheres, 232

ciliary neurotrophic factor (CNTF), 229

collagen microspheres, 232-233

double-walled microsphere delivery system, 233-234

fibroblast growth factor-2 (FGF-2), 229

fibroblast growth factor-2 (FGF-2)-loaded PLGA nanoparticles, 231-232

glial-derived neurotrophic factor (GDNF), 229

glial-derived neurotrophic factor (GDNF)-loaded PLGA microsphere, 232

hyaluronan and methylcellulose (HAMC), 231

hyaluronan and methylcellulose

(HAMC)/ poly(lactic-co-glycolic acid)

(PLGA) system, 231-232

hydrogels and hydrogel particles, $235 \mathrm{t}$

nerve growth factor (NGF), 228-229

nerve guidance channels (NGCs), 233

neurotrophin-3 (NT-3), 228-229

poly(lactic-co-glycolic acid) (PLGA), 230-231

progenitor cell differentiation, 228-229

sustained delivery, 230

systemic delivery, 229-230

VEGF, 229

VEGF and VEGF-loaded chitosan-dextran sulfate nanoparticles, 232-233

Neurotrophic factors

Schwann cells, 171-172

Neurotrophin

axonal regeneration pathway, 140

Neurotrophin-3 (NT-3), 228-229

New York University (NYU) impactor device, 260

$\mathrm{N}$ - $\gamma$-maleimidobutyryl-oxysuccinimide ester (GMBS), 114

Noncranial autonomic nervous system motor pathways, 16

0

Ohio State University (OSU) impactor, 260-261

Olfactory ectomesenchymal stem cells

(OE-MSCs), 204-205

Olfactory ensheathing cells (OECs)

cytoplasmic projections, 172

lamina propria, 172

markers, 172-173

mechanisms, 174 
Olfactory ensheathing cells (OECs) (Continued) neuronal growth, 173

phenotypes, 172-173

transplantation, 173-174

Oligodendrocyte-like cells, 187-188

Oligodendrocyte precursor cells (OPCs), 160, 169-170

Open field test, 267

P

Paracrine effect

embryonic stem cells (ESCs), 207

exosome-mediated delivery, 208-209

free delivery, 208

induced pluripotent stem cells, 208-210

mechanisms, 209f, 212

mesenchymal stem cells (MSCs), 206-207

therapeutic benefits, 205-206

therapeutic mediators, 210-211

Paraparesis, 3-4

Paraplegia, 3-4, 39-40

Parasecreting neurotrophic factors, 205

Parent aniline pentamer (PAP), 85-86

Partial transection models, 263

Particle replication in nonwetting templates (PRINT) technology, 142

Peripheral nervous system (PNS), 7, 157

Peripheral pathways recording electromyography (EMG), 26-28 motor conduction studies, 26-28 nerve excitability studies, 26-28 sensory conduction studies, $26-28$

Personal autonomy, 70

Pharmacological therapies

anti-NogoA antibody, 237

antithrombin therapy, 238

bioactive molecules, 225

biomaterial scaffolds, 224-225

cells, 225

Chicago sky blue (CSB), 236-237

chitosan, 238

dibutyryl cyclic adenosine monophosphate (dbcAMP), 237-238

ferulic acid, 236-237

flavopiridol, 236-237

hydrogels, 224-225

hydrogels and hydrogel particles. See Hydrogels

injectable hydrogel delivery, 225-228

localized delivery, 225, $226 \mathrm{f}$

methylprednisolone (MP) delivery, 234-236

minocycline delivery, 236

molecular therapies, 225 nanomaterials, 238

nano prostaglandin E1 (PGE1), 237

neurotrophic factor delivery, 228-229

neurotrophic factor (NTF) delivery. See Neurotrophic factor (NTF) delivery

superoxide dismutase (SOD), 238

systemic delivery, 225

Photochemically induced models, 265

pH-switch method, 137-138

Pia mater, 1

Plastifying extruders, 90-91

Pluronic F-127 hydrogels, 238

Poly(ethylene glycol) (PEG), 227-228

Poly(hydroxyalkanoates) (PHAs), 80

Poly(lactic acid) (PLA), 227-228

Poly(lactic-co-glycolic acid) (PLGA), 227-228, 230-231

nanoparticles

bioactive neurotrophin release, 140

nanomatrices, 138-139

stochastic model, 298-300

Polyacrylates, 86

Poly alkyl cyanoacrylates, 87

Polyanhydrides, 86-87

Polycaprolactone (PCL), 80-81

Poly(N-isopropylacrylamide) (PNIPAM) chains, 293-294

Polycondensation reaction, 136

Polydichlorophosphazene, 85-86

Polyether-ester, 84-85

Poly glycerol sebacic acid (PGS), 85

Polyglycolic acid, 298f

Poly (2-hydroxyethyl methacrylate) (PHEMA) hydrogels, 86, 109, 227-228

cell-adhesive peptides, 113-114

with charges, 110-112

cholesterol-modified superporous, 112-113, $112 \mathrm{f}$

macroporous poly(N-2-hydroxypropyl methacrylamide) (PHPMA) hydrogel, 109-110, $110 \mathrm{f}$ porosity

axonal growth, 115-116

brain-derived neurotrophic factor (BDNF),

114-115

gelatin porous sponges, 116-117

hydrogel infiltration, 115

modulus elasticity, 114-115

neurofilament staining, $115 \mathrm{f}$

rigid and soft hydrogels, $116 \mathrm{f}$

SIKVAV-modified PHEMA hydrogels, 114

superporous SIKVAV-modified P(HEMA-

AEMA) hydrogels, 114 
serotonin, 117

Poly 2-hydroxyethyl methacrylate (HEMA) hydrogel, 225-226

Polylactic acid, $298 \mathrm{f}$

Polymer chain relaxation, 287-288

Polymeric scaffolds, 211

Polymersomes

structure, 135-136, $135 \mathrm{f}$

syntheses, 137-138

Polyp-dioxanone (PDS), 84-85

Polyphosphazenes, 85-86

Polyphosphoesters (PPEs), 85

Polypropylene fumarate (PPF) scaffold, 87-88

Poly- $\beta$-hydroxybutyrate (PHB), 84

Polyvinyl alcohol (PVA), 88, $294 \mathrm{f}$

Posterior arthrodesis, 46

Posterior cord syndrome, 42

Preganglionic neuron, 16

Primary injury, 9f, 203

Primates

advantage, 252

contusion lesions, 253

nonhuman primate SCI models, 252-253

transection models, 253

Progressive posttraumatic cystic syringomyelia, 61

Pudendal sensory evoked potential, 25

Pulmonary embolism, 45

Purinergic signaling, 194

\section{Q}

Quantitative sensory test (QST), 26, 33

Quantum dots, 132-133

Quantum mechanics/molecular mechanics (QM/MM) methods, 280-282

$\mathbf{R}$

Radial glia (RG), 187

Radiological evaluation, 43-44

Ram, 90-91

Rat models

advantages and disadvantages, 250t-251t,

$$
\text { 251-252 }
$$

contusion model, 251

locomotor tests, 266-267

motor tests, 265-266

neuroanatomical differences, 251-252

physiological and pathological parameters, 251

sensorimotor tests, 268

sensory test, 267

spinal cord injury evaluation tests, $266 t$

Reactive injection molding, 93
Replica exchange, 280-282

Reservoir/monolithic system, 286-287

Retinal axons, 157

Retinoic acid- and embryoid body-based protocols, 158

Rexed laminae, 14-16

Rope climbing test, 268

Rotational molding, 93-94

Rubber elasticity theory, 291-293

\section{$S$}

Sacral injuries, animal model, 259

Salting out, 137

Scaffold micro/nanotopographical topography, 108

Scaffolds

aliphatic polyesters

axonal guidance, 108

brain and spinal cord, 107

cell adhesiveness, 108

chemically synthetized biomaterials, 106

degradability, 108

extracellular matrix (ECM), 106

function, 79

hydrophilic polymers, 106

mechanical properties, 107

micro/nanotopographical topography, 108

mode of application, 106-107

natural hydrogels

advantage, 118

extracellular matrix-based hydrogels, 119-121

hyaluronic acid hydrogel, 118-119

natural scaffolds, 106

parameters, 106

substrate stiffness, 107

synthetic hydrogels

degradable PHMA hydrogels, 117

PHEMA hydrogels. See Poly (2-hydroxyethyl

methacrylate) (PHEMA) hydrogels

synthetic materials, 109

tissue bridging, 106

tissue softening, 107

Schiff-Sherrington phenomenon, 41

Schwann cells (SCs), 157

autologous transplants, 172

cyclic adenosine monophosphate (cAMP)

modulation, 172

motor function, 171

myelinating function, 171

neurotrophic factors, 171-172

thoracic rat SCI model, 171-172

Secondary injury, 9f, 203 


\section{4}

\section{Index}

Secondary mechanism spinal cord injury models, 264-265

Secretome, 170-171

Self-determination, 70-71

Self-diffusion coefficients, 291-293

Semiempirical models, 284

Semithin, 87

Sensorimotor tests, 268

Sensory evoked potentials, 22-26

Sensory function, 15-16

Sensory nerve action potential (SNAP), 17-18

Sensory test, 267

Shellac resin, 79

Short T inversion recovery (STIR) images, 53, 57f

Signal to noise ratio (SNR), 50

Single-screw extruders, 90-91, 90f

Skilled forelimb reaching task, 265

Skin-derived precursors (SKPs), 171-172

Small ruminants, 253

Soft and rigid scaffolds, 105-122

Solvent casting, 94-95, 95

Solvent evaporation, 137

Somatosensory evoked potentials (SEPs)

International 10-20 System placement, 23-24

left median nerve stimulation, $23 \mathrm{f}$

lower limb, 24

recording montages, $23-24$

spinal pathway recording, 30-32, 31f

stimulation sites, 23-24

upper limb, 23-24

Sonic hedgehog (Shh), 229

Spatial encoding, 49-50

Spinal cord anatomy

functional anatomy, 14-16

gray matter, $14-16$

membranes, 1

organization, $1-2,3 f$

peripheral nerves, 1

somatotopic organization, $15 f$

autonomic nervous system, 16

corticospinal motor system, 14-15

sensory function, 15-16

spinal canal, 1, 2f

white matter, 14-16

Spinal cord injury (SCI)

acute phase, 7

acute spinal cord injury, 39

animal models. See Animal models

ASIA impairment scale, 6, $6 \mathrm{f}$

bladder control problems, 67

bowel control problems, 67

chronic stage, 7, 105 circulatory control problems, 67

classification, 39-42

clinical evaluation, 13

clinical examination protocol, 13-14

clinical sequelae, 13

clinical signs/symptoms, 13

comorbidity cost, 39

complete spinal cord lesion, 4, 40, 41f, 54

consequences, 3-5

definition, 67

economic impact, 223

ethical issues

animal models, 255-256

autonomy, 70-71

beneficence, $71-72$

justice, $68-70$

management direct and indirect costs, 68 etiological differences, 13

etiology, 40t

fluid-filled cyst, 224, $224 \mathrm{f}$

functionality, 223-224

incidence, 2-3, 4f

incomplete spinal cord lesion, 4, 41, 54

inflammation role, primary lesion, 9-10

inflammatory response, 203

irreversible nerve injury, 223

levels, 4, $5 \mathrm{f}$

limited central nervous system regeneration, 7-8

macroscopic damage, 53-54

management

anticoagulation prophylaxis, 45

blood pressure augmentation, 45

hospital management, 43, 44f

radiological evaluation, 43-44

steroids, 44

surgical options and timing, 45-47

microglia, 10, $10 \mathrm{f}$

motor and sensory disabilities, 105

neurophysiological techniques. See Neurophysiological techniques

nontraumatic causes, 2-3, 39

pharmacological therapies. See Pharmacological therapies

physiopathology, 7, 8f, 77

prevalence, 223

primary injury, 203

prognosis, 7

respiratory problems, 67

secondary injury, 203

sensory pathways, 13

sexual life problems, 67

skin sensation problems, 67 
spinal shock, 40

stem cell delivery, engineered biomaterial, 211 subacute phase, 7

therapeutic strategies, 105

time-scale phases, 53-54

traumatic causes, 2-3, 39

Spinal cord injury (SCI) regeneration

bacterial enzyme, 308

biomaterial-based delivery platform, 310-311

cell therapies. See Cell therapies

conventional pharmacological treatments, 307

ependyma stem cell niche. See Ependyma stem cell niche

modeling approaches

aliphatic polyesters, 297-300

coarse-grained models, 282-283

hydrogels. See Hydrogels

macroscale models. See Macroscale models

molecular dynamics (MD) simulations, 280-282

in vivo modeling, 301-302

nanomaterials. See Nanomaterials

nanoscaled delivery tools, 309

naturally occurring nanoparticles, 309-310

neuroregenerative agents, 308-309, 308f

novel engineered biocompatible scaffolds,

$$
\text { 307-308 }
$$

paracrine effect. See Paracrine effect

pharmacological therapies. See Pharmacological therapies

preclinical studies, 307

scaffolds. See Scaffolds

soft injectable polymeric matrices, 308

Spinal cord injury without radiographic abnormality (SCIWORA), 43-44, 58-60

Spinal pathway recording

contact heat evoked potentials (CHEPs), 33

dermatomal somatosensory evoked potentials (dSEPs), 32-33

impaired bladder function, 23

laser evoked potentials (LEPs), 33-34

motor evoked potential (MEP), 28, 29f-30f

quantitative sensory test (QST), 33

somatosensory evoked potentials (SEPs), 30-32, $31 \mathrm{f}$

transcranial magnetic stimulation (TMS), 28

Spinal shock, 13, 40, 54-55

Spinothalamic tracts, $15-16$

Spongy agarose nerve guidance scaffolds, 83

Static magnetization, 49-50

Steam autoclaving, 97

Stem cell-based therapies. See also Cell therapies cell differentiation and replacement, 204-205,

$$
212
$$

endogenous regenerative mechanisms, 204

engineered biomaterial for, 211

multi/pluripotent nature, 204-205

multitherapeutic ability, 203

neuroprotection and neuroregeneration, 205

nonhomogeneous origins, 212

origin and sources, $206 \mathrm{f}$

paracrine effect. See Paracrine effect

Sterilization, 97

Steroids, 44

Stochastic models, 284

Subacute progressive ascending myelopathy, 61

Subventricular zone (SVZ) stem cell niche,

$$
\text { 188-190 }
$$

Superoxide dismutase (SOD), 238

Supramaximal electrical nerve stimulation, 18

Supramolecular gels, 295-296

Surface erosion, 289

Surgical management

anterior arthrodesis, 46

contraindications, 46

decompression and stabilization, 47

laminectomy, 46

posterior arthrodesis, 46

Swim test, 267

Swine, 253

Sympathetic and parasympathetic systems, 16

Sympathetic skin response (SSR), 20

Synthetic hydrogels, 227-228

degradable PHMA hydrogels, 117

PHEMA hydrogels. See Poly (2-hydroxyethyl methacrylate) (PHEMA) hydrogels

T

Tactile sensory test, 267

Tetraparesis, 3-4

Tetraplegia, 3-4, 39-40

Tetraspanins, 209

Thermal thresholds, 33

Thoracic level injury, animal model, 259

Threshold tracking techniques, 19-20

Tissue softening, 107

Transcranial magnetic stimulation (TMS)

central motor conduction time (CMCT), 21-22

magnetic stimulating cap coil, $21 \mathrm{f}$

motor evoked potential (MEP), 21-22

recording electrodes, $21 \mathrm{f}$

spinal pathway recording, 28

Transection/laceration spinal cord injury models, 263 
Transient paralysis, 54-55

Transverse magnetization vector, 49-50

Triple-stimulation technique (TMT), 22

Turtles, 254-255

Twin-screw extruders, 90-91

Twin-screw extrusion method, 89-90

\section{U}

Umbilical cord mesenchymal stem cells (UC-MSCs), 168-169, 206-207, 210

University of British Columbia device, 263

University of Texas at Arlington distractor, 263-264

Upper motor neuron (UMN), 14-15

US National Nanotechnology Initiative, 129

V

Vascular endothelial growth factor (VEGF), 206-207, 227
Vehicle modification cost, 68-69

Ventral ramus, 14-15

Vestibulospinal pathways, 20

Volume-conducted potentials, 16

\section{W}

Water-miscible organic solvent, 137

Weight drop model, 260

Wharton's Jelly-derived human umbilical cord-MSCs (UC-MSCs), 168-169

White matter, 1-2, 3f, 14-16

$\mathrm{X}$

Xenopus laevis, 254

Z

Zebrafish, 254 